Thorsten Pieper

\title{
Wirkungsorientiertes Controlling staatlichen Handelns
}

Systematische Identifikation und Bewertung der gesamtgesellschaftlichen Wirkungen staatlichen Handelns 


\section{Thorsten Pieper}

\section{Wirkungsorientiertes Controlling staatlichen Handelns}

Im Rahmen der aktuellen Modernisierungsprozesse des öffentlichen Sektors wird zu selten nach den eigentlichen Wirkungen staatlichen Handelns gefragt, wodurch permanent die Gefahr besteht, die falschen - da unwirksamen - öffentlichen Maßnahmen zu realisieren. Vor diesem Hintergrund verfolgt der Autor in seiner Arbeit das Ziel, die Etablierung wirkungsorientierter Controllingprozesse zu unterstützen, um Entscheidungen in Politikund Verwaltung konsequenter als bisher auf Wirkungen auszurichten. Bislang gelingt es in der Regel nur unzureichend, die mit einer öffentlichen Maßnahme verbundenen Wirkungen zweifelsfrei zu ermitteln und sie darüber hinaus auch noch einer objektiven Bewertung zu unterziehen. An dieser Stelle setzt die Arbeit an, indem ein standardisiertes Instrumentarium vorgestellt wird, welches eine strukturierte Identifikation und Bewertung der durch staatliches Handeln ausgelösten Wirkungen erlaubt. Der bislang eher abstrakt diskutierte Wirkungsbegriff kann durch die vorgestellte Methodik der Wirkungsanalyse leichter in bestehende Controllingprozesse eingebunden werden.

Thorsten Pieper absolvierte ein Studium der Betriebswirtschaftslehre an der Universität Münster. Nach Studienabschluss war er im Rahmen eines Forschungsprojektes als Wissenschaftlicher Mitarbeiter am Lehrstuhl für Betriebswirtschaftslehre, insbesondere Controlling an der Universität Münster beschäftigt. Seitdem ist der Autor für eine Unternehmensberatung in Düsseldorf tätig und berät privatwirtschaftliche Unternehmen und öffentliche Institutionen bei der Implementierung von Controllingsystemen. 
Wirkungsorientiertes Controlling staatlichen Handelns 


\section{Beiträge zum Controlling}

Herausgegeben von Wolfgang Berens

Band 14

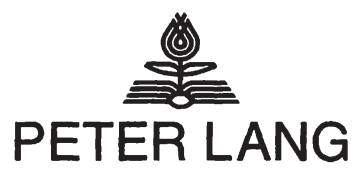

Frankfurt am Main - Berlin - Bern - Bruxelles - New York - Oxford - Wien

Thorsten Pieper - 978-3-631-75332-3

Downloaded from PubFactory at 01/11/2019 06:04:26AM

via free access 
Thorsten Pieper

\title{
Wirkungsorientiertes Controlling staatlichen Handelns
}

\author{
Systematische Identifikation \\ und Bewertung der gesamtgesellschaftlichen \\ Wirkungen staatlichen Handelns
}

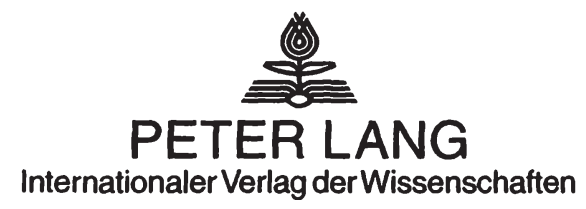

Thorsten Pieper - 978-3-631-75332-3

Downloaded from PubFactory at 01/11/2019 06:04:26AM 
Bibliografische Information der Deutschen Nationalbibliothek Die Deutsche Nationalbibliothek verzeichnet diese Publikation in der Deutschen Nationalbibliografie; detaillierte bibliografische Daten sind im Internet über <http://www.d-nb.de> abrufbar.

Open Access: The online version of this publication is published on www.peterlang.com and www.econstor.eu under the international Creative Commons License CC-BY 4.0. Learn more on how you can use and share this work: http://creativecommons.org/licenses/ by/4.0.

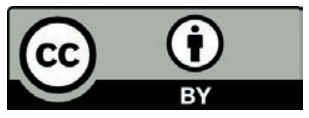

This book is available Open Access thanks to the kind support of ZBW - Leibniz-Informationszentrum Wirtschaft.

Zugl.: Münster (Westfalen), Univ., Diss., 2007

Gedruckt auf alterungsbeständigem, säurefreiem Papier.

\author{
D 6 \\ ISSN $1618-825 X$ \\ ISBN 978-3-631-57150-7 \\ ISBN 978-3-631-75332-3 (eBook) \\ (c) Peter Lang GmbH \\ Internationaler Verlag der Wissenschaften \\ Frankfurt am Main 2009 \\ Alle Rechte vorbehalten.
}

Das Werk einschließlich aller seiner Teile ist urheberrechtlich geschützt. Jede Verwertung außerhalb der engen Grenzen des Urheberrechtsgesetzes ist ohne Zustimmung des Verlages unzulässig und strafbar. Das gilt insbesondere für Vervielfältigungen, Übersetzungen, Mikroverfilmungen und die Einspeicherung und Verarbeitung in elektronischen Systemen.

Printed in Germany 123457

www.peterlang.de 


\section{Geleitwort}

Unter dem Leitbild des New Public Management sind in den letzten Jahren vielfältige Modernisierungsprozesse in der öffentlichen Verwaltung initiiert worden. Den einzelnen Reformansätzen ist dabei eine betriebswirtschaftliche Grundausrichtung gemein, mit dem Ziel, Flexibilität und Anpassungsfähigkeit des öffentlichen Sektors als Ganzes zu erhöhen, um so die zweckmäßige Verwendung öffentlicher Finanzmittel sicherzustellen. Eine entscheidende Rolle in diesem Modernisierungsprozess kommt dem Controlling zu, welches auf den unterschiedlichen Entscheidungsebenen in Politik und Verwaltung die dafür notwendige Transparenz herstellen soll.

In diesem Zusammenhang wird in der aktuellen Diskussion häufig problematisiert, dass sich bisherige Reformansätze meist der einseitigen Verbesserung der Wirtschaftlichkeit des Ressourceneinsatzes widmen, welches in der Regel mit Kostenreduzierungen gleichbedeutend ist. Hierbei wird allerdings eine wesentliche Perspektive vernachlässigt. $\mathrm{Zu}$ selten wird nach den eigentlichen Wirkungen staatlichen Handelns gefragt, wodurch permanent die Gefahr besteht, die falschen - da unwirksamen - öffentlichen Maßnahmen zu realisieren. Zur Sicherstellung einer zielkonformen Mittelverwendung muss der Staat sein Handeln daher systematisch an den resultierenden Wirkungen in der Gesellschaft ausrichten und sowohl eine hohe Wirksamkeit als auch die Effizienz realisierter Maßnahmen beachten. Dies sind die Herausforderungen, die sich an eine Weiterentwicklung des Controllings in der öffentlichen Verwaltung zukünftig stellen.

Vor diesem Hintergrund verfolgt die Arbeit von ThORSTEN PIEPER das Ziel, die Etablierung wirkungsorientierter Controllingprozesse zu unterstützen, um Entscheidungen in Politik und Verwaltung konsequenter als bisher auf Wirkungen auszurichten. Problematisch ist in diesem Zusammenhang allerdings, dass es häufig nur unzureichend gelingt, die mit einer öffentlichen Maßnahme verbundenen Wirkungen zweifelsfrei zu ermitteln und sie darüber hinaus auch noch einer objektiven Bewertung zu unterziehen. Dies ist aber eine unabdingbare Voraussetzung für die erfolgreiche Implementierung einer wirkungsorientierten Controlling-Konzeption. An dieser Stelle setzt die Arbeit von Herrn PIEPER an, indem ein standardisiertes Instrumentarium vorgestellt wird, welches eine strukturierte Identifikation und Bewertung der durch staatliches Handeln im gesellschaftlichen Umfeld ausgelösten Wirkungen erlaubt. Der bislang eher abstrakt diskutierte Wirkungsbegriff kann durch diesen Ansatz leichter in einen systematischen Controllingprozess eingebunden werden. Im Ergebnis besitzt eine derart gestaltete Controllingkonzeption das Potenzial, nicht nur die Wirtschaftlichkeit, sondern auch die Wirksamkeit der öffentlichen Mittelverwendung zu erhöhen.

An einem konkreten Beispiel wird zudem die Praxistauglichkeit der theoretisch erarbeiteten Inhalte überprüft. Im Rahmen einer Fallstudie zum staatlichen Flächenmanagements durch Bodenordnung wird durch Herrn PIEPER gezeigt, wie über eine systematische Identifikation und Bewertung der angestrebten Ziele und Wirkungen einerseits und einer Ermittlung der resultierenden Kosten andererseits, eine geschlossene und ausgewogene Darstellung des Verwaltungshandelns gelingen kann. Der Wertbeitrag des staatlichen Handelns kann in letzter Konsequenz im Sinne einer gesamtgesell- 
schaftlichen Wertschöpfung interpretiert und damit transparent gemacht werden. Durch das Controlling erhalten die verantwortlichen Akteure in Politik und Verwaltung auf diese Weise die notwendigen Informationen, öffentliche Maßnahmen so zu organisieren, dass sie eine möglichst hohe Wirksamkeit erzielen bzw. nur solche Maßnahmen zu realisieren, die konkrete Beiträge zur Steigerung des Gemeinwohls versprechen. Langfristig ist mit der Wirkungsorientierung die Hoffnung verbunden, dass die Relationen zwischen den verursachten Kosten und den erzielten Wirkungen öffentlicher Maßnahmen auch in der politischen Betrachtung stärker Berücksichtigung finden, so dass eine möglichst sinnvolle Verwendung der öffentlich bereitgestellten Mittel sichergestellt wird.

In diesem Zusammenhang freut es mich besonders, dass die im Rahmen der Fallstudie und weiteren Projekten durch Herrn PIEPER entwickelten Inhalte, Eingang in die strategischen Steuerungsprozesse mehrerer Bundesländer gefunden haben. Auf Grundlage einer edv-technischen Unterstützung werden dort Maßnahmen des staatlichen Flächenmanagements sowohl vor Einleitung als auch maßnahmenbegleitend grundsätzlich einer kombinierten Kosten- und Wirkungsanalyse unterzogen, um eine hohe Wirksamkeit der eingesetzten Finanzmittel und Ressourcen sicherzustellen. Die Implementierung wirkungsorientierter Controllingprozesse in der Praxis beweisen, dass eine Wirkungsorientierung, trotz der recht hohen Komplexität, auch im Rahmen standardisierter Controllingprozesse beherrschbar ist. Der Zugewinn an Transparenz und „eine neue Sicht der Dinge" sind es wert, diesen Weg des Verwaltungscontrollings konsequent weiter $\mathrm{zu}$ beschreiten. 


\section{Vorwort}

Die vorliegende Arbeit entstand während meiner Assistententätigkeit als wissenschaftlicher Mitarbeiter am Lehrstuhl für Betriebswirtschaftslehre, insb. Controlling sowie meiner Tätigkeit als Berater für die BMS Consulting GmbH, Düsseldorf und wurde im Sommersemester 2007 an der Westfälischen Wilhelms-Universität Münster als Dissertationsschrift angenommen. Besonderer Dank gilt in diesem Zusammenhang meinem akademischen Lehrer und Erstgutachter PROF. DR. WOLFGANG Berens. Die Möglichkeit zur Vereinbarkeit von Lehrstuhltätigkeiten, Beratungsaktivitäten und Promotion und die von ihm verfolgte Integration von Wissenschaft und Praxis haben sowohl die Arbeitszeit als auch die Promotionszeit zu einem lehrreichen Erlebnis werden lassen. Herrn PROF. DR. ALOYS PRINZ danke ich recht herzlich für die Übernahme des Zweitgutachtens.

Spezielle Anerkennung gilt weiterhin Herrn DR. THOMAS MOSIEK für den unermüdlichen Einsatz, den er für konstruktive Kritik und kreative Anregungen erbracht hat trotz seiner zeitintensiven Tätigkeit als Geschäftsführer der BMS Consulting GmbH. Für die anregende Diskussionen und die vielen hilfreichen Hinweise möchte ich zudem meinen Kollegen DR. ANDREAS RÖHRIG und DR. CHRISTIAN BUSCHHOFF ganz besonders danken. Für den Gedankenaustausch zur Überprüfung und praktischen Absicherung der Aussagen in der Fallstudie meiner Arbeit möchte ich ferner Herrn GEORG SEYER vom Umweltministerium des Landes Nordrhein-Westfalen sowie Herrn Prof. DR. AXEL LORIG vom Wirtschaftministerium des Landes Rheinland-Pfalz danken.

Gemeinsam mit Prof. Dr. WOLFGang Berens ist es den Gründern und Geschäftsführern der BMS Consulting GmbH DR. THOMAS MOSIEK und DR. ANDREAS SIEMES gelungen, ein liberales und zugleich produktives Arbeitsklima zu schaffen, welches immer wieder innovative Lösungskonzepte und darauf aufbauend pragmatische Beratungsansätze hervorbringt. Es freut mich ganz besonders, dass ich hierbei auch zukünftig weiter mitwirken kann. Insgesamt möchte ich allen Kollegen bei der BMS Consulting $\mathrm{GmbH}$ und dem Lehrstuhl für Betriebswirtschaftslehre, insbesondere Controlling für das außerordentlich gute Arbeitsklima danken.

Abschließend sei meinen Eltern und meiner Frau Kirsten für die Geduld, die immerwährende Unterstützung und nicht zuletzt für die notwendige Aufmunterung beim Schreiben der Arbeit ganz herzlich gedankt.

Bocholt, im Juli 2008

Dipl.-Kfm. Thorsten Pieper 
Thorsten Pieper - 978-3-631-75332-3

Downloaded from PubFactory at 01/11/2019 06:04:26AM

via free access 


\section{Inhaltsverzeichnis}

Abbildungsverzeichnis ................................................................................. XIII

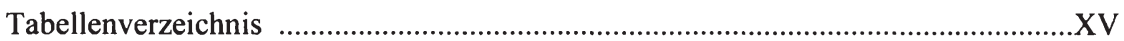

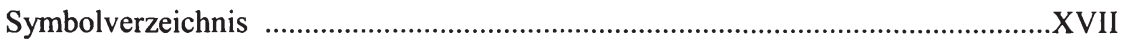

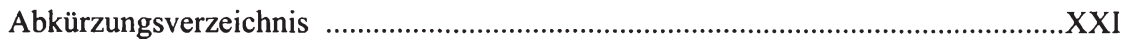

$1 \quad$ Einführung ................................................................................................... 1

1.1 Hintergrund und Problemaufriss ............................................................ 1

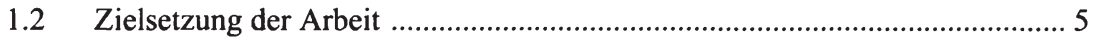

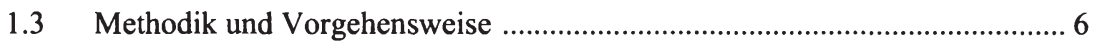

2 Wirkungsorientierte Steuerung staatlichen Handelns ............................ 9

2.1 Ausgewählte Aspekte zum staatlichen Handeln ......................................... 9

2.1.1 Ursachen, Aufgaben und Ziele der Staatstätigkeit ............................... 9

2.1.2 Organisation, Träger und Aufbau des öffentlichen Sektors................... 13

2.1.3 Instrumente und Ergebnisse staatlichen Handelns .............................. 17

2.1.4 Prozess öffentlicher Leistungserstellung ........................................... 19

2.1.5 Zur ökonomischen Bedeutung des öffentlichen Sektors ....................... 21

2.2 Zum Wandel der Steuerungsansätze im politisch-administrativen System .. 22

2.2.1 Bürokratische Steuerung und Kameralistik ......................................... 23

2.2.2 Modernisierungsleitlinien des New Public Management ...................... 24

2.2.2.1 Veränderungstreiber staatlichen Handelns....................................... 24

2.2.2.2 Modernisierungsbestrebung des deutschen Bürokratiemodells im Kontext internationaler Entwicklungen ....................................... 26

2.2.2.3 Konzept und Umsetzung der „Neuen Steuerung“ als deutsche Variante des New Public Management ............................................ 31

2.2.3 Ansatz der wirkungsorientierten Steuerung staatlichen Handelns.......... 34

2.2.3.1 Pfad zur wirkungsorientierten Steuerung ........................................ 34

2.2.3.2 Internationale Ansätze einer wirkungsorientierten Steuerung ............ 39

2.2.3.3 Chancen der Wirkungsorientierung ............................................... 45

2.2.3.4 Herausforderungen der Wirkungsorientierung ............................... 47

2.3 Führungsunterstützung wirkungsorientierter Steuerungsprozesse durch ein wirkungsorientiertes Controlling .................................................. 50

3 Wirkungsorientiertes Controlling staatlichen Handelns ......................... 55

3.1 Grundlagen des Controlling-Ansatzes .................................................. 55

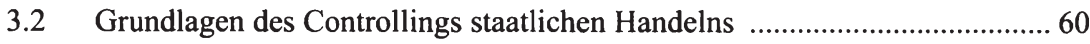


3.3 Wirkungsorientierte Controlling-Konzepte und Modelle .......................... 65

3.3.1 Zur Notwendigkeit eines ganzheitlichen Bezugsrahmens .....................6 65

3.3.2 Darstellung und Erläuterung ausgewählter Bezugsrahmen ....................66 66

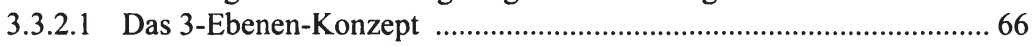

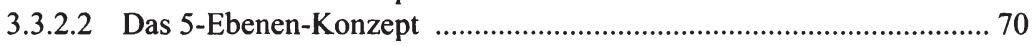

3.3.2.3 Das Ziel- und Ergebnisebenen-Modell öffentlicher Leistungserstellung .................................................................. 72

3.3.2.4 Das Erweiterte Ziel- und Ergebnisebenen-Modell öffentlicher Leistungserstellung .................................................................. 76

3.3.3 Vergleichende Bewertung der dargestellten Ebenenmodelle ................. 79

3.3.4 Implikationen des bisherigen Untersuchungsverlaufs .......................... 80

3.4 Instrumentelle Defizite in der systematischen Erfassung und Bewertung der Wirkungen staatlichen Handelns

4 Methodik der Wirkungsanalyse staatlichen Handelns 93

4.1 Modellbildung als Grundlage der weiteren Untersuchung ........................ 95

4.1.1 Wirkungen als wissenschaftliches Erkenntnisobjekt ........................... 95

4.1.1.1 Definitionsansätze und begriffliche Abgrenzung ............................ 95

4.1.1.2 Analyse von Ursache-Wirkungszusammenhängen aus wissenstheoretischer Perspektive................................................. 97

4.1.2 Konstruktion eines allgemeinen Beschreibungsmodells .................... 100

4.1.2.1 Beherrschung hochkomplexer Wirkungszusammenhänge der Realität durch Modellbildung .................................................. 100

4.1.2.2 Modellkonzeption der Wirkungen staatlichen Handelns ................. 101

4.1.2.3 Statische Aspekte der Modellkonzeption ....................................... 109

4.1.2.4 Dynamische Aspekte der Modellkonzeption ................................ 113

4.1.3 Erkenntnisgewinn für den Fortgang der Untersuchung ...................... 115

4.2 Abgrenzung der relevanten Analyseperspektiven .................................. 116

4.2.1 Bestehende Redundanzen in den Bewertungsebenen .......................... 116

4.2.2 Trennung der unterschiedlichen Analyseperspektiven ....................... 118

4.2.3 Analyseperspektiven und staatliche Aufgabenbereiche....................... 120

4.2.4 Instrumentelle Ausgestaltung der Analyseperspektiven ...................... 123

4.2.4.1 Output: Instrumentarium der Leistungsanalyse.............................. 123

4.2.4.2 Impact: Instrumentarium der Nutzenanalyse................................. 124

4.2.4.3 Outcome: Instrumentarium der Wirkungsanalyse ........................ 128

4.3 Systematik und Instrumentarium der Wirkungsanalyse ........................... 129

4.3.1 Vorgehenssystematik einer Wirkungsanalyse ................................... 129

4.3.2 Konzeptionsphase: Aufbau der Analysesystematik .......................... 134

4.3.2.1 Identifikation gesamtgesellschaftlicher Auswirkungen ................... 134

4.3.2.1.1 Pragmatisches Analysemodell der Auslöserebene .................... 134

4.3.2.1.2 Pragmatisches Analysemodell der Empfängerebene.................. 137

4.3.2.1.3 Abbildung eines gesamtgesellschaftlichen Wirkungsgefüges .... 140 
4.3.2.1.4 Instrumentelle Unterstützung der Wirkungsidentifikation ......... 143

4.3.2.2 Bewertung gesamtgesellschaftlicher Auswirkungen ...................... 146

4.3.2.2.1 Bewertungsperspektive einer Wirkungsanalyse ....................... 147

4.3.2.2.2 Systematik der Bewertungsformen........................................... 149

4.3.2.2.3 Instrumentelle Unterstützung der Wirkungsbewertung.............. 152

4.3.3 Anwendungsphase: Verfahren der Wirkungsrechnung ...................... 158

4.3.3.1 Instrumente der Wirkungsrechnung ............................................ 158

4.3.3.2 Funktionen im wirkungsorientierten Controllingprozess ................ 160

4.3.3.3 Ausgestaltung der Wirkungsrechnung .......................................... 162

4.3.3.3.1 Wirkungsprognose als ex-ante-Analyse ............................... 162

4.3.3.3.2 Wirkungsmonitoring als on-going-Analyse .......................... 163

4.3.3.3.3 Wirksamkeitsanalyse als ex-post-Analyse ............................... 164

4.3.4 Anpassungsphase: Initiierung kontinuierlicher Lernschleifen ............ 165

4.4 Institutionelle Ausgestaltung der Wirkungsanalyse ................................. 167

4.4.1 Institutionelle Ebenen des Wirkungscontrollings .............................. 167

4.4.2 Inhaltliche Ausgestaltung der Controllingebenen ................................ 169

4.4.2.1 Operativ-taktisches Wirkungscontrolling .................................... 169

4.4.2.2 Strategisches Wirkungscontrolling ......................................... 170

4.4.2.3 Politisches Wirkungscontrolling ................................................. 171

4.4.3 Integration von Ressourcen- und Wirkungsanalyse durch eine Wertschöpfungsbilanz ............................................................... 172

4.4.4 Systematisierung der praktischen Implementierungsansätze eines wirkungsorientierten Controllings

$5 \quad$ Fallstudie: Erstellung einer Nutzen- und Wirkungsanalyse des staatlichen Flächenmanagements durch Bodenordnung ..................... 179

5.1 Grundlagen des Flächenmanagements durch Bodenordnung .................... 180

5.1.1 Ziele und Aufgaben der ländlichen Bodenordnung ............................. 180

5.1.2 Gesetzliche Grundlagen und Verfahrensarten ................................ 183

5.1.3 Schematische Gegenüberstellung der Grundstücksverhältnisse ........... 186

5.1.4 Wesentliche Ablaufschritte eines Bodenordnungsverfahrens ............. 187

5.2 Nutzenanalyse: Impactbetrachtung des staatlichen Flächenmanagements durch Bodenordnung ............................................................................ 189

5.2.1 Ausgangssituation und Zielsetzung des Projektes ............................. 189

5.2.2 Aufbau und Konzeption der Kundenbefragung ................................ 190

5.2.3 Befragungsergebnisse zur Dienstleistungs- und Servicequalität .......... 193

5.2.4 Befragungsergebnisse zu Verfahrenszielen und Wirkungen ............... 196

5.3 Wirkungsanalyse in der Konzeptionsphase: Aufbau der Analyse- und

Bewertungsschemata ........................................................................... 201

5.3.1 Ausgangssituation und Zielsetzung des Projektes .............................. 201

5.3.2 Bodenordnungsverfahren als Dienstleistung des Landes

Rheinland-Pfalz 
5.3.3 Methodik der Wirkungsanalyse von Bodenordnungsverfahren ........... 205

5.3.4 Identifikation der Auswirkungen von Bodenordnungsverfahren........... 206

5.3.4.1 Gesetzlich intendierte Wirkungen der Bodenordnung ...................... 206

5.3.4.2 Inhaltsanalyse bestehender Forschungsergebnisse ........................... 210

5.3.4.3 Durchführung von Befragungen ..................................................... 215

5.3.5 Herleitung eines Wirkungsgefüges der Bodenordnung ....................... 216

5.3.5.1 Systematisierung der Auslöserebene .............................................. 216

5.3.5.2 Systematisierung der Empfängerebene ................................................ 217

5.3.5.3 Identifikation der relevanten Wirkungskomponenten......................... 218

5.3.5.3.1 Auswirkungen der Bodenordnung auf die Bürger ...................... 219

5.3.5.3.2 Auswirkungen der Bodenordnung auf die Wirtschaft.................. 227

5.3.5.3.3 Auswirkungen der Bodenordnung auf den Staat ......................... 231

5.3.5.3.4 Auswirkungen der Bodenordnung auf die Umwelt ..................... 236

5.3.5.4 Abbildung des gesamtgesellschaftlichen Wirkungsgefüges ............ 239

5.3.6 Bewertung der Wirkungskomponenten der Bodenordnung................... 240

5.3.6.1 Bewertung der Auswirkungen auf den Bürger ................................... 241

5.3.6.2 Bewertung der Auswirkungen auf die Wirtschaft.............................. 250

5.3.6.3 Bewertung der Auswirkungen auf den Staat ..................................... 256

5.3.6.4 Bewertung der Auswirkungen auf die Umwelt .................................. 261

5.3.7 Anwendung der Analyse- und Bewertungsschemata ........................... 262

5.4 Wirkungsanalyse in der Anwendungsphase: Wirkungsrechnung eines

exemplarisch ausgewählten Bodenordnungsverfahrens .............................. 263

5.4.1 Beschreibung des betrachteten Bodenordnungsverfahrens.................... 263

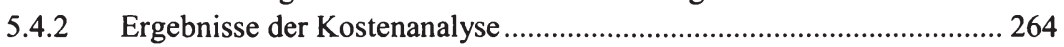

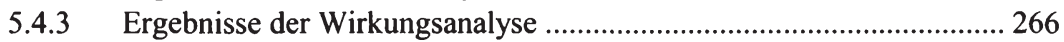

5.4.3.1 Identifikation der verfahrensspezifischen Auswirkungen ................. 266

5.4.3.2 Bewertung der verfahrensspezifischen Auswirkungen ..................... 268

5.4.4 Wertschöpfungsbilanz des Bodenordnungsverfahrens ......................... 272

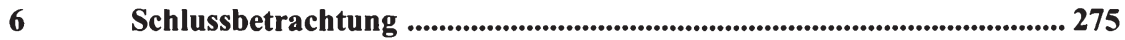

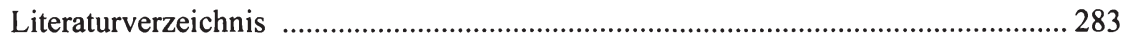

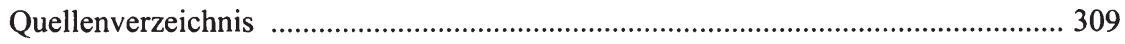




\section{Abbildungsverzeichnis}

Abbildung 1: Schematischer Überblick über die Struktur der Arbeit......................... 7

Abbildung 2: Systematisierung der Trägerstruktur ................................................. 16

Abbildung 3: Prozessmodell der öffentlichen Leistungserstellung .......................... 20

Abbildung 4: Statistische Daten des öffentlichen Sektors in Deutschland ............... 21

Abbildung 5: Veränderungstreiber staatlichen Handelns ....................................... 25

Abbildung 6: Internationale Reformansätze des NPM ............................................ 27

Abbildung 7: Vom Bürokratiemodell zum New Public Management ..................... 29

Abbildung 8: Stärken-Schwächen-Profile im Vergleich .......................................... 33

Abbildung 9: Steuerungsarten im politisch-administrativen System ........................ 34

Abbildung 10: Evolutionspfad der Steuerungsarten ................................................... 37

Abbildung 11: Internationale Reformansätze einer wirkungsorientierten Steuerung ..................................................................................... 39

Abbildung 12: Chancen der Wirkungsorientierung ................................................... 46

Abbildung 13: Herausforderungen der Wirkungsorientierung ..................................... 49

Abbildung 14: Notwendigkeit der Führungsunterstützung.......................................... 51

Abbildung 15: Gliederung des Führungssystems der Unternehmung ....................... 59

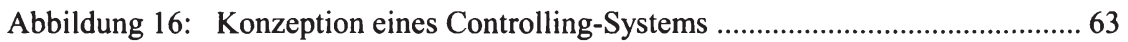

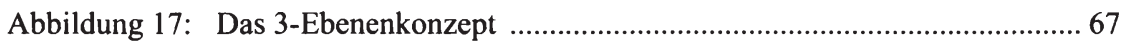

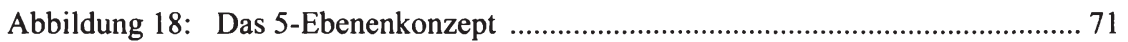

Abbildung 19: Ziel- und Ergebnisebenen öffentlicher Leistungserstellung ............... 73

Abbildung 20: Das Erweiterte Ziel- und Ergebnisebenenmodell

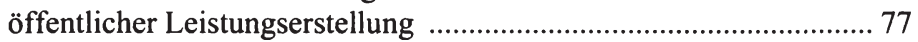

Abbildung 21: Controlling-Instrumente der Wirkungsebene ....................................... 85

Abbildung 22: Aufbau der weiteren Untersuchung ............................................... 93

Abbildung 23: Ebenen der 2-Sprachen-Theorie ………......................................... 99

Abbildung 24: Erster Schritt der Modellkonzeption................................................ 102

Abbildung 25: Zweiter Schritt der Modellkonzeption.............................................. 103

Abbildung 26: Dritter Schritt der Modellkonzeption................................................. 104

Abbildung 27: Vierter Schritt der Modellkonzeption ................................................. 105

Abbildung 28: Fünfter Schritt der Modellkonzeption ............................................. 106

Abbildung 29: Sechster Schritt der Modellkonzeption............................................... 107

Abbildung 30: Siebter Schritt der Modellkonzeption ................................................. 108

Abbildung 31: Achter Schritt der Modellkonzeption ............................................. 109

Abbildung 32: Komponenten der Wirkungsstärke ………................................... 110

Abbildung 33: Klassifizierung der Wirkungsinteraktionen....................................... 112

Abbildung 34: Dynamische Aspekte der Modellkonzeption ..................................... 114

Abbildung 35: Redundante Bewertungskreise........................................................ 117

Abbildung 36: Abgrenzung der relevanten Analyseperspektiven ........................... 119 
Abbildung 37: Analyseperspektiven staatlicher Aufgabenbereiche 121

Abbildung 38: Ansätze der Zufriedenheitsmessung 125

Abbildung 39: Durchführungsschritte einer Wirkungsanalyse. 130

Abbildung 40: Systematisches Modell der Auslöserebene..... 136

Abbildung 41:

Systematisches Modell der Empfängerebene 138

Abbildung 42: Wirkungsgefüge als Analysematrix 142

Abbildung 43: Abgrenzung der Bewertungsperspektive 147

Abbildung 44: Bewertungsformen der Wirkungsanalyse 150

Abbildung 45:

Unterstützende Verfahren der Wirkungsbewertung 153

Abbildung 46:

Anforderungen an Indikatoren

Abbildung 47:

Wirkungsrechnung im Controllingprozess 159

Abbildung 48:

Funktionen der Wirkungsrechnung 161

Abbildung 49:

Ebenen des Wirkungscontrolling. 168

Abbildung 50: Gesellschaftliche Wertschöpfungsbilanz 173

Abbildung 51: Systematisierung möglicher Implementierungsansätze. 175

Abbildung 52: Zie

Abbildung 53:

Ziele der Bodenordnung als Flächenmanagement. 181

Abbildung 54:

Verfahrensarten der Bodenordnung.... 183

Abbildung 55:

Grundstücksverhältnisse vor und nach Bodenordnung . 186

Abbildung 56: Prozessschritte eines Bodenordnungsverfahrens. 188

Abbildung 57: Konzeption und Rücklauf der Befragung.

Abbildung 58:

Beurteilung der Abwicklungsqualität 193

Abbildung 59:

Beurteilung der Bearbeitungsqualität 194

Abbildung 60:

Beurteilung der Servicequalität der Mitarbeiter

Abbildung 61:

Beurteilung der Globalzufriedenheit

Abbildung 62:

Die wichtigsten Verfahrensziele aus Kundensicht

Abbildung 63:

Portfoliodarstellung der relativen Zielerfüllung

Abbildung 64:

Portfoliodarstellung der relativen Zielerfüllung ... 200

Abbildung 65:

Struktur und Aufgaben der DLR 204

Abbildung 66:

Methodik der Wirkungsanalyse von 205

Abbildung 67:

Auslöserebene der Bodenordnung.

Abbildung 68:

Empfängerebene der Bodenordnung

218

Abbildung 69:

Gesellschaftliches Wirkungsgefüge der Bodenordnung..

Abbildung 70:

Eingesparte Transaktionskosten der Eigentümer. 241

Abbildung 71: Verfahrens- und Ausführungskosten der Bodenordnung 264 Gesellschaftliches Wirkungsgefüge des Verfahrens »Kaiserslautern-Ost-Mehlingen «.

Abbildung 72: Wertschöpfungsbilanz »Kaiserslautern-Ost-Mehlingen «........ 


\section{Tabellenverzeichnis}

Tabelle 1: Strukturelemente und Wirkungsbeziehungen der Modellkonzeption.... 116

Tabelle 2: Übersicht allgemeiner Wirkungsanalysen der Bodenordnung.......... 210

Tabelle 3: Übersicht spezifischer Wirkungsanalysen der Bodenordnung.......... 213

Tabelle 4: Übersicht potenzieller Katasternutzer............................... 236

Tabelle 5: Verfahrensbrief »Kaiserslautern-Ost-Mehlingen «..................... 263 
Thorsten Pieper - 978-3-631-75332-3

Downloaded from PubFactory at 01/11/2019 06:04:26AM

via free access 


\section{Symbolverzeichnis}

\begin{tabular}{|c|c|}
\hline$A_{k}$ & $\begin{array}{l}\text { Wirkungsbeitrag aus der Aktualisierung des Kataster- und } \\
\text { Grundbuch }\end{array}$ \\
\hline b & Beschleunigung in Jahren \\
\hline $\mathrm{BE}_{\mathrm{HW}}$ & Beschleunigungseffekt beim Hochwasserschutz \\
\hline $\mathrm{BESCH}$ & Beschäftigungseffekt durch Wegenetzinvestitionen \\
\hline BV & Bewirtschaftungsvorteil \\
\hline $\mathrm{BV}_{\text {Land }}$ & Bewirtschaftungsvorteil in der Landwirtschaft \\
\hline $\mathrm{BV}_{\text {Wald }}$ & Bewirtschaftungsvorteil in der Forstwirtschaft \\
\hline$B V_{\text {Wein }}$ & Bewirtschaftungsvorteil im Weinanbau \\
\hline BW & Barwert \\
\hline BW $W^{\text {Grundbuch }}$ & Barwert der Kostenersparnisse beim Grundbuchamt \\
\hline $\mathrm{BW}^{\mathrm{S}}$ & Barwert der vermiedenen Hochwasserschäden \\
\hline $\mathrm{BW}^{\mathrm{UK}}$ & Barwert der eingesparten Unterhaltungskosten \\
\hline d & monatliche Dauer der Unterhaltung der gem. Anlagen \\
\hline $\mathrm{E}$ & Entschädigungsleistungen \\
\hline$E^{\mathfrak{c}}$ & eingesparte Entschädigungsleistungen \\
\hline $\mathrm{E}_{\text {kalk }}$ & potenzielle Entschädigungen ohne Bodenordnung \\
\hline EP & Einsparpotenzial an Bewirtschaftungskosten \\
\hline $\mathrm{FBK}^{\mathrm{c}}$ & potenzielle Gesamtkosten der Flächenbereitstellung \\
\hline $\mathrm{FL}_{\mathrm{HW}}$ & Hochwassergefährdete Fläche in $\mathrm{km}^{2}$ \\
\hline $\mathrm{FL}_{\text {Komp }}$ & Kompensationsflächen in ha \\
\hline $\mathrm{FL}_{\text {neu }}$ & Neuvermessungsfläche pro ha \\
\hline $\mathrm{FL}_{\text {Trasse }}$ & Gesamtfläche eines Trassenprojektes in ha \\
\hline $\mathrm{Gb}$ & Kataster- und Notargebühren \\
\hline $\mathrm{Gb}^{\mathrm{e}}$ & eingesparte Kataster- und Notargebühren \\
\hline GK & Grunderwerbskosten \\
\hline $\mathrm{GK}^{\mathrm{e}}$ & eingesparte Grunderwerbskosten \\
\hline H & Honorare für Planungsleistungen im Wegebau \\
\hline $\mathrm{HK}_{\text {Wege }}$ & Herstellungskosten der Wirtschaftswege \\
\hline i & Diskontierungsrate bzw. -zinssatz \\
\hline$i_{L}$ & landwirtschaftlicher Kalkulationszinssatz \\
\hline I & Investitionssumme \\
\hline$I_{\text {Gesamt }}$ & Gesamtinvestitionssumme \\
\hline $\mathrm{I}_{\mathrm{Wegc}}$ & Investitionen in das regionale Wegenetz \\
\hline $\mathrm{I}_{\mathrm{j}}$ & Gesamtinvestitionen in Projekt $\mathrm{j}$ \\
\hline & Index für ein bestimmtes Projekt \\
\hline $\mathrm{k}$ & Index für die Vermessungsleistung \\
\hline $\mathrm{K}_{\mathrm{Bau}}$ & Baukosten der Projekte \\
\hline & $\begin{array}{r}\text { Thorsten Pieper - 978-3-631-75332-3 } \\
\text { wnloaded from PubFactory at 01/11/2019 06:04:26AM } \\
\text { via free access }\end{array}$ \\
\hline
\end{tabular}




\begin{tabular}{|c|c|}
\hline $\mathrm{K}_{\text {Bau }}^{\mathrm{c}}$ & eingesparte Baukosten der Projekte \\
\hline $\mathrm{K}_{\mathrm{Bo}}^{\mathrm{c}}$ & $\begin{array}{l}\text { eingesparte Baukosten durch spezielle Maßnahmen in der } \\
\text { Bodenordnung }\end{array}$ \\
\hline $\mathrm{KK}_{\mathrm{PD}}^{\mathrm{c}}$ & eingesparte Kontrollkosten des Agrarprüfdienstes \\
\hline KV & Anzahl der Kaufverträge im Grunderwerb \\
\hline $\mathrm{KV}_{\text {Jahr }}$ & Anzahl der Kaufverträge pro Mitarbeiter und Jahr \\
\hline $\mathrm{L}$ & Länge der langgestreckten Anlage in km \\
\hline Lw & Anzahl der Landwirte im Verfahrensgebiet \\
\hline NFL & landwirtschaftliche Nutzfläche in ha \\
\hline $\mathrm{NKF}_{\mathrm{j}}$ & Nutzen-Kosten-Faktor des Projektes j \\
\hline $\mathrm{p}$ & prozentualer Anteil des Planungshonorars \\
\hline PB & Personalbedarf für den Grunderwerb \\
\hline PK & Personal- und Sachkosten für den Grunderwerb \\
\hline $\mathrm{PK}^{\mathrm{e}}$ & eingesparte Personal- und Sachkosten \\
\hline PLK $_{\text {Wege }}$ & Planungskosten im Rahmen des Wirtschaftswegebaus \\
\hline RFL & Rebfläche in ha \\
\hline $\mathrm{S}$ & durchschnittlicher jährlicher Hochwasserschaden \\
\hline $\mathrm{S}^{\mathrm{e}}$ & jährliches Einsparpotenzial an Hochwasserschäden \\
\hline$S W_{\text {Wege }}$ & Sachwert des Wegenetzes \\
\hline $\mathrm{T}$ & Laufzeit in Jahren \\
\hline $\mathrm{t}$ & Laufindex der Zeit \\
\hline $\mathrm{Tk}^{\mathrm{e}}$ & eingesparte Transaktionskosten beim Flächenkauf \\
\hline $\mathrm{ts}_{\mathrm{gD}}$ & durchschnittlicher Tagessatz gehobener Dienst \\
\hline $\mathrm{ts}_{\mathrm{k}}$ & Tagessatz für Vermessungsleistung $\mathrm{k}$ \\
\hline $\mathrm{ts}_{\mathrm{mD}}$ & durchschnittlicher Tagessatz mittlerer Dienst \\
\hline $\mathrm{TV}_{\text {Wege }}$ & Touristischer Vorteil des Wegenetzes \\
\hline $\mathrm{TV}_{\text {Projckic }}$ & Touristischer Vorteil spezifischer Projekte \\
\hline UK & Unterhaltungskosten für gem. Anlagen \\
\hline $\mathrm{UK}^{\mathrm{e}}$ & eingesparte Unterhaltungskosten für gem. Anlagen \\
\hline $\mathrm{VA}_{\mathrm{Lw}}^{\mathrm{c}}$ & Verwaltungsaufwand der Prämienbeantragung \\
\hline $\mathrm{VA}_{\mathrm{PK}}^{\mathrm{e}}$ & Verwaltungsaufwand der Prämienkontrolle \\
\hline VFL & Verfahrensfläche in ha \\
\hline $\mathrm{VFL}_{\text {Komp }}$ & Kompensationsfläche im Verfahrensgebiet in ha \\
\hline VK & Vermessungskosten \\
\hline $\mathrm{VK}^{\mathrm{c}}$ & potenzielle Vermessungskostenersparnis \\
\hline $\mathrm{VK}_{\text {Fort }}$ & Kosten für Fortführungsvermessungen \\
\hline $\mathrm{VV}_{\text {Wege }}$ & Volkswirtschaftlicher Vorteil des Wegenetzes \\
\hline w & durchschnittliche Wachstumsrate der Baupreise \\
\hline WFL & Waldfläche in $\mathrm{m}^{2}$ \\
\hline $\mathbf{x}$ & Ursache \\
\hline
\end{tabular}




$\begin{array}{ll}x_{a} & \text { bestimmte staatliche Maßnahme als Ursache } \\ x_{\mathrm{a} 1} & \text { Ausgestaltung der Politik als Maßnahme } \\ x_{\mathrm{a} 2} & \text { administrativer Maßnahmenvollzug } \\ \mathrm{x}_{\mathrm{n}} & \text { andere staatliche Maßnahmen } \\ y & \text { Wirkung } \\ y_{n} & \text { gesellschaftliche Wirkungen staatlicher Maßnahmen } \\ y_{\mathrm{a}} & \text { intendierte Wirkung } \\ \mathrm{y}_{\mathrm{b}} & \text { Nebenwirkung } \\ \mathrm{y}_{\mathrm{b} 1} & \text { erwünschte Nebenwirkung } \\ \mathrm{y}_{\mathrm{b} 2} & \text { unerwünschte Nebenwirkung } \\ \mathrm{y}_{\mathrm{n}} & \text { primäre Wirkungen } \\ \mathrm{y}_{\mathrm{m}} & \text { sekundäre Folgewirkungen } \\ \mathrm{z}_{\mathrm{n}} & \text { exogene Effekte } \\ \mathrm{ZA} & \text { Zeitaufwand für Vermessungen pro ha in Tagen } \\ \mathrm{ZE}_{\mathrm{j}} & \text { Zinsertrag aus einer beschleunigten Projektrealisierung }\end{array}$


Thorsten Pieper - 978-3-631-75332-3

Downloaded from PubFactory at 01/11/2019 06:04:26AM

via free access 


\section{Abkürzungsverzeichnis}

\begin{tabular}{|c|c|}
\hline $3 \mathrm{E}$ & 3-Ebenen-Konzept \\
\hline $5 \mathrm{E}$ & 5-Ebenen-Konzept \\
\hline A & Autobahn \\
\hline ADD & Aufsichts- und Dienstleistungsdirektion \\
\hline AG & Aktiengesellschaft \\
\hline allg. & allgemein \\
\hline ARGE & Arbeitsgemeinschaft \\
\hline Aufl. & Auflage \\
\hline AUS & Australien \\
\hline $\mathrm{BAB}$ & Betriebsabrechnungsbogen \\
\hline Bd. & Band \\
\hline bearb. & bearbeitet \\
\hline BezReg & Bezirksregierung \\
\hline BFuP & Betriebswirtschaftliche Forschung und Praxis \\
\hline BSC & Balanced Scorecard \\
\hline bspw. & beispielsweise \\
\hline BWL & Betriebswirtschaftslehre \\
\hline bzw. & beziehungsweise \\
\hline ca. & circa \\
\hline CMR & California Management Review \\
\hline $\mathrm{CO}$ & Controlling \\
\hline DB & Der Betrieb \\
\hline DBW & Die Betriebswirtschaft \\
\hline DeGEval & Deutsche Gesellschaft für Evaluation \\
\hline DFG & Deutsche Forschungsgemeinschaft \\
\hline d.h. & das heißt \\
\hline Diss. & Dissertation \\
\hline DLR & Dienstleistungszentrum Ländlicher Raum \\
\hline d.ö.R. & des öffentlichen Rechts \\
\hline DVW & Deutscher Verein für Vermessungswesen e.V. \\
\hline EDV & Elektronische Datenverarbeitung \\
\hline e.G. & eingetragene Genossenschaft \\
\hline EntschR & Entschädigungsrichtlinie \\
\hline erw. & erweitert \\
\hline et al. & et alii \\
\hline etc. & et cetera \\
\hline EUR & Euro \\
\hline
\end{tabular}


e.V.

FAL

f.

ff.

GE

gem.

GFA

ggf.

$\mathrm{GmbH}$

GPRA

ha

Hrsg.

http

HWB

IAB

i.d.R.

i.e.S.

IMF

insb.

i.S.v.

IT

i.V.m.

i.w.S.

Jg.

Kap.

KEF

KGSt

KLR

krp

lfd.

LVO

Mgmt.

MRD

MWVLW

No.

NPM

$\mathrm{Nr}$.

NRW

NSM eingetragener Verein

Bundesforschungsanstalt für Landwirtschaft

folgende (Seite)

folgende (Seiten)

Grundstückseigentümer

gemeinschaftliche

Gesetzesfolgenabschätzung

gegebenenfalls

Gesellschaft mit beschränkter Haftung

Government Performance and Results Act

Hektar

Herausgeber

Hyper Text Transfer Protocol

Handwörterbuch Betriebswirtschaftslehre

Institut für Arbeitsmarkt- und Berufsforschung

in der Regel

im engeren Sinne

International Monetary Found

insbesondere

im Sinne von

Information Technologie

in Verbindung mit

im weiteren Sinne

Jahrgang

Kapitel

Konsolidierter Entwicklungs- und Finanzplan

Kommunale Gemeinschaftsstelle

Kosten- und Leistungsrechnung

Kostenrechnungspraxis

laufend

Landesverordnung

Management

Milliarden

Ministerium für Wirtschaft, Verkehr, Landwirtschaft und

Weinbau des Landes Rheinland-Pfalz

Number

New Public Management

Nummer

Nordrhein-Westfalen

Neues Steuerungsmodell 


\begin{tabular}{ll} 
NZ & Neuseeland \\
OECD & Organisation for Economic Co-operation and \\
o.g. & Development \\
o.J. & oben genannt \\
o.V. & ohne Jahrgang \\
pdf® & ohne Verfasser \\
PSA & Portable Document Format \\
RLP & Public Service Agreements \\
ROI & Rheinland-Pfalz \\
S. & Return on Investment \\
sog. & Seite \\
Sp. & so genannt \\
TG & Spalte \\
TÖB & Teilnehmergemeinschaft \\
TQM & Träger Öffentlicher Belange \\
TÜV & Total Quality Management \\
u. & Technischer Überwachungsverein \\
u.a. & und \\
usw. & und andere \\
v.Chr. & und so weiter \\
vgl. & vor Christus \\
Vol. & vergleiche \\
VOP & Volume \\
vs. & Verwaltung - Organisation - Personal \\
VTG & versus \\
wif & Vorstand der Teilnehmergemeinschaft \\
WiSt & Wirkungsorientierte Verwaltungsreform \\
WOV & Das Wirtschaftsstudium \\
WU & Wirkungsorientierte Verwaltungsführung \\
www & Wirtschaftsuniversität \\
z.B. & World Wide Web \\
ZfB & zum Beispiel \\
zfbf & Zeitschrift für Betriebswirtschaft \\
zfo & Zeitschrift für betriebswirtschaftliche Forschung \\
ZögU & Zeitschrift Führung + Organisation \\
z.T. & Zeitschrift für öffentliche und gemeinwirtschaftliche Un- \\
& zumen \\
\hline ing &
\end{tabular}


Thorsten Pieper - 978-3-631-75332-3

Downloaded from PubFactory at 01/11/2019 06:04:26AM

via free access 


\section{Einführung}

\subsection{Hintergrund und Problemaufriss}

Ein leistungsfähiger und effizienter öffentlicher Sektor ist für die Funktionsfähigkeit eines Staates, die Lebensqualität der Bevölkerung und die Wettbewerbsfähigkeit der Unternehmen von nicht zu unterschätzender Bedeutung. ' Staaten stehen als Anbieter von Standorten mittlerweile im weltweiten Wettbewerb um Unternehmensansiedlungen, Kapitalinvestitionen und Wissen. ${ }^{2}$ Die Flexibilität und Anpassungsfähigkeit staatlichen Handelns und der hiermit verbundene Einfluss auf relevante Standortfaktoren sind ein entscheidendes Wettbewerbselement. ${ }^{3}$ Die für den deutschen öffentlichen Sektor lange Zeit verlässlichen bürokratischen Steuerungssysteme - mit den vorherrschenden Prinzipien Ordnungsmäßigkeit und Rechtmäßigkeit ${ }^{4}-$ sind allerdings nicht bzw. nur begrenzt in der Lage, den gesteigerten Herausforderungen erfolgreich zu begegnen. ${ }^{5}$

Für Politik und öffentliche Verwaltung bestand und besteht daher die Aufgabe, die Zusammenarbeit im politisch-administrativen System neu zu justieren, um dessen Funktionsfähigkeit zu verbessern. Ausgangspunkt der Modernisierungswelle bildeten vorwiegend angelsächsische Länder, welche unter dem Stichwort New Public Management (NPM) ${ }^{6}$ am betriebswirtschaftlichen „Managerialismus“ orientierte Reformansätze einführten. ${ }^{7}$ Seit Beginn der frühen 90er Jahre wurden vergleichbare Reformansätze auch in Deutschland unter dem Begriff Neues Steuerungsmodell (NSM) erarbeitet. ${ }^{8}$ Ausgehend von der Kommunalverwaltung haben die Modernisierungsbestrebungen mittlerweile sämtliche Ebenen des öffentlichen Sektors erfasst und die entwickelten Konzepte werden schrittweise in die praktische Anwendung überführt. ${ }^{9}$

New Public Management stellt dabei jedoch kein in sich geschlossenes Gesamtkonzept dar, sondern eher ein Leitbild, welches verschiedene ökonomisch orientierte Reformelemente zusammenführt. ${ }^{10}$ Dazu gehört beispielsweise die Konzentration staatlicher

\footnotetext{
Vgl. stellvertretend Stiglitz (1999), S. 188 für diese in der Literatur weitestgehend unbestrittene Aussage.

Vgl. beispielsweise Baßeler/Heinrich/Utecht (2006), S. $558 \mathrm{ff}$.

Die Dynamik der Umweltentwicklungen geht in Deutschland einher mit einer finanzwirtschaftlichen Krise und einem wachsenden strukturellen Ungleichgewicht der staatlichen Haushalte. Vgl. hierzu ausführlich Sachverständigenrat (2005), S. $243 \mathrm{ff}$.

4 Bürokratische Steuerung beruht auf einer starken Betonung des normengerechten Vollzugs juristischer Rechtsvorschriften (z.B. Gesetze). Vgl. hierzu bspw. Wagener (1983) oder auch Reinermann (2000), S. 8.

5 Vgl. bspw. Frey (1994), S. 25; Reichard (1995), S. 60; Budäus (1998), S. 2; Schedler/Proeller (2003), S. 16.

6 Für eine nicht abschließende Aufzählung der unterschiedlichen, allerdings nicht immer deckungsgleichen Begrifflichkeiten im Reformkontext, welche häufig unter dem Stichwort New Public Management zusammengefasst werden vgl. Reinermann (2000), S. 5. Zum Begriff des NPM vgl. Hunold (2003), S. 21 ff. Vgl. Hood (1991) sowie mit einem guten Überblick der Reformelemente vgl. Schedler/Proeller (2000).

Vgl. KGSt (1991) und KGSt (1993) sowie eine hohe Anzahl anschließender konzeptioneller Arbeiten.

9 Die Kommunen haben ausnahmslos mit den Reformen begonnen, allerdings stark instrumentell geprägt, vgl. Zielinski (2000), S. I1. Insb. der Bund ist dagegen eher reformscheu, vgl. Bals/Reichhard (2000), S. 210.

10 Vgl. Schröter/Wollmann (2001), S. 80.
} 
Aufgaben auf Kernbereiche und die Auslagerung von Aufgaben an private Auftragnehmer, eine Stärkung der Markt- und Wettbewerbsorientierung sowie eine erhöhte Kundenorientierung der öffentlichen Verwaltung. ${ }^{11}$ Im Innenverhältnis des politischadministrativen Systems wird die Dezentralisierung der Verantwortung mit einer zielund ergebnisorientierten Steuerung anhand von Leistungsindikatoren verbunden. ${ }^{12}$ Kompetenzen und Verantwortlichkeiten über die Art und Weise der Leistungserstellung werden hierdurch auf den unteren Ebenen der Verwaltung vergrößert, wodurch die Wirtschaftlichkeit der Arbeitsweisen gefördert werden soll. ${ }^{13}$ Anstatt wie im Bürokratiemodell dezidierte rechtliche Vorschriften mit detaillierten Budgetbewilligungen in Form von Haushaltstiteln zu verknüpfen, agiert die politische Ebene im Konzept des NPM primär als Leistungsbesteller. Die Leistungserstellung erfolgt durch die öffentliche Verwaltung, die in Kontrakten an die Erfüllung konkreter Leistungsziele gebunden wird. ${ }^{14}$

Der inhaltlichen Ausgestaltung von Leistungszielen und Leistungskontrolle kommt im Reformkonzept des NPM daher besondere Bedeutung zu. Doch welche Größen sollen Gegenstand der Steuerung sein? Grundsätzlich ist es Aufgabe der öffentlichen Verwaltung, politisch festgelegte Ziele mit knappen Mitteln zu erreichen und dabei ein möglichst vorteilhaftes Verhältnis zwischen den eingesetzten Mitteln und dem Grad der Zielerreichung - im Sinne eines Wertschöpfungsbeitrags für die Gesellschaft - zu realisieren. ${ }^{15}$ Primäres Ziel staatlichen Handelns ist nicht das Erstellen einer bestimmten Anzahl von Leistungen (Produkte), sondern das Auslösen konkreter Wirkungen in der Gesellschaft. Nach Wirkungen zu fragen bedeutet beispielsweise, eine Arbeitsvermittlung nicht nach der Anzahl der geführten Beratungsgespräche zu beurteilen, sondern nach der Anzahl der vermittelten Arbeitssuchenden. Um eine effektive Steuerung im politisch-administrativen System zu gewährleisten, sollten letztlich nur die ausgelösten Wirkungen der Verwaltungsprodukte Gegenstand der Leistungsvereinbarungen und damit des Kontraktmanagements sein.

International wird daher zunehmend nach den Wirkungen der Verwaltungsleistungen gefragt. ${ }^{16}$ Korrespondierend hiermit erhält die Öffentlichkeit immer öfter Kenntnis über einen klar dokumentierbaren value for money für einzelne Leistungen des öffentlichen Sektors. In der deutschen Verwaltungspraxis spielt die Frage nach den angestrebten Wirkungen staatlichen Handelns ${ }^{17}$ bei der konkreten Ausgestaltung und Anwendung der Steuerungssysteme dagegen bislang kaum eine Rolle. ${ }^{18}$ Die Reformansätze konzentrieren sich bislang in erster Linie auf eine Verbesserung der Wirtschaftlichkeit des

11 Vgl. Reichard (1995), S. 64.

12 Vgl. Bucholtz (2001), S. 89; Schrödter/Wollmann (2001), S. 79 und Wollmann (2004a), S. 26.

13 Vgl. Schedler/Proeller (2000), S. 75.

14 Vgl. Naschold/Bogumil (2000), S. 88.

15 Vgl. Peffekoven (1996), S. 2.

16 Vgl. bspw. Kristensen/Groszyk/Bühler (2002) sowie Bühler (2002) und die dort angegebenen Beispiele.

17 Einige wenige Beispiele wirkungsorientierter Steuerungsprojekte nennt Brüggemeier (2004), S. 375.

18 Vgl. Brüggemeier (2004), S. 374. 
Ressourceneinsatzes, mit dem Ziel, die vielfältigen Verwaltungsleistungen so effizient wie möglich herzustellen. So ist in Deutschland ein starker Akzent auf die Einführung betriebswirtschaftlicher Instrumente wie Kostenrechnung, Produkthaushalt und Globalbudget zu verzeichnen, ohne dass die Wirkungen der hergestellten Verwaltungsprodukte bislang systematisch in den Steuerungsprozess einbezogen werden.

Worin könnte diese zögerliche Haltung begründet liegen? Neben einer allgemeinen Reformmüdigkeit, die nach Ansicht einiger Autoren die deutsche Verwaltungspraxis ergriffen hat, ${ }^{19}$ lassen sich in der aktuellen Diskussion weitere Problembereiche identifizieren. Fraglich bleibt, ob im System der Konkurrenzdemokratie die politisch Verantwortlichen überhaupt an einer Vorgabe transparenter Wirkungsziele, die ein realistisches Bild des Wirkungsgrades staatlichen Handelns zeichnen, interessiert sind. ${ }^{20}$ Kurzfristige politische Wettbewerbsziele werden die langfristige Steuerung von Wirkungszielen stets überlagern ${ }^{21}$, denn im Ergebnis geht es schlichtweg darum, Wahlen zu gewinnen. ${ }^{22}$ Mit klaren und messbaren Zielvorgaben bietet man sowohl der Opposition als auch dem Bürger eine offene Angriffsfläche. Zusätzlich bestehen methodische Probleme. Um klare und messbare Zielvorgaben zu erhalten, müssen komplexe Ursache-Wirkungszusammenhänge zweifelsfrei nachgewiesen und in objektive Indikatoren zur Operationalisierung und Messung der ausgelösten Wirkungen überführt werden. Entsprechende Instrumente fehlen bislang. Zuletzt ist eine systematische Wirkungsmessung mit nicht unerheblichen Kosten verbunden, so dass finanziellen Restriktionen in weiten Teilen des öffentlichen Sektors zum Verzicht auf derartige Projekte führen.

An einer wirkungsorientierten Steuerung führt langfristig allerdings kein Weg vorbei $\mathrm{zu}$ offensichtlich sind die damit verbundenen Vorteile. Ohne den Einbezug von Wirkungszielen in die Entscheidungsprozesse besteht permanent die Gefahr, die falschen (d.h. unwirksamen) Verwaltungsprodukte äußerst effizient herzustellen. ${ }^{23}$ Effizienzgewinne werden zu Lasten der Effektivität realisiert und führen in Summe zu Gemeinwohlverlusten für die Gesellschaft. Dabei zeigen erste Erfahrungen bei den reformierten Arbeitsagenturen, wie sich mit einer Steuerung über Leistungs- und Wirkungsziele, unterstützt durch ein sachgerechtes Controlling, erhebliche Verbesserungspotenziale realisieren lassen. ${ }^{24}$ Demgegenüber lassen sich zahlreiche Beispiele finden, welche die Gefahren einer mangelnden Analyse von Wirkungszusammenhängen dokumentieren. Sollte hier - insbesondere seitens der ausführenden Verwaltungsorgane - eine transparentere Darstellung der Wirkungsweisen gelingen, könnte dies langfristig auch zu einer Versachlichung politischer Entscheidungsprozesse führen.

\footnotetext{
19 Vgl. Jann (2004), S. 15 ff. und auf die dort verwiesenen Beiträge.

20 Vgl. Schatz (1991), S. 64 f.; Brinckmann (1995), S. 199 und Bogumil (2001), S. 124.

21 Vgl. Fiedler/Vernau (2000), S. 35 ff.

22 Vgl. Brüggemeier (1998), S. 235 ff.

23 Vgl. Brüggemeier (2004), S. 375.

24 Vgl. zum Steuerungsansatz Weber/Weise (2004), S. 361 ff. sowie zu ersten Ergebnissen o.V. (2006a), S. 20. 
Nach Ansicht von BRÜGGEMEIER sind die zuvor skizzierten politischen und methodischen Problembereiche der wirkungsorientierten Steuerung interdependent - kritischer Erfolgsfaktor ist die Lösung der methodischen Probleme. ${ }^{25}$ Gelingt die transparente Darstellung von Wirkungszusammenhängen, wird sich auch das politische System nicht mehr der Beteiligung am Zielbildungsprozess entziehen können. Eine Schlüsselrolle kommt in diesem Zusammenhang dem Verwaltungscontrolling zu. Controlling verstanden als Führungsunterstützung hat die Aufgabe, praktikable Instrumente bereitzustellen, mit denen sich die bestehenden methodischen Defizite überwinden lassen. Die Frage nach den Wirkungen des Verwaltungshandelns bilden damit den Ausgangspunkt für die Weiterentwicklung des Verwaltungscontrollings zu einem wirkungsorientierten Controlling staatlichen Handelns ${ }^{26}$ Dieses eher strategisch orientierte Verständnis von Verwaltungscontrolling hilft über die bisher eingesetzten binnenorientierten Steuerungsmethoden hinaus, Optimierungspotenziale nicht ausschließlich durch Kostenreduktionen zu erschließen. Es ist Aufgabe des politisch-administrativen Systems, das Verhältnis zwischen den eingesetzten Ressourcen und dem Grad der Wirkungszielerreichung zu optimieren. Durch die Bereitstellung entsprechender Methoden und Instrumente zur Wirkungsanalyse möchte die vorliegende Forschungsarbeit hierzu einen Beitrag leisten.

25 Vgl. Brüggemeier (2004), S. 385.

26 Vgl. u.a. Eichhorn/Wiechers (2001); Reichhard (2002), S. 36; Berens/Mosiek/Röhrig/Gerhardt (2004); Brüggemeier (2004); Röhrig (2008); KGSt (2000) und KGSt (2001). 


\subsection{Zielsetzung der Arbeit}

Vor dem Hintergrund der aufgezeigten Problemstellung ist es das Ziel der vorliegenden Arbeit, auf Grundlage modelltheoretischer Überlegungen ein praktikables Instrumentarium zur systematischen und strukturierten Analyse der durch staatliches Handeln im gesellschaftlichen Umfeld ausgelösten Wirkungen zu entwickeln. Damit möchte der Verfasser einen Beitrag leisten, die bestehenden methodischen Probleme und instrumentellen Defizite bei der Analyse der Wirkungen staatlicher Maßnahmen zumindest in Ansätzen zu überwinden. Aufgrund des ausgeprägten instrumentellen Charakters der Zielsetzung erscheint es zielführend, zur Problemüberwindung die betriebswirtschaftlich geprägte Perspektive des wirkungsorientierten Controllings zu nutzen. ${ }^{27}$ Die in diesem Forschungskontext erarbeiteten Grundlagen - mit den vorliegenden Modellen der Ziel- und Ergebnisebenen öffentlicher Leistungserstellung ${ }^{28}$ - eignen sich als Ausgangspunkt weiterer Überlegungen im Bereich der Wirkungsanalyse.

Die Herausforderung der vorliegenden Forschungsarbeit besteht in der methodischen Weiterentwicklung der Wirkungsanalyse staatlicher Maßnahmen. Um den politischadministrativen Steuerungsprozess zu unterstützen, müssen abstrakte politische Wirkungsziele durch die Auswahl geeigneter Indikatoren in operationale Handlungsanweisungen überführt werden. Dazu ist es notwendig, Kausalzusammenhänge zwischen Staatshandeln und den Wirkungen beim Bürger sowie in dessen Umfeld aufzudecken und diese theoretisch oder empirisch nachzuweisen. ${ }^{29}$ Zielsetzung der Arbeit ist, auf Basis modelltheoretischer Überlegungen eine Strukturierung der beteiligten Systemvariablen vorzunehmen, um die hochkomplexen Wirkungszusammenhänge der Realität beherrschbar zu machen. Die Beschreibung und Vereinfachung realer Zusammenhänge soll zur Ableitung eines verallgemeinerten Modellrahmens führen, welcher der Identifikation gesamtgesellschaftlich ausgelöster Wirkungen dient.

Sind Wirkungen identifiziert und kausal auf bestimmte staatliche Maßnahmen (Produkte) zurückzuführen, müssen in einem weiteren Schritt praktikable Messkonzepte für die Bewertung der Intensität dieser Wirkungen entwickelt werden. Hierbei sind, abhängig von der Wirkungsart, grundsätzlich unterschiedliche Messdimensionen denkbar, bis hin zu einer weitestgehend monetären Bewertung der gesamtgesellschaftlich ausgelösten Wirkungen. Zielsetzung der Arbeit ist hier, bestehende Theoriedefizite durch eine systematische Auseinandersetzung mit Wirkungsarten, Wirkungsdimensionen und möglichen Messverfahren zu überwinden. Weiterhin ist zu prüfen, wie die Ergebnisse einer Wirkungsanalyse in den laufenden Controllingprozess integriert werden können und damit der Steuerung im politisch-administrativen System zugänglich werden. Die politische Ebene ist expliziter Bestandteil der Analyse, da ein wirkungso-

27 Zu den unterschiedlichen Perspektiven des Controllings vgl. ausführlich Kapitel 3.1 dieser Arbeit.

28 Zum Gedanken des 3-E-Konzeptes vgl. grundlegend Budäus/Buchholtz (1997), S.322 ff. sowie die Erweiterung zum 5-E-Konzept vgl. Buschor (2002), S. 66. Eine modifizierte und erweiterte Darstellung findet sich bei Mosiek et al. (2003), S. 29 f. sowie den anschließenden Arbeiten von Röhrig (2008), S. 105 ff.

29 Vgl. bspw. Bühler (2002), S. 277 oder Schedler/Proeller (2003), S. 63 ff. 
rientiertes Management strategische Fragen aufwirft. ${ }^{30}$ Wirkungszielsetzung und die hiermit verbundene Prioritätensetzung ist immer eine politische Aufgabe. ${ }^{31}$

Mit der vorliegenden Arbeit wird ein pragmatisches Wissenschaftsziel verfolgt, der inhaltliche Fokus besteht in der praxistauglichen (Weiter-)Entwicklung von Methoden und Verfahren der Wirkungsanalyse. Daher sollen die zunächst deduktiv erarbeiteten Modelle und Verfahrensweisen in einem weiteren Schritt anhand eines praktischen Anwendungsfalles rückgekoppelt werden. Auf Grundlage eines konkreten Gesetzes und dem bestehenden Aufgabenvollzug einer öffentlichen Verwaltung ist der gesamte wirkungsorientierte Controllingprozess - mit dem analytischen Schwerpunkt der Wirkungsanalyse - aufzuzeigen.

\subsection{Methodik und Vorgehensweise}

Vor dem Hintergrund der ausgeführten Zielsetzungen dieser Arbeit (Kapitel 1) wird vom Verfasser ein zweistufiger Forschungsprozess angestrebt. Die erste Stufe dieses Prozesses orientiert sich an einer theoretisch ausgerichteten Grundlagenforschung. Sie hat zum Ziel, dass methodische Grundlagenwissen im Bereich der Wirkungsanalyse staatlichen Handelns zu verbreitern. Die zweite Stufe des Forschungsprozesses überführt die dort gewonnenen Erkenntnisse in einen praktischen Anwendungsfall. Die Identifikation und Bewertung von Wirkungszusammenhängen in einem spezifischen Leistungsbereich wird in diesem Zusammenhang als angewandte Forschung verstanden.

Im zweiten Kapitel werden zunächst die relevanten Grundlagen und Rahmenbedingungen des öffentlichen Sektors in Deutschland dargestellt. Anhand ausgewählter Aspekte wird dazu der Begriff staatliches Handeln näher spezifiziert und beschrieben. Ausgehend vom Bürokratiemodell wird der eingeleitete Prozess der Verwaltungsmodernisierung bis hin zur wirkungsorientierten Steuerung systematisch aufgearbeitet. Das Kapitel endet mit einer Würdigung der mit der Wirkungsorientierung verbundenen Chancen und Herausforderungen. Hierbei wird deutlich, dass wirkungsorientierte Steuerungsprozesse der Unterstützungsfunktion eines sachgerechten Verwaltungscontrollings bedürfen.

Das dritte Kapitel widmet sich zu Beginn den Grundlagen des Controlling-Ansatzes sowie den Besonderheiten des Verwaltungscontrollings. Im Hinblick auf die Einrichtung wirkungsorientierter Steuerungsprozesse wird die Weiterentwicklung des Verwaltungscontrollings zu einem wirkungsorientierten Controlling beschrieben. Als Bezugsrahmen für eine wirkungsorientierte Controlling-Konzeption dienen unterschiedliche in der Literatur vorgestellte Ebenenkonzepte. ${ }^{32}$ Für den weiteren Untersuchungsverlauf

\footnotetext{
30 Vgl. Berens/Mosiek/Röhrig/Gerhardt (2004), S. 329.

31 Vgl. bspw. Bühler (2002), S. 275.

32 Vgl. hierzu ausführlich Kapitel 3.3.2 dieser Arbeit.
} 
wird insbesondere auf eine Darstellung nach MOSIEK ET AL. und RÖHRIG zurückgegriffen. Eine eingehende Analyse dieser Modellkonzeptionen offenbart allerdings bestehende methodische und instrumentelle Gestaltungsdefizite bei der Identifikation und Bewertung der Wirkungen staatlichen Handelns.

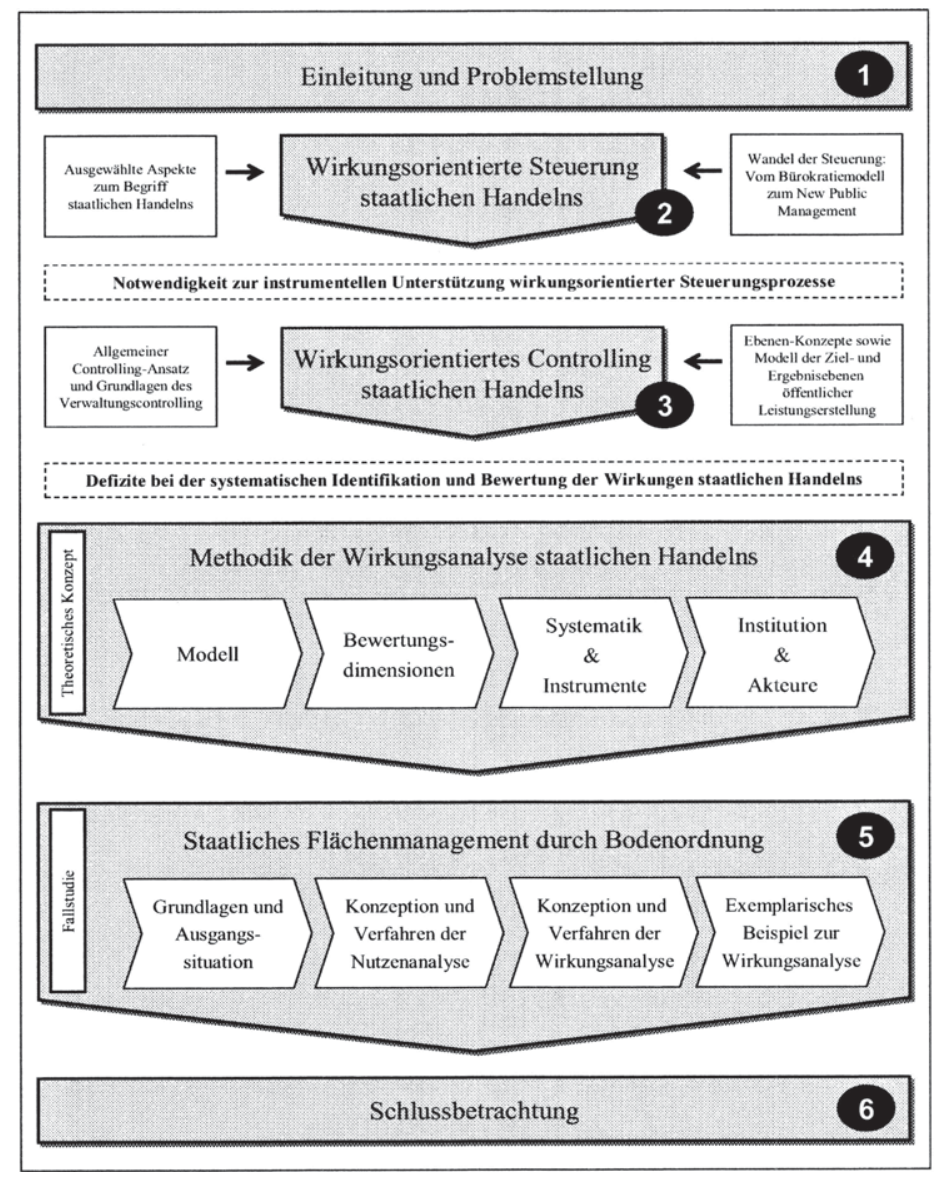

Abbildung 1: Schematischer Überblick über die Struktur der Arbeit

Die Aufgabe des vierten Kapitels besteht darin, einen Beitrag zur Überwindung der aufgezeigten methodischen Defizite zu leisten. Dazu wird im ersten Schritt zunächst ein allgemeines Beschreibungsmodell der Wirkungen staatlichen Handelns entwickelt, um die hochkomplexen Wirkungszusammenhänge der Realität beherrschbar zu machen. Durch die Strukturierung relevanter Systemvariablen kann so in einem zweiten 
Schritt die Abgrenzung der notwendigen Bewertungsdimensionen erfolgen. Anknüpfend wird dann eine eigene Systematik vorgestellt, die der Identifikation und Bewertung der gesamtgesellschaftlich ausgelösten Wirkungen dient. Den einzelnen Phasen dieses Vorgehensmodells werden entsprechende Strukturierungshilfen und Bewertungsinstrumente zugeordnet, die die Praktikabilität der Wirkungsanalyse gewährleisten sollen. In einem letzten Schritt wird diese Gesamtkonzeption wiederum in einen systematischen wirkungsorientierten Controllingprozess eingegliedert. Durch die Institutionalisierung unterschiedlicher Controllingebenen ist in diesem Zusammenhang den Informationsbedürfnissen der beteiligten Akteure Rechnung zu tragen.

Die vorliegende Fallstudie (Kapitel 5) stellt in diesem Kontext dar, wie auf Grundlage eines konkreten Gesetzes und dem Aufgabenvollzug durch die öffentliche Verwaltung adressatenbezogene Wirkungsbeiträge systematisiert, untersucht und bewertet werden können, um diese dem korrespondierenden Ressourcenverzehr gegenüberzustellen. Für den staatlichen Aufgabenbereich des Flächenmanagements durch Bodenordnung werden dazu die theoretisch erarbeiteten Inhalte zur Methodik der Wirkungsanalyse in einen realen wirkungsorientierten Controllingprozess überführt, um die Gesamtheit der Verwaltung wirkungsorientiert zu steuern.

Die Arbeit endet mit einer Schlussbetrachtung in Kapitel 6. Der Gesamtaufbau ist in Abbildung I zusammenfassend dargestellt. 


\section{Wirkungsorientierte Steuerung staatlichen Handelns}

Im Rahmen einer Forschungsarbeit wird ein Erkenntnisfortschritt angestrebt, welcher anschließend zu konkreten Handlungsempfehlungen im wissenschaftlich untersuchten Bereich führt. Zur Erzielung dieses Erkenntnisfortschritts ist zunächst eine angemessene Durchdringung des gewählten Forschungsgegenstandes notwendig. Dazu wird im Folgenden zunächst der Versuch unternommen, den Begriff staatliches Handeln als wissenschaftliches Erkenntnisobjekt näher zu beschreiben und zu erläutern. Durch eine systematische Analyse allgemeiner Rahmenbedingungen staatlichen Handelns sowie der bestehenden Steuerungsansätze im politisch-administrativen System ${ }^{33}$ soll eine , Inventur des Untersuchungsgegenstandes ${ }^{\text {"34 }}$ betrieben werden, um zunächst ein einheitliches Grundverständnis sicherzustellen. Dazu werden gezielt wichtige Kontextfaktoren des Forschungsgegenstandes in die Analyse integriert, um deren Bedeutung im weiteren Verlauf der Untersuchung auch angemessen Rechnung zu tragen.

\subsection{Ausgewählte Aspekte zum staatlichen Handeln}

\subsubsection{Ursachen, Aufgaben und Ziele der Staatstätigkeit}

Bevor der Untersuchungsgegenstand staatliches Handeln durch die Beschreibung organisatorischer Strukturen und eingesetzter Koordinationsinstrumente näher spezifiziert wird, sind zunächst die grundsätzlichen Ursachen, Aufgaben und Ziele staatlichen Handelns zu klären. Es stellt sich hier insbesondere die Frage, wieso staatliches Handeln bzw. die Einrichtung eines öffentlichen Sektors aus gesellschaftlicher Sicht überhaupt sinnvoll und nützlich ist.

Ausgangspunkt der Betrachtung sollen dazu die Überlegungen der Volkswirtschaftlehre zur Notwendigkeit staatlicher Aktivitäten bilden. Grundsätzlich ist unbestritten, dass privatwirtschaftlich organisierte Marktsysteme eine unter ökonomischen Gesichtspunkten optimale Ressourcenallokation gewährleisten - sofern ihre vollständige Funktionsfähigkeit vorliegt. ${ }^{35} \mathrm{Um}$ aber die Funktionsfähigkeit privater Märkte sicherzustellen, benötigen sowohl Gesellschaft als auch Wirtschaft einen staatlichen Ordnungsrahmen in Form einheitlicher rechtlicher Regularien. ${ }^{36}$ In demokratisch legitimierten

33 Zum Begriff des politisch-administrativen Systems vgl. die Ausführungen des Kapitels 2.1.2.

34 Amshoff (1993), S. 49.

35 Die Beschreibung der Leistungsfähigkeit privater Märkte geht bereits auf Adam Smith und seinem Paradigma der Unsichtbaren Hand zurück, vgl. Smith (1776), S. 371. Im Sinne der Pareto-Optimalität gelingt die bestmögliche Güterversorgung einer Gesellschaft - bei gegebener Ausstattung der Produktionsfaktoren wenn marktwirtschaftlich, d.h. über Angebot und Nachfrage gebildete Preise für Güter und Produktionsfaktoren koordiniert wird und auf allen Märkten vollständige Konkurrenz herrscht. Vgl. Stiglitz (1999).

36 Zur Notwendigkeit eines staatlichen Ordnungsrahmens vgl. ausführlich Cooter/Ulen (1988). Der Zusammenhang zwischen einer durchsetzungsfähigen staatlichen Ordnung und dem wirtschaftlichen Erfolg einer Gesellschaft wurde beim Zusammenbruch der ehemaligen Ostblockländer besonders deutlich. Die fehlende staatliche Ordnung führte hier zeitweise zu katastrophalen wirtschaftlichen Ergebnissen (vgl. IMF 1997) mit drastischen Reallohnverlusten, die ansonsten nur bei kriegerischen Einflüssen zu beobachten sind. Vgl. hierzu Frantzke (1999), S. 290. 
Staatsformen obliegt die Ausgestaltung dieses Ordnungsrahmens den Bürgern der Gesellschaft. Dabei sind Art, Ausmaß und Ausgestaltung des Ordnungsrahmens häufig Gegenstand heftiger gesellschaftlicher Kontroversen, welche sich insbesondere in unterschiedlichen politischen Positionen, beispielsweise zum Umfang der Einflussnahme des Staates, wieder finden. ${ }^{37}$

Die marktliche Koordination führt zwar unter gewissen Bedingungen zu einer optimalen Güterversorgung der Gesellschaft, allerdings häufig mit hohen Ungleichgewichten, so dass eine wichtige staatliche Funktion in einer angemessenen Einkommensumverteilung bestehen kann. ${ }^{38}$ Zusätzlich treten Unvollkommenheiten auf, in denen der Markt keine optimalen Ergebnisse liefert, da die Funktionsfähigkeit eingeschränkt ist - beispielsweise durch Wettbewerbsbeschränkungen. ${ }^{39}$ Hier hat der Staat die Aufgabe, durch Gesetze gegen Kartellbildung und unfairen Wettbewerb regulierend in den Marktmechanismus einzugreifen. ${ }^{40}$

Weiterhin gibt es bestimmte Güter, die auf privaten Märkten nicht angeboten werden, da ein sog. Marktversagen vorliegt. ${ }^{41}$ Hierbei handelt es sich um spezifische öffentliche Güter, die sich durch Nicht-Rivalität im Konsum und Nicht-Ausschließbarkeit vom Konsum auszeichnen. ${ }^{42}$ Im Gegensatz zu einem privaten Gut, etwa einem Kleidungsstück, können bei einem öffentlichen Gut, wie bspw. der Inneren Sicherheit, beliebig viele Konsumenten ohne gegenseitige Beeinträchtigung dieses Kollektivgut nutzen. Gleichzeitig ist es aus technischen oder ökonomischen Gründen unmöglich, potenzielle Nachfrager von der Nutzung dieses Gutes auszuschließen. ${ }^{43}$ Einem privaten Anbieter ist es daher unmöglich, seine Preisforderungen am Markt durchzusetzen und somit dieses Gut anzubieten, weshalb es Aufgabe des Staates ist, öffentliche Güter bereitzustellen. ${ }^{44}$

37 In vielen westlich orientierten demokratischen Staaten stehen sich dabei die gegensätzlichen Positionen des Neoliberalismus - mit dem sog. „Nachtwächterstaat" welcher ein hohes Maß an wirtschaftlicher Freiheit gewährt und sich nur auf notwendigste Aufgaben beschränkt und des Neosozialismus - mit einem Staat der sich deutlich stärker in die Belange der Bürger und Wirtschaft einmischt und ein hohes Maß an sozialer Sicherheit gewährt - gegenüber. Vgl. hierzu bspw. Schedler/Proeller (2003), S. 13 f.

38 Über das notwenige Ausmaß und die eingesetzten Methoden der Umverteilung bestehen meist vielfältige Meinungsverschiedenheiten, da sie regelmäßig auch mit einer Schwächung der Leistungsanreize verbunden sind. Vgl. Stiglitz (1999), S. 171.

39 Vgl. Peffekoven (1996), S. 36.

$40 \mathrm{Vgl}$. Baßeler/Heinrich/Utecht (2006), S.46 f. oder auch Stiglitz (1999), S. 173.

41 Zum Begriff und den Ursachen des Marktversagens vgl. Frantzke (1999), S. 293 ff.

42 Vgl. u.a. Zimmermann/Henke (1994), S. 42 f.; Peffekoven (1996), S. 37 oder Nowotny (1999), S. 36 ff.

43 In der Realität liegen hier häufig Mischformen vor. Beispielsweise spricht man von „unvollkommenen öffentlichen Gütern" oder von Gütern mit abnehmendem individuellen Nutzen ab einer bestimmten Anzahl von Konsumenten. Vgl. hier insbesondere Nowotny (1999), S. 36 ff. Aufgrund der unklaren Grenzziehung ist die Größenordnung der Bereitstellung öffentlicher Güter durch den Staat nicht unumstritten und Gegen-stand zahlreicher politischer Debatten. Vgl. beispielsweise Franzke (1999), S. 296.

44 Jeder potenzielle Nutzer könnte nämlich darauf hoffen, als „Trittbrettfahrer“ (free-rider) unentgeltlich in den Genuss der Güter zu kommen. Vgl. Peffekoven (1996), S. 38. 
Bei einer weiteren Gruppe von Gütern, die prinzipiell auch auf privaten Märkten angeboten werden können, kontrolliert der Staat aufgrund gesellschaftspolitischer Erwägungen Art und Ausmaß der Leistungserstellung. ${ }^{45}$ Dies ist einerseits bei meritorischen Gütern der Fall, wie bspw. im Gesundheitswesen, wo eine aus staatlicher Sicht unzureichende Menge des Gutes angeboten bzw. nachgefragt werden würde. Andererseits soll bei demeritorischen Gütern der Umfang der Bedürfnisbefriedigung, wie z.B. Zigarettenkonsum, durch staatliche Eingriffe verringert werden. ${ }^{46}$ Als Gründe für Eingriffe in die individuelle Konsumwahl werden verzerrte Präferenzen, falsche Informationen oder irrationale Entscheidungen der Bürger genannt. ${ }^{47}$ Dabei ist die Aufgabenwahrnehmung des Staates hier nicht unumstritten, da staatlicherseits ein mehr oder weniger an Leistung ,verordnet“ wird, als viele Bürger privat nachfragen würden. ${ }^{48}$

Damit verbunden sind sog. externe Effekte, die seit einigen Jahren insbesondere in den Mittelpunkt der umweltpolitischen Debatte gerückt sind. ${ }^{49}$ Güter mit negativen externen Effekten, wie z.B. Luftverschmutzung, werden häufiger produziert und nachgefragt, da sich nur ein Teil der tatsächlich entstehenden Kosten im Preis niederschlägt. Die Kosten der Umweltverschmutzung verbleiben als soziale bzw. volkswirtschaftliche Zusatzkosten und werden von der Gesellschaft insgesamt getragen. Dagegen werden Güter mit positiven externen Effekten, wie z.B. Erfindungen, weniger produziert, wenn nicht entsprechende Schutzrechte eine Entschädigung des Nutzens über den Preis garantieren. ${ }^{50}$

Die nicht abschließende Darstellung der volkswirtschaftlichen Ursachen staatlicher Betätigung zeigt die generelle Notwendigkeit eines öffentlichen Sektors. Art und Umfang der Aufgabenwahrnehmung sind allerdings häufig umstritten und daher Gegenstand gesellschaftlicher und politischer Kontroversen. Die eindeutige Abgrenzung zwischen privaten und öffentlichen Aufgaben gelingt trotz aller Bemühungen der Finanzwissenschaft nicht. ${ }^{51}$ Öffentliche Aufgaben werden daher durch dazu legitimierte politische Organe (z.B. Parlament) aus politischen Zielen ${ }^{52}$ abgeleitet und im öffentlichen Interesse wahrgenommen. ${ }^{53} \mathrm{Am}$ politischen Zielbildungsprozess ist eine Vielzahl unterschiedlicher Interessensgruppen beteiligt, es bestehen häufig Interessenskonflikte,

\footnotetext{
45 Vgl. Stiglitz (1999), S. 181.

46 Vgl. Peffekoven (1996), S. 38 f.

47 Vgl. Brümmerhoff (1988), S. 93.

48 Vgl. Peffekoven (1996), S. 38. Das Konzept widerspricht dem individualistischen Ansatz der Wohlfahrtstheorie, wenn ,... in die individuellen Präferenzen einfach deswegen eingegriffen wird, weil die [politischen] Entscheidungsträger ihre eigenen Präferenzen für besser halten ...“. Brümmerhoff (1988), S. 94.

49 Vgl. Franzke (1999), S. 296 ff. oder auch Baßeler/Heinrich/Utecht (2006), S. 46.

50 Vgl. Stiglitz (1999), S. 189.

51 Vgl. Eichhorn (2002), S. $760 \mathrm{f}$.

52 Um Orientierung für Handelnde zu geben, sind politische Ziele hinreichend genau nach Inhalt, Ausmaß und Zeitbezug zu bestimmen. Vgl. u.a. Diederich (1989), Sp. 1846; Reichard (1987), S. 33.

53 Vgl. Eichhorn (2002), S. 760.
} 
die i.d.R. durch Verhandlungen auszugleichen sind. ${ }^{54}$ Je abstrakter bzw. vager politische Ziele dabei formuliert sind, umso mehr sind sie zur Konsensbildung und Konfliktlösung geeignet, da sich tendenziell mehr Beteiligte mit ihnen identifizieren können. ${ }^{55}$

Staatliches Handeln ist daher regelmäßig an einem unscharf definierten und äußerst heterogenen Zielsystem ausgerichtet. ${ }^{56}$ Die zusätzlich hohe Komplexität der Zielstrukturen in der öffentlichen Verwaltung führt dazu, dass Zielsetzungen einem häufigen Wandel unterlegen sind und sich oft nur isoliert an bestimmten Projekten oder Programmen orientieren. ${ }^{57}$ Notwendig wären eine stärkere Vernetzung interdependenter Aufgabenfelder und die Bildung von Zielpräferenzen, um bestehende Zielkonflikte zwischen Aufgaben und Wirkungsweisen staatlichen Handelns aufzulösen.

Im Zielsystem der öffentlichen Verwaltung stehen sich inhaltlich zu unterscheidende Sach- und Formalziele ${ }^{58}$ gegenüber. $^{59}$ Während privatwirtschaftliche Unternehmen primär am Formalziel Gewinnerzielung ausgerichtet sind, ist staatliches Handeln primär auf eine Steigerung des Gemeinwohls bezogen. ${ }^{60}$ Der eher abstrakte Begriff des Gemeinwohls ist dabei durch politische Entscheidungen mit Inhalten zu versehen, das heißt, durch entsprechende sachliche Ziele zu konkretisieren. ${ }^{61}$ Sachziele beziehen sich demnach in erster Linie auf die mit den erstellten Leistungen verbundenen Wirkungen beim Leistungsempfänger. ${ }^{62}$ Als Formalziele des öffentlichen Sektors werden dagegen monetäre Ziele wie Kostendeckung, Haushaltsausgleich oder Liquiditätssicherung genannt. ${ }^{63}$ Durch die angespannte Haushaltslage wird die theoretisch gebotene Sachzieldominanz staatlichen Handelns faktisch durch budgetäre Zwänge bestimmt und damit verstärkt an den zuvor genannten Formalzielen ausgerichtet. ${ }^{64}$ Nicht am Gemeinwohl ausgerichtete Wirkungsziele sondern am Budget ausgerichtete Kostenziele sind für das politisch-administrative System zunehmend handlungsleitend.

$54 \mathrm{Zu}$ den beteiligten Interessensgruppen und ihren Beziehungen vgl. Oettle/Thiemeyer (1969), S. 6 oder auch Reichard (1987), S. 36 ff.

55 Vgl. Berens/Hoffjan (2004), S. 7; Braun (1988), S. 141 und Kirsch (1971), S. 157 f.

56 Vgl. u.a. Richter (2000), S. 17; Berens/Hoffjan (2004), S. 7. Das Zielsystem einer Organisation umfasst die Zielstruktur, also die Ordnung der Zielbeziehungen untereinander sowie die Festlegung von Zielinhalten, Zielausmaß und zeitlichem Bezug der Ziele. Vgl. u.a. Seifert (1998), S. 23 und Reichmann (1993), S. 37.

57 Vgl. Reichard (1987), S. 35.

58 Sachziele präzisieren den quantitativen, qualitativen und zeitlichen Erfüllungsgrad der erstellten Leistung, Formalziele messen das Ergebnis der Leistungserstellung und -verwertung in seiner monetären Konsequenz. Vgl. Berens/Hoffjan (2004), S. 7.

59 Vgl. Kosiol (1974), S. 223.

60 Vgl. Eichhorn (1997), S. 111 ; Richter (2000), S. 5; Brede (2001), S. 15.

${ }_{61}$ Vgl. Brecht (1999), S. 169.

62 Vgl. Siems (2005), S. 18.

63 Vgl. u.a. Promberger (1995), S. 166 ff. und Seifert (1998), S. 24 ff.

64 Vgl. Kückelhaus (1999), S. 1 und Berens/Hoffjan (2004), S. 8. 


\subsubsection{Organisation, Träger und Aufbau des öffentlichen Sektors}

Im bisherigen Verlauf der Untersuchung wurden die häufig verwendeten Begriffe staatliches Handeln, öffentlicher Sektor, öffentliche Verwaltung oder politischadministratives System eher intuitiv genutzt und ihr Bedeutungsgehalt nicht näher definiert. ${ }^{65}$ Dies soll an dieser Stelle nachgeholt werden, um sich im Anschluss näher mit dem Themenfeld der Organisation, der Träger und des Aufbaus des öffentlichen Sektors in Deutschland auseinanderzusetzen.

Der Begriff öffentliche Verwaltung ist aufgrund seiner Reichweite und Komplexität nicht klar definiert. ${ }^{66}$ Die öffentliche Verwaltung i.w.S. lässt sich nach EICHHORN als „Veranstaltung zur Wahrnehmung von Angelegenheiten von Gemeinwesen durch Sachverwalter" beschreiben. ${ }^{67}$ Diese weite Begriffsdefinition umfasst nicht nur die Exekutive als eine Gewalt neben Legislative und Judikative, sondern entgegen der Abgrenzung im öffentlichen Recht auch Parlamente und Gerichte. ${ }^{68}$ Verbreiteter ist dagegen das folgende Verständnis der öffentlichen Verwaltung: „Im politischen System der Gewaltenteilung steht die Verwaltung auf der Seite der Exekutive, und zwar als eine der Regierung zuarbeitenden und Entscheidung vollziehenden Instanz. ${ }^{669}$ Die öffentliche Verwaltung i.e.S. ist demnach in der Exekutive lokalisiert und umfasst jedes administrative Handeln im Rahmen gesetzten und gesprochenen Rechts im Auftrag politischer Entscheidungen. Eine idealtypische Grenzziehung zwischen Politik und Verwaltung darf mit diesem Definitionsansatz allerdings nicht verbunden sein, da in der Realität eine vielfältige Durchmischung zu beobachten ist. ${ }^{70}$

Die Vielzahl an Tätigkeiten und Institutionen der öffentlichen Verwaltung machen eindeutige Abgrenzungsversuche in funktionaler, materieller oder institutioneller Hinsicht schwierig. ${ }^{71}$ Beschreibungen orientieren sich daher eher an einer Darstellung charakteristischer Wesensmerkmale der öffentlichen Verwaltung - häufig verbunden mit einer Betonung der institutionellen Perspektive: ${ }^{72}$

- sie gehören nicht zur Gesetzgebung, Rechtsprechung oder Regierung

- sie erfüllen öffentliche Ziele und Aufgaben (Gemeinwohlorientierung) ${ }^{73}$

- es handelt sich um Organisationen im Eigentum des Staates, in öffentlichrechtlicher Trägerschaft (juristische Personen des öffentlichen Rechts)

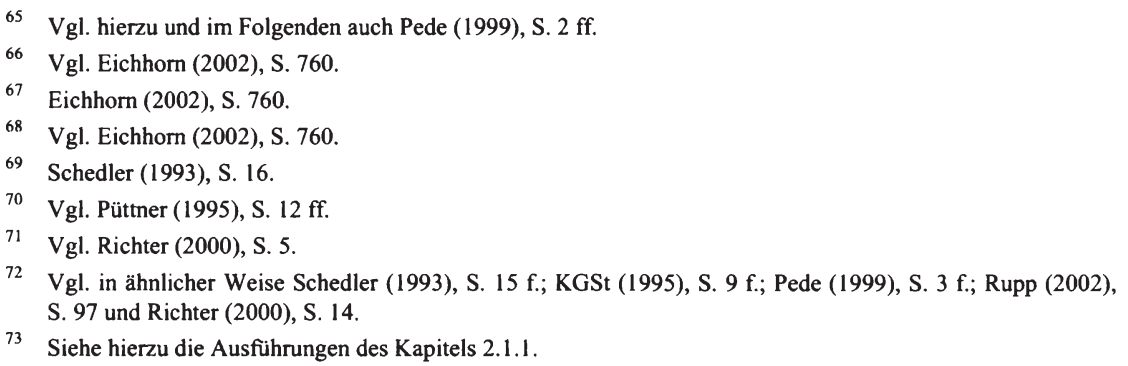


- die Handlungsgrundlage besteht in einem Auftrag des Gesetzgebers, der Entscheidungsspielraum ist politisch und rechtlich eingeschränkt

- Handlungen betreffen die Vorbereitung, Umsetzung und Kontrolle politischer Entscheidungen und auf diesen basierende Aktionen

- die erstellten Produkte sind i.d.R. keinem Marktdruck ausgesetzt

- Mitglieder sind Angehörige des öffentliche Dienstes

Der Begriff öffentlicher Sektor ist demgegenüber deutlich weiter gefasst und beschreibt einen Bereich, in dem Werte realisiert werden können, die außerhalb so nicht angemessen hervorgebracht werden. ${ }^{74}$ Er wird zur Abgrenzung von den anderen gesellschaftlichen Sektoren, dem Privaten Sektor und dem Dritten Sektor, verwendet und umfasst sämtliche Organe der Bundes-, Landes und Kommunalverwaltung, der Selbstverwaltung (z.B. Kammern, Hochschulen etc.) sowie sog. Verwaltungstrabanten in Privatrechtsform (z.B. DFG, Goetheinstitut) ${ }^{75}$

Der Begriff politisch-administratives System umfasst nicht nur den Staat im juristischen Sinne und seine Organe, sondern auch sämtliche politischen Akteure wie z.B. Parteien, die großen Einfluss auf Art und Inhalt der Entscheidungen der eigentlichen Staatsorgane haben und deshalb nicht unberücksichtigt bleiben dürfen. ${ }^{76}$

Der Begriff staatliches Handeln beschreibt im Verständnis der vorliegenden Arbeit die auf die Realisierung bestimmter Zielsetzungen gerichtete sowie zeitlich und logisch strukturierte Tätigkeit der unmittelbaren Staatsorgane. ${ }^{77}$

Die vorgenommenen Definitionen lassen deutlich werden, dass eine klare Abgrenzung der Begrifflichkeiten untereinander und zum privaten Sektor nicht immer eindeutig getroffen werden kann.$^{78}$ Es bestehen große Schnittmengen zwischen den ausgeführten Bedeutungsinhalten. Für die vorliegende Arbeit werden die erläuterten Begriffe daher hauptsächlich als Abgrenzung gegenüber dem privatwirtschaftlichen Bereich verwendet. Der Begriff staatliches Handeln wird dann verwendet, wenn der Verfasser eine zielgerichtete Tätigkeit der Staatsorgane beschreiben möchte. Der Begriff politischadministratives System betont dagegen das institutionelle Zusammenspiel von Politik und Verwaltung. Der Begriff öffentlicher Sektor soll die klare Abgrenzung zum Privatsektor sowie seine wirtschaftliche Bedeutung hervorheben. Der Begriff öffentliche Verwaltung beschreibt mit administrativen Aufgaben betraute Institutionen, die behördlichen Charakter im Sinne der zuvor genannten Merkmale aufweisen.

74 Vgl. Mc Kevitt/Lawton (1994), S. 58.

75 Vgl. Bogumil/Holtkamp/Wollmann (2003), S. 6.

76 Vgl. hierzu auch von Armin (1989), S. 41 zitiert aus Pede (1999), S. 2.

77 Zum Begriff der Handlung und der Handlungstheorie vgl. Kückelhaus (1999), S. $19 \mathrm{ff}$.

78 Vgl. hierzu sowie der im Folgenden getroffenen Abgrenzung der Begrifflichkeiten untereinander mit einem vergleichbaren Verständnis Pede (1999), S. 4. 
In der Literatur existiert eine Vielzahl weiterer Klassifizierungen, um das heterogene Tätigkeitsfeld der öffentlichen Verwaltung zu strukturieren und näher zu beschreiben. ${ }^{79}$ So werden beispielsweise in der juristischen Literatur die Eingriffsverwaltung, die in Rechte einzelner verbietend oder gebietend eingreifende Verwaltung und die Leistungsverwaltung, die Sachgüter oder Dienstleistungen gewährende Verwaltung, unterschieden. ${ }^{80}$ In einer inhaltlichen Gliederung unterteilen HESSE/ELLWEIN die Verwaltungstypen in: ${ }^{81}$

- Ordnungsverwaltung, welche Sicherheit und Ordnung aufrecht erhält und dafür in die (Freiheits-)rechte der Bürger eingreift (z.B. Polizei)

- Dienstleistungsverwaltung, zur Bearbeitung rechtlicher und politischer Aufträge gegenüber Einzelnen (Leistungsverwaltung) oder ganzer Bereiche, wie z.B. Wirtschaftsförderung (Lenkungsverwaltung) ${ }^{82}$

- Organisationsverwaltung, die interne (Vor-)leistungen für die Verwaltung selbst erbringt

- politische Verwaltung, als Schnittstelle zwischen Politik und Verwaltung

Neben den dargestellten Ansätzen kann eine Systematisierung nach Trägern weiteren Aufschluss über die Struktur der öffentlichen Verwaltung in Deutschland geben. Träger der öffentlichen Verwaltung sind juristische Personen mit eigener Rechtspersönlichkeit, die Körperschaften, Anstalten und Stiftungen des öffentlichen Rechts. ${ }^{83}$ Daneben existieren selbständige öffentliche Unternehmen ${ }^{84}$ ohne eigene Rechtspersönlichkeit wie das Sondervermögen ${ }^{85}$ (z.B. das bundesdeutsche Schienennetz) und der Eigenbetrieb $^{86}$ (z.B. der Landesbetrieb Straßen NRW) sowie der unselbständige Regiebe$\operatorname{trieb}^{87}$ (z.B. ein kommunales Krankenhaus). Die öffentliche Hand kann zudem Beteiligungen an privaten Unternehmen halten, wie Kapitalgesellschaften (z.B. die Deutsche Bahn AG) oder Personen des Privatrechts als Beliehene mit Aufgaben betrauen (z.B. TÜV). ${ }^{88}$

79 Vgl. z.B. Mayer (1972), S. 3; Kosiol (1976), S. 58 ff.; Lüder (1989), S. 1152 oder Maurer (2000), S. 6 ff. sowie Rupp (2002), S. 97 ff. mit einem Überblick ausgewählter Klassifizierungen.

80 Vgl. u.a. Forsthoff (1973), S. 23 und Becker (1997), S. 440.

81 Vgl. Hesse/Ellwein (1997), S. 343 f. sowie Rupp (2002), S. 97 f.

82 Zur Unterscheidung von Leistungs- und Lenkungsverwaltung vgl. Maurer (2000), S. 6 ff.

83 Vgl. Eichhorn (2002), S. 761.

84 Öffentliche Unternehmen sind Wirtschaftsbetriebe, die öffentlichen Zielen auf der Grundlage öffentlichen Eigentums unmittelbar nachgehen. Vgl. von Loesch (1997), S. 286.

85 Bei einem staatlichen Sondervermögen handelt es sich um eine aus der öffentlichen Verwaltung ausgegliederte Abteilung oder um einen ausgegliederten Vermögensgegenstand. Vgl. Jung (2002), S. 109.

${ }^{86}$ Ein Eigenbetrieb ist eine verselbständigte Anstalt mit eigener Betriebssatzung und kaufmännischem Rechnungswesen. Vgl. Hieber (1999), S. 37.

87 Unter einem Regiebetrieb versteht man eine unselbständige Wirtschaftseinheit ohne Rechtsfähigkeit, die über eigene Organe, eigenes Vermögen und ein eigenes Rechnungswesen verfügt und den Weisungen der Verwaltung unterliegt. Vgl. Jung (2002), S. 111 und Mühlenkamp (1994), S. 18.

${ }^{88}$ Vgl. Eichhorn (2002), S. 108. 
Bei Stiftungen handelt es sich um verselbständigte Vermögensmassen, die einem als gemeinnützig anerkannten Zweck gewidmet sind (z.B. Museen). Anstalten sind selbständige Verwaltungseinrichtungen zur Erfüllung öffentlicher Aufgaben von Dauer, zu deren Erledigung sie mit personellen und sachlichen Ressourcen ausgestattet sind (z.B. Sparkasse). Bei den Körperschaften sind die Gebiets- und Nichtgebietskörperschaften ${ }^{89}$ (z.B. Ärztekammer) zu unterscheiden. Die Gebietskörperschaften Bund, Länder und Gemeinden sind die eigentlichen Träger der öffentlichen Verwaltung, die sich ihrerseits wiederum der anderen Trägerstrukturen zur Wahrnehmung ihrer Angelegenheiten bedienen. ${ }^{90}$ Sie erfassen alle Personen ihres Gebietes und besitzen Gebietshoheit, d.h. die Gewalt, über alle Personen und Sachen ihres Gebietes hoheitlich zu bestimmen, sowie eine unmittelbar gewählte Vertretung, z.B. in Form des Bundestages, der Landtage, der Kreistage und Gemeinderäte (vgl. Abbildung 2). ${ }^{91}$

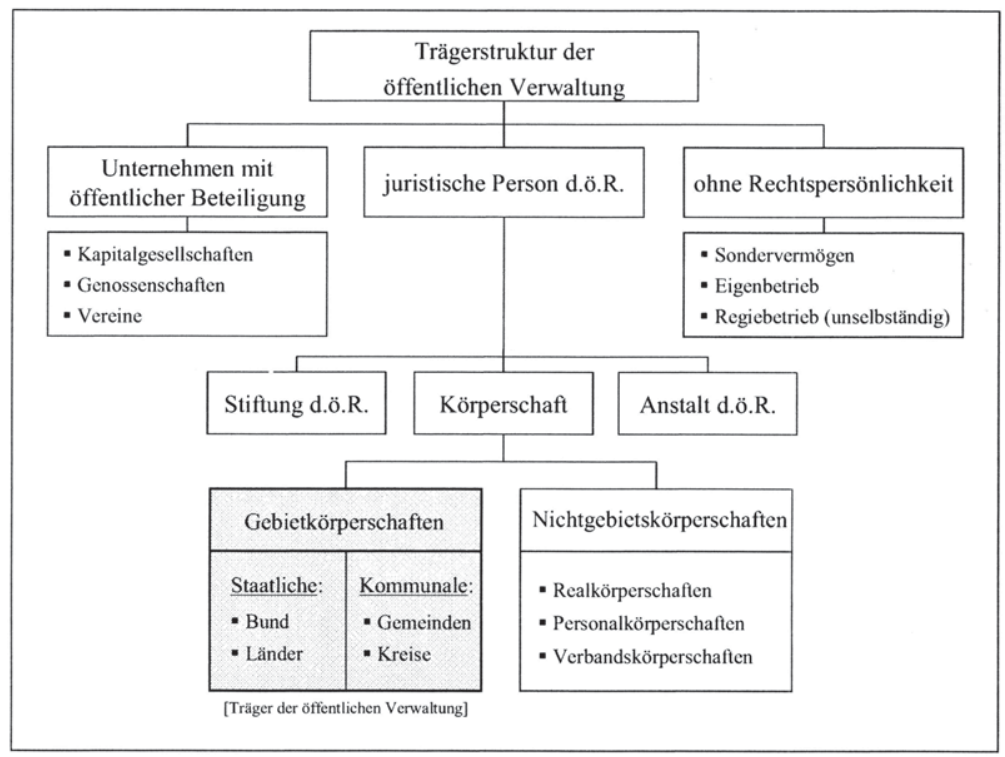

Abbildung 2: Systematisierung der Trägerstruktur ${ }^{92}$

Die öffentliche Verwaltung ist in ihrer äußeren Struktur dem demokratischen Herrschaftssystem entsprechend horizontal in die Gewaltenteilung von Legislative, Judikative und Exekutive eingebettet. Daneben besteht eine vertikale Gewaltenteilung, indem

89 Nichtgebietskörperschaften sind selbständige Verwaltungsträger, die ihre Aufgaben unter staatlicher Aufsicht wahrnehmen (Selbstverwaltung). Vgl. Schauer (1984), S. 79. Die Mitgliedschaft kann freiwillig oder erzwungen sein und gründet auf bestimmte Merkmale, z.B. die Berufsgruppe. Vgl. Richter (2000), S. 13. Vgl. Eichhorn (2002), S. 761.

92 Eigene Darstellung in Anlehnung an Richter (2000), S. 12 und Müller (2004), S. 61. 
die Verfassung Bund, Länder und Kommunen als je eigene politisch-administrative Ebene nennt. ${ }^{93}$ Die innere Struktur der Hauptverwaltungsebenen ist wiederum in mehrere Ebenen eingeteilt. Auf Bundes- und Landesebene ist die Verwaltung in die Ministerien $^{94}$, die Bundes- und Landesbehörden, Mittelbehörden ${ }^{95}$ und die örtlichen Behörden (Unterbehörden) geteilt. Auf kommunaler Ebene findet eine Trennung in Landschaftsverband, Kreise, kreisfreie Städte und kreisangehörige Gemeinden statt. ${ }^{96}$

Wesentliches Merkmal der Bundes- und Landesverwaltung, welche häufig auch als Staatsverwaltung bezeichnet wird, ist das Ressort- und Instanzenprinzip..$^{77}$ Die Verwaltung ist nach sachlichen Kriterien horizontal in einzelne Ressorts eingeteilt (z.B. Finanzministerium, Familienministerium etc.). Der Aufgabenvollzug innerhalb der Ressorts vollzieht sich, der vertikalen Strukturierung folgend, über mehrere Instanzen. In den Oberbehörden der Staatsverwaltung (z.B. Bundeskartellamt) sind spezielle Aufgaben der Ministerien ausgegliedert, die sich auf den gesamten Regierungsbezirk beziehen. ${ }^{98}$ Die Mittelinstanzen (z.B. Bezirksregierungen) sind zwischen Oberbehörde und örtlicher Behörde angesiedelt und in ihrem geografisch abgegrenzten Bereich für spezielle fachliche Aufgaben sowie Koordination und Aufsicht zuständig. ${ }^{99}$ Die Unterbehörden (z.B. Gesundheitsamt) stellen die operativen Einheiten des Verwaltungsvollzugs dar. Sie können durch Kommunalisierung auch Bestandteil der dritten Hauptebene, der kommunalen Selbstverwaltung sein. ${ }^{100}$ Hier bilden Gemeinden, zusammengefasst zu Kreisen, und die kreisfreien Städte die Verwaltungsebenen.

Zwischen den Hauptebenen der Verwaltung und den eingegliederten Behörden existiert eine Vielzahl von Beziehungen und Aufgabenverflechtungen. Das staatliche Handeln ist demnach durch eine äußerst verschachtelte Organisationsstruktur und hohe Uneinheitlichkeiten einzelner Vollzugsebenen geprägt, was sich teilweise in intransparenten Entscheidungsstrukturen niederschlägt.

\subsubsection{Instrumente und Ergebnisse staatlichen Handelns}

Dem Staat steht im Vergleich zur Privatwirtschaft hinsichtlich der Durchsetzung und Reichweite gesetzter Ziele ein breiteres Handlungsspektrum zu. ${ }^{101}$ Der Spielraum staatlichen Handelns spiegelt sich im Steuerungsinstrumentarium wider, welches unter-

\footnotetext{
93 Vgl. u.a. König (1997), S. 21 oder auch Lüder (1989), S. 1154.

94 Ministerien werden als oberste Bundes- oder Landesbehörden (bei Stadtstatten als Senate bezeichnet) von den jeweiligen Ministern geführt, welche damit sowohl dem politischen System (Regierung) als auch dem administrativen System (Verwaltung) angehören. Vgl. Thieme (1984), S. 189 ff. und Rupp (2002), S. 102.

95 In den kleineren Flächenstaaten existieren keine Mittelbehörden. Vgl. Bogumil (1999), S. 6.

96 Rupp (2002), S. 101.

97 Vgl. Rupp (2002), S. $101 \mathrm{f}$.

98 Vgl. Thieme (1984), S. 197.

99 Vgl. Eichhorn (2002), S. 761.

100 Vgl. Thieme (1984), S. 204.

101 Zu nennen wäre hier beispielsweise die Gesetzgebungskompetenz des Parlamentes.
} 
schiedliche Formen der Einflussnahme auf die individuellen Entscheidungspräferenzen der Bürger darstellt. ${ }^{102}$ Die Einflussnahme erfolgt dabei auf Grundlage von Gesetzen und ist durch entsprechende Mittelzuweisungen im Haushalt im zeitlichen Rahmen konkretisiert. ${ }^{103}$ Grundsätzlich lassen sich folgende Instrumente und Maßnahmen unterscheiden: ${ }^{104}$

- Absichtserklärungen und Apelle (als indirekte Form der Einflussnahme)

- Erlaubnisse (häufig an die Erfüllung bestimmter Bedingungen geknüpft)

- Besteuerung, Abgaben und Gebühren gemäß Kostenverursachung oder von unerwünschten Tatbeständen (um Anreize zu setzen)

- finanzielle Transfers (auch zur Befähigung Dritter)

- Gebote und Verbote (Gesetze, Erlasse, Bescheide etc.)

- unmittelbare Bereitstellung von Gütern und Dienstleistungen

Die aus staatlichem Handeln erzeugten Ergebnisse der öffentlichen Verwaltung werden als Verwaltungsleistung bezeichnet. ${ }^{105}$ Bei den Ergebnissen der Verwaltungstätigkeit sind Leistungen von Wirkungen zu unterscheiden ${ }^{106}$ Leistungen sind als Verwaltungsprodukte das unmittelbare Ergebnis staatlichen Handelns, betrachtet aus dem Blickwinkel eines externen Dritten. ${ }^{107}$ Wirkungen stellen demgegenüber die zielbezogenen Ergebnisse öffentlicher Leistungen für die Gesellschaft dar. ${ }^{108}$ Sie sind mittelbare Resultate einer oder eines Bündels von Leistungen beim Leistungsempfänger sowie im gesellschaftlichen Umfeld. ${ }^{109}$

102 „Eine wichtige Zielsetzung staatlichen Handelns ist es, in den von Marktversagen gekennzeichneten Bereichen den Markt durch die Bereitstellung öffentlicher Güter zu ersetzen. Die Ressourcenallokation für öffentliche Güter erfolgt ersatzweise über öffentliche Haushalte, die überwiegend steuerfinanziert sind und bei denen gewählte Gremien stellvertretend für das Individuum die Allokationsentscheidungen treffen. Folgen des Marktversagens können Zwangskonsum und Zwangsfinanzierung dieser Güter sein, die sich wiederum in der starken Betonung von Prinzipien wie Regelhaftigkeit, Nachvollziehbarkeit und Kontrollierbarkeit staatlichen Handelns niederschlagen. Beide Elemente, die Vertretung durch gewählte Gremien und die Installation zusätzlicher Kontrollmechanismen, sichern die demokratische Legitimation der Präferenzentscheidungen für bestimmte Güter, die der Einzelne an die Gemeinschaft abgegeben hat.“ Kiesel (2005), S. 11 f.

103 Vgl. Bräunig (2004), S. 311 ff.

104 Vgl. Mayntz (1978), S. 58 oder Kiesel (2005), S. 12

105 EICHHORN bezeichnet „Leistung“ als dualen Begriff, daher kann abweichend vom o.g. Verständnis Leistung nicht nur das Ergebnis einer Tätigkeit, sondern auch die Tätigkeit selbst - verstanden als Prozess - umschreiben. Vgl. Eichhorn (1991), S. 152.

106 In der Literatur ist der Ergebnis-Begriff öffentlicher Leistungserstellung nicht einheitlich definiert. Zum einen bezieht er sich nur auf das unmittelbare Leistungsergebnis - verstanden als Verwaltungsprodukt - zum anderen werden die eher mittelbaren Leistungsergebnisse - d.h. die in der Gesellschaft ausgelösten Wirkungen - mit in den Ergebnisbegriff einbezogen. Vgl. Schedler/Proeller (2003), S. 63.

$107 \mathrm{Vgl}$. Schedler/Proeller (2003), S. 63.

108 Vgl. Budäus/Buchholtz (1997), S. 329.

$109 \mathrm{Vgl}$. Schedler/Proeller (2000), S. 61. 
Die Produkte staatlichen Handelns weisen spezifische Charakteristika auf. Der Staat ist angehalten, in den von Marktversagen gekennzeichneten Bereichen tätig zu werden. Im Unterschied zu privaten Gütern und Dienstleistungen erfolgen Angebot und Nachfrage nicht über den Markt, sondern die Koordination ist hier Aufgabe des Staates. Bei öffentlich bereitgestellten Produkten kann der Marktmechanismus daher nicht einsetzen. Aufgrund der fehlenden Marktgängigkeit müssen Entscheidungen über Art und Umfang der öffentlichen Leistungen durch politische Entscheidungsorgane (z.B. Parlament) getroffen werden. ${ }^{110}$

Den öffentlichen Leistungen wird in der Literatur überwiegend Dienstleistungscharakter zugesprochen. ${ }^{11}$ Wesentliche Kennzeichen von Dienstleistungen sind deren Immaterialität $^{112}$, die Integration eines externen Faktors zur Erstellung ${ }^{113}$ und eine hieraus resultierende Nichtlagerfähigkeit. ${ }^{114}$ Die notwendige Beteiligung des Kunden am Produktionsprozess hat zur Konsequenz, dass Leistungserstellung und Leistungsabgabe zeitgleich stattfinden und zuvor eine Leistungsbereitstellung, als vordisponierte Bereitschaftskapazität, stattfinden muss. Aufgrund der Immaterialität kommt der Wahrnehmung des Kunden bei der Qualitätsbeurteilung eine hohe Bedeutung zu, da eine physische Produktqualität nicht bewertet werden kann. Die Heterogenität und Breite des staatlichen Leistungsspektrums äußern sich zudem in einer unterschiedlichen Standardisierbarkeit von Dienstleistungen, was eine einheitliche Leistungsbeurteilung erschwert. $^{115}$

\subsubsection{Prozess öffentlicher Leistungserstellung}

Die aus staatlichem Handeln erzeugten Ergebnisse der öffentlichen Verwaltung sind das Resultat eines Leistungserstellungsprozesses. Dieser Prozess kann als sequentielle Abfolge einzelner Leistungsteilschritte aufgefasst werden und soll im Folgenden anhand eines einfachen Modells kurz skizziert werden. Der Leistungserstellungsprozess lässt sich demnach, wie in Abbildung 3 dargestellt, in fünf unterschiedliche Phasen gliedern. ${ }^{116}$

110 Zum Begriff der fehlenden Marktgängigkeit bei öffentlichen Leistungen vgl. Siems (2005), S. 23 f.

111 Vgl. bspw. Chmielewicz (1985), S. 29 f.; Gornas (1992), S. 48; Schmidberger (1994), S. 11; Haiber (1997), S. 101 oder Buchholtz (2000), S. 21.

112 Das es sich bei Dienstleistungen immer um immaterielle Wirtschaftsgüter handelt ist nicht gänzlich unumstritten. Vgl. hierzu Niemand/Rassat (1997), S. 42. Teilweise werden zur Erstellung von Dienstleistungen materielle Güter als Trägermedium (z.B. Papier, Speicherkarte) eingesetzt. Vgl. Corsten (1990), S. 23.

113 Der externe Faktor besteht aus dem Leistungsnehmer oder aus Objekten, Tieren, Rechten und Informationen, die intensiv am Dienstleistungsprozess beteiligt sind und den Verlauf des Prozesses maßgeblich beeinflussen. Vgl. Corsten (1990), S. 35.

114 Zu den Abgrenzungskriterien von Dienstleistungen vgl. ausführlich Meffert/Bruhn (2003), S. 61 ff.

115 Bei nicht standardisierten Leistungen ist der Ermessenspielraum außerhalb von Gesetzen und Verfahrensvorschriften in der Regel deutlich höher und es bestehen interaktive Personenkontakte. Vgl. hierzu Schmidberger (1994), S. $252 \mathrm{f}$.

116 Vgl. zu den folgenden Ausführungen insbesondere Budäus/Buchholtz (1997), S. 328 f. 


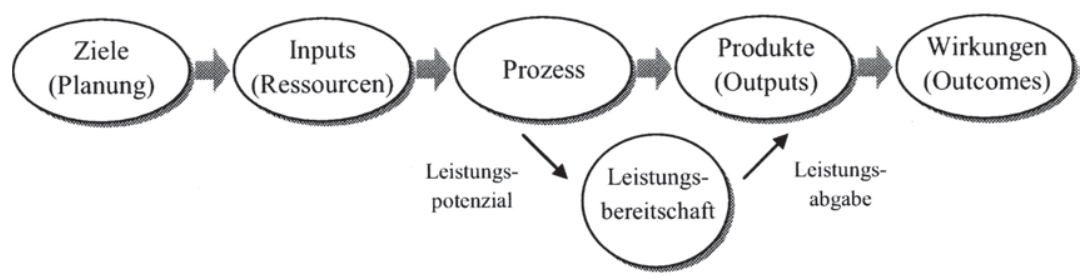

Abbildung 3: Prozessmodell der öffentlichen Leistungserstellung ${ }^{117}$

Ausgangspunkt der Betrachtung bilden die durch demokratische Auseinandersetzung in zuständigen Gremien gebildeten politischen Ziele ${ }^{118}$ Sie dienen als Grundlage für die folgenden Prozessschritte. Zur Erreichung der angestrebten Ziele werden Maßnahmen geplant, für deren Umsetzung der Einsatz von Ressourcen notwendig ist, welche als Inputs in den Prozess eingehen. ${ }^{119}$ Im anschließenden (Produktions-)Prozess findet die eigentliche Leistungserstellung statt. Entsprechend dem Dienstleistungscharakter öffentlicher Produkte ist dieser Prozessschritt zweistufig ausgebildet. Zunächst wird aus der Kombination innerbetrieblicher Ressourcen die Leistungsbereitschaft hergestellt. Erst durch Nachfrage des Abnehmers, der selbst externe Faktoren in den Produktionsprozess einbringt ${ }^{120}$, erfolgt die Leistungsabgabe in Form eines Produktes ${ }^{121}$. Produkte (Outputs) stellen damit das unmittelbare Ergebnis des Leistungsprozesses, wie z.B. die Erteilung einer Fahrerlaubnis, dar. Durch die unmittelbaren Leistungsergebnisse werden Wirkungen beim Leistungsempfänger oder in der Gesellschaft ausgelöst. ${ }^{122}$ Wirkungen sind die mittelbaren, erst langfristig erkennbaren Folgen des Leistungsprozesses, wie z.B. die Erhöhung der Verkehrssicherheit durch die staatliche Kontrolle der Fahrerlaubnis.

In der Realität läuft der dargestellte politisch-administrative Planungs- und Leistungsprozess weder zeitlich noch inhaltlich in diesen aufeinander folgenden Schritten ab. Ausgangspunkt sind häufig unklar definierte Zielsetzungen mit vielfältigen Rückkopplungen, bspw. von Wirkungen auf Produkte, Produkterstellung und politische Zielsetzungen. ${ }^{123}$ Weiterhin spielen Einflüsse externer Akteure und der Umwelt eine bedeu-

\footnotetext{
117 Eigene Darstellung in Anlehnung an Budäus/Buchholtz (1997), S. 329.

118 Zum Zielbildungsprozess im politisch-administrativen System vgl. Böllhoff/Wewer (2002), S. 147 ff.

119 Der Begriff Input umfasst alle notwenigen Ressourcen wie z.B. Personal, Sachmittel oder Dienstleistungen Dritter. Vgl. u.a. Kristensen/Grozyk/Bühler (2002), S. 10.

120 Zum Begriff ,externe Faktoren“ vgl. Corsten (1990), S. 35 f. bzw. die Ausführungen des Kapitels 2.1.3.

121 Produkte sind selbständige Leistungseinheiten der öffentlichen Verwaltung, verstanden als das unmittelbare, von Dritten sichtbare und damit messbare Ergebnis eines Bündels von Aktivitäten. Vgl. Schedler/Proeller (2003), S. 64 und S. 127 und die dort angegebene Literatur.

$122 \mathrm{Vgl}$. hierzu u.a. Schedler/Proeller (2003), S. 64. Outcomes sind die mit Hilfe von Outputs beabsichtigt oder auch unbeabsichtigt erzielten Wirkungen in einzelnen Politikbereichen oder der Gesellschaft als Ganzes. Vgl. Bühler (2002), S. 274.

123 Vgl. Haldemann (1998), S. 197.
} 
tende Rolle, so dass die klare Zuordnung, Messung und Bewertung von Leistungsergebnissen äußerst schwierig ist. ${ }^{124}$

\subsubsection{Zur ökonomischen Bedeutung des öffentlichen Sektors}

Der Staat spielt auch in Marktwirtschaften eine zentrale Rolle bei der Produktion und Verteilung von Gütern und Dienstleistungen und bei der Steuerung von Wirtschaftsprozessen. Der öffentliche Sektor übt einen großen Einfluss auf die Standortqualität einer Kommune, einer Region, eines Landes oder auch eines ganzen Wirtschaftsraumes aus. ${ }^{125}$ Durch die Organisation der Rahmenbedingungen privater Märkte ist direkt und indirekt auch die Wettbewerbsfähigkeit der Wirtschaft betroffen. Einerseits profitieren Unternehmungen direkt von den Leistungen des Staates, wie beispielsweise beim Ausbau der Infrastruktur. Andererseits besteht durch den Finanzbedarf des Staates und damit die Höhe von Steuern und Abgaben ein indirekter Einfluss auf wesentliche Standortfaktoren.

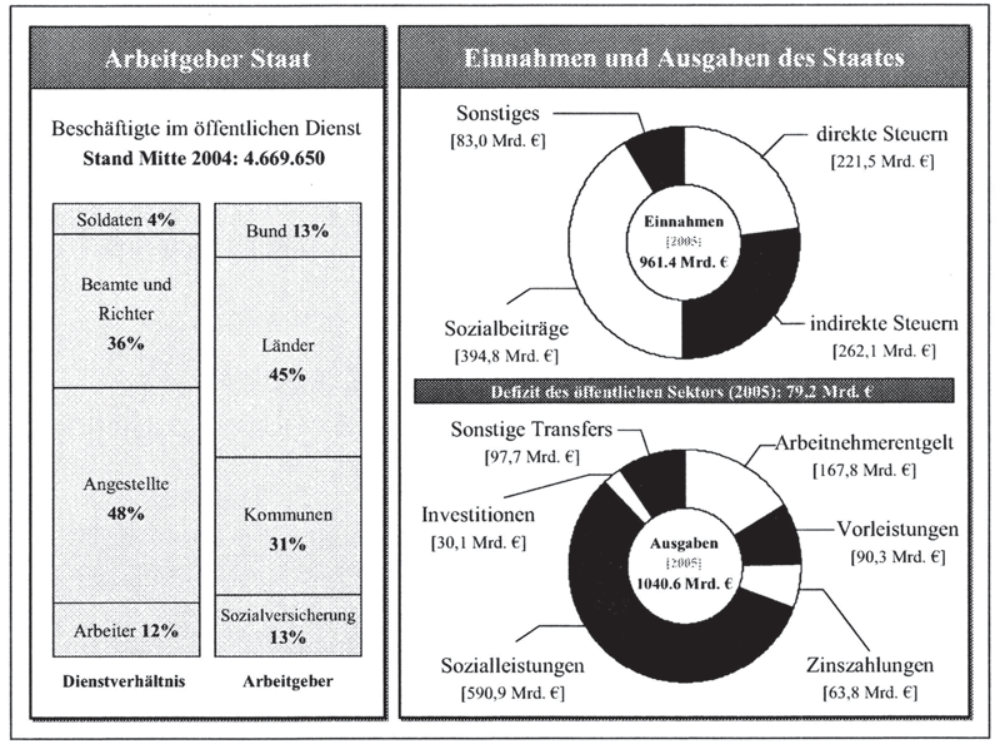

Abbildung 4: Statistische Daten des öffentlichen Sektors in Deutschland ${ }^{126}$

124 Vgl. Schedler (1996), S. 48 und Schedler/Proeller (2003), S. 64.

$125 \mathrm{Vgl}$. Teufel (1997), S. 9 oder auch Kniola (1998), S. 8.

126 Eigene Darstellung. Zu den finanzstatistischen und ökonomischen Daten vgl. Statistisches Bundesamt (2006) und Sachverständigenrat (2005). 
Die Bedeutung des öffentlichen Sektors in Deutschland lässt sich auch über finanzstatistische Daten belegen (vgl. Abbildung 4). Die zentrale Rolle des Staates wird deutlich, wenn man bedenkt, dass der Anteil der Staatsausgaben am gesamten Bruttoinlandsprodukt - die sog. Staatsquote ${ }^{127}$ - seit vielen Jahren ungefähr $50 \%$ beträgt (2005: 46,4\%). ${ }^{128}$ Damit wird hierzulande fast die Hälfte der verfügbaren Ressourcen der Gesellschaft direkt staatlich gelenkt. Analysiert man die Struktur von Einnahmen und Ausgaben des Staates ${ }^{129}$ so zeigt sich, dass die Sozialleistungen mit nahezu $60 \%$ der Gesamtausgaben deutlich dominieren. Steuereinnahmen und Sozialbeiträge können das Gesamtvolumen der Ausgaben bereits seit vielen Jahren nicht decken, im Kalenderjahr 2005 betrug das Defizit knapp 80 Mrd. $€$. Die Bedeutung des öffentlichen Sektors wird auch in der Funktion des Arbeitgebers deutlich. Mitte des Jahres 2004 hatten Bund, Länder, Gemeinden und die Träger der Sozialversicherung ca. 4,67 Millionen Beschäftigte. Dies entspricht ungefähr 13,5\% aller abhängigen Erwerbstätigen der Bundesrepublik Deutschland. ${ }^{130}$

\subsection{Zum Wandel der Steuerungsansätze im politisch-administrativen System}

Im Rahmen der bisherigen Untersuchung wurden ausgewählte Rahmenbedingungen staatlichen Handelns vorgestellt, um eine einheitliche Auffassung des Forschungsobjektes sicherzustellen. Durch die Identifizierung und Analyse zentraler Eigenschaften konnte zunächst eine Abgrenzung und Charakterisierung des Untersuchungsgegenstandes vorgenommen werden. Auffällig ist, dass staatliches Handeln trotz der immensen Bedeutung für zahlreiche Standortfaktoren in der bisherigen wissenschaftlichen Betrachtung durch die Betriebswirtschaftslehre nur eine untergeordnete Rolle spielt. ${ }^{131}$

Zielsetzung der vorliegenden Arbeit ist es, sich systematisch mit den Wirkungen staatlichen Handelns auseinanderzusetzen, um sie einer verbesserten Steuerung zugänglich zu machen. Daher wird der nächste Schritt der Untersuchung den derzeitigen Wandel der Steuerungsansätze im politisch-administrativen System analysieren. Um hier ein grundlegendes Verständnis zu erzielen, sollen ausgehend vom bürokratisch geprägten Steuerungsansatz (Kapitel 2.2.1) die Leitideen einer Steuerung im Sinne des New Public Management bzw. des Neuen Steuerungsmodells (Kapitel 2.2.2) bis hin zu einem konsequent wirkungsorientierten Steuerungsansatz (Kapitel 2.2.3) vorgestellt werden.

\footnotetext{
127 Zum Sektor Staat werden nach Konvention der Volkswirtschaftlichen Gesamtrechnung die Gebietskörperschaften (insb. Bund, Länder, Gemeinden) und die Sozialversicherungsträger (insb. Renten-, Kranken-, Unfall-, Pflege- und Arbeitslosenversicherung) zusammengefasst. Vgl. Baßeler/Heinrich/Utecht (2006) S. 378. Seit Beginn der 80er Jahre liegt die Staatsquote in Deutschland zwischen $45 \%$ und $50 \%$. Sie hatte ihren vorläufigen Höchstpunkt im Jahr 1996 mit 49,3\% und ist seit einigen Jahren rückläufig mit aktuell (2005) 46,4\%. Vgl. hierzu ausführlich Sachverständigenrat (2005), S. 241.

$129 \mathrm{Zu}$ den finanzstatistischen Daten des öffentlichen Sektors vgl. Sachverständigenrat (2005).

$130 \mathrm{Vgl}$. Statistisches Bundesamt (2006).

131 Vgl. bspw. Eichhorn (1997), S. 23.
} 


\subsubsection{Bürokratische Steuerung und Kameralistik}

Seit dem späten 19. Jahrhundert sind Bürokratie als administratives Steuerungsmodell und Kameralistik als Instrument der Finanzmittelallokation die dominierenden Systeme im deutschen Staatswesen. ${ }^{132}$ Zwar befindet sich die öffentliche Verwaltung in Deutschland durch die im Zuge des New Public Management eingeleiteten Reformbemühungen seit Beginn der 90er Jahre im Umbruch ${ }^{133}$, doch trotz dieser Anstrengungen ist in Deutschland noch immer die bürokratische Steuerung als der vorrangige Koordinationsmechanismus in der öffentlichen Verwaltung vorherrschend. ${ }^{134} \mathrm{Er}$ wird ergänzt um die Kameralistik als Planungs- und Rechnungswesen, welches der Verwaltung im Haushalt finanzielle Verfügungsrechte auf Grundlage politischer Entscheidungen zuweist. ${ }^{135}$

Die Beschreibung der kennzeichnenden Merkmale von Bürokratie geht auf MAX WEBER und sein Modell des „bürokratischen Verwaltungsstabes“ zurück. ${ }^{136}$ Wesentliche Merkmale des Bürokratiemodells sind demnach: ${ }^{137}$

- festgelegte Hierarchien und Kompetenzen mit Instanzenzug (einschl. Befehl und Gehorsam)

- eine auf Spezialisierung beruhende Arbeitsteilung mit stark zentralisierter Entscheidungskompetenz

- Einhaltung fester Kommunikationswege (Dienstwege) sowie Schriftlichkeit und Aktenkundigkeit des Handelns

- formalisiertes Handeln durch detaillierte Vorschriften und Weisungen

- lebenslängliche Anstellung und Aufstieg (Laufbahnprinzip) mit hierarchisierten Lohnstrukturen

Im heutigen Sprachgebrauch ist Bürokratie weitestgehend negativ belegt. In diesem Zusammenhang darf aber nicht vergessen werden, dass bürokratische Strukturen seit mehr als hundert Jahren maßgeblich zur Etablierung unserer liberalen und demokratischen Verfassungsordnung beigetragen haben. ${ }^{138}$ Patriarchalische Herrschaft und subjektive Willkür sollten abgelöst und durch die korrekte, personenunabhängige, nach-

132 Vgl. Reichard (1995), S. 58 oder auch Kiesel (2005), S. 9.

13.3 Siehe hierzu die Ausführungen des folgenden Kapitels 2.2.2.

134 Vgl. u.a. Budäus (2005), S. 83; Richter (2000), S. 24 und Reichard (1995), S. 59.

135 Vgl. Bräunig (2004), S. 311 f. Dabei zeigt die hohe Gliederungstiefe der Haushaltsansätze den Wunsch nach einer besonders engen Bindung der Verwaltung an den politischen Willen. Vgl. Kiesel (2005), S. 21.

136 Vgl. Weber (1976).

137 Vgl. u.a. Weber (1976); Eichhom (1991), S. 153; Reichard (1995), S. 57; Mayntz (1997), S. 110; Budäus (1998), S. 1 f.; Richter (2000), S. 24 und Schedler/Proeller (2003), S. 16.

$138 \mathrm{Vgl}$. Schedler/Proeller (2003), S. 15 f. Bürokratische Steuerung stellte zum Zeitpunkt ihrer Konzeption eine erhebliche Verbesserung gegenüber der ursprünglichen Feudalherrschaft dar, die vor allem die einzelwirtschaftlichen Interessen der Feudalherren bediente. Vgl. Kiesel (2005), S. 17. 
vollziehbare und sachbezogene Umsetzung vorgegebener Regeln ersetzt werden. ${ }^{139}$ Entsprechend zeichnet sich das klassische Bürokratiemodell durch ein hohes Maß an Berechenbarkeit, Kontinuität und Stabilität aus, weshalb die deutsche Verwaltung im internationalen Vergleich eine hohe Verfahrens- und Rechtssicherheit aufweist. ${ }^{140}$

Rechtmäßigkeit und Kontrollierbarkeit staatlichen Handelns, wie sie im Rahmen bürokratischer Strukturen zu erwarten sind, stellen nach wie vor unverzichtbare Bedingungen des demokratischen Verfassungsstaates dar. ${ }^{141}$ Doch die Rahmenbedingungen und das gesellschaftspolitische Umfeld, auf die seinerzeit die Verwaltungsstrukturen im Sinne des Bürokratiemodells zugeschnitten wurden, sind mit den heutigen Herausforderungen nicht mehr zu vergleichen. ${ }^{142}$ Die Anforderungen dynamischer Umwelten und Marktentwicklungen und die rapide abnehmende Finanzierbarkeit von bisher öffentlich wahrgenommenen Aufgaben haben den Handlungsdruck massiv erhöht. ${ }^{143}$ Hierauf gründet der in den letzten Jahren eingeleitete Paradigmenwechsel von bürokratischer Verwaltungssteuerung zum New Public Management - mit einer zusätzlichen Betonung der Wirtschaftlichkeit und der Wirkungsorientierung staatlichen Handelns. ${ }^{144}$

\subsubsection{Modernisierungsleitlinien des New Public Management}

\subsubsection{Veränderungstreiber staatlichen Handelns}

Um die Leitideen des New Public Management zu verstehen, ist es wichtig, sich zunächst mit den Herausforderungen zu beschäftigen, denen sich der öffentliche Sektor national wie international - zu stellen hat. Die Veränderungstreiber staatlichen Handelns wurden von BUDÄUS systematisch analysiert und werden hier als Umweltfaktoren des derzeitigen Modernisierungsprozesses aufgefasst. ${ }^{145}$ Diese Veränderungstreiber lassen sich folgendermaßen charakterisieren:

- Die digitale Revolution wird durch neuere Entwicklung der Informations- und Kommunikationstechnik auch weiterhin Arbeitsformen, Arbeitsteilung, Märkte und Gesellschaft revolutionieren. Die neuen Wege der Informationsverarbeitung erleichtern Verfügbarkeit und Delegation von Wissen und ersetzen bürokratische Merkmale wie Hierarchie, Spezialisierung und Dienstweg zunehmend durch Planungs- und Kontrollkonzepte. ${ }^{146}$ Prägend ist in diesem Zusammenhang auch die Kombination mit anderen wissenschaftlichen Disziplinen, wie z.B. im Bereich der Medizintechnik. Hierdurch ergeben sich Rückwirkungen - etwa hinsichtlich der Lebenserwartung der Bürger - denen sich staatliches Handeln ebenfalls zu stellen hat.

\footnotetext{
139 Vgl. Budäus (1998), S. I.

140 Vgl. Heun (1989), S. 106 und Kiesel (2005), S. 19.

141 Vgl. Frey (1994), S. 23 und Reichard (1995), S. 59.

142 Vgl. Reichard (1995), S. 59.

143 Vgl. Budäus (1998), S. 2.

144 Vgl. Reinermann (1993), S. 2; Reinermann (2000), S. 8 und Schedler/Proeller (2003), S. 18.

145 Vgl. hierzu und im Folgenden Budäus (2005), S. 76 ff. und die dort angegebene Literatur.

146 Vgl. Laux (1993), S. 342 f. und Schedler/Proeller (2003), S. 26 f.
} 
- Ein weiterer Änderungstreiber ist die Globalisierung, d.h. die zunehmende Internationalisierung und Liberalisierung nationaler Märkte zu einem einheitlichen Weltmarkt. ${ }^{147}$ Konsequenz der Globalisierung ist ein sich verschärfender Wettbewerb auf allen Ebenen, auf Gütermärkten, auf Kapitalmärkten, auf Arbeitsmärkten und letztlich auch auf der Ebene von Standorten und Staaten. Staaten stehen als Anbieter von Standorten mittlerweile im weltweiten Wettbewerb um Unternehmensansiedlungen, Kapitalinvestitionen und Wissen. Staatliches Handeln und der hiermit verbundene Einfluss auf Standortfaktoren ${ }^{148}$ sind ein entscheidendes Wettbewerbselement, gehen aber einher mit einem generellen Autonomieverlust nationaler Wirtschaftspolitik.

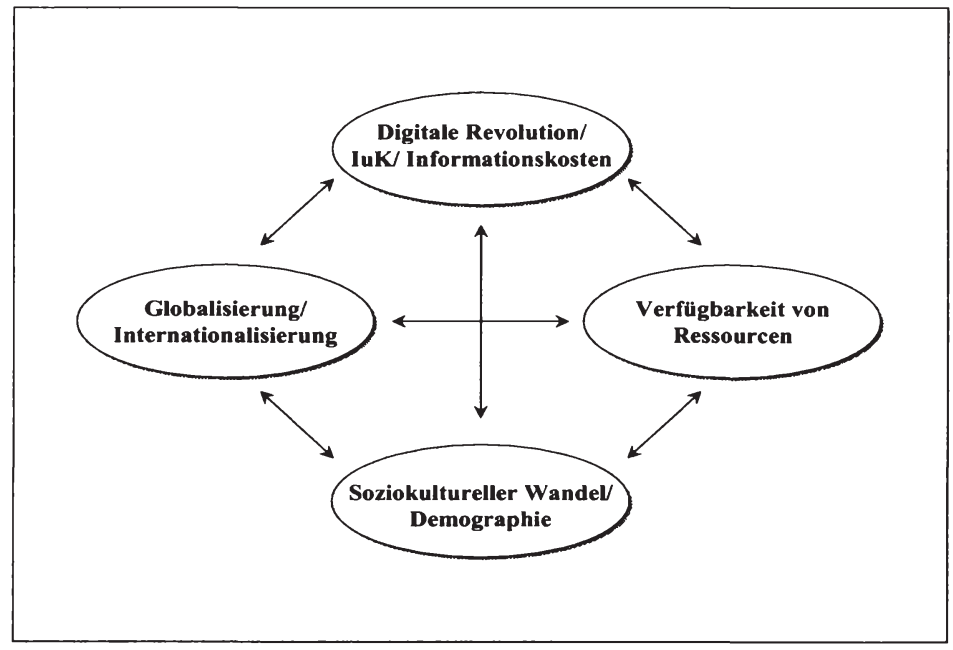

Abbildung 5: Veränderungstreiber staatlichen Handelns ${ }^{149}$

- Der dritte Einflussbereich betrifft den soziokulturellen Wandel mit einer wachsenden Individualisierung der Gesellschaft und einer Vielzahl kleiner partikularisierter Interessenzirkel. ${ }^{150}$ Die Entwicklung ist verbunden mit einem gewandelten Staatsverständnis und einer Zersplitterung des Rechts, so dass Anspruchsgruppen zunehmend Einzelfallgerechtigkeit suchen. ${ }^{151}$ Staatliches Handeln sieht sich daher mit

147 Zum Begriff der Globalisierung und der hiermit verbundenen Konsequenzen für den privaten und öffentlichen Sektor vgl. ausführlich Baßeler/Heinrich/Utecht (2006), S. $558 \mathrm{ff}$.

148 Wesentliche Standortfaktoren sind die Bedingungen der Beschaffung von Produktionsfaktoren (Qualifikation und Kosten des Faktors Arbeit, Kapitalkosten, Energiekosten etc.), Bedingungen des Absatzes (Marktgröße, Kaufkraftniveau etc.) und die Rahmenbedingungen nationaler Wirtschaftpolitik (Steuern und Subventionen, Infrastruktur, Rechtssicherheit etc.). Vgl. Baßeler/Heinrich/Utecht (2006), S. 562 f.

149

Budäus (2005), S. 77.

150

Vgl. Budäus (1995), S. 13 f.

$151 \mathrm{Vgl}$. Schedler/Proeller (2003), S. 26 und Kiesel (2005), S. 25. 
Problemen wie einer Entsolidarisierung der Gesellschaft oder der egoistischen Inanspruchnahme des Gemeinwesens konfrontiert. Zusätzlich führt die demographische Entwicklung in den nächsten Jahrzehnten zu einer deutlichen Verschiebung der Altersstruktur. Dies erfordert Veränderungen an den gesellschaftlichen Strukturen des Zusammenlebens und geht einher mit einer sinkenden Anzahl erwerbstätiger Bürger bei einer nahezu konstanten Bevölkerung.

- Hierdurch wird sich der vierte Veränderungstreiber, die Verfügbarkeit von Ressourcen in der Gesellschaft, zukünftig noch verschärfen. Dies betrifft zum einen finanzielle Ressourcen. Bereits heute beträgt die Verschuldung der öffentlichen Haushalte in Deutschland ungefähr 1,5 Billionen $€$ und das jährliche strukturelle Defizit aktuell knapp $80 \mathrm{Mrd}$. $€{ }^{152}$ Staatliches Handeln steht damit langfristig unter erheblichen finanziellen Restriktionen. Zum anderen wird die demographische Entwicklung auch zum Rückgang immaterieller Ressourcen, wie dem zur Verfügung stehenden Humankapital, führen.

Das bürokratische Verwaltungsmodell, das lange Zeit den staatlichen und gesellschaftlichen Erfordernissen entsprach, zeigt in der veränderten Umwelt zunehmend Schwächen und Dysfunktionalitäten. ${ }^{153}$ Es gilt für den Großteil der öffentlichen Verwaltung als zu langsam, zu segmentiert, zu starr und zu teuer. ${ }^{154}$ Nun gab es in Deutschland bereits seit geraumer Zeit vereinzelte Reformaktivitäten, die im Grunde jedoch erfolglos blieben. Erst die durch die deutsche Einheit ausgelöste Finanzkrise zu Beginn der 90er Jahre hat schließlich einen tiefgreifenden Reformprozess im politisch-administrativen System ausgelöst. ${ }^{155}$

\subsubsection{Modernisierungsbestrebung des deutschen Bürokratiemodells im Kontext internationaler Entwicklungen}

Die Bestrebungen zur Modernisierung des deutschen Bürokratiemodells zu Beginn der 90er Jahre sind im Kontext internationaler Entwicklungen zu verstehen. Dort setzten vergleichbare Reformprozesse deutlich früher ein und dienten damit als Vorbild für die Entwicklungen hierzulande (vgl. Abbildung 6). Ausgangspunkt war eine Krise der öffentlichen Sektoren in vielen OECD-Staaten Ende der 70er Jahre. Die öffentlichen Haushalte hatten mit finanziellen Schwierigkeiten zu kämpfen und die Staatsapparate wurden von den Bürgern als ineffizient, bürokratisch und unflexibel angesehen. ${ }^{156}$ Infolge dessen entwickelten sich in diesen Staaten verwaltungspolitische Reformstrategien, die sich stark an den betriebswirtschaftlich geprägten Management-Praktiken privater Unternehmen orientierten. ${ }^{157}$ Die verschiedenen Strategien formten sich urs-

\footnotetext{
152 Vgl. Sachverständigenrat (2005), S. 236 ff.

$153 \mathrm{Vgl}$. Schedler/Proeller (2003), S. 31.

154 Reichard (1995), S. 60.

$155 \mathrm{Vgl}$. Reichard (1995), S. 62 f.

156 Vgl. Aucoin (1995), S. 1; Borins/Grüning (1998), S. 12 und Grüning (2000), S. 11 f.

157 Vgl. Peters/Savoie (1995), S. 419 f.; Budäus/Grüning (1998), S. 6 und Grüning (2000), S. 14.
} 
prünglich zu Beginn der 80er Jahre in Ländern mit angelsächsischer Verwaltungstradition (Großbritannien, USA, Australien, Neuseeland) zu einem gemeinsamen Reformstrang, bevor sich vor allem auch in den skandinavischen Staaten und den Niederlanden - wenn auch in unterschiedlicher Ausprägung - vergleichbare Reformbewegungen herausbildeten. ${ }^{158}$ Die Reformbestrebungen in der deutschen Verwaltung erfolgten zeitlich danach, erst zu Beginn der 90er Jahre und werden häufig unter dem Begriff „Neues Steuerungsmodell“" subsumiert. ${ }^{159}$ Als deutsche Variante der internationalen Reformansätze wurde der Modernisierungsprozess insbesondere von den Kommunen bzw. der kommunalen Gemeinschaftsstelle (KGSt) vorangetrieben, angelehnt an das Modellvorbild der niederländischen Stadt Tilburg. ${ }^{160}$

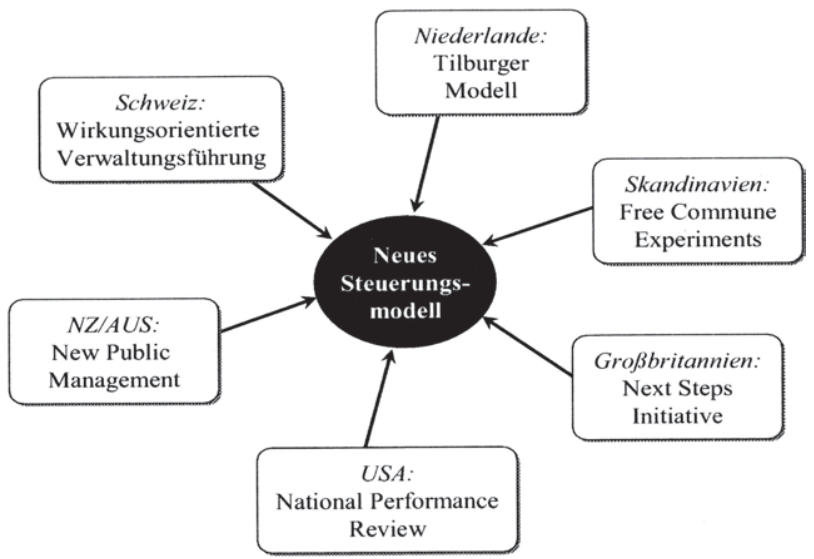

\section{Abbildung 6: Internationale Reformansätze des NPM ${ }^{161}$}

Bemerkenswert spät, erst zu Beginn der 90er Jahre, begann auch eine wissenschaftliche Aufarbeitung der bereits vielfach in der internationalen Verwaltungspraxis implementierten Reformansätze. ${ }^{162}$ Der internationale Modernisierungsprozess öffentlicher Verwaltungen war damit vor allem eine Praktiker-Bewegung, die von Politikern ini-

158 Schröter/Wollmann (2002), S. 63. Ausgangspunkt der Entwicklung waren die tiefgreifenden gesellschaftlichen Reformen durch Margret Thatcher in Großbritannien, die auch eine grundsätzliche Reform der öffentlichen Verwaltung einschlossen. Vgl. hierzu Wollmann (2002), S. 494 ff. Andere angelsächsische Länder wie Kanada, Australien und Neuseeland folgten dem Beispiel Großbritanniens. Parallel zum Reformprozess der sog. „Westminster-Systeme“, die im Wesentlichen von nationalen Regierungen ausging, kam es in den USA zunächst zu verbreiteten Reformen auf kommunaler Ebene. Erst später wurde die Reform der amerikanischen Bundesverwaltung (Reinventing Government) eingeleitet. Vgl. Borins/Grüning (1998), S. 13.

159 Vgl. Jann (2002), S. 74.

$160 \mathrm{Vgl}$. Reichard (1995), S. 69 bzw. KGSt (1991) und KGSt (1993).

161 Eigene Darstellung in Anlehnung an Schedler (1996), S. 11.

162 Vgl. Budäus/Grüning (1998), S. 6 und Grüning (2000), S. 15. 
tiiert und gefördert wurde. ${ }^{163}$ Doch trotz der Vielzahl von Ländern, unterschiedlicher politischer und administrativer Kulturen, unterschiedlichen Zeitpunkten und teilweise abweichenden Problemsituationen, zeigte die wissenschaftliche Analyse und Kategorisierung der einzelnen Umsetzungsstrategien einen außerordentlichen Gleichklang zentraler Merkmale und Reformbestandteile. ${ }^{164}$ Für das Bündel dieser Reformstrategien hat sich in der wissenschaftlichen Diskussion seitdem der Begriff „New Public Management (NPM)“ durchgesetzt. ${ }^{165}$

Dem grundsätzlichen Anspruch nach zielt das NPM-Konzept darauf ab, das öffentliche Verwaltungshandeln unter ein neues Leitbild zu stellen: Im Vordergrund steht die effiziente und effektive Aufgabenwahrnehmung ${ }^{160}$, mit mehr Eigenverantwortung aller Beteiligten und einer Betonung der Steuerungskompetenz des Marktes bzw. wettbewerblicher Strukturen - ohne jedoch die Bedeutung des öffentlichen Sektors für moderne Gesellschaften grundsätzlich in Abrede zu stellen. ${ }^{167}$ Als Praktiker-Ansatz gründet New Public Management auf kein geschlossenes theoretisches Konzept bzw. folgt einem methodisch einheitlichen Modell, sondern ist vielmehr als eine Kombination unterschiedlicher Ansätze und Methoden zu verstehen. ${ }^{168}$ Das Gesamtkonzept NPM lässt sich demnach in verschiedene regionale Reformtypen aufspalten, mit unterschiedlichen inhaltlichen Schwerpunkten und Umsetzungsstrategien. ${ }^{169}$ Die Abgrenzung und Strukturierung des Verständnisses von NPM als Sammelbegriff für die im Detail zu unterscheidenden internationalen Reformtypen kann nach BUDÄUS auf drei Ebenen erfolgen - mit jeweils zentralen Reformmerkmalen. ${ }^{170}$

Die erste Ebene betrifft ein grundsätzlich gewandeltes Rollenverständnis vom produzierenden zum gewährleistenden Staat, der nur noch sog. Kernaufgaben selbst wahrnimmt. ${ }^{171}$ Aufgrund der öffentlichen Finanzkrisen und wachsendem Zweifel an der Problemlösungskompetenz des Staates ist eine generelle Tendenz zum Zurückdrängen

163 Vgl. Budäus/Grüning (1998), S. 6.

164 Vgl. hierzu ausführlich Grüning (2000), S. 16 und die dort angegebene Literatur.

165 Vgl. u.a. Schröter/Wollmann (2002), S. 63 oder Grüning (2000), S. 15. Der Begriff „New Public Management" wurde dabei durch Christopher Hood (1991) aus dem neuseeländischen bzw. australischen Raum in die Verwaltungswissenschaften getragen. Vgl. Schedler (1996), S. 11.

166 Es geht hier um einen „Paradigmenwechsel“ vom bürokratischen Verwaltungshandeln zum NPM - vom normengerechten Vollzug und von Ordnungsmäßigkeit in der Bürokratie zu einer Betonung von Zweck und Mitteln, der Wirtschaftlichkeit und Wirksamkeit des Verwaltungshandelns. Vgl. Reinermann (2000), S. 8.

$167 \mathrm{Vgl}$. Schröter/Wollmann (2002), S. 63 oder auch Naschold (1995), S. 10.

168 Vgl. bspw. Naschold (1995), S. 7 f.; Reichard (1996), S. 248 oder Budäus/Grüning (1998), S. 7.

169 Vgl. Reichard (1994), S. 154; Naschold (1995), S. 8 und Reichard (1995), S. 64.

170 Vgl. hierzu und im Folgenden Budäus (1998), S. 3 sowie Abbildung 7.

171 In der Staatskonzeption des „Gewährleistungsstaates“ wird der Entscheid über die Aufgabenbreite und die ideologische Ausgestaltung des Staates (neo-liberaler vs. Sozial- und Wohlfahrtsstaat) entkoppelt von Fragen der Gestaltung des Service Public und der Aufgabenwahmehmung. Die Aufgabenbreite wird durch politische Instanzen in demokratischen Verfahren festgelegt. Bei der Aufgabenerfüllung trägt der Staat in allen öffentlichen Aufgabenbereichen die Gewährleistungsverantwortung, erbringt aber lediglich sog. Kernaufgaben des Staates selbst. Vgl. Schedler/Proeller (2003), S. 35. Zum Konzept des Gewährleistungsstaates vgl. ausführlich Schedler/Proeller (2003), S. 31 ff. 
des Staates zu verzeichnen. ${ }^{172}$ Bisher staatlich wahrgenommene Aufgaben werden zunehmend hinterfragt und durch Budgetkürzungen, Neuzuschnitt des Leistungsprogramms, Privatisierungen oder neuere Organisationsformen, wie z.B. Private Public Partnership $^{173}$, abgebaut (vgl. Abbildung 7).

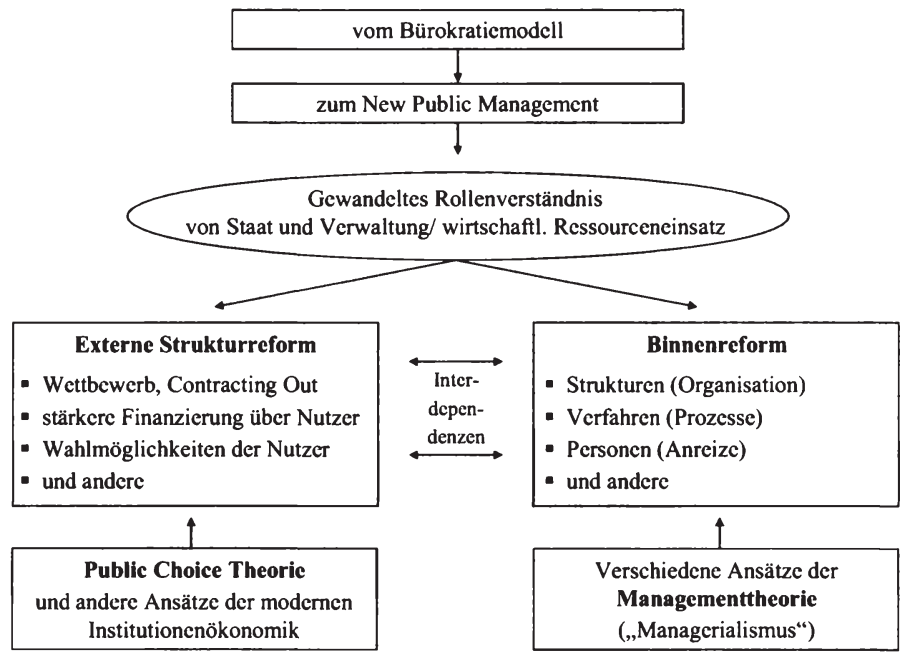

\section{Abbildung 7: Vom Bürokratiemodell zum New Public Management ${ }^{174}$}

Die zweite Ebene bezieht sich auf Reformstrategien, die vor allem die externen Strukturen und Handlungsbedingungen des öffentlichen Sektors als Ganzes betreffen. Diese externe Strukturreform umfasst schwerpunktmäßig Ansätze zur Schaffung von Wettbewerbsbedingungen, eine stärkere Finanzierung öffentlicher Güter und Dienstleistungen durch die Nutzer sowie die Schaffung von Wahlmöglichkeiten der Nutzer. ${ }^{175}$ Hierzu werden Aufgaben, die primär die Bereitstellung eines Gutes gewährleisten, von Aufgaben, die primär die Produktion eines Gutes beinhalten, getrennt. Durch Leistungsverträge („Contracting“) der gewährleistenden Einheiten mit öffentlichen oder privaten Produzenten entsteht bei der Vergabe Wettbewerb zwischen den interessierten Auftragnehmern. ${ }^{176}$ Hiermit ist auch ein Umdenken in Bezug auf die Rolle des Bürgers verbunden, der als Kunde der Verwaltung Leistungen in entsprechender Qualität empfängt und in geeigneten Bereichen hierfür Leistungsentgelte entrichtet. ${ }^{177}$

\footnotetext{
172 Vgl. Budäus/Grüning (1998), S. 7 und Grüning (2000), S. 16-17.

173 Zum Konzept des Private Public Partnership vgl. ausführlich Budäus/Eichhorn (1997).

174 Budäus/Grüning (1998), S. 8.

175 Budäus (1998), S. 4.

176 Vgl. Budäus/Grüning (1998), S. 7.

177 Vgl. Mintzberg (1996), S. 76 f. und Grüning (2000), S. 18.
} 
Die dritte Ebene betrifft die Binnenreform und befasst sich mit der Modernisierung der internen Strukturen, Verfahren und dem personalen Verhalten. Merkmal der Binnenreform ist eine Anpassung und Übertragung betriebswirtschaftlicher Instrumente aus der Privatwirtschaft auf die öffentliche Verwaltung. ${ }^{178}$ So wird das öffentliche Rechnungswesen um eine Kosten-, Leistungs- und Vermögensrechnung erweitert und damit vom Geldverbrauchskonzept zum Ressourcenverbrauchskonzept umgebaut. ${ }^{179}$ Weiterhin wird eine stärkere Dezentralisierung angestrebt, mit einem autonomen Handlungs- und Entscheidungsspielraum einzelner Einheiten. Detaillierte Regelungen und Weisungen werden durch Leistungsvereinbarungen, differenzierte Haushaltsansätze durch Globalbudgets ersetzt. Hierdurch befinden sich Fach- und Ressourcenkompetenz in einer Hand. ${ }^{180}$ Die strukturellen Veränderungen erfordern zusätzlich eine verstärkte Aktivierung der Mitarbeiter durch kooperative Führungsstile und ein flexibles Personalmanagement mit individuellen Beförderungen und Leistungsprämien. ${ }^{181}$

Obwohl NPM eine Praktiker-Bewegung darstellt, besteht in der Literatur weitestgehend Einigkeit über die grundlegenden theoretischen Ansätze. ${ }^{182}$ Die externen Strukturreformen lassen sich demnach vor allem auf die Public Choice-Theorie ${ }^{183}$ und andere Ansätze der modernen Institutionenökonomie ${ }^{184}$ zurückführen. Die Binnenmodernisierung basiert dagegen auf der Übertragung privater Management-Konzepte ${ }^{185}$ und der hiermit verbundenen theoretischen Ansätze. ${ }^{186}$ In diesem Zusammenhang sind zudem die vielfältigen Interdependenzen zwischen externer Strukturreform und Binnenmodernisierung zu beachten. Beispielsweise wird die Schaffung leistungsfähiger Verwal-

178 Vgl. Reichard (1995), S. 64 f.

179 Vgl. hierzu ausführlich Lüder (2001) und Bräunig (2004), S. 309 ff.

180 Vgl. Eichhorn (1999), S. 10 f.

181 Vgl. beispielsweise Müller (1995), S. 16 f. oder Reichard (2001), S. 21 f.

$182 \mathrm{Vgl}$. hierzu die Diskussion bei Borins/Grüning (1998), S. 18 ff. oder Grüning (2000), S. 25 ff.

183 Die Public Choice-Theorie beschäftigt sich mit dem Entscheidungsverhalten in Politik und Verwaltung. In der Analyse stehen rationale, nutzenmaximierende Individuen im Vordergrund, die ihr Verhalten an den eigenen Präferenzen, den vorhanden Anreizstrukturen und dem daraus folgenden Nutzenkalkül ausrichten. Im Ergebnis zeigt sich damit ein pessimistisches Bild des bestehenden politisch-administrativen Systems. Der "politische Markt" begünstigt demnach Partikularinteressen zu Lasten des Gemeinwohls und notwendige Problemlösungen werden durch kurzfristige Machterhaltungsstrategien blockiert. Noch kritischer werden die Wirkungsweisen öffentlicher Bürokratien bewertet, die frei von Konkurrenzdruck agierend Qualitätsaspekte und Kostenbewusstsein vernachlässigen. Auch ist eine wirksame Führung der Verwaltung durch die Mandatsträger nicht möglich, da spezielles „Dienstwissen“ über Kosten und Leistungen fehlt. Auf Grundlage der Public Choice-Theorie wurden daher Reformansätze erarbeitet, um die aufgezeigten Steuerungsdefizite zu beseitigen. Vgl. Schröter/Wollmann (2002), S. 65 f. oder grundlegend Niskanen (1971) und Downs (1967).

184 Ein Großteil der Organisationsmaximen des New Public Management leitet sich aus institutionenökonomischen Überlegungen ab, die als Transaktionskostenanalyse, Prinzipal-Agenten-Theorie und Theorie der Verfügungsrechte diskutiert werden. Vgl. Schröter/Wollmann (2002), S. 66.

185 In diesem Zusammenhang auch häufig als „Managerialismus“ bezeichnet. Grundsätzlich wird öffentliches Handeln auch als ein Produktionsprozess verstanden, so dass sich privatwirtschaftliches Managementwissen situativ auf den öffentlichen Sektor übertragen lässt, so dass die rechtliche Steuerung des Verwaltungshandelns zunehmend durch eine ökonomische Steuerung ergänzt werden kann. Vgl. Pede (1999), S. 29.

186

Vgl. Reichard (1995), S. 64 und Budäus/Grüning (1998), S. 7.

Thorsten Pieper - 978-3-631-75332-3 
tungen durch Wettbewerb einen systematischen Einbezug von Managementwissen erfordern. $^{187}$

Für das politisch-administrative System ist die Steuerungslogik des New Public Management letztendlich mit einer Veränderung des Rollenverständnisses verbunden. ${ }^{188}$ Parlamente und politische Gremien sollen nur noch das „Was“, d.h. die strategischen Ziele, vorgeben und die Verwaltung bestimmt dann das „Wie“ der Ausführung der vorgegebenen Ziele. ${ }^{189}$ Ein idealtypischer Prozess im Sinne des NPM hat demnach folgende Struktur: Das Parlament konkretisiert zunächst die zu erbringenden Leistungen und die zu erreichenden Wirkungen in einem Leistungsauftrag und stellt dafür ein Globalbudget zur Verfügung. Exekutive und Verwaltungsspitze entscheiden anschließend über die operative Umsetzung, d.h. die bestmögliche Form der Aufgabenerledigung. Anhand von Leistungs- und Wirkungsindikatoren prüft das Parlament abschließend, ob die im Leistungsauftrag vereinbarten Ziele auch wirklich erreicht wurden. ${ }^{190}$

\subsubsection{Konzept und Umsetzung der „Neuen Steuerung“ als deutsche Variante des New Public Management}

Das von der KGSt entwickelte „Neue Steuerungsmodell“ stellt den Entwurf eines NPM-Konzeptes für Deutschland dar und steht als Leitbild stellvertretend für die Vielzahl der - im Detail sicherlich zu unterscheidenden - Modernisierungsbestrebungen in deutschen Verwaltungen. ${ }^{191}$ Nachdem zu Beginn/Mitte der 90er Jahre zunächst die Kommunen begonnen haben, Elemente des Neuen Steuerungsmodells (NSM) umzusetzen, zogen schrittweise auch die Länder und der Bund nach. ${ }^{192}$ Dem Leitbild des NPM folgend, ist die Neubewertung der Staatsaufgaben und die Neuorganisation der Aufgabenerledigung durch staatliche und kommunale Institutionen das Hauptziel der deutschen Reformbemühungen. ${ }^{193}$ Entsprechend den Veröffentlichungen der KGSt ${ }^{194}$ werden dabei häufig die folgenden Kernelemente des Neuen Steuerungsmodells unterschieden: ${ }^{195}$

- Erforderlich ist der Aufbau einer dezentralen Führungs- und Organisationsstruktur mit klar abgegrenzten Verantwortungsbereichen, dezentraler Bewirtschaftung der Ressourcen und einer Steuerung über Leistungsergebnisse. Hierzu wird ein Kontraktmanagement zwischen den unterschiedlichen Hierarchieebenen eingerichtet, mit

\footnotetext{
187 Vgl. Budäus (1998), S. 5.

188 Vgl. bspw. Budäus/Grüning (1998), S. 7 oder Schedler/Proeller (2003), S. 54 ff.

$189 \mathrm{Vgl}$. Schedler/Proeller (2003), S. 54.

190 Zum Steuerungsprozess im politisch-administrativen System vgl. auch Bühlmeier et al. (2001), S. $111 \mathrm{ff}$.

191 Vgl. Jann (2002), S. $74 \mathrm{ff}$.

192 Vgl. Bühler (2002), S. 273.

193 Vgl. Naschold/Bogumil (2000).

194 Vgl. grundlegend KGSt (1993) sowie die nachfolgenden Veröffentlichungen zum Neuen Steuerungsmodell. Zur Konzeption eines verallgemeinerten Neuen Steuerungsmodells vgl. Bertelsmann (2005).

195 Vgl. hierzu bspw. Reichard (1995), S. 69 ff.; Jann (2002), S. 77 ff. oder Bogumil/Kuhlmann (2004), S. 52 f.
} 
verbindlichen Absprachen über die zu erbringenden Leistungen und die dafür zur Verfügung stehenden Mittel (Budget). Diese Absprachen sind sowohl zwischen der politischen Führung, der Verwaltung und den Fachbereichen, als auch innerhalb der Verwaltung zwischen Leitung und Mitarbeitern zu treffen. ${ }^{196} \mathrm{Im}$ Rahmen globaler Budgets erhalten die dezentralen Bereiche ausreichend Handlungsspielräume für den zweckentsprechenden Einsatz der Ressourcen (Personal, Sachmittel etc.) zur Erreichung der vereinbarten Ergebnisziele. Notwendige Unterstützung erhält dieser Regelkreis durch einen zentralen Steuerungs- und Controllingbereich, zur Koordination, Analyse und Überprüfung von Leistungsplanung und Leistungsergebnissen.

- Weiterhin ist die Einführung einer Outputsteuerung und der hierzu erforderlichen betriebswirtschaftlichen Instrumente notwendig. Zentrale Bedeutung kommt in diesem Zusammenhang der Definition von Produkten zu. ${ }^{197}$ Die einzelnen Aktivitäten der Verwaltung werden zu einer überschaubaren Zahl von Leistungspaketen - den Produkten - zusammengefasst und sollen die Arbeitsergebnisse der Verwaltung aus Kundensicht beschreiben. Der Produktkatalog dient als Grundlage für den outputorientierten Haushalt, d.h. die Koppelung von Produktbudgets als gewünschtes Leistungsergebnis mit den dafür notwendigen Ressourcen. Die Einführung einer Kostenund Leistungsrechnung ermöglicht den Ausweis produktbezogener Gesamtkosten sowie weiterer Daten, wie Mengen- und Qualitätsangaben. Ein durchgängiges Qualitätsmanagement erfasst systematisch die Bürgererwartung an die Verwaltungsprodukte und stellt so die Kundenorientierung sicher.

- Die neuartigen Organisationsstrukturen und Steuerungsinstrumente müssen letztlich durch Wettbewerb aktiviert werden. Mit Hilfe von Ausschreibungen, Markttests und Outsourcing sollen die dezentralen (Fach-)Einheiten der öffentlichen Verwaltung mit privaten oder gemeinnützigen Anbietern um Aufträge konkurrieren. Bei spezifischen öffentlichen Gütern kann aufgrund monopolistischer Angebotsstrukturen kein echter Marktdruck entstehen, so dass hier durch Betriebs- und Leistungsvergleiche (Benchmarking ${ }^{198}$ ) sog. Wettbewerbssurrogate eingeführt werden sollten.

Der derzeitige Umsetzungsstand der Verwaltungsreformen in Deutschland lässt sich nach JANN nur äußerst schwer bewerten, da bislang nur wenige verlässliche empirische Studien existieren. „Offenkundig ist bislang nur, dass die Veränderungsintensität der Reformen von unten nach oben abnimmt. Auf der Ebene der Kommunen ist am meisten passiert und sind Strukturen am ehesten im Wandel, Länder und Bund sind, nicht zuletzt aufgrund ihrer Aufgabenschwerpunkte und der unterschiedlichen Relevanz von Reformtreibern, stabiler. “ ${ }^{* 199}$

196 Im Ergebnis wird auch im Neuen Steuerungsmodell eine klare Verantwortungsabgrenzung zwischen Politik („Was?") und Verwaltung (,Wie?") angestrebt.

197 Produkte bilden die zentralen Anknüpfungspunkte für alle weiteren Informationen. Mit Produkten werden Mengen, Kosten, Erlöse, Qualitätsindikatoren, Ziele, Wirkungen und Prioritäten verbunden. Ein Produkt ist Bestandteil eines Produktkatalogs, der das Leistungsspektrum eines abgegrenzten Verantwortungsbereiches abbildet und so auch die Verbindung zum Ressourceninput (Budget) darstellt. Vgl. Kiesel (2005), S. 32.

Zum Benchmarking in der öffentlichen Verwaltung vgl. detailliert Burr/Seidlmeier (1998), S. 55 ff.

199 Jann (2004), S. 15. 
Besonders schwierig ist eine Einschätzung, ob mit den eingeleiteten Reformen nach dem Leitbild des NSM das Ziel einer Verbesserung der Steuerungsfähigkeit des politisch-administrativen Systems erreicht werden konnte. Es ist festzustellen, dass die deutsche Reformentwicklung sowohl auf kommunaler Ebene als auch auf staatlicher Ebene nicht alle Reformelemente des NSM systematisch einbezieht. Aber selbst für die kommunale Ebene gilt, „die Realisierung der Kernmodells ist, gemessen an den Zielvorgaben, weit hinter den Reformabsichten zurückgeblieben, so dass zu diesem Zeitpunkt zumindest von einem 'relativen' Scheitern der Bemühungen auszugehen ist" ${ }^{\text {". }}{ }^{200}$ Die oftmals isolierte Einführung und Anwendung von operativen Instrumenten, wie z.B. der Kosten- und Leistungsrechnung, führen häufig zu einer einseitigen Fixierung auf Einsparungen, so dass die eigentlich entscheidenden Kriterien der Qualität und der Wirkung staatlichen Handelns nur nachrangig berücksichtigt werden. ${ }^{201}$ Es fehlt vielfach ein strategisches Management, welches stärker auf die Wirksamkeit und Effektivität von politischen Entscheidungen gerichtet ist. ${ }^{202}$ Der im NSM vollzogene Perspektivenwechsel von der bürokratischen Steuerung des Ressourcen-Inputs zur OutputSteuerung über Produkte kann daher nur ein Zwischenziel darstellen. Um eine effektive Steuerung im politisch-administrativen System zu gewährleisten, können letztlich nur die ausgelösten Wirkungen der Verwaltungsprodukte Gegenstand der Steuerung sein.

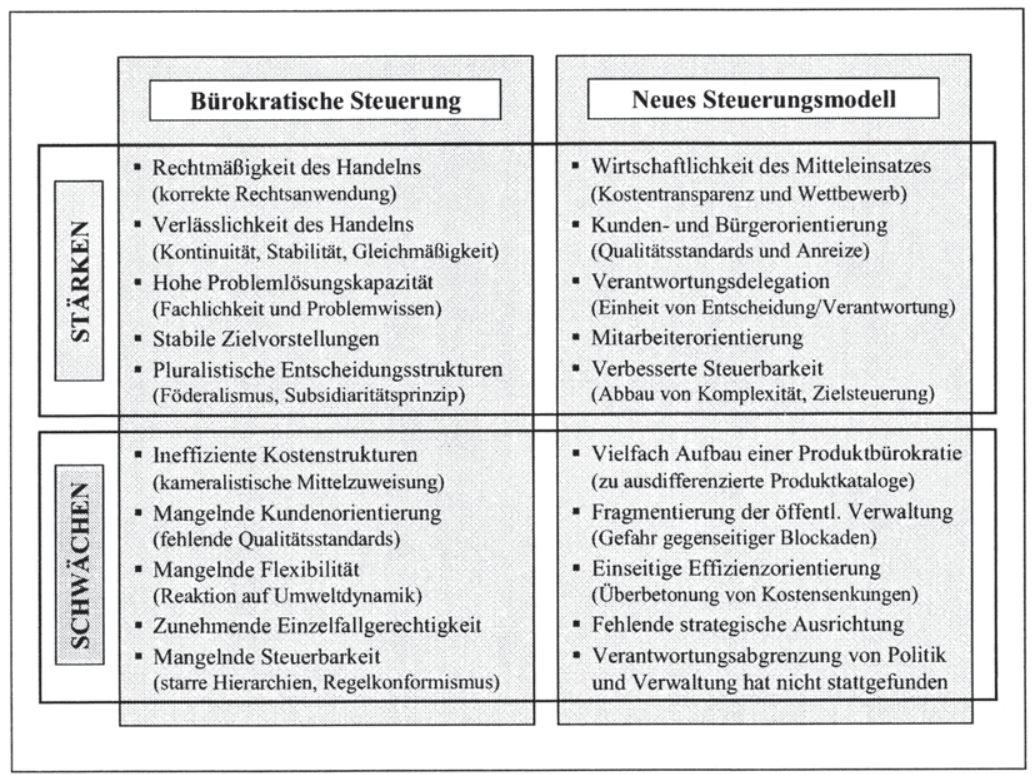

Abbildung 8: Stärken-Schwächen-Profile im Vergleich

200 Bogumil/Kuhlmann (2004), S. 58.

201 Vgl. Speier (2002), S. 169 ff. und Klages (2003), S. 7.

202 Vgl. Bühler (2002), S. 274. 


\subsubsection{Ansatz der wirkungsorientierten Steuerung staatlichen Handelns}

\subsubsection{Pfad zur wirkungsorientierten Steuerung}

Der Steuerungsprozess im politisch-administrativen System erfolgt über die Funktionen Planung, Umsetzung und Kontrolle. ${ }^{203}$ Der Steuerungsprozess bezieht sich dabei auf das Prozessmodell der öffentlichen Leistungserstellung. ${ }^{204}$ Auf Grundlage von politischen Zielen werden Maßnahmen bzw. Produkte geplant und die dafür erforderlichen Mittel bereitgestellt. Durch die administrative Leistungserstellung werden Mittel verbraucht, Produkte erstellt und Wirkungen in der Gesellschaft ausgelöst. Die Kontrolle der tatsächlichen Leistungsergebnisse führt zu Lernprozessen und damit zu einer Rückkopplung zur Planung, wodurch ein geschlossener Managementkreislauf entsteht. (vgl. Abbildung 9).

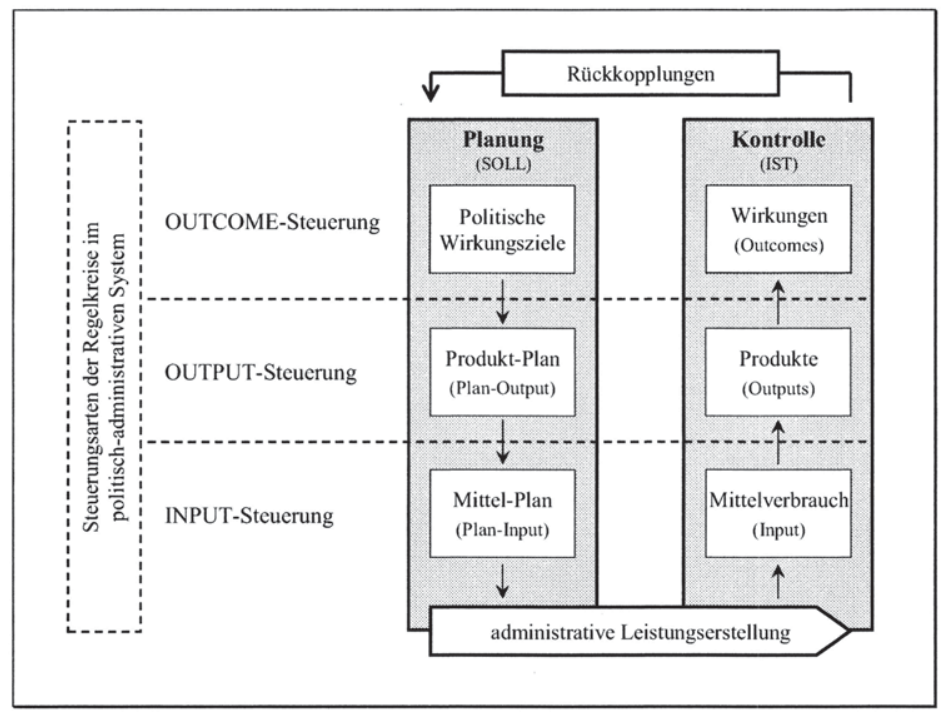

Abbildung 9: Steuerungsarten im politisch-administrativen System ${ }^{205}$

Das Handeln im politisch-administrativen System vollzieht sich über drei ineinander greifende Regelkreise ${ }^{206}$, in denen führende, d.h. steuernde und ausführende, d.h. gesteuerte Einheiten, zu unterscheiden sind. ${ }^{207}$ Der erste Regelkreis betrifft ausschließlich das politische System - mit dem Bürger als Wähler und damit steuernde Einheit und

Vgl. Ulrich/Sidler (1977), S. 50.

gl. hierzu ausführlich Kapitel 2.1.4.

Eigene und deutlich erweiterte Darstellung auf Grundlage von Mäder/Schedler (1994), S. 58.

Der Begriff Regelkreis bezieht sich auf die kybernetische Systemtheorie mit den Funktionsprinzipien Steuerung und Regelung zur Erklärung stabiler dynamischer Systeme. Vgl. grundlegend Teubner (2002), S. 690.

$207 \mathrm{Zu}$ den Regelkreisen im politisch-administrativen System vgl. ausführlich Kiesel (2005), S. 45 ff. 
dem Parlament bzw. der Regierung als ausführende Einheit. Ergebnis dieses Regelkreises ist das Zustandekommen des politisch Gewollten. Der zweite Regelkreis verbindet das politische System mit dem administrativen System. Die Regierung konkretisiert das politisch Gewollte durch verbindliche Ziele und Maßnahmen und beauftragt die Verwaltung mit der Leistungserstellung. Der dritte Regelkreis bezieht sich auf das administrative System und beschreibt das Verhältnis der Verwaltungsführung als steuernde Einheit zu den nachgeordneten Fachdiensten und Ämtern als ausführende Einheit. ${ }^{208}$

Der zuvor beschriebene Managementkreislauf bezieht sich im System der verbundenen Regelkreise insbesondere auf den zweiten und dritten Regelkreis, d.h. die Verbindung von Politik und Verwaltung sowie der Binnensteuerung innerhalb der Verwaltung. ${ }^{209}$ Die Art der Planung, Umsetzung und Kontrolle ist dabei abhängig von der Art der Steuerung, die für ausführende Verwaltungseinheiten vorgesehen ist. Sie wird durch die führenden Einheiten festgelegt und kann sich sowohl auf den Input, den Output als auch den Outcomes des Leistungsprozesses beziehen. ${ }^{210}$

Die inputorientierte Steuerung auf Basis von Finanzmitteln ist die klassisch reglementierte Steuerungsart des bürokratischen Verwaltungsmodells sowie des kameralen Haushaltswesens. ${ }^{211}$ Den ausführenden Einheiten werden die Inputs wie z.B. finanzielle Mittel und Personal detailliert zugeteilt, mit denen sie dann die gesetzlich vorgeschriebenen Aufgaben zu erfüllen haben. Die führende Einheit kontrolliert anschließend den normengerechten Vollzug und die sachgerechte Mittelverwendung. Der Output und die damit verbundenen Wirkungen sind in diesem Steuerungsmodell nicht Gegenstand der Betrachtung, wodurch problematische Anreizstrukturen entstehen. Für die ausführenden Einheiten der Verwaltung ist eine effiziente und effektive Ressourcenbewirtschaftung nicht attraktiv, da realisierte Mitteleinsparungen mit dauerhaften Budgetkürzungen ,bestraft“ werden. ${ }^{212}$ Sowohl Wirtschaftlichkeit als auch Wirksamkeit des Mitteleinsatzes sind in diesem Steuerungsansatz daher als eher gering zu bewerten.

Aufgabe der öffentlichen Verwaltung ist allerdings nicht das Tätigen bestimmter Ausgaben, sondern die Erzielung von Ergebnissen. ${ }^{213}$ Zentrales Element der Reformen im Sinne des NPM ist daher die Verschiebung der Steuerungslogik vom Ressourceninput

208 Sowohl der beschriebene Managementkreislauf als auch das System der in einander greifenden Regelkreise wurde in diesem Zusammenhang als idealtypischer Prozess und stark vereinfacht dargestellt. Die in der Realität verlaufenden Planungs-, Leistungs- und Kontrollprozesse verlaufen weder zeitlich noch inhaltlich in diesen klaren Schritten ab, sondern sind geprägt von gegenseitigen Rückkopplungen und externen Effekten.

Die wissenschaftliche Auseinandersetzung mit dem Beziehungsgeflecht im ersten Regelkreis, innerhalb des politischen Systems, ist Gegenstand der Policy-Forschung und damit nicht im Fokus der Betrachtung dieser Arbeit. Zur Policy-Forschung vgl. grundlegend bspw. Parsons (1995) oder Schubert/Bandelow (2003).

210 Unterschiede liegen insbesondere in der Frage, welche Daten zu Dokumentationszwecken erhoben werden müssen und welche Informationen für die Steuerung notwendig sind. Vgl. Mosiek et al. (2003), S. 28.

211 Vgl. Schedler/Proeller (2003), S. 117 bzw. die Ausführungen des Kapitels 2.1.1.

212 Vgl. Schedler/Proeller (2003), S. 62.

213 Vgl. Kristensen/Grozyk/Bühler (2002), S. 9. 
zu den Leistungsergebnissen. ${ }^{214}$ In der deutschen Reformvariante, dem NSM, sind daher die Produkte der Verwaltung als Leistungsergebnisse Gegenstand des Steuerungsprozesses. ${ }^{215}$ Bei der output-orientierten Steuerung erhalten die ausführenden Einheiten die finanziellen Mittel in Form von Globalbudgets gekoppelt an Leistungsvereinbarungen mit quantitativen und qualitativen Produktvorgaben. Die steuernde Einheit überwacht anschließend die Einhaltung der Vorgaben anhand von Produkt- und Kosteninformationen. ${ }^{216}$ Die Verantwortung für die Art der Mittelverwendung und damit für eine effiziente Produkterstellung liegt nun auf der ausführenden Ebene. Verbunden mit der Kostentransparenz kann die Wirtschaftlichkeit des Ressourceneinsatzes in diesem Steuerungsansatz deutlich verbessert werden. ${ }^{217}$

Die möglichst effiziente Erstellung von öffentlichen Leistungen stellt allerdings keinen Selbstzweck dar, denn die hierdurch ausgelösten Wirkungen sind das eigentliche Ziel der Leistungserstellung. ${ }^{218}$ Grundsätzlich sollte sich staatliches Handeln daher an Wirkungen orientieren, da die Aufgabe erst dann erfüllt ist, wenn die erwünschten Wirkungen in der Gesellschaft eingetreten sind. ${ }^{219}$ Die Analysen zum NSM zeigen allerdings, dass beim output-orientierten Steuerungsansatz - auch in einer idealtypischen Ausprägung - eine einseitige Betonung auf Effizienzgesichtspunkte vorherrscht. ${ }^{220}$ Der Output wird nicht wirkungsbezogen hinterfragt und es besteht die Gefahr, dass man „das Falsche richtig (im Sinne von effizient) macht“. ${ }^{221}$ Ein solcher, eher strategisch geprägter Managementansatz, welcher die Wirksamkeit staatlichen Handelns systematisch einbezieht, fehlt in der deutschen Reformlandschaft bislang noch völlig. ${ }^{222}$

Nach SCHEDLER/PROELLER stellt die Produktorientierung konzeptionell gesehen lediglich eine Art „Zwischenstufe“ auf dem Weg zur Wirkungsorientierung dar. ${ }^{223}$ Beide Steuerungsansätze sind aber nicht als Alternativen anzusehen, sondern als sich ergänzende Betrachtungsperspektiven aufzufassen. ${ }^{24}$ Hiermit wird der in der nachfolgenden Abbildung 10 dargestellte Entwicklungspfad hin zur Outcome-Steuerung beschrieben, welcher sowohl eine hohe Wirtschaftlichkeit als auch eine hohe Wirksamkeit des Ressourceneinsatzes gewährleisten kann.

Möglicher Ansatzpunkt eines wirkungsorientierten Managements ist einerseits die strategische Gesamtplanung staatlicher Aufgabenbereiche sowie andererseits die Binnensteuerung innerhalb der Verwaltung durch die Ergänzung der Leistungsvereinbarungen

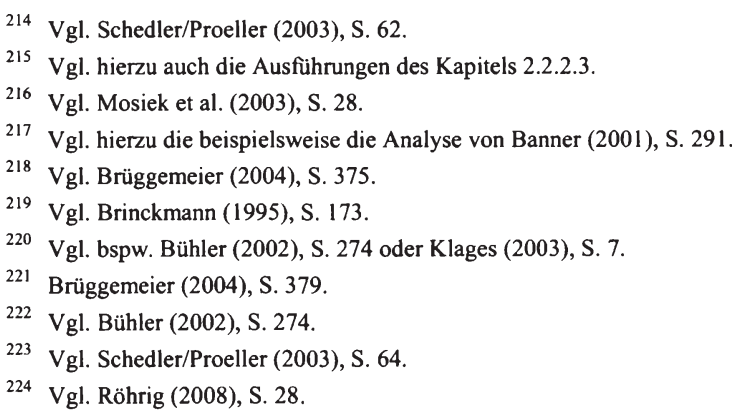


um Wirkungsinformationen. ${ }^{225}$ Ein wirkungsorientiertes Management der Verwaltung durch die Politik über die gesamte Breite der öffentlichen Aufgaben kann im Rahmen einer strategischen Gesamtplanung vollzogen werden. Damit eröffnet sich das Potenzial, die bisher weitgehend nebeneinander stehenden Prozesse von Politikformulierung, Ressourcenallokation und Evaluation politischer Programme in einen systematischen Managementprozess zusammenzuführen, da Wirkungen als Bindeglied zwischen diesen Disziplinen fungieren. ${ }^{226}$

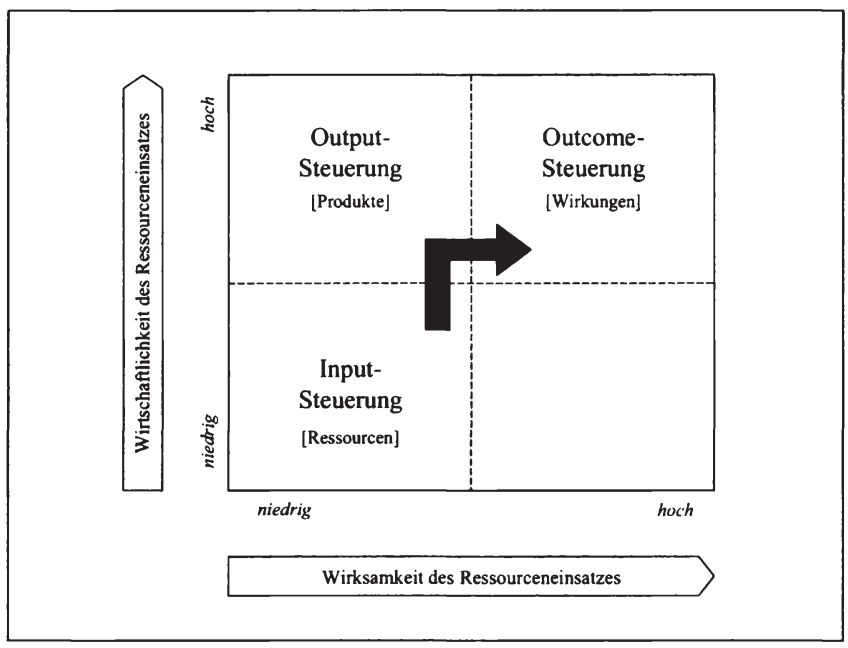

Abbildung 10: Evolutionspfad der Steuerungsarten

Insbesondere für die deutschen Verwaltungsreformen könnte ein derart gestalteter strategischer Managementansatz für mehr Klarheit in Bezug auf die Rollenverteilung zwischen Politik und Verwaltung beitragen. ${ }^{227}$ In letzter Konsequenz bedeutet wirkungsorientierte Steuerung, dass die Politik der Verwaltung nur noch konkrete Wirkungsziele vorgibt. Die Auswahl der geeigneten Maßnahmen und Programme (Produkte) zur Zielerreichung obliegt dann der ausführenden Ebene, d.h. der Verwaltung. ${ }^{228}$ Die Mit-

225 Vgl. Röhrig (2008), S. 28. Die beiden Ansatzpunkte beziehen sich damit auf die zuvor beschriebenen Regelkreise im politisch-administrativen System. Die strategische Gesamtplanung setzt an der Schnittstelle zwischen Politik und Verwaltung an und führt im Idealfall auch zu Rückkopplungen in das politische System, d.h. die Bürger erhalten mehr Transparenz über die Wirksamkeit staatlichen Handelns und der dafür eingesetzten Ressourcen. Analog der Leistungsvereinbarung können Wirkungsinformationen auch systematisch in die eher operative Binnensteuerung innerhalb der Verwaltung einbezogen werden.

226 Vgl. Bühler (2002), S. 276.

227 Vgl. Bühler (2002), S. 278.

228 Erst ein derart gestalteter Managementprozess vollzieht die im Rahmen des NPM geforderte klare Rollenteilung zwischen Politik und Verwaltung: Parlamente und politische Gremien sollen nur noch das „Was“, d.h. die strategischen Ziele, vorgeben und die Verwaltung bestimmt dann das „Wie“ der Ausfürung der vorgegebenen Ziele. Vgl. Schedler/Proeller (2003), S. 54. 
telzuweisungen im Haushalt würden entsprechend direkt an das Erreichen der gewünschten Wirkungsziele gekoppelt. ${ }^{229} \mathrm{Im}$ Vergleich zum Status Quo erhält die Verwaltung im wirkungsorientierten Steuerungsansatz einen deutlich größeren bzw. erweiterten Handlungs- und Gestaltungsspielraum. Die politische Kontrolle der WirkungsZielerreichung sowie der dafür erstellten Produkte muss daher durch ein entsprechend ausgebautes Instrumentarium der Führungsunterstützung ermöglicht werden.

Die zweite Ebene des wirkungsorientierten Steuerungsansatzes betrifft die eher operativ ausgerichtete Binnensteuerung der Verwaltung. Im Rahmen der laufenden Steuerung von öffentlichen Maßnahmen und Programmen kann die systematische Integration von Wirkungsinformationen dazu beitragen, dass der Gestaltungsspielraum der öffentlichen Verwaltung bei der Leistungserstellung nicht nur hinsichtlich der Wirtschaftlichkeit, sondern auch hinsichtlich der Wirksamkeit der Produkte genutzt wird. Die „Dinge richtig tun“ heißt eben nicht nur, sie effizient, sondern auch möglichst effektiv zu tun. ${ }^{230}$ Idealerweise werden dazu Wirkungsvereinbarungen zwischen den führenden und ausführenden Stellen der Verwaltung getroffen, wobei die geschlossenen Leistungskontrakte explizit wirkungsorientiert ausgestattet sind ${ }^{231}$ Dies betrifft insbesondere auch Verträge mit externen Auftragnehmern. Diese sollten eine Umschreibung der Wirkungen und Wirkungsvorgaben, Regeln zur Bezahlung des Auftragnehmers bei Zielerreichung sowie Bestimmungen über Kontrolle, Aufsicht und Sanktionen bei Nichteinhaltung enthalten. ${ }^{232}$ Der Weg zur Zielerreichung bleibt wiederum dem Auftragnehmer überlassen - innerhalb der gesetzlich bzw. vertraglich definierten Grenzen. Der Auftragnehmer trägt das Risiko der Nichterreichung der vereinbarten Wirkungsziele, wodurch der Anreiz besteht, mit möglichst geringem Ressourceneinsatz möglichst wirksame Produkte zu erstellen.

Sieht man von einigen isolierten Ansätzen ${ }^{233} \mathrm{ab}$, gibt es in Deutschland bislang noch wenig praktische Erfahrungswerte mit einem systematischen wirkungsorientierten Steuerungsansatz entsprechend dem zuvor beschriebenen Modell. ${ }^{234}$ In einigen Ländern ist man der deutschen Reformentwicklung wiederum einen Schritt voraus, dort wird die Steuerung staatlichen Handelns zunehmend an den Wirkungen ausgerichtet. ${ }^{235} \mathrm{Im}$ Folgenden werden daher ausgewählte internationale Praxisansätze des wirkungsorien-

\footnotetext{
229 Vgl. Osbome/Gaebler (1992), S. $160 \mathrm{ff}$.

230 Brüggemeier (2004), S. 388.

231 Vgl. Röhrig (2008), S. 28. Mit wirkungsorientierten Steuerungsgrößen (z.B. Vermittlungsquote von arbeitsfähigen Sozialhilfeempfängern als Wirkung spezifischer öffentlicher Maßnahmen) kann oft viel besser an das jeweilige professionelle Selbstverständnis der Mitarbeiter angeknüpft werden, als dies mit rein output- oder input-orientierten Steuerungsgrößen möglich ist. Vgl. Brüggemeier (2004), S. 388.

$232 \mathrm{Vgl}$. Schroeder/Kettiger (2001), S. $18 \mathrm{f}$.

233 BRÜGglimeléR nennt einige isolierte Projekte im Bereich der Sozialverwaltung, Jugendhilfe und Wirtschaftsförderung [vgl. z.B. Kroker-Stille/Kölling (2001); Beck (2002); Türke (2002)] bzw. umfassendere Konzepte auf Basis des Balanced-Scorecard-Ansatzes [Scherer/Alt (2002); Horvath/Kühnle (2002); Mosiek et al. (2003)]. Vgl. hierzu Brüggemeier (2004), S. 375.

234 Zu dieser Aussage vgl. bspw. Bühler (2002), S. 274 oder Brüggemeier (2004), S. 375.

235 Vgl. Kristensen/Groszyk/Bühler (2002), S. 8.
} 
tierten Managements kurz vorgestellt, um hieraus Implikationen für den weiteren Untersuchungsverlauf abzuleiten.

\subsubsection{Internationale Ansätze einer wirkungsorientierten Steuerung}

Da es sich bei wirkungsorientierten Steuerungsansätzen auch in der internationalen Praxis noch um ein recht neues Thema handelt, gibt es bislang nur sehr wenige Veröffentlichungen hierzu, so dass eine Bewertung der Umsetzungserfolge zum jetzigen Zeitpunkt sehr schwierig ist. ${ }^{236}$ Eine ausgewählte Darstellung internationaler Ansätze des ,outcome-focused management" hat beispielsweise die OECD vorgenommen. ${ }^{237}$ Hier zeigt sich, dass insbesondere Länder, die auch Vorreiter der generellen NPMReformen waren, gegen Ende der 90er Jahre begonnen haben, verstärkt wirkungsorientierte Informationen in die bestehenden Steuerungssysteme zu integrieren. Nach einer aktuellen Studie der OECD verwenden immerhin die Hälfte von dreißig befragten Mitgliedsländern nach eigenen Angaben kombinierte Output- und Outcome-Daten im Rahmen des politisch-administrativen Planungs- und Kontrollprozesses. ${ }^{238}$

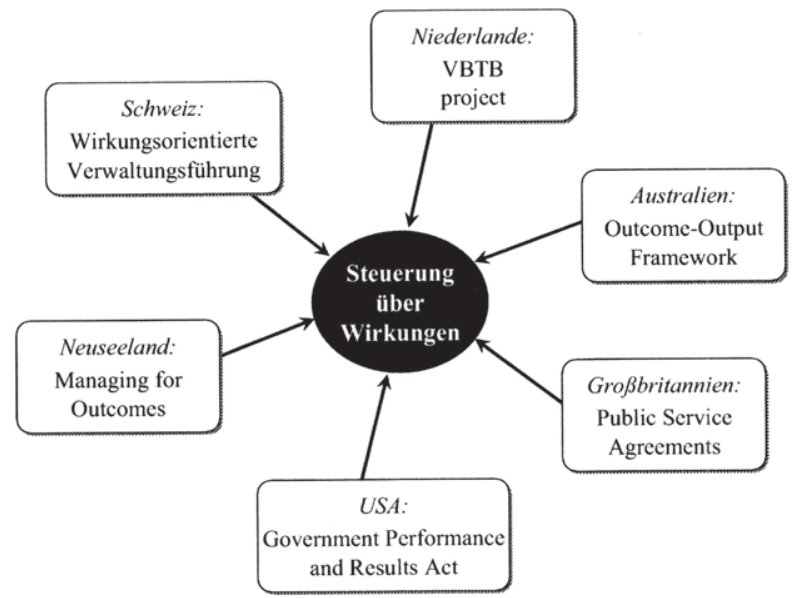

Abbildung 11: Internationale Reformansätze einer wirkungsorientierten Steuerung

Entsprechend Abbildung 11 werden ausgewählte internationale Reformansätze der Steuerung anhand von Wirkungsinformationen kurz vorgestellt:

236 Vgl. hierzu auch Liner et al. (2001), S. 4.

$237 \mathrm{Vgl}$. OECD (2002) und mit einer zusammenfassenden Übersicht ausgewählter Länderberichte Kristensen/Groszyk/Bühler (2002), S. 17 ff.; sowie eine aktuelle Studie der OECD, vgl. Curristine (2005), S. $87 \mathrm{ff}$.

238 Vgl. Curristine (2005), S. 89 f. 
- In der Schweiz sind auf allen administrativen Ebenen umfassende Ansätze zur Reorganisation der Steuerungsabläufe unter dem Stichwort Wirkungsorientierte Verwaltungsführung (WOV) durchgeführt worden. ${ }^{239}$ Im Gegensatz zur deutschen Reformentwicklung war damit neben der Produktorientierung von Beginn an - zumindest konzeptionell - auch eine verstärkte Konzentration auf eine wirkungsorientierte Führung verbunden. ${ }^{240}$ Als beispielhaft und besonders erfolgreich gilt in diesem $\mathrm{Zu}$ sammenhang das Reformprojekt des Kantons Zürich unter dem Titel wif! (wirkungsorientierte Verwaltungsreform). ${ }^{241}$ Zentrales Instrument der strategischen Planung ist hier der sog. Konsolidierte Entwicklungs- und Finanzplan (KEF), welcher die strategischen Ziele und Leistungsgruppen der nächsten vier Jahre benennt. ${ }^{242}$ Als Grundlage der Steuerung der Direktionen erfolgt die Gliederung des KEF institutionell, d.h. aufbauend auf den Ämtern in einer Direktion. ${ }^{243}$ Inhaltlich werden die Leistungsgruppen entsprechend des 5-E-Konzepts ${ }^{244}$ weiter spezifiziert, mit messbaren Zielvorgaben in Form von Indikatoren auf der Wirkungs-, Leistungs- und Wirtschaftlichkeitsebene.

- Die Niederlande haben im Jahr 1999 mit dem VBTB-Projekt ${ }^{24}$ eine Reform des Haushaltswesens eingeleitet, die jedes Ministerium beim Haushaltsentwurf dazu verpflichtet, mit dem Budgetansatz drei zentrale Fragestellungen zu beantworten: Was wollen wir erreichen? Was werden wir tun, um es zu erreichen? Welche Kosten werden dafür entstehen? ${ }^{246}$ Ein entsprechender Budgetentwurf wurde dem Parlament erstmals für das Fiskaljahr 2002 präsentiert. ${ }^{247}$ Jedes Ministerium definiert darin zentrale politische Handlungsfelder mit den jeweiligen zentralen Zielsetzungen, objektiviert durch entsprechende Wirkungsindikatoren. ${ }^{248}$ Für jedes Handlungsfeld werden die geplanten Programme und Produkte und das dafür erforderliche Budget angegeben. Anhand des jährlichen Haushaltsberichtes überprüft das Parlament, ob gesetzte Ziele erreicht, Programme umgesetzt und das Budget eingehalten wurde. ${ }^{249}$

${ }^{239} \mathrm{Vgl}$. Schedler (1996), S. 13.

${ }^{240}$ Vgl. Reichard (2002), S. 25 f.

${ }^{241}$ Vgl. Rieder (2006), S. 24. Als Hauptgrund für den vergleichsweise erfolgreichen Verlauf des Modernisierungsprozesses im Kanton Zürich verweist RILDLR auf einen klaren Promoter, der Einführung und Umsetzung vorangetrieben hat und auch die erforderliche Breitenwirkung erzielen konnte.

${ }^{242}$ Der KEF wird durch die Regierung und die Direktionen jährlich erstellt und aktualisiert. In Form einer sog. rollenden Planung wird jedes Jahr ein neuer Plan für die jeweils nächsten vier Jahre verabschiedet, inhaltliche Änderungen zum Vorjahr werden dabei kenntlich gemacht.

243 Vgl. econcept (2002), S. 12 f.

${ }^{244}$ Vgl. wif! (2002), S. 9 und Buschor (2002), S. 66.

245 VBTB-Projekt: "Van Beleidsbegroting Tot Beleidsverantwoording“ kann übersetzt werden mit „vom politischen Haushalt zur politischen Verantwortung". Vgl. Conings (2006), S. 10.

246 Vgl. Kristensen/Groszyk/Bühler (2002), S. 23 oder Sterk/Bouckaert (2006), S. 7.

247 Vgl. Kristensen/Groszyk/Bühler (2002), S. 23.

248 Im Durchschnitt hat jedes Ministerium zehn politische Handlungsfelder definiert, wofur vom Parlament ein entsprechendes Budget bereitgestellt wird. Vgl. Sterk/Bouckaert (2006), S. 7. Die Anzahl der Haushaltsposten hat sich hierdurch von ca. 800 auf ungefähr 140 reduziert. Vgl. IOFEZ (2004), S. 11.

249 Vgl. Sterk/Bouckaert (2006), S. 7 f. 
- In Australien wurde mit dem Fiskaljahr 1999-2000 erstmals ein sog. OutcomeOutput Budgeting and Reporting Framework eingeführt. ${ }^{250}$ Äußerst konsequent werden dabei die politischen Ziele durch die Regierung in konkrete Wirkungsziele überführt und mit den jährlichen Budgetansätzen verknüpft. ${ }^{251}$ Die Ministerien sind für die Auswahl wirksamer Verwaltungsprodukte verantwortlich, die ihrerseits in Quantität, Qualität und Preis festgelegt werden. Anhand vorab definierter Indikatoren wird anschließend die Effizienz der Output-Erstellung und die Effektivität der Outputs zur Erreichung der Wirkungsziele überprüft. ${ }^{252}$

- Seit 1998 wird die nationale Verwaltung in Großbritannien durch die Einführung der Public Service Agreements (PSA) wirkungsorientiert gesteuert. ${ }^{253}$ PSA werden für jedes Ministerium für eine Zeitperiode von drei Jahren vereinbart, sie enthalten ein Oberziel (aim), das in verschiedene Unterziele (objectives) heruntergebrochen wird. Für letztere werden dann ein oder mehrere Outcome-Performance-Kennzahlen entwickelt und mit Sollwerten (targets) versehen. ${ }^{254}$ Der Zielerreichungsgrad wird laufend überprüft und kann öffentlich nachvollzogen werden. ${ }^{255}$ In sog. Service Delivery Agreements vereinbart jedes Ministerium, wie es seine Ziele erreichen will, einschließlich der dafür notwendigen Produkte und Programme. ${ }^{256}$ Zuletzt hat die strategische Steuerung durch das Joined-Up-Programm ${ }^{257}$ eine weitere Stärkung und Neuausrichtung erfahren. Viele politische Themenbereiche bzw. Problemstellungen sind organisations- und sektorübergreifend adressiert und müssen entsprechend bearbeitet werden. Die bestehende Fragmentierung soll überwunden werden, indem auf Regierungsebene „Cross Cutting Issues“ zentral bestimmt und dort koordiniert sowie problem-/themenzentriert gesteuert werden. ${ }^{258}$

- Strategische Steuerung ist in den USA eng mit der Gesetzgebung des Government Performance und Results Act (GPRA) verbunden, der noch unter der ClintonRegierung eingeführt wurde. ${ }^{259}$ Dieses Gesetz verpflichtet alle Fachbereiche und Behörden eine eher langfristig orientierte strategische Planung vorzunehmen sowie

250 Vgl. Chan et al. (2002), S. 35.

251 Vgl. Sterk/Bouckaert (2006), S. 6.

252 Vgl. Chan et al. (2002), S. $36 \mathrm{ff}$.

253 Vgl. Ellis/Mitchell (2002), S. $111 \mathrm{ff}$.

254 Zu Beginn der Reform im Jahr 1998 waren allerdings nur 11\% der targets echte Outcome-Ziele, in der deutlichen Mehrzahl handelte es sich noch um Prozess- und Produktziele. Für den Zeitraum 2001-2004 konnte der Anteil der Outcome-Ziele aber auf 67\% stark erhöht werden. Vgl. Ellis/Mitchell (2002), S. 111.

Das HM Treasury (Finanzministerium) veröffentlicht die laufende Messung der Ziele und des Zielerreichungsgrades im Internet, sowohl die PSA der einzelnen Ministerien als auch sog. PSA Performance Table können dort abgerufen werden. Die Zielkaskade ist dabei bis auf die Mitarbeiterebene heruntergebrochen, so dass zu jedem Unterziel (objective) ein zuständiger Mitarbeiter genannt wird. Vgl. HM Treasury (2006).

256 Vgl. Ellis/Mitchell (2002), S. $116 \mathrm{ff}$.

257 Vgl. hierzu Pollitt (2003), S. 34 ff. oder Proeller (2006), S. 16.

258 Dazu wurden sog. „Units“ eingerichtet, die die Koordination übernehmen. Vgl. Proeller (2006), S. 16.

259 Vgl. Proeller (2006), S. 15. Zuletzt wurde der GPRA ergänzt durch das „Program Assessment Rating Tool (PART)“ des Office of Management and Budget, welches zusätzliche Datenanforderungen, wie auch Wirkungsinformationen, an alle Bundesbehörden stellt. Vgl. Hatry et al. (2003), S. 5. 
zur operativen Steuerung jährliche, auf ein Fiskaljahr bezogene „PerformancePlans“ und „Performance-Reports“ zu verfassen. ${ }^{260}$ Die strategische Steuerung erfolgt in Form einer rollenden 5-Jahres-Planung, die alle drei Jahre aktualisiert wird. Die Planung wird dazu in sog. „Mission-Statements“ verfasst, sieht Leistungs- und Wirkungsziele sowie entsprechende Indikatoren vor und soll Stakeholder systematisch in den Planungsprozess einbeziehen. ${ }^{261}$

- In Neuseeland wurde im Zuge der NPM-Reformen ein rigides System des Kontraktmanagements, verbunden mit einem konsequent output-orientierten Steuerungsansatz, eingeführt. Bereits in den 90er Jahren wurde dieses System um eine strategische Komponente ergänzt. ${ }^{262}$ Vom Kabinett wurden politikfeldbezogene, organisationsübergreifende „Strategic Results Areas“ definiert, die Ministerien spezifizierten in sog. „Key Results Areas“ ihre strategischen Beiträge zu diesen regierungsweiten Zielsetzungen. ${ }^{263}$ Aufgrund der mangelnden Konkretisierung der strategischen Planung diente der Ansatz allerdings eher als themenbezogene Diskussionsbasis zwischen den ausführenden Einheiten und blieb deshalb erfolglos. ${ }^{264}$ Mit dem aktuellen Programm Managing for Outcomes sollen die regierungsweiten Wirkungsziele daher stärker in den bestehenden Planungsprozess integriert werden. ${ }^{265}$ Jedes Ministerium erstellt dazu ein "Statement of Intent" mit einer mehrjährigen strategischen Planung, welche darlegt, wie und womit einzelne Verwaltungseinheiten zu den Wirkungszielen beitragen. ${ }^{266}$ Insbesondere ist aufzuzeigen, wie durch eine Umfeldanalyse die besten Alternativen bzw. Produkte zur Zielerreichung ausgewählt wurden. Die Überprüfung des tatsächlichen Beitrags findet im Rahmen des jährlichen Reportings statt. Dabei geht es nicht um die Zuweisung von Verantwortung, sondern um praktikable Wege längerfristige Wirkungsziele im öffentlichen Management angemessen zu berücksichtigen. ${ }^{267}$

Es wurden beispielhaft sechs internationale Ansätze zur strategischen Steuerung des öffentlichen Sektors auf Basis von Wirkungsinformationen vorgestellt. Im Weiteren soll allerdings keine detaillierte Analyse der jeweiligen Stärken und Schwächen dieser Ansätze erfolgen. Modernisierungsprozesse im öffentlichen Sektor müssen immer vor dem Hintergrund unterschiedlicher Regierungsstrukturen und -systeme (bspw. zentralstaatlich vs. föderal) sowie auch bestehender kultureller Unterschiede gesehen wer-

\footnotetext{
260 Vgl. Groszyk (2002), S. 129.

261 Vgl. Proeller (2006), S. 15.

262 Vgl. Kibblewhite/Ussher (2002), S. 85 ff.

263 Vgl. Proeller (2006), S. 14.

264 Das Programm wurde abgebrochen, da keine strategische Schwerpunktsetzung erreicht und gleichzeitig die Beteilung an Strategic Results Areas bzw. Key Results Areas vielfach nur zur Begründung zusätzlichen Ressourcenbedarfs genutzt wurde; vgl. Proeller (2006), S. 14. Die Ergebnisse nach zehn Jahren Erfahrungen mit dem Output-Outcome-Framework zeigen, dass die Informationen über Outcomes so allgemein gehalten sind, dass sie im Prinzip bedeutungslos sind; vgl. Norman (2006), S. 8 und die dort angegebene Literatur.

265 Vgl. Proeller (2006), S. 14.

266 Vgl. hierzu ausführlich Norman (2006), S. $10 \mathrm{f}$.

267 Vgl. Kibblewhite/Ussher (2002), S. 91; Norman (2006), S. 10 oder Proeller (2006), S. 14.
} 
den. ${ }^{268}$ Weiterhin erschwert die erst kurze Implementierungsdauer verlässliche Evaluationsergebnisse. Man befindet sich auch international vielfach noch in einer Phase des Experimentierens. Vielmehr stellt sich für die weitere Diskussion zunächst die Frage nach bereits erkennbaren Mustern und Zusammenhängen, die sich aus den ersten praktischen Umsetzungserfahrungen ableiten lassen. Hieraus sollen Implikationen für den deutschen Modernisierungsprozess und die mit einer wirkungsorientierten Steuerung verbundenen Chancen und Herausforderungen gewonnen werden.

Grundsätzlich lässt sich feststellen, dass in den betrachteten Ländern - trotz bestehender Schwierigkeiten bzw. Herausforderungen - in der Regel eine positive Gesamtbilanz gezogen wird und das wirkungsorientierte Steuerungsansätze einen Beitrag zur Steigerung der Wirksamkeit staatlichen Handelns leisten können. ${ }^{269}$ Dabei wird die Notwendigkeit, der einseitigen Effizienzorientierung der output-basierten Steuerung durch die systematische Integration von Wirkungsinformationen entgegenzuwirken, durchgehend betont, was damit als Motor der Entwicklung verstanden werden kann. Auch in Bezug auf Mitarbeitermotivation und Ausgestaltung der Anreizstrukturen wird einer strategischen Planung, die nicht nur die Wirtschaftlichkeit, sondern auch die Wirksamkeit des eigenen Handelns in Betracht zieht, erhebliche Bedeutung zugesprochen. Die bestehenden Ansätze werden international konsequent verfolgt und weiterentwickelt. ${ }^{270}$

Wichtige Gemeinsamkeiten des internationalen Modernisierungsprozesses sind insbesondere in der Verknüpfung von Output- und Outcome-Steuerung zu sehen. ${ }^{271}$ Die strategische Ausrichtung an den Wirkungen des Verwaltungshandelns kann als Weiterentwicklung auf funktionsfähige Systeme des Kontraktmanagements, der Zielorientierung und des Performance-Measurement aufbauen. ${ }^{272}$ Der bisher auf Outputs basierende Steuerungsprozess wird quasi um die Wirkungsdimension erweitert. Dazu werden im Rahmen einer strategischen Planung längerfristige Wirkungsziele, meist für die nächsten drei bis fünf Jahre, vereinbart. Die Planung der jährlichen Programme und Produkte erfolgt nun in Verbindung mit den gesetzten Wirkungszielen und wird diesen zugeordnet. Durch ein etabliertes Performance-Measurement findet nach der Leistungserstellung eine Überprüfung der Zielerreichung in Bezug auf Outputs und Outcomes statt, wodurch wiederum Lernprozesse angestoßen werden.

268 Vgl. hierzu ausführlich Wollmann (2002), S. $489 \mathrm{ff}$.

269 Vgl. mit demselben Ergebnis Proeller (2006), S. 19. In einer Kurzanalyse vergleicht Prollleir die Stärken und Schwächen der strategischen Steuerungsansätze in den Ländern Neuseeland, USA, Großbritannien, Irland und Skandinavien/Finnland.

270 Mit dem Joined-Up-Programm in Großbritannien und dem Managing for Outcomes in Neuseeland sind die bereits bestehenden wirkungsorientierten Steuerungsansätze in die zweite Entwicklungsstufe getreten, mit dem Ziel, erkannte Schwächen abzustellen und die strategische Steuerung schrittweise zu verbessern.

271 Hier bestätigt sich die bereits zuvor auf theoretischer Basis getroffene Feststellung, dass ein wirkungsorientierter Steuerungsansatz im Rahmen eines Evaluationspfades zu verwirklichen ist. Output- und OutcomeSteuerung stellen demnach sich ergänzende Betrachtungsperspektiven dar.

272 Vgl. ebenso Proeller (2006), S. 20. 
Die betrachteten Beispiele weisen allerdings auch erhebliche Unterschiede auf. So sind manche Ansätze als eher zentrales, einheitliches Steuerungssystem ausgelegt (bspw. in Großbritannien), wohingegen im amerikanischen Beispiel ein eher dezentraler, wenig zentral koordinierter Steuerungsansatz gewählt wurde. ${ }^{273}$ Die horizontale Koordination sektorübergreifender Themenstellungen über verschiedene Ministerien wurde durch das Joined-Up-Programm in Großbritannien aktuell sogar noch deutlich gestärkt. ${ }^{274}$ Auch kann zwischen „harten“ und „,weichen“ Ansätzen unterschieden werden ${ }^{275}$, wobei Erstere einen hohen Grad der Konkretisierung aufweisen, d.h. Wirkungen werden durch geeignete Indikatoren operationalisiert und es werden verbindliche Zielniveaus und Zeiträume zur Zielerreichung festgelegt. Dementsprechend liegt hier ein hoher Fokus auf Kontrolle und Abweichungsahndung, während „weiche Ansätze“ weniger konkrete Zielsetzungen treffen und der Fokus eher auf Lerneffekte gerichtet ist. Eng damit verbunden ist die Zuweisung von Verantwortung. Während in Großbritannien Wirkungsziele bis auf die Ebene einzelner Mitarbeiter heruntergebrochen werden, trägt das Verwaltungsmanagement in Neuseeland keine direkte Verantwortung für das Erreichen bestimmter Wirkungsziele. ${ }^{276}$

Die internationale Analyse zeigt zudem zwei zentrale Herausforderungen, die im Zusammenhang mit wirkungsorientierten Steuerungsansätzen nach wie vor bestehen. Einerseits ist der Einbezug der politischen Ebene in die strategischen Steuerungsprozesse meist wenig ausgeprägt, d.h. strategische Steuerung ,passiert“ maßgeblich auf der Stufe der Verwaltung und entsprechende Anstrengungen werden dort initiiert und vorangetrieben. ${ }^{277}$ Die Vorgabe von Wirkungszielen durch die Politik, d.h. die Beantwortung der Frage, welche Wirkungen durch die öffentliche Leistungserstellung erzielt werden sollen, bleibt häufig unkonkret bzw. offen. Es besteht eine mangelnde Bereitschaft der Verantwortungsträger, sich dahingehend überprüfen zu lassen. ${ }^{278}$ Andererseits bestehen nach wie vor erhebliche methodische und instrumentelle Defizite beim Wirkungsmanagement. Die Operationalisierung von Wirkungen durch geeignete Indikatoren sowie die verursachungsgerechte Verknüpfung zwischen erstellten Produkten und erzielten Wirkungen sind häufig problematisch. ${ }^{279}$ Daher sind Wirkungsziele vielfach ohne entsprechende Messindikatoren hinterlegt ${ }^{280}$ oder es werden Output- oder Prozessindikatoren herangezogen, da diese leichter messbar sind. ${ }^{281}$

\footnotetext{
273 Vgl. auch Proeller (2006), S. 19.

274 Neuseeland betont im Modernisierungsprozess ebenfalls die Notwendigkeit einer horizontalen Koordination von sektorübergreifenden Themenstellungen. Vgl. bspw. Norman (2006), S. 8 ff.

275 Zur Bezeichnung von „harten“ und „weichen“ Ansätzen in diesem Kontext vgl. Proeller (2006), S. 19.

276 In Neuseeland wird dies mit der Tatsache begründet, dass externe Effekte häufig maßgeblichen Einfluss auf die Entstehung von Wirkungen im gesellschaftlichen Umfeld ausüben. Die Beeinflussbarkeit des Verwaltungsmanagements zur Erreichung bestimmter Wirkungsziele ist demnach nur begrenzt, so dass die Zuweisung von Verantwortung hierfür unterbleibt.

277 Vgl. Proeller (2006), S. 19.

278 Vgl. Naschold et al. (1996), S 54 und Budäus (1999a), S. 63.

279 Vgl. Budäus (1999a), S. 63.

280 Siehe für das Beispiel der Schweiz eine Aufstellung bei Rieder (2006), S. 26.

281 Siehe hierzu beispielhaft die Erfahrungen in Großbritannien oder Neuseeland.
} 
Auch international findet im Rahmen der Haushaltsberatungen bisher keine direkte Kopplung der Finanzmittelallokation an die aus der Politikformulierung konkretisierten Wirkungsziele statt. Vielmehr stehen mit der strategischen Steuerung und der Budgetierung zwei Managementprozesse häufig noch recht unverbunden nebeneinander. Wirkungsinformationen werden zwar teilweise mit in die Haushaltsberatungen eingebracht, doch inwieweit diese von der politischen Ebene wirklich als Entscheidungsgrundlage genutzt werden, ist derzeit noch schwer zu beurteilen. Erste Einschätzungen und Berichte zeigen ein bislang eher gering ausgeprägtes Interesse der politischen Ebene, so dass sich wirkungsorientiertes Management bislang hauptsächlich auf der Verwaltungsebene vollzieht und entwickelt.

\subsubsection{Chancen der Wirkungsorientierung}

Die bisherigen Erkenntnisse aus der theoretischen Analyse wirkungsorientierter Steuerungsprozesse und die Implikationen aus den ersten internationalen Umsetzungserfahrungen sollen im Folgenden auf den deutschen Modernisierungsprozess übertragen werden. Obwohl allgemein anerkannt wird, dass die Erreichung beabsichtigter gesellschaftlicher Wirkungen die Zwecksetzung und damit die Legitimationsbasis von Politik und Verwaltung ist, bleibt die Frage jedoch regelmäßig unbeantwortet, warum Wirkungsziele in der Praxis so selten Eingang in den politisch-administrativen Politikformulierungs- und Politikdurchführungsprozess finden. ${ }^{282}$ Die Argumente, die für die Etablierung eines wirkungsorientierten Steuerungsansatzes sprechen und die hiermit verbundenen Chancen den politisch-administrativen Prozess sowohl effizient als auch effektiv zu steuern, sollen im Folgenden zusammenhängend dargestellt werden.

Zentrales Element der Wirkungsorientierung ist die deutlich gesteigerte Transparenz in politisch-administrativen Abläufen. Fehlt die Transparenz bezüglich der Wirkungen, kann dies nach BRÜGGEMEIER ansonsten zu gefährlichen Zielverschiebungen führen. ${ }^{28 .}$ Im Rahmen der öffentlichen Leistungserstellung wird der Output zwar effizient erstellt, weil die Wirkungen des Outputs aber unberücksichtigt bleiben, erfüllt dieser unter Umständen gar nicht die beabsichtigten Zielvorstellungen der Politik. Es besteht dann die Gefahr, entweder Effizienzgewinne zu Lasten der Effektivität zu realisieren oder Effizienzverluste aufgrund einer Vernachlässigung der Effektivität zu erleiden (vgl. auch Abbildung 12). ${ }^{284}$

Transparenz schafft zudem die Voraussetzung für die Zuweisung von Verantwortung und zwar für Politik und Verwaltung. Die Verwaltung hat beim Vollzug ihrer Aufgaben und bei der Konkretisierung der politischen und gesetzlichen Vorgaben regelmäßig

282 Vgl. Mosiek/Gerhardt (2003), S. 288.

283 Vgl. hierzu ausführlich Brüggemeier (2004), $379 \mathrm{ff}$.

284 BRÜGGLMEIIR nennt als Beispiel den Verzicht der Kommunen auf wirksame Verkehrssicherheitsmaßnahmen um Bußgeldeinnahmen zu erhöhen. Ein weiteres Beispiel ist die einseitige Fokussierung auf eher marginale Einsparungen im Rahmen des Leistungsprozesses in der Sozialverwaltung, statt einer Senkung des viel umfangreicheren Transfervolumens (z.B. Verzicht auf Missbrauchskontrollen aufgrund hoher Kosten). 
einen nicht unerheblichen Gestaltungsspielraum. ${ }^{285}$ Wirkungsorientierung schafft die Voraussetzungen, dass die Verwaltung sich nicht nur der Wirkungen ihrer erstellten Produkte bewusst ist, sondern auch steuernd auf die Wirksamkeit ihres Handelns Einfluss nimmt. Die Verwaltung trägt somit eine Teilverantwortung für das Erreichen der Wirkungsziele. ${ }^{286}$ In diesem Zusammenhang ist auch die im internationalen Kontext häufig betonte Steigerung der Motivation der Mitarbeiter zu verstehen. Mit der Vereinbarung wirkungsorientierter Zielsetzungen kann oftmals viel besser an das jeweilige Selbstverständnis der Mitarbeiter appelliert werden, als dies mit rein output- oder input-orientierten Steuerungsgrößen möglich ist. ${ }^{287}$

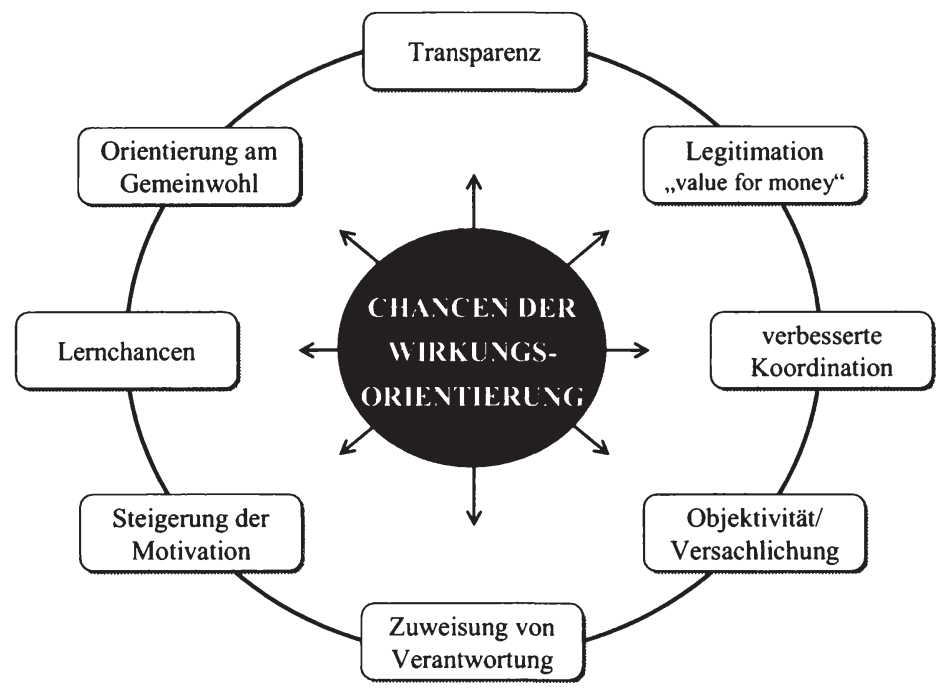

Abbildung 12: Chancen der Wirkungsorientierung

Die Politik trägt die Hauptverantwortung für das Erreichen gesellschaftlicher Wirkungsziele sowie für die Ausrichtung staatlichen Handelns am Oberziel der Gemeinwohlförderung. Durch die gesteigerte Transparenz steigt zum einen die Verantwortung der Politik gegenüber dem Wähler, da die Erreichung der politisch geforderten Ziele gesellschaftlich überprüft werden kann. ${ }^{288}$ Zum anderen erhalten politische Instanzen objektivere Informationen darüber, welche Wirkungen durch ihre Entscheidungen ausgelöst wurden. Im Rahmen von Rückkopplungsprozessen ermöglicht dies eine ständige Verbesserung durch Erkenntnis- und Lernfortschritte aller beteiligten Akteure. ${ }^{289}$

\footnotetext{
285 Vgl. Mosiek et al. (2003), S. 29.

286 Vgl. Hatry (1999), S. 5.

287 Vgl. Brüggemeier (2004), S. 388.

288 Vgl. Röhrig (2008), S. 44.

289 Vgl. Hill (2000), S. 37.
} 
Da Wirkungen häufig politikfeldübergreifend adressiert sind, fördert ein besseres Verständnis der Wirkungszusammenhänge die horizontale Koordination und Kooperation unterschiedlicher Handlungsfelder und Verwaltungseinheiten. ${ }^{290}$ BRÜGGEMEIER spricht von institutionenübergreifenden „Wirkungskoalitionen“, die geschlossen werden, um angestrebte Outcomes besser zu erreichen. ${ }^{291}$ Letztlich, so seine Hoffnung, kann die Wirkungsorientierung sogar zu einer Versachlichung und Entideologisierung politischer Diskussionen führen, welche Mittel oder Maßnahmen sich zur Erreichung gesellschaftlicher Ziele (bspw. Abbau der Arbeitslosigkeit) besonders gut eignen. ${ }^{292}$

Die zunehmenden finanziellen Restriktionen der öffentlichen Hand setzen Politik und Verwaltung zusätzlich unter Handlungsdruck, die Wirkungen ihres Handelns aufzuzeigen und sich zu rechtfertigen. Als Legitimation gegenüber dem Bürger kann die Wirksamkeit öffentlicher Maßnahmen nachgewiesen und so ein "Gegenwert" für gezahlte Steuern und Abgaben (,value for money“) dokumentiert werden. ${ }^{293}$ Besteht darüber hinaus die Notwendigkeit, Leistungen zu kürzen, ist eine Priorisierung der Aufgaben anhand der erzielten Wirkungen möglich. ${ }^{294}$ Unterschiedliche Programme und Produkte sind anhand eines Nutzen-Kosten-Verhältnisses vergleichbar. ${ }^{295}$

\subsubsection{Herausforderungen der Wirkungsorientierung}

Mit den erläuterten Chancen wurde die Notwendigkeit, politisch-administrative Prozesse auch in Deutschland wirkungsorientiert zu steuern, deutlich nachgewiesen. $\mathrm{Zu}$ groß wäre bei einem Verzicht der entgangene Nutzen für unsere Gesellschaft, insbesondere vor dem Hintergrund, dass die Funktionsfähigkeit des öffentlichen Sektors von erheblicher Bedeutung für die Wettbewerbsfähigkeit der gesamte Volkswirtschaft ist. ${ }^{296}$ Ein wirkungsorientiertes Management steht trotz der Chancen vor einer Reihe von Schwierigkeiten und Problemen - welche im Rahmen dieser Arbeit allerdings als Herausforderungen der Wirkungsorientierung aufgefasst werden sollen. Im Rahmen der internationalen Recherche wurden der Einbezug der politischen Ebene und die Überwindung methodischer Defizite als wesentliche Herausforderungen der Wirkungsorientierung identifiziert. ${ }^{297}$ In einem aktuellen Beitrag zum deutschen Modernisierungsprozess bezeichnet BRÜGGEMEIER dies als „Methodenprobleme im Schatten der Konkurrenzdemokratie“ “. ${ }^{298}$ Die hieraus resultierenden Herausforderungen sollen im Folgenden näher untersucht und systematisch aufgearbeitet werden.

\footnotetext{
290 In ähnlicher Weise auch Bühler (2002), S. 276.

291 Vgl. Brüggemeier (2004), S. 381.

292 Vgl. Brüggemeier (2004), S. 381.

293 Vgl. Bühler (2002), S. 276.

294 Vgl. Mosiek et al. (2003), S. 29.

$295 \mathrm{Vgl}$. Kristensen/Groszyk/Bühler (2002), S. 12.

$296 \mathrm{Vgl}$. hierzu die Ausführungen des Kapitels 2.1.1. und 2.1.5.

297 Vgl. Kapitel 2.2.3.2.

298 Vgl. Brüggemeier (2004), S. 382.
} 
Mit der Wirkungsorientierung sind eine Reihe keineswegs trivialer methodischer Probleme verbunden. ${ }^{299}$ Zunächst können Outcomes meist weniger gut beschrieben werden als Outputs. Es ist deshalb schwieriger, aufwendiger und kostspieliger, Wirkungen zu messen. ${ }^{300}$ Dies betrifft insbesondere die Operationalisierung der Ziele und die Messung der Zielerreichung mithilfe geeigneter Indikatoren. Zwischen Indikator und Wirkung muss dazu ein schlüssiges Modell stehen, das die Verbindung und Beschreibung der angestrebten Wirkung durch den Indikator nachvollziehbar macht. ${ }^{301}$ Dies wird in der Realität immer zu mehr oder weniger deutlichen Unschärfen führen, bzw. recht komplexe Indikatorensysteme erfordern. Zu viele Indikatoren könnten allerdings die Aufnahmefähigkeit von Politikern und Verwaltungsmanagern überfordern, so dass die Akzeptanz eines derartigen Steuerungssystems nicht gegeben ist. ${ }^{302}$

Methodisch problematisch ist auch die Fundierung der Ursache-Wirkungsbeziehung zwischen den eingesetzten Mitteln, den erstellten Outputs und den erzielten Outcomes. ${ }^{303}$ Hier besteht meist implizit eine hypothetische Kausalbeziehung, die zunächst verifiziert werden muss. Es ist aber vielfach unmöglich oder mit einem außerordentlich hohen Forschungsaufwand verbunden, eine bestimmte Wirkung zu messen und die festgestellte Veränderung dann noch auf eine bestimmte Ursache (d.h. ein staatliches Programm oder Produkt) zurückzuführen. ${ }^{304}$ Meist werden Outcomes durch eine Vielzahl externer Faktoren beeinflusst, den konkreten Beitrag eines staatlichen Produktes $\mathrm{zu}$ isolieren ist oftmals schwierig. Hinzu kommt das Problem, dass zwischen dem Verwaltungs-Output und seinen ausgelösten Wirkungen nicht selten ein time lag von mehreren Jahren besteht. ${ }^{305}$ Dies erschwert zusätzlich die zeitnahe Kontrolle des Zielerreichungsgrades und damit die Steuerung anhand von Wirkungszielen.

Den einzelnen Akteuren im politisch-administrativen System Verantwortung für das Erreichen von Wirkungszielen zuzuweisen, ist eine der zentralen Chancen der Wirkungsorientierung - zugleich aber auch eine wichtige Herausforderung. Zum einen wird angeführt, dass die Kontrollierbarkeit von Outcomes für Verwaltungen geringer ist als die von Outputs, da externe Faktoren die Wirkungszielerreichung beeinflussen. ${ }^{306}$ Zum anderen bleibt aufgrund der beschriebenen methodischen Probleme die Messung der Zielerreichung oft unscharf. Ohne objektive und verlässliche Daten Ergebnisverantwortung zuzuweisen und hieran noch entsprechende Sanktions- oder Belohnungssysteme zu koppeln, ist allerdings äußerst problematisch. Weiterhin erschwe-

\footnotetext{
299 Vgl. hierzu und im Folgenden u.a. Brüggemeier (2004), S. 382 ff.

300 Vgl. Bühler (2002), S. 277.

301 Vgl. Schenker-Wicki (1996), S. 166 ff.

302 Vgl. Bühler (2002), S. 277.

303 Vgl. Schedler/Proeller (2003), S. 63 ff.

304 Vgl. Schedler/Proeller (2003), S. 64.

305 Brüggemeier (2004), S. 382.
}

$306 \mathrm{Vgl}$. Irwin (1996), S. 23. Daher werden in Neuseeland Verwaltungsmanager weiterhin für das Erreichen von Output-Zielen verantwortlich gemacht. Outcomes dienen eher als strategische Steuerungsgrößen, die den Outputs vorgeschaltet sind und damit eine generelle Richtung vorgeben, ohne konkrete Zielerreichungsgrade zu messen und zu verantworten. Vgl. Norman (2006), S. 8 ff. 
ren die bereits beschriebenen Wirkungskoalitionen aus mehreren Akteuren und Verwaltungseinheiten die Zuweisung von Verantwortung. Lassen sich die Beiträge der einzelnen Akteure nicht verlässlich isolieren, besteht hier immer die Gefahr einer kollektiven Unverantwortlichkeit (vgl. zusammenfassend Abbildung 13).

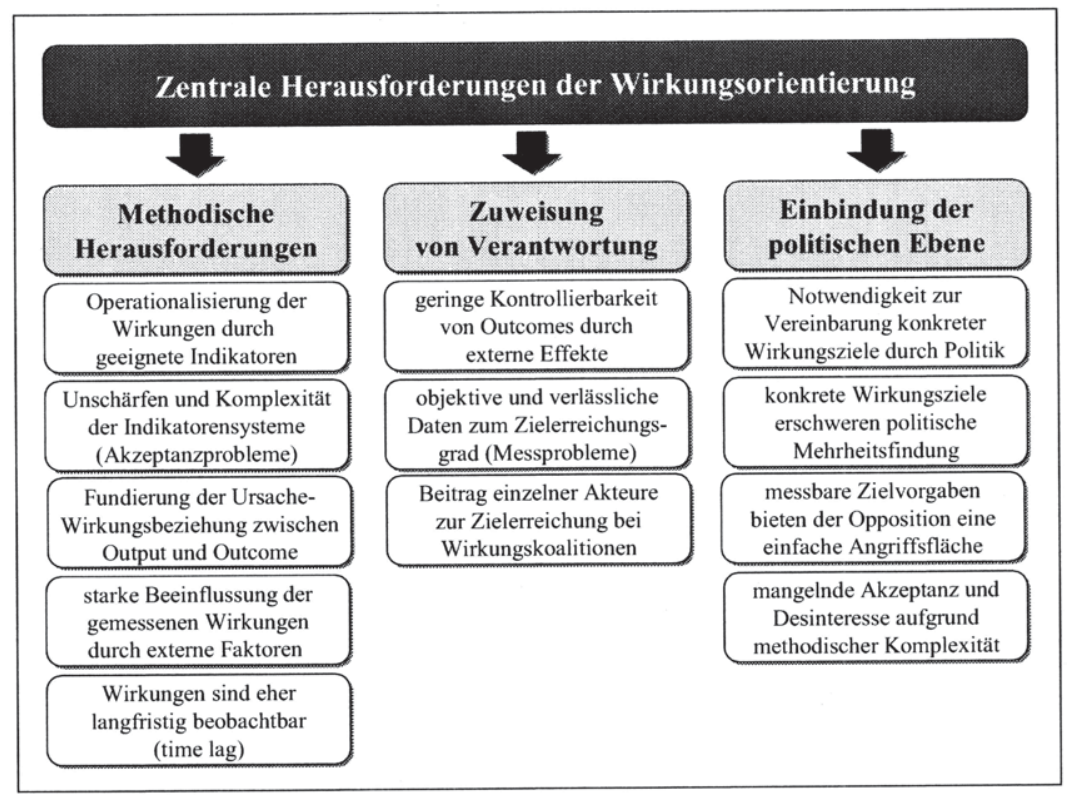

Abbildung 13: Herausforderungen der Wirkungsorientierung

Grundvoraussetzung für die Funktionsfähigkeit der wirkungsorientierten Verwaltungsführung ist ein deutlich abgestimmtes Zielsystem zwischen Politik und Verwaltung sowie eine darauf basierende trennscharfe Aufgabenteilung. ${ }^{307}$ Politiker geraten damit allerdings in eine Dilemmasituation, die man als Zielfalle bezeichnen könnte: Einerseits müssen Politiker gegenüber der Verwaltung klare Wirkungsziele formulieren, andererseits erschwert der Zwang zur Formulierung und Verständigung operationaler Ziele die politische Mehrheitsfindung (Zwang zur Interessenbündelung). ${ }^{308}$ Mit klaren und messbaren Zielvorgaben wird der politischen Opposition zudem eine einfache Angriffsfläche geboten, sollten gesetzte Ziele verfehlt werden. Aus Sicht einer politischen Rationalität erscheint es daher fraglich, ob die mit der Wirkungsorientierung verbundene Transparenz und Verantwortungszuweisung für die eigene Position wirk-

307 Vgl. Röhrig (2008), S. 30. MOSIEK/GERHARDT fordem, dass eine klare strategische Ausrichtung erkennbar sein muss. Vgl. Mosiek/Gerhardt (2003), S. 289.

308 Vgl. Brüggemeier (2004), S. 383. 
lich vorteilhaft ist. ${ }^{309}$ Unter den Bedingungen der Konkurrenzdemokratie werden die eher langfristigen Wirkungsziele stets durch kurzfristige politische Wettbewerbsziele überlagert. ${ }^{310}$ Hierin scheint die auch international zu beobachtende Zurückhaltung bis hin zum Desinteresse der politischen Ebene begründet zu liegen, weshalb die Wirkungsorientierung bisher maßgeblich auf der Ebene der Verwaltung initiiert und vorangetrieben wird. $^{311}$

Die Feststellung, dass die Chancen der Wirkungsorientierung zu groß sind, als das man hierauf verzichten könnte, macht es daher notwendig, die aufgeführten Problembereiche als zu lösende und überwindbare Hausforderungen zu begreifen. Es ist folglich eine der wesentlichen Aufgaben der betriebswirtschaftlich geprägten Verwaltungsforschung, sich näher mit wirkungsorientierten Steuerungssystemen auseinanderzusetzen und entsprechende Methoden, Instrumente und Konzepte zu entwickeln, mit denen den genannten Problembereichen begegnet werden kann. Vieles spricht dafür, dass in diesem Zusammenhang die Weiterentwicklung des Verwaltungscontrollings zu einem wirkungsorientierten Controlling ganz entscheidende Bedeutung zukommt. ${ }^{312}$

\subsection{Führungsunterstützung wirkungsorientierter Steuerungsprozesse durch ein wirkungsorientiertes Controlling}

Zur Etablierung eines funktionsfähigen Wirkungsmanagements lassen sich aus den vorangegangenen Überlegungen die nachfolgenden Voraussetzungen ableiten. $\mathrm{Zu}-$ nächst zeigen die internationalen Beispiele, dass die wirkungsorientierte Steuerung des politisch-administrativen Systems auf einer funktionsfähigen Output-Steuerung aufbauen sollte. In geeigneten Bereichen kann dann eine schrittweise Umstellung der handlungsleitenden Steuerungsinformationen von Produkte auf Wirkungen erfolgen. Wesentliche Voraussetzung ist zudem die Existenz eines eindeutigen Zielsystems mit der Formulierung von Wirkungszielen und Zielvereinbarungen als Ausdruck des politischen Willens und damit der Beantwortung der Frage, welche Wirkungen durch die öffentliche Leistungserstellung erzielt werden sollen. ${ }^{313}$ Die Herausforderung besteht vor allem darin, auch auf der politischen Ebene Akzeptanz für transparente Wirkungsziele zu gewinnen und so eine feste Einbindung in den gesamten Steuerungsprozess zu erreichen, obwohl genau dies der politischen Rationalität vielfach zu widersprechen scheint. ${ }^{314}$ Daneben ist auch eine breite Akzeptanz unter den Verwaltungsmitarbeitern

309 „Ob unser System der politischen Verantwortlichkeit mit einer wachsamen Opposition, den Wahl- und Wahlkreisinteressen der Volksvertreter und der Verflechtung von Parteien und Interessengruppen in der Lage ist, ein realistisches Bild des Wirkungsgrades staatlichen Handelns zu ertragen, bleibt die offene Frage“" Brinckmann (1995), S. 199. In ähnlicher Weise auch Schatz (1991), S. 64 f. und Bogumil (2001), S. 124.

$310 \mathrm{Vgl}$. Fiedler/Vernau (2001), S. 35 ff.

311 Vgl. Proeller (2006), S. 19.

312 Vgl. hierzu bspw. Reichard (2002), S. 36; Heinz (2000); Eichhorn/Wiechers (2001); KGSt (2000); KGSt (2001); Mosiek/Gerhardt (2003); Berens et al. (2004); Brüggemeier (2004) und Röhrig (2008).

313 Vgl. bspw. Schröder/Kettiger (2001), S. 21; Mosiek/Gerhard (2003), S. 289 oder Röhrig (2008), S. 30 ff.

314 Siehe hierzu auch die vorangegangen Ausführungen des Kapitels 2.2.3.4. 
erforderlich, damit diese die Wirkungsinformationen in ihrer Entscheidungsfindung berücksichtigen und so die bestehenden Handlungsspielräume nutzen, um möglichst wirksame Produkte zu erstellen. ${ }^{315}$

Eine weitere zentrale Voraussetzung für ein funktionierendes Wirkungsmanagement ist die objektive Erfassung der Zielerreichung. Die methodischen Herausforderungen betreffen einerseits die Identifikation der durch die Verwaltungsprodukte ausgelösten Wirkungen sowie andererseits die Bestimmung des Zielerreichungsgrades durch die Festlegung geeigneter Messindikatoren. ${ }^{316}$ Eng damit verbunden ist die dritte wesentliche Voraussetzung, die Schaffung von Transparenz und Verantwortlichkeit. Hier zeigt sich auch die hohe Interdependenz zwischen den einzelnen Herausforderungen: Es ist erst möglich, den Akteuren in Politik und Verwaltung Verantwortung für das Erreichen von Wirkungszielen zuzuweisen, wenn zum einen objektive Messmethoden hierfür bereitstehen und zum anderen die beteiligten Akteure auch die Bereitschaft zeigen, ihre Handlungen auf Wirkungsziele auszurichten (vgl. Abbildung 14).

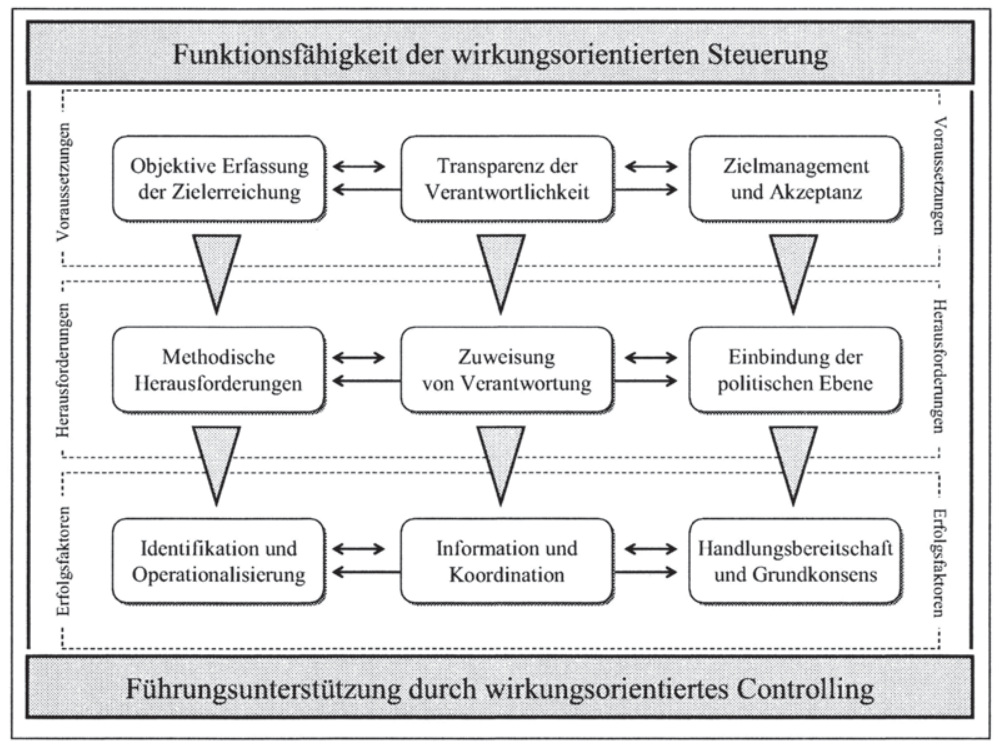

Abbildung 14: Notwendigkeit der Führungsunterstützung

Die Komplexität wirkungsorientierter Steuerungsprozesse sowie die damit verbundenen Voraussetzungen machen deutlich, dass es zur Etablierung der Wirkungsorientierung einer sachgerechten Führungsunterstützung bedarf. Hierzu sind die bestehenden, im Zuge der NPM-Reformen eingerichteten Controllingsysteme zu einem wirkungso-

315 Vgl. Caiden/Caiden (1999), S. 141.

$316 \mathrm{Vgl}$. auch hierzu die detaillierten Ausführungen des Kapitels 2.2.3.4. 
rientierten Controlling weiterzuentwickeln. ${ }^{317}$ Dieses eher strategisch orientierte Verständnis von Verwaltungscontrolling hilft über die bisher eingesetzten binnenorientierten Steuerungsmethoden hinaus, Optimierungspotenziale nicht ausschließlich durch Kostensenkungen zu erschließen, sondern vielmehr das Verhältnis zwischen eingesetzten Ressourcen und dem Grad der Wirkungszielerreichung zu optimieren. ${ }^{318}$

Als Erfolgsfaktoren eines derart gestalteten Controllingsystems lassen sich aus den bisherigen Überlegungen insbesondere drei Implikationen ableiten: Ein wirkungsorientiertes Verwaltungscontrolling muss erstens den Zielfindungsprozess aller beteiligten Akteure unterstützen; es hat zweitens die Aufgabe, die mit dem Verwaltungshandeln verbunden Wirkungen zu identifizieren sowie geeignete Messverfahren zur Dokumentation der Zielerreichung zu entwickeln; und es muss drittens Informationen zum Wirkungsbeitrag einzelner Verwaltungsleistungen bereitstellen sowie koordinierend die institutionenübergreifenden Wirkungskoalitionen unterstützen.

Für den weiteren Forschungsprozess zur wirkungsorientierten Steuerung des politischadministrativen Systems zeichnen sich demnach zwei wesentliche Entwicklungsrichtungen ab. Die erste Entwicklungsrichtung betrifft insbesondere die Möglichkeiten zur Einbindung der politischen Ebene. Unter Berücksichtigung der spezifischen Anreizstrukturen des politischen Regelkreises (politische Rationalität) ist zu untersuchen, wie die Akzeptanz der politischen Akteure für wirkungsorientierte Steuerungsprozesse und ihre Bereitschaft zur Mitwirkung vergrößert werden kann. Die Erarbeitung und Entwicklung entsprechender Lösungswege für dieses Problemfeld ist primär Aufgabe der Policy-Forschung. ${ }^{319}$

Die zweite Entwicklungsrichtung betrifft insbesondere die methodischen Herausforderungen der Wirkungsorientierung sowie die konkrete Ausgestaltung der Führungsunterstützung durch ein wirkungsorientiertes Controlling. Hier müssen aus der eher betriebswirtschaftlich geprägten Perspektive des Verwaltungscontrollings entsprechende Modelle, Methoden und Instrumente entwickelt und bereitgestellt werden, so dass eine sachgerechte Führungsunterstützung sowohl operativer als auch strategischer Steuerungsprozesse im politisch-administrativen System erfolgen kann. Die Weiterentwicklung des bestehenden Verwaltungscontrollings zu einer Gesamtkonzeption des wirkungsorientierten Controllings bedarf daher zukünftig einer außerordentlich hohen Aufmerksamkeit der Controllingforschung im öffentlichen Sektor.

Der weitere Untersuchungsverlauf der vorliegenden Arbeit widmet sich genau dieser Problemstellung und beschäftigt sich mit der Gesamtkonzeption eines wirkungsorientierten Controllings. Dies betrifft einerseits die Fragestellung nach der detaillierten und konkreten Ausgestaltung eines integrierten Bezugsrahmens, der in der Lage ist, den wirkungsorientierten Steuerungsprozess übersichtlich zu strukturieren sowie geeignete Bewertungskriterien zuzuweisen und zu verdeutlichen, wie die Planungs-, Durchfüh-

317 Vgl. Brüggemeier (2004), S. 387.

318 Vgl. Pieper et al. (2006), S. 245.

319 Zur Policy-Forschung vgl. grundlegend bspw. Parsons (1995) oder Schubert/Bandelow (2003). 
rungs- und Kontrollkreisläufe von Politik und Verwaltung ineinander übergehen. Andererseits müssen in einen derartigen Bezugsrahmen geeignete und praktikable Controllinginstrumente integriert werden, die sämtliche Planungs-, Durchführungs- und Kontrollkreisläufe auf allen Bewertungsebenen unterstützen. 
Thorsten Pieper - 978-3-631-75332-3

Downloaded from PubFactory at 01/11/2019 06:04:26AM

via free access 


\section{Wirkungsorientiertes Controlling staatlichen Handelns}

Im Gegensatz zu privatwirtschaftlichen Unternehmen ist die Institutionalisierung eines Controllings in der öffentlichen Verwaltung bislang weit weniger fortgeschritten. „Der Henker mit dem Taschenrechner" lautet die Schlagzeile einer Wochenzeitschrift aus dem Jahr 2004 über eine Controlling-Beratung im öffentlichen Sektor. ${ }^{320}$ Dieses pointierte Zitat steht stellvertretend für eine immer noch weit verbreitete Skepsis in der öffentlichen Verwaltung gegenüber betriebswirtschaftlich orientierten Koordinationsinstrumenten und deren Anwendung im Zuge des Verwaltungscontrollings.

Durch die Reformbemühungen des öffentlichen Sektors im Kontext des New Public Management seit Beginn der 90er Jahre hat das Controlling auch in der öffentlichen Verwaltung stark an Bedeutung gewonnen und in weiten Teilen eine erste praktische Umsetzung erfahren. ${ }^{321}$ Verwaltungscontrolling wird bis heute allerdings sehr einseitig wahrgenommen und häufig nur mit dem Instrument der Kosten- und Leistungsrechnung gleichgesetzt. Die einseitige Effizienzorientierung des NPM-Ansatzes begünstigt sicherlich ein Controllingverständnis im öffentlichen Sektor, welches vielfach mit Kontrolle, insbesondere Kostenkontrolle und Kostensenkung umschrieben wird. Macht aber Controlling, entsprechend einem eher privatwirtschaftlich orientierten Verständnis, auch in der öffentlichen Verwaltung den Anspruch einer umfassenden Führungsunterstützung geltend, müssen zukünftig strategische Aspekte, d.h. insbesondere Aspekte der Wirkungsorientierung, in den Ansätzen des Verwaltungscontrollings deutlich stärkere Beachtung finden.

Zielsetzung dieses Kapitels ist es, die bestehenden Konzeptionen und Ansätze eines wirkungsorientierten Controllings vorzustellen und auf ihre Eignung zur ganzheitlichen Unterstützung wirkungsorientierter Steuerungsprozesse zu unter-suchen. Um dazu ein einheitliches Grundverständnis sicherzustellen, wird der gesamte Entwicklungspfad zum wirkungsorientierten Controlling nachgezeichnet. $\mathrm{Zu}$ Beginn widmen sich die Ausführungen kurz dem allgemeinen, eher privatwirtschaftlich orientierten Controlling-Ansatz (Kapitel 3.1), um darauf aufbauend die Grundlagen des Verwaltungscontrollings zu erläutern (Kapitel 3.2). Anschließend werden unterschiedliche Ansätze zum wirkungsorientierten Controlling eingehend vorgestellt (Kapitel 3.3), um sie abschließend auf mögliche Defizite zu untersuchen (Kapitel 3.4).

\subsection{Grundlagen des Controlling-Ansatzes}

Das Entstehen institutionalisierter Controllingaufgaben im heutigen Sinne geht auf die industrielle Entwicklung in den USA in der zweiten Hälfte des 19. Jahrhunderts zurück, wo erstmals sog. „Comptroller« in Unternehmen mit überwiegend finanzwirtschaftlichen Aufgaben betraut wurden. ${ }^{322}$ Vielfach wird der eigentliche Ursprung des

\footnotetext{
320 Vgl. hierzu Berens/Hoffjan (2004), S. 1.

321 Vgl. Pook/Tebbe (2002), S. 42.

322 Vgl. Jackson (1949), S. 7 f.; Horváth (2001), S. 28 f. und Weber (2004), S. 9 f.
} 
Controllings aber im staatlichen Bereich gesehen, bereits im 15. Jahrhundert beschäftigte das englische Könighaus sog. "Countroller«, die Aufzeichnungen über ein- und ausgehende Gelder und Güter machten. ${ }^{323}$ In Deutschland hat das Controlling erst seit der zweiten Hälfte der 50er Jahre zunehmend Beachtung gefunden. ${ }^{324}$ Empirische Studien zeigen, dass das Controlling heute in privatwirtschaftlichen Unternehmen weit verbreitet ist ${ }^{325}$, es aber auch ein sehr heterogenes Feld individueller Ausgestaltungen und Aufgabenwahrnehmungen gibt. ${ }^{326}$

Die Problematik des Controlling-Begriffs ergibt sich aus der Tatsache, dass Controlling eine Entwicklung der Praxis ist und erst zeitversetzt verschiedenste wissenschaftliche Deutungen erfahren hat. ${ }^{327}$ In der Literatur existiert daher keine einheitliche Begriffsdefinition und es bestehen deutliche Erkenntnislücken bei der theoretischen Fundierung des „Praxisphänomens“ Controlling. ${ }^{328}$ WEBER weist darauf hin, dass zunächst zwei grundsätzliche Perspektiven unterschieden werden müssen: Die Begriffe »Controller" und »Controllership « bezeichnen den Stelleninhaber und das von ihm wahrgenommene Aufgabenbündel, der Begriff "Controlling" bezeichnet demgegenüber eine spezielle Führungs- und Managementfunktion, die von verschiedenen Aufgabenträgern wahrgenommen werden kann. ${ }^{329}$ Es lassen sich demnach eine institutionelle und eine funktionale Sichtweise unterscheiden. Während sich die funktionale Perspektive mit den Aufgaben, Tätigkeiten und Handlungen des Controllings beschäftigt, betrachtet die institutionelle Perspektive die Handlungsträger und ihre Motive. ${ }^{330}$

Der funktionale Begriff "Controlling" leitet sich aus dem amerikanischen Verb "to control« ab. ${ }^{331}$ Dies sollte allerdings nicht einseitig mit dem deutschen Wort »kontrollieren « übersetzt werden, sondern auch andere Bedeutungsinhalte wie einschränken, überwachen, beherrschen, regeln oder steuern berücksichtigen. ${ }^{332}$ Empirisch wird Controlling häufig mit dem Bild eines Navigationssystems beschrieben, welches den Steuermann (Führung) unterstützt. ${ }^{333}$ Die induktive Herleitung einer kontextunabhängigen Controllingfunktion aus der Praxis lässt allerdings kein charakteristisches, von anderen

\footnotetext{
323 Vgl. dazu ausführlicher Weber (2004), S. 9. LiNGNAU sieht den Ursprung des Controllings sogar um $2.500 \mathrm{v}$. Chr. in Ägypten, wo staatliche Stellen die Überwachung des Pyramidenbaus übernahmen bzw. 500 v. Chr. in Rom, wo Quästoren ein Aufgabenspektrum Inne hatten, welches dem heutigen Controllingverständnis bereits stark ähnelt. Vgl. Lingnau (1998), S. 278.

324 Vgl. Küpper (2005), S. 1.

325 Nach einer Studie aus dem Jahr 1988 verfügen bereits knapp 75\% der befragten Unternehmen über eine Controlling-Stelle, bei Unternehmen mit mehr als 1.000 Beschäftigten sind es $86 \%$, bei Unternehmen mit mehr als 10.000 Beschäftigten sogar 96\%. Vgl. Küpper/Winckler/Zhang (1990), S. 439.

326 Eine Übersicht empirischer Studien für Deutschland findet sich bei Horváth (2001), S. 57 ff.

327 Vgl. Mosiek (2002), S. 8.

328 Vgl. Rupp (2002), S. 7 und Berens/Bertelsmann (2002), Sp. 281.

329 Vgl. Weber (2004), S. 5 f.

330 Vgl. Scherm/Pietsch (2001), S. 207.

331 Vgl. Steinle/Bruch (2003), S. 6.

332 Vgl. Klatt/Dietrich (1983), S. 131 und Rupp (2002), S. 8.

333 Vgl. hierzu Bramsemann/Köster (1998), S. 13.
} 
betrieblichen Funktionen klar abgrenzbares Aufgabenspektrum, erkennen ${ }^{334}$ und erscheint aufgrund der Vermengung von funktionalen und organisatorischen Aspekten problematisch. ${ }^{335}$

Aus theoretischer Sicht wird Controlling nach vorherrschender Meinung als zukunftsorientierte Führungsunterstützung aufgefasst, welche die Phasen des Planungs-, Kontroll-, Informations- und Koordinationsprozesses integriert und somit weit über ein vergangenheitsorientiertes „Kontrollieren“ hinausgeht. ${ }^{336}$ Aufgabe des Controllings ist es, die Führung bei ihrer zweckorientierten Steuerungs- und Lenkungsaufgabe und den damit verbundenen Entscheidungen zu entlasten. Diese Unterstützung erfolgt als Serviceleistung in Form einer Bereitstellung entscheidungsrelevanter Informationen, die durch zweckorientiert Aggregation und Analyse von Daten gewonnen werden. ${ }^{337}$ Controlling lässt sich demgemäß als Beschaffung, Aufbereitung, Analyse und Kommunikation von Daten zur Vorbereitung zielsetzungsgerechter Entscheidungen bezeichnen. ${ }^{338}$ Entscheidungsfindung und -durchsetzung sind demgegenüber originäre Führungsaufgaben. Dem Controlling obliegt es in diesem Zusammenhang, die Rationalität der Führung sicherzustellen. ${ }^{339}$

Im wissenschaftlichen Diskussionsprozess zum Controlling-Verständnis besteht aber nach wie vor Uneinigkeit über die inhaltliche Struktur eines Bezugsrahmens, der sämtliche funktionalen Aspekte des Controlling-Begriffes systematisch integriert. Daher haben sich in der Literatur unterschiedliche Controlling-Konzeptionen herausgebildet, die nach KÜPPER derzeit drei inhaltlichen Entwicklungsrichtungen zugeordnet werden können. ${ }^{340}$ Diese sollen im Folgenden kurz vorgestellt werden:

- Die gewinn- bzw. ergebniszielorientierte Controlling-Konzeption stellt die Notwendigkeit in den Mittelpunkt, dem übergeordneten formalen Gewinnziel des Unternehmens zuwiderlaufende Bereichsziele zu erkennen und auf das Oberziel abzustimmen. ${ }^{341}$ Das Erfolgsziel stellt die Deduktionsbasis dar, aus der sich die controllingrelevanten Aufgaben ableiten lassen. ${ }^{342}$ Diese traditionelle Konzeption stellt damit die operativ-taktische Gewinnausrichtung in den Mittelpunkt der Überlegungen, die Betrachtung strategischer Erfolgspotenziale wird nicht originär dem Controlling zugerechnet. ${ }^{343} \mathrm{Zu}$ diesem Ansatz ist kritisch anzumerken, dass die Gewinnzielaus-

\footnotetext{
334 Vgl. Küpper (2005), S. 11. Demgegenüber führt WEBER aus einem induktiven Ansatz die Rationalitätssicherung der Führung als originären Inhalt und Kern des Controllings an. Vgl. Weber (2004), S. $48 \mathrm{ff}$.

335 Vgl. Mosiek (2002), S. 9.

336 Vgl. Berens/Bertelsmann (2002), Sp. 281.

337 Vgl. Berens/Hoffjan (2004), S. 5.

338 Vgl. Berens/Hoffjan/Strack (1995), S. 144 und Mosiek (2002), S. 18.

339 Vgl. Weber (2004), S. 55.

340 Vgl. hierzu und im Folgenden ausführlich Küpper (2005), S. 15 ff.

341 Vgl. zur ausführlichen Erläuterung des Ansatzes Pfohl/Zettelmeyer (1987), S. 150 ff. Vgl. in der jüngeren Literatur zu dieser Konzeption auch Franz (2004).

342 Vgl. Pfohl/Zettelmeyer (1987), S. 149.

343 Vgl. Küpper (2005), S. 15.
} 
richtung selbst noch keine eigenständige Problemstellung des Controlling bildet, da sie auch von anderen Führungsteilsystemen verfolgt wird. Es stellt damit für das Controlling ein maßgebliches, aber kein charakterisierendes oder von anderen Führungsbereichen abgrenzendes Merkmal dar. ${ }^{344}$

- Die führungsprozessbezogene Controlling-Konzeption wird insbesondere von WEBER und SCHÄFFER vertreten und sieht die Kernaufgabe des Controlling in der Sicherstellung einer angemessenen Rationalität der Führung. ${ }^{345}$ Grundlage ist die Gliederung des Führungsprozesses in die Phasen Willensbildung, Willensdurchsetzung, Ausführung und Kontrolle - die Rationalitätssicherungsfunktion des Controlling bezieht sich auf alle Phasen des Führungszyklus. ${ }^{346}$ Die Funktion der Rationalitätssicherung dient demnach primär der Unterstützung des Managements, welches die „Verantwortung für die Führung des Unternehmens“"347 trägt. Hieraus lassen sich drei Typen von Controlleraufgaben ableiten: ${ }^{348}$ Entlastungsaufgaben mit einer reinen Zulieferfunktion zur effizienten Datenversorgung der Führung (bspw. Berichtswesen), Ergänzungsaufgaben bei denen durch spezifisches Fach- und Methodenwissen die Handlungen des Managements aus der Perspektive des Controllers geprüft werden und Begrenzungsaufgaben, bei denen der Controller darauf achtet, dass Manager innerhalb des ihnen gesteckten Rahmens bleiben und nicht opportunistisch handeln. Die zentrale kritische Frage bei diesem Ansatz ist, inwieweit Rationalitätssicherung eine eigenständige Funktion darstellt, die anderen betriebswirtschaftlichen Funktionen nicht zukommt und dadurch charakteristisch für das Controlling sein kann. ${ }^{349}$

- Bei den koordinationsorientierten Controlling-Konzeptionen lassen sich u.a. drei wesentliche Ansätze hinsichtlich des Koordinationsaspektes bzw. der Reichweite der Koordination unterscheiden. Bei der informationsorientierten ControllingKonzeption wird „das Controlling als zentrale Einrichtung der Informationswirtschaft ${ }^{\star 350}$ verstanden. Der Ansatz fokussiert auf die Koordination von Informations-

344 Die Gewinnzielausrichtung ist in vielen erwerbswirtschaftlichen Unternehmen schon bisher für die Planung, Steuerung und Kontrolle sowie das Informationssystem bestimmend. Es ist nicht zu erkennen, dass mit dem Controlling zu den bestehenden Führungssystemen ein weiteres hinzutreten muss, das im Unterschied zu diesen Führungssystemen die Zielausrichtung bewirkt. Aus diesem Grund stellt die Gewinnzielausrichtung keine eigenständige zusätzliche Problemstellung dar. Vgl. hierzu ausführlich Küpper (2005), S. 17.

Vgl. hierzu ausführlich Weber (2004), S. 47 ff.; Weber/Schäffer (1999), S. 731 ff. Mit der reflexionsorientierten Controlling-Konzeption wurde von PIETSCH und SCHERM eine weitere führungsprozessbezogene Controlling-Konzeption in die Diskussion eingebracht, auf die im Folgenden allerdings nicht näher eingegangen wird. Vgl. hierzu ausfuihrlich Pietsch/Scherm (2001) sowie Pietsch (2003). Vgl. Weber/Schäffer (1999), S. 734 ff. Weber (2004), S. 38. Vgl. hierzu ausfuihrlich Weber (2004), S. 40 ff. und die dort angegebene Literatur.

349 Vgl. Küpper (2005), S. 19. KÜPPLR bemängelt in den folgenden Ausführungen die mangelnde Spezifität des Rationalitätsbegriffs, weshalb es kein typisches Merkmal für eine bestimmte Funktion sein kann. Zusätzlich erschwert die unklare Trennung zwischen Funktion und organisatorischer Gestaltung in diesem Ansatz die Kennzeichnung typischer Merkmale der Controllingfunktion. Vgl. Küpper (2005), S. $19 \mathrm{f}$. 
bedarf und Informationsbeschaffung. ${ }^{351}$ Darüber hinaus besteht eine wichtige Controllingfunktion in einer differenzierten adressatenbezogenen Aufbereitung und Bereitstellung von Informationen. ${ }^{352}$ Deutlich erweitert wird das Blickfeld durch die planungs- und kontrollorientierte Konzeption nach HORVÁTH. ${ }^{353}$ Hier wird die Funktion des Controlling ,in der ergebnisorientierten Koordination von Planung und Kontrolle sowie Informationsversorgung ${ }^{6354}$ gesehen. ${ }^{355}$ Die umfangreichste koordinationsorientierte Controlling-Konzeption stellt KÜPPER vor. ${ }^{356}$ Demnach besteht die Controlling-Funktion im Kern in der Koordination des Führungsgesamtsystems zur Sicherstellung einer zielgerichteten Lenkung. ${ }^{357}$ Als wichtige Bestandteile des Führungssystems werden das Zielsystem, die Organisation, die Planung und Kontrolle, das Personalführungssystem und das Informationssystem genannt. ${ }^{358}$ Controlling richtet sich jedoch nicht auf den Leistungsvollzug selbst, dies bleibt unverändert eine originäre Führungsaufgabe, sondern verbindet innerhalb des Führungssystems deren einzelne Subsysteme miteinander (vgl. auch Abbildung 15). ${ }^{359}$

Führungssystem der Unternehmung

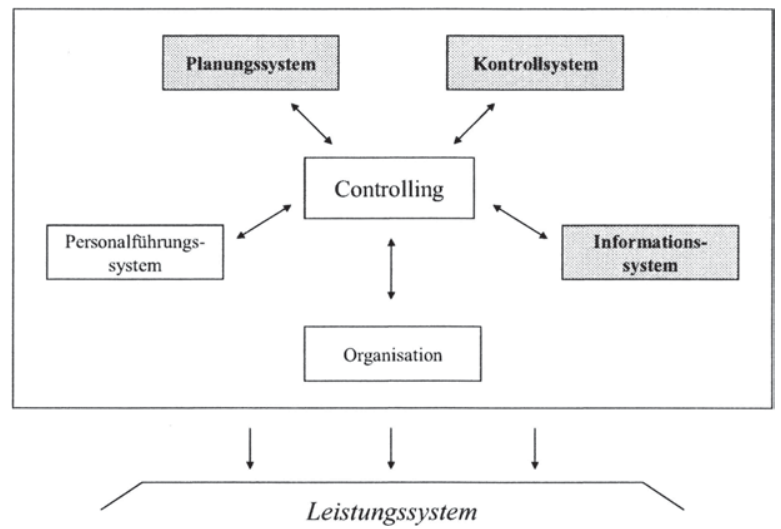

Abbildung 15: Gliederung des Führungssystems der Unternehmung ${ }^{360}$

351 Vgl. Müller (1974), S. 145 ff.

352 Vgl. Mosiek (2002), S. 10.

$353 \mathrm{Vgl}$. Horváth (2001), S. $150 \mathrm{ff}$.

354 Horváth (2001), S. 150.

355 In der planungs- und kontrollorientierten Konzeption wird ein weiterreichendes Koordinationsproblem zugrunde gelegt, als in der informationsorientierten Konzeption. Es wird hier deutlicher sichtbar, dass es sich um die Koordination von Führungsteilsystemen handelt. Vgl. Küpper (2005), S. 27.

356 Vgl. grundlegend Küpper (1988), S. 168 ff. sowie Küpper (2005), S. 28 ff.

357 Vgl. Küpper/Weber/Zünd (1990), S. 282 und Küpper (2005), S. 27.

$358 \mathrm{Vgl}$. Küpper (2005), S. 29.

359 Berens/Hoffjan (2004), S. 6.

360 Leicht modifizierte Darstellung in Anlehnung an Küpper (2005), S. 30. 
Der inhaltlich strukturierende Bezugsrahmen der Controlling-Konzeptionen bestimmt die situative Ausgestaltung der Controlling-Institution, die in Abhängigkeit relevanter Kontextfaktoren unterschiedliche Ausprägungen annehmen kann. Entsprechend einer qualitativen Interpretation von WEBER liegt der Schwerpunkt der Controller-Aufgaben in den Bereichen Planung, Kontrolle und Informationsversorgung. ${ }^{361}$ Zur Verrichtung der Koordinationsaufgaben bedient sich das Controlling einer Vielzahl von Instrumenten, wie Planungs-, Kontroll- und Informationsinstrumenten (z.B. Kostenrechnung, Abweichungsanalysen) sowie Instrumenten zur übergreifenden Koordination (z.B. Budgetierung). ${ }^{362}$

\subsection{Grundlagen des Controllings staatlichen Handelns}

Entsprechend den herausgearbeiteten Grundlagen zum allgemeinen, eher privatwirtschaftlich geprägten Controlling-Ansatz, folgt die Arbeit einem Controllingverständnis mit einer Schwerpunktsetzung auf Koordinationsaufgaben in den Bereichen Planung, Kontrolle und Informationsversorgung. In einem weiteren Schritt ist dieser allgemeine Controlling-Ansatz um die spezifischen Rahmenbedingungen im politisch-administrativen System zu erweitern. Diese Kontextfaktoren sollen im Folgenden zusammenfassend kurz dargestellt werden: ${ }^{363}$

- komplexes und heterogenes Zielsystem mit einer Betonung von Sachzielen gegenüber Formalzielen sowie resultierenden Operationalisierungsproblemen

- komplexe Organisations- und Trägerstruktur des öffentlichen Sektors mit hoher Spezialisierung und Zentralisation einzelner Verwaltungsaufgaben

- Dienstleistungscharakter und fehlende Marktgängigkeit des öffentlichen Leistungsprogramms erschweren Ergebnismessbarkeit und Leistungsbeurteilung

- schrittweise Ablösung der bürokratischen Verhaltens- und Führungsstruktur durch den Modernisierungsprozess unter dem Leitbild des NPM mit einer Betonung betriebswirtschaftlich orientierter Koordinationsinstrumente

Die geschilderten Besonderheiten führen somit zu einer spezifischen Anpassung und Ausgestaltung der Controlling-Konzeption. Die Notwendigkeit eines Controllings im politisch-administrativen System ergibt sich einerseits aus der Komplexität und Vielschichtigkeit der Strukturen und des Leistungsprogramms sowie andererseits aus den komplexen Interdependenzen zwischen Politik und Verwaltung. Hierdurch entsteht für Führungsfunktionen ein großer Bedarf an steuerungsrelevanter Information und Koordination. ${ }^{364}$ Weiterhin werden durch den eingeleiteten Modernisierungsprozess in der öffentlichen Verwaltung auf Rechtsnormen basierende bürokratische Verfahren zu-

\footnotetext{
361 Vgl. Weber (2004), S. $99 \mathrm{ff}$. und $307 \mathrm{ff}$.

$362 \mathrm{Vgl}$. Berens/Hoffjan (2004), S. 7.

363 Siehe hierzu Kapitel 2.1 und Kapitel 2.2 dieser Arbeit sowie prägnant Berens/Hoffjan (2004), S. 9.

364 Vgl. Heinz (2000), S. 14 und Budäus/Buchholtz (1997), S. 323.
} 
nehmend durch ein ergebnisorientiertes Managementmodell substituiert. ${ }^{365}$ Die Reformen begründen einen erheblich erweiterten Handlungs- und Entscheidungsspielraum eher dezentralisierter Organisationseinheiten - verbunden mit der Ergebnisorientierung ergibt sich der faktische Bedarf eines Controllings in der öffentlichen Verwaltung. Controlling ist damit ein wesentlicher Bestandteil des Reformprozesses. ${ }^{366}$ Aufgrund der Unterschiede und Spezifika des politisch-administrativen Systems ist ein einfaches „Überstülpen“ privatwirtschaftlicher Controlling-Instrumente auf den öffentlichen Sektor jedoch nicht möglich. ${ }^{367}$ Grundsätzlich erfordert die Auseinandersetzung mit den vorgestellten Controlling-Konzeptionen die Identifikation relevanter Merkmale, die sich auf ein Controlling des staatlichen Handelns anpassen lassen.

Die Ergebniszielorientierung des Controlling kann sich prinzipiell auf alle Formen von Oberzielen beziehen. ${ }^{368}$ Im Gegensatz zur Privatwirtschaft handelt es sich dabei aber nicht um Gewinn- oder Rentabilitätsziele, sondern die Gemeinwohlausrichtung des öffentlichen Sektors führt zu einer Dominanz von Sach-zielen. Für das politischadministrative System stellen daher die Effektivität und die Effizienz des staatlichen Handelns - unter Beachtung der rechtlichen Grundsätze - die obersten Ergebnisziele dar. ${ }^{369}$ Entsprechend dem Ansatz wird damit die operativ-taktische Steuerung eines wirksamen und wirtschaftlichen Verwaltungshandelns betont.

Das Verständnis von Controlling als Sicherstellung einer angemessen Führungsrationalität kann sich im politisch-administrativen System sowohl auf die Ebene der Verwaltungsführung, als auch auf die politische Ebene beziehen. Der Führungszyklus beinhaltet hier allerdings eine deutlich komplexere Struktur, was insbesondere die Beachtung einer speziellen politischen Rationalität erforderlich macht und es somit einer Übersetzungsfunktion zwischen Politik und Verwaltung bedarf. ${ }^{370}$ Aus dem zugrunde liegenden Controllingverständnis ergibt sich damit die Notwendigkeit zu einem strategischen Controlling, welches eher der administrativen Ebene zuzuordnen ist und einem politischen Controlling, welches eher der politischen Ebene zuzuordnen ist. Auch im öffentlichen Sektor kommen dem Controlling somit Entlastungs-, Ergänzungs- und Begrenzungsaufgaben zu.

Im Rahmen der koordinationsorientierten Ansätze werden Controllingaufgaben als zielgerichtete Koordination des Führungsgesamtsystems betont. Die außerordentlich hohe Komplexität von Zielen, Strukturen und Programmen sowie die hohe Anzahl unterschiedlicher Akteure im politisch-administrativen System unterstreichen auch hier den erheblichen Koordinationsbedarf. Entsprechend zielt Controlling als spezifische,

365 Vgl. zum „Neuen Steuerungsmodell“ ausführlich Kapitel 2.2.2.3. und die dort angegebene Literatur.

366 Vgl. hierzu bspw. Hill (1996), S. 233; Budäus/Buchholtz (1997), S. 322; Budäus (2001), S. 27 und Budäus (2002a), S. 207.

367 Vgl. Klingebiel (1999), S. 378 und Berens/Bücker/Finken (1998), S. 373.

$368 \mathrm{Vgl}$. Küpper/Weber/Zünd (1990), S. 282 f.

369 Vgl. Richter (2000), S. 32.

370 Zur Ausgestaltung der Übersetzungsfunktion zwischen politischer und administrativer Rationalität vgl. Schedler/Proeller (2003), S. 54 ff. 
flächendeckende Funktion im öffentlichen Sektor darauf ab, die Führungs- bzw. Managementfunktionen Planung, Organisation, Personal, Kontrolle in und zwischen den dezentralen Einheiten zu koordinieren. ${ }^{371}$ Die spezifische Ausgestaltung des Controllingsystems wird dann durch das jeweilige Informations- und Koordinationsinteresse der Führungsebene sowie der Führungskraft bestimmt. ${ }^{372}$ Hierdurch entsteht die Notwendigkeit $\mathrm{zu}$ einem adressatenorientierten Berichtswesen mit differenzierten $\mathrm{Be}-$ richtsebenen sowie unterschiedlichen Verdichtungsgraden und Berichtsperioden. ${ }^{373}$

Die Auseinandersetzung mit den eher privatwirtschaftlich geprägten ControllingKonzeptionen und ihre Übertragung auf die Besonderheiten des politisch-administrativen Systems zeigen sowohl die Notwendigkeit als auch die grundsätzliche Eignung des Controlling-Ansatzes für den öffentlichen Sektor. Dabei erscheint es für den weiteren Untersuchungsverlauf wenig zielführend, die „richtige“ Controlling-Konzeption zu bestimmen, sondern es müssen vielmehr die unterschiedlichen Aspekte der vorgestellten Ansätze miteinander verknüpft werden. Um nachfolgend ein gemeinsames Grundverständnis für den Rahmen dieser Arbeit sicherzustellen, soll unter dem Controlling staatlichen Handelns daher , die Beschaffung, Aufbereitung, Analyse und Kommunikation von Daten zur Vorbereitung zielsetzungsgerechter Entscheidungen "verstanden werden. ${ }^{374}$

Dieses recht weit gefasste Controlling-Verständnis umfasst sowohl die maßgeblichen Elemente der zuvor geschilderten Controlling-Konzeptionen, als auch die Möglichkeit, die bereits im zweiten Kapitel herausgearbeiteten Anforderungen an die Wirkungsorientierung des Controlling zu berücksichtigen. ${ }^{375}$ Die Definition beinhaltet insbesondere die Herstellung von Transparenz, d.h. die Effizienz und Effektivität sowie hiermit verbundene Gestaltungsmöglichkeiten herauszuarbeiten und so zielsetzungsgerechte Entscheidungen der Führung zu unterstützen. Zur Bestimmung der Effektivität kommt dem Controlling die Aufgabe der Identifikation und Operationalisierung der Wirkungen staatlichen Handelns zu. Hier schafft Controlling die notwendigen Voraussetzungen zur Zuweisung von Verantwortung, auch für das Erreichen gesetzter Wirkungsziele. Das Controlling unterstützt so den komplexen Zielbildungsprozess im politischadministrativen System. Als eine Art Übersetzungsfunktion zwischen politischer und administrativer Rationalität bildet das Controlling die notwendige Verbindung zwischen den im neuen Steuerungsmodell getrennten Ebenen Politik und Verwaltung. Eng hiermit verbunden ist auch die Koordination der Führungsteilsysteme und die Abstimmung der Subziele. Durch die Beschaffung, Aufbereitung, Analyse und Kommunikation von (Wirkungs-)Daten erfüllt das Controlling eine Servicefunktion und stellt so die adressatengerechte Informationsversorgung aller beteiligten Akteure sicher.

\footnotetext{
371 Vgl. Budäus (2002a), S. 206.

372 Vgl. Pook/Tebbe (2002), S. 43 f.

373 Vgl. hierzu auch Buchholtz (2001), S. $28 \mathrm{ff}$.

374 Vgl. Berens/Hoffjan/Strack (1995), S. 144 und Mosiek (2002), S. 18.

$375 \mathrm{Vgl}$. hierzu ausführlich Kapitel 2.3 dieser Arbeit.
} 
Generell kann festgestellt werden, dass ein Controlling staatlichen Handelns als Führungsunterstützung sämtliche Reformelemente der Neuen Steuerung unterstützt ${ }^{376}$ und so für den Entwicklungspfad zur wirkungsorientierten Steuerung ein unverzichtbares Reformelement darstellt. In Anlehnung an HOFFJAN wird das Grundgerüst eines solchen Controllingsystems vorgestellt (vgl. Abbildung 16). ${ }^{377}$ Systemtheoretisch handelt es sich beim Controlling um ein Lenkungssystem mit einem Steuerungselement (top down) und einem Regelungselement (bottom up). Die Grundstruktur orientiert sich dabei an der Unterscheidung einer strategischen und einer operativen Koordinationsund Steuerungsebene.

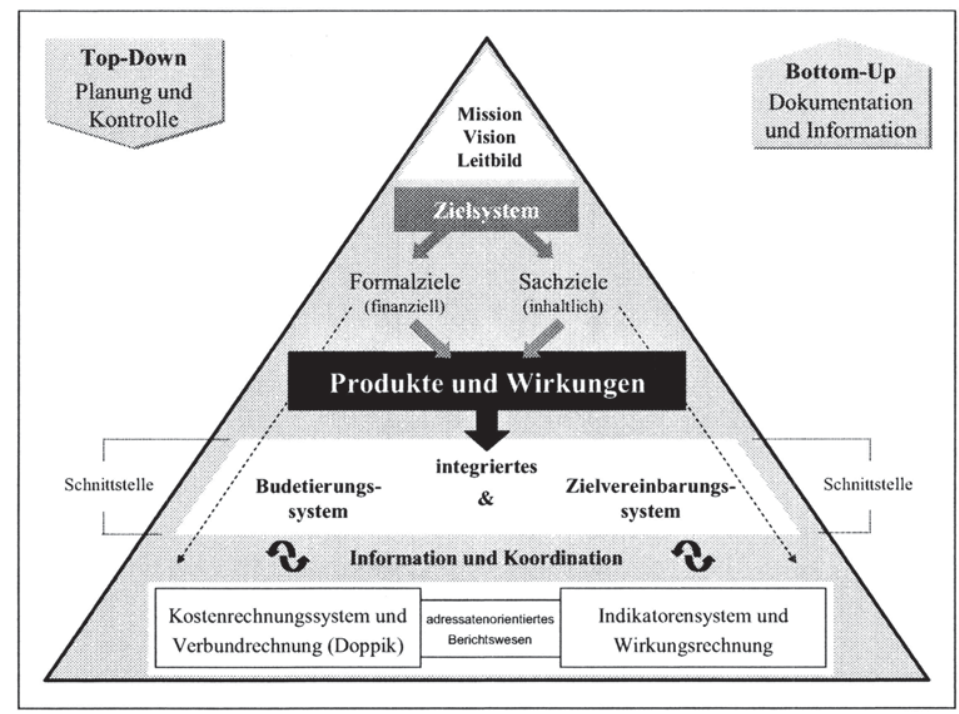

Abbildung 16: Konzeption eines Controlling-Systems ${ }^{378}$

Das strategische Controlling erfolgt grundsätzlich in einem längeren Zeithorizont und hat die Effektivitätssteigerung zum Ziel. ${ }^{379}$ Hier sind zwei Perspektiven zu unterscheiden: Auf der politischen Ebene werden entsprechend dem Leitbild einer öffentlichen Einrichtung die zu erfüllenden Sachziele vorgegeben und um die mit den aktuellen finanziellen Möglichkeiten abgestimmten Formalziele ergänzt. Das strategische Controlling muss dabei die Planung politischer Ziele unterstützen und entsprechende Instrumente und Informationen, auch über Entwicklungen im gesellschaftlichen Umfeld, bereitstellen. ${ }^{380}$ Die zweite Perspektive geht von gegebenen Zielen aus und betrifft die

\footnotetext{
376 Vgl. Budäus (2002a), S. 207 und Günther/Niepel/Schill (2002), S. 221.

377 Vgl. hierzu im Folgenden auch Hoffjan (1998), S. 161 ff. sowie Berens/Hoffjan (2004), S. $10 \mathrm{f}$.

378 Erweiterte Darstellung in Anlehnung an Hoffjan (1998), S. 161 und Röhrig (2008), S. 53.

379 Vgl. Richter (2000), S. 395.

380 Vgl. Brüggemeier (1998), S. 82 ff. oder Heinz (2000), S. 99 ff.
} 
Analyse der Zielerreichung, d.h. die Beantwortung der Frage, ob „die richtigen Dinge getan" werden. ${ }^{381}$

Über Produkte (Output-Steuerung) und Wirkungen (Outcome-Steuerung) erfolgt die Verknüpfung der Zieldimension mit den dafür einzusetzenden Ressourcen. Im Rahmen eines integrierten Budgetierungssystems werden die Wirkungs- und Leistungsziele an die Finanzziele gekoppelt. Im Idealfall geben die politischen Entscheidungsträger der Verwaltung nur noch die Ziele vor und weisen gleichzeitig ein Globalbudget zu. Im Gegensatz zum bestehenden Haushaltswesen sorgt die Zuordnung von Finanzmitteln auf leistungs- bzw. wirkungsbezogene Zielgrößen für eine optimale Allokation der begrenzten Ressourcen. ${ }^{382}$ Der Verzicht der Legislative auf die inputorientierte Detailsteuerung bedeutet allerdings zunächst einen Machtverlust der Politik. ${ }^{383}$ Damit dieser Machtverlust kompensiert wird, muss das Verwaltungshandeln durch ein wirkungsorientiertes Controlling „kontrollierbarer“ werden. ${ }^{384}$ Ein adressatenorientiertes Berichtswesen sollte daher die politische Ebene einbeziehen und die Verwaltung so die gesellschaftlichen Wirkungen ihres Handelns dokumentieren. ${ }^{385}$

Das operative Controlling ist eher kurzfristig orientiert und beantwortet primär die Frage, ob „die Dinge richtig getan“ werden. ${ }^{386}$ Darunter fällt beispielsweise die Schaffung und Verbesserung der Führungsinformationssysteme, die Unterstützung der operativen Planung und Budgetierung sowie die operative Überwachung des Betriebsgeschehens. ${ }^{387}$ Als ein wichtiges operatives Controlling-Instrument kann die Kosten- und Leistungsrechnung Ressourcenverbräuche sowie die Leistungserstellung transparent machen und damit Kostenbewusstsein erzeugen. ${ }^{388}$ Kostensenkungs- und Leistungssteigerungspotenziale werden offen gelegt, so dass die Effizienz des Verwaltungshandelns gesteigert werden kann. Über ein Indikatorensystem und unterstützende Kennzahlen soll die Operationalisierung und Abbildung der Sachziele erfolgen ${ }^{389}$ Die indikatorengestützte Konkretisierung der Sachziele ist Voraussetzung dafür, dass Leistungsmenge und Leistungsqualität erfasst werden können. Ein wirkungsorientiertes Controlling benötigt darüber hinaus den Aufbau einer systematischen Wirkungsrechnung, welche die Wirkungen der Verwaltungsprodukte erfasst. Auch wenn Wirkungsinformationen eher bei strategischen Entscheidungen eine Rolle spielen, sind Aufbau und Pflege der Wirkungsrechnung eher dem operativen Verwaltungscontrolling zuzurechnen. ${ }^{390}$

\footnotetext{
381 Vgl. Budäus (2002b), S. 391.

382 Vgl. Berens/Hoffjan (2004), S. 10.

384 Vgl. Budäus (2002b), S. 392.

385 Vgl. Mosiek et al. (2003), S. 28.

386 Vgl. Brüggemeier (1998), S. 97.

387 Vgl. hierzu ausführlich Richter (2000), S. 75 ff.

$388 \mathrm{Vgl}$. Günther/Niepel/Schill (2002), S. 220.

389 Vgl. Männel (1988), S. 853.

390 Vgl. Richter (2000), S. 99 ff.
}

383 In einer Demokratie ist ein wichtiges Recht der Legislative das Budgetrecht. Nur die politisch gewählten Vertreter dürfen über die Verwendung von Steuergeldern entscheiden. Vgl. Budäus (2002b), S. 392. 
In der vorgestellten Controlling-Systematik kommt der primäre Charakter der Führungsunterstützung des Controllings zum Ausdruck. Das wirkungsorientierte Controlling versorgt in einem so verstanden Kontext das Verwaltungsmanagement und die Politik mit allen relevanten Informationen hinsichtlich des Erreichungsgrades gesetzter Ziele, insbesondere der Wirkungen. ${ }^{391}$ Bisherige Schwächen der Rückkopplung im Hinblick auf den Maßnahmenerfolg sowie die fehlende Verbindung zur Haushaltsplanung bzw. Mittelzuteilung können durch die Systemintegration von Kosten- und Wirkungsrechnung behoben werden. ${ }^{392}$ Damit wird zum einen der Politik ermöglicht, im Rahmen einer strategischen Planung die Gesamtheit der Verwaltung wirkungsorientiert zu führen, zum anderen kann innerhalb der Verwaltung zwischen unterschiedlichen Ebenen wirkungsorientiert gesteuert werden. ${ }^{393}$

Die bisherigen eher abstrakten Ausführungen zum Grundgerüst eines wirkungsorientierten Controllings im politisch-administrativen System sollen im weiteren Untersuchungsverlauf durch die Darstellung ganzheitlicher Bezugsrahmen wirkungsorientierter Controlling-Konzepte näher spezifiziert werden.

\subsection{Wirkungsorientierte Controlling-Konzepte und Modelle}

\subsubsection{Zur Notwendigkeit eines ganzheitlichen Bezugsrahmens}

Die wesentliche Aufgabe des wirkungsorientierten Controllings ist es, das Führungsgesamtsystem bei der Erreichung der Organisationsziele durch Koordinationsleistungen zu unterstützen. Der Koordinationsbedarf innerhalb des politisch-administrativen Führungssystems ist insbesondere von der Ausgestaltung des Steuerungsprozesses mit den wesentlichen Funktionen Planung, Umsetzung und Kontrolle abhängig. ${ }^{394}$ Die Steuerung des politisch-administrativen Systems muss sich in diesem Zusammenhang auf ein Prozessmodell der öffentlichen Leistungserstellung ${ }^{395}$ beziehen. Hieraus resultiert die Notwendigkeit, das zuvor vorgestellte Grundgerüst einer wirkungsorientierten Controlling-Konzeption durch einen ganzheitlichen Bezugsrahmen weiter auszudifferenzieren.

In einem ganzheitlichen Bezugsrahmen kann der Managementprozess öffentlicher Leistungserstellung mit seinen einzelnen Prozessschritten erfasst werden. Den verschiedenen Prozessebenen lassen sich geeignete Bewertungskriterien des Zielerreichungsgrades zuordnen. Zur Ermittlung der Zielerreichung werden im Rahmen des Controllings regelmäßig sachgerechte Instrumente angewendet. Die notwendigen Aufgaben und Instrumente des strategischen und operativen Controllings sind daher ebenfalls in den Bezugsrahmen zu integrieren. Weiterhin sollten die unterschiedlichen Be-

\footnotetext{
391 Vgl. Röhrig (2008), S. 52.

392 Vgl. auch Berens/Hoffjan (2004), S. 11.

393 Vgl. Röhrig (2008), S. 53 bzw. die Ausführungen des Kapitels 2.2.3.1.

394 Vgl. hierzu ausführlich Kapitel 2.2.3.1.

395 Vgl. hierzu auch beispielhaft das Prozessmodel in Kapitel 2.1.4. dieser Arbeit.
} 
teiligungsrollen und das spezifische Informationsinteresse der relevanten Akteure des politisch-administrativen Systems Berücksichtigung finden. Dergestalt kann durch einen geeigneten Bezugsrahmen die strukturierte und transparente Abbildung der komplexen Sachverhalte einer wirkungsorientierten Controlling-Konzeption gelingen.

Die folgenden Ausführungen beschäftigen sich mit den relevanten wirkungsorientierten Controlling-Konzeptionen bzw. Modellen. Ihrer chronologischen Erscheinung in der Literatur folgend, wird zunächst das 3-Ebenen-Konzept ${ }^{396}$ und das 5-EbenenKonzept $^{397}$ und anschließend das Modell der Ziel- und Ergebnisebenen öffentlicher Leistungserstellung ${ }^{398}$ sowie das Erweiterte Modell der Ziel- und Ergebnisebenen öffentlicher Leistungserstellung ${ }^{399}$ vorgestellt.

\subsubsection{Darstellung und Erläuterung ausgewählter Bezugsrahmen}

\subsubsection{Das 3-Ebenen-Konzept}

Grundlage der folgenden Ausführungen ist das in Anlehnung an BUSCHOR ${ }^{400}$ von BUDÄUS/BUCHHOLTZ entwickelte 3-Ebenen-Konzept (3-E-Konzept). ${ }^{401}$ Das 3-E-Konzept wurde als „Bezugsrahmen für ein Verwaltungscontrolling“ entwickelt, welches konzeptionell ,sowohl den auf das Gemeinwohl gerichteten Zielbezug der Wirtschaftlichkeit als auch die Wirkungen des Verwaltungshandelns auf Bürger und Gesellschaft" berücksichtigt. ${ }^{402}$ Der Ansatz soll damit dem Umbruch im öffentlichen Sektor Rechnung tragen und einen systematischen Zugang zur ökonomischen Bewertung des Verwaltungshandelns gewährleisten. ${ }^{403}$

Die Entwicklung des 3-E-Konzeptes basiert auf einem Querschnittsvergleich ausgewählter internationaler Ansätze der politischen Erfolgskontrolle aus den Bereichen Performance Measurement $^{404}$, externe Auditierung ${ }^{405}$ und Programmevaluation ${ }^{406}$. Trotz unterschiedlicher Anwendungsbereiche lassen sämtliche Ansätze eine „Konvergenz

396 Vgl. Budäus/Buchholtz (1997), S. $323 \mathrm{ff}$.

397 Vgl. Buschor (2002), S. $65 \mathrm{ff}$.

$398 \mathrm{Vgl}$. Mosiek et al. (2003), S. 28 ff. und Mosiek/Gerhardt (2003), S. $289 \mathrm{ff}$.

399 Vgl. Röhrig (2008), S. 105 ff.

400 Zum „Vierebenenkonzept“"vgl. Buschor (1993), S. 238.

$401 \mathrm{Vgl}$. hierzu und im Folgenden Budäus/Buchholtz (1997), S. $323 \mathrm{ff}$.

402 Budäus/Buchholtz (1997), S. 323 und S. 326.

403 Vgl. Budäus/Buchholtz (1997), S. 322 und Röhrig (2008), S. 69.

404 Unter Performance Measurement kann ein organisationsinterner Ablauf zur Bestimmung der Leistungsfähigkeit und der Leistung einer Organisation verstanden werden. Dazu sind das Finden von Leistungsmaßstäben bzw. -zielen, die Auswahl von geeigneten Kennzahlen und Indikatoren, die Beurteilung der Ergebnisse hinsichtlich Zielerreichung sowie die Überprüfung der gesetzten Ziele notwendig. Vgl. Sturm (2000), S. 62 f.

405 Unter Externer Auditierung kann ein prozessunabhängiger Überwachungsvorgang anhand definierter Bezugsgrößen durch organisationsfremde Personen verstanden werden. Vgl. Pede (1999), S. 6 f.

406 Unter Evaluation kann die systematische Anwendung sozialwissenschaftlicher Forschungsmethoden zur Beurteilung der Konzeption, Ausgestaltung, Umsetzung und des Nutzens sozialer Interventionsprogramme verstanden werden. Vgl. Rossi (1998), S. 3 oder auch Stockmann (2004), S. 13 ff. 
hinsichtlich der konzeptionellen Grundlagen“ erkennen ${ }^{407}$ Die Synopse erfolgt dabei auf „relativ hohen Abstraktionsniveau, um die vielfältigen Aspekte des öffentlichen Leistungsprozesses umfassend abbilden zu können" ". ${ }^{408}$ Konkret analysiert der Beitrag das jeweils zugrunde liegende Prozessmodell und die zugeordneten Bewertungskriterien. ${ }^{409}$ Zielsetzung von BUDÄUS/BUCHHOLTZ war es, aus den vielfältigen Ansätzen eine Basiskonzeption zu entwickeln, die als ,geeignetes Referenzmodell auch für weitere Forschungsarbeiten" herangezogen werden kann. ${ }^{410}$

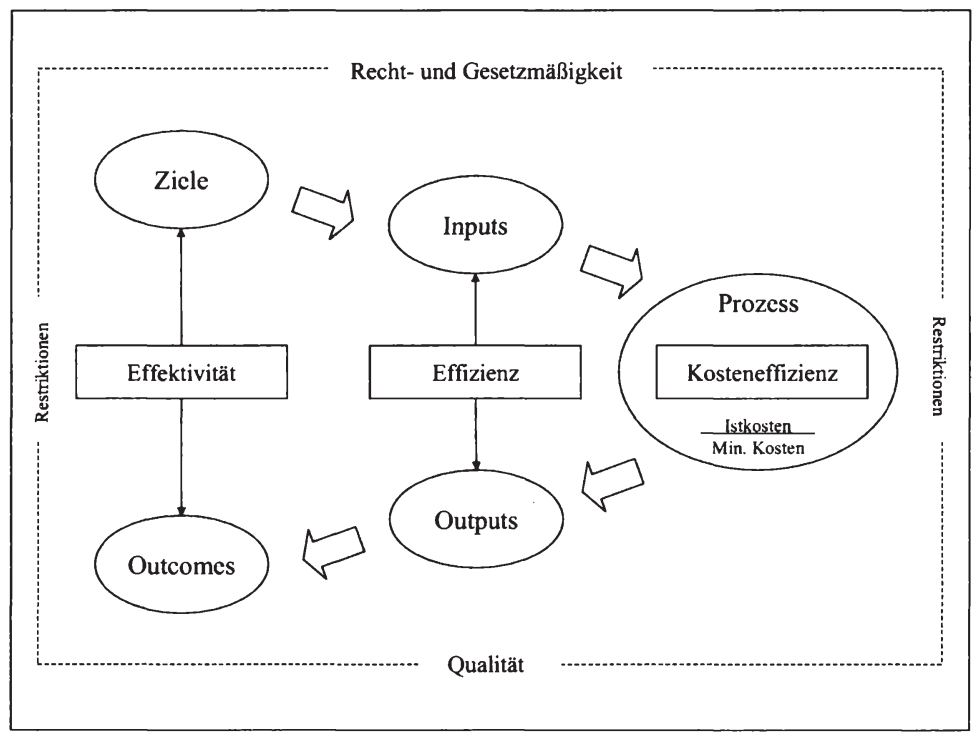

Abbildung 17: Das 3-Ebenenkonzept ${ }^{411}$

Im Sinne der Basiskonzeption des 3-E-Konzeptes werden die Prozessebene, die Leistungsebene und die Wirkungsebene mit den Bewertungskriterien Kosteneffizienz, Effizienz und Effektivität unterschieden. ${ }^{412}$ Als Nebenbedingung (Restriktionen) gilt die Einhaltung von Qualitätsstandards und die Recht- bzw. Gesetzmäßigkeit des Verwaltungshandelns. Gleichzeitig werden über die einzelnen Ebenen die öffentlichen Ziele, Inputs, Prozesse, Outputs und Outcomes als Prozessschritte und formale Bewertungs-

407 Budäus/Buchholtz (1997), S. 327.

408 Budäus/Buchholtz (1997), S. 327.

$409 \mathrm{Zu}$ den Ansätzen vgl. insbesondere die Abbildungen 2 und 3 bei Budäus/Buchholtz (1997), S. $327 \mathrm{f}$.

410 Budäus/Buchholtz (1997), S. 327.

411 Vgl. Budäus/Buchholtz (1997), S. 332.

412 Das 3-E-Konzept entstammt als Terminologie dem anglo-amerikanischen Raum. Der deutsche Begriff „Kosteneffizienz" kann in diesem Zusammenhang mit dem englischen Begriff „Economy“ übersetzt werden. The three E's: Economy, Efficiency and Effectiveness. Vgl. Buschor/Lüder (1994), S. 182. 
maßstäbe des Verwaltungshandelns abgebildet. ${ }^{413}$ Abbildung 17 verdeutlicht den Zusammenhang zwischen den einzelnen Prozessschritten, den Prozessebenen und den zugeordneten Bewertungskriterien.

Ausgangspunkt des Modells sind die Ziele der öffentlichen Verwaltung, welche den Handlungsrahmen für die nachfolgenden Prozessschritte und gleichzeitig die Basis für die Messung der Zielerreichung, d.h. der Effektivität, bilden. ${ }^{414}$ Zur Zielerreichung werden Maßnahmen abgeleitet, die einen Ressourceneinsatz als Input erfordern. Der eigentliche Produktionsprozess kombiniert die eingesetzten Ressourcen (Produktionsfaktoren) zur Erstellung der öffentlichen Produkte. Als Produktionsleistung bzw. erkennbares Ergebnis des Verwaltungshandelns erhält man anschließend die Outputs. ${ }^{415}$ Den letzten Prozessschritt stellen die Outcomes, verstanden als ,zielbezogene Wirkungen von öffentlichen Leistungen auf den Bürger und die Gesellschaft", dar. ${ }^{416}$ Diese lassen sich in einer Modellerweiterung nach BUDÄUS/BUCHHOLTZ zudem in subjektive Outcomes und objektive Outcomes unterscheiden. ${ }^{417}$ Wirkungen können demnach „,sowohl subjektiv vor dem Hintergrund individueller Ziele der Leistungsnutzer als auch objektiv in Bezug auf die am Gemeinwohl orientierten Zielsetzungen" betrachtet werden. $^{418}$

Das 3-E-Konzept beinhaltet weiterhin formale Bewertungskriterien zwischen den Ebenen des dargestellten Gesamtprozesses öffentlicher Leistungserstellung. Auf der Prozessebene werden die öffentlichen Leistungen erstellt, wodurch entsprechend dem hierfür notwendigen Ressourcenverbrauch Kosten anfallen. ${ }^{419}$ Die Kosteneffizienz leitet sich aus der Interpretation der Wirtschaftlichkeit nach dem Minimalprinzip ab: Bei gegebenem Output soll der Produktionsprozess zu geringstmöglichen Kosten erfolgen. ${ }^{420}$ Rechnerisch wird damit das Verhältnis von Istkosten zu Sollkosten i.S.v. minimalen Kosten beschrieben. ${ }^{421}$ Auf der Leistungsebene misst die Effizienz das Verhältnis des Outputs zum eingesetzten Input. Sie ist damit Ausdruck der mengenmäßigen bzw. wertmäßigen Wirtschaftlichkeit der Leistungserstellung. ${ }^{422}$ Im öffentlichen Sektor lässt

\footnotetext{
413 Vgl. Budäus/Buchholtz (1997), S. 326 und Budäus (1999), S. 55.

414 Vgl. hierzu auch den in Kapitel 2.1.4 dargestellten Prozess der öffentlichen Leistungserstellung.

415 In einer Erweiterung ihres Modells differenzieren BUDÄUS/BUCHHOLTZ bei der Erstellung der Outputs zwischen Leistungsbereitschaft und Leistungsabgabe. In die Schaffung der Leistungsbereitschaft gehen zunächst ausschließlich innerbetriebliche Ressourcen ein, demgegenüber erfordert die Leistungsabgabe, dass der Nutzer selbst externe Faktoren mit in den Produktionsprozess einbringt. Vgl. hierzu ausführlich Kapitel 2.1.4 dieser Arbeit.

416 Budäus/Buchholtz (1997), S. 329.

417 Vgl. Budäus/Buchholtz (1997), S. 328.

418 Budäus/Buchholtz (1997), S. 329.

419 Vgl. Brühlmeier et al. (2001), S. 73.

420 Vgl. Seifert (1998), S. 43 und Buchholtz (2001), S. 52.

421 Vgl. Budäus/Buchholtz (1997), S. 330.

422 Die betriebswirtschaftliche Effizienz umfasst die mengenmäßige Wirtschaftlichkeit (Produktivität), also das Verhältnis vom mengenmäßigen Ertrag und mengenmäßigen Einsatz von Produktionsfaktoren, und die wertmäßige Effizienz, die das Verhältnis von in Geldeinheiten bewerteten Faktoreinsatzmengen und Outputmengen bestimmt. Vgl. Seifert (1998), S. 42 und Siems (2004), S. 28.
} 
sich der Output i.d.R. nur zuverlässig durch nicht-monetäre Größen beschreiben, so dass den in Kosten bewerteten Inputs meist der mengenmäßige Output gegenübergestellt wird. ${ }^{423}$

Für das wirkungsorientierte Verwaltungsmanagement und -controlling ist insbesondere die Wirkungsebene relevant. Hier werden die politisch gewollten Ziele mit den tatsächlich eingetretenen Wirkungen verglichen ${ }^{424}$ Die Effektivität beschreibt dabei das Verhältnis von Outcomes zu Zielen, sie ist also „eine Maßgröße dafür, inwieweit die Wirkungen des Verwaltungshandelns zur Erreichung der Ziele beigetragen" haben. ${ }^{425}$ Problematisch ist die Messung der Effektivität aufgrund der vielfältigen Herausforderungen bei der Operationalisierung von Wirkungszielen und der Bestimmung des Zielerreichungsgrades. ${ }^{426}$ Daher werden von BUDÄUS/BUCHHOLTZ zwei ergänzende Kennzahlen der Wirkungsebene vorgeschlagen: Die Kosteneffektivität als das Verhältnis von Input zu Outcome und die Output-Effektivität als das Verhältnis von Output zu Outcome. ${ }^{427}$

Mit dem Prozessmodell und den Bewertungskriterien bietet das 3-E-Konzept einen ganzheitlichen Bezugsrahmen zur Beurteilung des Handelns der Verantwortlichen im politisch-administrativen Prozess. Die einzelnen Betrachtungsebenen sind dabei nicht unabhängig voneinander, weshalb nur eine Gesamtbetrachtung sinnvoll ist: Während für die Formulierung der Effektivitätsziele überwiegend die Politik aber auch die Verwaltungsführung verantwortlich ist, stellt die Effizienzebene, also die Festlegung von Maßnahmen und Produkten sowie entsprechender Budgets, die eigentliche Schnittstelle zwischen Verwaltung und Politik dar. Für eine kosteneffiziente Produkterstellung im Rahmen des Produktionsprozesses ist weitgehend die Verwaltung zuständig. ${ }^{428}$ Damit ist das 3-E-Konzept nicht nur ein reines Dokumentations-, sondern auch ein Planungsmodell, in welchem Zielgrößen festgelegt werden, die über das Ergebnis des Leistungserstellungsprozesses einer Zielerreichungskontrolle zugeführt werden können. ${ }^{429}$

423 Vgl. Budäus/Buchholtz (1997), S. 330.

424 Vgl. Haldemann (1998), S. 199.

425 Budäus/Buchholtz (1997), S. 330.

426 Vgl. hierzu ausführlich Kapitel 2.2.3.4.

427 Die Kosteneffektivität beschreibt das Verhältnis von Kosten (als Input) und ausgelösten Wirkungen im Sinne eines „value for money“. Die Output-Effektivität dient als Maßstab dafür, mit welchen Leistungen welche Wirkungen erzielt werden. Vgl. Budäus/Buchholtz (1997), S. 330; ähnlich auch Nullmeier (1998), S. 318.

428 Vgl. Budäus (2000a), S. 74 und Siems (2004), S. 31.

${ }^{429}$ Vgl. Budäus (2000b), S. 19 f. und Röhrig (2008), S. 72. 


\subsubsection{Das 5-Ebenen-Konzept}

Eine Weiterentwicklung des Referenzmodells von BUDÄUS/BUCHHOLTZ mit einer stärkeren Ausdifferenzierung der Betrachtungsebenen stellt das 5-Ebenen-Konzept (5E-Konzept) nach BUSCHOR dar. ${ }^{430}$ Entwickelt wurde es im Zuge der Schweizer Verwaltungsreform wif $^{431}$ des Kantons Zürich. ${ }^{432}$ Ein Kernelement der wirkungsorientierten Verwaltungsreform bildet das Verwaltungscontrolling. Mit dessen Einführung sollen der Regierungsrat und das Parlament als politische Führungsorgane des Staates durch eine wachsende Transparenz des öffentlichen Leistungsprozesses gestärkt werden. Die Planung und Steuerung der staatlichen Tätigkeiten soll demnach nicht nur nach rechtsstaatlichen Prinzipien, sondern vermehrt auch anhand betriebswirtschaftlicher Kriterien erfolgen. ${ }^{433}$

Als Orientierungsrahmen für den Aufbau des Controllings dient das 5-Ebenen-Modell. Hiermit wird die Zielsetzung verfolgt, in transparenter Form die Beziehungen zwischen den verschiedenen, stufengerechten Controllingaufgaben darzustellen. ${ }^{434}$ Es handelt sich dabei um ein Führungsmodell, das auf fünf Ebenen abstellt. Wenn eine öffentliche Aufgabe bzw. ein staatliches Programm erfüllt wird, müssen nach BUSCHOR fünf zentrale Fragen beantwortet werden: ${ }^{435}$

- Wer tut was?

- Wozu tut er/sie das?

- Was leistet er/sie um das „Wozu“ zu erreichen?

- Wie setzt er/sie die Ressourcen ein?

- Wie viel Mittel werden ihm/ihr zur Verfügung gestellt?

Um eine entscheidungsorientierte Beurteilung der einzelnen Aspekte staatlicher Leistungserstellung zu ermöglichen, sind demnach Aussagen zur Steuerungsform, Effektivität, Effizienz, Wirtschaftlichkeit und Finanzierbarkeit notwendig. Aussagen zu diesen Bewertungskriterien des öffentlichen Leistungserstellungsprozesses erhält man,

${ }^{430} \mathrm{Vgl}$. Buschor (2002), S. $65 \mathrm{f}$.

${ }^{431}$ Unter dem Titel wif! (Wirkungsorientierte Verwaltungsreform) hat der Regierungsrat des Kantons Zürich im Jahr 1995 ein umfassendes Reformprojekt zur Modernisierung der öffentlichen Institutionen eingeleitet. Innerhalb von zwei Legislaturperioden wurden dazu bis zum Jahr 2003 mehr als 60 Einzelprojekte initiiert und weitestgehend abgeschlossen. Die Ziele, die durch das Reformprojekt verfolgt wurden, waren zum einen eine verstärkte Kunden- und Bürgerorientierung und eine Verbesserung der Effizienz bzw. Wirtschaftlichkeit. Daneben wurde eine verstärkte Zielorientierung mit Trennung von strategischer und operativer Führung sowie insgesamt eine Verbesserung eingesetzter Steuerungsprozesse und -instrumente angestrebt. Letztlich sollen durch ein aussagekräftiges Controlling mit Informationen über Leistungen und Wirkungen die Steuerungsfähigkeit der öffentlichen Einrichtungen erhöht werden. Vgl. wif! (2002), S. 3 ff. und wif! (2006) mit einer ausführlichen Dokumentation der einzelnen Reformbausteine. Für eine Evaluation der Verwaltungsreform im Kanton Zürich vgl. econcept (2002).

${ }^{432} \mathrm{Vgl}$. hierzu ausführlich Kapitel 2.2.3.2 und auf die dort verwiesene Literatur.

433 Vgl. wif! (2002), S. 9 f.

434 Vgl. Buschor (2002), S. 65

${ }^{435}$ Buschor (2002), S. 65. 
wenn man den Orientierungsrahmen als Kreislauf interpretiert, mit SOLL-Werten auf der linken und IST-Werten auf der rechten Seite des Modells (siehe nachstehende $A b$ bildung 18). ${ }^{436}$ Controlling wird so als Teil des Führungsprozesses verstanden und schließt die gesamte Regelung des Prozesses der Zielfestlegung, Planung, Steuerung und Berichterstattung in Verwaltung, Regierung und Parlament ein. ${ }^{437}$

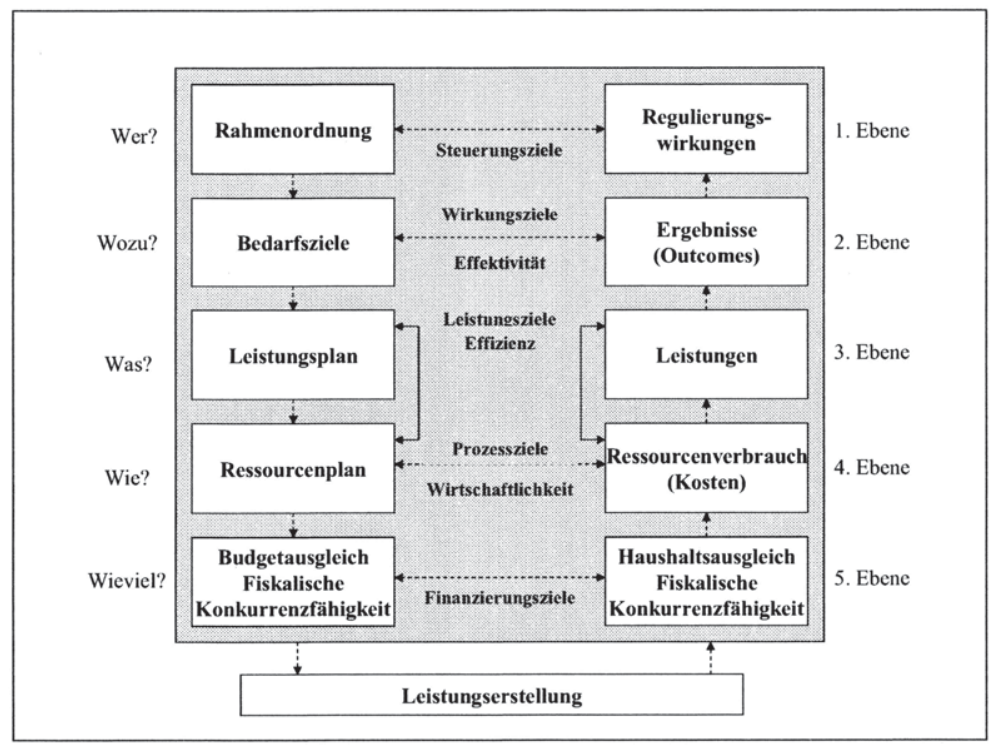

Abbildung 18: Das 5-Ebenenkonzept ${ }^{438}$

Gemäß dem 5-E-Konzept beschäftigt sich die Rahmenordnung zunächst mit der bestmöglichen Trägerschaft einer staatlichen Aufgabe. Auf der 2. Ebene erfolgt die Hinterfragung der Bedarfs- bzw. Wirkungsziele, um Aussagen zur Effektivität zu gewinnen. Mit Hilfe des Leistungs- und Ressourcenplans sollen die Effizienz und Wirtschaftlichkeit der Leistungserstellung sichergestellt werden. Die Effizienz beantwortet die Frage, ob Menge und Qualität der öffentlichen Leistung im Verhältnis zu den Leistungszielen angemessen sind. Die Wirtschaftlichkeit überprüft, ob Leistungen mit möglichst geringen Kosten erbracht wurden. ${ }^{439}$ Auf der letzten Ebene wird die Finanzierbarkeit der staatlichen Leistung vor dem Hintergrund der verfügbaren Mittel beantwortet. Die konsequente Anwendung des 5-E-Konzeptes im Rahmen des Controllings gewährleis-

$436 \mathrm{Vgl}$. Buschor (2002), S. $65 \mathrm{f}$.

437 Vgl. wif? (2002), S. 8.

438 Vgl. wif! (2002), S. 9.

439 Im Gegensatz zum 3-E-Konzept umfasst der Wirtschaftlichkeitsbegriff bei BUSCHOR als Oberbegriff nicht verschiedene Effizienzkriterien, sondern steht gleichbedeutend mit der Kostenwirtschaftlichkeit. 
tet so eine permanente Auseinandersetzung mit den relevanten Fragestellungen und Bewertungskriterien öffentlicher Leistungserstellung. ${ }^{440}$

Zur Überprüfung der genannten Fragestellungen kommen unterschiedliche Controllinginstrumente zum Einsatz, die sich den einzelnen Betrachtungsebenen zuordnen lassen. ${ }^{41}$ So werden von BUSCHOR in einer modifizierten Darstellung des 5-E-Konzeptes die nachfolgenden Instrumente vorgeschlagen. ${ }^{442}$ Der Bereich der Wirkungssteuerung und die Frage nach der optimalen Steuerungsform (z.B. Eigenerstellung durch Verwaltung oder Vertrag mit privatem Anbieter) erfordern den Einsatz aufwendiger sozialwissenschaftlicher Methoden, wie Evaluationen. ${ }^{443}$ Der Bereich der Verwaltungs- oder Betriebssteuerung und der Bereich der Ressourcenoptimierung verlangen zwingend Instrumente der Leistungsmessung und Qualitätssicherung sowie Kostenrechungssysteme. Abschließend erfordert die Einhaltung von Budgetvorgaben den Betrieb der konventionellen Formen der Haushaltsführung. Ergänzt um eine fortlaufende Planung und Berichterstattung können so alle relevanten Daten Eingang in den Steuerungskreislauf des politisch-administrativen Systems finden.

Im Vergleich zum 3-E-Konzept nach BUDÄUS/BUCHHOLTZ handelt es sich beim 5Ebenenmodell um einen deutlich erweiterten Bezugsrahmen einer wirkungsorientierten Controllingkonzeption. So wurde von BUSCHOR aus den abgeleiteten Fragestellungen die Anzahl der Betrachtungsebenen und der zugeordneten Bewertungskriterien von drei auf fünf erhöht. Dies betrifft einerseits die Integration einer notwendigen Rahmenordnung und andererseits die Trennung der Kostenebene von der Finanzebene. Weiterhin wird durch die Gegenüberstellung von SOLL-Werten und IST-Werten der Kreislaufcharakter des Modells betont. In einer modifizierten Darstellung wurde zudem eine explizite Zuordnung wichtiger Controllinginstrumente vorgenommen.

\subsubsection{Das Ziel- und Ergebnisebenen-Modell öffentlicher Leistungserstellung}

In Anlehnung an BUDÄUS/BUCHHOLTZ und BUSCHOR stellen MOSIEK ET AL. mit dem Modell der Ziel- und Ergebnisebenen öffentlicher Leistungserstellung eine modifizierte und erweiterte Darstellung der Ebenenkonzepte vor. ${ }^{444}$ Die Modellkonzeption ist im Rahmen eines Pilotprojektes im Bereich des "Wirkungsorientierten Controllings « für die Bezirksregierung in Münster entwickelt worden. ${ }^{45}$ Das Modell dient als ganzheitlicher Bezugsrahmen für wirkungsorientierte Controlling-Konzeptionen in der öffentli-

\footnotetext{
$440 \mathrm{Vgl}$. Buschor (2002), S. $65 \mathrm{f}$.

441 Vgl. hierzu und im Folgenden Buschor (2002), S. 65 f.

442 Vgl. Buschor (2002), S. 66.

443 Zur Methodik der Evaluation vgl. ausführlich Kapitel 3.4.

444 Vgl. Mosiek et al. (2003), S. 28 ff. und Mosiek/Gerhardt (2003), S. $289 \mathrm{ff}$.

445 Bei dem Pilotprojekt handelt es sich um die Entwicklung einer wirkungsorientierten Controlling-Konzeption für den Vollzug des Bundeserziehungsgeldgesetzes in der Versorgungsverwaltung NRW. Vgl. hierzu sowie zu dem im Rahmen des Projektes entwickelten Modell der Ziel- und Ergebnisebenen öffentlicher Leistungserstellung auch ausführlich BMS Consulting (2004).
} 
chen Verwaltung. Neben der Herstellung von Transparenz, verbesserte Koordinationsmöglichkeiten und Führungsinformationen sollen durch den Bezugsrahmen sowohl die Effizienz als auch die Effektivität öffentlicher Leistungserstellung herausarbeitet sowie Gestaltungsmöglichkeiten aufzeigt werden. ${ }^{446}$ „Das Controlling versorgt in einem so verstanden Kontext, im Sinne einer Führungsunterstützungsfunktion, das Verwaltungsmanagement und die Politik mit allen relevanten Informationen“" ${ }^{447}$

In der Darstellung und Erläuterung des Ziel- und Ergebnisebenenmodells folgen die Autoren dem stufenweisen Aufbau des Bezugsrahmens. ${ }^{448}$ Dieser ist in der nachfolgenden Abbildung 19 dargestellt. Die erste Ebene bildet das immer noch in vielen deutschen Verwaltungsbetrieben dominierende kameralistische Rechnungswesen, über das eine implizit inputorientierte Steuerung per Mittelzuweisung vollzogen wird und retrospektiv die Mittelverwendung dokumentiert werden kann. ${ }^{449}$ Daraus resultieren auf der einen Seite die Finanzziele, die auf der anderen Seite den Finanzergebnissen gegenübergestellt werden, um im Rahmen der Liquiditätssteuerung das Ziel der Budgetkonformität herzustellen.

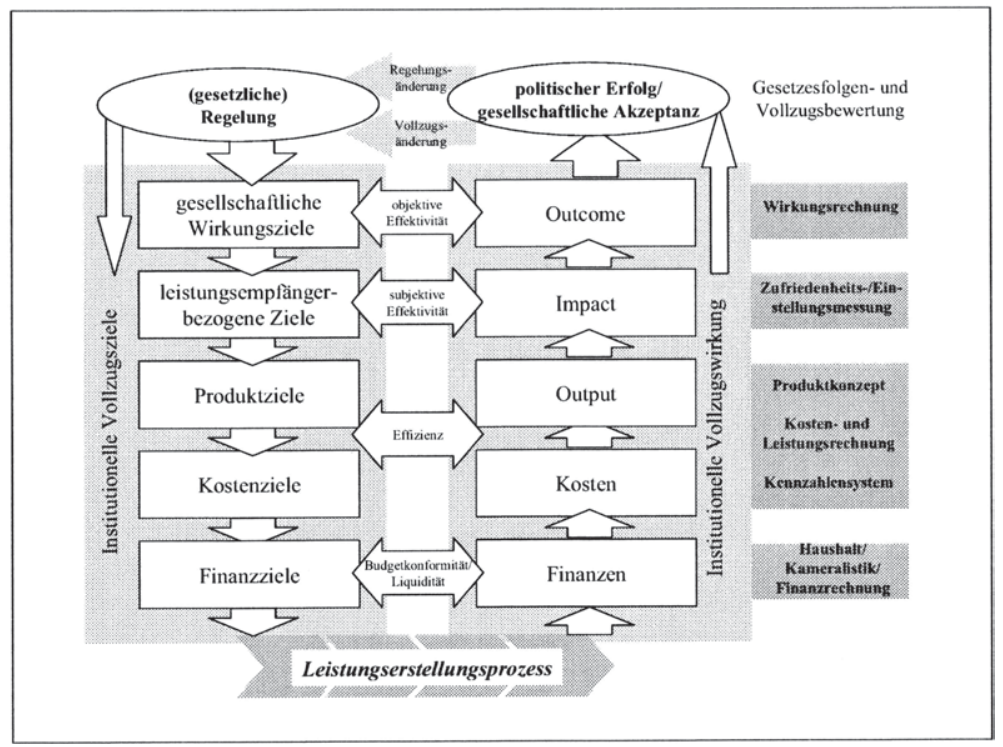

Abbildung 19: Ziel- und Ergebnisebenen öffentlicher Leistungserstellung ${ }^{450}$

446 Vgl. Pook/Fischer (2002), S. 46 und Berens et al. (2004), S. 329.

447 Berens et al. (2003), S. 329.

448 Vgl. Mosiek et al. (2003), S. 28 ff. und BMS Consulting (2004), S. 31 ff.

449 Vgl. Mosiek et al. (2003), S. 28.

450 Vgl. Mosiek et al. (2003), S. 29 und Mosiek/Gerhardt (2003), S. 289. 
Ergänzend zu diesem auf dem Geldverbrauchskonzept basierenden Rechnungswesen wenden sich immer mehr Verwaltungen Ressourcenverbrauchskonzepten zu, indem sie ein kaufmännisches Rechnungswesen und/oder Kostenrechungssysteme einführen. ${ }^{451}$ Insbesondere die Einführung eines Kostenrechungssystems ermöglicht eine zweckorientierte Zuordnung der Kosten zu den Orten ihrer Entstehung - den Kostenstellen sowie zu zuvor definierten Leistungsergebnissen - den Produkten als Kostenträger. Der Vergleich von Kostenzielen und Kostenergebnissen ermöglicht dann für Kostenstellen und Produkte die Beurteilung der Kosteneffizienz.

Im Rahmen eines Produktkonzeptes muss dazu in der öffentlichen Verwaltung allerdings zunächst der Dienstleistungsoutput ${ }^{452}$ in Form von Produkten abgegrenzt und beschrieben werden. Auf dieser Basis kann anhand von Kennzahlen, die das Verwaltungsergebnis kennzeichnen, neben der Kostensteuerung auch eine Leistungsdokumentation in Form von Mengen- und Qualitätsindikatoren vollzogen werden. Die zugehörigen Ziele werden über die Ebene der Produktziele abgebildet, während die Ergebnisse als Output im Modell dargestellt werden. Eine über quantitative und qualitative Kennzahlen operationalisierte Leistungsbewertung stellt damit die Grundlage zur Beurteilung der Bearbeitungseffizienz sowie schließlich für eine outputorientierte Verwaltungssteuerung dar.

Die Besonderheiten der öffentlichen Verwaltung erfordern es aus Sicht von MOSIEK ET AL. auf der Wirkungsebene zwischen dem Impact und den Outcomes zu unterscheiden. ${ }^{453}$ Grundsätzlich werden auf der Wirkungsebene politisch gewollte Ziele mit den tatsächlich eingetretenen Wirkungen verglichen. ${ }^{454}$ Die subjektive Wahrnehmung der adressierten Leistungsempfänger - der Impact - kann jedoch in vielen Fällen von den gesamtgesellschaftlich relevanten Wirkungen - den Outcomes - abweichen. Will man jedoch einerseits die durch die integrative Leistungserstellung bedingte Beeinflussbarkeit des Leistungsergebnisses und andererseits auch die für die Wirkungen bedeutsame Funktion der Zufriedenheit des Leistungsempfängers einer eigenen Analyse zugänglich machen, bietet sich ein separater Ausweis in Form einer Impact-Betrachtung an. ${ }^{455}$ Der Vergleich leistungsempfängerbezogener Zielsetzungen mit dem tatsächlichen Impact führt so zum Bewertungskriterium der subjektiven Effektivität.

Die Befriedigung der subjektiven Kundenbedürfnisse einzelner Leistungsempfänger kann jedoch nur ein Zwischenziel staatlichen Handelns darstellen. In letzter Konsequenz stellen die gesamtgesellschaftlich erzielten Wirkungen bzw. der Outcome und damit die objektive Effektivität des öffentlichen Leistungserstellungsprozesses das re-

\footnotetext{
451 Mosiek et al. (2003), S. 28.

452 Zum Dienstleistungscharakter der öffentlichen Leistungserstellung vgl. Kapitel 2.1.4.

453 Vgl. Mosiek et al. (2003), S. 28. BuDÄUS/BuCHHOLTZ unterscheiden in den erläuterten Erweiterungen zum 3-E-Konzept ebenfalls zwischen einem subjektiven und einem objektiven Outcome, ohne jedoch diesen Sachverhalt explizit im Ebenenmodell abzubilden. Vgl. hierzu die Ausführungen des Kapitels 3.3.2.1 bzw. Budäus/Buchholtz (1997), S. 328.

455 BMS Consulting (2004), S. 36.
} 
levante Bewertungskriterium dar. ${ }^{456}$ Dazu sehen die Autoren den Einsatz von Wirkungsrechnungen vor, indem anhand spezifischer Wirkungsindikatoren die Zielgerechtigkeit und damit die objektive Effektivität einer staatlichen Maßnahme sowie der flankierenden Aufgabenwahrnehmung bewertet werden. Im Idealfall können über derartige Analysen die Wirkungstreiber identifiziert werden, um diese einer Steuerung zugänglich $\mathrm{zu}$ machen und somit schließlich eine wirkungsorientierte Verwaltungssteuerung ermöglichen. ${ }^{457}$

Der Grundsatz der Recht- und Gesetzmäßigkeit staatlichen Handelns ist über eine weitere Ebene im Modell abgebildet. Die oberste Ebene der Zielhierarchie bilden die politischen und gesetzlichen Rahmenvorgaben, welche über die nachfolgenden Zielebenen einer zunehmenden Ausführungskonkretisierung bedürfen und letztlich den Prozess der Leistungserstellung auslösen. Die Überprüfung der Zielerreichung über die verschiedenen Ergebnisebenen und die Analyse der mit der Maßnahme verbundenen Wirkungen gewährleisten eine objektive Information der politischen Instanzen. Der Wirkungskreis schließt sich, wenn es gelingt, die gesellschaftliche Akzeptanz bzw. den politischen Erfolg einer Maßnahme transparent und bewertbar zu machen. Durch eine Gesetzesfolgen- und Vollzugsbewertung wird es der politischen Ebene ermöglicht, auf Grundlage objektiver Kriterien, die notwendigen Anpassungen an den gesetzlichen Regelungen und am Vollzugsmodell vorzunehmen. ${ }^{458}$

Die Hierarchie der Ziel- und Ergebnisebenen zeigt, dass ein konsequentes Verwaltungscontrolling die Wirkungsebene nicht ignorieren darf, auch wenn die in der Verwaltung dominierende Ausführungsfunktion vielfach eine Fokussierung auf operative Aspekte der Leistungserstellung impliziert. Neben der theoretischen Abgrenzung des operativen und strategischen Controllings zeigt die prozessorientierte Zusammenführung der Ziel- und Ergebnisebenen, unter Berücksichtigung wirkungsorientierter politischer Vorgaben, wie Steuerungskreise von Verwaltung und Politik ineinander übergehen. Innerhalb „eines solchen Modells kann es gelingen, den Realisations- und Wirkungsprozess zu strukturieren, sowie Instrumente zur Abbildung und Steuerung zuzuordnen“. 459

Im Vergleich zu den Konzepten nach BUDÄUS/BUCHHOLTZ und BUSCHOR handelt es sich beim Modell der Ziel- und Ergebnisebenen von MOSIEK ET AL. um den differenzierteren Bezugsrahmen für ein wirkungsorientiertes Controlling. So wurde durch die Trennung von Impact und Outcome eine weitere Bewertungsebene explizit in den Modellrahmen eingeführt, wodurch auf der Wirkungsebene zusätzliche Analysen möglich sind. Weiterhin stellt die Betonung der Zielhierarchie mit einer zunehmenden Ausführungskonkretisierung über die einzelnen Betrachtungsebenen eine klare Verbindung zu

\footnotetext{
456 Vgl. Mosiek et al. (2003), S. 28 f.

457 Zum wirkungsorientierten Steuerungsansatz im politisch-administrativen System vgl. ausführlich Kapitel 2.2.3.1 dieser Arbeit, zu den damit verbunden Chancen und Herausforderungen vgl. detailliert Kapitel 2.2.3.3 und Kapitel 2.2.3.4 dieser Arbeit.

458 Zu den Verfahren der Gesetzesfolgenabschätzung vgl. Böhret/Konzendorf (2000), S. 9 ff.

459 Berens et al. (2004), S. 330.
} 
den unterschiedlichen Steuerungsarten des politisch-administrativen Prozesses ${ }^{460}$ her. Im Modellrahmen gelingt es somit, die Steuerungsansätze nicht als Alternativen, sondern als sich ergänzende Betrachtungsperspektiven auf dem Evolutionspfad zur Wirkungsorientierung abzubilden ${ }^{461}$ Auch die Zuordnung notwendiger Controllinginstrumente zu den einzelnen Bewertungsebenen erscheint bei MOSIEK ET AL. klarer strukturiert und insgesamt differenzierter vorgenommen.

\subsubsection{Das Erweiterte Ziel- und Ergebnisebenen-Modell öffentlicher Leistungs- erstellung}

Eine nochmals modifizierte und erweiterte Darstellung der Modellkonzeption von MOSIEK ET AL. wurde schließlich von RÖHRIG mit dem Erweiterten Ziel- und Ergebnisebenen-Modell vorgestellt. ${ }^{462}$ In einer Analyse ausgewählter Ebenenmodelle identifiziert RÖHRIG in den bestehenden Konzeptionen für das wirkungsorientierte Controlling noch einige Schwachstellen. ${ }^{463}$ Als Ergebnis der Analyse kann festgehalten werden, dass das Ziel- und Ergebnisebenenmodell von MOSIEK ET AL. dem „Idealbild eines integrierten Bezugsrahmens am Nähesten kommt" - aber noch Weiterentwicklungsbedarf besteht. ${ }^{464}$ Zur Beseitigung der identifizierten Schwachstellen dient es RÖHRIG daher als maßgebliche Grundlage für die Ableitung einer eigenen ControllingKonzeption. Im Folgenden werden deshalb nur die in diesem Zusammenhang vorgenommenen Erweiterungen und Modifikationen am Ziel- und Ergebnisebenen-Modell dargestellt.

Eine wesentliche Erweiterung betrifft die Integration ganzheitlicher Qualitätsdimensionen in den Bezugsrahmen. „Das Zusammenführen umfassender Aspekte des zugrunde liegenden Qualitätsmanagementverständnisses für das politisch-administrativen System mit der prozessorientierten Sichtweise bisheriger Controlling-Modelle verdeutlicht den Anspruch zur Schaffung eines integrierten Bezugsrahmens". ${ }^{465}$ Die zweite wesentliche Erweiterung betrifft die Differenzierung des Leistungserstellungsprozesses - hier verstanden als der eigentliche Produktionsprozess öffentlicher Leistungserstellung - als eine Kombination von internen und externen Inputfaktoren in einer Wertekette. ${ }^{466}$

Modifikationen wurden einerseits bei der Fusion der unteren beiden Ziel- und Ergebnisebenen des Bezugsrahmens vorgenommen, um hier die zunehmende Konvergenz des öffentlichen Rechnungswesens zu berücksichtigen. Andererseits wurde die ImpactEbene eingeschränkt, da „die Erfüllung leistungsempfängerbezogener Ziele nicht in

\footnotetext{
$460 \mathrm{Zu}$ den unterschiedlichen Steuerungsarten vgl. ausführlich Kapitel 2.2.3.1.

461 Vgl. hierzu auch Abbildung 9 auf Seite 37 sowie Abbildung 10 auf Seite 40 dieser Arbeit.

462 Vgl. hierzu und im Weiteren ausführlich Röhrig (2008), S. $105 \mathrm{ff}$.

463 Vgl. Röhrig (2008), S. 99 ff.

464 Röhrig (2008), S. 104.

465 Röhrig (2008), S. 105.

466 Zum Instrument der Wertekette vgl. Porter (1999), S. 66 ff.
} 
allen Fällen zweckmäßig erscheint" ${ }^{467}$ Eine zusätzliche Erweiterung hat der Modellrahmen auch hinsichtlich der Zuweisung weiterer Controlling-Instrumente erfahren. Abbildung 20 stellt das Erweiterte Ziel- und Ergebnisebenen-Modell öffentlicher Leistungserstellung als Bezugsrahmen für ein wirkungsorientiertes Controlling im politisch-administrativen System dar.

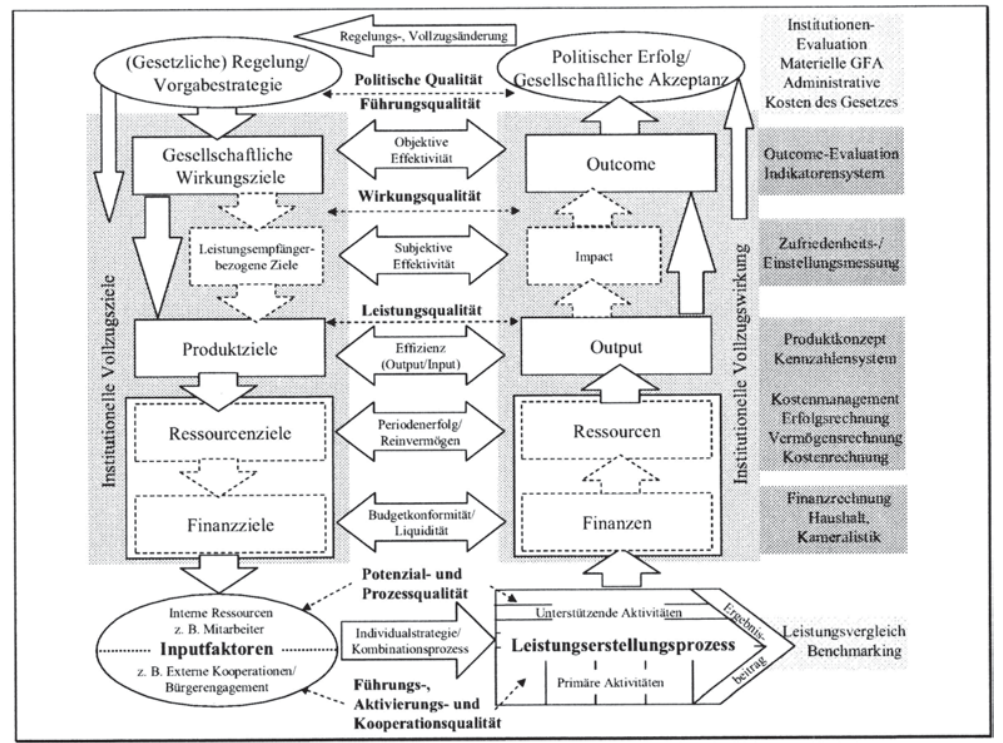

\section{Abbildung 20: Das Erweiterte Ziel- und Ergebnisebenenmodell öffentlicher Leistungserstellung ${ }^{468}$}

Auffällig ist zunächst die Vielzahl an unterschiedlichen Qualitätsdimensionen, die in der Modellkonzeption von RÖHRIG Berücksichtigung finden. ${ }^{469}$ Diese lassen sich grob drei unterschiedlichen Bereichen zuordnen:

- Der erste Bereich betrifft den eigentlichen Leistungserstellungsprozess. Die Potenzial- und Prozessqualität bezieht sich auf die internen Ressourcen des öffentlichen Sektors. Hiermit wird einerseits das Potenzial an sachlichen und personellen Ressourcen sowie der organisatorischen Strukturen beschrieben und andererseits die Fähigkeit, diese im Prozess der Leistungserstellung effizient einzusetzen. Die Akti-

467 Röhrig (2008), S. 106.

468 Vgl. Röhrig (2008), S. 109.

469 RÖHRIG definiert in diesem Zusammenhang ,Qualität als die Gesamtheit von Merkmalen und Eigenschaften einer öffentlichen Aufgabe bzw. eines öffentlichen Aufgabenvollzugs, welche dazu geeignet sind, explizit oder implizit gegebene Erfordernisse zu erfüllen“; Röhrig (2008), S. 79 in Anlehnung an Broekmate/Dahrendorf/Dunker (2001), S. $36 \mathrm{f}$. 
vierungs- und Kooperationsqualität richtet sich demgegenüber auf die externen Inputfaktoren, d.h. inwieweit es gelingt, die Bürger zur aktiven Teilnahme am Leistungsprozess zu aktivieren bzw. nützliche Kooperationen mit externen Akteuren ${ }^{470}$ einzugehen. Zusätzlich ist im Modellrahmen die Führungsqualität ausgewiesen, welche die Fähigkeit der Verwaltungsführung beschreibt, interne und externe Inputfaktoren zielgerichtet einzusetzen.

- Der zweite Bereich betrifft die Qualitätsdimensionen der Ergebnisebene. Im Prozessmodell öffentlicher Leistungserstellung sind hier insbesondere das direkte Leistungsergebnis, d.h. der Output sowie die mit dem Output verbundenen Wirkungen zu unterscheiden. Entsprechend integriert RöHRIG auf der Produktebene die Leistungsqualität und auf der Wirkungsebene die Wirkungsqualität in den Bezugsrahmen.

- Letztlich wird der Prozess öffentlicher Leistungserstellung erheblich durch politische Vorgaben beeinflusst. „Die politische Qualität sowie die damit zusammenhängende politische Erfolgskontrolle beinhalten u.a. Aussagen, inwieweit - unabhängig von der eigentlichen Leistungserstellung - politische Vorgaben und Programme dazu geeignet sind, beabsichtigte Veränderungen in der Gesellschaft zu erzielen“". 471

Eine deutliche Erweiterung hat die Modellkonzeption auch auf der Ebene der Leistungserstellung - verstanden als der eigentliche „Produktionsprozess“ zur Erfüllung der öffentlichen Aufgabe ${ }^{472}$ - erfahren. In Anlehnung an das Modell der Wertekette aus der Privatwirtschaft nimmt RÖHRIG auch für den öffentlichen Sektor eine Differenzierung in primäre Aktivitäten und unterstützende Aktivitäten der Leistungserstellung vor. ${ }^{473}$ Das Modell der Wertekette erlaubt in diesem Zusammenhang die Identifikation von Wert- und Ergebnistreibern, um entsprechend des Handlungs- und Entscheidungsspielraums der Beteiligten den Ergebnisbeitrag auf den unterschiedlichen Ergebnisebenen zu optimieren. Aufgrund des Dienstleistungscharakters öffentlicher Leistungen $^{474}$ ist der Produktionsprozess zudem als eine Kombination interner Ressourcen (z.B. Mitarbeiter) mit externen Faktoren (z.B. Bürger) zu verstehen. Das Ergebnis der Leistungserstellung ist somit nicht nur vom administrativen Ressourceninput, sondern auch von der Einbindung und Beteiligung externer Faktoren abhängig.

Eine weitere Ausdifferenzierung hat das Erweiterte Ziel- und Ergebnisebenenmodell letztlich auch beim zugeordneten Controllinginstrumentarium erfahren. Für die operative Ebene des eigentlichen Leistungserstellungsprozesses werden von RÖHRIG der Einsatz von Leistungsvergleichen und Benchmarking ${ }^{475}$ vorgeschlagen. ${ }^{476}$ Hierdurch

\footnotetext{
470 In diesem Zusammenhang ist beispielsweise auf die Private Public Partnership-Modelle zu verweisen. Vgl. für einen Überblick Schedler/Proeller (2003), S. 219 ff. bzw. ausführlich Roschmann (2005), S. 36 ff.

471 Röhrig (2008), S. 109 f. in Anlehnung an Wegener (2002), S. 89 f.

472 Vgl. Röhrig (2008), S. 110.

473 Vgl. Röhrig (2008), S. $110 \mathrm{ff}$.

$474 \mathrm{Vgl}$. hierzu ausführlich Kapitel 2.1.3 und Kapitel 2.1.4.

475 Zum Benchmarking in der öffentlichen Verwaltung vgl. Burr/Seidlmeier (1998), S. 55 ff.

476 Vgl. Röhrig (2008), S. $110 \mathrm{ff}$.
} 
kann im Wege laufender, auf Kennzahlen gestützter intra- und inter-organisatorischer Leistungsvergleiche ein (Quasi-)Wettbewerb um eine möglichst hohe Bearbeitungseffizienz erreicht werden. ${ }^{47}$ Auf der Finanz- und Ressourcenebene greift RöHRIG die aktuellen Entwicklungen im öffentlichen Rechungswesen auf. ${ }^{478}$ Das im öffentlichen Sektor immer noch vorherrschende Haushaltswesen der Kameralistik ${ }^{479}$ wird zunehmend durch ein doppisches Rechnungswesen ${ }^{480}$ ergänzt bzw. abgelöst. Zusätzlich wird mit dem Einsatz einer Kosten- und Leistungsrechnung die Ressourcenbetrachtung vervollständigt, indem durch Zurechnung sämtlicher Kosten auf die Produkte der Verwaltung die Grundlage für eine umfassende Kostentransparenz geschaffen wird.

Für die Wirkungsebene und die Ebene der Gesetzgebung empfiehlt RöHRIG den Einsatz sozialwissenschaftlicher Methoden der Evaluation. ${ }^{481}$ Einem weiten Begriffsverständnis folgend ist "Evaluation the systematic investigation of the merit or worth of an object (program) for the purpose of reducing uncertainty in decision making" ${ }^{482}$ Evaluationen, die auf die Wirkungen eines staatlichen Programms bezogen sind, bezeichnet man auch als Outcome-Evaluation. Zur Unterstützung der Wirkungsanalyse kommen dazu häufig Indikatorensysteme zum Einsatz. Auf der Ebene der Gesetzgebung spricht man dagegen von einer Institutionen-Evaluation sowie dem ebenfalls unterstützenden Instrument der Gesetzesfolgenabschätzung. ${ }^{483}$

\subsubsection{Vergleichende Bewertung der dargestellten Ebenenmodelle}

Die Unterschiede zwischen den vorgestellten Modellkonzeptionen wurden bereits ausführlich thematisiert und sollen hier nochmals zusammengefasst und entsprechend gewürdigt werden. Mit dem 3-Ebenen-Konzept sowie dem 5-Ebenen-Konzept nach BUDÄUS/BUCHHOLTZ und BUSCHOR sowie den Modellen der Ziel- und Ergebnisebenen nach MOSIEK ET AL. und RÖHRIG wurden umfassende Bezugsrahmen für ein wirkungsorientiertes Controlling dargestellt und erläutert. Durch eine kurze vergleichende Bewertung der Konzeptionen ist demnach das geeignete Modell für den Fortgang der Untersuchung auszuwählen.

477 Vgl. Schuster (2001), S. $201 \mathrm{ff}$.

$478 \mathrm{Vgl}$. Röhrig (2008), S. $115 \mathrm{ff}$.

479 Die Kameralistik ist eine periodisierte Einnahmen/Ausgabenrechnung und delegiert Verfügungsrechte über finanzielle Ressourcen in Form inhaltlich abgegrenzter Kapitel und Titel. Die Mittel dürfen ausschließlich für den bezeichneten Zweck, für die angegebene Höhe und nur bis zum Ende des Haushaltsjahres in Anspruch genommen werden. Die Zurechnung von monetären Größen auf Perioden erfolgt durch das Zahlungsprinzip, d.h. einzelne Zahlungsvorgänge werden sowohl chronologisch als auch sachspezifisch nach Haushaltsstellen erfasst.

480

Grundsätzlich kann man zwischen dem Verfahren der erweiterten Kameralistik und einem doppischen Rechnungswesen unterscheiden. Das doppische Rechnungskonzept folgt einem dreistufigen Aufbau in Form einer integrierten Ergebnis-, Finanz- und Vermögensrechnung, welche systematisch miteinander verbunden sind. Vgl. hierzu ausführlich Lüder (1999) und Lüder (2001) sowie Budäus (2002b), S. 389 ff.

Vgl. Röhrig (2008), S. 139 ff.

482

Mertens (1998), S. 219; zur Diskussion unterschiedlicher Definitionen vgl. Stockmann (2004), S. 13 ff.

483 Vgl. hierzu ausführlich Böhret/Konzendorf (2001); Konzendorf (2005) oder auch Bräunlein (2004). 
Mit ihrer Synopse wichtiger internationaler Ansätze der politischen Erfolgskontrolle haben BUDÄUS/BUCHHOLTZ zunächst die Grundlagen für die nachfolgenden Arbeiten geschaffen. Durch ihre übersichtliche Darstellung unterschiedlicher Prozessmodelle öffentlicher Leistungserstellung sowie zugeordneter Bewertungskriterien wurde zunächst ein Referenzmodell entwickelt und damit ein einheitlicher Diskussionsstand im Forschungsbereich ermöglicht. Eine deutliche Erweiterung dieses Bezugsrahmens hat BUSCHOR mit dem 5-Ebenen-Konzept vorgenommen. Als Grundgerüst der schweizerischen Verwaltungsmodernisierung wurde von ihm einerseits der Kreislaufcharakter betont - Planung, Umsetzung und Kontrolle mit einer entsprechenden Rückkopplung zur Planung. Andererseits wurden mit der Rahmenordnung sowie der Trennung von Finanz- und Kostenebene zwei weitere Ebenen in den Modellrahmen integriert.

Die anschließenden Arbeiten von MOSIEK ET AL. haben zu einer weiteren Ausdifferenzierung der Wirkungsebene geführt. So wurde mit der Trennung des Leistungsimpact "verstanden als subjektive Kundenbedürfnisse" vom Outcome "verstanden als eher objektive gesamtgesellschaftliche Vollzugswirkung« eine weitere Analyseebene explizit in das Modell eingefügt. Zudem berücksichtigt der stufenweise Aufbau des Bezugsrahmens die unterschiedlichen Steuerungsansätze des politisch-administrativen Systems. Eine zusätzliche Erweiterung des Modellrahmens hat zuletzt RÖHRIG vorgenommen, indem von ihm insbesondere unterschiedliche Qualitätsdimensionen sowie die Ebene des eigentlichen Leistungserstellungsprozesses in den bestehenden Modellrahmen integriert wurden.

Für den weiteren Verlauf der Untersuchung wird daher das Modell der Ziel- und Ergebnisebenen nach MOSIEK ET AL. als geeigneter Bezugsrahmen für ein wirkungsorientiertes Controlling herangezogen. Soweit notwendig, findet an ausgewählten Stellen eine Berücksichtigung der Erweiterungen von RÖHRIG statt. Sowohl der systematische Aufbau dieser Modellkonzeption als auch der Umfang und Differenzierungsgrad der berücksichtigten Analyseperspektiven mit den jeweils zugeordneten Bewertungsinstrumenten erscheinen hier im Vergleich am umfassendsten vorgenommen. Der Modellrahmen ist damit am ehesten geeignet, die aus der wirkungsorientierten Steuerung resultierenden inhaltlichen und methodischen Herausforderungen an die Ausgestaltung eines wirkungsorientierten Controllings zu bewältigen.

\subsubsection{Implikationen des bisherigen Untersuchungsverlaufs}

Bevor sich die nachfolgenden Ausführungen detailliert mit der instrumentellen Ausgestaltung der einzelnen Ziel- und Ergebnisebenen des wirkungsorientierten Controllings auseinandersetzen, soll der bisherige Zwischenstand der Untersuchung zur Wirkungsorientierung staatlichen Handelns an dieser Stelle kurz zusammengefasst werden. Die Realisierung eines wirkungsorientierten Steuerungsansatzes ist für das politisch-administrative System mit vielfältigen Chancen verbunden. ${ }^{484} \mathrm{Im}$ Zuge der „Neuen Steuerung“" wurde daher auch in Deutschland der Pfad zur Wirkungsorientierung

$484 \mathrm{Zu}$ den Chancen der Wirkungsorientierung vgl. ausführlich Kapitel 2.2.3.3. 
eingeschlagen. ${ }^{485}$ Neben dem Output der staatlichen Leistungserstellung werden zunehmend auch die hiermit verbundenen Impacts und Outcomes in das Zentrum des Interesses rücken und es wird vermehrt nach der eigentlichen Wirksamkeit des staatlichen Ressourceneinsatzes gefragt. Das die Wirkungsorientierung bislang faktisch noch keinen Eingang in die bestehenden Steuerungskreisläufe von Politik und Verwaltung gefunden hat, ist auf bisher ungelöste Problembereiche zurückzuführen. Im Rahmen dieser Arbeit wurden sowohl methodische Herausforderungen ${ }^{486}$, als auch die klare Zuweisung von Verantwortlichkeiten ${ }^{487}$ sowie die Einbindung der politischen Ebene ${ }^{488}$ als zentrale Problembereiche der Wirkungsorientierung identifiziert. ${ }^{489}$ Der Erfolg der wirkungsorientierten Steuerung als praxistaugliches Konzept und die Realisierung der damit verbundenen Chancen liegt demnach in einer schrittweisen Überwindung der aufgezeigten Problemstellungen.

Ein hohes Potenzial - zumindest die methodischen Herausforderungen ansatzweise zu überwinden - wird in diesem Zusammenhang dem Verwaltungscontrolling zugesprochen. Die Frage nach den Wirkungen staatlichen Handelns bildet damit den Ausgangspunkt zur Weiterentwicklung des Verwaltungscontrollings zu einem wirkungsorientierten Controlling. ${ }^{490}$ Die im Zuge des New Public Management häufig betonte Schlüsselfunktion des Controllings für den Modernisierungsprozess in der öffentlichen Verwaltung wird hierdurch weiter untermauert. ${ }^{491}$ Als Instrument der Führungsunterstützung hat Controlling im politisch-administrativen System somit auch die Aufgabe, sachgerechte und praktikable Methoden bereitzustellen, die eine systematische Analyse und Steuerung der Wirkungen staatlichen Handelns erlauben.

Mit den vorgestellten Bezugsrahmen eines wirkungsorientierten Controllings gelingt es zunächst, den komplexen Prozess öffentlicher Leistungserstellung sowie die relevanten Betrachtungsebenen und Bewertungskriterien angemessen zu strukturieren. Fraglich ist nun, inwieweit das auf den einzelnen Ziel- und Ergebnisebenen bereitgestellte Control-

485 Der Pfad zur Wirkungsorientierung wurde in Kapitel 2.2.3.1 aufgezeigt.

486 Die methodischen Probleme betreffen einerseits die Schwierigkeit, komplexe Ursache-Wirkungszusammenhänge zweifelsfrei nachzuweisen und auf die Produkte staatlichen Handelns zurückzuführen. Andererseits müssen objektive Indikatoren zur Operationalisierung und Messung der ausgelösten Wirkungen gefunden werden.

487 Häufig sind realisierte Wirkungen auf mehrere Ursachen zurückzuführen, da einerseits staatliche Wirkungskoalitionen aus mehreren Verwaltungsbreichen und andererseits auch externe Effekte dafür verantwortlich sein können. Die insgesamt geringe Kontrollierbarkeit von Wirkungen erschwert damit die eindeutige $\mathrm{Zu}$ weisung von Verantwortung.

488 Fraglich bleibt, inwieweit die politisch Verantwortlichen im System der Konkurrenzdemokratie überhaupt an einer Vorgabe transparenter und messbarer Wirkungsziele, die ein realistisches Bild des Wirkungsgrades staatlichen Handelns zeichnen, interessiert sind. Grundsätzlich bieten klare und messbare Ziele sowohl der Opposition als auch dem Bürger bei Zielverfehlung eine offene Angriffsfläche, was einem langfristigen politischen Erfolg eventuell eher im Wege steht. Vgl. hierzu auch Fiedler/Vernau (2000), S. 35 ff.

Zur systematischen Aufarbeitung der Problembereiche der Wirkungsorientierung vgl. auch Kapitel 2.2.3.4.

490 Vgl. u.a. Eichhorn/Wiechers (2001); Reichhard (2002), S. 36; Berens/Mosiek/Röhrig/Gerhardt (2004); Brüggemeier (2004); Röhrig (2008); KGSt (2000) und KGSt (2001).

491 Vgl. Budäus (2002a), S. 207. 
ling-Instrumentarium die beteiligten Akteure dazu in die Lage versetzt, die Bewertungsebenen des Leistungsprozesses sachgerecht abzubilden. Der nächste Untersuchungsschritt besteht demnach in einer Analyse, ob die instrumentelle Ausgestaltung der Ebenenmodelle den Informationserfordernissen der Akteure im politisch-administrativen System angemessen Rechnung tragen kann oder hier noch Defizite bestehen.

\subsection{Instrumentelle Defizite in der systematischen Erfassung und Bewer- tung der Wirkungen staatlichen Handelns}

Das Modell der Ziel- und Ergebnisebenen öffentlicher Leistungserstellung nach MOSIEK ET AL. weist insgesamt fünf unterschiedliche Bewertungsebenen auf, denen jeweils unterschiedliche Controllinginstrumente zugeordnet sind. ${ }^{492}$ Diese Controllinginstrumente lassen sich grob in drei unterschiedliche Kategorien einteilen. Auf der Ebene des Ressourcenverbrauchs sind dies Bewertungsinstrumente zur Finanz- und Kostenkontrolle. Auf der Produktebene werden Instrumente zur Leistungsanalyse eingesetzt und auf der Wirkungsebene sind Instrumente zur Bewertung leistungsempfängerbezogener und gesellschaftlicher Wirkungsziele notwendig. Im Folgenden sollen die jeweils einsetzbaren Instrumentarien vorgestellt und auf ihre Tauglichkeit für einen systematischen wirkungsorientierten Controllingprozess hin überprüft werden.

Auf der Ebene des Ressourcenverbrauchs werden im öffentlichen Sektor bereits vielfältige Anstrengungen unternommen. Mit der Umstellung auf das doppische Rechnungswesen befindet sich das gesamte öffentliche Haushaltswesen derzeit in einem tiefgreifenden Reformprozess. ${ }^{493}$ Das aktuell noch vorherrschende kameralistische Rechnungswesen wird in den nächsten Jahren schrittweise abgelöst. ${ }^{44}$ Hierdurch gelingt auch in öffentlichen Haushalten erstmals eine periodengerechte Abgrenzung der Aufwendungen sowie die Gegenüberstellung des gesamten Vermögens und der gesamten Schulden - beispielsweise durch die Berücksichtigung von Pensionsrückstellungen für verbeamtete Mitarbeiter. Dies führt zu einer transparenten Dokumentation der Finanzierungsverhältnisse des Haushaltes, so dass nicht wie bei der Kameralistik der Anreiz besteht, laufende Betriebsausgaben zu Lasten zukünftiger Generationen zu finanzieren. ${ }^{495} \mathrm{Im}$ Ergebnis wird damit die Grundlage für eine wirtschaftliche Steuerung des staatlichen Mitteleinsatzes - auch im Sinne einer intergenerativen Gerechtigkeit geschaffen.

Gleichzeitig wurde bereits in vielen Bereichen der öffentlichen Verwaltung eine Kosten- und Leistungsrechnung zur differenzierten Erfassung und Darstellung des Ressourcenverbrauchs eingeführt. ${ }^{496}$ Die Kosteninformationen dienen einerseits der mittelfristigen Planung des notwendigen Ressourceneinsatzes, andererseits der Kontrolle

\footnotetext{
492 Vgl. hierzu ausführlich Kapitel 3.3.2.3.

$493 \mathrm{Vgl}$. hierzu beispielsweise Bräunig (2004), S. 309 ff. oder auch Berens et al. (2005), S. $887 \mathrm{ff}$.

494 Zum gegenwärtigen Umstellungsprozess in den einzelnen Bundesländern vgl. Reichard (2004), S. $87 \mathrm{ff}$.

495 Vgl. Bräunig (2004), S. 312 und Eichhorn (1987), S. 56.

496 Zum derzeitigen Umsetzungstand der KLR in der öffentlichen Verwaltung vgl. Bogumil/Kuhlmann (2004). 
der Kostenwirtschaftlichkeit sowie der Dokumentation von Kostendeckungsgraden, beispielsweise zur Gebührenkalkulation. ${ }^{497}$ Die hiermit geschaffene Transparenz der Kostensituation stellt zudem zuverlässige Daten für make-or-buy-Entscheidungen zur Verfügung. ${ }^{498}$

Die Kostenartenrechnung bietet die Möglichkeit, kalkulatorische Kostenbestandteile in die Betrachtung aufzunehmen, wie zum Beispiel kalkulatorische Abschreibungen oder kalkulatorische Zinsen. Im Rahmen einer Kostenstellenrechnung können innerbetriebliche Verantwortungsbereiche abgegrenzt werden, um den Werteverzehr der Ressourcen verursachungsgerecht zuzurechnen und beispielsweise durch Soll-Ist-Vergleiche Kostentransparenz zu schaffen. Mit Hilfe der Kostenträgerrechnung kann der Ressourcenverzehr schließlich den Produkten der öffentlichen Verwaltung ${ }^{499}$ zugeordnet werden und damit Informationen darüber bereitstellen, welche Leistungsergebnisse (Outputs) die Kostenentstehung verursacht haben. Diese Informationen sind nicht nur für die Steuerung des politisch-administrativen Systems relevant, sondern prägen auch das Verhalten der Akteure in Verwaltung, Politik und Öffentlichkeit - letztlich kann „ohne Kosteninformationen kein Kostenbewusstsein entstehen“. 500

Voraussetzung für den Einsatz einer Kostenträgerrechnung ist ein Produktkonzept, welches auf der Ebene des Outputs die Aktivitäten einer öffentlichen Verwaltung zu Produkten zusammenfasst. Bei Produkten handelt es sich um die kleinsten selbständigen Leistungs- bzw. Dienstleistungseinheiten, die von Kunden der Verwaltung genutzt werden können. ${ }^{501}$ Durch die Bildung übergeordneter Produktgruppen und Produktbereiche lässt sich eine Produkthierarchie abbilden, die im Ergebnis einen Gesamtüberblick über das gesamte Leistungsspektrum der öffentlichen Verwaltung ermöglicht. ${ }^{502}$

Neben der Erhebung von produktbezogenen Kostendaten durch die KLR, erlauben Kennzahlensysteme die quantitative Erfassung relevanter Leistungsdaten. ${ }^{503}$ Kennzahlen sind i.d.R. direkt messbar und beziehen sich auf wichtige behördliche Tatbestände

497 Vgl. Röhrig (2008), S. 120 f. und Siems (2005), S. 56 ff.

$498 \mathrm{Vgl}$. Schedler/Proeller (2003), S. 205.

499 Bei den Produkten der öffentlichen Verwaltung handelt es sich überwiegend um Dienstleistungen, vgl. hierzu ausführlich Kapitel 2.1.3.

500 Beyer/Kienzle (2005), S. 353; vgl. auch Röhrig (2008), S. 117.

501 Als selbständig gilt eine Leistungseinheit, wenn sie ohne Bezug einer weiteren Leistung einen Nutzen stiftet. Vgl. Schedler/Proeller (2003), S. 127.

502 Vgl. Bähr (2002), S. 41.

503 Eine über quantitative und qualitative Kennzahlen operationalisierte Leistungsbewertung stellt die Grundlage für die outputorientierte Verwaltungssteuerung dar. Die Anwendung von Kennzahlen bietet jedoch vielfach die Gefahr, das regelmäßig komplexe Leistungsergebnis nicht umfassend und damit nicht steuerungskonform abzubilden. Daher müssen neben direkt messbaren Kennzahlen häufig auch Indikatoren erhoben werden, um nicht direkt wahrnehmbare bzw. nicht direkt messbare Tatbestände abzubilden. Neben Bestandsindikatoren, welche Umfeldzustände dokumentieren, vermag der ergänzende Einsatz von Aktivitätsindikatoren (Ergebnis pro Zeiteinheit) für eine effiziente Produkterstellung erfolgskritische Ereignisse zu dokumentieren. Darüber hinaus können anhand von Qualitätsindikatoren Aussagen über die Sachgerechtigkeit, Zeitgerechtigkeit und Wirtschaftlichkeit getroffen werden, was gleichsam in Verbindung mit Aktivitätsindikatoren den Output der Verwaltung determiniert. Vgl. hierzu BMS Consulting (2004), S. 35; auch Röhrig (2008), S. 127 f. 
und stellen diese in konzentrierter Form dar. In dieser Weise werden die relevanten quantitativen und qualitativen Aspekte der Verwaltungsprodukte erfasst. Über die Produkte der Verwaltung lässt sich idealerweise auch eine Verbindung zur Wirkungsebene (Impact und Outcomes) herstellen, indem strategische Fragestellungen nach den Wirkungszielen bzw. den tatsächlich erreichten Wirkungen beantwortet werden. ${ }^{504}$

Mit der Kosten- und Leistungsrechnung sowie den verbundenen Produktkonzepten und Kennzahlensystemen sind bereits wichtige Instrumente des Verwaltungscontrollings in weiten Teilen der öffentlichen Verwaltung eingeführt. ${ }^{505}$ Insbesondere auf kommunaler Ebene ist die Modernisierung auch in Deutschland mittlerweile weit fortgeschritten. Die staatlichen Ebenen der Bundesländer sowie des Bundes sind insgesamt etwas zurückhaltender - befinden sich vielfach jedoch auch in einem konsequenten Modernisierungsprozess. ${ }^{506}$ Weiterhin ist auch die beschriebene Umstellung des Rechnungsund Haushaltswesens zumindest auf kommunaler Ebene schon auf den Weg gebracht, so dass durch die Ablösung der Kameralistik in den nächsten Jahren eine deutliche Verbesserung der Transparenz des Rechenwerks eintreten wird. ${ }^{507}$

Während auf der Ebene des Ressourcenverbrauchs und des Verwaltungsoutputs bereits ein hoher Umsetzungsgrad wichtiger Controllinginstrumente festzustellen ist, sind vergleichbare Maßnahmen auf der Wirkungsebene nicht zu verzeichnen. Strategische Fragestellungen nach den Wirkungszielen sowie den tatsächlich erreichten Wirkungen der öffentlichen Verwaltung bleiben damit regelmäßig unbeantwortet. In Deutschland konzentrieren sich die Bemühungen bislang erst auf einige wenige Pilotprojekte zur Wirkungsorientierung. ${ }^{508}$ Sogar die internationalen Vorreiter-Nationen des New Public Management befinden sich bei wirkungsorientierten Steuerungsansätzen häufig noch in einem experimentellen Stadium. ${ }^{509}$ Die bislang mangelnde Umsetzung wirkungsorientierter Ansätze in der Verwaltungspraxis lassen Defizite des bestehenden Controlling-Instrumentariums auf der Wirkungsebene vermuten - theoretische Methodendefizite haben so anscheinend praktische Umsetzungsdefizite zur Folge.

Zur Analyse und Steuerung der Wirkungen staatlichen Handelns empfehlen MOSIEK ET AL. und RÖHRIG in ihren Modellkonzeptionen sowohl Verfahren der Einstellungsund Kundenzufriedenheitsmessung zur Bewertung des Leistungsimpact, als auch Evaluationen und Wirkungsrechnungen zur Bewertung der Outcomes. ${ }^{510}$ Die Perspektive des Impact - verstanden als die subjektive Anspruchserfüllung des Leistungsempfängers durch die Verwaltung ${ }^{511}$ - und die Perspektive der Outcomes - verstanden als die

\footnotetext{
504 In gleicher Weise auch Balzer (2005), S. 425.

$505 \mathrm{Vgl}$. Bogumil/Kuhlmann (2004).

506 Vgl. Jann (2004), S. 15. Für ein Vergleich der Bundesländer vgl. Reichard (2004), S. 87 ff. sowie für den Modernisierungsprozess des Bundes vgl. Jann (2004), S. $100 \mathrm{ff}$.

507 Vgl. Budäus (2004), S. 82 ff. oder Reichard (2004), S. 87 ff.

508 Vgl. Brüggemeier (2004), S. 375, der einige ausgewählte Projekte nennt.

$509 \mathrm{Vgl}$. hierzu die Ausführungen des Kapitels 2.2.3.2.

$510 \mathrm{Vgl}$. Mosiek et al. (2004), S. 28 f. sowie Röhrig (2008), S. 105 ff.

511 Vgl. Röhrig (2008), S. 130.
} 
objektiv ausgelösten gesamtgesellschaftliche Wirkungen ${ }^{512}-$ sind in diesem Zusammenhang nicht als alternative, sondern als sich ergänzende Betrachtungsebenen anzusehen. Fraglich ist, inwieweit sich die genannten Instrumente in einen systematischen wirkungsorientierten Controllingprozess einbinden lassen (vgl. Abbildung 21).

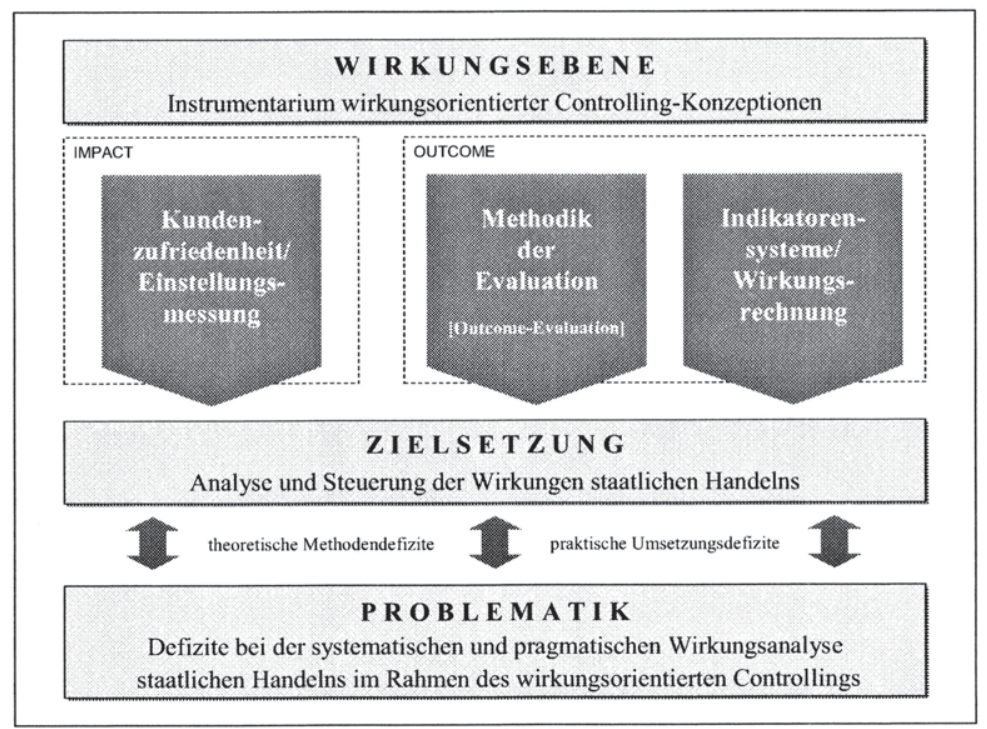

Abbildung 21: Controlling-Instrumente der Wirkungsebene

Verfahren der Einstellungs- und Kundenzufriedenheitsmessung sind insbesondere für solche Verwaltungseinheiten von Bedeutung, die Serviceleistungen für den Bürger erbringen und dadurch Ansatzpunkte zur Stärkung der Kundenorientierung in den Vordergrund rücken, der Leistungsimpact stellt hier eine wichtige Bewertungsdimension staatlichen Handelns dar. Versteht man den Bürger als Kunden und damit zugleich auch als Auftraggeber der öffentlichen Verwaltung, sollten Serviceleistungen an den spezifischen Kundenbedürfnissen ausgerichtet werden. Als Kunde ist der Bürger in der Lage, die erbrachten Leistungen sachgerecht $\mathrm{zu}$ beurteilen und gegebenenfalls konstruktive Kritik zu üben bzw. Verbesserungsvorschläge zur Optimierung der Serviceleistung vorzubringen. ${ }^{513}$

Als besonders wichtige Merkmale öffentlicher Serviceleistungen werden von Kunden beispielsweise die Öffnungs- und Sprechzeiten, die Schnelligkeit der Vorgangsbearbeitung und die Verständlichkeit der schriftlichen Unterlagen genannt. ${ }^{514}$ Die Auswahl

\footnotetext{
512 Vgl. Berens et al. (2004), S. 331.

513 Vgl. Röhrig (2008), S. 132.

514 Vgl. Meyer/Dornach (2001), S. 117.
} 
und Relevanz einzelner Merkmale ist allerdings stark von der Art der öffentlichen Leistung abhängig und fallweise entsprechend anzupassen. Zur Erhebung der Kundenzufriedenheit kommen häufig Kunden- $b z w$. Bürgerbefragungen zum Einsatz, teilweise wird auch ein Beschwerdemanagement eingerichtet. Wichtig ist in diesem Zusammenhang eine kontinuierliche Rückkopplung des Leistungsprozesses durch den Kunden, um vorhandene Optimierungspotenziale frühzeitig zu erschließen. Um die Impactanalyse in einen systematischen wirkungsorientierten Controllingprozess zu überführen, sollten die Befragungsergebnisse schließlich zu einem überschaubaren Set steuerungsrelevanter Impact-Indikatoren verdichtet werden, die unter vertretbarem Aufwand einer regelmäßigen Messung unterzogen werden können. ${ }^{515}$

Zur Beurteilung der Outcomes, d.h. der durch den öffentlichen Leistungsprozess gesamtgesellschaftlich ausgelösten Wirkungen, sollen der Empfehlung nach insbesondere Wirkungsrechnungen bzw. entsprechende Indikatorensysteme sowie die Methodik der Evaluation herangezogen werden. ${ }^{516}$ Eine Wirkungsrechnung erfasst die Auswirkungen der Verwaltungstätigkeit, d.h. der staatlichen Produkte und Programme, und setzt sie in Beziehung zu den politischen Zielen, die damit verfolgt wurden. ${ }^{517}$ Bei einer Wirkungsrechnung handelt es sich demnach um ein Indikatorensystem, das die Wirkung staatlichen Handelns abbildet. Mittels Indikatoren können nicht direkt messbare Sachverhalte ansatzweise messbar gemacht werden und man erhält ein vereinfachtes Abbild der Realität. ${ }^{518}$ Durch geeignete Indikatoren kann so die objektive Effektivität und damit der Zielerreichungsgrad des Verwaltungshandelns auf der Ebene der Outcomes bestimmt werden. ${ }^{519}$ Voraussetzung für aussagekräftige Informationen im Rahmen der Wirkungsrechnung ist aber die Existenz klarer und messbarer Wirkungsziele. ${ }^{520}$

Doch an methodisch ungenügenden Zieldefinitionen scheitert in der Regel der verbreitete Einsatz einer Wirkungsrechnung. Insbesondere auf politischer Ebene besteht nur eine äußerst geringe Bereitschaft zur Vorgabe klarer und messbarer Zielvorgaben, da die hiermit verbundene Transparenz und Verantwortungszuweisung vielfach der politischen Rationalität widerspricht. ${ }^{521}$ Daneben besteht eine weitere Schwäche darin, dass eine Wirkungsrechnung keine kausalorientierten Überlegungen anstellt. Dies bedeutet, dass selbst bei der Existenz klarer und messbarer Wirkungsziele nur die hiermit verbundenen intendierten Auswirkungen in der Gesellschaft durch das Indikatorensystem erfasst würden. Sämtliche vom staatlichen Produkt ausgelösten Neben- und Folgewirkungen auf die gesamtgesellschaftliche Wohlfahrt würden nicht beachtet, was faktisch eine unzulässige Verkürzung der Wirksamkeitsbetrachtung darstellt. ${ }^{522} \mathrm{Um}$ entspre-

\footnotetext{
515 Vgl. Röhrig (2008), S. 136.

516 Vgl. beispielsweise Buschor (2002), S. 66; Mosiek et al. (2004), S. 28 f. und Röhrig (2008), S. 136 ff.

517 Vgl. Schedler/Proeller (2003), S. 207.

518 Vgl. Hill (2002), S. 65.

519 Vgl. Röhrig (2008), S. 154.

$520 \mathrm{Vgl}$. Schedler/Proeller (2003), S. 207.

$521 \mathrm{Vgl}$. Brüggemeier (2004), S. 383 oder Fiedler/Vernau (2001), S. 35 ff. und insbesondere Kapitel 2.2.3.4.

522 Vgl. hierzu ausführlich Kapitel 4.1.1.1 dieser Arbeit.
} 
chende kausalorientierte Überlegungen vorzunehmen, wird daher ergänzend das Instrumentarium der Evaluation empfohlen. ${ }^{523}$

Bei Evaluationen handelt es sich um ein Instrument der Erfolgskontrolle, das Informationen über staatliches Handeln bereitstellt. ${ }^{524}$ Dem Wortsinn nach bedeutet Evaluation die Beurteilung eines Gegenstandes oder Sachverhaltes. ${ }^{525}$ Im Rahmen der schweizerischen Verwaltungsmodernisierung hat sich in den letzten Jahren ein Begriffsverständnis durchgesetzt, dass mit Evaluation die wissenschaftliche und empirisch gestützte Beurteilung der Konzeption, des Vollzugs und der Wirksamkeit staatlicher Maßnahmen beschreibt. ${ }^{526}$

Diesem weiten Begriffsverständnis folgend, beziehen sich Evaluationen - vergleichbar den vorgestellten Controlling-Bezugsrahmen - auf sämtliche Bewertungsebenen des öffentlichen Leistungsprozesses. Dies würde auch die Ressourcen- und Produktebene mit einschließen. Damit weist der Evaluationsbegriff starke Überschneidungen zum wirkungsorientierten Controllingbegriff dieser Arbeit auf - auch wenn das Controlling stärker begleitend und führungsunterstützend angelegt ist. ${ }^{527}$ Für den weiteren Untersuchungsverlauf ist demnach notwendig, das Evaluationsverständnis entsprechend dem vorliegenden Kontext weiter zu spezifizieren.

Im Rahmen der vorliegenden Arbeit wird daher eine enge Auslegung des Evaluationsbegriffs gewählt, wonach sich Evaluationen primär auf die Wirkungserfassung beziehen. ${ }^{528}$ Evaluationen stellen somit ein Controllinginstrument der Wirkungsebene dar. Der Kern der Evaluation liegt in der Untersuchung der Wirksamkeit öffentlicher Maßnahmen und Programme, was einer „Beurteilung und Bewertung staatlicher Maßnahmen und Programme mit wissenschaftlichen Methoden ${ }^{\circ 529}$ gleichkommt. ${ }^{530}$ Unter Evaluation kann demnach die sozialwissenschaftlich orientierte und methodisch reflektierte Forschung über die tatsächlichen Wirkungszusammenhänge staatlicher Maßnahmen

523 „Im Ergebnis können in der Kombination spezifischer Verfahren der Evaluation sowie dem zielgerichteten Einsatz von Wirkungsindikatoren die Probleme einer Wirkungsoperationalisierung unter bestimmten Voraussetzungen deutlich reduziert werden"; Röhrig (2008), S. 139 f. In ähnlicher Weise fordert auch BUDÄUS einen kombinierten Einsatz von Evaluationen und Indikatorensystemen. Vgl. Budäus (1999b), S. 336.

Vgl. Bussmann (1995), S. $345 \mathrm{ff}$.

525 Wenn jemand z.B. überprüft, ob gebratenes Fleisch besser schmeckt als rohes oder wenn jemand ausprobiert, ob bestimmte Pflanzen essbar sind oder nicht, dann führt er eine Evaluation durch. Evaluationen sind ein wichtiges Instrument zur Generierung von Erfahrungswissen. Vgl. Stockmann (2004), S. 13.

Vgl. Buschor (2002).

$527 \mathrm{Zu}$ Überschneidung der Begrifflichkeiten bei Instrumenten der Erfolgskontrolle vgl. Kissling-Näf/Knoepfel (1993), S. 277 ff. oder auch Bussmann (1995), S. 345 ff. Die Gesamtheit der Informationsinstrumente wird im deutschsprachigen Raum häufig unter dem Begriff „Controlling“ zusammengefasst. Insbesondere im angelsächsischen Sprachgebiet wird dafür in der Regel der Begriff „Evaluation“ verwendet.

528 Vgl. Rossi/Freeman (1993). „Evaluationen haben immer mit der Erfassung von Wirkungen staatlichen Handelns zu tun". Klöti (1997), S. 46.

529 Klöti (1997), S. 39.

530 Vgl. Röhrig (2008), S. 141. 
verstanden werden. ${ }^{531}$ Dergestalt erfolgt durch die Untersuchung von Kausalzusammenhängen eine Zurechnung der Outcomes auf die Produkte der Verwaltung. Zur besseren Abgrenzung der Begrifflichkeiten wird bei entsprechend angelegten Evaluationen im Folgenden von Outcome-Evaluationen gesprochen. ${ }^{532}$

Zur Analyse der Wirkungen staatlichen Handelns findet demnach die Methodik der Outcome-Evaluation Eingang in den Bezugsrahmen des wirkungsorientierten Controllings. Hinsichtlich der Phasen des politisch-administrativen Prozesses kann eine Outcome-Evaluation grundsätzlich drei verschiedene Analyseperspektiven ${ }^{533}$ einnehmen: $^{534}$

- ex-ante: In der Phase der Maßnahmenplanung werden mutmaßliche künftige Wirkungen abgeschätzt bzw. zu erwartende negative Auswirkungen antizipiert, um die Gestaltung der Maßnahme zu unterstützen. ${ }^{535}$

- on-going: In der Phase der Maßnahmendurchführung findet die Überprüfung von Zwischenergebnissen statt, um frühzeitig notwendige Anpassungen bzw. die Korrektur von Fehlentwicklungen vorzunehmen.

- ex-post: Nach Abschluss der Maßnahme werden die ausgelösten Wirkungen möglichst vollständig erfasst und bewertet, um Kenntnis von der Wirksamkeit der Maßnahme zu erlangen sowie ein tieferes Verständnis der Wirkungszusammenhänge zu erhalten. ${ }^{536}$

Eine weitere Differenzierung wird auch hinsichtlich der Trägerform der OutcomeEvaluation vorgenommen. Grundsätzlich kann man zwischen den Formen der internen Selbstevaluation sowie der externen Fremdevaluation unterscheiden. ${ }^{537}$ Während die interne Selbstevaluation von den beteiligten Akteuren bzw. der ausführenden Verwaltungseinheit selbst erstellt wird, ist die Anfertigung durch wissenschaftliche For-

531 Vgl. Haldemann/Marek (2001), S. 41.

${ }^{532}$ Zum Begriff der Outcome-Evaluation sowie einer Abgrenzung zu anderen Evaluationstypen vgl. auch Wollmann (2004b), S. 205 ff. WOLLMANN unterscheidet Evaluationstypen danach, ,welche Reichweite die Evaluation haben soll“". Wollmann (2004b), S. 207. Man spricht von Institutionen-Evaluation, wenn der Verlauf und das Ergebnis institutioneller Veränderungen beurteilt werden soll. Auf veränderte Handlungsprozesse und -muster beziehen sich die Performanz-Evaluation bzw. Prozess-Evaluation. Evaluationen können auch auf die verwaltungsökonomische Effizienz, insbesondere erzielte Kostenreduktionen im Sinne eines „saving of money " durch Reformprojekte abstellen. Output-Evaluationen bewerten dagegen die hierdurch erzielten (materiellen) Leistungsverbesserungen. Darüber hinausreichende verteilungspolitische „systemische" Veränderungen in der Gesellschaft werden im Rahmen von Outcome-Evaluationen beurteilt. Vgl. Wollmann (2004b), S. 205 ff.

533 Eine übersichtliche Darstellung der Synthese unterschiedlicher Evaluationsansätze und Analyseperspektiven findet sich bei Röhrig (2008), S. 143.

534 Vgl. hierzu Stockmann (2004), S. 15 ff.; in ähnlicher Weise auch Bussmann (1995), S. 35 f.

535 BUSSMANN bezeichnet diese als prospektive Evaluationen, vgl. Bussmann (1995), S. 35. Evaluationen im Rahmen der Maßnahmenformulierung haben formativen Charakter, d.h. sie sind aktiv-gestaltend, prozessorientiert, konstruktiv und kommunikationsfördernd angelegt. Vgl. Stockmann (2004), S. 17.

536 Solche Evaluationen haben i.d.R. summativen Charakter, d.h. sie sind zusammenfassend, bilanzierend und ergebnisorientiert. Vgl. Stockmann (2004), S. 17.

537 Vgl. Vedung (1999), S. 104 f. 
schungseinrichtungen und spezialisierte Beratungsgesellschaften kennzeichnend für die externe Fremdevaluation. Die Wahl der Trägerschaft sollte von der grundlegenden Zielsetzung der Evaluation abhängig gemacht werden. ${ }^{538}$ Will der Auftraggeber $R e$ chenschaft über eine staatliche Maßnahme ablegen, sprechen die höhere Objektivität und Glaubwürdigkeit für die externe Fremdevaluation. Ist dagegen die lernorientierte Verbesserung der Steuerungsprozesse das Ziel, können die fachspezifischen Kenntnisse der Mitarbeiter auch im Rahmen der internen Selbstevaluation genutzt werden.

Allerdings werden von den Vertretern der Evaluationsforschung stets die Anforderungen an die fundierten fachlichen Methodenkenntnisse betont, die zur Durchführung einer qualitativ gesicherten Evaluation notwendig sind. ${ }^{539}$ Hierdurch ergeben sich automatisch Einschränkungen für die Anwendung der internen Selbstevaluation. Evaluatoren benötigen demnach tiefgehende Kenntnisse sowohl über das zu untersuchende Fachgebiet als auch über die sozialwissenschaftlichen Methoden der Evaluationsforschung. ${ }^{540}$ Die methodische Komplexität resultiert aus dem verbreiteten Einsatz empirischer Forschungstechniken, Evaluationen sind häufig als Sozialexperimente angelegt. Hierdurch erhalten wissenschaftliche Qualitätskriterien wie Objektivität, Validität und Reliabilität ein hohes Gewicht - pragmatische Aspekte sind von geringerer Bedeutung.

Im Zuge der Professionalisierung der Evaluationsforschung werden bereits seit den 70er Jahren in den USA Kriterienkataloge entwickelt, mit denen die Qualität von Evaluationen sichergestellt werden soll. ${ }^{541}$ In Anlehnung an diese Kriterienkataloge hat die Deutschen Gesellschaft für Evaluation (DeGEval) eigene Qualitätsstandards erarbeitet. ${ }^{542}$ Demzufolge sollen Evaluationen die vier grundlegenden Eigenschaften der Nützlichkeit, Durchführbarkeit, Fairness und Genauigkeit aufweisen: ${ }^{543}$

- Die Nützlichkeitsstandards sollen sicherstellen, dass sich der Zweck einer Evaluation am Informationsbedürfnis der vorgesehenen Nutzer ausrichtet.

- Die Durchführbarkeitsstandards sollen gewährleisten, dass eine Evaluation sowohl realistisch und gut durchdacht als auch kostenbewusst angelegt wird.

- Die Fairnessstandards regeln, dass in einer Evaluation diplomatisch und respektvoll mit betroffenen Akteuren umgegangen wird sowie mögliche Interessenkonflikte offen gelegt werden.

\footnotetext{
$538 \mathrm{Vgl}$. hierzu und im Folgenden Vedung (1999), S. $106 \mathrm{ff}$.

539 Vgl. bspw. Klöti (1997), S. 44; Bussmann (1995), S. 348; Stockmann (2004), S. 20 f.; Wollmann (2004b), S. 211 ff.; Widmer (2004), S. 83 ff.; Vedung (1999), S. 104 ff. oder Schedler/Proeller (2003), S. 208.

540 Vgl. Klöti (1997), S. 44 ff. „Evaluationen sind deshalb schon fast definitionsgemäß multidisziplinäre Forschungsvorhaben. Einzelne Forscherinnen und Forscher sind immer weniger in der Lage, im Alleingang Evaluationen durchzuführen, selbst wenn sie über beträchtliche Erfahrung verfügen. Gefragt ist immer mehr Teamarbeit.“ Klöti (1997), S. 44.

541 Vgl. Stockmann (2004), S. 22.

542 Vgl. DeGEval (2002).

543 Vgl. hierzu Stockmann (2004), S. 22 f.; DeGEval (2002), S. 8 f. oder ausführlich Widmer (2004), S. 92 ff.
} 
- Die Genauigkeitsstandards sollen dafür sorgen, dass eine Evaluation gültige und nachprüfbare Information bzw. Ergebnisse hervorbringt, Vorgehensweise und Informationsquellen sind zu verdeutlichen.

Nach STOCKMANN hat sich der Schwerpunkt der Evaluationsdiskussion in den letzten Jahren allerdings vom methodischen Rigorismus hin zu mehr Pragmatismus verschoben. ${ }^{544}$ Das Kriterium der Nützlichkeit von Evaluationsergebnissen für Auftraggeber und Stakeholder wird zunehmend in den Vordergrund gestellt. ${ }^{545}$ Dies ist vor allem auf die Erkenntnis der Evaluationsforschung zurückzuführen, dass eine konzeptionelle „Verschlankung“ der Evaluation, deren Initiierung und Durchführung politisch, zeitlich und finanziell erst ermöglicht. ${ }^{546}$ Für Outcome-Evaluationen gilt dies umso mehr, da es sich aufgrund der beschriebenen methodischen Problembereich ${ }^{547}$ häufig um sehr aufwendige und komplexe Untersuchungen handelt. Aus der Perspektive des wirkungsorientierten Controllings ist die aktuelle Entwicklung zu weniger Wissenschaftlichkeit und mehr Pragmatismus bei der Durchführung von Evaluationsstudien sicherlich zu begrüßen, da hiermit die Grundlagen geschaffen werden, erzielte Ergebnisse auch in die Steuerungskreisläufe von Politik und Verwaltung zu überführen.

Doch ändert dies wenig an den - zumindest bislang - bestehenden praktischen Umsetzungsdefiziten der Outcome-Evaluation. Der BUNDESRECHNUNGSHOF stellt in einer Studie aus dem Jahr 1998 fest, dass in der öffentlichen Verwaltung systematische Erfolgskontrollen und Wirkungsuntersuchungen, die auch nicht-intendierte Effekte berücksichtigen und staatliche Maßnahmen einer Ursache-Wirkungsanalyse unterziehen, nahezu komplett fehlen. ${ }^{548}$ Und STOCKMANN ergänzt kritisch, dass, wenn in einem Politikfeld wie z.B. dem Bildungsbereich häufiger Evaluationsstudien durchgeführt wurden, die Ergebnisse meist widersprüchlich und wenig umsetzungsorientiert sind. Weiterhin sei zu bemängeln, dass Evaluationen meist sektoral und unabhängig voneinander angelegt wurden, eine Konsolidierung über verschiedene Politikfelder hinweg fehlt bislang völlig. ${ }^{549}$ WOLLMANN stellt daher fest, dass der Stand der Outcome-Evaluation - national wie international - als defizitär einzuschätzen ist, die Wirkungsdimension bleibt in Studien fast durchweg ausgeblendet, sind Aussagen anzutreffen, sind sie eher generell und ,weich“ formuliert. ${ }^{550}$

In ihren Thesen zur Zukunft der strategischen Steuerung greift SCHENKER-WICKI die notwendige Entwicklungsrichtung der Evaluationsforschung auf: „Für die konkrete

\footnotetext{
$544 \mathrm{Vgl}$. Stockmann (2004), S. 22.

545 Über alle theoretischen und methodischen Fragen hinweg, besteht Einigkeit darin, dass es darauf ankommt, dass Evaluationsergebnisse im politischen Prozess genutzt werden. Vgl. hierzu Chelimsky (1995), S. 8.

$546 \mathrm{Vgl}$. Wollmann (2004b), S. 210. THOLNIG plädiert daher für den eher ,pragmatischen“ Evaluationsansatz der Quasi-Evaluation. „This is a perfectly good way to start. There is no surer way of stifling evaluation at birth than to confine it in the ghetto of methodology". Thoenig (2003) zitiert bei Wollmann (2004b), S. 210.

547 Vgl. hierzu die Ausführungen des Kapitels 2.2.3.4.

$548 \mathrm{Vgl}$. Bundesrechnungshof (1998), S. $22 \mathrm{ff}$.

549 Vgl. Stockmann (2004), S. 28.

550 Vgl. Wollmann (2004b), S. 223.
} 
Ausgestaltung einzelner Politikbereiche [...] interessiert nämlich nicht in erster Linie, wie gut oder effizient eine Verwaltungseinheit arbeitet, sondern ob Leistungen, die vom Staat erbracht werden, auch die tatsächlich gewünschte Wirkung zeigen und zur allgemeinen Wohlfahrt beitragen. In diesem Bereich liegt allerdings noch vieles im Argen: Es werden zwar immer wieder Evaluationen durchgeführt - die klassischerweise zur Überprüfung von Policies eingesetzt werden - doch handelt es sich dabei in der Regel um nicht standardisierte Prüfungen, deren Daten nicht weiterverwendet werden können. Im Gegensatz zu den standardisierten Auditmodellen in der Betriebswirtschaft werden bei einer Evaluation in der Regel für ein bestimmtes Programm oder bestimmte Projekte immer wieder neue und individuelle Lösungen gesucht. Dies ist möglich, da die Theorie die Verwendung unterschiedlicher Bezugsrahmen für ein und dieselbe Problematik erlaubt, was allerdings in der Folge zu widersprüchlichen Aussagen über eine Programmwirkung führen kann und kaum einen systematischen Erkenntnisgewinn zulässt. Aus diesem Grund sind in Politikbereichen zusätzliche Anstrengungen zur Standardisierung - zum Beispiel der Einsatz von standardisierten Leistungsprüfungen - notwendig". 551

Für die instrumentelle Ausgestaltung eines wirkungsorientierten Controllings auf der Wirkungsebene bleiben also folgende Defizite festzuhalten. Standardisierte Evaluationen auf der Wirkungsebene sind bereits durch den Umstand begrenzt, dass - zumindest bislang - überhaupt nur in sehr geringer Anzahl echte Outcome-Evaluationen durchgeführt wurden. Sind entsprechende Evaluationen verfügbar, handelt es sich in der Regel um wissenschaftlich geprägte Einzelfalluntersuchungen durch externe Institute, die meist wenig systematischen Erkenntnisgewinn zulassen und sich somit auch nur begrenzt für eine wirkungsorientierte Steuerung eignen. Hier besteht jedoch die Hoffnung, dass durch eine fortlaufende Standardisierung und mehr Pragmatismus in der methodischen Anlage künftig eine gesteigerte Nutzung externer Evaluationsergebnisse erfolgen kann. Doch die Nachteile solcher externen Evaluationen, der hohe Kostenund Zeitaufwand, bleiben grundsätzlich bestehen, weshalb sich eine wirkungsorientierte Verwaltungssteuerung in der Breite nicht hierauf stützen kann. ${ }^{552}$ Hier muss das wirkungsorientierte Controlling anknüpfen und zukünftig sachgerechte und praktikable Methoden bereitstellen, die eine systematische Analyse und Steuerung der Wirkungen staatlichen Handelns erlauben.

Neben der eher extern geprägten Outcome-Evaluation muss also im Rahmen des wirkungsorientierten Controllings ein selbständig anwendbares, bislang faktisch noch nicht bestehendes Instrumentarium zur Wirkungsanalyse staatlichen Handelns entwickelt werden. Die Aufgabe besteht hier sicherlich nicht darin, „das Rad neu zu erfinden“, sondern eher in einer sinnvollen Fortentwicklung bestehender Ansätze der Wirkungsrechnung und der Outcome-Evaluation. Insbesondere ist es notwendig, die Wirkungsrechnung bzw. Indikatorensysteme mit den eher kausalorientierten Überlegungen der Evaluation zu verknüpfen. In ähnlicher Weise kommt auch BRÜGGEMEIER in sei-

551 Schenker-Wicki (2005).

552 Vgl. Schedler/Proeller (2003), S. 198. 
nem Beitrag zum wirkungsorientierten Controlling zu dem Schluss, dass nun „eine gute Gelegenheit ist, dass sich partiell verwandte Konzepte wie "Controlling" und "Evaluation" endlich einander annähern und sich wechselseitig befruchten" ${ }^{553}$ Ebenso schließt sich WOLLMANN dieser Sichtweise an: „Angesichts der strategischen Bedeutung, die in der neueren NPM- und NSM-inspirierten Verwaltungsreformdiskussion den Verfahren des verwaltungsinternen Controlling beigemessen wird, verdienen Varianten der internen Evaluation besondere Aufmerksamkeit" ${ }^{\text {“54 }}$

Im Vergleich zu den genannten Defiziten der externen Outcome-Evaluation für einen systematischen Controllingprozess, sollte sich das Instrumentarium der Wirkungsanalyse durch folgende Kriterien auszeichnen:

- praktikables Instrumentarium, welches im Rahmen der internen Controllingprozesse Anwendung finden kann

- Einbindung in einen kontinuierlichen und systematischen Controllingprozess, welcher regelmäßig steuerungsrelevante Daten zur Verfügung stellt

- möglichst hohe Standardisierbarkeit und damit ein angemessenes Verhältnis zwischen entstehenden Kosten und dem Steuerungsnutzen

- Strukturierungshilfen bereitstellen, um eine Konsolidierung bzw. Verdichtung über verschiedene Steuerungsebenen zu ermöglichen

Die genannten Aspekte stellen damit den Rahmen für den weiteren Verlauf der Untersuchung dar. Durch die Weiterentwicklung bestehender Ansätze der Evaluationsforschung, insbesondere der Outcome-Evaluation, soll das nachfolgende Kapitel somit einen Beitrag leisten, ein geeignetes Instrumentarium zur Wirkungsanalyse staatlichen Handelns zu entwickeln.

553 Brüggemeier (2004), S. 386.

554 Wollmann (2004b), S. 211. 


\section{Methodik der Wirkungsanalyse staatlichen Handelns}

Die Forderung, durch die Wirkungssteuerung die Wirksamkeit des staatlichen Ressourceneinsatzes sicherzustellen, kann aufgrund der genannten Defizite, auch durch die bestehenden Ansätze des wirkungsorientierten Controllings, derzeit nicht vollständig erfüllt werden. Daher ist es das Ziel der weiteren Untersuchung, ein praktikables Instrumentarium zur systematischen Analyse der durch staatliches Handeln im gesellschaftlichen Umfeld ausgelösten Wirkungen zu entwickeln. Grundsätzlich wird hiermit ein eher pragmatisches Wissenschaftsziel verfolgt. Die instrumentelle Erweiterung des wirkungsorientierten Controllings auf der Wirkungsebene soll praktischen Steuerungsnutzen im politisch-administrativen System entfalten. Zugleich hat die weitere Untersuchung aber auch den Charakter einer eher theoretisch orientierten Grundlagenforschung, da eine möglichst allgemeingültige Übertragbarkeit der Ergebnisse auf sämtliche Bereiche staatlichen Handelns angestrebt wird. Der weitere Untersuchungsaufbau ist in der nachfolgenden Abbildung dargestellt.

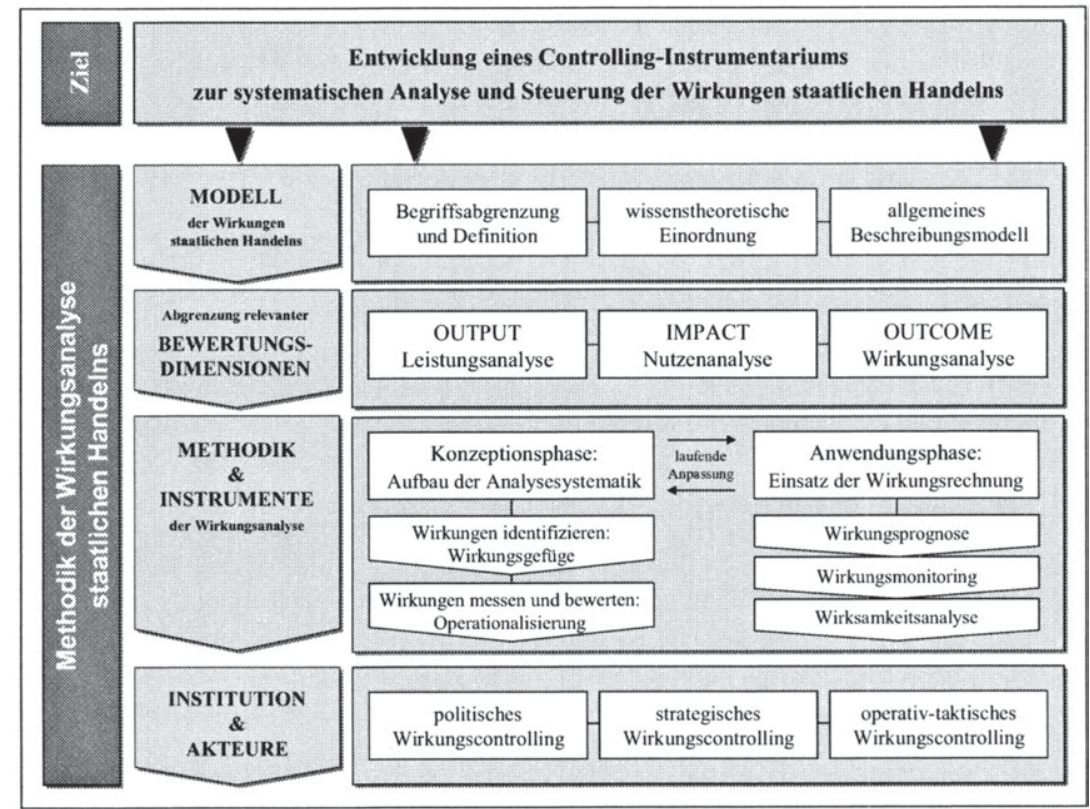

Abbildung 22: Aufbau der weiteren Untersuchung

Der nächste Schritt der Untersuchung besteht darin, zunächst ein allgemeingültiges Beschreibungsmodell der Wirkungen staatlichen Handelns zu entwickeln. Dies ist notwendig, um die hochkomplexen Wirkungszusammenhänge der Realität beherrschbar zu machen und die relevanten Betrachtungsobjekte für den weiteren Untersuchungsverlauf abzugrenzen. In diesem Zuge erfolgen auch die eindeutige Definition 
des Wirkungsbegriffes sowie die notwendige wissenstheoretische Einordnung von Ursache-Wirkungsbeziehungen. Durch das Beschreibungsmodell können zunächst die theoretischen Defizite der Literatur bezüglich einer Strukturierung der staatlich ausgelösten Wirkungen beseitigt werden.

Danach widmen sich die Ausführungen den unterschiedlichen Bewertungsdimensionen einer Wirkungsanalyse. In diesem Zusammenhang ist problematisch, dass die in den Ebenenmodellen unterstellte eindeutige Abgrenzung von Output, Impact und Outcome in der Realität so nicht existiert. Die einzelnen Modellebenen lassen sich häufig nicht trennscharf bestimmen und weisen nicht unerhebliche Redundanzen auf. Um Wirkungen zu analysieren bzw. pragmatische Vorschläge zur instrumentellen Ausgestaltung der einzelnen Bewertungsdimensionen - Leistungsanalyse, Nutzenanalyse und Wirkungsanalyse - zu unterbreiten, müssen die bestehenden Redundanzen durch die Schaffung eindeutiger Analyseperspektiven daher soweit wie möglich aufgelöst werden.

Im nachfolgenden Schritt wird dann ein pragmatischer Vorschlag zur Ausgestaltung und Methodik der Wirkungsanalyse staatlichen Handelns unterbreitet. Auf Grundlage des entworfenen Beschreibungsmodells werden eine Vorgehenssystematik sowie das notwendige Instrumentarium zur Strukturierung und Bewertung der Outcomes entwickelt. Im Rahmen dieses Vorgehensmodells sind grundsätzlich zwei Phasen zu unterscheiden. In einer sog. Konzeptionsphase erfolgt zunächst die Identifikation der ursächlich auf das staatliche Handeln zurückzuführenden Wirkungen sowie eine Operationalisierung und Bewertung der einzelnen Wirkungskomponenten. In der sog. Anwendungsphase erfolgt dann der eigentliche operative Einsatz der in der Konzeptionsphase aufgebauten Wirkungsrechnung. Hier können abhängig vom Einsatzzeitpunkt eine Wirkungsprognose, das Wirkungsmonitoring und die Wirksamkeitsanalyse unterschieden werden. Durch Rückkopplungen aus dem operativen Einsatz erfolgt zudem eine laufende Anpassung der angewendeten Analysesystematik.

Im letzten Schritt werden schließlich Vorschläge zur Institutionalisierung eines systematischen und durchgängigen Wirkungscontrollings unterbreitet. Abhängig von den beteiligten Akteuren sowie den unterschiedlichen Informationsbedürfnissen sind auch unterschiedliche Aufgabenstellungen und Verdichtungsgrade wirkungsorientierter Controllingdaten erforderlich. Demzufolge sind für das politisch-administrative System ein politisches Controlling, ein strategisches Controlling und ein operatives Controlling zu unterscheiden. 


\subsection{Modellbildung als Grundlage der weiteren Untersuchung}

\subsubsection{Wirkungen als wissenschaftliches Erkenntnisobjekt}

\subsubsection{Definitionsansätze und begriffliche Abgrenzung}

Bislang ist in der Literatur kein einheitliches Verständnis bzw. keine allgemeingültige Definition des Begriffes "Wirkung" zu beobachten. Dabei beschäftigen sich fast sämtliche Wissenschaftsdisziplinen in irgendeiner Weise mit der Erforschung von Wirkungen. Auffällig ist, dass in den einzelnen Wissenschaftsdisziplinen teilweise sogar ein völlig divergierendes Wirkungsverständnis vorherrscht. Nachfolgende Aufstellung gibt daher vorab einen kurzen Überblick über ausgewählte Definitionsansätze:

- In der Physik wird Wirkung als physikalisch skalare Größe der Dimensionen Energie mal Zeit oder Impuls mal Länge definiert. ${ }^{55 s}$

- In der Elektrotechnik ist Wirkung definiert als die Beeinflussung einer Größe, der beeinflussten Größe, durch eine oder mehrere andere Größen, der verursachenden Größe. ${ }^{556}$

- In der Informatik ist Wirkung definiert als die Veränderung des Zustands oder der Prozesse eines Systems. ${ }^{557}$

- In der Pharmazie wird Wirkung definiert als die erwünschten Effekte eines Wirkstoffes, unerwünschte Effekte werden als Nebenwirkungen bezeichnet. ${ }^{558}$

- Die Philosophie definiert Wirkung als ein Geschehen, das ohne ein anderes Geschehen, die Ursache, nicht stattfände. Nach dem Gesetz der Kausalität muss jedes Geschehen im naturgesetzlichen, geschichtlichen oder rechtlichen Zusammenhang eine Ursache haben. ${ }^{559}$

Mit einem eher allgemeinen und damit die unterschiedlichen Sichtweisen verbindenden Begriffsverständnis definiert SCHARPF Wirkung als „die Veränderung eines Sachverhaltes durch die Veränderung eines anderen". ${ }^{560}$ Unter Wirkung kann also das $R e$ sultat einer Ursache verstanden werden. Bezogen auf das staatliche Handeln stellen Wirkungen somit die ursächlich auf den öffentlichen Leistungserstellungsprozess zurückzuführenden Resultate dar. ${ }^{561}$ Umgangssprachlich wird der Wirkungsbegriff häufig mit ähnlichen Begriffen wie Effekt, Einwirkung, Auswirkung, Wirksamkeit oder auch Einflussnahme synonym verwendet. ${ }^{562}$

\footnotetext{
$555 \mathrm{Vgl}$. Brockhaus (2006c), S. 168.

556 Vgl. DIN (1997), DIN-Norm 19226.

$557 \mathrm{Vgl}$. Kramer/Neulau (1998), S. $11 \mathrm{f}$.

558 Vgl. Brockhaus (2006a), S. 442 und Brockhaus (2006b), S. 363.

559 Vgl. Brockhaus (2006c), S. 168.

560 Scharpf (1982), S. 92.
}

561 In einem weiten Begriffsverständnis könnte der im Rahmen des öffentlichen Leistungserstellungsprozesses benötigte Ressourceninput so auch als Wirkung aufgefasst werden. Der benötigte Ressourceninput ist jedoch nicht Bestandteil des Wirkungsbegriffes staatlichen Handelns dieser Arbeit, da dieser im Modell der Zielund Ergebnisebenen separat ausgewiesen wird.

562 Zu synonymen Begriffen des Wirkungsbegriffes vgl. auch Duden (2002), S. 1054. 
Daher wird für den weiteren Verlauf der Untersuchung eine Abgrenzung der Begrifflichkeiten vorgenommen. Die Begriffe Effekt, Auswirkung und Einwirkung werden dem Wirkungsbegriff inhaltlich untergeordnet, sie stellen somit unterschiedliche Bewertungsdimensionen von »Wirkungen« dar. Mit Effekt werden die unmittelbaren, d.h. direkt ersichtlichen bzw. nachweisbaren Wirkungen der Leistungserbringung bezeichnet. ${ }^{563}$ Einwirkungen geben die Wirkungen des Verwaltungshandelns so wieder, wie sie durch die Adressaten subjektiv empfunden werden - dies entspricht dem Impact. ${ }^{564}$ Auswirkungen stellen demgegenüber die eher mittelbaren Wirkungen der Leistung auf Gesellschaft und Umwelt dar - dies entspricht dem Outcome. ${ }^{565}$

Der Begriff der Wirksamkeit impliziert hingegen das Vorhandensein eines Erfolgsmaßstabes. Eine Maßnahme wird als wirksam bezeichnet, wenn sie beabsichtigte Wirkung erzielt bzw. mit Erfolg wirkt. ${ }^{566}$ Bezogen auf die Wirkungen staatlichen Handelns ergeben sich somit zwei unterschiedliche Bewertungsmöglichkeiten:

- Einerseits kann die Wirksamkeit staatlichen Handelns anhand vorab definierter Wirkungsziele bewertet werden - im Sinne eines Soll-Ist-Vergleichs.

- Andererseits kann die Wirksamkeit staatlichen Handelns aber auch anhand des Beitrags zur allgemeinen Wohlfahrt, verstanden als gesamtgesellschaftlicher Wertschöpfungsbeitrag für die Gesellschaft, bewertet werden. ${ }^{57}$

Die Wahl des Erfolgsmaßstabes hat direkte Konsequenzen für die Untersuchungsanlage einer Wirkungsanalyse. Grundsätzlich müssen sich beide Perspektiven nicht gegenseitig ausschließen, es ändern sich allerdings der Betrachtungswinkel und der notwendige Analyseumfang. Während sich die Wirkungsanalyse im ersten Fall primär auf den Zielerreichungsgrad der explizit vereinbarten Programmziele konzentriert, werden im zweiten Fall auch eventuell unbeabsichtigte Nebenwirkungen in die Analyse einbezogen. ${ }^{568}$ Damit ist die zweite Analyseperspektive breiter angelegt und schließt sowohl beabsichtigte als auch unbeabsichtigte Wirkungen mit ein. Da beide Arten von Wirkungen relevant sind, wird letzterer Betrachtungsstandpunkt in dieser Arbeit eingenommen.

${ }^{563}$ Vgl. Schröder/Kettiger (2001), S. 8. Der Effekt ist also eine durch eine bestimmte Ursache eindeutig hervorgerufene Wirkung.

${ }^{564}$ Unter dem Begriff Einflussnahme als Wirkung wird ebenfalls das Einwirken auf das subjektive Empfinden von Leistungsempfängern verstanden. Beide Begriffe sind somit synonym zu verwenden.

565 Vgl. Schröder/Kettiger (2001), S. 8 oder auch Schedler/Proeller (2003), S. 207 f. die ebenfalls die Begrifflichkeiten Einwirkung und Auswirkungen in einem vergleichbaren Kontext verwenden.

566 Vgl. Duden (2002), S. 1054.

567 Wirksamkeit verstanden als gesamtgesellschaftliche Wertschöpfung, beschreibt die Differenz zwischen den erzielten Wirkungen und den hierfür eingesetzten und bewerteten Ressourcen. Vgl. hierzu Mosiek/Gerhardt (2003), S. 293 f. oder auch Pieper et al. (2006), S. 245.

568 In der Evaluationsforschung werden ebenfalls ein zielorientierter Ansatz und der sog. „goal-free“-Ansatz unterschieden, der von SCRIVEN in die Diskussion gebracht wurde. Vgl. Klöti (1997), S. 47 bzw. grundlegend zum ,goal-free“-Ansatz vgl. Scriven (1973). Mit einer sehr ausführlichen Diskussion der unterschiedlichen Evaluationsmodelle vgl. auch Vedung (1999), S. $30 \mathrm{ff}$. 
Der Arbeit liegt somit folgende Definition einer Wirkungsanalyse zugrunde: Die Wirkungsanalyse staatlichen Handelns ist die Untersuchung und Bewertung der Wirkungsweise und Wirksamkeit öffentlicher Programme bzw. Produkte. ${ }^{569}$ Die gewählte Definition ist sehr weitreichend und deckt alle denkbaren Wirkungen ab. Es wäre dem weiten Spektrum staatlichen Handelns auch nicht angemessen, einen engeren Standpunkt einzunehmen. ${ }^{570}$

Neben der Beurteilung der Wirksamkeit besteht die zweite wesentliche Aufgabe der Wirkungsanalyse also in einer Untersuchung der Wirkungsweise. Entsprechend einer Outcome-Evaluation obliegt es auch der Wirkungsanalyse, die Kausalstrukturen zwischen staatlichem Handeln und den dadurch entstehenden Wirkungen in der Gesellschaft aufzudecken. So erfolgt eine Zurechnung ausgelöster Wirkungen auf die Produkte der Verwaltung. Die Identifikation und Beschreibung von Ursache-Wirkungszusammenhängen zwischen den Produkten der öffentlichen Leistungserstellung und den gesamtgesellschaftlich ausgelösten Wirkungen ist angesichts multifaktorieller Beziehungen sowie vielfältiger äußerer Einflussfaktoren sehr diffizil. Weiterhin können zum Nachweis kausaler Zusammenhänge i.d.R. keine kontrollierten Experimente vergleichbar dem Laborexperiment in den Naturwissenschaften - eingesetzt werden. Dies erschwert die Anwendung rein experimenteller Forschungsmethoden in den Sozialwissenschaften grundsätzlich. Die folgenden Ausführungen setzen sich daher zunächst mit der wissenstheoretischen Erkenntnisperspektive dieser Arbeit auseinander.

\subsubsection{Analyse von Ursache-Wirkungszusammenhängen aus wissenstheoreti- scher Perspektive}

Kausalität bezeichnet die Beziehung zwischen Ursache und Wirkung, also die ursächliche Verbindung zweier Ereignisse. ${ }^{571}$ Dabei sind Ursache und Wirkung korrelativ aufeinander bezogen: Keine Ursache ohne Wirkung und keine Wirkung ohne Ursache. ${ }^{572}$ Während die Korrelation allerdings nur den statistischen Zusammenhang zweier Ereignisse beschreibt, sucht Kausalität - als wissenschaftliches Prinzip - nach ursächlichen Erklärungen, d.h. nach einem Ursache-Wirkungszusammenhang. ${ }^{573}$ Um Ursache-Wirkungszusammenhänge zu analysieren, muss man sich infolgedessen differenziert mit dem sozialwissenschaftlichen Konzept der Kausalität auseinandersetzen.

„Die meisten der heute lebenden Wissenschaftstheoretiker, insbesondere die kritischen Rationalisten, deren Auffassung viele Ökonomen akzeptieren, sind der Meinung, dass man niemals eine sichere Basis des Wissens finden wird. ${ }^{\text {"574 }}$ Die wissenstheoretische

\footnotetext{
569 Mit einem vergleichbaren Definitionsansatz für den Bereich der Wirkungsanalyse von Technologie- und Gründerzentren vgl. Behrendt (1995), S. 8.

570 Zum Spektrum staatlichen Handelns vgl. die Ausführungen des Kapitels 2.1.

571 Lateinisch „causa“ = „die Ursache“.

572 Vgl. Brockhaus (2006), S. 561.

573 Vgl. Brockhaus (2006), S. 561.

574 Gans/Marggraf (1997), S. 7.
} 
Erkenntnisperspektive des sog. Kritischen Rationalismus wurde Mitte der 30er Jahre von POPPER begründet. ${ }^{575}$ Alles Wissen ist demnach rein hypothetisch, nicht beweisbar wahr oder falsch. Da es sicheres faktisches Wissen nicht gibt, können die Erfahrungswissenschaften daher nur fehlbares Wissen über die Welt produzieren. ${ }^{576}$ Damit Wissenschaft die Erkenntnisse über die Wirklichkeit verbessern kann, müssen systematisch die Schwächen des bisherigen Wissens erkundet werden. „Erfahrungswissenschaftliches Wissen muss also ständig kritisiert werden, um zu besseren Erkenntnissen zu gelangen. Nur über eine negative Auslese kann man sich der Wahrheit annähern, ohne zu wissen, ob man sie erreicht. “577

Diese Sichtweise entspricht dem gängigen Wissenschaftsparadigma, wonach man sich den wahren Gesetzen annähern kann, sie aber niemals sicher und vor allem nie in einem ersten Entwurf gefunden hat. Postulierte Kausalzusammenhänge zwischen Ursache und Wirkung stellen lediglich potenzielle Zusammenhänge dar. Nach den Prinzipien des kritischen Rationalismus ist Kausalität also nicht messbar, es kann lediglich versucht werden, Hypothesen über Ursache-Wirkungsbeziehungen $\mathrm{zu}$ falsifizieren. ${ }^{578}$ Werden die Hypothesen ${ }^{579}$ in einer Reihe von Überprüfungen nicht falsifiziert, können die Hypothesen als bewährt akzeptiert werden. Aus wissenstheoretischer Perspektive gibt es somit keine hinreichende, sondern nur eine notwendige Bedingung für Kausalität. Besteht zwischen Ursache und Wirkung ein kausaler Zusammenhang, so sind die folgenden notwendigen Bedingungen erfüllt: ${ }^{580}$

- Empirische Korrelation: Ursache und Wirkung zeigen eine gemeinsame Variation.

- Zeitliche Asymmetrie: Zwischen der Variation der Ursache- und Wirkungsvariablen kommt es zu einer Zeitverzögerung.

- Keine exogenen Effekte: ${ }^{581}$ Die Variation von Ursache und Wirkung wird nicht durch exogene Effekte beeinflusst.

- Theoretische Begründung: Die Kausalhypothese ist theoretisch begründet.

In den Naturwissenschaften testet man die Gültigkeit von Hypothesen, indem man immer wieder die Verknüpfung von Ursache und Wirkung empirisch überprüft. In Form

575 Vgl. hierzu grundlegend Popper (2005) in aktueller Auflage oder auch Albert (1980).

576 Vgl. Gans/Marggraf (1997), S. 8.

577 Gans/Marggraf (1997), S. 8.

$578 \mathrm{Vgl}$. Haenecke (2002), S. 171.

579 Unter einer Hypothese versteht man eine widerspruchsfreie Aussage, deren Geltung nur vermutet ist und die eine wissenschaftliche Annahme darstellt, um zu beobachtende Sachverhalte zu erklären.

580 Vgl. hierzu auch Hildebrandt (1992), S. 7 sowie Hildebrandt (1999), S. 48 f.

581 In diesem Zusammenhang wird in der Literatur auch häufig von externen Effekten gesprochen. Der Bedeutungsgehalt des Begriffs »externe Effekte« ist allerdings stark durch die Volkswirtschaftslehre geprägt, welche hiermit Auswirkungen einer Aktivität auf Dritte beschreibt, ohne dass dafür eine Kompensation erfolgt. Ein häufig verwendetes Beispiel sind die negativen Umweltbelastungen des Autoverkehrs, ohne dass hierfür eine direkte Entschädigung gezahlt werden muss. Daher wird in bewusster Abgrenzung zu diesem Begriff im Folgenden von exogenen Effekten gesprochen, womit eine Einflussnahme von Außen bzw. durch Dritte gemeint ist. 
von Laborexperimenten können empirische Korrelation, zeitliche Asymmetrie sowie der Einfluss exogener Effekte kontrolliert und damit i.d.R. zweifelsfrei nachgewiesen werden. Die Theorie bzw. die theoretische Begründung der Hypothese erfährt so durch die Konfrontation mit der Wirklichkeit zunehmend empirische Evidenz oder wird bei Falsifikation verworfen.

In den Sozialwissenschaften sind entsprechende Laborexperimente zur experimentellen Überprüfung vermuteter Kausalzusammenhänge faktisch nicht möglich. Zum einen erschweren subjektive personengebundene Einflüsse sowie die mangelnde Isolierung exogener Effekte eine vergleichbare Untersuchungsanlage. Versucht man diese Schwächen zumindest teilweise zu beheben, erfordert dies üblicherweise einen erheblichen zeitlichen und finanziellen Aufwand. Zum anderen können die in den Sozialwissenschaften zu untersuchenden Sachverhalte meist nicht direkt empirisch überprüft bzw. beobachtet werden. Dies macht eine Operationalisierung von theoretischen Konstrukten ${ }^{582}$ durch geeignete messbare Indikatoren notwendig. ${ }^{583}$ Ein solches theoretisches Konstrukt ist beispielsweise die »menschliche Intelligenz«, die man durch das Indikatorensystem des »Intelligenzquotienten« zu operationalisieren versucht. Abbildung 23 fasst die bisherigen wissenstheoretischen Erkenntnisse nochmals zusammen.

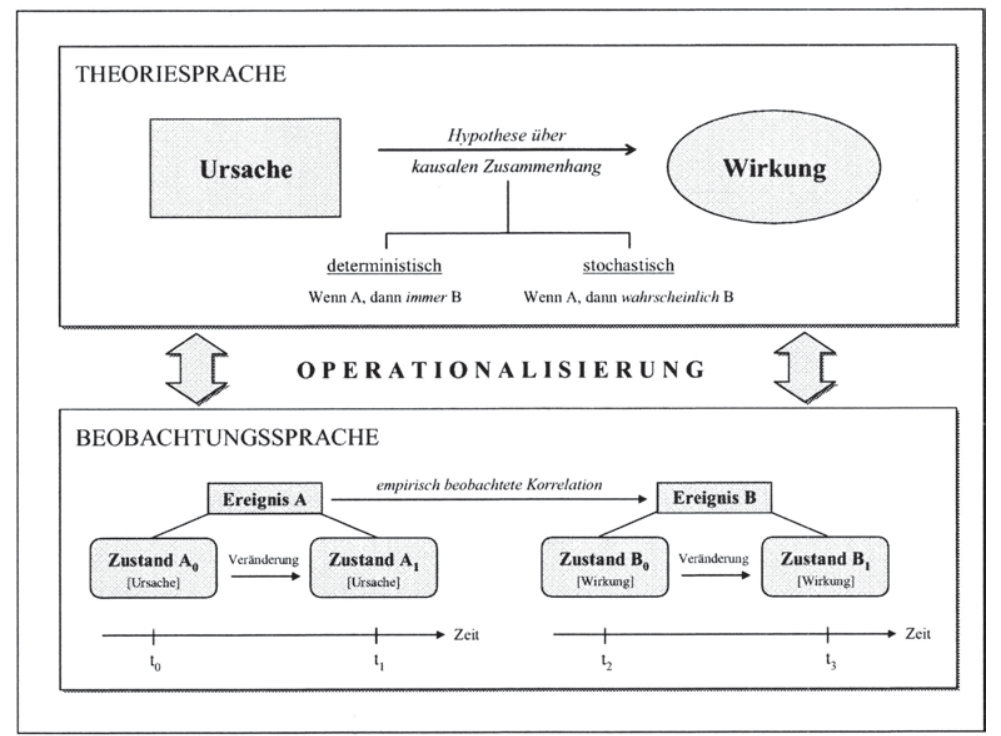

Abbildung 23: Ebenen der 2-Sprachen-Theorie

582 Ein theoretisches Konstrukt ist gedanklicher Natur und beschreibt einen nicht direkt beobachtbaren Sachverhalt innerhalb einer wissenschaftlichen Theorie. Vgl. Jenner (2000), S. 327.

583 Vgl. Jenner (2000), S. 327 f. 
Demnach kann zwischen den beiden Ebenen der Beobachtungssprache und der theoretischen Sprache unterschieden werden. Die Beobachtungssprache beschreibt Größen, die unmittelbar messbar sind. Die Theoriesprache hingegen beschreibt mit Hilfe theoretischer Konstrukte abstrakte Charakteristika eines Untersuchungsobjektes, die zur Erklärung der Beobachtung notwendig sind, die aber in der Realität nicht unmittelbar erfasst werden können. ${ }^{54}$ Bezogen auf staatliches Handeln bedeutet dies, dass theoretisch begründbare Vermutungen (Hypothesen) über die Verbindung des Verwaltungsoutput (als Ursache) sowie resultierenden Impacts und Outcomes (als Wirkungen) angestellt werden. Outputs, Impacts und Outcomes müssen als theoretische Konstrukte durch geeignete Indikatoren operationalisiert werden. Anhand dieser Indikatoren können die Zustandsveränderungen des Ereignisses A als Ursache und des zeitlich nachgelagerten Ereignisses B als Wirkung empirisch überprüft werden. So erfolgt auch im Rahmen der Wirkungsanalyse eine schrittweise Validierung vermuteter Kausalzusammenhänge zwischen staatlichem Handeln und den hierdurch ausgelösten Wirkungen in der Gesellschaft.

Die hier grundsätzlich angestellten wissenstheoretischen Überlegungen sollen im Folgenden durch die Konstruktion eines allgemeinen Beschreibungsmodells der Wirkungen staatlichen Handelns weitergeführt werden.

\subsubsection{Konstruktion eines allgemeinen Beschreibungsmodells}

\subsubsection{Beherrschung hochkomplexer Wirkungszusammenhänge der Realität durch Modellbildung}

Die empirische Überprüfung kausaler Zusammenhänge zwischen den Produkten staatlichen Handelns und den gesamtgesellschaftlich ausgelösten Wirkungen ist angesichts multifaktorieller Beziehungen sowie vielfältiger exogener Einflussfaktoren äußerst diffizil. Staatliches Handeln erfolgt in der Realität in hochkomplexen und dynamischen Systemen und wird bei unvollkommenem Informationsstand durchgeführt. ${ }^{585}$ Derartige Systeme zeichnen sich durch folgende relevante Eigenschaften aus: ${ }^{586}$

- In komplexen Systemen bestehen zwischen den Systemelementen vielfältige nichtlineare, nicht-additive Verknüpfungen. Als Konsequenz dieser Eigenschaft lassen sich die auf einzelne staatliche Maßnahmen zurückgehenden Wirkungen nicht separieren (Multikausalität). Nicht die isolierte Wirkungsanalyse einzelner Produkte, sondern erst die vernetzte und simultane Analyse sämtlicher staatlicher Produktbereiche sowie exogener Einflussfaktoren würde eindeutige Einsichten in das resultierende Verhalten des Gesamtsystems vermitteln.

\footnotetext{
$584 \mathrm{Vgl}$. Haenecke (2002), S. 173.

585 Ein System bezeichnet ein Gebilde, dessen wesentliche Elemente so aufeinander bezogen sind und in einer Weise wechselwirken, dass sie als zweckgebundene Einheit angesehen werden können und sich in dieser Hinsicht gegenüber der sie umgebenden Umwelt auch abgrenzen. Vgl. hierzu grundlegend Luft (2006).

586 Vgl. in ähnlicher Weise auch Adam (2000), S. 1 ff. sowie Adam (1998), S. 10 ff.
} 
- In dynamischen Systemen besteht nicht nur zu einem Zeitpunkt eine Verbundwirkung; vielmehr existieren daneben zeitübergreifende Beziehungen. Staatliche Maßnahmen lösen sowohl heute als auch in Zukunft Wirkungen aus. Durch jede Maßnahme verändert sich der Zustand des Gesamtsystems, woraus in der Folge weitere Änderungen resultieren, die wiederum durch zukünftige Maßnahmen beeinflusst werden. Als Konsequenz dieses intertemporalen Zusammenhangs lassen sich den einzelnen staatlichen Produkten meist nur Ketten von Wirkungen zuordnen (Kausalketten).

- Staatliches Handeln erfolgt grundsätzlich bei einem unvollkommenen Informationsstand. Die Informationsdefizite beziehen sich einerseits auf künftige Handlungsmöglichkeiten und Daten, von denen die Wirkungen heutiger Maß-nahmen auch abhängen. Andererseits erschweren vielfältige subjektive und personengebundene Einflüsse den Aufbau eindeutiger Wirkungsketten, die zur Beurteilung des Gesamtsystems erforderlich wären.

Die skizzierten Systemeigenschaften der Wirkungen staatlichen Handelns zeigen sehr deutlich die hochkomplexen Zusammenhänge der Realität auf. Für das wirkungsorientierte Controlling bzw. die Methodik der Wirkungsanalyse stellt sich damit die Frage nach einer sinnvollen Strukturierung der interdependenten Problemstellung. Nach ADAM ist es keine erfolgsversprechende Strategie, die vielfältigen statischen und dynamischen Beziehungen zwischen den Systemelementen simultan abbilden zu wollen. Der Komplexitätsgrad wäre sehr schnell nicht mehr zu bewältigen. ${ }^{587}$ Das schlechtstrukturierte - da viel zu komplexe - Gesamtproblem muss daher in möglichst gut strukturierte Teilprobleme gegliedert werden, wobei den wesentlichen Beziehungen zwischen relevanten Systemelementen dennoch Rechnung getragen wird. ${ }^{588}$

Zur Strukturierung der aufgezeigten Problemstellung soll auf Grundlage der Systembetrachtung ein möglichst allgemeingültiges Beschreibungsmodell der Wirkungen staatlichen Handelns erarbeitet werden. Modelle können als Abbilder oder Repräsentation von Ausschnitten der Realität verstanden werden. ${ }^{59}$ Beschreibungsmodelle strukturieren das modellierte Problem, indem sie eine geordnete Anzahl faktischer Aussagen über die Realität machen. ${ }^{590}$

\subsubsection{Modellkonzeption der Wirkungen staatlichen Handelns}

Zielsetzung dieses Kapitels ist die Konzeption eines möglichst allgemeingültigen Beschreibungsmodells der Wirkungen staatlichen Handelns. Mit diesem Modell soll zunächst die Strukturierung der komplexen Problemstellung gelingen, um darauf auf-

\footnotetext{
587 Vgl. Adam (2000), S. 2. ADAM betrachtet den Komplexitätsgrad von betrieblichen Investitionsentscheidungen, die auch vielfältige statische und dynamische Interdependenzen aufweisen.

588 Vgl. Adam (2000), S. 2 f.

589 Vgl. Berens /Delfmann (1995), S. 23.

$590 \mathrm{Vgl}$. Berens /Delfmann (1995), S. 27.
} 
bauend geeignete Instrumentarien der Wirkungsanalyse bereitzustellen. Das Modell erweitert die von KıÖTI geschaffenen Grundlagen zur wissenschaftlichen Untersuchungsanlage einer Outcome-Evaluation. ${ }^{591}$ In einem ersten Schritt widmen sich die Ausführungen statischen Aspekten, anschließend den dynamischen Aspekten der Wirkungen staatlichen Handelns.

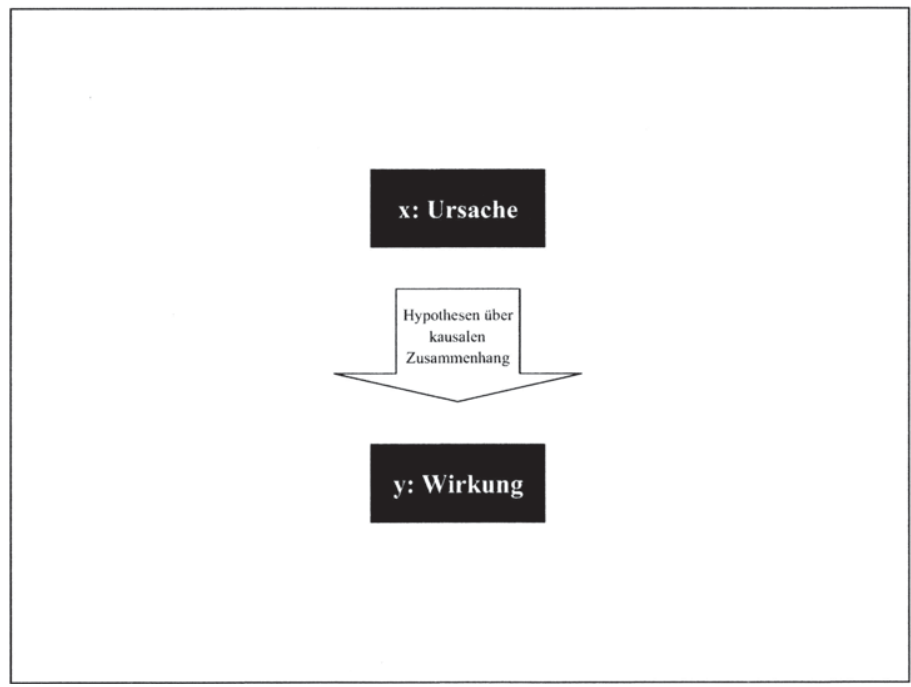

Abbildung 24: Erster Schritt der Modellkonzeption

Die Modellkonzeption basiert auf den wissenstheoretischen Erkenntnissen über die Beziehung zwischen Ursache und Wirkung. ${ }^{592}$ In Form von Hypothesen werden Vermutungen über einen kausalen Zusammenhang zwischen Ursache und Wirkung angestellt. Die Ursache wird in diesem Zusammenhang als erklärende, die Wirkung als zu erklärende Variable aufgefasst (siehe Abbildung 24):

- $\mathrm{x}=$ Ursache als unabhängige (erklärende) Variable

- $y=$ Wirkung als abhängige (zu erklärende) Variable

Übertragen in den Kontext des staatlichen Handelns bedeutet dies, dass Vermutungen darüber bestehen, dass durch eine bestimmte staatliche Maßnahme unterschiedliche Wirkungen in der Gesellschaft ausgelöst werden:

- $\mathrm{x}_{\mathrm{a}}=$ staatliche Maßnahme als erklärende Variable

- $\mathrm{y}_{\mathrm{n}}=$ gesamtgesellschaftliche Wirkungen als zu erklärende Variablen

\footnotetext{
$591 \mathrm{Vgl}$. im Folgenden grundlegend Klöti (1997), S. 48 ff.

592 Vgl. hierzu ausführlich Kapitel 4.1.1.2.
} 
Zur Analyse der Wirkungen wird untersucht, ob und in welchem Ausmaß räumlich und zeitlich identifizierbare Veränderungen in der gesellschaftlichen Wirklichkeit auftreten, die sich möglicherweise auf die öffentliche Maßnahme zurückführen lassen. ${ }^{593}$ Zur Beobachtung dieser Veränderungen wird im Folgenden der Begriff des ,gesamtgesellschaftlichen Wirkungsgefüges" verwendet. ${ }^{594}$

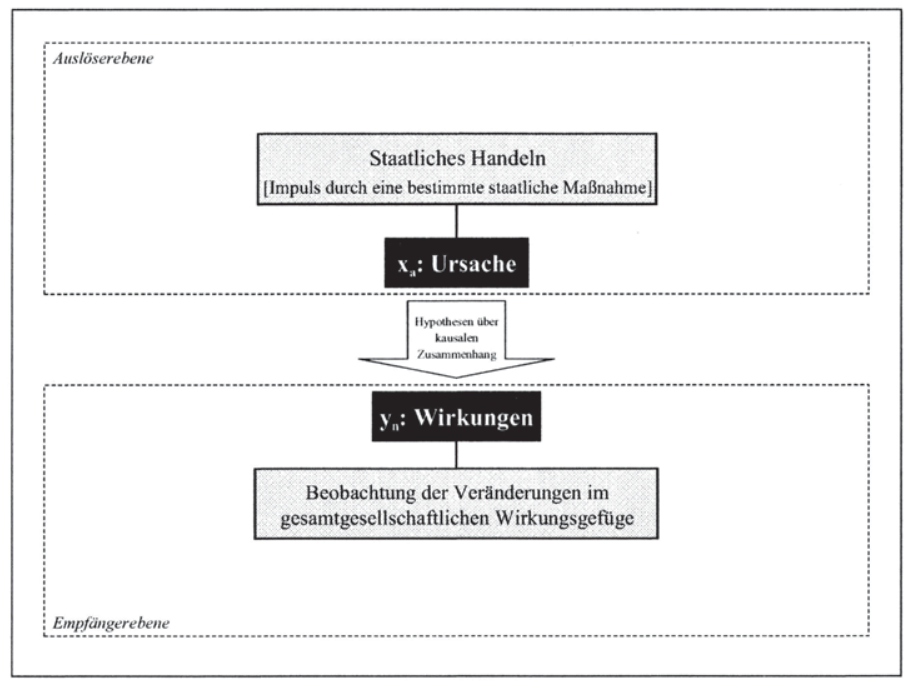

Abbildung 25: Zweiter Schritt der Modellkonzeption

Abbildung 25 zeigt weiterhin, dass innerhalb der Modellkonzeption zwei unterschiedliche Systemebenen voneinander abgegrenzt werden können. Demnach wird das staatliche Handeln einer Auslöserebene zugeordnet, die hierdurch in der Gesellschaft ausgelösten Wirkungen werden einer Empfängerebene zugeordnet. Ein Beispiel für dieses bislang recht einfache Modell ist die staatliche Maßnahme "Tempolimit«, mit dem vermutlich unter anderem die Wirkungen »weniger Abgase« und »weniger Unfälle« verbunden sind.

Dieses einfache Modell entspricht jedoch in keiner Weise den komplexen Zusammenhängen der Realität. Die erste Problematik besteht darin, dass auf das gesamtgesellschaftliche Wirkungsgefüge gleichzeitig eine Vielzahl unterschiedlicher staatlicher Maßnahmen einwirken (siehe Abbildung 20). Die zu beobachtenden bzw. empirisch nachgewiesenen Wirkungen könnten daher auch auf andere staatliche Maßnahmen zu-

593 Vgl. Klöti (1997), S. 49.

594 Der Begriff des Wirkungsgefüges stammt aus der Verhaltensforschung und wird dort zur Analyse ganzheitlicher Zusammenhänge bzw. des Zusammenwirkens unterschiedlicher Einflussfaktoren verwendet. Vgl. hierzu Brockhaus (2006), S. 168. 
rückzuführen sein. Solche erklärenden Einflussvariablen müssen im Rahmen einer Wirkungsanalyse möglichst separiert werden:

- $\mathbf{x}_{\mathrm{n}}=$ andere staatliche Maßnahmen als erklärende Variablen

So könnte in der Fortführung des obigen Beispiels nicht nur die staatliche Maßnahme "Tempolimit« für die Reduzierung der Abgaswerte verantwortlich sein, sondern auch die "Verschärfung der Abgasnormen« für Neufahrzeuge. Und die Reduzierung der Unfallzahlen ist bspw. nicht nur auf die Einführung eines »Tempolimits « zurückzuführen, sondern eventuell auch auf das Aufstellen »zusätzlicher Hinweisschilder«.

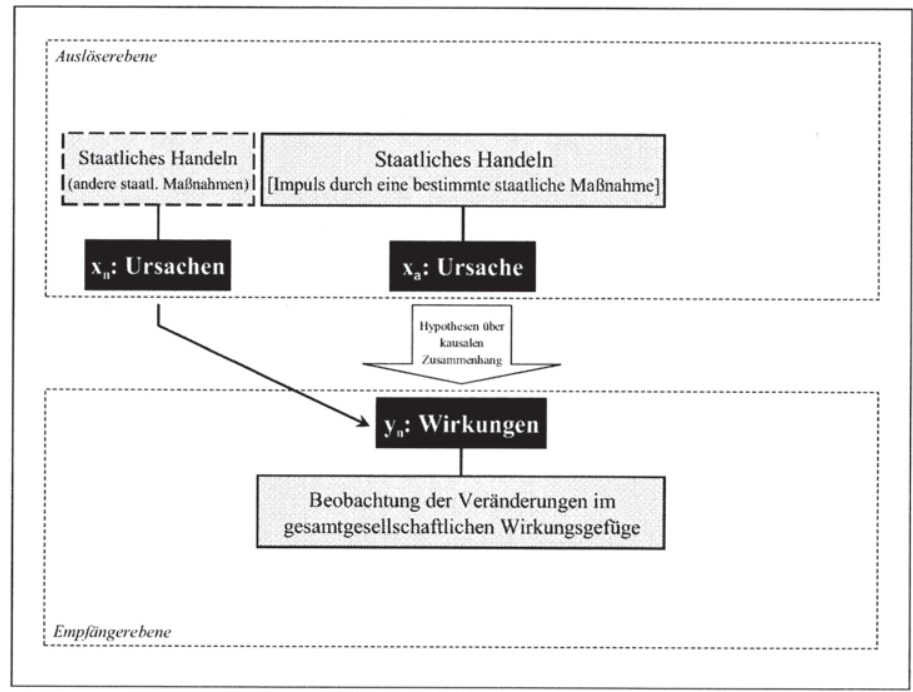

Abbildung 26: Dritter Schritt der Modellkonzeption

Komplizierend kommt hinzu, dass neben den staatlichen Maßnahmen eine Vielzahl weiterer Faktoren für die Veränderungen im gesamtgesellschaftlichen Wirkungsgefüge verantwortlich sein könnte. Solche Faktoren werden im Folgenden als exogene Effekte bezeichnet und gehen ebenfalls als unabhängige Variablen in die Modellkonzeption ein:

- $\mathrm{z}_{\mathrm{n}}=$ exogene Effekte als erklärende Variablen

Daher ist es notwendig, die Auslöserebenen zu trennen und zwischen staatlichen Maßnahmen einerseits und allen sonstigen gesellschaftlichen Einflussfaktoren andererseits 
zu unterscheiden. ${ }^{595}$ Entsprechend müssen auch in der Wirkungsanalyse die exogenen Effekte separiert werden.

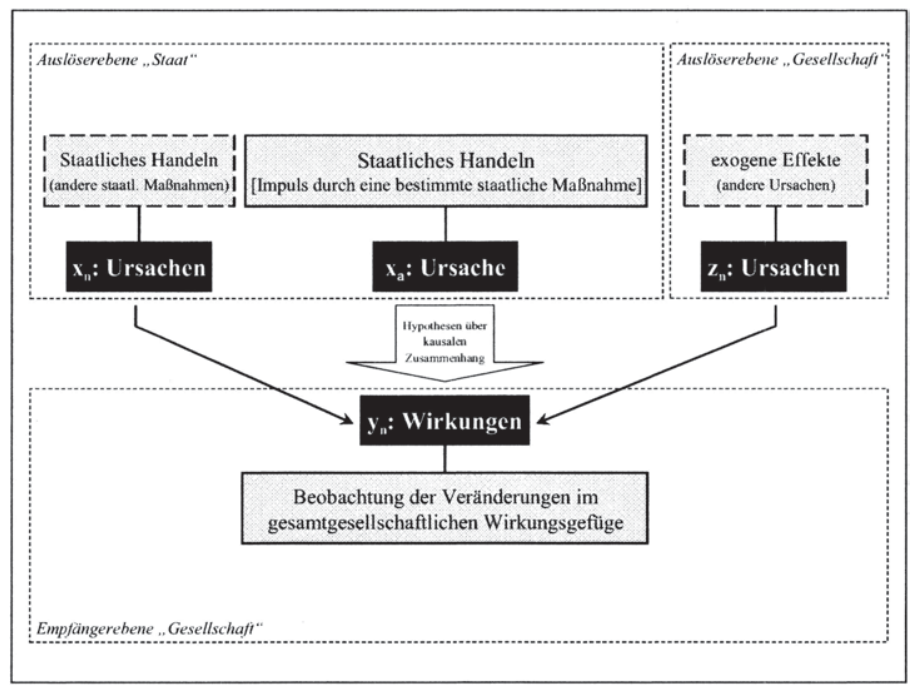

Abbildung 27: Vierter Schritt der Modellkonzeption

Bezogen auf das obige Beispiel sind beobachtete Wirkungen unter Umständen gar nicht auf die staatlichen Maßnahmen zurückzuführen, sondern auf exogene Effekte. So hat eventuell eine "Verbesserung der Fahrzeugtechnik" zu den reduzierten Abgaswerten und rückläufigen Unfallzahlen geführt. Nicht staatliches Handeln, sondern allgemeine gesellschaftliche Entwicklungen wären damit Auslöser beobachteter Veränderungen im Wirkungsgefüge.

Für die Untersuchungsanlage einer Wirkungsanalyse bzw. die Beurteilung der Wirksamkeit staatlicher Maßnahmen ist der zugrunde liegende Bewertungsmaßstab entscheidend. ${ }^{596}$ Die Analyse kann sich einerseits nur an einem explizit definierten Zielsystem $^{597}$ des politisch-administrativen Systems ausrichten ${ }^{598}$ oder andererseits auch die vorher nicht beabsichtigten Wirkungen außerhalb der Zieldimension in die Analyse

595 Unter Gesellschaft wird in diesem Zusammenhang die Verbundenheit aller Lebewesen in einen abgrenzbaren Lebenszusammenhang (z.B. Nation) verstanden - hierauf ist staatliches Handeln primär gerichtet.

$596 \mathrm{Vgl}$. hierzu auch die Ausführungen des Kapitels 4.1.1.1.

597 Problematisch ist in diesem Zusammenhang die Abstimmung des staatlichen Zielsystems. Theoretisch könnte im politisch-administrativen System ein vollständig abgestimmtes Zielsystem für sämtliche staatliche Handlungsbereiche existieren. Doch in der Realität ist man von diesem Idealzustand weit entfernt. Zwar existieren in abgegrenzten staatlichen Handlungsbereichen mittlerweile recht klare Zielsetzungen, doch findet nur eine mangelnde Abstimmung zwischen unterschiedlichen Sektoren statt. Dies kommt durch die Darstellung einer brüchigen Verbindung in Abbildung 28 zum Ausdruck.

598 Diese Vorgehensweise ist im Rahmen der klassischen Outcome-Evaluation sehr verbreitet. 
einbeziehen. Für eine umfassende Wirkungsanalyse staatlichen Handelns sind aber sowohl an Zielen orientierte Wirkungen, als auch die nicht intendierten Nebenwirkungen relevant:

- $\mathrm{y}_{\mathrm{a}}=$ (intendierte) Wirkung als zu erklärende Variablen

- $\mathrm{y}_{\mathrm{b}}=$ (nicht intendierte) Nebenwirkung als zu erklärende Variablen

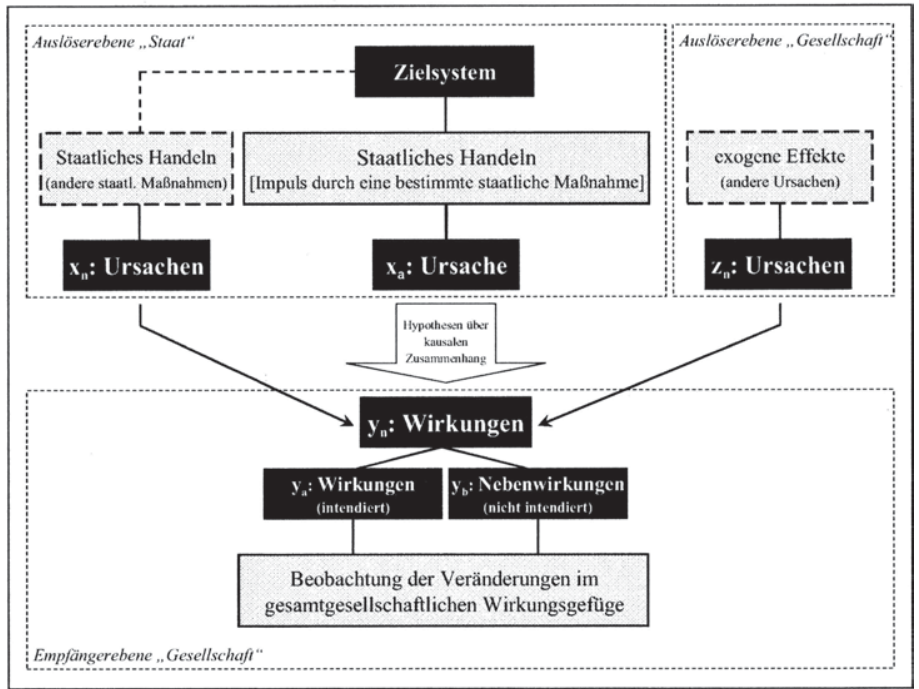

Abbildung 28: Fünfter Schritt der Modellkonzeption

Im Beispiel könnten zusätzlich zu den intendierten Wirkungen der »Abgasreduzierung« und »Unfallvermeidung « auch Nebenwirkungen des »Tempolimits« beobachtet werden, wie "weniger Staubildung auf verkehrsreichen Strecken« oder ein »Ausweichen des Verkehrs auf Nebenstraßen und Schleichwege«. Eine genauere Betrachtung dieser Nebenwirkungen zeigt zudem, dass hier zwei verschiedene Arten zu unterscheiden sind. So kann die Vermeidung von Staubildung positiv bewertet und das Ausweichverhalten des Verkehrs auf Nebenstrecken negativ bewertet werden. Die Wertung, ob es sich um positive oder negative Nebenwirkungen handelt, muss vor dem Hintergrund der Zielsetzungen des politisch-administrativen Systems vorgenommen werden. Wie Abbildung 29 zeigt, können sich die Nebenwirkungen daher als erwünscht oder als unerwünscht herausstellen. ${ }^{599}$ In entsprechender Weise sind sie in der Wirkungsanalyse zu berücksichtigen:

- $\mathrm{y}_{\mathrm{bl}}=$ erwünschte Nebenwirkung als zu erklärende Variablen

- $\mathrm{y}_{\mathrm{b} 2}=$ unerwünschte Nebenwirkung als zu erklärende Variablen

599 VLDUNG unterscheidet in diesem Zusammenhang zwischen günstigen Nebeneffekten und schädlichen Nebeneffekten. Vgl. Vedung (1999), S. 48. 


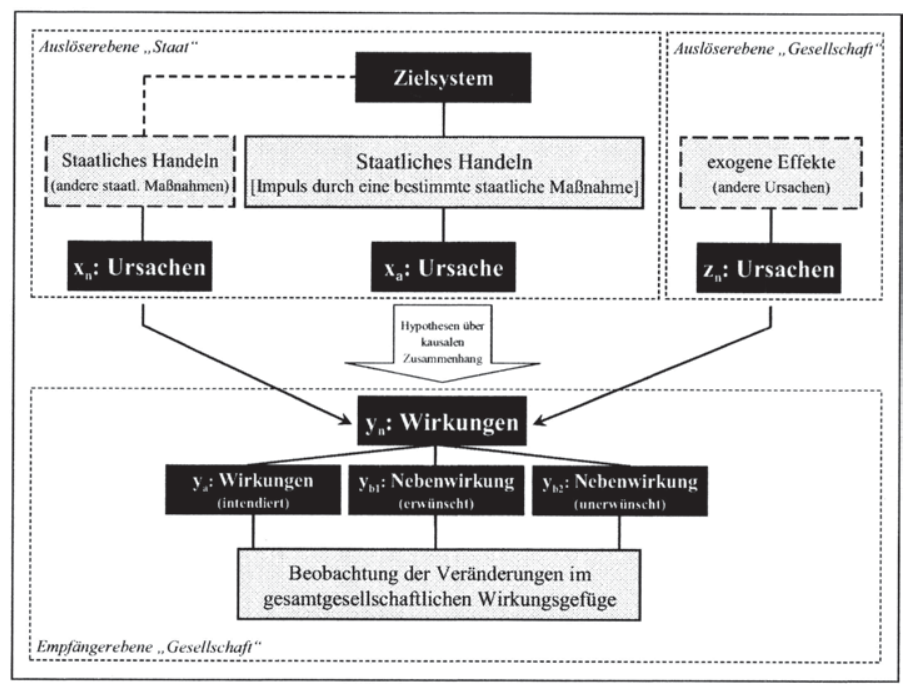

Abbildung 29: Sechster Schritt der Modellkonzeption

Die Aufgabe der Wirkungsanalyse besteht allerdings nicht nur darin, einen Zusammenhang zwischen einer staatlichen Maßnahme und den hierdurch ausgelösten Wirkungen herzustellen. Eine weitere wesentliche Herausforderung der Wirkungsorientierung besteht darin, den Akteuren im politisch-administrativen System Verantwortung zuzuweisen ${ }^{600}$ Verfehlt eine staatliche Maßnahme die Zielsetzungen oder werden Anknüpfungspunkte für Verbesserungen gesucht, muss man die betrachtete Maßnahme als unabhängige Variable weiter aufschlüsseln.

Hier sind im politisch-administrativen System grundsätzlich zwei Teilvariablen zu unterscheiden ${ }^{601}$ Verantwortung trägt einerseits die politische Ebene durch die Ausgestaltung der Politik, beispielsweise in Form von Gesetzen. ${ }^{602}$ Andererseits sind ebenso der administrative Maßnahmenvollzug und die hieraus entstehenden Produkte der öffentlichen Verwaltung für das Gelingen politischer Vorgaben verantwortlich: ${ }^{603}$

- $\mathrm{x}_{\mathrm{a} 1}=$ Ausgestaltung der Politik als erklärende Variable (Gesetze)

- $\mathrm{x}_{\mathrm{a} 2}=$ administrativer Vollzug als erklärende Variable (Produkte)

Wenn im Beispiel der staatlichen Maßnahme "Tempolimit« die beobachteten Wirkungen hinter den Erwartungen zurückbleiben, lautet die zu untersuchende Fragestellung,

$600 \mathrm{Vgl}$. die detaillierten Ausführungen des Kapitels 2.2.3.4.

601

Vgl. hierzu auch Klöti (1997), S. 52.

602 Vgl. Linder/Peters (1989), S. 631 ff.

603 KLÖTI weist in diesem Zusammenhang zu Recht darauf hin, dass hier Rückkopplungen zu beachten sind. So ist es denkbar, dass das Policy-Design selbst bereits so ausgestaltet ist, das ein sinnvoller Vollzug entweder unmöglich oder zumindest erschwert wird (programmierte Vollzugsdefizite). Vgl. Klöti (1997), S. 52. 
ob dies entweder auf die Unwirksamkeit der gesetzlichen Regelung zurückzuführen ist - die Reduktion der zulässigen Höchstgeschwindigkeit könnte zu gering ausgefallen sein; oder ob der mangelnde administrative Vollzug für die Unwirksamkeit verantwortlich ist, da die Einhaltung des Tempolimits nur unzureichend durch die öffentliche Verwaltung kontrolliert wurde.

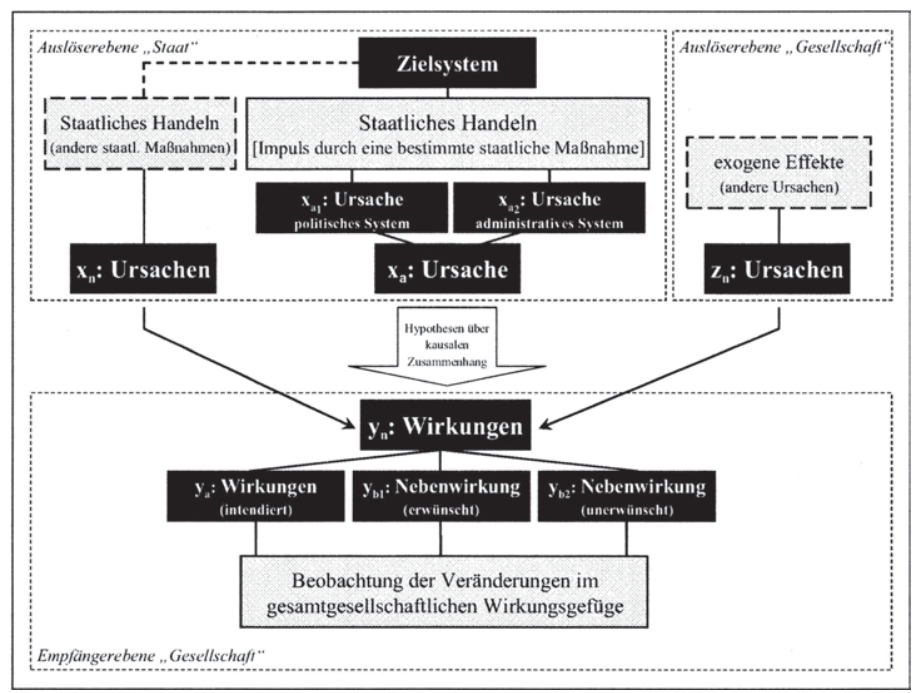

Abbildung 30: Siebter Schritt der Modellkonzeption

Wirkungen bzw. die Veränderungen im gesamtgesellschaftlichen Wirkungsgefüge stellen für sich betrachtet eigene Ereignisse dar, die wiederum hieraus folgende Ereignisse kausal begründen können. Demnach ergibt sich eine Kausalkette bzw. Wirkungskette, bei der jede Wirkung selbst wieder Ursache neuer Wirkungen ist. Im Rahmen der Modellkonzeption muss daher zwischen den primären Wirkungen, welche direkt durch die staatliche Maßnahme ausgelöst werden und sekundären Folgewirkungen, welche durch die primären Wirkungen ausgelöst werden, unterschieden werden:

- $\mathrm{y}_{\mathrm{n}}=$ (primäre) Wirkungen als zu erklärende Variablen

- $y_{m}=$ (sekundäre) Folgewirkungen als zu erklärende Variablen

Wirkungsketten sind in der Regel mehrstufig, d.h. aus sekundären Folgewirkungen erwachsen als eigenständiges Ereignis erneut tertiäre Folgewirkungen, so dass sich dieser Prozess beliebig weit fortführen lässt. Weiterhin ist $\mathrm{zu}$ beachten, dass auch sämtliche Folgewirkungen wiederum als intendierte (sekundäre) Wirkung oder als erwünschte bzw. unerwünschte (sekundäre) Nebenwirkung eingestuft werden können. 


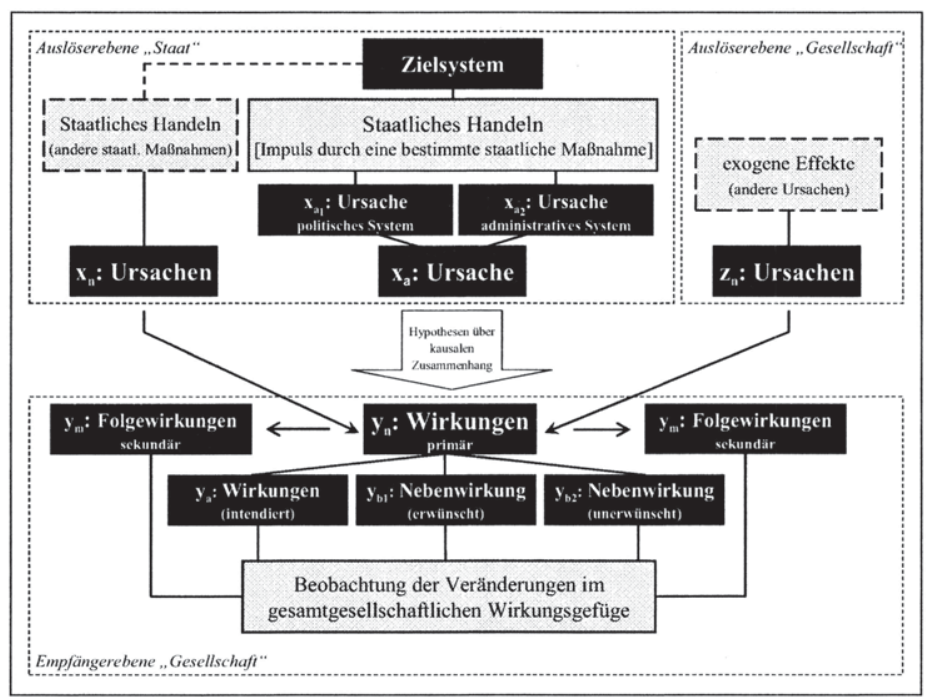

Abbildung 31: Achter Schritt der Modellkonzeption

Mögliche Folgewirkungen des »Tempolimits« könnten z.B. die »langfristige Verringerung von Atemwegserkrankungen « durch die Reduzierung der Abgasbelastung und »Einsparungen im Gesundheitssektor« durch den Rückgang der Unfallzahlen sein. In diesem einfachen Beispiel wird bereits deutlich, dass die Betrachtung von Wirkungsketten aufeinander folgende Ereignisse betrifft und damit sachliche und zeitliche Interdependenzen zwischen Ursache und Wirkungen eine wichtige Rolle spielen. Nachdem zunächst die Abgrenzung und Strukturierung der wesentlichen Elemente der Modellkonzeption erfolgt ist, beschäftigen sich die weiteren Ausführungen mit den statischen und dynamischen Aspekten der Beziehungen zwischen den enthaltenen Elementen.

\subsubsection{Statische Aspekte der Modellkonzeption}

Aufgabe der Wirkungsanalyse ist es, die Wirkungsweise staatlichen Handelns zu analysieren. Da offensichtlich immer mehrere staatliche Maßnahmen und eine Vielzahl exogener Effekte gleichzeitig Auslöser bestimmter Wirkungen in der Gesellschaft sind, bzw. beobachtete Veränderungen im gesamtgesellschaftlichen Wirkungsgefüge sich immer auf mehrere erklärende Faktoren zurückführen lassen, ist eine detaillierte Untersuchung der Zusammenhänge und Beziehungen von besonderer Bedeutung. In der statischen Betrachtung spielen insbesondere die drei nachfolgenden Effekte eine wesentliche Rolle: ${ }^{604}$

604 Vgl. hierzu Wilde (1989), S. 56 und Daschmann (1993), S. 6. Beide Autoren analysieren die Beziehungen und Zusammenhänge von unternehmensbezogenen Erfolgsfaktoren auf das Erfolgspotenzial eines Unternehmens. Die allgemeinen Erkenntnisse zu den statischen Aspekten des Zusammenhangs zwischen Erfolgsfaktor und Erfolgspotenzial werden im Folgenden auf die Wirkungen staatlichen Handelns übertragen. 
- Analyse der Wirkungsrelevanz einer staatlichen Maßnahme

- Analyse der Wirkungsstärke der staatlichen Maßnahme anhand der Wirkungselastizität, -variabilität und -determiniertheit

- Analyse der Wirkungsinteraktion der betrachteten staatlichen Maßnahme mit anderen staatlichen Maßnahmen sowie exogenen Effekten

Die Analyse der Wirkungsrelevanz beschreibt die einer beobachteten Wirkung zugrunde liegenden ursächlichen Faktoren. Im Idealfall lässt sich die Wirkung nur auf einen auslösenden Faktor bzw. eine bestimmte staatliche Maßnahme zurückführen, so dass die Wirkung nur dieser Maßnahme zugerechnet werden kann. In der Realität ist aber meist eine Vielzahl unterschiedlicher Faktoren potentiell relevant, so dass alle Faktoren in die Analyse einbezogen werden müssen. Im Beispiel des "Tempolimits" besitzt neben dieser öffentlichen Maßnahme sicherlich eine Vielzahl weiterer Faktoren Wirkungsrelevanz für den beobachteten »Rückgang von Abgasen und Unfallzahlen«.

Die Analyse der Wirkungsstärke schließt sich an die Analyse der Wirkungsrelevanz an. Im Rahmen der statischen Wirkungsanalyse wird die Stärke des Zusammenhangs zwischen dem unabhängigen Faktor - der betrachteten staatlichen Maßnahme - und dem abhängigen Faktor - der ausgelösten Wirkung - näher betrachtet. Die Wirkungsstärke lässt sich wiederum auf drei Komponenten zurückführen (siehe auch Abbildung 32). ${ }^{605}$

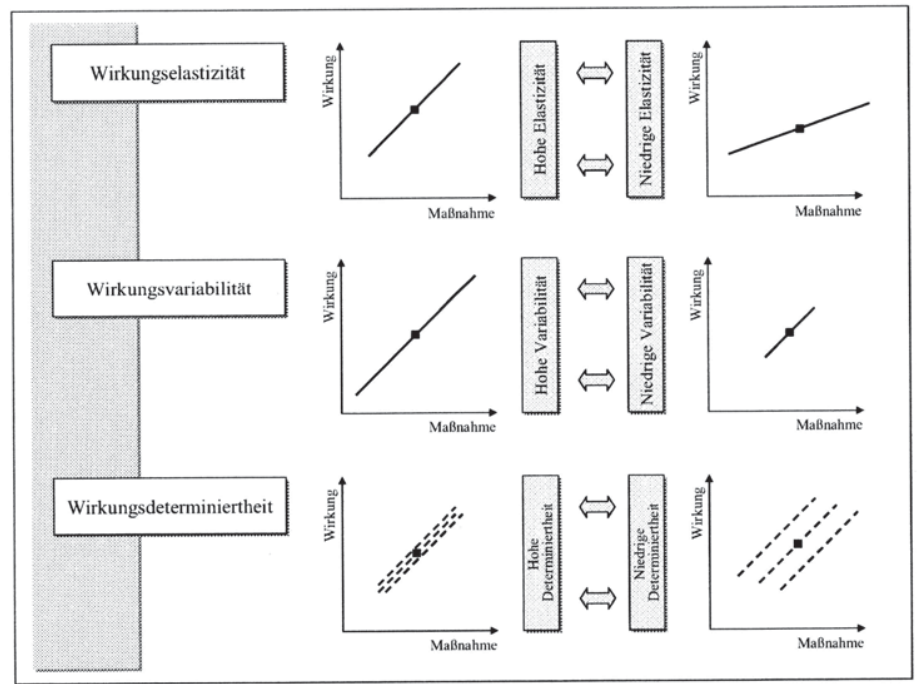

Abbildung 32: Komponenten der Wirkungsstärke ${ }^{600}$

605 Zu den Komponenten vgl. auch Wilde (1989), S. 56 ff.

606 In Anlehnung an Wilde (1989), S. 57, der in ähnlicher Weise die Komponenten der Wirkungsstärke von unternehmensbezogenen Erfolgsfaktoren und dem Erfolgspotenzial darstellt. 
Die einzelnen Komponenten der Wirkungsstärke werden nachfolgend kurz erläutert:

- Die Wirkungselastizität einer staatlichen Maßnahme beschreibt die Höhe beobachtbarer Veränderungen einer Wirkung, die sich aus einer Variation in der Leistungserstellung ergeben, d.h. Menge oder Intensität der betrachteten öffentlichen Maßnahme werden erhöht oder verringert. Der entsprechende Zusammenhang kann auch als „Hebelwirkung“ interpretiert werden. Beispielsweise ist vorstellbar, dass eine Verschärfung des »Tempolimits« aufgrund hoher Wirkungselastizität zwar zu einer deutlichen »Reduzierung der Abgaswerte« führt, die »Unfallzahlen« sich aufgrund geringer Wirkungselastizität zur Geschwindigkeitsbegrenzung aber nur unwesentlich reduzieren.

- Die Wirkungsvariabilität beschreibt die grundsätzliche Bandbreite, in der Menge oder Intensität der Maßnahmengestaltung überhaupt verändert werden können. Bei konstanter Elastizität werden die Möglichkeiten, Veränderungen im gesellschaftlichen Wirkungsgefüge hervorzurufen, wesentlich durch diese Bandbreite des staatlichen Handlungsspektrums bestimmt. Diese Bandbreite ist daher gleichbedeutend mit den zur Verfügung stehenden Mitteln der Akteure im politisch-administrativen System, steuernd auf die Gesellschaft Einfluss zu nehmen. So zeichnet sich das Handlungsspektrum bei der Festlegung eines »Tempolimits« zunächst durch eine hohe Wirkungsvariabilität aus, da jede Geschwindigkeitsbegrenzung grundsätzlich in Frage kommt - faktisch ist der Bereich sinnvoller Tempolimite jedoch klar begrenzt.

- Die Wirkungsdeterminiertheit einer Maßnahme beschreibt die Bestimmtheit oder auch Wahrscheinlichkeit beobachtbarer Veränderungen einer Wirkung infolge von Variationen in der Leistungserstellung. Bei einer hohen Determiniertheit führen zusätzliche Maßnahmen recht zuverlässig zu den beabsichtigten Veränderungen im gesellschaftlichen Wirkungsgefüge - doch staatliches Handeln zeigt häufig stochastische Wirkungszusammenhänge. ${ }^{607}$ So zeichnet sich ein »Tempolimit« tendenziell durch eine eher geringe Wirkungsdeterminiertheit aus, da die Einführung einer Geschwindigkeitsbegrenzung an verschiedenen Strecken nicht immer die gleiche Wirkung entfaltet.

Die Kombination dieser drei Faktoren erklärt die Wirkungsstärke einer staatlichen Maßnahme auf bestimmte Wirkungen in der Gesellschaft. Bislang wurde die Beziehung zwischen staatlicher Maßnahme und ausgelöster Wirkung als linearer Zusammenhang dargestellt (siehe Abbildung 32). Grundsätzlich sind jedoch ganz unterschiedliche Verläufe des Wirkungszusammenhangs denkbar, wie zum Beispiel progressive oder degressive Wirkungsverläufe oder auch Sprung- oder Sättigungs-Effekte. ${ }^{608}$ So ist davon auszugehen, dass eine schrittweise »Verschärfung des Tempolimits« keinen li-

$607 \mathrm{Vgl}$. hierzu auch die Ausführungen des Kapitels 4.1.1.2.

$608 \mathrm{Vgl}$. Wilde (1989), S. 58. WILDE hat die in der Literatur gefundenen Beispiele für Wirkungsbeziehungen zwischen einem Erfolgsfaktor (als unabhängige Variable) und Erfolgspotenzial (als abhängige Variable) zusammengestellt. 
nearen Zusammenhang zur »Reduzierung der Unfallzahlen« aufweist. Vielmehr dürfte der Wirkungszusammenhang eher degressiv verlaufen, d.h. jede erneute Verschärfung führt nur noch zu einem entsprechend geringeren »Rückgang der Unfallzahlen«.

In der Realität besitzt eine Vielzahl unterschiedlicher Faktoren Wirkungsrelevanz und beeinflusst in jeweils unterschiedlicher Wirkungsstärke die beobachteten Veränderungen im gesellschaftlichen Wirkungsgefüge. Es ist also davon auszugehen, dass zwischen diesen auslösenden Faktoren vielfältige Interdependenzen bestehen, welche im Folgenden als Wirkungsinteraktionen bezeichnet werden. Es lassen sich prinzipiell vier denkbare Klassifizierungen dieser Interaktionsbeziehungen unterscheiden, die in $A b$ bildung 33 zusammenfassend dargestellt sind. ${ }^{609}$

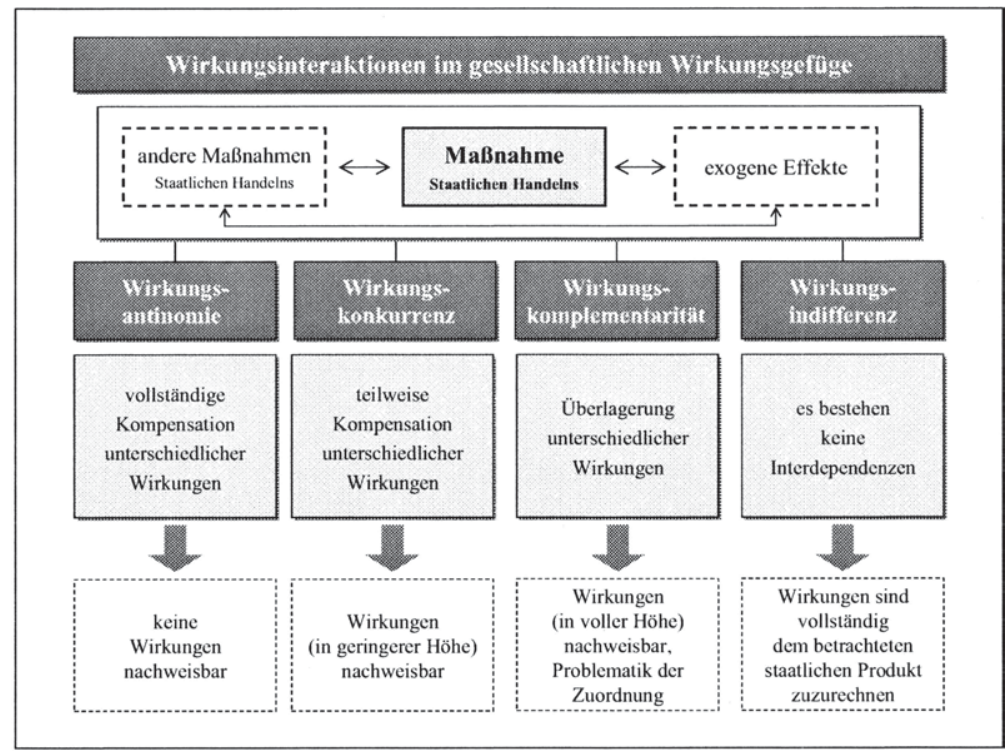

Abbildung 33: Klassifizierung der Wirkungsinteraktionen

Die Begrifflichkeiten beschreiben, wie sich die auslösenden Faktoren staatlichen Handelns sowie exogene Effekte, bzw. die hierdurch verursachten Wirkungen, zueinander verhalten. ${ }^{610}$ So kann der Eintritt einer neuen Wirkung die bestehenden Wirkungszusammenhänge in der Gesellschaft verändern. Im Rahmen der Wirkungsanalyse staatlichen Handelns müssen demnach die entsprechenden Wechselbeziehungen beachtet werden:

609 SCHMITTING/SikMES nehmen eine vergleichbare Klassifizierung der Wechselbeziehungen unterschiedlicher Risiken für die Konzeption eines Risikomanagementsystems vor. Vgl. Schmitting/Siemes (2003), S. 535 f.

$610 \mathrm{Zu}$ den verwendeten Begrifflichkeiten vgl. auch Schmitting/Siemes (2003), S. 535 f. 
- In diesem Zusammenhang spricht man von Wirkungsantinomie, wenn sich unterschiedliche Wirkungen vollständig kompensieren, so dass im Endeffekt keine beobachtbaren Veränderungen im gesellschaftlichen Wirkungsgefüge eintreten - positive und negative Wirkungen heben sich gegenseitig auf. So könnte zum Beispiel die durch die Geschwindigkeitsbegrenzung beabsichtigte Abgasreduzierung aufgrund eines gesellschaftlichen Trends zu höheren Motorisierungen der Fahrzeuge vollständig aufgehoben werden.

- Wirkungskonkurrenz liegt vor, wenn sich die gegenläufigen Effekte nicht vollständig aufheben. In der Realität ist diese Konstellation sehr häufig zu beobachten. Zwar werden die beabsichtigten Wirkungen in der Gesellschaft ausgelöst, doch aufgrund unerwünschter Nebenwirkungen und exogener Effekte teilweise kompensiert und damit verringert. Problematisch ist, dass sogar staatliches Handeln selbst häufig Wirkungskonkurrenz aufweist, da sektorale Zielsetzungen nur ungenügend aufeinander abgestimmt sind. So könnten beispielsweise umweltpolitische Erwägungen zur Verschärfung von Geschwindigkeitsbegrenzungen führen, aber wirtschaftspolitische Erwägungen zu einer steuerlichen Begünstigung des Schwerlastverkehrs mit den entsprechend negativen Nebenwirkungen für die Umwelt.

- Dagegen beschreibt die Wirkungskomplementarität eine Wechselbeziehung, bei der sich die unterschiedlichen Effekte lediglich überlagern. Wirkungen werden in voller Höhe in der Gesellschaft wirksam, problematisch ist allerdings die Zuordnung zu einem bestimmten auslösenden Faktor. In Bezug auf staatliches Handeln wird in diesem Zusammenhang auch häufig von „Mitnahmeeffekten“ gesprochen. Bestimmte staatliche Leistungen sind demnach gar nicht notwendig, da auch ohne staatlichen Anreiz die entsprechenden Wirkungen in der Gesellschaft eingetreten wären.

- Im Falle einer Wirkungsindifferenz bestehen keine Wechselbeziehungen zwischen unterschiedlichen Ereignissen, so dass sämtliche ausgelösten Wirkungen in voller Höhe der staatlichen Maßnahme zugerechnet werden können.

Nachdem mit der Wirkungsrelevanz, der Wirkungsstärke und der Wirkungsinteraktion die wesentlichen statischen Aspekte der Wirkungsanalyse charakterisiert wurden, sind im Folgenden die dynamischen Aspekte Gegenstand einer näheren Betrachtung.

\subsubsection{Dynamische Aspekte der Modellkonzeption}

Angesichts der teilweise sehr langen - theoretisch betrachtet sogar endlosen - Wirkungsketten wird deutlich, dass der Zeitfaktor in der Wirkungsanalyse staatlichen Handelns eine wichtige Rolle spielt. Daher ist es von besonderer Bedeutung, auch die dynamischen Aspekte der Modellkonzeption strukturiert herauszuarbeiten. Grundsätzlich ist zu beachten, dass die zuvor erläuterten statischen Aspekte der Wirkungsrelevanz, Wirkungsstärke und Wirkungsinteraktion auch im Zeitablauf dynamischen Veränderungen unterworfen sein können. Die entsprechend statisch analysierten Wir- 
kungszusammenhänge sind daher in periodischen Zeitabständen auf ihre Beständigkeit hin erneut zu überprüfen.

Darüber hinaus lassen sich die nachfolgenden dynamischen Aspekte einer Wirkungsanalyse separieren:

- Analyse der mehrstufigen Wirkungsketten staatlichen Handelns ${ }^{611}$

- Analyse der Wirkungsverzögerung zwischen staatlicher Leistungserstellung und dem Eintritt einer Wirkung

- Analyse der Wirkungsdauer nach Wirkungseintritt

Im Rahmen der Modellkonzeption wurde eine Wirkungskette als Abfolge kausal verknüpfter Ereignisse beschrieben, bei der jede Wirkung selbst wieder Ursache einer neuer Wirkung ist. Ursache und Wirkung sind zeitlich strikt aufeinander folgende Ereignisse $^{612}$, so dass eine Wirkungskette eine mehrstufige dynamische Ereignisreihenfolge darstellt (siehe Abbildung 34). Wird der Bezug zu einer staatlichen Maßnahme als Ausgangsursache dieser Kette hergestellt, kann demnach zwischen primären Wirkungen und wiederum hieraus resultierenden Folgewirkungen unterschieden werden.

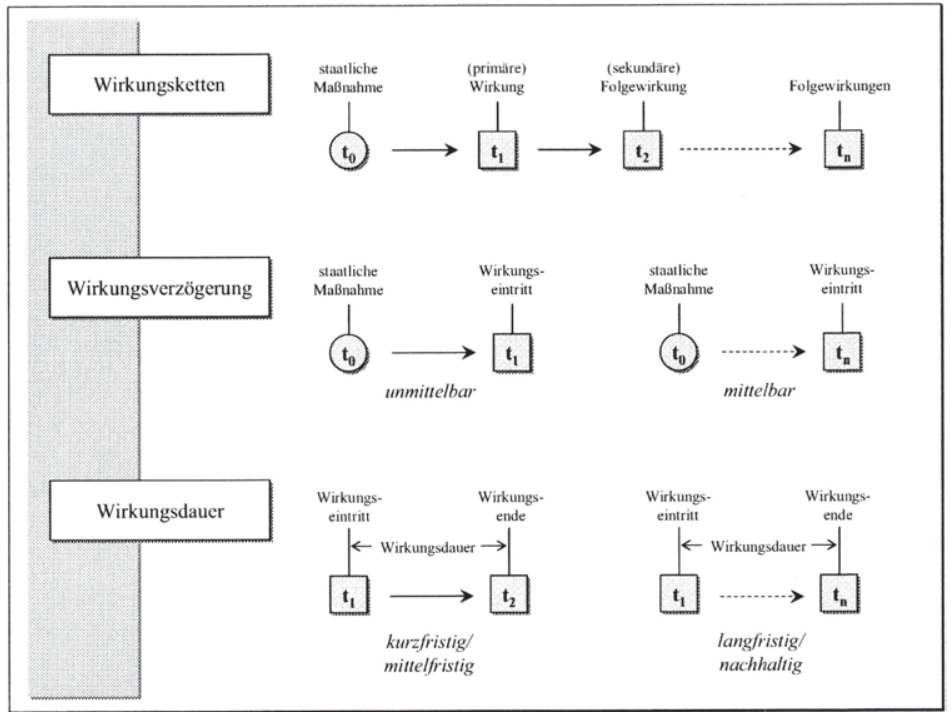

Abbildung 34: Dynamische Aspekte der Modellkonzeption

611 Zum Begriff der Wirkungskette vgl. auch Klöti (1997), S. 52 f.

612 Vgl. hierzu die wissenstheoretischen Ausführungen des Kapitels 4.1.1.2. 
Einen weiteren dynamischen Aspekt der vorgestellten Modellkonzeption stellt die Wirkungsverzögerung zwischen der staatlichen Leistungserstellung und dem tatsächlichen Eintritt der Wirkung in der Gesellschaft dar. Eine solche Verzögerung wird teilweise auch als time lag bezeichnet. ${ }^{613}$ Hinsichtlich der Ausprägung der Wirkungsverzögerung lassen sich unmittelbare Wirkungen, deren Eintritt direkt auf die Leistungserstellung folgt und mittelbare Wirkungen, die erst mit einigen Monaten oder Jahren Verzögerung im gesellschaftlichen Wirkungsgefüge sichtbar werden, unterscheiden (vgl. hierzu $A b$ bildung 34).

Als letzter wesentlicher Aspekt der dynamischen Wirkungsanalyse ist die Wirkungsdauer zu nennen. Die Wirkungsdauer beschreibt den zeitlichen Fortbestand einer Wirkung nach dem Wirkungseintritt - unabhängig vom Wirkungsverlauf bzw. der Wirkungsstärke. ${ }^{614}$ Die Wirkungsdauer beantwortet damit die Frage, wie lange eine bestimmte Wirkung in der Gesellschaft erkennbar bzw. wahrnehmbar bleibt. Hinsichtlich der Ausprägung der Wirkungsdauer lassen sich kurz- bis mittelfristige Wirkungen mit einem eher kürzeren Zeithorizont und langfristige bzw. nachhaltige Wirkungen mit einem theoretisch unbegrenzten Zeithorizont unterscheiden (vgl. auch hierzu Abbildung 34).

\subsubsection{Erkenntnisgewinn für den Fortgang der Untersuchung}

Nachfolgend sind die bisherigen Erkenntnisse zur modellhaften Abbildung der Wirkungen staatlichen Handelns nochmals übersichtlich zusammengestellt (vgl. Tabelle 1). Die vielfältigen Strukturelemente sowie die statischen und dynamischen Aspekte der Wirkungsbeziehungen zeigen deutlich, wie schwierig es ist, die hochkomplexen Zusammenhänge der Realität beherrschbar zu machen.

Das entwickelte Beschreibungsmodell soll in diesem Zusammenhang einen Beitrag leisten, die für eine Wirkungsanalyse relevanten Elemente und Wirkungsbeziehungen sinnvoll zu strukturieren und voneinander abzugrenzen. Mit Hilfe des Modells kann so eine vollständige Analyse und Beschreibung des Wirkungsverlaufs im gesamtgesellschaftlichen Wirkungsgefüge gelingen. Als umfassender Untersuchungsansatz ist dabei der Wirkungsverlauf für alle Wirkungen, einschließlich erwünschter und unerwünschter Nebenwirkungen sowie wesentlicher Folgewirkungen, nachzuzeichnen. Fraglich ist nun, wie die in den Ebenenmodellen unterschiedenen Bewertungsdimensionen der Wirkungsebene (Impact und Outcome) Berücksichtigung finden. ${ }^{615}$ Aufgrund der Redundanzen in den Bewertungsdimensionen ist im Folgenden zunächst eine eindeutige Abgrenzung der Analyseperspektiven vorzunehmen.

\footnotetext{
613 Vgl. Brüggemeier (2004), S. 382; bezogen auf die Erfolgsfaktorenforschung auch Daschmann (1993), S. 6.

614 Es ist beispielsweise vorstellbar, dass sich eine Wirkung im Zeitverlauf deutlich abschwächt. Dennoch beschreibt die Wirkungsdauer einen zeitlichen Rahmen, solange noch relevante Effekte im gesamtgesellschaftlichen Wirkungsgefüge messbar sind.

615 Vgl. Mosiek et al. (2003), S. 28 ff. und auch Röhrig (2008), S. 105 ff.
} 


\section{Strukturelemente der Modellkonzeption}

- eine staatliche Maßnahme als auslösender Faktor

- andere staatliche Maßnahmen als mögliche auslösende Faktoren

- exogene Effekte als mögliche auslösende Faktoren

- Wirkungen als beobachtbare Veränderungen im gesellschaftlichen Wirkungsgefüge

- intendierte, am Zielsystem ausgerichtete Wirkungen staatlichen Handelns

- zusätzliche erwünschte und unerwünschte Nebenwirkungen

- aus eingetretenen Wirkungen resultierende Folgewirkungen

\section{Aspekte der Wirkungsbeziehungen}

STATISCH

DYNAMISCH

- Wirkungsrelevanz

- Wirkungsstärke

- Wirkungsinteraktion

- Wirkungskette

- Wirkungsverzögerung (time lag)

- Wirkungsdauer

Tabelle 1: Strukturelemente und Wirkungsbeziehungen der Modellkonzeption

\subsection{Abgrenzung der relevanten Analyseperspektiven}

\subsubsection{Bestehende Redundanzen in den Bewertungsebenen}

Die Modellkonzeption der Wirkungen staatlichen Handelns unterscheidet zwischen einer auslösenden und einer empfangenden Ebene, wobei unter den wesentlichen Strukturelementen vielfältige statische und dynamische Wirkungsbeziehungen bestehen. Der auslösenden Ebene lassen sich die Produkte staatlichen Handelns bzw. der Output zuordnen, der empfangenden Ebene die in der Gesellschaft ausgelösten Wirkungen. Die Charakteristik staatlichen Handelns bringt es mit sich, dass hierbei mit dem Impact und dem Outcome zwei unterschiedliche Wirkungsdimensionen von Relevanz sind. ${ }^{616}$ Beide Dimensionen sind in diesem Zusammenhang nicht als alternative, sondern als sich ergänzende Bewertungsebenen anzusehen:

- Die Perspektive des Impact - Wirkungen verstanden als die subjektive Anspruchserfüllung des Leistungsempfängers. ${ }^{617}$

- Die Perspektive des Outcome - Wirkungen verstanden als die objektiv ausgelösten gesamtgesellschaftlichen Wirkungen. ${ }^{618}$

Entsprechend dem Modell der Ziel- und Ergebnisebenen öffentlicher Leistungserstellung sind für ein wirkungsorientiertes Controlling beide Analyseebenen explizit zu

\footnotetext{
${ }^{616} \mathrm{Vgl}$. hierzu grundlegend Budäus/Buchholtz (1997), S. 328.

617 Vgl. Röhrig (2008), S. 130.

${ }^{618} \mathrm{Vgl}$. Berens et al. (2004), S. 331.
} 
berücksichtigen. ${ }^{619}$ Im Rahmen der Analyse der Wirkungen staatlichen Handelns sind beide Bewertungsdimensionen demnach klar voneinander getrennt $\mathrm{zu}$ betrachten. In diesem Zusammenhang ist jedoch problematisch, dass die im Ebenenmodell unterstellte eindeutige Abgrenzung von Impact und Outcome in der Realität so nicht existiert. Weiterhin ist auch die bislang unterstellte eindeutige Abgrenzung zwischen der Leistungs- und Wirkungsebene in der Realität so nicht existent. Die einzelnen Ziel- und Ergebnisebenen lassen sich nicht trennscharf bestimmen und weisen nicht unerhebliche Redundanzen auf.

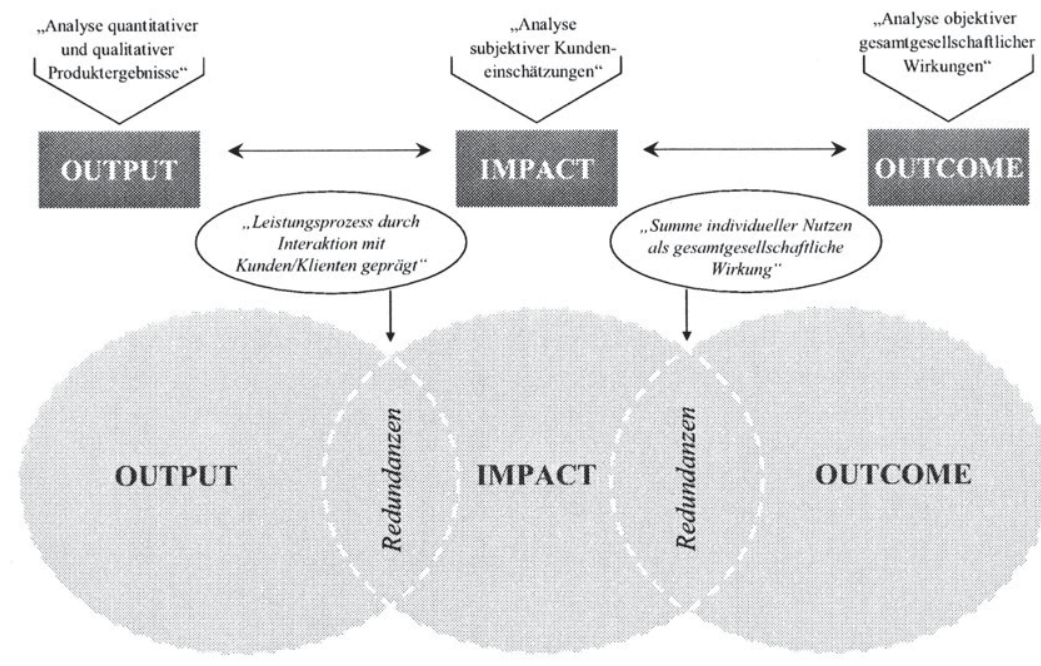

\section{LEISTUNGSEBENE}

\section{WIRKUNGSEBENE}

\section{Abbildung 35: Redundante Bewertungskreise}

So kann auf der einen Seite keine eindeutige Trennung zwischen der Leistungs- und der Wirkungsebene bzw. der Auslöserebene und der Empfängerebene getroffen werden, da keine klare Grenze zwischen dem Output und dem Impact der öffentlichen Leistungserstellung gezogen werden kann. Dies ist darauf zurückzuführen, dass öffentliche Leistungen überwiegend Dienstleistungscharakter aufweisen, ${ }^{620}$ was eine notwendige Beteiligung des Kunden am Erstellungsprozess zur Konsequenz hat. ${ }^{621}$ Hierdurch werden die Ergebnisse der Leistungserstellung, d.h. die quantitativen und vor allem die qualitativen Produktergebnisse des staatlichen Handelns, aktiv durch den Kunden be-

619 Vgl. Mosiek et al. (2003), S. 28 ff. und für das erweiterte Modell auch Röhrig (2008), S. 105 ff.

620 Vgl. Chmielewicz (1985), S. 29 f.; Gornas (1992), S. 48; Schmidberger (1994), S. 11; Haiber (1997), S. 101 und Buchholtz (2000), S. 21 oder auch die Ausführungen des Kapitels 2.1.3 dieser Arbeit.

621 Vgl. Schedler/Proeller (2003), S. 61. 
einflusst. Aufgrund dieser Interaktionsbeziehung kommt der subjektiven Kundenwahrnehmung bei der Qualitätsbeurteilung der Leistungsergebnisse eine besondere Bedeutung zu.

Die Kundenwahrnehmung ist daher bei der Analyse und Bewertung quantitativer und qualitativer Produktergebnisse unbedingt zu berücksichtigen und damit einerseits dem Bewertungskreis des Output bzw. der Leistungsebene zuzurechnen. Gleichzeitig berührt die Kundenwahrnehmung als subjektive Anspruchserfüllung der Leistungsempfänger aber auch die Wirkungsebene und wäre damit andererseits dem Bewertungskreis des Impact zuzurechnen. Die Bewertung der öffentlichen Leistungserstellung als Interaktionsbeziehung zwischen Kunde und Verwaltung weist demnach erhebliche Redundanzen auf. Zur Verdeutlichung dieses Sachverhaltes sind die Ebenen Output und Impact in Abbildung 35 als sich überlappende Bewertungskreise dargestellt.

Auf der anderen Seite kann keine trennscharfe Abgrenzung innerhalb der Wirkungsebene erfolgen, da keine klare Grenze zwischen dem Impact und dem Outcome der öffentlichen Leistungserstellung gezogen werden kann. Problematisch ist, dass mit den Outcomes eine objektive Bewertung gesellschaftlich ausgelöster Wirkungen erfolgen soll. Doch die Analyse der individuellen Nutzenvorstellungen der Leistungsempfänger sind in ihrer Gesamtheit eine bedeutende Teilmenge der gesamtgesellschaftlich ausgelösten Wirkungen, so dass auch hier eine deutliche Überschneidung in den Bewertungsebenen zu verzeichnen ist.

\subsubsection{Trennung der unterschiedlichen Analyseperspektiven}

Um Wirkungen zu analysieren bzw. pragmatische Vorschläge zur instrumentellen Ausgestaltung der einzelnen Bewertungsdimensionen zu unterbreiten, müssen die bestehenden Redundanzen durch die Schaffung eindeutiger Analyseperspektiven daher soweit wie möglich aufgelöst werden. Hierzu wird im Folgenden eine eindeutige Abgrenzung der dargestellten Bewertungskreise vorgenommen, indem den betrachteten Kreisen eine eindeutige akteursbezogene Bewertungsperspektive zugeordnet wird.

Der erste Bewertungskreis betrifft die Analyse der Leistungen staatlichen Handelns die Leistungsanalyse. Die öffentlichen Leistungen werden dazu im Rahmen eines Produktkonzeptes als Outputs abgegrenzt und definiert. ${ }^{622}$ Mit Hilfe von Kennzahlen und Indikatoren kann anschließend die Analyse der quantitativen und qualitativen Produktergebnisse stattfinden. Die Leistungsanalyse hat in diesem Zusammenhang die Aufgabe, wichtige behördliche Tatbestände in konzentrierter Form darzustellen. Insbesondere bei den qualitativen Produktergebnissen gibt es allerdings Überschneidungen zum Impact. Innerhalb des ersten Bewertungskreises sollen daher nur Analysen aus verwaltungsinterner Perspektive erfolgen. Folglich würden zur Bewertung qualitativer Leistungsergebnisse auch nur intern messbare Qualitätsindikatoren zum Einsatz kommen, wie z.B. die durchschnittliche Dauer einer Vorgangsbearbeitung.

622 Vgl. Mosiek et al. (2003), S. 28. 
Der zweite Bewertungskreis beschreibt die Analyse der Nutzen staatlichen Handelns die Nutzenanalyse. ${ }^{623}$ Die Nutzenanalyse hat die Aufgabe, den Impact der staatlichen Leistungserstellung zu bewerten und betrifft damit die Analyse subjektiver Kundenbedürfnisse. Zur Erhebung der Kundenzufriedenheit kommen häufig Befragungen zum Einsatz. Als Referenzgröße dienen die subjektiven Bedürfnisse und Erwartungen der Kunden an die staatliche Leistungserstellung, welche vor dem Hintergrund der jeweiligen individuellen Nutzenvorstellungen erfolgen. Innerhalb des zweiten Bewertungskreises werden demnach die Einwirkungen auf den Kunden aus einer individuellen personalen Perspektive analysiert. Die Nutzenanalyse weist die bereits beschriebenen Überschneidungen zum Output und zu den Outcomes auf. So haben die Ergebnisse einer Nutzenanalyse einerseits Relevanz für die qualitativen Aspekte des Leistungsoutput. Andererseits können die erhobenen Einwirkungen bei entsprechend großer Stichprobe auch zur Bewertung der gesellschaftlichen Auswirkungen herangezogen werden.

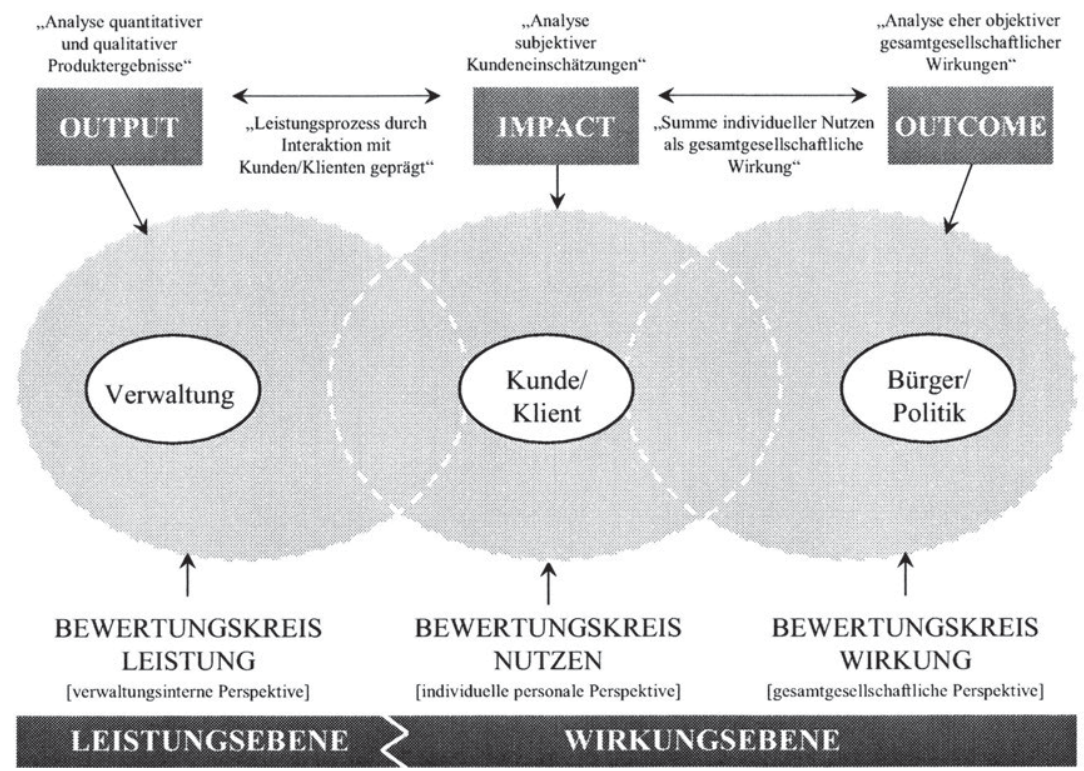

Abbildung 36: Abgrenzung der relevanten Analyseperspektiven

623 Eine Nutzenanalyse bezieht sich im Verständnis dieser Arbeit ausschließlich auf die Untersuchung der subjektiven Ansprucherfüllung der Adressaten staatlicher Leistungserstellung. Vgl. in ähnlicher Weise Schedler/Proeller (2003), S. 208. Dies ist von einem Nutzenbegriff zu trennen, der - z.B. im Rahmen einer Nutzen-Kostenanalyse - auch die gesellschaftlichen Auswirkungen staatlichen Handelns beinhaltet. Die Analyse der gesellschaftlichen Auswirkungen ist im Verständnis dieser Arbeit der Wirkungsanalyse vorbehalten. 
Der dritte Bewertungskreis betrifft die Analyse der Wirkungen staatlichen Handelns. Mit den Outcomes werden die objektiven Auswirkungen im gesamtgesellschaftlichen Wirkungsgefüge erhoben. Als Referenz dienen hier entweder politische Zielsetzungen oder auch die allgemeinen Erwartungen der Bürger an das staatliche Handeln - verstanden als Beitrag zur allgemeinen Wohlfahrt. Innerhalb des dritten Bewertungskreises werden die Auswirkungen auf die Gesellschaft demnach aus einer gesamtgesellschaftlichen Perspektive betrachtet. In dieser Hinsicht bestimmen die Bürger über die Bereiche und das Ausmaß staatlichen Handelns und damit letztlich auch über die hierdurch ausgelösten Wirkungen in der Gesellschaft. Das Bürgerinteresse ist dem Kundeninteresse damit vorangestellt, denn diese legen gleichsam fest, wer Kunde der öffentlichen Verwaltung sein darf und welche Leistungen er dabei beanspruchen kann. ${ }^{624}$

\subsubsection{Analyseperspektiven und staatliche Aufgabenbereiche}

Die zuvor eingeführten Bewertungskreise können demnach als unterschiedliche Analyseperspektiven aufgefasst werden, die die öffentliche Leistungserstellung aus einem jeweils anderen Blickwinkel betrachten. Grundsätzlich lassen sich sämtliche staatlichen Aufgabenbereiche aus allen drei Perspektiven - Leistungsanalyse, Nutzenanalyse und Wirkungsanalyse - bewerten. In diesem Zusammenhang ist jedoch zu beachten, dass abhängig vom staatlichen Aufgabenbereich, eine bestimmte Analyseperspektive bzw. eine Kombination der Analyseperspektiven sinnvoller und damit auch vorzuziehen ist. Auf Basis verschiedener Charakteristika wird nachfolgend eine grobe Typisierung staatlicher Leistungen vorgenommen, woraus sich unter Umständen Hinweise auf eine besonders relevante Analyseperspektive gewinnen lassen (siehe Abbildung 36). ${ }^{625}$

Die erste Typisierung beschreibt staatliche Aufgaben mit weitestgehend standardisiertem Charakter. Im Fokus der öffentlichen Leistungserstellung steht häufig eine gesetzestreue und regelkonforme Erfüllung der übertragenen Aufgaben - vielfach betrifft dies auch hoheitliche Anliegen. Die öffentliche Verwaltung besitzt während des Aufgabenvollzugs einen eher geringen Gestaltungsspielraum, stattdessen ist eine möglichst gleichartige Leistungserstellung mit möglichst einheitlichen Leistungsergebnissen wichtig. Tendenziell handelt es sich dabei um repetitive Tätigkeiten, die Interaktionsbeziehung zum Kunden bzw. Klienten ${ }^{626}$ ist meist gleichförmig ausgeprägt, weshalb der Analyse individueller Kundenbedürfnisse eine eher geringe Bedeutung zukommt.

624 Zur Abgrenzung von Bürger und Kunde vgl. auch Schedler/Proeller (2003), S. 61.

625 Im Gegensatz zu KIESEL werden nachfolgend drei Typen öffentlicher Dienstleistungen unterschieden. In Anlehnung an WEBER/SCHÄFFLR unterscheidet KIESEL in ihrem Beitrag zur wirkungsorientierten Steuerung einer Landesverwaltung zwei Typen von staatlichen Dienstleistungen. Beim Typ I handelt es sich um weitestgehend standardisierte Aufgaben, mit entsprechend geringen Freiheitsgraden der öffentlichen Verwaltung. Typ II beschreibt hingegen eher schöpferische und kreative Dienstleistungen, die zusätzlich durch hohe Unsicherheiten bezüglich des Leistungsergebnisses geprägt sind. Vgl. Weber/Schäffer (2002), S. 7 und Kiesel (2005), S. $14 \mathrm{ff}$.

${ }^{626}$ Die Bezeichnung Klient soll im Gegensatz zum Kundenbegriff verdeutlichen, dass das Gegenüber der Verwaltung nicht immer als gleichberechtigter Partner entgegentritt, sondern vielmehr auf Leistungen der Verwaltung angewiesen bzw. diesen auch zwangsweise ausgesetzt ist. Vgl. Schedler/Proeller (2003). S. 60. 
Bei entsprechenden Aufgaben ist eine quantitative und qualitative Bewertung der Leistungsergebnisse aus verwaltungsinterner Analyseperspektive üblicherweise ausreichend, so dass hier die Leistungsanalyse staatlichen Handelns besondere Relevanz besitzt. Eine ergänzende Nutzen- oder Wirkungsanalyse verspricht nur einen begrenzten zusätzlichen Erkenntnisgewinn. Das wirkungsorientierte Controlling unterstützt bei diesem Aufgabentypus die Erzielung effizienter Leistungsergebnisse. Dazu werden produktbezogene Kosten- und Leistungskennzahlen erhoben und ergänzt um intern messbare Qualitätsindikatoren. Beispiele für entsprechende staatliche Aufgabenbereiche sind die Ausgabe von Bescheinigungen, Bescheiden und Erlaubnissen (wie z.B. eines Reisepasses).
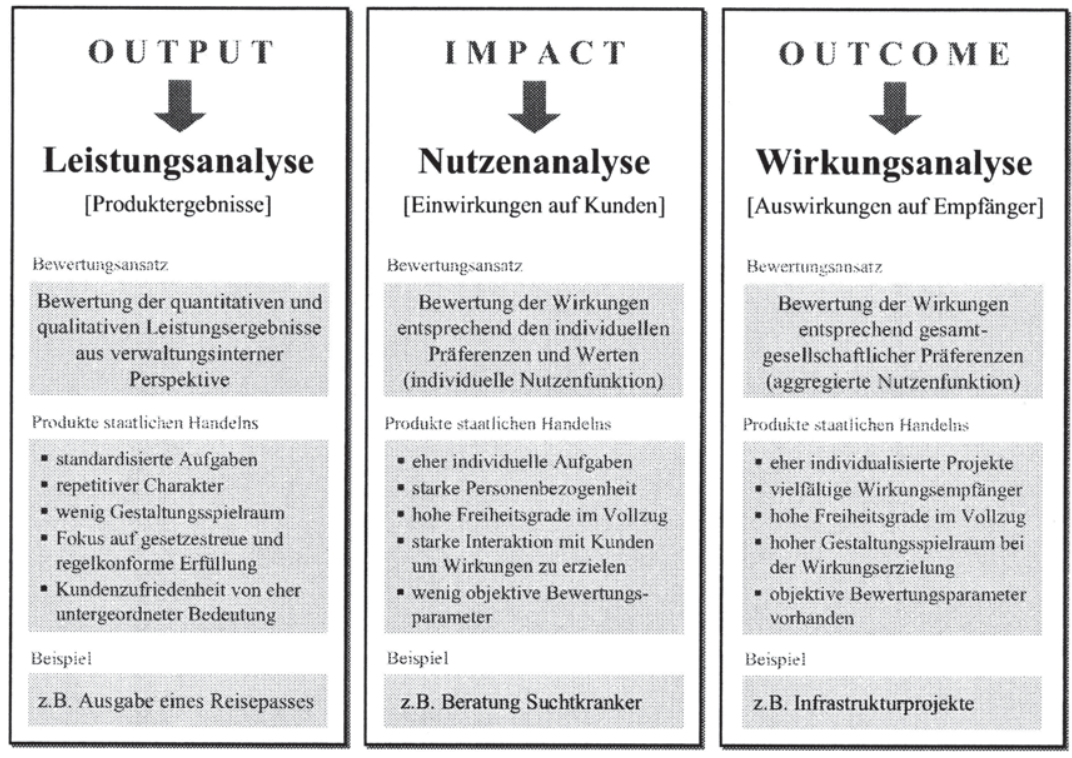

\section{Abbildung 37: Analyseperspektiven staatlicher Aufgabenbereiche}

Der zweite Typus bezieht sich auf eher individuelle staatliche Aufgaben, die durch hohe Freiheitsgrade im Leistungserstellungsprozess charakterisiert sind. Weiterhin zeichnen sich diese Aufgaben durch eine äußerst starke Interaktionsbeziehung zum Kunden bzw. Klienten und damit durch eine hohe Personenbezogenheit der öffentlichen Leistung aus. Die Kunden sind in starkem Maße in den Leistungserstellungsprozess eingebunden, wodurch eine hohe Variabilität der Leistungsergebnisse besteht. Nicht selten handelt es sich dabei um eher sozial orientierte Aufgaben, für die sich nur wenige objektive Bewertungsparameter finden lassen. 
Bei entsprechenden Aufgaben kommt der Wirkungsbewertung als Einwirkung auf einzelne Kunden bzw. Klienten besondere Bedeutung zu. Im Rahmen der Nutzenanalyse staatlichen Handelns werden die Wirkungen anhand der individuellen Präferenzen und Werte beurteilt. Zur Erzielung effektiver Leistungsergebnisse ist es für das wirkungsorientierte Controlling daher unerlässlich, die Impactperspektive in die Analyse einzubeziehen und im Rahmen eines systematischen Controllingprozesses steuerungsrelevante Daten zur Verfügung zu stellen. Erst hierdurch werden die notwendigen Lernprozesse über die Wirkungsweisen des staatlichen Handelns beim Kunden angestoßen und die hohen Freiheitsgrade beim Vollzug lassen sich für eine zielsetzungsgerechte Wirkungssteuerung nutzen. Abhängig vom staatlichen Aufgabenbereich bietet sich gegebenenfalls eine ergänzende Leistungsanalyse an, um über produktbezogene Kennzahlen auch eine Kontrolle der Leistungseffizienz zu gewährleisten. Beispiele für entsprechende staatliche Aufgabenbereiche sind insbesondere Betreuungs-, Beratungsund Bildungsleistungen (z.B. die Beratung Suchtkranker).

Hiervon zu unterscheiden ist der dritte Aufgabentypus, welcher ebenfalls durch hohe Freiheitsgrade im Leistungserstellungsprozess gekennzeichnet ist und damit über hohen Gestaltungsspielraum bei der Wirkungserzielung verfügt. Es handelt sich hierbei um kreative und schöpferische Dienstleistungen in Form individueller Projekte. Im Gegensatz zum vorherigen Aufgabentypus haben diese Projekte häufig eine Vielzahl unterschiedlicher Wirkungsempfänger, so dass Auswirkungen in vielfältigen gesellschaftlichen Bereichen zu verzeichnen sind. Im Unterschied zu eher personenbezogenen Aufgaben kann i.d.R. auch auf eine ausreichende Anzahl objektiver Bewertungsparameter zurückgegriffen werden.

Bei solchen Aufgaben sollte die Wirkungsanalyse staatlichen Handelns zum Einsatz kommen, um eine möglichst umfassende Bewertung der gesellschaftlich ausgelösten Wirkungen vorzunehmen. Als Referenz dienen hier die allgemeinen Erwartungen der Bürger an das staatliche Handeln bzw. die politischen Zielsetzungen - verstanden als eine Art aggregierte Nutzenfunktion der gesamtgesellschaftlichen Wohlfahrt. Im Rahmen des wirkungsorientierten Controllings wird so die Perspektive des Outcome ausgefüllt, um eine effektive Steuerung der staatlichen Leistungsergebnisse zu ermöglichen. Bestehende Gestaltungsspielräume in der Leistungserstellung können gezielt zur Verbesserung der gesamtgesellschaftlichen Wohlfahrt genutzt werden. Häufig bietet es sich zudem an, ergänzend eine Leistungsanalyse und unter Umständen auch eine Nutzenanalyse durchzuführen, um sämtliche Ziel- und Ergebnisebenen im Controllingansatz zu berücksichtigen. Beispiele für entsprechende staatliche Aufgaben sind insbesondere planerische oder gestalterische Tätigkeiten (wie z.B. Infrastrukturprojekte).

Wie bereits zuvor angedeutet, zeigen die Ausführungen zu den grob unterschiedenen Aufgabentypen, dass die Wahl einer bestimmten Analyseperspektive für jeden staatlichen Handlungsbereich individuell entschieden werden muss. Hierzu sollte die vorgenommene Typisierung und Charakterisierung eine erste Orientierungshilfe geben. Während grundsätzlich bei sehr klar strukturierten Aufgaben der Einsatz der Leistungsanalyse ausreichend erscheint, erhöht sich mit steigendem Komplexitätsgrad der 
staatlichen Aufgabenstellung auch die Notwendigkeit, ergänzend eine Nutzen- bzw. Wirkungsanalyse durchzuführen. Eine Nutzenanalyse bietet sich dann an, wenn eine hohe Personenbezogenheit der öffentlichen Leistung vorliegt. Der Einsatz der Wirkungsanalyse ist insbesondere bei breiten gesellschaftlichen Empfängerebenen sinnvoll und zusätzlich vom Vorhandensein objektiver Bewertungsparameter abhängig.

Im Modell der Ziel- und Ergebnisebenen öffentlicher Leistungserstellung bzw. bei der Ausgestaltung eines wirkungsorientierten Controllings ist demnach darauf $\mathrm{zu}$ achten, dass nicht für alle Produkte staatlichen Handelns die durchgängige Anwendung sämtlicher Modellebenen sinnvoll erscheint. Auswahl und instrumentelle Ausgestaltung der Analyseperspektiven muss für jeden öffentlichen Aufgabenbereich neu entschieden werden. Die Entscheidung wird einerseits sicherlich von den speziellen Informationserfordernissen der Akteure im politisch-administrativen System sowie andererseits von der generellen Eignung der öffentlichen Aufgabenstellung abhängig sein. Die folgenden Ausführungen geben Hinweise zur instrumentellen Ausgestaltung der einzelnen Analyseperspektiven - Leistungsanalyse, Nutzenanalyse und Wirkungsanalyse.

\subsubsection{Instrumentelle Ausgestaltung der Analyseperspektiven}

\subsubsection{Output: Instrumentarium der Leistungsanalyse}

Aufgabe der Leistungsanalyse ist die quantitative und qualitative Bewertung der Leistungsergebnisse aus verwaltungsinterner Perspektive. Das Instrument dazu bildet die Leistungsrechnung. ${ }^{627}$ Dem Aufbau einer Leistungsrechnung geht zuvor die Definition von Produkten voraus. Hierbei handelt es sich um selbstständige Dienstleistungseinheiten einer öffentlichen Verwaltung. Die Leistungsrechnung versucht, möglichst sämtliche Produkte flächendeckend über die gesamte Verwaltung quantitativ zu erfassen und diese Daten systematisch auszuwerten. Durch die Verknüpfung mit Ergebnissen der Kostenrechnung lassen sich so produktbezogene Kosten- und Leistungskennzahlen ermitteln. ${ }^{62}$ Auf dieser Basis kann anhand von Kennzahlen, die das Verwaltungsergebnis beschreiben, neben einer Kostensteuerung sowie Kosten- und Leistungsvergleichen $^{629}$ auch eine umfassende Leistungsdokumentation vollzogen werden. ${ }^{630}$

Kennzahlen bzw. Kennzahlensysteme stellen ein zielführendes Instrument zur Ermittlung von Outputgrößen dar. ${ }^{631}$ Dabei handelt es sich um Messgrößen, die behördliche Sachverhalte und Zusammenhänge zahlenmäßig quantifizieren und in konzentrierter

\footnotetext{
627 Vgl. Schedler/Proeller (2003), S. 207.

628 Auf der Ebene des Outputs lassen sich produktbezogene Kosten- und Mengendaten zusammenführen, so dass in der Regel für sämtliche Produkte der öffentlichen Verwaltung Stückkosten ermittelt werden können.

629 In diesem Zusammenhang sind einerseits Längsschnittvergleiche vorstellbar, andererseits kann bei entsprechend einheitlicher Definition von Produkten und Kennzahlen auch ein Querschnittsvergleich zwischen verschiedenen Organisationseinheiten oder Verwaltungen stattfinden. Vgl. bspw. Balzer (2005), S. 426.

630 Vgl. Mosiek et al. (2003), S. 28.

631 Vgl. Röhrig (2008), S. 128.
} 
Form Informationen hierüber bereitstellen. ${ }^{632}$ In diesem Zusammenhang wird häufig zwischen absoluten Kennzahlen und Verhältniskennzahlen unterschieden. Absolute Kennzahlen messen den Wert einer Leistungsgröße zu einem bestimmten Zeitpunkt bzw. für einen bestimmten Zeitraum. ${ }^{633}$ Verhältniskennzahlen bilden die Beziehung zweier isolierter Kennzahlen zueinander ab und verfügen oftmals über einen höheren Informationsgehalt ${ }^{634}$ Mittels des Einsatzes von Kennzahlen oder Kennzahlensystemen kann eine sachgerechte Bewertung der quantitativen Leistungsergebnisse eines öffentlichen Produktes gelingen, problematisch bleibt aber die Abbildung der Qualitätsdimension.

Zur Bewertung qualitativer Leistungsergebnisse müssen im Rahmen der Leistungsanalyse intern messbare Qualitätsindikatoren herangezogen werden. ${ }^{635} \mathrm{Im}$ Gegensatz zu Kennzahlen bilden Indikatoren Sachverhalte ab, die selbst nicht direkt quantitativ messbar sind ${ }^{636}$ Sie stellen damit empirisch messbare Hilfsgrößen dar, um theoretisch vermutete Zusammenhänge zu operationalisieren. ${ }^{637}$ Aus verwaltungsinterner Perspektive können mit Hilfe von Indikatoren wichtige produktbezogene Qualitätsmerkmale der öffentlichen Leistung abgebildet werden, die sich in unterschiedlichen Attributen eines Produktes ausdrücken.

\subsubsection{Impact: Instrumentarium der Nutzenanalyse}

Aufgabe der Nutzenanalyse ist es, die kundenbezogene Qualitätsdimension zu bewerten. ${ }^{638}$ Während produktbezogene Qualitätsmerkmale mit Hilfe von Indikatoren auch aus verwaltungsinterner Perspektive abgebildet werden können, versucht die Nutzenanalyse die subjektive Qualitätswahrnehmung der Kunden bzw. Klienten direkt zu erfassen. Dies betrifft insbesondere die mit der öffentlichen Leistungserstellung verbundenen Einwirkungen auf die Leistungsempfänger - den Nutzen. Hierzu gehört einerseits die allgemeine Kundenzufriedenheit, andererseits aber auch angestrebte Veränderungen im Kunden- bzw. Klientenverhalten oder in den Fähigkeiten der Kunden ${ }^{639}$ Die Bewertung erfolgt entsprechend den jeweiligen individuellen Bedürfnissen der Kunden bzw. Klienten. Als Bewertungskriterium dient das Erfolgsmaß der subjektiven Effektivität, d.h. der Umfang, in dem leistungsempfängerorientierte Zielsetzungen durch das staatliche Handeln erreicht werden konnten. ${ }^{640}$

\footnotetext{
632 Vgl. Bähr (2002), S. 72 f.

633 Ein absoluter Kennzahlenwert ist beispielsweise die Anzahl ausgestellter Bescheinigungen pro Quartal.

634 Eine Verhältniskennzahl ist demgegenüber die Kosten pro ausgestellte Bescheinigung.

635 Hiervon zu trennen ist die Bewertung der Qualitätsdimension im Rahmen einer Nutzenanalyse, die eine Bewertung des Leistungsimpact als subjektive Anspruchserfüllung durch Kunden bzw. Klienten vornimmt.

636 Vgl. Jenner (2000), S. 327 f.

637 Bei einem Indikator besteht damit kein zwingender Zusammenhang zur gemachten Aussage in Form einer eindeutigen „1:1-Beziehung“. Vgl. hierzu ausführlich Kapitel 4.1.1.2.

638 Zur Abgrenzung der Qualitätsdimensionen im öffentlichen Sektor vgl. Schedler/Proeller (2003), S. 69 f.

$639 \mathrm{Vgl}$. Schedler/Proeller (2003), S. 70.

640 Vgl. Röhrig (2008), S. 130.
} 
Das dominierende Instrument der Nutzenanalyse stellen Kundenbefragungen dar. ${ }^{641}$ Die Auswertung der Befragungsergebnisse gibt Auskunft über die Zufriedenheit der Kunden bzw. Klienten mit der öffentlichen Leistung. Zufriedenheit kann in diesem Zusammenhang als das subjektive Ergebnis eines komplexen Prozesses im Sinne eines Erwartungs-Wahrnehmungs-Vergleichs (Soll-Ist-Vergleich) verstanden werden. ${ }^{642}$ Den Maßstab zur Beurteilung der wahrgenommenen Situation (Ist-Größe) bilden dabei die Erwartungen bzw. das individuelle Anspruchsniveau (Soll-Größe) des Kunden. Das Erfüllen oder Übertreffen der in die öffentliche Leistung gesetzten Erwartungen führt demnach zu Zufriedenheit, ein Nichterfüllen führt hingegen zu Unzufriedenheit.

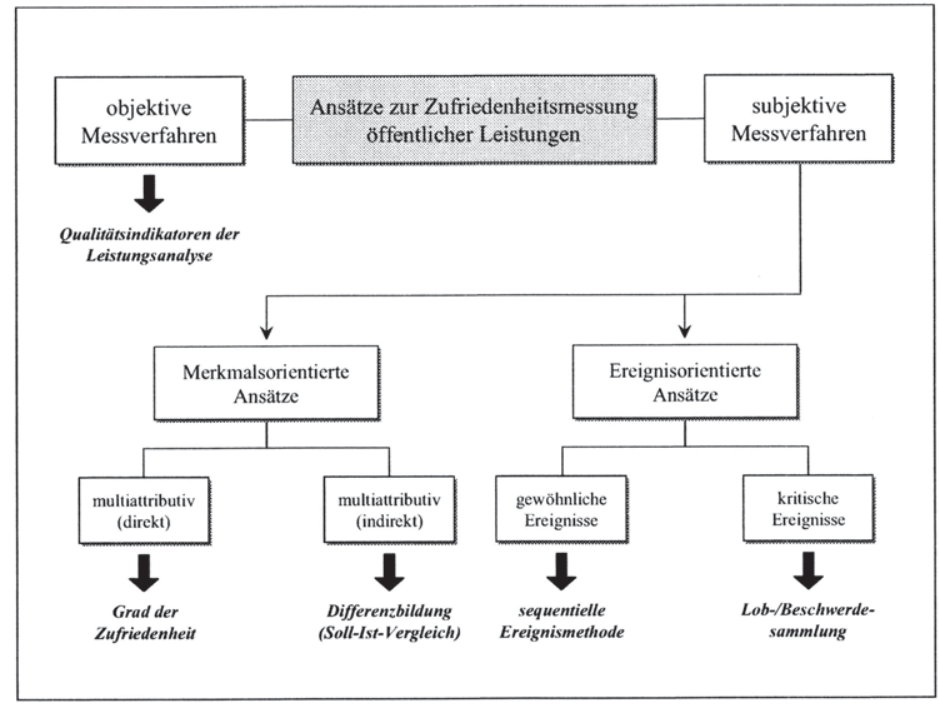

Abbildung 38: Ansätze der Zufriedenheitsmessung ${ }^{643}$

In der Literatur lassen sich verschiedene Verfahren zur Zufriedenheitsmessung finden, welche in Abbildung 37 im Überblick dargestellt sind. ${ }^{644}$ Es kann zunächst zwischen objektiven Verfahren und subjektiven Verfahren unterschieden werden. Objektive Verfahren versuchen die Zufriedenheit durch Qualitätsindikatoren zu messen, die einen hohen Bezug zur Kundenzufriedenheit aufweisen. Solche Indikatoren kommen beim wirkungsorientierten Controlling im Rahmen der Leistungsanalyse zur Bewertung qua-

\footnotetext{
641 Vgl. Mosiek/Gerhardt (2003), S. 291.

642 Entsprechend der in der Marketing-Literatur vielfach untersuchten Kundenzufriedenheit wird die Zufriedenheit von Kunden bzw. Klienten im öffentlichen Sektor dem Confirmation/Disconfirmation-Paradigma folgend als Ergebnis eines komplexen Soll-Ist-Vergleichs verstanden, der mehrere Dimensionen umfasst. Zur Diskussion des Confirmation/Disconfirmation-Paradigmas vgl. Homburg/Rudolph (1997), S. 38 f.

Eigene Darstellung in Anlehnung an Mosiek (2002), S. 234

644 Vgl. hierzu grundlegend Bruhn (2007), S. 87 ff.
} 
litativer Leistungsmerkmale zur Anwendung. Problematisch ist die Validität objektiver Verfahren, da regelmäßig offen bleibt, inwieweit die Indikatoren wirklich mit der Kundenzufriedenheit in Verbindung stehen. Bei subjektiven Verfahren erfolgt hingegen eine individuelle Erfassung der Zufriedenheit der Kunden bzw. Klienten.

Subjektive Ansätze lassen sich wiederum in merkmals- und ereignisorientierte Verfahren differenzieren. ${ }^{645}$ Die ereignisorientierten Methoden fokussieren auf solche Standard- oder Schlüsselerlebnisse eines Dienstleistungsprozesses, die aus Kundensicht als besonders qualitätswahrnehmungsrelevant erachtet werden. ${ }^{646}$ Die Zufriedenheitsermittlung kann hierbei entweder an gewöhnlichen Ereignissen oder an kritischen Ereignissen ansetzen. So werden beispielsweise mit der sequenziellen Ereignismethode gewöhnliche Ereignisse entlang einer Interaktionsbeziehung zwischen Kunde und Verwaltung identifiziert. Die Kunden müssen bezüglich dieser Ereignisse mittels offener Fragen eine Bewertung ihrer Wahrnehmung vornehmen, worüber eine Analyse ihres Qualitätsempfindens erfolgen kann. Demgegenüber fokussiert beispielsweise eine Lob- und Beschwerdesammlung auf kritische Ereignisse. Hierbei geben die Kunden Auskunft über Ereignisse der Interaktionsbeziehung, die sie als besonders kritisch erlebt haben. Kritisch bedeutet in diesem Zusammenhang, dass mit den Ereignissen starke positive Empfindungen und Zufriedenheit oder umgekehrt starke negative Empfindungen und Unzufriedenheit verbunden werden.

Bei ereignisorientierten Verfahren handelt es sich um eher qualitative Methoden, die keine standardisierte Auswertung erhobener Daten erlauben. Insofern sind sie für eine regelmäßige und repräsentative Bewertung des Leistungsimpact im Rahmen eines systematischen wirkungsorientierten Controllingprozesses grundsätzlich weniger geeignet. Sie sind vielmehr als Ergänzung der stärker standardisierten merkmalsorientierten Verfahren anzusehen, da sich hierüber detaillierte und handlungsrelevante Informationen über die Kundenbeziehungen gewinnen lassen. Nicht selten bieten solche Informationen wertvolle Hinweise für bestehende Optimierungspotenziale. Die Aufgabe des Controllings besteht darin, Kanäle zur gezielten Aufnahme entsprechender Hinweise zu schaffen und die Informationen auf ihre Handlungsrelevanz hin zu analysieren.

Im Gegensatz zu der auf spezielle Kundenkontaktsituationen bezogenen ereignisorientierten Messung der Kundenzufriedenheit fokussieren merkmalsorientierte Verfahren auf die Bewertung einzelner Leistungselemente, woraus sich wiederum die wahrgenommene Gesamtdienstleistungsqualität ableiten lässt. ${ }^{647}$ Wesentlich bei merkmalsorientierten Verfahren ist die Vorgehensweise im Sinne des Multiattributansatzes, da hierdurch konkrete Anhaltspunkte für die Beseitigung subjektiver Qualitätsmängel gewonnen werden können. ${ }^{648}$ Dabei ist zwischen einer direkten und einer indirekten Erhebungsmethode zu unterscheiden. Bei der indirekten Zufriedenheitsmessung erfolgt

\footnotetext{
645 Vgl. im Folgenden Mosiek (2002), S. 233 ff.

646 Vgl. Meffert/Bruhn (2003), S. 229.

647 Vgl. Meffert/Bruhn (2003), S. 220.

648 Vgl. Mosiek (2002), S. 236.
} 
eine separate Erhebung von Kundenerwartungen (Soll-Komponente) und tatsächlich wahrgenommener Leistung (Ist-Komponente) mit anschließender Differenzbildung. ${ }^{649}$ Alternativ kann die wahrgenommene Differenz zwischen Soll und Ist aber auch durch eine direkte Frage nach der Zufriedenheit des Kunden erhoben werden.

Für das wirkungsorientierte Controlling ist insbesondere das direkte merkmalsorientierte Verfahren von Relevanz. Hierbei handelt es sich um einen weitestgehend standardisierten und bewährten Ansatz, der eine repräsentative Bewertung des Leistungsimpact ermöglicht. Die Erhebung kann sowohl in strukturierten Interviews oder bei großen Kundengruppen auch in Form einer schriftlichen Befragung erfolgen. Im Rahmen der Nutzenanalyse kann so ein überschaubares Set wichtiger Qualitäts- und Zufriedenheitsmerkmale unter vertretbarem Aufwand einer regelmäßigen Messung unterzogen werden. Der hohe Standardisierungsgrad ermöglicht zudem einen Vergleich der Ergebnisse im Zeitablauf sowie unter verschieden Verwaltungseinheiten.

Für eine Nutzenanalyse ist auch die genaue Definition und Abgrenzung der relevanten Kundengruppen einer Verwaltungseinheit notwendig. Hierbei ist darauf $\mathrm{zu}$ achten, dass in vielen Bereichen des öffentlichen Sektors nicht nur ein Kundenkontakt zum Bürger besteht, sondern auch andere gesellschaftliche Akteure in einen kundenorientierten Ansatz einbezogen werden müssen. Grundsätzlich stellen sämtliche Interaktionsbeziehungen zu externen Akteuren oder Anspruchsgruppen Kundenkontakte dar, so dass sich die Definition relevanter Kundengruppen an diesen Interaktionsbeziehungen orientieren kann. Als Beispiele relevanter Kundengruppen der öffentlichen Verwaltung wären Unternehmen, Verbände, Gremien, Interessensvertretungen oder auch andere Verwaltungseinheiten zu nennen. Häufig besitzt eine öffentliche Verwaltung mehrere relevante Kundengruppen. Dies ermöglicht der Nutzenanalyse überdies interessante Auswertungen und Querschnittsvergleiche verschiedener Kundengruppen.

Bei der konkreten Ausgestaltung merkmalsorientierter Erhebungen sind zudem unterschiedliche Vorgehensweisen denkbar. Zum einen kann die Zufriedenheit mittels einzelner Merkmale global (bereichsbezogen) oder leistungsspezifisch für eine Interaktionsbeziehung ermittelt werden. Zum anderen können einzelne Interaktionsphasen anhand mehrerer Merkmale bewertet werden ${ }^{650}$ Einige Beispiele solcher Leistungsmerkmale listen MEYER/DORNACH auf: ${ }^{651}$

- Öffnungs- und Sprechzeiten sowie Erreichbarkeit

- Dauer und Schnelligkeit der Vorgangsbearbeitung

- Verständlichkeit schriftlicher Unterlagen

- Freundlichkeit, Sachverstand und Engagement der Mitarbeiter

- Verlässlichkeit der Aussagen und Nachvollziehbarkeit von Entscheidungen

649 Diese Vorgehensweise weist allerdings zahlreiche methodische und messtechnische Probleme auf, wodurch die Aussagequalität beschränkt wird. Vgl. hierzu ausführlich Homburg/Rudolph (1997).

${ }^{650} \mathrm{Vgl}$. Mosiek (2002), S. 236.

651 Auflistung in Anlehnung an Meyer/Dornach (2001), S. 117. 
Neben diesen Leistungsmerkmalen, die eher die Kundenorientierung und Servicequalität einer öffentlichen Verwaltung betreffen, sollte eine Nutzenanalyse auch immer wirkungsbezogene Merkmale abfragen. Wirkungsbezogene Merkmale können sich sowohl an spezifischen Zielsetzungen einzelner Leistungsempfänger, als auch an gesamtgesellschaftlichen Zielen orientieren. Hier treten die bereits ausgeführten Überschneidungen in den Bewertungsdimensionen auf. Die subjektiven Nutzenvorstellungen einzelner Leistungsempfänger können bei ausreichender Repräsentativität der Erhebung auch zur Bewertung gesamtgesellschaftlicher Auswirkungen herangezogen werden. ${ }^{652}$ Dies ist allerdings Aufgabe der nachfolgend zu erläuternden Wirkungsanalyse. Bei wirkungsbezogenen Merkmalen handelt es sich um äußerst spezifische Fragestellungen, sie müssen deshalb für jeden Verwaltungs- bzw. Aufgabenbereich sowie für jede Kundengruppe separat ermittelt werden.

Oftmals lassen sich aus den Auswertungsergebnissen einer Zufriedenheitsanalyse konkrete bedürfnisorientierte Verbesserungsmaßnahmen zur Optimierung der Kundenbeziehung ableiten. Für eine zusammenfassende und übersichtliche Analyse der festgestellten Handlungsnotwendigkeiten schlägt RöHRIG den Einsatz eines Handlungsportfolios vor. ${ }^{653}$ Hierbei handelt es sich um eine dreidimensionale Portfoliodarstellung, in der sämtliche Stärken und Schwächen einer Verwaltung in Bezug auf die Erfüllung der Bürgerwünsche gebündelt werden. Als Dimensionen werden die »Zufriedenheit des Kunden« mit der wahrgenommenen "Wichtigkeit der gleichen Leistung« aus Kundensicht gegenübergestellt. Zusätzlich wird die Dimension »Zusatzkosten/Realisierbarkeit« hinzugefügt, um die Maßnahmen auch realistisch priorisieren zu können. Die Gesamtdarstellung des Instrumentes erlaubt der Verwaltungsführung anschließend die zielführende Auswahl der notwendigen Verbesserungsmaßnahmen.

\subsubsection{Outcome: Instrumentarium der Wirkungsanalyse}

Aufgabe der Wirkungsanalyse ist es, die gesamtgesellschaftlichen Auswirkungen der staatlichen Leistungserstellung zu bewerten. Hierzu wird der kombinierte instrumentelle Einsatz von Wirkungsrechnungen bzw. Wirkungsindikatoren und der Methodik der Evaluation, insbesondere der Outcome-Evaluation, empfohlen. ${ }^{654}$ Jedoch scheitert der verbreitete Einsatz von Wirkungsrechnungen in der Regel bereits an den methodisch ungenügenden Zieldefinitionen, da auf politischer Ebene nur eine äußerst geringe Bereitschaft zur Vorgabe klarer und messbarer Zielvorgaben besteht. Zusätzlich werden durch das Indikatorensystem nur die intendierten Auswirkungen des staatlichen Handelns erfasst, was eine unzulässige Verkürzung der Wirkungsanalyse bedeuten würde.

Im Rahmen einer Outcome-Evaluation werden zwar im Idealfall sämtliche Auswirkungen des staatlichen Handelns auf das gesamtgesellschaftliche Wirkungsgefüge analysiert und auf die Produkte der Verwaltung zugerechnet. Doch handelt es sich hierbei

\footnotetext{
652 Vgl. Kapitel 4.2.1.

653 Vgl. hierzu ausführlich Röhrig (2008), S. 133 ff.

654 Vgl. Budäus (1999b), S. 336 und Röhrig (2008), S. 154.
} 
meist um wissenschaftlich geprägte Einzelfalluntersuchungen durch externe Institute, die zudem mit einem hohen Kosten- und Zeitaufwand verbunden sind und einen äußerst geringen Standardisierungsgrad aufweisen. ${ }^{655}$ Zur instrumentellen Anwendung im Rahmen eines kontinuierlichen und systematischen Controllingprozesses ist die Outcome-Evaluation daher nicht geeignet. Die wirkungsorientierte Verwaltungssteuerung wird sich daher in der Breite nicht auf Outcome-Evaluationen stützen können. ${ }^{656}$

Zur instrumentellen Ausgestaltung der Wirkungsanalyse staatlichen Handelns sollen daher im nachfolgenden Kapitel 4.3 alternative Vorschläge zur Methodik der Wirkungsanalyse unterbreitet werden. Die Vorschläge zur Systematik und zum Instrumentarium der Wirkungsanalyse sollen sich dabei auf die bereits gewonnenen Erkenntnisse zur Modellkonzeption der Wirkungen staatlichen Handelns stützen. ${ }^{657}$ Einerseits sind mit den intendierten Wirkungen, erwünschten und unerwünschten Nebenwirkungen und den Folgewirkungen die wesentlichen Strukturelemente der Modellkonzeption abzubilden. Andererseits sind möglichst sämtliche statischen und dynamischen Aspekte der Wirkungsbeziehungen im gesellschaftlichen Wirkungsgefüge zu beachten.

Das Ausmaß der Komplexität realer Wirkungszusammenhänge macht es gleichzeitig erforderlich, ein möglichst praktikables Instrumentarium bereitzustellen. Die relevanten Elemente und Wirkungsbeziehungen zwischen auslösender Ebene und empfangender Ebene müssen angemessen strukturiert werden, um sie dauerhaft einer systematischen Analyse zugänglich zu machen. Es ist von entscheidender Bedeutung, durch die Wirkungsanalyse steuerungsrelevante Informationen zu den Auswirkungen staatlichen Handelns in den internen wirkungsorientierten Controllingprozesses einzubinden. Durch einen möglichst hohen Standardisierungsgrad in der Vorgehensweise kann so die regelmäßige Auswertung wirkungsorientierter Controllingdaten erfolgen. Gleichzeitig verhilft eine angemessene Strukturierung auch zur Konsolidierung bzw. Verdichtung der Wirkungsinformationen über die unterschiedlichen Steuerungsebenen im politisch-administrativen System.

\subsection{Systematik und Instrumentarium der Wirkungsanalyse}

\subsubsection{Vorgehenssystematik einer Wirkungsanalyse}

Aufgabe und Zielsetzung des vorliegenden Kapitels ist es, einen pragmatischen Vorschlag zur Systematisierung und instrumentellen Ausgestaltung von Wirkungsanalysen zu unterbreiten. Auf Grundlage der gewonnen Erkenntnisse im Rahmen des entworfenen Beschreibungsmodells der Wirkungen staatlichen Handelns wird nun zunächst ein mögliches Vorgehensmodell der Wirkungsanalyse detailliert beschrieben.

\footnotetext{
$655 \mathrm{Zu}$ den instrumentellen Defiziten der Wirkungsrechnung und der Outcome-Evaluation zur systematischen Erfassung und Bewertung der Wirkungen staatlichen Handelns vgl. ausführlich Kapitel 3.4 dieser Arbeit.

$656 \mathrm{Vgl}$. Schedler/Proeller (2003), S. 198.

657 Vgl. zusammenfassend Kapitel 4.1.3.
} 
Im Rahmen dieses Vorgehensmodells sind grundsätzlich zwei Phasen zu unterscheiden. In einer sog. Konzeptionsphase erfolgt zunächst die Identifikation der ursächlich auf das staatliche Handeln (Produkt/ Programm) zurückzuführenden Wirkungen sowie in einem zweiten Schritt die Operationalisierung und Bewertung der einzelnen Wirkungskomponenten. In der sog. Anwendungsphase erfolgt dann der eigentliche operative Einsatz der in der Konzeptionsphase aufgebauten Analysesystematik. Hier werden abhängig vom Einsatzzeitpunkt im Rahmen des Controllingprozesses die Wirkungsprognose, das Wirkungsmonitoring und die Wirksamkeitsanalyse unterschieden. Durch kontinuierliche Rückkopplungsschleifen aus dem operativen Einsatz sollte zudem eine laufende Anpassung der verwendeten Analyse- und Bewertungsschemata erfolgen (vgl. hierzu Abbildung 39).

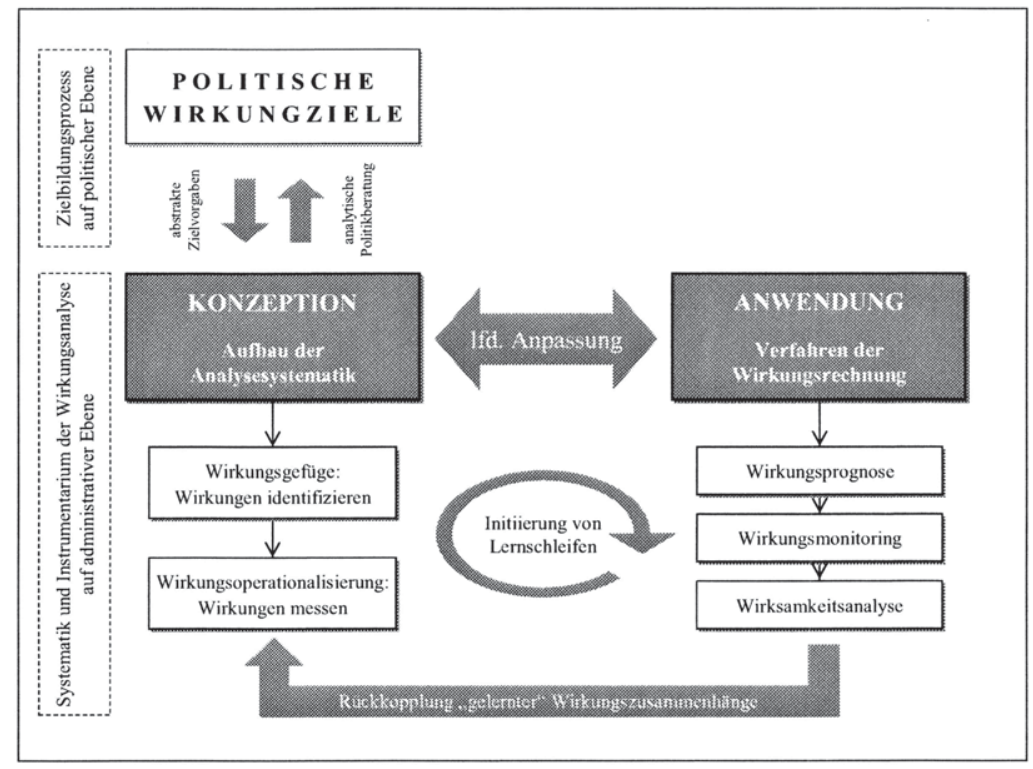

Abbildung 39: Durchführungsschritte einer Wirkungsanalyse

Die erste Säule der Wirkungsanalyse bildet die Konzeptionsphase. Innerhalb der Konzeptionsphase hat das wirkungsorientierte Controlling die Aufgabe, eine geeignete Analysesystematik zur Bewertung der gesamtgesellschaftlich ausgelösten Wirkungen aufzubauen. Hierbei handelt es sich um einen zweistufigen Bewertungsprozess. Der erste Schritt dieses Prozesses besteht darin, für einen abgegrenzten staatlichen Handlungsbereich das gesamtgesellschaftliche Wirkungsgefüge zu konkretisieren. Das Wirkungsgefüge dient der Identifikation sämtlicher durch das staatliche Handeln ausgelöster Wirkungen. Es enthält zunächst Hypothesen über die potenziell durch das staatliche Programm in der Gesellschaft ausgelösten Wirkungen. In der anschließenden Anwendungsphase werden diese hypothetischen Wirkungen immer wieder mit den realen Be- 
obachtungen und Erfahrungen abgeglichen, wodurch eine schrittweise Validierung des Wirkungsgefüges gelingt. Ein dergestalt beschriebenes Wirkungsgefüge unterstützt die Akteure im politisch-administrativen System, sich die durch das staatliche Handeln hervorgerufenen Veränderungen in der Gesellschaft vollständig bewusst zu machen.

Der zweite Schritt des Bewertungsprozesses besteht dann in einer Operationalisierung der einzelnen Wirkungskomponenten durch geeignete Indikatoren. Hierdurch wird die bislang eher abstrakte Beschreibung der Veränderungen im Wirkungsgefüge messbar gemacht, bis hin zu einer monetären Quantifizierung einzelner Wirkungsbeiträge. Gelingt es so, einzelne Wirkungskomponenten weitestgehend bewertbar zu machen, eröffnet dies den Akteuren im politisch-administrativen System nicht nur die Chance, substantielle Vorstellungen über die ausgelösten Wirkungen zu entwickeln, sondern diese auch mit konkreten Zielerreichungsgraden zu versehen. In diesem Kontext wird die Unterstützungsfunktion des wirkungsorientierten Controllings deutlich, indem wirkungsorientierte Steuerungsprozesse durch eine objektive und verlässliche Bewertung ausgelöster Wirkungen überhaupt erst ermöglicht werden.

Grundlage für den Aufbau der Analysesystematik in der Konzeptionsphase bilden die mit einem bestimmten Handlungsprogramm verbundenen politischen Zielsetzungen. Die politische Ebene intendiert mit ihren Handlungen regelmäßig spezifische gesellschaftliche Einzelwirkungen zur Steigerung des Gemeinwohls. ${ }^{658}$ Idealtypisch besteht im Rahmen eines wirkungsorientierten Steuerungsansatzes eine eindeutige Rollenverteilung zwischen Politik und Verwaltung. Auf Basis eines abgestimmten Zielsystems gibt die Politik der Verwaltung nur noch konkretisierte Wirkungsziele vor. ${ }^{69}$ Die Auswahl der geeigneten Programme bzw. Produkte zur Zielerreichung obliegt dann der ausführenden Ebene, d.h. der öffentlichen Verwaltung. ${ }^{660}$

Doch diese idealtypische Rollenverteilung zwischen politischer und administrativer Ebene wird aufgrund unterschiedlicher Problemstellungen im Zielbildungsprozess in der Realität so nicht in jedem Fall gelingen. ${ }^{661}$ Zum einen besteht grundsätzlich eine geringe Bereitschaft der Politik, der Verwaltung objektiv messbare Wirkungsziele vorzugeben. Es widerspricht der politischen Rationalität, da konkrete Wirkungsziele einerseits eine offene Angriffsfläche bieten und andererseits die Konsensbildung zur Mehrheitsfindung erschweren. ${ }^{662}$ Zum anderen besitzt die öffentliche Verwaltung auch eine ,informatorische Machtposition“ gegenüber der Politik, die in der relativen Nähe zu den Leistungsempfängern begründet liegt. Vielfach kann die administrative Ebene daher viel besser beurteilen, welche Wirkungen durch staatliches Handeln in der Gesellschaft ausgelöst wurden - insbesondere wenn alle wichtigen Neben- und Folgewirkungen mit berücksichtigt werden sollen.

\footnotetext{
658 Vgl. Pieper et al. (2006), S. 245.

659 Zur Ausgestaltung eines idealtypischen wirkungsorientierten Steuerungsansatzes vgl. Kapitel 2.2.3.1.

660 Vgl. beispielsweise Bühler (2002), S. 278 oder Schedler/Proeller (2003), S. 54.

$661 \mathrm{Vgl}$. hierzu die zentralen Herausforderungen der Wirkungsorientierung in Kapitel 2.2.3.4.

662 Vgl. Brüggemeier (2004), S. 383.
} 
Dies hat für die Konzeption einer Wirkungsanalyse zur Konsequenz, dass das wirkungsorientierte Controlling in der Regel nicht auf ein politisch abgestimmtes Wirkungszielsystem aufsetzen kann. Die Wirkungsorientierung muss daher maßgeblich auf administrativer Ebene initiiert und vorangetrieben werden. ${ }^{663}$ Für die öffentliche Verwaltung ergibt sich demnach zur Durchführung einer Wirkungsanalyse die Notwendigkeit, durch geeignete Mittel die abstrakten politischen Zielformulierungen zu konkretisieren und sämtliche Kausalzusammenhänge zwischen Staatshandeln und den hierdurch ausgelösten Veränderungen im gesellschaftlichen Umfeld aufzudecken. ${ }^{664}$ Auf diese Weise entsteht faktisch ein kombinierter Abstimmungsprozess zwischen politischer und administrativer Ebene. Abstrakte politische Zielvorgaben müssen auf administrativer Ebene konkretisiert und operationalisiert sowie um wichtige Nebeneffekte ergänzt werden, um ein vollständiges Bild innerhalb des Wirkungsgefüges zu zeichnen. Anschließend werden diese Informationen der politischen Ebene zurückgespiegelt, wodurch eine Form der analytischen Politikberatung durch die Verwaltung stattfindet (vgl. Abbildung 39). Geht man davon aus, dass entsprechend bewährte Wirkungsinformationen im politischen Zielbildungsprozess schrittweise Berücksichtigung finden, könnte dies letztlich sogar zu einer Versachlichung und Entideologisierung politischer Diskussionen führen. ${ }^{665}$

Eine entsprechend gestaltete Vorgehensweise ist allerdings auch mit nicht unerheblichen Gefahren verbunden. Die öffentliche Verwaltung wird mit der Wirkungsanalyse an den bestehenden Leistungen ansetzen, wodurch die Gefahr einer nachträglichen Legitimierung des vorhandenen Produktprogramms besteht. Aufbauend auf den vorgenommenen Produktdefinitionen wird im Rahmen des Controllings nach den hierdurch ausgelösten Wirkungen gesucht. Rein theoretisch bedarf ein wirkungsorientiertes Management aber einer Zielbildung, bei der zunächst gefragt wird, welche Wirkungen überhaupt angestrebt werden sollen, unabhängig vom bestehenden Leistungsspektrum. Diese Vorgehensweise wäre radikaler und würde die Chancen erhöhen, bestehende Leistungen einzustellen, da Wirkungen entweder weniger wichtig sind oder mit anderen Mitteln erreicht werden können. ${ }^{66}$ Es ist allerdings nicht zu erwarten, dass auf administrativer Ebene ein entsprechend isolierter Zielbildungsprozess stattfinden kann.

Aus den genannten Gründen erscheint es zur Zeit gleichfalls nicht wahrscheinlich, dass seitens der politischen Ebene die Vorgabe eines abgestimmten und konkretisierten Wirkungszielsystems erfolgt. Demnach gibt es momentan keine reale Alternative zur beschriebenen Vorgehensweise zur Konzeption einer Wirkungsanalyse, die auf administrativer Ebene an den bestehenden Verwaltungsprodukten ansetzt. Wichtig ist vielmehr, sich im Rahmen des Controllingprozesses und beim Aufbau der Analysesystematik dieser Gefahr bewusst zu sein. Sämtliche getroffenen Hypothesen zu Wirkungs-

\footnotetext{
663 Vgl. Proeller (2006), S. 19.

664 Vgl. Pieper et al. (2006), S. 245.

$665 \mathrm{Zu}$ den Chancen der Wirkungsorientierung vgl. auch Kapitel 2.2.3.3.

666 Vgl. Heinz (2000), S. 99.
} 
zusammenhängen sind besonders kritisch zu hinterfragen bzw. die Bewertungsansätze zur Operationalisierung von Wirkungen entsprechend vorsichtig zu wählen.

Die zweite Säule der Wirkungsanalyse bildet die Anwendungsphase. Nach dem Aufbau der Analysesystematik für einen bestimmten staatlichen Produktbereich findet innerhalb der Anwendungsphase die eigentliche operative Erfolgskontrolle des Verwaltungsoutputs im Rahmen eines systematischen Controllingprozesses statt. In Anlehnung an die bislang in der Literatur verwendeten Begrifflichkeiten wird dies als $\mathrm{Ver}$ fahren der Wirkungsrechnung bezeichnet, wobei jedoch zu beachten ist, dass der Umfang der Analyse hier deutlich über das bisherige Literaturverständnis hinausgeht (vgl. hierzu Abbildung 39). ${ }^{667}$ Abhängig vom Einsatzzeitpunkt im Rahmen des Controllingprozesses kann die Wirkungsrechnung wiederum in die Wirkungsprognose, das Wirkungsmonitoring und die Wirksamkeitsanalyse unterteilt werden.

Aufgabe der Wirkungsprognose ist die Abschätzung mutmaßlicher Wirkungen, die durch ein zukünftig zu erstellendes Produkt ausgelöst werden. Grundlage der Wirkungsprognose bildet die in der Konzeptionsphase entworfene Analysesystematik. Eine Prognose erfordert zahlreiche Annahmen zur Art und Weise der Produkterstellung und den damit verbundenen Wirkungszusammenhängen. Hierdurch entstehen natürlich hohe Unsicherheiten bezüglich der gewonnenen Ergebnisse, was bezogen auf den frühen Bewertungszeitpunkt aber unvermeidbar ist. Zielsetzung einer Wirkungsprognose soll daher die grobe Abschätzung möglicher Wirkungsweisen ohne aufwändige spezifische Analysen sein. Das wirkungsorientierte Controlling kann so sehr schnell Einschätzungen zu steuerungsrelevanten Sachverhalten treffen, um Fehlentscheidungen zu vermeiden.

Dagegen besteht die Aufgabe des Wirkungsmonitoring in der begleitenden Wirkungsanalyse während der Leistungserstellung. Dies soll insbesondere eine Feinsteuerung des Vollzugs durch die zuständigen Verwaltungsmitarbeiter ermöglichen, um bestehende Handlungsspielräume bei der Wirkungszielerreichung nutzbar zu machen. Das Verfahren eignet sich daher vor allem bei längerfristigen Projekten, bei denen das wirkungsorientierte Controlling den Mitarbeitern kontinuierlich steuerungsrelevante Daten bereitstellt.

Die Aufgabe der Wirksamkeitsanalyse besteht dann in der eigentlichen Erfolgskontrolle nach Produkterstellung, mit dem Ziel, Kenntnis über die tatsächlich ausgelösten Wirkungen sowie deren Wirksamkeit zu erhalten. Das Controlling schafft damit die

${ }^{667}$ Im bisherigen Literaturverständnis beschreibt eine Wirkungsrechnung die Messung intendierter politischer Wirkungszielvorgaben mit Hilfe geeigneter quantitativer Indikatoren. Vgl. beispielsweise Schedler/Proeller (2003), S. 207. Im Kontext dieser Arbeit werden Erweiterungen vorgenommen, die zum einen das Zielsystem betreffen und zum anderen das Bewertungsniveau einzelner Wirkungskomponenten. So werden bei der Wirkungsidentifikation nicht nur politisch intendierte Wirkungen, sondern auch sämtliche Neben- und Folgewirkungen berücksichtigt, da es sich auch hier um reale Veränderungen im gesamtgesellschaftlichen Wirkungsgefüge handelt, die ursächlich auf das staatliche Produkt zurückgehen. Hinsichtlich des Bewertungsniveaus besteht die Erweiterung in einer Operationalisierung, welche Wirkungen mit Hilfe von Quantifizierungsansätzen auch eine monetäre Wertigkeit zuweist. 
Voraussetzung, im Rahmen wirkungsorientierter Steuerungsprozesse Verantwortung zuzuweisen, bis hin zu einem differenzierten Ausweis des Beitrags einzelner Akteure zur Zielerreichung. Gleichzeitig dienen entsprechende Informationen auch der Legitimation gegenüber dem Bürger, indem die Wirksamkeit öffentlicher Maßnahmen nachgewiesen und ein „Gegenwert“ für die öffentlich eingesetzten Mittel dokumentiert werden kann.

Fundierte Kenntnisse über tatsächlich eingetretene Wirkungen sowie das zunehmende Verständnis der Wirkungszusammenhänge erfordern kontinuierliche Rückkopplungsschleifen aus dem operativen Einsatz. Hierdurch kann eine laufende Anpassung der verwendeten Analysesystematik erfolgen, ausgebildet als Lernschleife zwischen der Konzeptions- und Anwendungsphase (vgl. Abbildung 39). Im Rahmen derartiger Rückkopplungsprozesse ermöglicht dies durch den Erkenntnis- und Lernfortschritt aller beteiligten Akteure eine ständige Verbesserung der verwendeten Analyse- und Bewertungsschemata. Auf diese Weise handelt es sich auch bei der Wirkungsanalyse um einen geschlossenen Controllingkreislauf.

\subsubsection{Konzeptionsphase: Aufbau der Analysesystematik}

\subsubsection{Identifikation gesamtgesellschaftlicher Auswirkungen}

Den ersten Abschnitt im Rahmen der Vorgehenssystematik bildet die Konzeptionsphase. Aufgabe der Konzeptionsphase ist die Bereitstellung des notwendigen Analyserasters zur Identifikation und Bewertung der Wirkungen staatlichen Handelns, welches in der anschließenden Anwendungsphase Eingang in die operativen Controllingprozesse finden kann. Um sämtliche gesamtgesellschaftlichen Auswirkungen zu erfassen, müssen im vorliegenden Kapitel 4.3.2.1 daher zunächst geeignete Strukturierungshilfen zur Wirkungsidentifikation entwickelt werden. Der zweite Schritt der Konzeptionsphase besteht dann in der Auswahl geeigneter Bewertungsansätze zur Operationalisierung der einzelnen Wirkungskomponenten, welche im nachfolgenden Kapitel 4.3.2.2 ausführlich behandelt werden.

Auf Grundlage der Modellkonzeption der Wirkungen staatlichen Handelns werden in den anschließenden Ausführungen Vorschläge für pragmatische Analysemodelle der Auslöserebene (Kapitel 4.3.2.1.1) und Empfängerebene (Kapitel 4.3.2.1.2) sowie zur Abbildung des gesamtgesellschaftlichen Wirkungsgefüges als übersichtliche Matrixdarstellung (Kapitel 4.3.2.1.3) unterbreitet. Die Ausführungen dieses Kapitels enden mit einer Darstellung unterstützender Instrumente zur Wirkungsidentifikation (Kapitel 4.3.2.1.4).

\subsection{Pragmatisches Analysemodell der Auslöserebene}

Die Modellkonzeption der Wirkungen staatlichen Handelns unterscheidet zwischen einer auslösenden und einer empfangenden Ebene, wobei unter den wesentlichen Strukturelementen vielfältige statische und dynamische Wirkungsbeziehungen beste- 
hen. ${ }^{668}$ Der auslösenden Ebene ist der gesamte Wertschöpfungsprozess staatlichen Handelns zuzuordnen, beginnend mit den gesetzlichen Reglungen durch die Legislative bis hin zur Leistungserstellung der staatlichen Produkte bzw. des Outputs durch die öffentliche Verwaltung.

Ausgangspunkt dieses Wertschöpfungsprozesses bilden politisch abgestimmte Zielsetzungen und die hiermit intendierten Wirkungen in der Gesellschaft, auf dessen Grundlage die Leistungserstellung durch die öffentliche Verwaltung erfolgt. ${ }^{669}$ Die mangelnde Festsetzung eines politisch abgestimmten Zielsystems verlangt innerhalb des öffentlichen Wertschöpfungsprozesses allerdings eine zunehmende Ausführungskonkretisierung abstrakter Wirkungsziele durch die öffentliche Verwaltung. ${ }^{670}$ Hierbei entstehen für die beteiligten Akteure im politisch-administrativen System teilweise erhebliche Spielräume zur Steigerung des Gemeinwohls bzw. zur Erzielung spezifischer gesellschaftlicher Einzelwirkungen. Im Rahmen der Wirkungsanalyse muss daher eine angemessene Strukturierung des öffentlichen Wertschöpfungsprozesses erfolgen, um den beteiligten Akteuren Verantwortung für ihr Handeln zuweisen zu können.

Die im bestehenden Modellansatz getroffene Unterscheidung von politischer und administrativer Ebene ${ }^{671}$ als Verantwortungsträger für das Erreichen von Wirkungszielen erscheint für den Fortgang der Untersuchung nicht ausreichend. Für den Einsatz der Wirkungsanalyse soll daher im Folgenden ein erweiterter Vorschlag zur Differenzierung der staatlichen Auslöserebene unterbreitet werden. Dies erfolgt in Anlehnung an die dezentral organisierten Entscheidungsstrukturen im New Public Management. ${ }^{672}$ Demnach lassen sich im öffentlichen Leistungs- und Wertschöpfungsprozess vier wesentliche Aufgaben und Entscheidungsträger unterscheiden. Das wirkungsorientierte Controlling erhält so die Möglichkeit, den einzelnen Akteuren gezielt Verantwortung für das Erreichen von Wirkungszielen zuzuweisen und gleichzeitig Verbesserungsvorschläge im Rahmen der vollzogenen Leistungsprozesse zu unterbreiten. Abbildung 40 stellt die Zusammenhänge übersichtlich dar.

Die höchste Ebene im Modell stellen die Leistungsbesteller dar. In einer parlamentarischen Demokratie bildet die Legislative bzw. das Parlament als Entscheidungsträger die Spitze des Wertschöpfungsprozesses. Die Leistungsbesteller sind für den öffentlichen Aufgabenumfang und die grundsätzliche Verwendung der hierfür eingesetzten Einnahmen verantwortlich. Zur Regelung der Leistungserstellungsprozesse auf den nachfolgenden Ebenen stehen dem Parlament als normative Instrumente beispielsweise die Verfassung, Gesetze oder sonstige Normen zur Verfügung. Damit verfügt die Legislative als Leistungsbesteller üblicherweise über den höchsten Gestaltungsspielraum,

668 Vgl. hierzu ausführlich Kapitel 4.1.

$669 \mathrm{Vgl}$. hierzu auch die vorgestellten Ebenenmodelle öffentlicher Leistungserstellung in Kapitel 3.3.

670 Vgl. Pieper et al. (2006), S. 245.

671 Die Politik trägt Verantwortung für die Ausgestaltung der Gesetze, die Verwaltung für den administrativen Vollzug der gesetzlichen Regelungen.

672 Zur Dezentralisierung der Führungs- und Organisationsstruktur im New Public Management vgl. ausführlich Schedler/Proeller (2003), S. $79 \mathrm{ff}$. 
die gewünschten Änderungen im gesamtgesellschaftlichen Wirkungsgefüge hervorzurufen. Ausgangspunkt der Überlegungen bilden die gesellschaftlichen Bedürfnisse bzw. der konkrete Bedarf der Bürger und deren politische Wertzumessung.

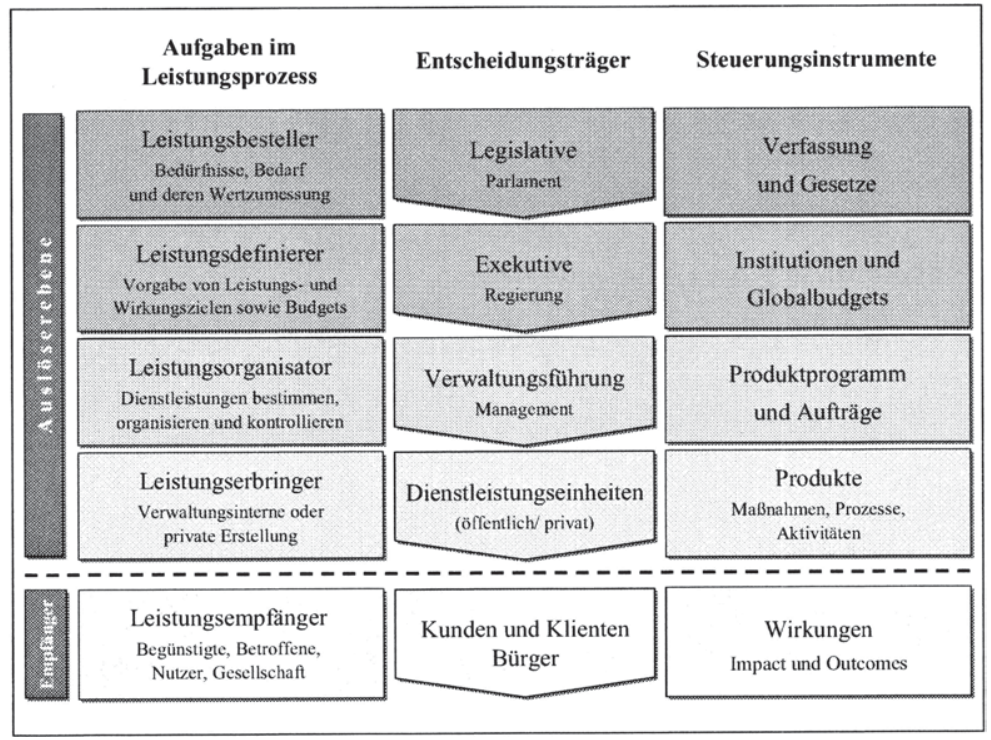

Abbildung 40: Systematisches Modell der Auslöserebene ${ }^{673}$

Auf der zweiten Ebene des Modells sind die Leistungsdefinierer angesiedelt. Als Entscheidungsträger fungiert hier die Exekutive, insbesondere die Regierung mit den zugehörigen Ministerien. Die Leistungsdefinierer stellen in der parlamentarischen Demokratie häufig das Bindeglied zwischen der politischen und administrativen Ebene dar, da ein nicht unwesentlicher Teil der Ministerien auch politisch tätig ist. ${ }^{674}$ Auf dieser Stufe des Wertschöpfungsprozesses erfolgt bereits eine erste Konkretisierung der häufig eher abstrakten politischen Wertzumessungen durch die Vorgabe von allgemeinen Wirkungszielen. Gleichzeitig verantwortet die Regierung die detaillierte Zuteilung des Budgets für die nachgelagerten Behörden und Ämter. Hierdurch verfügt die Regierung als Leistungsdefinierer üblicherweise über einen hohen Spielraum bei der Gestaltung und Priorisierung öffentlicher Aufgaben, mit den entsprechenden Auswirkungen auf das gesamtgesellschaftliche Wirkungsgefüge.

Die dritte Ebene des Modells bilden die Leitungsorganisatoren mit der administrativen Verwaltungsführung als Entscheidungsträger. Als Leistungsorganisatoren sind die Verwaltungsspitzen für das Management öffentlicher Produkte und deren Vollzug

673 Eigene Darstellung in Anlehnung an Müller (2005), S. 12.

674 Vgl. Schedler/Proeller (2003), S. 94. 
durch die nachgelagerten Dienstleistungseinheiten verantwortlich. Abhängig vom regierungsseitig zugestandenen Freiheitsgrad besitzt die Verwaltungsführung bei der Ausgestaltung des öffentlichen Produktprogramms und den administrativen Vollzugsprozessen einen nicht unwesentlichen Gestaltungsspielraum, wodurch wiederum Auswirkungen auf das gesamtgesellschaftliche Wirkungsgefüge bestehen. Dies erfordert auf dieser Ebene die weitere Konkretisierung des Wirkungszielsystems, bis hin zu einer möglichst weitgehenden operativen Operationalisierung der Wirkungsbeiträge einzelner Verwaltungsprodukte.

Die vierte und letzte Ebene des Modells bilden schließlich die Leistungserbringer. Als Entscheidungsträger fungieren hier die öffentlichen Dienstleistungseinheiten in Form von Behörden und Ämtern oder entsprechend beauftragte private Dienstleistungsunternehmen. Die einzelnen Verwaltungseinheiten sind für die Leistungserstellung bestimmter staatlicher Produkte zuständig. Zur Erstellung dieser Produkte müssen in der Regel eine Vielzahl administrativer Prozesse durchlaufen werden, in denen die Verwaltungsmitarbeiter unterschiedliche Maßnahmen ergreifen und Aktivitäten ausführen. Im Rahmen dieser Tätigkeiten besitzen auch die Verwaltungsmitarbeiter einen gewissen Gestaltungsspielraum, auf gesellschaftliche Auswirkungen Einfluss zu nehmen. Der Umfang dieses Spielraums ist dabei stark von den spezifischen Charakteristika des untersuchten staatlichen Produktes abhängig. ${ }^{675}$

Die Leistungserbringer stehen in direkter Verbindung zur Ebene der Leistungsempfänger. Als Leistungsempfänger ziehen die Kunden und Klienten der öffentlichen Verwaltung spezifischen Nutzen aus der staatlichen Leistungserstellung (Impact). Gleichzeitig sind auf der Empfängerebene die vielfältigsten gesellschaftlichen Bereiche von den Auswirkungen der staatlichen Leistungserstellung begünstigt bzw. betroffen (Outcomes). Als Entscheidungsträger der Empfängerebene beurteilen die Bürger der Gesellschaft die Auswirkungen im gesellschaftlichen Wirkungsgefüge und nehmen in der parlamentarischen Demokratie als Leistungsbesteller gleichzeitig Einfluss auf deren Gestaltung. Im nachfolgenden Kapitel wird analog zu den Ausführungen der Auslöserebene ein Analysemodell für die Empfängerebene der 'Wirkungen staatlichen Handelns' vorgestellt.

\subsection{Pragmatisches Analysemodell der Empfängerebene}

Der empfangenden Ebene in der Modellkonzeption der Wirkungen staatlichen Handelns sind sämtliche in der Gesellschaft ausgelösten Wirkungen zugeordnet. Wirkungen wurden definiert als die beobachtbaren Veränderungen im gesamtgesellschaftlichen Wirkungsgefüge, verstanden als räumlich und zeitlich identifizierbare Änderungen der gesellschaftlichen Wirklichkeit, die sich auf öffentliche Maßnahmen zurückführen lassen. ${ }^{676} \mathrm{Im}$ bestehenden Modellansatz sind Wirkungen mit Bezug zu den Zielsetzungen in intendierte Wirkungen sowie erwünschte und unerwünschte Nebenwir-

675 Für eine grobe Typisierung staatlicher Aufgabenbereiche vgl. Kapitel 4.2.3 dieser Arbeit.

676 Vgl. hierzu ausführlich Kapitel 4.1. 
kungen unterteilt. Weiterhin wurde eine Unterscheidung in direkt durch das staatliche Handeln ausgelöste primäre Wirkungen und wiederum hieraus resultierende Folgewirkungen getroffen.

Um eine systematische Wirkungsanalyse im gesellschaftlichen Wirkungsgefüge zu ermöglichen, ist die vorgenommene Differenzierung der bisherigen Modellelemente nicht ausreichend. Für das wirkungsorientierte Controlling wird daher zusätzlich eine geeignete Strukturierungshilfe zur Wirkungsidentifikation benötigt. Im Folgenden soll dazu ein pragmatischer Vorschlag unterbreitet werden, der eine möglichst redundanzfreie Systematisierung der unterschiedlichen gesellschaftlichen Empfängerebenen beabsichtigt. Zur Analyse der objektiven Auswirkungen staatlichen Handelns erfolgt dazu eine mehrstufige Unterteilung der Gesamtgesellschaft in unterschiedliche Subsysteme, denen jeweils spezifische Wirkungsbeiträge zugeordnet werden können. Der Differenzierungsgrad der Wirkungsanalyse ist demnach davon abhängig, ob die Betrachtung auf der Makroebene der Gesamtgesellschaft oder auf der Mikroebene mit einer entsprechend hohen Strukturierung der Teilsysteme ansetzt. In Abbildung 41 sind die unterschiedenen gesellschaftlichen Ebenen zunächst übersichtlich dargestellt.

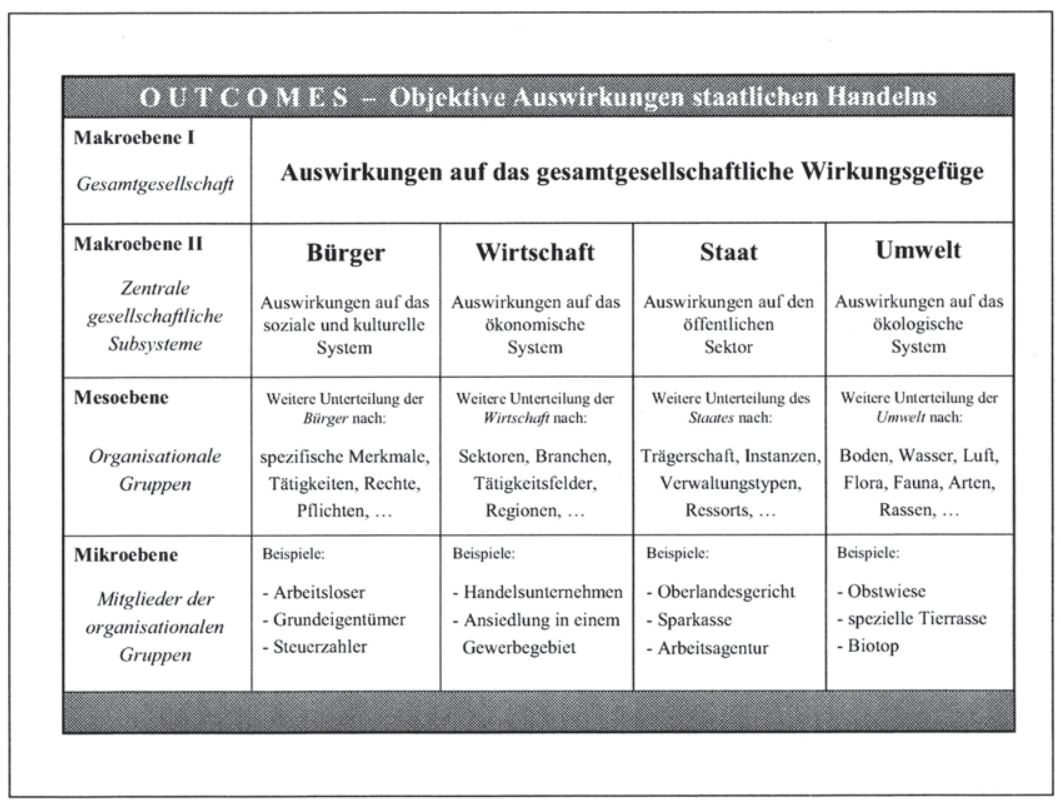

Abbildung 41: Systematisches Modell der Empfängerebene

Auf der obersten Makroebene sind sämtliche Auswirkungen auf das gesamtgesellschaftliche Wirkungsgefüge erfasst. Verbleibt die Wirkungsanalyse auf dieser Ebene, besteht faktisch kein Unterschied zur bisherigen Modellkonzeption. Soll dagegen eine 
empfängerbezogene Systematisierung der Wirkungsbeiträge erfolgen, muss die Gesamtgesellschaft in zentrale Subsysteme unterteilt werden. Als Strukturierungshilfe wird hierzu die Unterteilung der Makroebene in die zentralen gesellschaftlichen Subsysteme »Bürger«, "Wirtschaft«, "Staat» und »Umwelt« vorgeschlagen:

- Die Sphäre der Bürger beinhaltet die Auswirkungen des staatlichen Handelns auf das soziale und kulturelle System. ${ }^{677}$ Hiermit sind insbesondere die in der Gesellschaft lebenden Menschen gemeint sowie von diesen wahrgenommene Tätigkeiten, Rechte und Pflichten im Zusammenleben.

- Die Sphäre der Wirtschaft beschreibt dagegen Auswirkungen auf das ökonomische System. Hierunter fallen in erster Linie sämtliche privatrechtliche Unternehmungen und Wirtschaftsbetriebe sowie damit in Verbindung stehende Dienste und Güter.

- Die Sphäre des Staates umfasst die Rückwirkungen des staatlichen Handelns auf den öffentlichen Sektor. Die Größe und Bedeutung des öffentlichen Sektors ${ }^{678}$ führt in der Regel zu vielfältigen Auswirkungen der öffentlichen Leistungserstellung auf andere staatliche Handlungsbereiche.

- Die Sphäre der Umwelt beinhaltet schließlich sämtliche Wirkungen innerhalb des ökologischen Systems. In erster Linie umfasst die Sphäre damit die außermenschliche Natur, wobei hierunter sowohl die belebte Natur (z.B. Pflanzen und Tiere) als auch die unbelebte Natur (z.B. Steine, Flüssigkeiten, Gase) zu fassen sind. ${ }^{679}$

Auf der Mesoebene findet eine weitere Aufgliederung der vier zentralen gesellschaftlichen Subsysteme in jeweils zu unterscheidende organisationale Gruppen statt. In der Regel bestehen hier zahlreiche Möglichkeiten bzw. Kriterien, nach denen die weitere Systematisierung erfolgen kann. Grundsätzlich ist zu beachten, dass sich auf dieser Ebene Interdependenzen zwischen den Gruppen kaum vermeiden lassen. Die Entscheidung für eine bestimmte Art der Strukturierung muss daher vom konkreten Erkenntnisinteresse der Wirkungsanalyse und von den speziellen Erfordernissen des zu untersuchenden staatlichen Handlungsbereichs abhängig gemacht werden. In Abbildung 41 sind Beispiele möglicher Systematisierungskriterien aufgeführt.

So kann eine Gruppierung der Sphäre »Bürger« nach ganz unterschiedlichen Kriterien der hierunter gefassten Personen erfolgen. Mögliche Gliederungskriterien wären zum Beispiel spezifische Merkmale oder auch bestimmte ausgeübte Tätigkeiten der Bürger. Für den staatlichen Handlungsbereich einer Arbeitsagentur würde beispielsweise die Gruppe der Arbeitslosen näher betrachtet, für die Finanzverwaltung dagegen die Gruppe der Steuerzahler. Grundsätzlich können auch innerhalb einzelner Gruppierungen wieder Abstufungen vorgenommen werden, beispielsweise nach Dauer der Arbeitslosigkeit (z.B. Langzeitarbeitslose) oder nach der Höhe des Steueraufkommens (z.B.

677 Der Begriff ,soziales System“ ist hier nicht deckungsgleich mit dem Begriffsverständnis der soziologischen Systemtheorie zu verstehen, sondern betrifft Menschen und ihr Zusammenleben in der Gesellschaft.

679 Zur Trennung von biotischer (belebter) und abiotischer (unbelebter) Natur vgl. Schaefer (2003). 
Gutverdiener). Entsprechend dieser Vorgehensweise können im Rahmen einer Wirkungsanalyse die relevanten Gruppierungen in den gesellschaftlichen Subsystemen abgegrenzt und anschließend detailliert untersucht werden.

Die Mikroebene umfasst schließlich die einzelnen Mitglieder der auf der Mesoebene unterteilten organisationalen Gruppen. Damit stellt sie die tiefste und zugleich detaillierteste Untersuchungsebene einer Wirkungsanalyse dar. Die Auswirkungen staatlichen Handelns werden dann mit direktem Bezug auf einzelne Personen, Unternehmen, öffentliche Einrichtungen oder natürliche Objekte analysiert.

Auch wenn innerhalb des vorgestellten Strukturierungsansatzes i.d.R. keine komplett überschneidungsfreie Zuordnung der einzelnen Wirkungskomponenten gelingen kann, sollten den verschiedenen Sphären, Gruppierungen und Abstufungen - soweit möglich - sämtliche Einzelwirkungen zugeordnet werden. Die konkrete Auswahl der Systematisierungskriterien und Gliederungstiefe ist abhängig vom Erkenntnisinteresse der Wirkungsanalyse. Grundsätzlich besteht bei der Gliederungstiefe die Möglichkeit, bis auf die Mikroebene vorzudringen und einzelne Gruppenmitglieder detailliert zu betrachten. Gleichzeitig ist innerhalb des vorgestellten Analyseschemas aber auch eine eher aggregierte Sichtweise möglich, auf der Wirkungsinformationen verdichtet dargestellt sind.

Der mehrstufige Aufbau des Analyseschemas verschafft so eine gewisse Flexibilität bei der Zusammenführung der Wirkungsinformationen unterschiedlicher staatlicher Produktbereiche. Dergestalt könnte durch eine Konsolidierung der Wirkungsinformationen in der Verwaltungsspitze eine bessere Abstimmung der Auswirkungen unterschiedlicher staatlicher Handlungsbereiche gelingen. Dies erhöht die Transparenz und Nachvollziehbarkeit von Produktentscheidungen auf administrativer Ebene. Gleichzeitig sollten entsprechend verdichtete Wirkungsinformationen auch auf politischer Ebene von Interesse sein. Im nachfolgenden Kapitel werden die vorgestellten Analysemodelle der Auslöser- und Empfängerebene durch die Abbildung eines gesamtgesellschaftlichen Wirkungsgefüges zusammengeführt.

\subsection{Abbildung eines gesamtgesellschaftlichen Wirkungsgefüges}

Zur Abbildung des gesamtgesellschaftlichen Wirkungsgefüges kann eine übersichtliche Darstellung in Form einer Analysematrix gewählt werden. Bei dieser Matrix handelt es sich um eine zweidimensionale Abbildung, welche die zuvor behandelten Modelle der Auslöser- und Empfängerebene sinnvoll zueinander in Beziehung setzt. Als ein wichtiges Instrument der Wirkungsanalyse unterstützt die Analysematrix die Identifikation relevanter Wirkungsbeiträge in der Gesellschaft. Gleichzeitig kann die übersichtliche Darstellungsform aber auch für die zusammenfassende Dokumentation und Information der insgesamt durch das staatliche Handeln ausgelösten Wirkungen genutzt werden. Zum vollständigen Aufbau des Wirkungsgefüges müssen dazu die nachfolgenden Schritte durchgeführt werden. 
Im ersten Schritt erfolgt die Auswahl des zu untersuchenden staatlichen Produktbereichs. Hierzu sollte das Analysemodell der Auslöserebene herangezogen werden, um die an der Leistungserstellung beteiligten Wertschöpfungsstufen und Akteure des politisch-administrativen Systems in die Untersuchung einzubeziehen ${ }^{680}$ Der notwendige Analyseumfang bemisst sich in diesem Zusammenhang nach der ausgewählten Ebene. Sind die gesellschaftlichen Auswirkungen eines gesamten staatlichen Produktprogramms oder Gesetzesbereiches Gegenstand der Wirkungsanalyse, ist der notwendige Untersuchungsumfang tendenziell umfassender. Erfolgt die Wirkungsanalyse demgegenüber auf der tieferen Stufe einzelner öffentlicher Maßnahmen oder Aktivitäten, ist der Umfang entsprechend geringer.

Im zweiten Schritt muss eine Systematisierung und Strukturierung der gesellschaftlichen Empfängerebene vorgenommen werden, wozu das Analysemodell der Empfängerebene zum Einsatz kommt. Die konkrete Auswahl der geeigneten Gliederungskriterien für die zentralen gesellschaftlichen Subsysteme muss hierbei vom zu untersuchenden staatlichen Produktbereich abhängig gemacht werden. Die Wahl der geeigneten Gliederungstiefe - Makro-, Meso- oder Mikroebene - ist wiederum von der im ersten Schritt gewählten Breite des Untersuchungsansatzes abhängig. Um die gesellschaftlichen Auswirkungen einzelner behördlicher Aktivitäten zu untersuchen, kann durchaus eine Gliederungstiefe bis hin zur Mikroebene sinnvoll sein. Bei der Analyse ganzer Produktprogramme sollte allerdings eine stärker aggregierte Sichtweise auf der Mesoebene gewählt werden. Grundsätzlich gewährt das Modell der Empfängerebene hier eine recht hohe Flexibilität in der spezifischen Anlage einer Wirkungsanalyse.

Im dritten Schritt erfolgt die Zusammenführung der beiden bislang getrennt betrachteten Analyseebenen zum gesamtgesellschaftlichen Wirkungsgefüge. Um die Matrixdarstellung zu erzeugen, wird die vertikale Dimension durch die Auslöserebene und die horizontale Dimension durch die Empfängerebene abgebildet. Die relevanten Wirkungskomponenten des untersuchten staatlichen Handlungsbereiches werden nun entsprechend der gewählten Systematisierung in die Matrix eingeordnet (vgl. hierzu $A b$ bildung 42).

In einem vierten Schritt müssen die Strukturelemente sowie die statischen und dynamischen Aspekte der Modellkonzeption der Wirkungen staatlichen Handelns berücksichtigt werden. Die Strukturelemente ermöglichen zunächst die Unterscheidung von intendierten Wirkungen sowie von erwünschten und unerwünschten Nebenwirkungen. Zusätzlich sind die durch die primären Wirkungen ausgelösten Folgewirkungen in das Wirkungsgefüge aufzunehmen. ${ }^{681}$ Mit den entsprechenden Informationen gelingt so ein transparenter Ausweis sämtlicher Auswirkungen im gesellschaftlichen Wirkungsgefüge - auch entsprechend dem Bezug zur staatlichen Maßnahme bzw. Programm.

${ }^{680}$ Die detaillierte Betrachtung der einzelnen, am staatlichen Leistungserstellungsprozess beteiligten Wertschöpfungsstufen und Akteure ermöglicht zudem den Ausweis des korrespondierenden Ressourcenverzehrs, indem sämtliche hierfür notwendige Kostenbestandteile in die Analyse einbezogen werden.

${ }^{681}$ Zur Modellkonzeption der Wirkungen staatlichen Handelns vgl. ausführlich Kapitel 4.1.2 sowie zusammenfassend Kapitel 4.1.3. 
Weiterhin sind die statischen Aspekte der Modelkonzeption beim Aufbau der Untersuchungsanlage zu beachten. ${ }^{682}$ Grundsätzlich dürfen im Wirkungsgefüge nur Wirkungen abgebildet werden, für die das betrachtete Produkt bzw. die analysierte staatliche Maßnahme Wirkungsrelevanz besitzt - d.h. sie üben einen ursächlichen Einfluss auf die beobachtbaren gesellschaftlichen Auswirkungen aus. Die Aufnahme in das Wirkungsgefüge sollte zudem von der spezifischen Wirkungsstärke abhängig gemacht werden. ${ }^{683}$ Demnach sollten nur Wirkungen aufgenommen werden, bei denen das staatliche Produkt einen deutlich erkennbaren und relevanten Einfluss auf deren Entstehung ausübt.

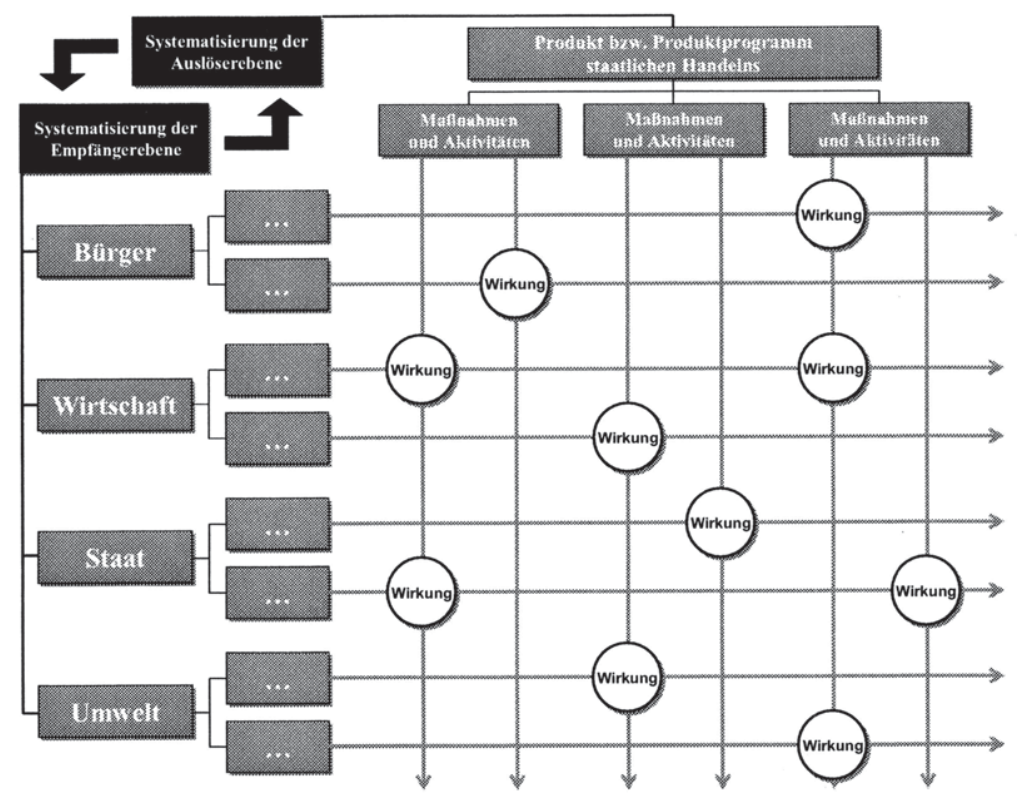

Abbildung 42: Wirkungsgefüge als Analysematrix

In diesem Zusammenhang ist allerdings problematisch, dass in der Realität häufig eine Vielzahl unterschiedlicher Faktoren gleichzeitig Wirkungsrelevanz besitzt und dies in jeweils unterschiedlicher Wirkungsstärke. Demnach bestehen mit den beschriebenen Formen der Wirkungsinteraktion vielfältige Interdependenzen zwischen den auslösenden Faktoren - d.h. zwischen dem betrachteten staatlichen Produkt, anderen staatlichen Produkten und sonstigen exogenen Faktoren. Die beobachtbaren Veränderungen im

${ }^{682} \mathrm{Zu}$ den statischen Aspekten der Modellkonzeption vgl. ausführlich Kapitel 4.1.2.3 dieser Arbeit.

${ }^{683}$ Die Wirkungsstärke beschreibt die Stärke des Zusammenhangs zwischen dem unabhängigen Faktor, d.h. dem staatlichen Produkt und den abhängigen Faktoren, d.h. den hierdurch ausgelösten Wirkungen. Die Wirkungsstärke lässt sich dabei auf die drei Komponenten Wirkungselastizität, Wirkungsvariabilität und Wirkungsdeterminiertheit zurückführen. Vgl. Kapitel 4.1.2.3. 
gesamtgesellschaftlichen Wirkungsgefüge lassen sich aufgrund von Kompensationen und Überlagerungseffekten häufig nicht eindeutig nachweisen bzw. zuordnen. Für eine sachgerechte Wirkungsanalyse ist es allerdings notwendig, diese Effekte - soweit möglich - zu isolieren und nur Wirkungsbestandteile in das Wirkungsgefüge aufzunehmen, die sich eindeutig dem betrachteten staatlichen Produkt zurechnen lassen. Gleichzeitig sollten die bestehenden Kompensationen und Überlagerungseffekte gesondert erfasst werden, da sie bezogen auf das betrachtete Produkt potenzielle Wirkungen darstellen. Die hierdurch gewonnene Transparenz über die potenziellen Auswirkungen staatlichen Handelns könnte helfen, die in der Realität immer wieder zu beobachtenden Mitnahmeeffekte zu vermeiden sowie gegenläufige Effekte im staatlichen Produktangebot zu beseitigen.

Schließlich sind auch die dynamischen Aspekte der Modelkonzeption im Rahmen der Wirkungsidentifikation zu beachten. ${ }^{684}$ Als kausal verknüpfte Ereignisse werden auch durch Wirkungen ausgelöste Folgewirkungen in das Wirkungsgefüge aufgenommen und damit mehrstufige Wirkungsketten abgebildet. Für eine vollständige Wirkungsidentifikation ist allerdings problematisch, wenn Wirkungen erst mittelbar zu beobachten sind und Wirkungsverzögerungen auftreten. Diese 'time lags' zwischen staatlicher Leistungserstellung und dem Wirkungseintritt erschweren die Analyse, da die empirische Überprüfung des Sachverhaltes unter Umständen erst mit mehrjähriger Verzögerung, nach Wirkungseintritt, erfolgen kann. Demgegenüber ist die Untersuchung der Wirkungsdauer, d.h. ob Wirkungen nur kurzfristig in der Gesellschaft auftreten oder nachhaltig bestehen bleiben, nicht unbedingt für die Identifikation, sondern eher für eine sachgerechte Wirkungsbewertung, relevant.

Beim Aufbau eines gesamtgesellschaftlichen Wirkungsgefüges steht jede Wirkungsanalyse vor spezifischen methodischen Herausforderungen. Insbesondere die vielfältigen Interdependenzen zwischen unterschiedlichen auslösenden Faktoren macht eine verlässliche Isolierung zurechenbarer Wirkungsbestandteile grundsätzlich schwierig. Inwieweit sich die hier theoretisch erarbeiteten Analysemodelle und Strukturierungshilfen im Umgang mit diesen Herausforderungen bewähren, muss schließlich eine Erprobung in praktischen Anwendungsfällen zeigen. Für die Ausgestaltung einer wirkungsorientierten Controllingkonzeption unterstreicht dies die Notwendigkeit, kontinuierliche Rückkopplungsschleifen aus der praktischen Anwendung vorzusehen. Die folgenden Ausführungen zeigen, welche unterstützenden Instrumente das Controlling darüber hinaus zur Wirkungsidentifikation einsetzen kann.

\subsection{Instrumentelle Unterstützung der Wirkungsidentifikation}

Mit der Abbildung eines gesamtgesellschaftlichen Wirkungsgefüges wurde ein praktikables Instrumentarium entwickelt, welches das Controlling bei der Strukturierung und systematischen Analyse der Wirkungen staatlichen Handelns unterstützt. Offen bleibt bislang, welche zusätzlichen unterstützenden Instrumente das Controlling zur Wir-

$684 \mathrm{Zu}$ den dynamischen Aspekten der Modellkonzeption vgl. ausführlich Kapitel 4.1 .2.4. 
kungsidentifikation nutzen kann. Theoretisch betrachtet enthält das Wirkungsgefüge Kausalhypothesen über die gesellschaftlichen Auswirkungen des untersuchten staatlichen Handlungsbereiches. Entsprechend des gewählten Forschungsparadigmas der Wirkungsanalyse müssen diese vermuteten Auswirkungen des staatlichen Handelns nicht nur theoretisch begründbar sein, sondern auch schrittweise empirisch validiert werden. ${ }^{685}$ Demnach hat das wirkungsorientierte Controlling die Aufgabe, umfangreiche Erkenntnisse über die Veränderungen in der gesellschaftlichen Wirklichkeit zu gewinnen. Zur instrumentellen Unterstützung der Wirkungsidentifikation muss sich das Controlling daher den Methoden der empirischen Sozialforschung bedienen. Grundsätzlich stehen dazu die drei nachfolgenden Verfahren der Datenerhebung zur Auswahl, die im Weiteren kurz vorgestellt werden: ${ }^{686}$

- Befragungen

- Beobachtungen

- Inhalts- und Dokumentenanalysen

Die in den Sozialwissenschaften bei weitem am häufigsten angewandte Datenerhebungsmethode stellen Befragungen dar. ${ }^{687}$ Die Befragung gilt nach wie vor als das Standardinstrument bei der Ermittlung von Fakten, Wissen, Meinungen, Einstellungen oder Bewertungen im zu untersuchenden Anwendungsbereich. ${ }^{688}$ Bei der Befragung werden im Rahmen eines kommunikativen Prozesses Informationen gesammelt. ${ }^{689}$ Grundsätzlich sind dabei unterschiedliche Vorgehensweisen denkbar. Nach der Form der Durchführung unterscheidet man zwischen "persönlichen Interviewbefragungen«, »Telefoninterviews« und "schriftlichen Befragungen«. In Abhängigkeit von der Teilnehmerzahl handelt es sich entweder um Einzel- oder Gruppenbefragungen. ${ }^{690}$

Ein weiteres wichtiges Unterscheidungskriterium ist der Strukturierungsgrad der Befragung. Insbesondere bei den mündlich durchgeführten Interviewsituationen handelt es sich um einen sozialen Prozess, mit wechselseitigen Wahrnehmungen und Orientierungen. ${ }^{691}$ Durch die Strukturierung des Interviews mit einem Leitfaden oder durch die Vorgabe eines standardisierten Fragebogens lässt sich die Befragung so gestalten, dass valide und substantielle Ergebnisse zu erwarten sind. Abhängig vom notwendigen Stichprobenumfang und den damit verbundenen Erhebungskosten wird bei einer entsprechend stark strukturierten Erhebung dann auch häufig der schriftliche Durchfüh-

\footnotetext{
685 Vgl. hierzu ausführlich Kapitel 4.1.1.2.

${ }^{686}$ Zur vorgenommenen Systematisierung der Methoden empirischer Sozialforschung vgl. Schnell/Hill/Esser (1999), S. $297 \mathrm{ff}$. oder auch Laatz (1993), S.103 ff.

687 Ungefähr 90\% aller Daten in den empirischen Sozialwissenschaften werden mit dieser Methode erhoben. Vgl. Bortz/Döring (2006), S. 236.

$688 \mathrm{Vgl}$. Schnell/Hill/Esser (1999), S. 299.

689 Vgl. Laatz (1993), S. 103.

690 Diese Unterscheidung besitzt insbesondere bei einem mündlichen Durchführungsweg Relevanz, vgl. Laatz (1993), S. 103.

691 Vgl. hierzu und im Folgenden Schnell/Hill/Esser (1999), S. 300 ff.
} 
rungsweg ${ }^{692}$ oder das Telefoninterview angewandt. Dagegen bietet sich eine wenig strukturierte Interviewsituation eher dann an, wenn das Untersuchungsobjekt in seinen Dimensionen noch nicht klar umrissen ist und weiterer Klärungsbedarf besteht. Solche stark am informellen Gespräch orientierte Vorgehensweisen können sich sowohl auf die Befragung von Einzelpersonen (z.B. in „Expertengesprächen“), als auch auf die Diskussion in Gruppen (,Gruppendiskussion“) beziehen. ${ }^{693}$

Für das Verfahren der Wirkungsanalyse und die Wirkungsidentifikation sind insbesondere Expertengespräche ein wichtiges Instrument zur explorativen Erkundung möglicher Wirkungen im gesellschaftlichen Umfeld. So können Vertreter oder Interessenvertreter der unterschiedlichen gesellschaftlichen Empfängergruppen direkt nach den Auswirkungen des staatlichen Handelns befragt werden. In gleicher Weise können auch Einzel- oder Gruppenbefragungen in Form von Mitarbeitergesprächen stattfinden, da diese direkt an der öffentlichen Leistungserstellung beteiligt sind. Das wirkungsorientierte Controlling muss in diesem Zusammenhang nur die jeweiligen spezifischen Interessenslagen der Befragten beachten und einen möglichst neutralen Standpunkt einnehmen. Zur Überprüfung und Validierung möglicher Auswirkungen ist dagegen eine eher standardisierte Befragung mittels Fragebogen zu wählen, wobei die Leistungsempfänger bzw. Betroffenen um eine konkrete Beurteilung der einzelnen Auswirkungen gebeten werden.

Ein weiteres wichtiges Instrument der empirischen Sozialforschung stellen Beobachtungen dar. Im Allgemeinen wird dies als die „ursprünglichste“ Datenerhebungstechnik betrachtet. ${ }^{694}$ Im Vergleich zur Befragung handelt es sich dabei um das Sammeln von Erfahrungen in einem nichtkommunikativen Prozess mit Hilfe sämtlicher Wahrnehmungsmöglichkeiten. ${ }^{69}$ Der Übergang von einer Alltagsbeobachtung zur wissenschaftlichen Beobachtung ist vor allem dadurch gekennzeichnet, dass das Verfahren kontrolliert und systematisch abläuft und die gesammelten Beobachtungsinhalte nach einem vorab definierten Schema strukturiert werden. ${ }^{696}$ Je nach Kenntnisstand der beobachteten Personen vom Beobachtungsvorgang unterscheidet man zwischen »offener Beobachtung« und »verdeckter Beobachtung«. Ist der Beobachter Interaktionspartner

692 Neben den Erhebungskosten bei entsprechend umfangreichen Stichproben, werden als weitere inhaltliche und methodische Vorteile der schriftlichen Befragung insbesondere das ,ehrlichere“ und „überlegtere“ Antwortverhalten der Befragten genannt. Durch die häufig zugesicherte Anonymität ist das Antwortverhalten glaubwürdiger und Interviewfehler können vermieden werden. Demgegenüber besteht jedoch ein wichtiger Nachteil in der üblicherweise recht hohen Ausfallquote, da nur ein Teil der Fragebögen zurückgeschickt wird. Die Datenerhebungssituation ist damit nicht mehr vollständig kontrollierbar und es können Stichprobenverzerrungen auftreten, da nur ein bestimmter Personenkreis (z.B. höheres Bildungsniveau, Interesse an der Thematik etc.) an der Befragung teilnimmt. Zudem besteht auch kein Einblick in die „Ernsthaftigkeit“" des Antwortverhaltens beim Ausfüllen des Fragebogens. Zu einer umfangreichen Diskussion der mit schriftlichen Befragungen verbundenen Vor- und Nachteile vgl. Bortz/Döring (2006), S. $256 \mathrm{ff}$.

693 Zu einer Übersicht der möglichen Formen mündlicher Befragung vgl. Schnell/Hill/Esser (1999), S. 301.

694 Vgl. hierzu Schnell/Hill/Esser (1999), S. $358 \mathrm{ff}$.

695 Vgl. Laatz (1993), S. 169.

696 Die Form der wissenschaftlichen Beobachtung wird daher auch als systematische Beobachtung bezeichnet. Vgl. Cranach/Frenz (1969), S. 269. 
der beobachteten Personen, spricht man von einer "teilnehmenden Beobachtung «, ansonsten von der »nicht-teilnehmenden Beobachtung $«{ }^{697}$

Bei der Durchführung einer Wirkungsanalyse können Beobachtungen - vor allem die explorative Untersuchung - von bisher wenig analysierten gesellschaftlichen Bereichen unterstützen. Dennoch muss für jeden Einzelfall entschieden werden, ob Wirkungstatbestände besser durch eine Befragung oder durch systematisches Beobachten erschlossen werden können. Dabei wird das wirkungsorientierte Controlling in vielen gesellschaftlichen Bereichen nicht auf das Instrument der Beobachtung verzichten können - z.B. bei komplexen Sachverhalten. Diese können von den beteiligten Akteuren weder angemessen wahrgenommen werden, noch können sie detailliert darüber berichten. Teilweise können verbale Auskünfte auch nicht eingeholt werden (wie z.B. bei Kindern) bzw. nur das Verhalten der Akteure ist für die Untersuchung von Interesse. ${ }^{698}$

Als drittes Instrument der empirischen Sozialforschung werden schließlich Inhaltsund Dokumentenanalysen angeführt. ${ }^{699}$ Hierbei werden schriftliche und nichtschriftliche Dokumente aller Art als Quellen für die Beschreibung und Erklärung von Sachverhalten verwendet und ihre Inhalte einer Analyse unterzogen. Im Gegensatz zur Befragung und einigen Beobachtungsverfahren besteht ein Vorteil des Instrumentes darin, dass sich die untersuchten Individuen oder Kollektive der Untersuchungssituation nicht bewusst sind und keine entsprechenden Reaktionseffekte zeigen. Auch eine Wirkungsanalyse kann meist auf umfangreiches dokumentarisches Material zurückgreifen, welches Hinweise auf mögliche gesellschaftliche Auswirkungen beinhaltet. Wichtig ist, die herangezogenen Quellen kritisch auf ihre Verlässlichkeit hin zu überprüfen.

\subsubsection{Bewertung gesamtgesellschaftlicher Auswirkungen}

Nach Abschluss der Wirkungsidentifikation besteht der zweite Schritt der Konzeptionsphase in der Entwicklung geeigneter Bewertungsansätze zur Operationalisierung der einzelnen im Wirkungsgefüge dargestellten Wirkungskomponenten. Dazu wird in den nachfolgenden Ausführungen zunächst die relevante Bewertungsperspektive abgegrenzt, die der Wirkungsanalyse üblicherweise zugrunde liegt (Kapitel 4.3.2.2.1). Anschließend wird eine Systematik der Bewertungsformen vorgestellt (Kapitel 4.3.2.2.2). In Bezug zu diesem Bewertungshintergrund enden die Ausführungen des Kapitels mit einer übersichtlichen Darstellung der unterschiedlichen unterstützenden Instrumente der Wirkungsbewertung (Kapitel 4.3.2.2.3).

\footnotetext{
697 Vgl. hierzu Schnell/Hill/Esser (1999), S. 361 f.

698 Zu den aufgeführten Anwendungsfällen der Beobachtung vgl. Friedrichs (1973), S. 274.

699 Vgl. Laatz (1993), S. 207 ff.
} 


\subsection{Bewertungsperspektive einer Wirkungsanalyse}

Bevor die Wirksamkeit einer öffentlichen Maßnahme durch eine geeignete Operationalisierung sämtlicher Wirkungsbeiträge beurteilt werden kann, muss zunächst die relevante Bewertungsperspektive der Wirkungsanalyse abgegrenzt werden. Wirkungen sind definiert als die beobachtbaren Veränderungen im gesamtgesellschaftlichen Wirkungsgefüge - verstanden als räumlich und zeitlich identifizierbare Änderungen der gesellschaftlichen Wirklichkeit, die sich auf die öffentliche Maßnahmen zurückführen lassen. ${ }^{700}$ Problematisch sind in diesem Zusammenhang die vielfältigen Interdependenzen zwischen unterschiedlichen auslösenden Faktoren. Wirkungen können von anderen Faktoren ausgelöst worden sein, aber gleichzeitig mit der staatlichen Maßnahme auftreten.

Für die Wirkungsanalyse wollen wir also wissen, wie die Ergebnisse ausgesehen hätten, wenn die staatliche Maßnahme nicht gewesen wäre, und vergleichen diesen Fall mit dem der vorhandenen Maßnahme. Im amerikanischen Raum wird dazu die Terminologie Policy on - policy off verwendet: Dieser Vergleich liegt der Wirkungsbewertung zugrunde. Um die Wirksamkeit einer öffentlichen Maßnahme zu erfassen, muss der Zustand „mit Maßnahme“ mit dem Zustand verglichen werden, der „ohne Maßnahme“ bestehen würde. ${ }^{701}$ Wesentlich für die konkrete Ausgestaltung einer Wirkungsanalyse ist demnach das Mit-und-Ohne-Prinzip. ${ }^{702}$

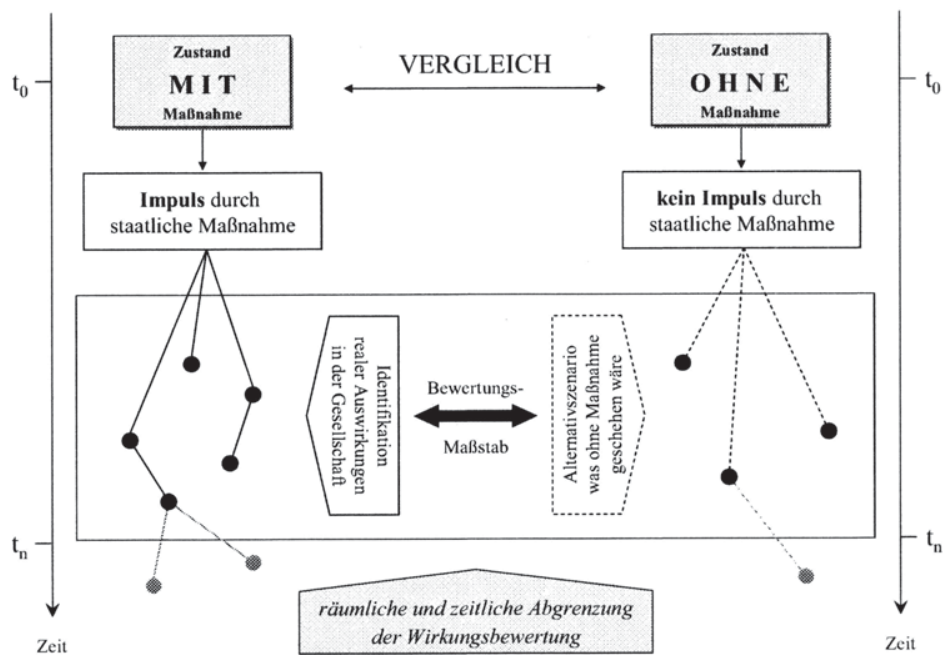

Abbildung 43: Abgrenzung der Bewertungsperspektive

$700 \mathrm{Vgl}$. hierzu ausfuihrlich Kapitel 4.1.

$701 \mathrm{Vgl}$. Bussmann (1995), S. 346.

702 Zum Begriff des Mit-und-Ohne-Prinzips in der Nutzen-Kosten-Analyse vgl. Hanusch (1994), S. 6. 
Bei einer Abgrenzung der Analyseperspektive ist demzufolge zu beachten, dass nur die Unterschiede zwischen zwei alternativen Entwicklungen - mit und ohne öffentliche Maßnahme - verglichen werden dürfen. Ein reiner Zeitpunktvergleich vor und nach Durchführung der staatlichen Maßnahme ist damit nicht zulässig, da hier auch Wirkungen bewertet würden, die ohne die staatliche Maßnahme aufträten. ${ }^{703}$ Die Abgrenzung der relevanten Bewertungsperspektive einer Wirkungsanalyse ist in Abbildung 43 veranschaulicht.

Für eine sachgerechte Abgrenzung der Bewertungsperspektive müssen die tatsächlichen Auswirkungen der öffentlichen Maßnahme mit denen einer alternativen Bezugsgruppe verglichen werden, die anzeigt, was geschehen wäre, wenn die betrachtete Maßnahme nicht initiiert oder eine andere Maßnahme durchgeführt worden wäre. ${ }^{704}$ Wären in beiden Situationen alle anderen Faktoren weitestgehend identisch, können darauf folgende gesellschaftliche Unterschiede der betrachteten Maßnahme zugeschrieben werden. Ein solcher Quervergleich unter realen Bedingungen setzt allerdings voraus, dass eine äquivalente gesellschaftliche Kontrollgruppe besteht oder zu Untersuchungszwecken eingerichtet werden kann. ${ }^{705}$ Die Wirkungsanalyse wird sich in der Regel aber nicht auf solche experimentellen und quasi-experimentellen Designs stützen können, da äquivalente gesellschaftliche Vergleichsgruppen entweder nicht vorhanden sind, oder die Einrichtung einer Kontrollgruppe zu aufwendig bzw. nicht zulässig ist. ${ }^{706}$

Alternativ kann im Rahmen der Wirkungsanalyse auch ein Alternativszenario entworfen werden, welches die tatsächlich aufgetretenen Wirkungen mit den Wirkungen vergleicht, die aufgetreten wären, hätte man die staatliche Maßnahme nicht initiiert. Da die Wirkungen im Szenario allerdings nie tatsächlich aufgetreten sind, sind sie nicht wirklich, d.h. sie sind irreal. Die Differenz zwischen den tatsächlichen und den irrealen Auswirkungen entspricht dann den Auswirkungen, die der betrachteten staatlichen Maßnahme zurechenbar sind. ${ }^{707}$

Hierbei handelt es sich um eine recht pragmatische Vorgehensweise, die auch für das wirkungsorientierte Controlling in der Breite anwendbar erscheint. Sicherlich ist ein solches Vorgehen für den Nachweis der Kausalbeziehung zwischen staatlichem Handeln und den gesellschaftlichen Wirkungen weniger leistungsfähig. Doch sollte dieser Nachteil für eine praxistaugliche Anwendung der Wirkungsanalyse in Kauf genommen

\footnotetext{
703 Dies wäre ein Längsschnittvergleich im Sinne einer Vorher-Nachher-Betrachtung.

704 Vgl. Vedung (1999), S. 142.

705 BUSSMANN schlägt in diesem Zusammenhang die Einrichtung „föderalistischer Labors“ vor, denen sich das politisch-administrative System zu Analysezwecken bedienen kann. Vgl. Bussmann (1995), S. 346.

706 So wäre beispielsweise die Vergabe von Subventionen an zufällig ausgewählte Empfänger - während andere Personen in identischen Situationen diese Zuwendungen nicht erhalten - rechtlich nicht zulässig. Diese Bedingungen sind beim staatlichen Handeln aus ethischen, politischen, sozialen und rechtlichen Gründen sehr häufig gegeben. Zu experimentellen und quasi-experimentellen Designs in der Evaluationsforschung vgl. auch Klöti/Widmer (1997), S. 195.

$707 \mathrm{Vgl}$. in ähnlicher Weise für die Evaluationsforschung Vedung (1999), S. $142 \mathrm{ff}$.
} 
werden. Wichtig ist daher, ein möglichst konservatives Alternativszenario zu entwerfen, das die Auswirkungen des eigenen Handelns nicht überschätzt.

Der Geltungsbereich einer Wirkungsanalyse muss zudem in räumlicher und zeitlicher Hinsicht abgegrenzt werden (vgl. Abbildung 43). ${ }^{708}$ Was die geographische Abgrenzung betrifft, ist für die Wirkungsanalyse staatlichen Handelns in der Regel eine nationale Abgrenzung zielführend. Abhängig vom staatlichen Handlungsbereich ist unter Umständen auch eine Analyse im internationalen Rahmen durchzuführen, insbesondere wenn außenpolitische Ausstrahlungen erfasst werden müssen. Sofern sich Auswirkungen öffentlicher Projekte nur auf bestimmte Regionen beschränken, bietet sich selbstverständlich eine Untersuchung nur auf dieser lokal begrenzten Ebene an.

Der Zeitraum, für den die Auswirkungen öffentlicher Maßnahmen betrachtet werden, sollte sich am gewöhnlichen Planungshorizont des untersuchten staatlichen Handlungsbereichs orientieren. Bei der Wahl des zeitlichen Analysezeitraums sollte man zudem prüfen, ob aufgrund von Wirkungsverzögerungen auch erst mittelbar mit relevanten gesellschaftlichen Veränderungen zu rechnen ist und mit der Wirkungsdauer auch die Nachhaltigkeit von Auswirkungen beachten. ${ }^{709}$ In der Regel wird man die zeitliche Analyse daher auf terminierte Zeiträume beschränken, die sich beispielsweise am Zeitraum einer Generation orientiert bzw. eine Dauer von ungefähr 25 bis 30 Jahren umfasst.

\subsection{Systematik der Bewertungsformen}

Durch die Bildung eines Alternativszenarios sowie die räumliche und zeitliche $\mathrm{Ab}$ grenzung des Geltungsbereiches ist die relevante Bewertungsperspektive der Wirkungsanalyse vollständig definiert. Im Folgenden müssen die im gesamtgesellschaftlichen Wirkungsgefüge enthaltenen Auswirkungen der betrachteten öffentlichen Maßnahme bewertet werden. Hier lassen sich in Abhängigkeit von Messbarkeit und Messniveau verschiedene Bewertungsformen der Wirkungsanalyse unterscheiden.

Das Messniveau unterscheidet zwischen Auswirkungen, die sich monetär bewerten lassen und solchen, die keiner monetären Bewertung zugänglich sind. Viele Auswirkungen staatlichen Handelns werden sich jedoch einer sinnvollen monetären Quantifizierung entziehen, da sich der Kausalbezug zur Maßnahme entweder nicht eindeutig abgrenzen lässt oder die Wirkung sehr subjektiven Wertvorstellungen unterliegt. ${ }^{710}$ Ein weiteres Problem stellt die vielfach sehr lückenhafte Verfügbarkeit der notwendigen Daten und Bewertungsgrundsätze dar, um Wirkungsbeiträge monetär zu quantifizieren. Ein vollständiges Schließen dieser Lücken durch das Controlling wird häufig nicht möglich sein. 
In Anlehnung an ECKSTEIN wird im Folgenden daher auch zwischen tangiblen und intangiblen Wirkungsbeiträgen differenziert. ${ }^{71}$ Tangible und intangible Wirkungen einer staatlichen Maßnahme lassen sich nach dem Kriterium der Messbarkeit unterscheiden. Der Begriff "Tangible» umfasst dabei alle Wirkungsdeterminanten, die sich in irgendeiner Form quantifizieren lassen, entweder monetär oder in anderen messbaren Größen. Alle anderen Wirkungen sind demgegenüber als »Intangible« zu bezeichnen und lassen sich nur mit Hilfe qualitativer Angaben verbal umschreiben. ${ }^{712}$ Damit können Wirkungsbewertungen teilweise monetarisierend, teilweise quantifizierend und teilweise nur qualifizierend erfolgen (vgl. Abbildung 44).

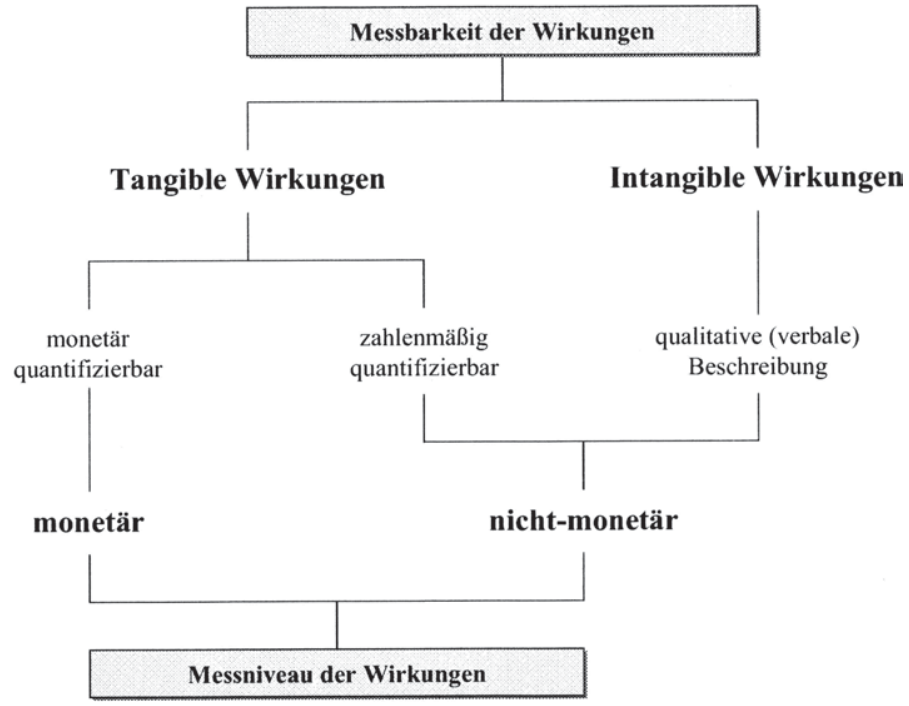

Abbildung 44: Bewertungsformen der Wirkungsanalyse

Im konkreten Fall wird es allerdings oftmals umstritten bleiben, was als tangibel und was als intangibel anzusehen ist. Eine eindeutige Grenze zwischen tangiblen und intangiblen Wirkungen lässt sich nicht exakt bestimmen, die Übergänge verlaufen hier fließend. Ob ein zunächst für intangibel gehaltener Effekt im Laufe einer Wirkungsanalyse tatsächlich noch quantifiziert werden kann, ist häufig eine Frage der verfügbaren wissenschaftlichen und technischen Möglichkeiten und auch des zeitlichen und finanziellen Aufwands, den das Controlling dafür zu leisten bereit ist. ${ }^{713}$ Entscheidend ist nur, dass sowohl die tangiblen als auch die intangiblen Wirkungen einer Maßnahme vollständig erfasst und beschrieben werden.

\footnotetext{
711 Vgl. Eckstein (1961), S. 51.

712 Vgl. hierzu auch Pieper et al. (2006), S. 250.

713 Vgl. Hanusch (1994), S. 10.
} 
Um die Wirkungen einer öffentlichen Maßnahme monetär zu quantifizieren, müssen die erwünschten und unerwünschten gesellschaftlichen Auswirkungen bewertet und in monetären Äquivalenten erfasst werden. Teilweise liegen für bestimmte gesellschaftliche Wirkungen direkt originär monetäre Messgrößen vor, beispielsweise wenn der Staat direkt geldliche Zuwendungen an Leistungsempfänger zahlt oder staatliches Handeln zu eindeutigen Vermögensänderungen führt. Vielfach müssen Wirkungen allerdings durch abgesicherte Näherungs- oder Umrechnungsverfahren erst monetarisiert werden. Hierzu sind die im Wirkungsgefüge erfassten Wirkungsbeiträge jeweils durch geeignete monetäre Bewertungsansätze zu operationalisieren, so dass den einzelnen Wirkungskomponenten ein gesellschaftlich akzeptierter Marktpreis zugeordnet wird. $^{714}$

Sind die monetär quantifizierbaren Wirkungsbeiträge auf diese Weise bewertet, kann in einem weiteren Schritt eine Summierung sämtlicher positiver und negativer Auswirkungen erfolgen. Aufgrund von auftretenden Wirkungsverzögerungen und der unterschiedlichen Wirkungsdauer der einzelnen Wirkungsbeiträge sind die verschiedenen Bewertungszeitpunkte allerdings zuvor in temporaler Hinsicht zu homogenisieren. Ebenso wie in der privatwirtschaftlichen Investitionsrechnung bezeichnet man den Vorgang der zeitlichen Homogenisierung als Diskontierung. ${ }^{715}$ Als gemeinsamer Betrachtungszeitpunkt bietet sich dazu, abhängig vom Zeitpunkt der Wirkungsanalyse, entweder der Beginn oder das Ende der betrachteten öffentlichen Maßnahme an. Die Bestimmung eines sachgerechten Zinssatzes zur Diskontierung ${ }^{716}$ ist für die Wirkungsanalyse von nicht unerheblicher Bedeutung, denn dieser impliziert immer die Gefahr des 'Schönrechnens' und damit auch die Gefahr falscher Handlungsempfehlungen. ${ }^{717}$

Eine dergestalt vorgenommene Bilanzierung aller positiven und negativen Wirkungen kann nach MOSIEK/GERHARDT auch für eine vollständige Integration von Ressourcenund Wirkungsbetrachtung genutzt werden. ${ }^{718}$ In einer sog. gesellschaftlichen Wertschöpfungsbilanz werden die Gesamtkosten der staatlichen Leistungserstellung den monetarisierten Auswirkungen gegenübergestellt, um auf diese Weise einen Wertschöpfungssaldo zu ermitteln. ${ }^{719}$ Der Vorteil einer monetären Bewertung gesellschaftlicher Auswirkungen liegt in der schnellen Vergleichbarkeit und Gesamtbeurteilung der betrachteten öffentlichen Maßnahme - auch im Sinne eines dokumentierten »value for money«. Problematisch sind allerdings die notwendigen Prämissen in den Bewer-

$714 \mathrm{Zu}$ den verschiedenen Bewertungsansätzen zur Monetarisierung der Auswirkungen staatlichen Handelns vgl. Kapitel 4.3.2.2.4.

715 Vgl. Hanusch (1994), S. 97.

716 Die Bestimmung einer sachgerechten Diskontierungsrate kommt in vielen volkswirtschaftlichen Fragestellungen eine erhebliche Bedeutung $\mathrm{zu}$, weshalb darüber in der Literatur eine intensive und teilweise recht kontroverse Diskussion geführt wird. Nach HANUSCH lässt sich der Stand der Diskussion auf drei Ansätze reduzieren, die die Abzinsung entweder über eine soziale Zeitpräferenzrate oder einer sozialen Opportunitätskostenrate bzw. einer Synthese aus beiden Raten abbilden wollen. Vgl. Hanusch (1994), S. $97 \mathrm{ff}$.

717 Vgl. Hoffjan (1999), S. 245 und Berens/Hoffjan (2004), S. 213 f.

718 Vgl. Mosiek/Gerhardt (2003), S. 293 f.

719 Vgl. Mosiek/Gerhardt (2003), S. 293 f. oder auch Röhrig (2008), S. 175 ff. 
tungsmaßstäben, die zu gravierenden Verzerrungen in den Ergebnissen führen können. Im Rahmen einer Wirkungsanalyse muss das Controlling daher besonders vorsichtige Bewertungsansätze wählen, um ein eher konservatives Gesamtergebnis mit einer möglichst geringen Fehlertoleranz auszuweisen.

Gelingt eine monetäre Bewertung nicht, können Auswirkungen alternativ auch durch die Zuordnung anderer Messwerte quantifiziert werden. ${ }^{720}$ Dazu sind die einzelnen Wirkungsbeiträge jeweils durch geeignete Wirkungsindikatoren zu operationalisieren. Operationalisierungen bestehen aus der Angabe von genauen Messanweisungen, mit denen die theoretisch formulierten Wirkungsbeschreibungen des Wirkungsgefüges durch direkt messbare Indikatoren beobachtbar gemacht werden. ${ }^{721}$ Das Messen der Ausprägungen des Indikators ermöglicht dann in der Regel einen begründeten Rückschluss auf die nicht direkt messbare Zielgröße der Wirkung. Wirkungsindikatoren stellen damit Abbildungsgrößen dar, mit denen die Effektivität, und damit der Zielerreichungsgrad des staatlichen Handelns auf der Wirkungsebene beurteilt werden kann. ${ }^{722}$ In dieser Weise unterstützen sowohl Indikatoren, als auch mit Hilfe von Indikatoren gebildete Kennzahlen das wirkungsorientierte Controlling und damit letztlich auch die wirkungsorientierte Steuerung des politisch-administrativen Systems.

Die intangiblen Wirkungen sollten zumindest so präzise wie möglich verbal beschrieben und den anderen Ergebnissen der Wirkungsanalyse in Form einer Argumentenbilanz beigefügt werden. Deren Wertung ist dann letztlich den Entscheidungsträgern im politisch-administrativen System überlassen. Nicht selten handelt es sich gerade bei intangiblen Wirkungen um außerordentlich wichtige Wirkungsbeiträge, die eine hohe politische Wertzumessung erfahren.

\subsection{Instrumentelle Unterstützung der Wirkungsbewertung}

Auch beim zweiten Analyseschritt, der sachgerechten Wirkungsbewertung, kann das Controlling auf eine Vielzahl unterstützender Instrumente zur Wirkungsbewertung zurückgreifen kann. Der Strukturierung der Bewertungsformen folgend, lassen sich hier insbesondere Bewertungsansätze zur monetären Quantifizierung und Wirkungsindikatoren zur quantitativen Operationalisierung der Auswirkungen staatlichen Handelns unterscheiden. Zusätzlich sind die intangiblen Auswirkungen verbal argumentativ zu beschreiben (siehe Abbildung 45).

Die nachfolgenden Ausführungen widmen sich zunächst der Erläuterung unterschiedlicher monetärer Bewertungsansätze. Hierbei ist zu beachten, dass sich der Gesamt-

${ }^{720}$ Unter Messen wird allgemein die Zuordnung von Zahlen („Messwerten“) zu Objekten gemäß festgelegter Regeln verstanden. Vgl. Schnell/Hill/Esser (1999), S. 132.

721 Indikatoren kommen immer dann zum Einsatz, wenn sich die zu messende Realität wegen ihrer Vielschichtigkeit einer umfassenden und objektiv nachprüfbaren Erfassung entzieht. Vgl. Haiber (1997), S. 400. Bei den Auswirkungen staatlichen Handelns handelt es sich in der Regel um theoretische Konstrukte, die durch geeignete Indikatoren operationalisiert werden müssen. Vgl. ausführlich Kapitel 4.1.1.2 dieser Arbeit.

722 Vgl. Röhrig (2008), S. 153 f. 
wert einer Wirkung aus unterschiedlichen Komponenten zusammensetzen kann. ${ }^{723}$ Grundsätzlich sollte eine möglichst vollständige Bewertung erfolgen. Doch aufgrund der relativen Marktnähe gelingt dies vor allem bei Wirkungskomponenten, die die gegenwärtige bzw. zukünftige konsumtive oder produktive Nutzung der öffentlichen Leistungserstellung beschreiben.

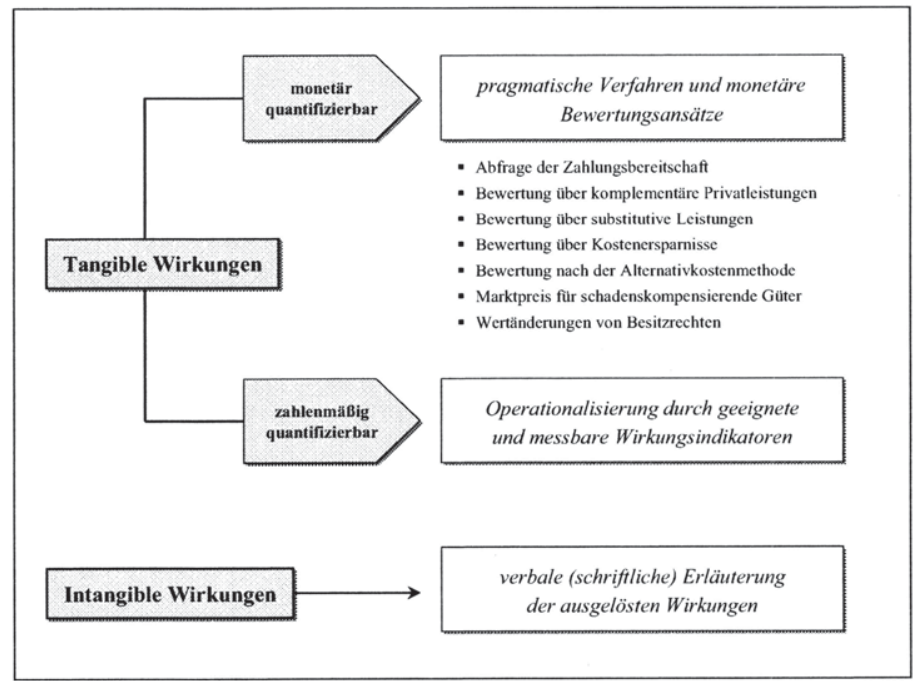

Abbildung 45: Unterstützende Verfahren der Wirkungsbewertung

\section{Pragmatische Verfahren und monetäre Bewertungsansätze}

Um die Auswirkungen öffentlicher Maßnahmen monetär zu quantifizieren, finden sich insbesondere in der Literatur zur Nutzen-Kosten-Analyse unterschiedliche Verfahrensweisen und Bewertungsansätze. ${ }^{724}$ Diese Bewertungsansätze können grundsätzlich auch im Rahmen einer Wirkungsanalyse Anwendung finden, so dass die gebräuchlichsten Methoden im Folgenden kurz vorgestellt werden:

- Eine mögliche Methode, näherungsweise einen Marktpreis zu ermitteln, liegt in der Abfrage individueller Zahlungsbereitschaften für öffentliche Leistungen. ${ }^{725}$ Wie in der Marktforschung werden die Präferenzen der Leistungsempfänger für ein be-

723 Der ökonomische Gesamtwert setzt sich aus einem Nutzungswert, einem Optionswert und einem Existenzwert zusammen. Der Nutzungswert beinhaltet eine Wertzumessung, welcher sich auf die gegenwärtige konsumtive oder produktive Nutzung öffentlicher Leistungen durch die Gesellschaft bezieht. Der Optionswert beinhaltet das Recht einer zukünftigen Nutzung. Dagegen bezieht sich der Existenzwert auf den marktunabhängigen Wert des grundsätzlichen Ressourcenerhalts, wie z.B. der Natur. Vgl. bspw. Baumgärtner (2003).

724 Vgl. hierzu und im Folgenden detailliert Hanusch (1994), S. 76 ff.

$725 \mathrm{Vgl}$. hierzu grundlegend Johansson (1993). 
stimmtes öffentliches Produkt bzw. eine bestimmte Wirkung über Befragungen ermittelt. ${ }^{726}$ Bei positiven Wirkungen werden die Begünstigten direkt nach dem Betrag gefragt, den sie für die erhaltenen Vorteile maximal zu zahlen bereit sind; bei negativen Wirkungen nach der monetären Mindestentschädigung, die sie als Betroffene dafür erwarten. Dieser Bewertungsansatz weist jedoch zwei gewichtige Schwächen auf. Einerseits können die Befragten bei den weitestgehend kostenlos bereitgestellten öffentlichen Produkten häufig keine eindeutigen Angaben zur tatsächlichen Zahlungsbereitschaft machen. Andererseits ist mit einem strategischen Antwortverhalten der Befragten zu rechnen, wobei die Wertzumessung bewusst über- oder unterschätzt wird, da man sich persönliche Vorteile hiervon verspricht. ${ }^{727}$

- Eine Bewertung über komplementäre Privatleistungen versucht die Wertzumessung eines öffentlichen Produktes indirekt über die Wahlhandlungen der Nutzer zu ermitteln. ${ }^{728}$ Diese freiwilligen Wahlhandlungen sind einer Bewertung über den Marktpreis ausgesetzt und entsprechen damit annährend dem Mindestwert des öffentlichen Produktes bzw. einzelner Wirkungsbeiträge, da die Privatleistungen in komplementärer Beziehung zum staatlichen Angebot stehen (,Schattenpreise“). Einen besonders wichtigen Schattenpreis stellen die Anfahrtskosten dar, die private Nutzer aufbringen, um ein öffentliches Produkt zu erhalten oder zu konsumieren. ${ }^{729}$ Der Gesamtwert des öffentlichen Produktes bemisst sich folglich nach den aggregierten Anfahrtskosten sämtlicher Nutzer. Die Schwäche dieses Bewertungsansatzes liegt vor allem darin, dass mit der komplementären Privatleistung immer nur die Wertuntergrenze eines Produktes oder einer Wirkung angegeben werden kann. Der tatsächliche gesellschaftliche Wert wird damit systematisch unterschätzt.

- Ein weiteres Verfahren stellt die Bewertung über substitutive Privatleistungen dar. ${ }^{730}$ Zur Bewertung des öffentlichen Produktes wird auf bereits existierende Privatleistungen zurückgegriffen, die mit dem staatlichen Angebot vergleichbar sind oder sogar in substitutiver Beziehung dazu stehen. Sind die Marktpreise des privaten Vergleichsobjektes bekannt, können diese Informationen zur Bewertung des öffentlichen Produktes herangezogen werden. Bei Anwendung dieser Methodik ist allerdings Vorsicht angebracht, da eine ausreichende Ähnlichkeit zwischen dem öffentlichen und dem privaten Produkt bestehen muss. Schon kleine Unterschiede in der Produktausstattung können merkliche Abweichungen in der Zahlungsbereitschaft der Nutzer hervorrufen, so dass es sich tendenziell eher um eine Wertobergrenze handelt. ${ }^{731}$

726 Zur konkreten Ausgestaltung der Befragungsmethoden vgl. Diamond/Hausman (1994), S. 45 ff.

727 Die Gefahr besteht vor allem deshalb, weil öffentliche Produkte in der Regel aus allgemeinen Steuermitteln finanziert werden und damit der Finanzierungsbeitrag des Einzelnen von einer kaum merklichen Größenordnung ist. Vgl. Hanusch (1994), S. 77.

728 Vgl. hierzu grundlegend Andel (1977).

729 Praktische Anwendung findet das Konzept der Anfahrtskosten insbesondere bei der Bewertung öffentlicher Einrichtungen aus dem Erholungs-, Freizeit- oder Kulturbereich, wie z.B. einem Naturpark.

730 Vgl. hierzu grundlegend Faber (1986).

731 Vgl. Hanusch (1994), S. 83. 
- Eine Bewertung über Kostenersparnisse kann erfolgen, wenn staatliche Maßnahmen bzw. deren Auswirkungen zu Ressourceneinsparungen bei privaten Haushalten, Unternehmen oder anderen öffentlichen Einrichtungen führen. ${ }^{732}$ Dazu sind zunächst die Art der Einsparung und ihr mengenmäßiger Umfang zu bestimmen, um deren monetären Wert anhand der vorgefundenen Marktpreise zu quantifizieren. Kostenersparnisse beziehen sich einerseits auf direkt beobachtbare Ressourceneinsparungen, wie zum Beispiel reduzierte Betriebs-kosten. Andererseits handelt es sich dabei aber auch um vermiedene Kosten zur Behebung von ansonsten aufgetretenen Schäden. Ein weiterer wichtiger Wirkungsbeitrag sind Zeitersparnisse in Folge von öffentlichen Maßnahmen, wie zum Beispiel durch Verkehrsprojekte. Diese Zeitersparnisse können entweder anhand von Lohnsätzen oder anhand von individuellen Wertzumessungen der Begünstigten bewertet werden. ${ }^{733}$

- Eine Bewertung nach der Alternativkostenmethode basiert auf der Überlegung, dass durch die öffentliche Maßnahme eine ansonsten notwendige Maßnahme im privaten oder öffentlichen Sektor überflüssig geworden ist. Die Bewertung der Maßnahme kann sich demnach an den Kosten des irrelevant gewordenen Alternativvorhabens orientieren. Dem Opportunitätsgedanken folgend, darf hierbei allerdings nur die kostengünstigste gleichwertige Alternative berücksichtigt werden.

- Um die negativen Auswirkungen einer öffentlichen Maßnahme zu quantifizieren, können zur Bewertung die Marktpreise für schadenskompensierende Güter herangezogen werden. ${ }^{734}$ Hat eine öffentliche Maßnahme negative Auswirkungen auf davon Betroffene, stellen die Marktpreise der erforderlichen Gegenmaßnahmen zur Behebung der Negativeinflüsse einen guten Wertmaßstab dar. Beispielsweise kann der gestiegenen Lärmbelästigung der Anwohner bei einem Verkehrsinfrastrukturprojekt durch den Einbau schalldämmender Fenster entgegengewirkt werden, wodurch entsprechende Kosten entstehen. Problematisch ist, dass die Kosten der Schadenskompensation den wirklichen Umfang der negativen Auswirkung häufig nur unvollständig erfasst und damit unterschätzt. Andererseits ist aber auch eine Überschätzung möglich, wenn die betreffenden Güter nicht nur der Schadenskompensation dienen, sondern zusätzlich auch andere Zwecke erfüllen. ${ }^{735}$

- Schließlich sind öffentliche Vorhaben in der Regel mit positiven oder negativen Auswirkungen auf den Wert von Besitzrechten verbunden. ${ }^{736}$ Die Veränderungen in den Marktpreisen von Vermögenswerten entsprechen damit auch näherungsweise den positiven oder negativen Auswirkungen der öffentlichen Maßnahme auf die Besitzrechte. Problematisch ist, dass der Wert von Besitzrechten, etwa einem Grund-

732 Unter der Prämisse einer vollständigen Konkurrenz ist der Marktpreis von Produktionsfaktoren in allen Verwendungen gleich, so dass der Wert der freigesetzten Ressourcen genau dem Wert in alternativen Verwendungen entspricht.

733 Zur Bewertung von Zeitersparnissen vgl. ausführlich Beesley (1965).

734 Vgl. hierzu grundlegend Andel (1977).

735 Vgl. Hanusch (1991), S. 91.

736 Vgl. hierzu grundlegend Arnold (1980). 
stück oder Gebäude, durch eine Vielzahl unterschiedlicher Faktoren und Eigenschaften bestimmt wird. Preisänderungen könnten damit einerseits auch auf qualitative Veränderungen dieser Faktoren zurückzuführen sein, andererseits sind Preisänderungen zu einem beträchtlichen Teil vom allgemeinen Angebot-Nachfrageverhalten abhängig.

Zusammenfassend lässt sich festhalten, dass die Wirkungsanalyse auf vielfältige Methoden und Ansätze zur monetären Quantifizierung von Wirkungsbeiträgen zurückgreifen kann. Die konkrete Auswahl und Ausgestaltung des Bewertungsansatzes muss vom Controlling situationsspezifisch und in Abhängigkeit vom jeweiligen Wirkungsbeitrag entschieden werden. Der vorgenommene methodische Überblick kann für die praktische Anwendung zunächst eine gute Orientierungshilfe leisten.

\section{Operationalisierung durch messbare Wirkungsindikatoren}

Gelingt die monetäre Bewertung der Wirkungsbeiträge nicht, können Auswirkungen alternativ durch die Zuordnung von Wirkungsindikatoren quantifiziert werden. Bei Wirkungen handelt es sich in der Regel um theoretische Konstrukte, die durch geeignete Indikatoren zunächst operationalisiert werden müssen. ${ }^{737}$ Unter Operationalisierung versteht man die Angabe von genauen Messanweisungen, welche die theoretisch formulierten Wirkungsbeschreibungen durch die Zuordnung von Zahlen ${ }^{738}$ (,Messwerten") beobachtbar machen. ${ }^{739}$ Zwischen dem ausgewählten Indikator und der zu messenden Wirkung muss daher ein relevanter Zusammenhang bestehen, der unter pragmatischen Gesichtspunkten entweder durch eine begründete Vermutung oder empirische Anzeichen nachgewiesen werden kann. ${ }^{740}$ Das Messen der Ausprägungen des Wirkungsindikators ermöglicht dann in der Regel einen begründeten Rückschluss auf die nicht direkt messbare Wirkung.

Wirkungsindikatoren stellen damit vereinfachte Abbildungsgrößen dar, mit denen die Effektivität, und damit der Zielerreichungsgrad des staatlichen Handelns auf der Wirkungsebene beurteilt werden kann. ${ }^{741}$ Nach HOFFJAN lässt sich die Informationsversorgungsfunktion von Indikatoren in unterschiedliche Teilaufgaben gliedern. ${ }^{742}$ Aufgrund der Operationalisierungsfunktion haben Indikatoren die Aufgabe, nicht wahrnehmungsfähige Sachverhalte zu präzisieren und einer quantitativen Analyse zugänglich

737 Vgl. hierzu ausführlich Kapitel 4.1.1.2.

738 In einem sehr weiten Begriffsverständnis kann unter Messen auch die Zuordnung von Symbolen zu Objekten nach festen Regeln verstanden werden. Vgl. Laatz (1993), S. 51. Für die Ausgestaltung der Wirkungsanalyse ist eine entsprechende Operationalisierung allerdings nicht zielführend, da eine Messung zumindest auf einer Ordinalskala erfolgen sollte. Erst hierdurch wird eine Rangordnung möglich, die zumindest eine Verbesserung oder Verschlechterung der Auswirkungen anzeigen kann und damit auch steuerungsrelevante Informationen bereitstellt.

$739 \mathrm{Vgl}$. Schnell/Hill/Esser (1999), S. 132.

740 Vgl. Haiber (1997), S. 118.

741 Vgl. Röhrig (2008), S. 153 f.

742 Vgl. hierzu und im Folgenden Hoffjan (1998), S. 289 f. 
zu machen. Die Messfunktion ermöglicht eine direkte Messung des Indikators und damit indirekt des Sachverhaltes. Zudem kann die Vorgabe von Sollwerten angestrebte Wirkungsziele verdeutlichen und durch die Motivationsfunktion motivierend auf die beteiligten Mitarbeiter wirken. In dieser Weise unterstützen Indikatorensysteme das wirkungsorientierte Controlling und damit letztlich auch die wirkungsorientierte Steuerung des politisch-administrativen Systems.

Um diese Funktionen erfüllen zu können, werden vielfältige Anforderungen an Wirkungsindikatoren gestellt. Die konkrete Auswahl von Indikatoren für einzelne Wirkungsbeiträge muss grundsätzlich situationsspezifisch erfolgen und ist stark von der betrachteten öffentlichen Maßnahme abhängig. Aufgrund der ausgeprägten Steuerungsrelevanz von Wirkungsindikatoren lassen sich aber allgemeine Anforderungskriterien formulieren, welche die Eignung der eingesetzten Indikatoren beschreiben (vgl. Abbildung 46). ${ }^{743}$

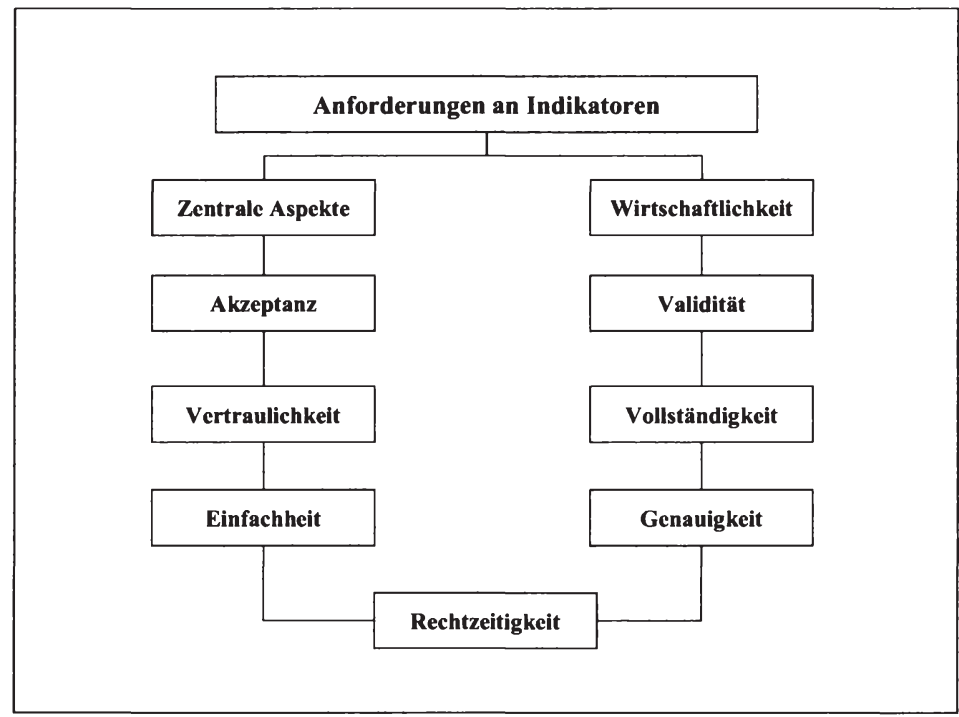

Abbildung 46: Anforderungen an Indikatoren ${ }^{744}$

Entsprechend dieser Anforderungskriterien müssen Wirkungsindikatoren in der Lage sein, die zentralen Aspekte eines Sachverhaltes zu messen. Darüber hinaus sollen Indikatoren valide Informationen zu sämtlichen Auswirkungen im gesellschaftlichen Wirkungsgefüge bereitstellen. Hier spielen Aspekte wie Verlässlichkeit und Genauigkeit in der Widergabe des zugrunde liegenden Sachverhaltes eine wichtige Rolle. Daher ist situativ abzuwägen, inwieweit ein Indikator oder aber mehrere Indikatoren notwendig

743 Vgl. im Folgenden Buschor (2002), S. 63 ff.; Hill (2002), S. 67 ff. und Röhrig (2008), S. 158 ff.

744 Vgl. Röhrig (2008), S. 159. 
sind, um einen Sachverhalt adäquat und damit vollständig abzubilden. Gleichzeitig sollte ein Indikator aber auch möglichst einfach und nachvollziehbar sein. Ansonsten besteht die Gefahr, dass die erhobenen Wirkungsindikatoren nicht auf die notwendige Akzeptanz bei den beteiligten Mitarbeitern stoßen und damit nicht der zugesprochenen Motivationsfunktion gerecht werden können. ${ }^{745}$ Daher sind zudem Vertraulichkeitsaspekte zu berücksichtigen, da Wirkungsindikatoren sich auch auf Sachverhalte beziehen, die unter Umständen Persönlichkeitsschutzvorschriften betreffen. Für die Funktionsfähigkeit wirkungsorientierter Controllingprozesse ist weiterhin die Rechtzeitigkeit der Informationsbereitstellung ein zentrales Anforderungskriterium und nicht zuletzt auch die Erhebungswirtschaftlichkeit der Indikatoren zu beachten.

Die Vielfalt der genannten Anforderungen offenbart zahlreiche Zielkonflikte zwischen den einzelnen Kriterien, so dass grundsätzlich nur ein möglichst ausgewogenes Verhältnis angestrebt werden kann. Dies macht deutlich, dass eine Bewertung von Wirkungen durch Wirkungsindikatoren in vielen Fällen mit großen Herausforderungen verbunden ist, welche das Controlling situationsspezifisch zu lösen hat.

\section{Verbale (schriftliche) Erläuterung intangibler Wirkungen}

Sämtliche intangiblen Wirkungen sollten im Rahmen einer Wirkungsanalyse zumindest so präzise wie möglich verbal beschrieben werden. Nicht selten handelt es sich gerade bei den intangiblen Wirkungen um außerordentlich wichtige Wirkungsbeiträge, die eine hohe politische Wertzumessung erfahren und auch gesetzlich kodifiziert sind.

Es ist daher von besonderer Bedeutung, auch intangible Wirkungsbeiträge einer umfassenden qualitativen Analyse zu unterziehen. Hierbei kann eine vorgegebene Auswertungs- und Analysestrategie ein sinnvolles unterstützendes Instrument darstellen. Diese Strategie zeichnet sich durch eine systematische Vorgehensweise aus, bei der für einzelne Wirkungskomponenten zunächst relevante Untersuchungskriterien herausgearbeitet werden. Mit den Kriterien verfügt man über eine Art qualitatives Raster, mit dessen Hilfe eine möglichst objektive und präzise Beschreibung und schließlich auch Bewertung der intangiblen Auswirkungen gelingen kann. Die Ergebnisse der qualitativen Analyse sind den quantitativen Ergebnissen der Wirkungsanalyse in Form einer Argumentenbilanz beizufügen. Deren abschließende Bewertung ist dann letztlich den Entscheidungsträgern im politisch-administrativen System überlassen.

\subsubsection{Anwendungsphase: Verfahren der Wirkungsrechnung}

\subsubsection{Instrumente der Wirkungsrechnung}

Den zweiten Abschnitt der Wirkungsanalyse bildet die Anwendungsphase. Nach dem Aufbau der erforderlichen Analysesystematik zur Identifikation und Bewertung der gesamtgesellschaftlichen Wirkungen einer öffentlichen Maßnahme erfolgt innerhalb

745 Vgl. Hoffjan (1998), S. 291 f. 
der Anwendungsphase die eigentliche operative Erfolgskontrolle des Verwaltungsoutputs im Rahmen eines systematischen Controllingprozesses. ${ }^{746}$ In Anlehnung an die bislang in der Literatur verwendeten Begrifflichkeiten wird dies im Folgenden als Instrumentarium der Wirkungsrechnung bezeichnet. ${ }^{747}$ Wie in Abbildung 47 dargestellt, können in Abhängigkeit vom Einsatzzeitpunkt im Rahmen des Controllingprozesses die Wirkungsprognose, das Wirkungsmonitoring und die Wirksamkeitsanalyse unterschieden werden.

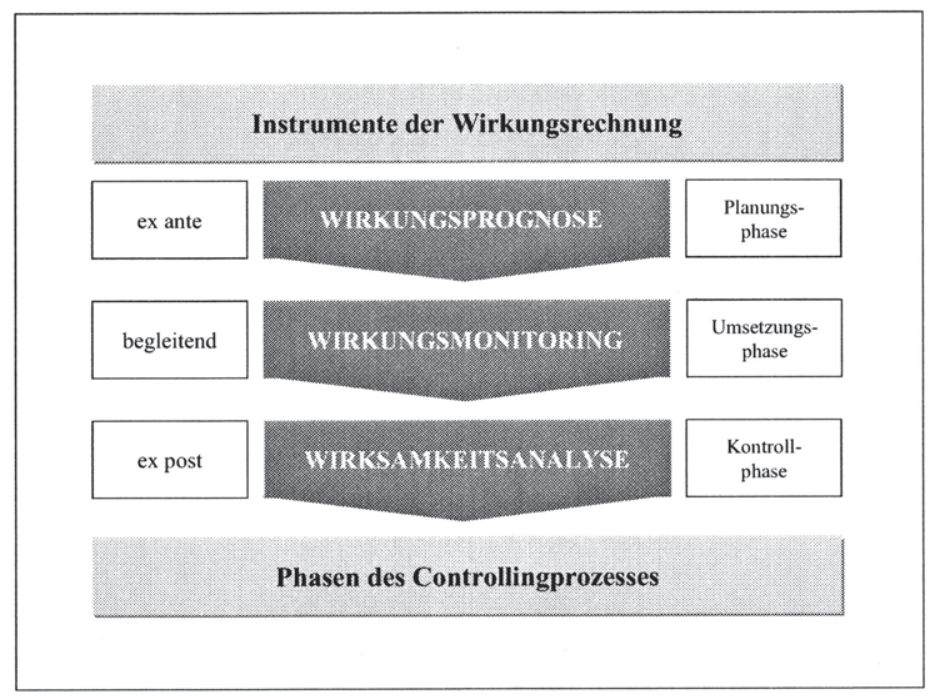

Abbildung 47: Wirkungsrechnung im Controllingprozess

Das Instrumentarium der Wirkungsrechnung orientiert sich dabei an den unterschiedlichen Phasen eines wirkungsorientierten Controllingprozesses. Die wesentliche Aufgabe des wirkungsorientierten Controllings ist es, das Führungsgesamtsystem bei der Erreichung der Organisationsziele durch Koordinationsleistungen zu unterstützen. ${ }^{748}$ Der wirkungszielbezogene Koordinationsbedarf innerhalb des politisch-administrativen Führungssystems ist insbesondere von der Ausgestaltung des Steuerungsprozesses mit den wesentlichen Funktionen Planung, Umsetzung und Kontrolle abhängig. ${ }^{749}$

Entsprechend unterstützt das wirkungsorientierte Controlling die Phase der Planung einer staatlichen Maßnahme bzw. eines öffentlichen Produktes mit der Durchführung einer Wirkungsprognose. Aufgabe der Wirkungsprognose ist die Abschätzung und

\footnotetext{
$746 \mathrm{Vgl}$. hierzu auch die Vorgehenssystematik der Wirkungsanalyse in Kapitel 4.3.1.

747 Vgl. stellvertretend Schedler/Proeller (2003), S. 207 f.

$748 \mathrm{Zu}$ den Aufgaben einer wirkungsorientierten Controllingkonzeption vgl. Kapitel 3.2.

$749 \mathrm{Vgl}$. hierzu ausführlich Kapitel 2.2.3.1.
} 
Bewertung der mutmaßlichen Auswirkungen, die durch das zukünftig zu erstellende Produkt ausgelöst werden. Grundlage einer Wirkungsprognose ist die in der Konzeptionsphase entworfene Analysesystematik. Die Perspektive der Analyse zur eigentlichen operativen Leistungserstellung ist ex-ante angelegt, wobei das politisch-administrative Führungssystem mit den notwendigen Daten zur Realisierung einer wirksamen Produkt- und Programmplanung versorgt wird.

Dagegen besteht die Aufgabe des Wirkungsmonitoring in der begleitenden Wirkungsanalyse während der operativen Leistungserstellung. Es ist demnach in der Phase der Umsetzung einer staatlichen Maßnahme angesiedelt. Innerhalb eines kontinuierlichen Prozesses sammelt und bewertet das wirkungsorientierte Controlling fortlaufend Informationen über den bisherigen Maßnahmenverlauf und die bis dahin realisierten Zwischenergebnisse. Das Führungssystem erhält so die Möglichkeit zur wirksamen Feinsteuerung des administrativen Vollzugs bei der Umsetzung und Ausführung von laufenden Maßnahmen.

Die Aufgabe der Wirksamkeitsanalyse besteht schließlich in der eigentlichen operativen Erfolgskontrolle nach dem Maßnahmenvollzug. Entsprechend ist es der Phase der Kontrolle im politisch-administrativen Steuerungsprozess zuzuordnen. Anhand der entworfenen Analysesystematik identifiziert und bewertet das wirkungsorientierte Controlling möglichst umfassend die durch die staatliche Maßnahme ausgelösten Veränderungen in der Gesellschaft. Die eingenommene Analyseperspektive zur operativen Leistungserstellung ist damit ex-post angelegt. Auf Grundlage der durch das Controlling aufbereiteten wirkungsbezogenen Informationen erlangt das Führungssystem detaillierte Kenntnis über die tatsächliche Wirksamkeit der Maßnahme und die durch das staatliche Handeln beeinflussten Wirkungszusammenhänge in der Gesellschaft.

\subsubsection{Funktionen im wirkungsorientierten Controllingprozess}

Die mit der Wirkungsprognose, dem Wirkungsmonitoring und der Wirksamkeitsanalyse vorgestellten Instrumente der Wirkungsrechnung sind grundsätzlich auch isoliert voneinander anwendbar. Versteht sich das wirkungsorientierte Controlling allerdings als umfassende Funktion der Führungsunterstützung, erscheint es notwendig, den gesamten Prozess der öffentlichen Leistungserstellung durch die Aufbereitung der entsprechenden wirkungsbezogenen Informationen abzubilden. Erst hierdurch ist gewährleistet, dass die hiermit verbundenen Vorteile für die Steuerung des politischadministrativen Systems auch nutzbar gemacht werden. ${ }^{750}$ Generell können der Wirkungsrechnung in diesem Zusammenhang vier unterschiedliche Funktionen zugeschrieben werden (vgl. Abbildung 48) ${ }^{751}$ Diese sind natürlich nicht trennscharf abgegrenzt, sondern jeweils eng miteinander verbunden.

\footnotetext{
$750 \mathrm{Zu}$ den vielfältigen Chancen der Wirkungsorientierung vgl. Kapitel 2.2.3.3.

751 Zu den Funktionen der Evaluation vgl. in ähnlicher Weise Stockmann (2004), S. 17 ff.
} 
"Erkenntnis und Lernen" ist eine bedeutende Funktion der Wirkungsrechnung. Mit Hilfe der Wirkungsrechnung werden entscheidungsrelevante Daten über die gesamtgesellschaftlichen Auswirkungen staatlichen Handelns aufbereitet und damit transparent gemacht. Die auf diese Weise gesammelten Informationen können anschließend für Steuerungsentscheidungen genutzt werden, beispielsweise bei der Gestaltung öffentlicher Maßnahmen oder zur Vermeidung von Fehlwirkungen.

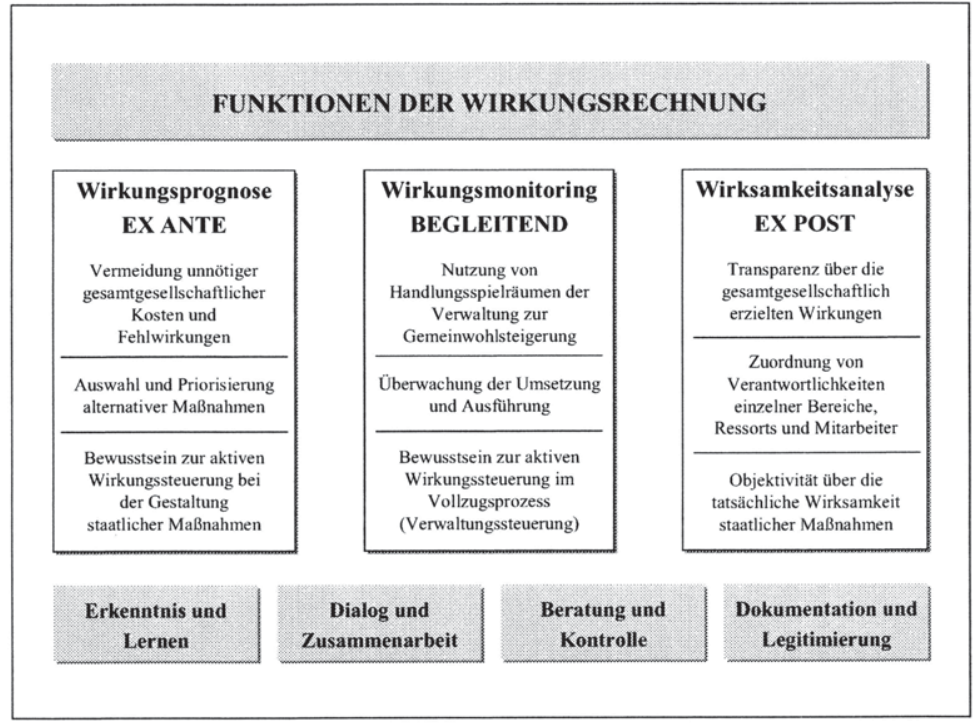

Abbildung 48: Funktionen der Wirkungsrechnung

Eine weitere wichtige Funktion besteht in »Dialog und Zusammenarbeit«. Die Wirkungsrechnung stellt Informationen bereit, die den Dialog zwischen den unterschiedlichen Akteuren des politisch-administrativen Systems auf eine neue Grundlage stellt. Auf der Basis objektiver wirkungsbezogener Daten wird unter Umständen eine Versachlichung des Diskussionsprozesses über die Wirksamkeit alternativer Vorgehensweisen und Maßnahmen eintreten. Zudem lässt sich auch die Zusammenarbeit der beteiligten Akteure verbessern, indem transparent aufgezeigt wird, wie erfolgreich die Zusammenarbeit verlief und wo Defizite auftraten, um daraus Konsequenzen für die Gestaltung der weiteren Zusammenarbeit zu ziehen.

Darüber hinaus handelt es sich bei »Beratung und Kontrolle« um eine weitere wichtige Funktion der Wirkungsrechnung. Durch die laufende Überwachung der Umsetzung und Ausführung erhalten die beteiligten Akteure Entscheidungshilfen für die Steuerung der Maßnahmendurchführung und können gegebenenfalls frühzeitig notwendige Korrekturen vornehmen. Gleichzeitig schafft die Wirkungsrechnung die Voraussetzung, im Rahmen wirkungsorientierter Steuerungsprozesse Verantwortung zuzuweisen 
- bis zu einem differenzierten Ausweis des Beitrags einzelner Akteure zur Zielerreichung. Direkt und indirekt ist so auch eine Form der Kontrolle mit der Wirkungsrechnung verbunden.

Abschließend ist noch die "Dokumentation und Legitimierung" als eine maßgebliche Funktion der Wirkungsrechnung zu nennen. Die gewonnene Datenbasis bietet die Möglichkeit, nachprüfbar zu belegen, mit welchem Ressourceneinsatz die entsprechenden Wirkungen in der Gesellschaft erzielt wurden. Gleichzeitig dienen entsprechende Informationen auch der Legitimation gegenüber dem Bürger, indem die Wirksamkeit oder der Wirkungsgrad öffentlicher Maßnahmen nachgewiesen und ein „Gegenwert" für die öffentlich eingesetzten Mittel dokumentiert werden kann. Dergestalt unterstützt die Wirkungsrechnung die Verantwortlichen im politisch-administrativen System bei der Auswahl und Priorisierung alternativer staatlicher Maßnahmen.

\subsubsection{Ausgestaltung der Wirkungsrechnung}

\subsection{Wirkungsprognose als ex-ante-Analyse}

Aufgabe der Wirkungsprognose ist die Abschätzung und Bewertung der mutmaßlichen Auswirkungen, die durch eine bestimmte staatliche Maßnahme zu erwarten sind. Inhaltlich ist dabei eine eher grobe Abschätzung möglicher Wirkungsweisen angestrebt, ohne zusätzlich spezifische und damit kosten- und zeitintensive Analysen durchführen zu müssen. Entsprechend umgesetzt, lässt sich die Wirkungsprognose auch in einen systematischen Controllingprozess einbinden, indem vor Produkterstellung (ex-ante) regelmäßig eine Abschätzung der mutmaßlichen Auswirkungen durchlaufen wird.

Die für die Leistungserstellung zuständigen Verwaltungsmitarbeiter formulieren die Wirkungsprognose in Zusammenarbeit mit dem Controlling. Dazu können sie auf die in der Konzeptionsphase entworfene Analysesystematik zurückgreifen. Die Analysesystematik besteht einerseits aus dem gesamtgesellschaftlichen Wirkungsgefüge des Produktbereiches. Dabei handelt es sich quasi um eine Art Katalog möglicher Wirkungsweisen und Auswirkungen, welcher zur Identifikation der mutmaßlichen Auswirkungen des betrachteten Produktes herangezogen wird. Andererseits hält die Analysesystematik für die möglichen Auswirkungen auch spezifische Bewertungsansätze bereit. Mit Hilfe dieser Bewertungsansätze kann die quantitative oder qualitative Abschätzung der Intensität der zu erwartenden Wirkungsbeiträge erfolgen. Gleichzeitig liegen hiermit transparente und meist auch objektiv messbare Wirkungsziele vor, welche Eingang in den weiteren Controllingprozess finden.

Eine Wirkungsprognose erfordert zahlreiche Annahmen und Prämissen zur Art und Weise der Produkterstellung und den hierdurch ausgelösten Wirkungen in der Gesellschaft. Demzufolge entstehen natürlich Unsicherheiten bezüglich der gewonnen Ergebnisse, was bezogen auf den frühen Bewertungszeitpunkt aber grundsätzlich nicht zu vermeiden ist. Um dennoch eine möglichst hohe Prognosevalidität zu gewährleisten, sollten die erforderlichen Einschätzungen durch die zuständigen Verwaltungsmitarbei- 
ter vorgenommen werden, da diese sicherlich über die höchste Produktnähe und meist auch über die beste Produktkenntnis verfügen. Dem Controlling kommt im Rahmen der Wirkungsprognose daher eine eher beratende und unterstützende Funktion zu. Allerdings müssen die von den Verwaltungsmitarbeitern getroffenen Einschätzungen vom Controlling immer kritisch überprüft werden, da ansonsten die Gefahr des 'Schönrechnens' besteht, beispielsweise um Produktentscheidungen positiv zu beeinflussen.

Die wesentliche Zielsetzung der Wirkungsprognose besteht in der Vermeidung unnötiger gesellschaftlicher Kosten und Fehlwirkungen. Staatliche Maßnahmen und Projekte, die im Rahmen der Wirkungsprognose keine ausreichende Wirksamkeit nachweisen, können demnach zugunsten anderer Projekte zurückgestellt werden. Eine Wirkungsprognose unterstützt auf diese Weise die Auswahl und Priorisierung alternativer Maßnahmen bei knappen Ressourcen. Anders als bisher, wird die Entscheidung zur Produkterstellung nicht mehr oder weniger willkürlich, sondern anhand weitestgehend transparenter und objektiver Wirkungskriterien getroffen. Wichtig ist zudem, dass die zuständigen Mitarbeiter ein Bewusstsein zur aktiven Wirkungssteuerung bei der Gestaltung öffentlicher Maßnahmen entwickeln. Häufig lässt sich schon durch die Veränderung weniger Stellschrauben die Wirksamkeit einer staatlichen Maßnahme stark erhöhen.

\subsection{Wirkungsmonitoring als on-going-Analyse}

Die Aufgabe des Wirkungsmonitoring besteht in einer begleitenden Wirkungsanalyse während der operativen Leistungserstellung. Dies soll insbesondere die Feinsteuerung des Maßnahmenvollzugs durch die zuständigen Verwaltungsmitarbeiter ermöglichen, um die Handlungsspielräume bei der Wirkungszielerreichung nutzbar zu machen. Das Verfahren ist daher vor allem bei längerfristigen Projekten von Relevanz, wobei das Controlling den zuständigen Mitarbeitern kontinuierlich steuerungsrelevante Informationen bereitstellt.

Das Controlling sammelt und bewertet fortlaufend Informationen über den bisherigen Verlauf der Leistungserstellung und berichtet innerhalb vorab definierter Zeitabstände über die bis dahin realisierten Zwischenergebnisse (on-going). Die zuständigen Mitarbeiter erhalten so die Möglichkeit zur wirksamen Feinsteuerung des Leistungserstellungsprozesses. Gleichzeitig erhält das Führungssystem Informationen über den spezifischen Zielerreichungsgrad und kann gegebenenfalls zusätzliche Maßnahmen einleiten. Mit dem Fortschreiten der Leistungserstellung findet auch eine zunehmende Verdichtung der Datenlage statt, indem die zahlreichen Annahmen der Wirkungsprognose schrittweise durch die tatsächlich realisierten Werte des Vollzugsprozesses ersetzt werden können.

Die wesentliche Zielsetzung des Wirkungsmonitoring besteht in der konsequenten Nutzung vorhandener Handlungsspielräume der Verwaltung bei der Erzielung positiver Wirkungen in der Gesellschaft. Auch hier spielt das Bewusstsein der Mitarbeiter zur 
aktiven Wirkungssteuerung während des administrativen Vollzugsprozesses eine wichtige Rolle. Gleichzeitig hat das Controlling die Aufgabe, anhand der vorab definierten Zielsetzungen, die Umsetzung und Ausführung der Maßnahme zu überwachen und Abweichungen zu berichten.

\subsection{Wirksamkeitsanalyse als ex-post-Analyse}

Die Aufgabe der Wirksamkeitsanalyse besteht schließlich in der eigentlichen operativen Erfolgskontrolle nach erfolgter Produkterstellung (ex-post). Inhaltlich wird eine möglichst umfassende Kenntnis der tatsächlich ausgelösten Wirkungen angestrebt sowie das Verständnis der zugrunde liegenden Wirkungszusammenhänge. Die Bewertung der einzelnen Auswirkungen schafft die Voraussetzung zur Beurteilung der Wirksamkeit der betrachteten Maßnahme und bildet damit den Schlusspunkt des maßnahmenbezogenen Controllingprozesses.

Zur Identifikation und Bewertung der einzelnen Auswirkungen bedient sich das Controlling wiederum der in der Konzeptionsphase erstellten Analysesystematik. Innerhalb des Wirkungsgefüges werden die durch die staatliche Maßnahme ausgelösten Veränderungen in der Gesellschaft dokumentiert. Problematisch für die Durchführung einer Wirksamkeitsanalyse sind allerdings auftretende Wirkungsverzögerungen zwischen staatlicher Leistungserstellung und Wirkungseintritt. Diese erschweren eine zeitnahe Identifikation der tatsächlichen Auswirkungen. Hier wird das Controlling auch weiterhin mit sachgerechten Annahmen operieren müssen. Die endgültige Beurteilung der Maßnahmenwirksamkeit erfolgt dann auf Grundlage der zugeordneten Bewertungsansätze. Weiterhin können in Bezug auf die im Rahmen der Wirkungsprognose gesetzten Wirkungszielsetzungen umfangreiche Abweichungsanalysen vorgenommen werden.

Die wesentliche Zielsetzung der Wirksamkeitsanalyse besteht darin, umfassende Transparenz über die gesamtgesellschaftlichen Auswirkungen der betrachteten staatlichen Maßnahme zu gewinnen. Die Bewertung der realisierten Wirkungsbeiträge erzeugt Objektivität über die tatsächliche Wirksamkeit der Maßnahme. Das Controlling schafft damit die Voraussetzung, im Rahmen wirkungsorientierter Steuerungsprozesse Verantwortung zuzuweisen. Gleichzeitig dienen entsprechende Informationen auch der Legitimation gegenüber dem Bürger, indem ein 'Gegenwert' für die öffentlich eingesetzten Mittel dokumentiert wird.

Mit den Erkenntnissen der Wirksamkeitsanalyse schließt sich der methodische Controllingkreislauf der Wirkungsanalyse. Die 'gelernten Wirkungszusammenhänge der Realität' durchlaufen in einer Anpassungsphase eine Rückkopplungsschleife zur verwendeten Analysesystematik. 


\subsubsection{Anpassungsphase: Initiierung kontinuierlicher Lernschleifen}

Durch die Initiierung kontinuierlicher Lernschleifen aus der operativen Anwendung entsteht bei der Wirkungsanalyse ein geschlossener Controllingkreislauf. Die im Rahmen der Wirkungsrechnung gesammelten Erfahrungen über tatsächlich eingetretene Wirkungen sowie das zunehmende Verständnis der Wirkungszusammenhänge erfordern eine ständige Rückkopplung dieser Erkenntnisse zur Konzeptionsphase. Hierdurch kann eine laufende Anpassung der verwendeten Analysesystematik erfolgen, mit dem Ziel, durch den Erkenntnis- und Lernfortschritt aller beteiligten Akteure eine schrittweise Verbesserung der verwendeten Analyse- und Bewertungsschemata zu erreichen.

Übersetzt in einen Forschungskontext handelt es sich bei dem aufgebauten Wirkungsgefüge um ein spezifisches Wirkungsmodell für den betrachteten staatlichen Handlungsbereich. Die dort postulierten Zusammenhänge zwischen der öffentlichen Maßnahme und den ausgelösten Wirkungen in der Gesellschaft stellen aber lediglich potenzielle Zusammenhänge dar. ${ }^{752}$ Die im Wirkungsgefüge formulierten Hypothesen zu potenziellen Auswirkungen und die Eignung der zugeordneten Bewertungsansätze müssen sich daher durch eine empirische Überprüfung schrittweise an der Realität bewähren. Entsprechend der vorgestellten Vorgehenssystematik der Wirkungsanalyse erfolgt diese empirische Überprüfung im Rahmen der Anwendungsphase.

Innerhalb der Anwendungsphase werden die Annahmen des Wirkungsmodells durch die Wirkungsrechnung fortlaufend der Realität ausgesetzt, indem eine Vielzahl praktischer Anwendungsfälle als staatliche Produkte die Analysesystematik durchlaufen. Unter Umständen ergeben sich für das Controlling hieraus Hinweise, dass vermutete kausale Zusammenhänge empirisch nicht nachvollziehbar sind. Weiterhin besteht in der Gesellschaft ein stetiger Wandel der Umweltbedingungen. Diese rufen wiederum Änderungen in den Wirkungszusammenhängen zwischen der öffentlichen Maßnahme und der Gesellschaft hervor, so dass getroffene Annahmen nicht mehr gültig sind. $\mathrm{Zu}$ dem zeigt sich die generelle Angemessenheit und Eignung der verwendeten Bewertungsansätze und Wirkungsindikatoren. Die Aufgabe der Anwendungsphase besteht in einer systematischen Sammlung und Auswertung dieser 'gelernten Wirkungszusammenhänge der Realität' und in einer kontinuierlichen Rückkopplung dieser Verbesserungshinweise zur Konzeptionsphase.

Auf diese Weise erfolgt durch den geschlossenen Controllingkreislauf der Wirkungsanalyse eine schrittweise Validierung der verwendeten Analyse- und Bewertungsschemata. Gleichzeitig verbessert sich auch die Verlässlichkeit und damit die Qualität der gewonnenen Ergebnisse. Eine Wirkungsanalyse soll vor allem eine sorgfältige Analyse und Bewertung staatlichen Handelns sein. ${ }^{753}$ Daher muss die Wirkungsanalyse bestimmten Qualitätsansprüchen, wie beispielsweise einer systematischen Datenerhebung

\footnotetext{
752 Zur Analyse von Ursache-Wirkungszusammenhängen aus wissenstheoretischer Perspektive vgl. auch Kapitel 4.1.1.2 dieser Arbeit.

753 Zu dieser Aussage in Bezug auf die Evaluation vgl. auch Vedung (1999), S. $11 \mathrm{f}$.
} 
und der gewissenhaften Anwendung von Bewertungsansätzen, gerecht werden. Die schrittweise Verbesserung der Analysesystematik in der Anpassungsphase sollte sich dazu an Qualitätskriterien orientieren. In Anlehnung an die Genauigkeitsstandards der Evaluationsforschung werden im Folgenden wichtige allgemeine Qualitätskriterien einer Wirkungsanalyse aufgeführt: ${ }^{754}$

- Einnahme einer unparteiischen Analyseperspektive, die mögliche Wirkungen objektiv und neutral beurteilt. Dementsprechend sind Vorkehrungen gegen den Einfluss persönlicher Gefühle und Vorlieben zu treffen.

- Angemessene Beachtung des Analysekontext, damit äußere Einflussfaktoren identifiziert werden können.

- Die Verwendung verlässlicher Informationsquellen, so dass die Angemessenheit der genutzten Informationen und Daten eingeschätzt werden kann.

- Die Verwendung valider Informationen, damit die Gültigkeit der gewonnenen Daten in Bezug auf die staatliche Maßnahme sichergestellt ist.

- Die Verwendung reliabler Informationen, so dass die Zuverlässigkeit der gewonnenen Daten in Bezug auf die staatliche Maßnahme sichergestellt ist.

- Die gesammelten quantitativen und qualitativen Informationen einer systematischen Analyse und Überprüfung unterziehen, um gefundene Fehler korrigieren zu können.

- Begründete Schlussfolgerungen und Interpretation der aufbereiteten Informationen, die getroffene Annahmen nachvollziehbar darstellt.

- Verwendung verlässlicher Bewertungsansätze zur Operationalisierung. ${ }^{755}$

- Wahl eines angemessenen Diskontierungssatzes bei der monetären Bewertung von Wirkungsbeiträgen.

Bei den vorgestellten Qualitätskriterien handelt es sich um einen eher allgemein formulierten Katalog wichtiger Hinweise. Dieser zeigt auf, worauf bei der Erarbeitung und Überprüfung der grundlegenden Analyse- und Bewertungsschemata einer Wirkungsanalyse zu achten ist. Die Qualität einer Wirkungsanalyse lässt sich in der Regel nicht anhand klar definierter Kriterien beurteilen, sondern erfordert immer eine intensive Begutachtung des speziellen Anwendungsfalles und der dort verwendeten Methodik. Um dennoch von Zeit zu Zeit eine intensive Qualitätskontrolle der verwendeten Analyse- und Bewertungssystematik vorzunehmen, kann an dieser Stelle auf das Instrumentarium der externen Evaluation zurückgegriffen werden. In Form einer eher wissenschaftlich geprägten Untersuchung werden die getroffenen Annahmen zum Wirkungsmodell durch externe Institute überprüft. ${ }^{756}$ Nachteil ist der hohe Kosten- und Zeitaufwand für derartige Untersuchungen, weshalb sie sich nur zur Ergänzung der Wirkungsanalyse und des hiermit verbundenen Controllingprozesses eignen.

\footnotetext{
754 Vgl. hierzu DeGEval (2002) sowie Widmer (2004), S. 96 ff.

$755 \mathrm{Vgl}$. hierzu auch die gestellten Anforderungen an Wirkungsindikatoren in Kapitel 4.3.2.2.4.

756 Zur Methodik der Evaluation vgl. Kapitel 3.4.
} 
Ein weiterer nicht zu unterschätzender Faktor der Qualitätskontrolle ist die angemessene Kommunikation der Analyseergebnisse. Einerseits erfordert dies ein sachgerechtes Berichtswesen im politisch-administrativen System, welches über die Grenzen der leistungsverantwortlichen Verwaltungseinheit hinausgeht. Zudem kann auch eine periodische Veröffentlichung der Ergebnisse erfolgen, beispielsweise in Form eines Jahresberichtes. Auf diese Weise erhalten die Leistungsempfänger, interessierte Bürger, Experten und Fachleute oder auch Kritiker des Verwaltungsbereiches, Gelegenheit, sich mit den Ergebnissen der Wirkungsanalyse auseinanderzusetzen. In diesem Zusammenhang ist davon auszugehen, dass die in den Berichten beschriebenen Auswirkungen konkret benannter öffentlicher Maßnahmen sowie die dazu getroffenen Annahmen und Bewertungsansätze kritisch hinterfragt werden. Eine unrealistische Darstellung oder Überschätzung der erreichten Wirkungen würde dann eher Unverständnis hervorrufen und eben nicht zur Legitimierung staatlichen Handelns beitragen.

Im nachfolgenden Kapitel widmen sich die Ausführungen schließlich der Institutionalisierung der Wirkungsanalyse innerhalb der bestehenden Controllingsysteme. Hierbei sind insbesondere die wesentlichen institutionellen Ebenen des Verwaltungscontrollings im politisch-administrativen System zu unterscheiden. In diesem Kontext spielen die speziellen Informationserfordernisse der beteiligten Akteure und entsprechend ausgestaltete Berichtswege eine besondere Rolle.

\subsection{Institutionelle Ausgestaltung der Wirkungsanalyse}

\subsubsection{Institutionelle Ebenen des Wirkungscontrollings}

Die institutionelle Ausgestaltung eines wirkungsorientierten Controllings muss sich an den jeweiligen Informationserfordernissen der Entscheidungsträger im politischadministrativen System orientieren. Auf der Ebene der operativen Leistungserstellung durch Leistungsorganisatoren und Leistungserbringer ist das operativ-taktische Wirkungscontrolling zur Sicherstellung eines wirksamen und wirtschaftlichen Verwaltungshandelns angesiedelt. Controlling bezieht sich im politisch-administrativen System zusätzlich auf die Exekutive und die Legislative. Der Führungszyklus beinhaltet hier allerdings eine komplexe Struktur, was insbesondere die Beachtung einer speziellen politischen Rationalität erforderlich macht. ${ }^{757}$ Hieraus ergibt sich die Notwendigkeit zur Abgrenzung eines strategischen Controllings, welches eher der administrativen Regierungsebene zuzuordnen ist und eines politischen Controllings, welches eher der politischen Ebene zuzuordnen ist (vgl. Abbildung 49). ${ }^{758}$

Das operativ-taktische Wirkungscontrolling ist damit eindeutig auf der administrativen Ebene angesiedelt. Es stellt das Bindeglied zwischen der Verwaltungsführung und den ausführenden Verwaltungseinheiten sowie den für die Leistungserstellung zuständigen

757 Zur Ausgestaltung der Übersetzungsfunktion zwischen politischer und administrativer Rationalität vgl. ausführlich Schedler/Proeller (2003), S. $54 \mathrm{ff}$.

$758 \mathrm{Zu}$ den Grundlagen des Controllings staatlichen Handelns vgl. Kapitel 3.2. 
Mitarbeitern dar. Auch das strategische Wirkungscontrolling ist der administrativen Ebene zuzuordnen und zwar als Bindeglied zwischen dem als Leistungsorganisator tätigen Verwaltungsmanagement und den regierungsseitigen Leistungsdefinierern. Dagegen richtet sich das politische Wirkungscontrolling in erster Linie an die politische Ebene der Legislative, bis hin zum Bürger und stellt wiederum das Bindeglied zur Exekutive dar. Die Übergänge zwischen den Controllingebenen sind inhaltlich nicht trennscharf, sondern verlaufen in der Regel fließend.

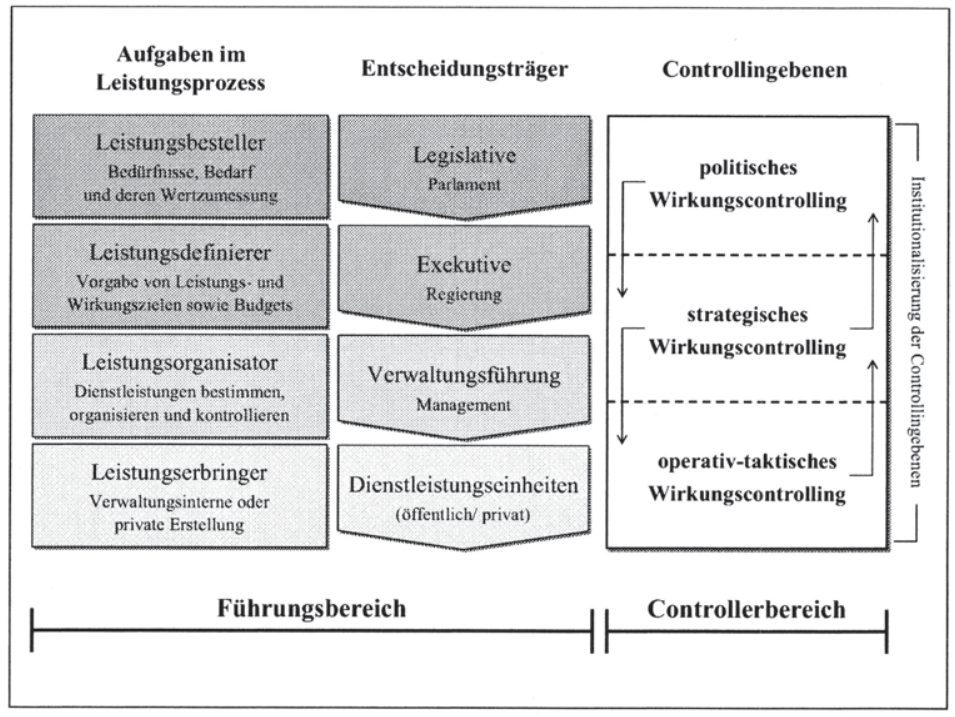

Abbildung 49: Ebenen des Wirkungscontrolling

Strukturell würde sich damit idealtypisch ein dreistufiger Aufbau des Wirkungscontrollings ergeben. Das operativ-taktische Wirkungscontrolling wäre auf der Ebene der ausführenden Verwaltungseinheiten angesiedelt, beispielsweise als Stabstelle der Behördenleitung. Das strategische Wirkungscontrolling wäre dagegen auf der Ebene der übergeordneten Oberbehörden und/ oder Ministerien zu finden, beispielsweise in den jeweils zuständigen Fachreferaten. ${ }^{79}$ Das politische Wirkungscontrolling sollte dementsprechend in Form eines zentralen Steuerungsdienstes auf Regierungsebene ausgebildet sein, wobei die Hauptaufgabe in der wirkungsbezogenen Koordination der vielfältigen Teilbereiche besteht. ${ }^{760}$ Grundsätzlich sollten beim strukturellen Aufbau eines Wirkungscontrollings aufwändige Parallelorganisationen möglichst vermieden werden. Die anfallenden Aufgaben sind vielmehr in die bestehenden Strukturen des

\footnotetext{
759 Die konkrete Ausgestaltung der Controllingebenen ist immer von der Größe und Struktur des betrachteten Verwaltungsbereiches abhängig.

760 Vgl. Egli/Käch (1995), S. 177 und Schedler/Proeller (2003), S. 86.
} 
Verwaltungscontrollings einzugliedern. Das wirkungsorientierte Controlling unterstützt auf diese Weise alle Entscheidungsträger des öffentlichen Leistungsprozesses und koordiniert die im Sinne des NPM verstärkt dezentral arbeitenden Verwaltungsbereiche. Das gesamte Controlling-System ist als ein vernetzter Steuerungskreislauf aufgebaut. Im Zusammenspiel von mehr oder weniger konkretisierten Zielsetzungen zur Planung und Kontrolle (top down) und einem sachgerechten Berichtswesen zur Dokumentation und Information (bottom up) nimmt die Controllingfunktion eine an Steuerungsinteressen orientierte Maklerinstanz ein, um die gegensätzlichen Interessen der Führungsinstanzen miteinander zu verbinden. ${ }^{761}$

Versteht sich Controlling als Führungsunterstützung jeder Verantwortungsebene des politisch-administrativen Systems, ist die inhaltliche Ausgestaltung der Controllingebenen vom spezifischen Informations- und Koordinationsinteresse der jeweiligen Entscheidungsträger abhängig. ${ }^{762}$ Hierdurch ergeben sich differenzierte Berichtsebenen mit unterschiedlichen Verdichtungsgraden und unterschiedlichen Berichtsperioden. Die notwendige und beherrschbare Dichte wirkungsorientierter Informationen wird auf der jeweils höheren Ebene abnehmen, da der Umfang an betrachteten Verwaltungseinheiten und öffentlichen Leistungen zunimmt. Hierauf muss sich das wirkungsorientierte Verwaltungscontrolling bei der Ausgestaltung und Aufbereitung von Wirkungsdaten einstellen. Zur inhaltlichen Ausgestaltung der Controllingebenen hinsichtlich der Anwendung und Nutzung von Wirkungsanalysen geben die nachfolgenden Ausführungen einen Überblick.

\subsubsection{Inhaltliche Ausgestaltung der Controllingebenen}

\subsubsection{Operativ-taktisches Wirkungscontrolling}

Das operativ-taktische Wirkungscontrolling ist eher kurzfristig orientiert und beantwortet primär die Frage, ob ,die Dinge richtig getan“ werden. ${ }^{763}$ Es hat die Aufgabe, die Auswirkungen des staatlichen Handelns zu analysieren. Dazu ist der Aufbau einer systematischen Wirkungsrechnung notwendig, welche die Wirkungen der Verwaltungsprodukte untersucht. ${ }^{764}$ Auch wenn Wirkungsinformationen insbesondere für strategische Entscheidungen eine Rolle spielen, sind Aufbau, Pflege und Betrieb der Wirkungsrechnung eher dem operativ-taktischen Wirkungscontrolling zuzurechnen.

Die Anwendung der Wirkungsrechnung erfolgt in enger Abstimmung zwischen dem Controlling und den für die Leistungserstellung zuständigen Mitarbeitern. Auf Grundlage der in der Konzeptionsphase erstellten Analyse- und Bewertungsschemata ist es die Aufgabe des operativen Controllings, die Auswirkungen der Verwaltungstätigkeit systematisch zu erfassen und zu bewerten. Sind die zurechenbaren Auswirkungen der

\footnotetext{
761 Zur Controllingfunktion als Maklerinstanz vgl. Brüggemeier (1998), S. 289 ff.

762 Vgl. Pook/Tebbe (2002), S. 43 f.

763 Vgl. Brüggemeier (1998), S. 97.

764 Vgl. hierzu Kapitel 4.3.3.
} 
Verwaltungsleistungen durch die Wirkungsrechnung dokumentiert, sollte das wirkungsorientierte Controlling diese Informationen für das interne und externe Berichtswesen nutzen. Die Daten zu den gesellschaftlichen Auswirkungen des eigenen Handelns müssen entsprechend den spezifischen Informationsbedürfnissen der Entscheidungsträger aufbereitet werden. Gleichzeitig sind die Daten auch für die internen Steuerungssysteme nutzbar, beispielsweise durch die Bildung von speziellen Wirkungskennzahlen oder durch die Integration in eine Balanced Scorecard. ${ }^{765}$

Objektivere Informationen über Wirkungen schaffen Transparenz und damit die Voraussetzungen für die Zuweisung von Verantwortung. Die ausführende Verwaltungsebene hat beim Vollzug ihrer Aufgaben sowie bei der Konkretisierung der politischen und gesetzlichen Vorgaben regelmäßig einen nicht unerheblichen Gestaltungsspielraum. ${ }^{766}$ Das operativ-taktische Wirkungscontrolling schafft die Voraussetzungen, dass die Verwaltung sich nicht nur der Wirkungen ihrer erstellten Produkte bewusst ist, sondern auch steuernd auf die Wirksamkeit ihres Handelns Einfluss nimmt. In diesem Zusammenhang ist auch eine Steigerung der Motivation der zuständigen Mitarbeiter zu erwarten. Mit wirkungsorientierten Steuerungsgrößen kann oftmals viel besser an deren Selbstverständnis appelliert werden, als dies mit rein output- oder inputorientierten Steuerungsgrößen möglich ist. ${ }^{767}$

Neben dem internen Steuerungsnutzen des betrachteten Verwaltungsbereiches werden die Wirkungsinformationen durch das Berichtswesen auch den übergeordneten Führungsebenen zugänglich gemacht. Hier finden die Daten Eingang in das strategische Wirkungscontrolling, wo sie entsprechend den Informationsbedürfnissen dieser Steuerungsebene stärker verdichtet werden. Die Schnittstellen zwischen dem operativtaktischen und dem strategischen Controlling verlaufen hier fließend. So ist insbesondere beim Aufbau bzw. bei der kontinuierlichen Anpassung der Analyse- und Bewertungsschemata in der Konzeptions- und Anpassungsphase eine intensive Zusammenarbeit der Controllingebenen vorzusehen. Der intensive Austausch über die gesamtgesellschaftlichen Auswirkungen und die grundlegenden Wirkungszusammenhänge soll auf allen Führungsebenen ein tiefes Verständnis der Kausalzusammenhänge staatlichen Handelns ermöglichen.

\subsubsection{Strategisches Wirkungscontrolling}

Das strategische Controlling verfolgt grundsätzlich einen längeren Zeithorizont und betrifft primär die Beantwortung der Frage, ob ,die richtigen Dinge getan“ werden. ${ }^{768}$ Die Aufgabe des strategischen Wirkungscontrollings besteht vor allem darin, die Wirk-

${ }^{765}$ Zur Balanced Scorecard im Allgemeinen vgl. Kaplan/Norton (1996). Zum Einsatz der Balanced Scorecard im Rahmen einer wirkungsorientierten Controlling-Konzeption vgl. Mosiek et al. (2003), S. 34 und Röhrig (2008), S. $180 \mathrm{ff}$.

766 Vgl. Mosiek et al. (2003), S. 29.

${ }^{767} \mathrm{Vgl}$. Brüggemeier (2004), S. 388.

768 Vgl. Budäus (2002b), S. 391. 
samkeit des staatlichen Mitteleinsatzes sicherzustellen, wenn den mit der operativen Leistungserstellung beauftragten Verwaltungseinheiten die Budgets zugeteilt werden. Das strategische Wirkungscontrolling fungiert damit als wichtiges Bindeglied zwischen der Regierung als Leistungsdefinierer und dem Management der operativ tätigen Verwaltungseinheiten als Leistungsorganisatoren. In der Outcome-Steuerung erfolgt die Verknüpfung der Zieldimension mit den dafür einzusetzenden Ressourcen über Wirkungen. Im Rahmen eines integrierten Budgetierungssystems werden die regierungsseitigen Wirkungsziele an die Finanzziele gekoppelt. ${ }^{769}$

Die Aufgabe des strategischen Wirkungscontrollings besteht insbesondere in einer Bündelungsfunktion. Die verdichteten Wirkungsinformationen der nachgeordneten Verwaltungseinheiten bzw. des operativ-taktischen Wirkungscontrollings zur Produkterstellung werden auf der Regierungsebene gesammelt und ausgewertet. Einerseits unterstützen die produktbezogenen Wirkungsinformationen die Leistungsdefinierer dabei, Wirkungszielsetzung zukünftig weitestgehend anhand operationaler Kriterien festlegen zu können. Andererseits sind Wirkungen häufig politikfeldübergreifend adressiert. Hier fördert das strategische Wirkungscontrolling ein besseres Verständnis der Wirkungszusammenhänge sowie die horizontale Koordination und Kooperation unterschiedlicher Handlungsfelder der Regierung. Dergestalt können beispielsweise Wirkungskoalitionen zwischen unterschiedlichen Ministerien geschlossen werden, um die angestrebten Outcomes besser zu erreichen bzw. die vielfach zu beobachtenden Mitnahmeeffekte zu vermeiden. ${ }^{770}$

Hier durchbricht das strategische Wirkungscontrolling wiederum die Grenze zu einem politischen Wirkungscontrolling. Die Abstimmung unterschiedlicher politischer Handlungsfelder zur Sicherstellung eines wirksamen Ressourceneinsatzes und die entsprechende Berücksichtigung in den Haushaltsansätzen ist vor allem eine politische Aufgabe. Die Politik trägt die Hauptverantwortung für das erreichen der gesellschaftlichen Wirkungsziele und wird hierbei vom politischen Wirkungscontrolling unterstützt.

\subsubsection{Politisches Wirkungscontrolling}

Das politische Wirkungscontrolling muss die Planung politischer Ziele unterstützen, hierfür hat es Informationen bereitzustellen. Bei der wirkungsorientierten Gesamtplanung des Haushaltes muss sich das Controlling insbesondere an die Legislative wenden. In einer Demokratie liegt das Budgetrecht bei der Legislative, nur politisch gewählte Vertreter dürfen über die Verwendung der Steuergelder im Haushalt entscheiden. ${ }^{771}$ Der idealtypische wirkungsorientierte Steuerungsprozess sieht vor, dass die politischen Entscheidungsträger der Verwaltung nur noch Wirkungsziele vorgeben und die Erreichung dieser Ziele mit der Zuweisung eines Globalbudgets verknüpfen. Dies würde zunächst einen Machtverlust für die Politik bedeuten, da sie auf eine inputorien-

\footnotetext{
769 Vgl. hierzu auch die vorgestellte Konzeption eines Controlling-Systems in Kapitel 3.2.

770 Vgl. auch Brüggemeier (2004), S. 381.

771 Vgl. Budäus (2002b), S. 392.
} 
tierte Detailsteuerung verzichtet. Dieser Machtverlust ließe sich aber durch das politische Wirkungscontrolling ausgleichen, da das Verwaltungshandeln „kontrollierbarer“ wird. ${ }^{772}$

Doch diese idealtypische Rollenverteilung zwischen politischer und administrativer Ebene wird aufgrund der unterschiedlichen Problemstellungen im Zielbildungsprozess in der Realität meist nicht gelingen. ${ }^{733}$ Zum einen widerspricht es der politischen Rationalität der Verwaltung konkret messbare Wirkungsziele vorzugeben. Zum anderen besitzt die öffentliche Verwaltung auch eine ,informatorische Machtposition“" gegenüber der Politik, die in der relativen Nähe zu den Leistungsempfängern begründet liegt. Vielfach kann die administrative Ebene viel besser beurteilen, welche Wirkungen durch staatliches Handeln in der Gesellschaft ausgelöst wurden.

Das Controlling muss sich in Form eines politischen Wirkungscontrollings dennoch der politischen Ebene zuwenden. Mittels eines durchgängig gestalteten Berichtswesens werden die Wirkungsinformationen der operativen Verwaltungsebene im jeweils gewünschten Verdichtungsgrad den (Fach-)Politikern zugänglich gemacht. Die Verwaltung hat die Möglichkeit, die gesellschaftliche Wertschöpfung ihres Handelns zu dokumentieren. ${ }^{74}$ Das politische Wirkungscontrolling betreibt in dieser Form zunächst hauptsächlich analytische Politikberatung mit Wirkungsinformationen. Damit bietet es zunächst nur einen groben Rahmen für den politischen Zielbildungsprozess. Gleichwohl bleibt weiterhin erfolgskritisch, dass die bereitgestellten Wirkungsinformationen in den politischen Entscheidungsprozessen auch angemessen Berücksichtigung finden.

\subsubsection{Integration von Ressourcen- und Wirkungsanalyse durch eine Wert- schöpfungsbilanz}

Die Integration von Ressourcen- und Wirkungsbetrachtung eröffnet dem politischadministrativen System unterschiedliche Optionen, das gesamtgesellschaftliche $\mathrm{Ge}$ meinwohl zu steigern. Durch die Institutionalisierung der Ebenen des Wirkungscontrollings werden die Akteure in die Lage versetzt, ein aktives Wirkungsmanagement zu betreiben und den eigenen Gestaltungsspielraum - in Kenntnis der zuvor analysierten Wirkungszusammenhänge - im Sinne einer gesellschaftlichen Wertschöpfung optimal zu nutzen.

Die Differenz zwischen den gesamtgesellschaftlich ausgelösten Wirkungen und den dafür eingesetzten Vorleistungen wird als gesellschaftliche Wertschöpfung bezeichnet. ${ }^{775}$ Die gesellschaftliche Wertschöpfung kann damit als eine Art Gesamtbeurteilung einer staatlichen Maßnahme und der zugehörigen Prozesse im politisch-administrativen System interpretiert werden. Als Darstellungsform bietet sich eine Gegenü-

\footnotetext{
772 Vgl. Budäus (2002b), S. 392.

$773 \mathrm{Vgl}$. hierzu die zentralen Herausforderungen der Wirkungsorientierung in Kapitel 2.2.3.4.

774 Vgl. Mosiek et al. (2003), S. 28.

775 Vgl. Röhrig (2008), S. 175.
} 
berstellung des Ressourcenverzehrs und der korrespondierenden Auswirkungen in Form einer Bilanz an (vgl. Abbildung 50).

\section{W E R T S C H ÖP F U N G S B I L A N Z}

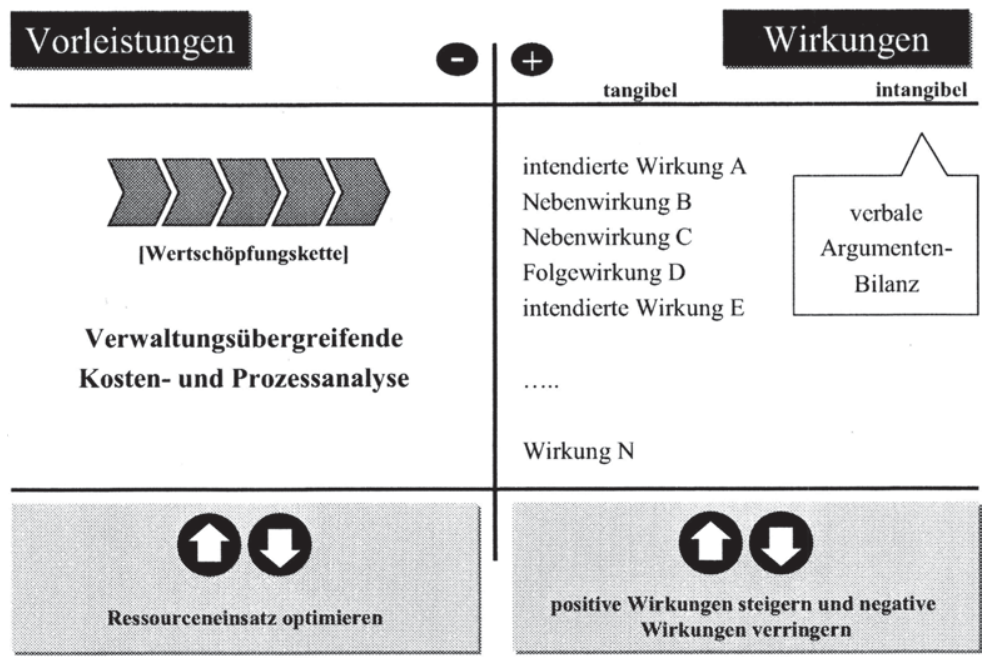

[Nutzung der Gestaltungspotenziale]

Abbildung 50: Gesellschaftliche Wertschöpfungsbilanz ${ }^{776}$

Der Begriff der "gesellschaftlichen Wertschöpfung" erscheint in diesem Kontext angemessen, da eine Verbindung von staatlichem Ressourceninput und den hierdurch ausgelösten Wirkungen hergestellt werden soll. In diesem Zusammenhang ist allerdings wichtig, den Wertschöpfungsbegriff vom klassischen betriebswirtschaftlichen ${ }^{777}$ und volkswirtschaftlichen ${ }^{778}$ Begriffsverständnis zu trennen. Bezogen auf das wirkungsorientierte Controlling staatlichen Handelns errechnet sich der Betrag der Wertschöpfung aus dem Wert, den das politisch-administrative System für die Gesellschaft schafft, abzüglich der Vorleistungen, die in die eigenen Prozesse eingehen. ${ }^{779}$ Es stellt in dieser Weise auch einen geeigneten Maßstab für die Effektivität und Wirksamkeit staatlichen Handelns dar.

\footnotetext{
776 Erweiterte Darstellung in Anlehnung an Mosiek/Gerhardt (2003), S. 293 und Röhrig (2008), S. 177.

777 Im betriebswirtschaftlichen Verständnis errechnet sich die Wertschöpfung aus der Produktionsleistung (zum Marktwert) abzüglich der Vorleistungen für eigene Prozesse. Vgl. Schierenbeck (2003), S. 64 und $620 \mathrm{ff}$.

${ }^{778}$ Im volkswirtschaftlichen Verständnis errechnet sich die Nettowertschöpfung als Summe aller Faktoreinkommen, d.h. aller Löhne und Gehälter, Gewinne der Unternehmen, Mieten und Pachten sowie der Zinsen. Vgl. Hanusch/Kuhn/Cantner (2002), S. 244.

779 Vgl. Mosiek/Pieper/Kasten (2006), S. 16.
} 
So kann beispielsweise für einen staatlichen Produktbereich oder eine bestimmte öffentliche Maßnahmen eine Wertschöpfungsbilanz erstellt werden, indem die bewerteten Vorleistungen monetären und nicht-monetären Wirkungskennziffern gegenübergestellt werden. Auf der linken Seite der Bilanz werden sämtliche Stufen der betrachteten staatlichen Wertschöpfungskette abgebildet. Dazu ist eine verwaltungsübergreifende Kosten- und Prozessanalyse notwendig, um den gesamten staatlichen Ressourceninput zu erfassen. Auf der rechten Bilanzseite werden sämtliche Auswirkungen abgebildet. Die intangiblen Wirkungskomponenten können in Form einer zusätzlichen Argumentenbilanz erfasst werden. Eine Betrachtung im Zeitverlauf erlaubt unter Beachtung der zu Grunde liegenden Wirkungshypothesen eine genaue Studie der Wirkungsrichtungen, ihrer Intensitäten und ihrer Beeinflussbarkeit. ${ }^{780}$

In dieser Weise eröffnet die Integration von Ressourcen- und Wirkungsbetrachtung Gestaltungspotenziale zur Steigerung der gesellschaftlichen Wertschöpfung. Grundsätzlich können die Gestaltungspotenziale sowohl auf der Vorleistungsseite als auch auf der Wirkungsseite der Bilanz ansetzen. Einerseits kann die gesellschaftliche Wertschöpfung durch eine Optimierung des staatlichen Ressourceneinsatzes, z.B. durch Reduktion der Kosten, erhöht werden. Andererseits kann eine Erhöhung der Wertschöpfung durch Ausweitung positiver Wirkungen bzw. Vermeidung negativer Wirkungen erfolgen. Wichtig ist nur, dass sich die beteiligten Akteure ihres jeweiligen Gestaltungsbeitrags bewusst sind.

\subsubsection{Systematisierung der praktischen Implementierungsansätze eines wirkungsorientierten Controllings}

Bei der Einführung und Umsetzung eines wirkungsorientierten Steuerungsansatzes bzw. bei der Implementierung eines wirkungsorientierten Controllings sind unterschiedliche Vorgehensweisen denkbar. Diese Vorgehensweisen sollen im Folgenden anhand von zwei zentralen Kriterien strukturiert und damit in vier grundsätzlich zu unterscheidende Implementierungsansätze der Wirkungsorientierung abgegrenzt werden. In Abbildung 51 ist ein Portfolio der verschiedenen Ansätze dargestellt.

Das erste Abgrenzungskriterium beinhaltet die 'Zuweisung von Verantwortung'. Hiermit ist gemeint, inwieweit die handelnden Akteure des politisch-administrativen Systems für das Erreichen bzw. Verfehlen von Wirkungen oder Wirkungszielen tatsächlich Verantwortung tragen. Die internationalen Beispiele zeigen ${ }^{781}$ dass in einigen Ländern aufgrund der 'relativen Unkontrollierbarkeit' der exogenen Effekte hiervon abgesehen wird. Da der Zielerreichungsgrad einzelner Akteure daher nicht völlig zweifelsfrei ausgewiesen werden kann, werden die Sanktions- und Belohnungssysteme auch nicht mit Wirkungen verknüpft.

\footnotetext{
780 Vgl. Mosiek/Gerhardt (2003), S. 293 f.

781 Vgl. hierzu ausführlich Kapitel 2.2.3.2 und an dieser Stelle insbesondere den »Länderbericht Neuseeland». 
Beim zweiten Abgrenzungskriterium handelt es sich um die 'Schaffung von Transparenz' bezüglich der gesamtgesellschaftlichen Auswirkungen der betrachteten staatlichen Maßnahme. Dieses Kriterium beschreibt, wie umfassend die Identifikation und Bewertung der relevanten Wirkungsbeiträge im Rahmen der Wirkungsanalyse vorgenommen wird. Sind Wirkungen nur unvollständig erfasst sowie hauptsächlich verbal erläutert, ist die Transparenz eher niedrig. Im Gegensatz dazu spricht man von einer hohen Transparenz, wenn die gesellschaftlichen Auswirkungen möglichst vollständig erfasst sind und weitestgehend durch Indikatoren oder monetäre Ansätze bewertet werden.

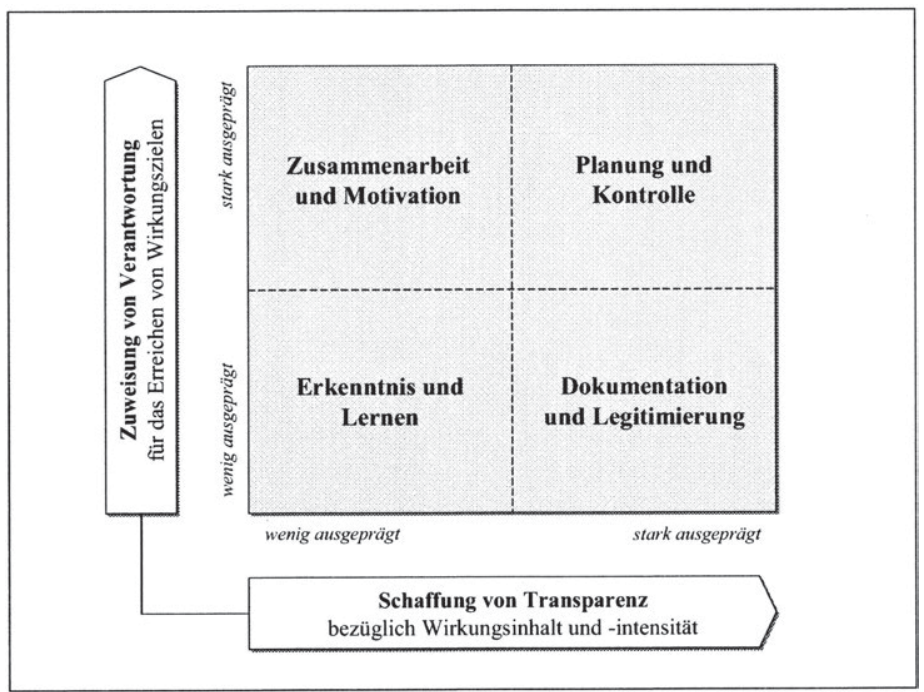

Abbildung 51: Systematisierung möglicher Implementierungsansätze

Beide Abgrenzungskriterien systematisieren die vier unterschiedlichen Ansätze bei der praktischen Umsetzung der Wirkungsorientierung. Die vorgenommene Systematisierung erinnert in dieser Weise auch an die bereits vorgestellten Funktionen der Wirkungsrechnung. ${ }^{782}$ Die praktischen Implementierungsansätze lassen sich dabei wie folgt charakterisieren:

- Der Implementierungsansatz »Erkenntnis und Lernen« weist einen niedrigen Umsetzungsstand bei 'Transparenz' und 'Verantwortung' auf. Vergleichbar einem strategischen Leitbild soll die Wirkungsorientierung durch eine eher weiche Beschreibung wesentlicher Wirkungsfelder des betrachteten Verwaltungsbereiches erreicht werden. Dergestalt handelt es sich zunächst um eine strategische Orientierungshilfe für Verwaltungsführung und Mitarbeiter, mit der Zielsetzung, mehr über die gesellschaftlichen Wirkungen des eigenen Handelns zu erfahren. 
- Der Ansatz »Dokumentation und Legitimierung" weist dagegen einen hohen Umsetzungsstand bei der 'Transparenz', allerdings einen niedrigen Umsetzungsstand bei der 'Zuweisung von Verantwortung', auf. Wichtigstes Ziel der Wirkungsanalyse ist hier der Nachweis der Wirksamkeit öffentlicher Maßnahmen. Die finanziellen Restriktionen der öffentlichen Haushalte setzen die Verwaltung unter Handlungsdruck, die Wirkungen ihres Handelns aufzuzeigen und sich zu rechtfertigen. Im Rahmen einer regelmäßigen Wirksamkeitsanalyse ${ }^{783}$ kann gegenüber politisch Verantwortlichen und dem Bürger der entsprechende „Gegenwert“ für gezahlte Steuern und Abgaben (,value for money") dokumentiert werden - beispielsweise in Form einer Wertschöpfungsbilanz. ${ }^{784}$

- Im genau umgekehrten Fall weist der Ansatz "Zusammenarbeit und Motivation« einen niedrigen Umsetzungsstand bei der 'Transparenz' und einen hohen Umsetzungsstand bei der 'Zuweisung von Verantwortung' auf. Zwar erfolgt die Erfassung und Beschreibung der Wirkungen vielfach noch anhand eher weicher Kriterien, dennoch wird versucht, diese Kriterien in die Abläufe der handelnden Verwaltungsmitarbeiter $\mathrm{zu}$ integrieren. In diesem Zusammenhang wird insbesondere die Steigerung der Motivation der Mitarbeiter betont, da mit wirkungsorientierten Steuerungsgrößen oftmals viel besser das Selbstverständnis der Mitarbeiter angesprochen wird, als dies mit rein produktbezogenen Kennzahlen möglich ist. ${ }^{785}$

- Der Implementierungsansatz »Planung und Kontrolle« weist bei beiden Kriterien einen hohen Umsetzungsstand auf, sowohl bei der 'Transparenz' als auch bei der 'Zuweisung von Verantwortung'. Die ausgelösten gesamtgesellschaftlichen Wirkungen des betrachteten Verwaltungsbereiches werden möglichst vollständig erfasst und weitestgehend durch konkret messbare Wirkungsindikatoren operationalisiert. Dies schafft die notwendigen Voraussetzungen, bereits vor der operativen Leistungserstellung im Rahmen einer Wirkungsprognose konkrete Zielsetzungen und Planwerte für Wirkungen zu erarbeiten. Nach der Produkterstellung erfolgen die Kontrolle der tatsächlich eingetretenen Wirkungen sowie eine Analyse der Soll-IstAbweichungen. Dergestalt können den handelnden Akteuren im politisch-administrativen System klare Verantwortlichkeiten zugewiesen werden. Die Kopplung der Wirkungsziele mit entsprechenden Sanktions- und Belohnungssystemen soll schließlich die Wirksamkeit des staatlichen Handelns und damit des öffentlichen Mitteleinsatzes sicherstellen.

Die Charakterisierung der praktischen Umsetzungsmodelle zeigt, dass in der Regel eine schrittweise Implementierung wirkungsorientierter Steuerungsansätze angemessen erscheint. Bei der Wirkungsorientierung handelt es sich demnach um einen längerfristigen Entwicklungspfad, der im betrachteten Verwaltungsbereich stufenweise Verbreitung finden muss. Bevor man den Mitarbeitern Verantwortung für das Erreichen bestimmter Wirkungen zuweist, müssen die zugrunde liegenden Analyse- und Bewer-

783 Zur inhaltlichen Ausgestaltung der Wirksamkeitsanalyse vgl. Kapitel 4.3.3.3.3 dieser Arbeit.

784 Vgl. hierzu auch Kapitel 4.4.3.

785 Vgl. Hatry (1999), S. 5 und Brüggemeier (2004), S. 388 sowie die Ausführungen des Kapitels 2.2.3.3. 
tungsschemata eine hohe Evidenz aufweisen. Der betrachtete Verwaltungsbereich und das wirkungsorientierte Controlling sollte zunächst umfangreiche Erfahrungen mit den identifizierten Wirkungen sowie den zugrunde liegenden Operationalisierungen und Bewertungsansätzen sammeln. Erst bei hinreichender Bewährung der erarbeiteten Wirkungshypothesen trifft die Wirkungsorientierung auf die notwendige Akzeptanz der Akteure im politisch-administrativen System - sowohl bei den Mitarbeitern und der Verwaltungsführung, als auch bei der Politik und dem interessierten Bürger.

Die nachfolgenden Ausführungen beschäftigen sich ausführlich mit der adressatenbezogenen Systematisierung und Bewertung der Wirkungsbeiträge des staatlichen Flächenmanagements durch Bodenordnung. Die im Rahmen dieser Arbeit theoretisch erarbeiteten Inhalte zum wirkungsorientierten Controlling und zur Wirkungsanalyse staatlichen Handelns werden so in Form einer Fallstudie an einem praktischen Anwendungsfall auf ihre Eignung überprüft. Entsprechend den Zielsetzungen dieser Arbeit wird die Anwendung der Wirkungsanalyse in einem spezifischen staatlichen Leistungsbereich in diesem Zusammenhang als angewandte Forschung verstanden. ${ }^{786}$ 
Thorsten Pieper - 978-3-631-75332-3

Downloaded from PubFactory at 01/11/2019 06:04:26AM

via free access 


\section{Fallstudie: Erstellung einer integrierten Nutzen- und Wirkungs- analyse des staatlichen Flächenmanagements durch Bodenordnung}

Das Kapitel 5 beschäftigt sich ausführlich mit einem Anwendungsbeispiel zum wirkungsorientierten Controlling. Die vorliegende Fallstudie stellt dar, wie auf Grundlage eines konkreten Gesetzes und dem Aufgabenvollzug durch die öffentliche Verwaltung adressatenbezogene Wirkungsbeiträge systematisiert, untersucht und bewertet werden können. Für den staatlichen Aufgabenbereich des Flächenmanagements durch Bodenordnung werden dazu die theoretisch erarbeiteten Inhalte zur Methodik der Nutzenund Wirkungsanalyse in einen realen wirkungsorientierten Controllingprozess überführt, um die ausführende Flurbereinigungsverwaltung wirkungsorientiert zu steuern. ${ }^{787}$

Die nachfolgend beschriebene Fallstudie zur Nutzen- und Wirkungsanalyse des staatlichen Flächenmanagements durch Bodenordnung basiert maßgeblich auf zwei Projekten zur Thematik des wirkungsorientierten Controllings. Beim ersten Projekt handelt es sich um eine gesamtgesellschaftliche Wertschöpfungsanalyse von Bodenordnungsverfahren nach $\$ 87$ FlurbG (Unternehmensflurbereinigung) der Verwaltung für Agrarordnung in Nordrhein-Westfalen. ${ }^{788}$ Das zweite Projekt hat die Entwicklung und Einführung eines Konzeptes zur Wirkungsanalyse und -prognose für Bodenordnungsverfahren in Rheinland-Pfalz zum Gegenstand. ${ }^{789}$

Die Ausführungen dieses Kapitels stellen Auszüge aus den Abschlussberichten und Veröffentlichungen mit den dokumentierten Projektergebnissen dar. Die Projektleitung oblag jeweils dem Unternehmen BMS CONSULTING, die Umsetzung erfolgte in Kooperation mit dem LEHRSTUHL FÜR FINANZWISSENSCHAFT II der Westfälischen WilhelmsUniversität Münster. Im Rahmen der Projektbearbeitung wurde ein interdisziplinärer Ansatz verfolgt, wobei Analysen aus betriebswirtschaftlicher und volkswirtschaftlicher Perspektive vorgenommen wurden. ${ }^{790}$ Der Verfasser dieser Arbeit war in diesem Kontext verantwortlich für die Durchführung der betriebswirtschaftlichen Analysen und die Erstellung der wissenschaftlichen Abschlussdokumentationen.

$\mathrm{Zu}$ den wesentlichen Dienstleistungen im Bereich des staatlichen Flächenmanagements gehört die Durchführung von Bodenordnungsverfahren. Dabei handelt es sich um Verfahren zur Neuordnung und Zusammenlegung ländlichen Grundbesitzes nach dem Flurbereinigungsgesetz, wobei nach dem Gesetz verschiedene Verfahrensarten unterschieden werden. ${ }^{791}$ Flurbereinigungsverfahren zeichnen sich durch eine sehr langfristige und individuelle Abwicklung aus und haben damit den Charakter staatli-

787 Die Begriffe »Bodenordnung« und »Flurbereinigung« werden im Folgenden synonym verwendet.

788 Für die vollständige Dokumentation des Abschlussberichtes vgl. BMS Consulting (2005). Eine komprimierte Darstellung der relevanten Projektergebnisse findet sich auch bei Seyer/Pieper/Kasten/Mosiek (2006) und bei Pieper et al. (2006), S. 246 ff.

789

Für eine vollständige Dokumentation der ersten Projektphase und der bis dahin erzielten Zwischenergebnisse vgl. Mosiek/Pieper/Kasten (2006) sowie in einer komprimierten Darstellung Lorig/Kasten/Mosiek/Pieper (2006), S. $458 \mathrm{ff}$.

$790 \mathrm{Vgl}$. BMS Consulting (2005), S. 32 ff. und Mosiek/Pieper/Kasten (2006), S. 18 ff.

$791 \mathrm{Vgl}$. hierzu das Flurbereinigungsgesetz (FlurbG). 
cher Projekte. Der umfangreiche Adressatenkreis und die außerordentlich hohe Verfahrenskomplexität bzw. -spezifität erschweren bislang eine transparente Darstellung der relevanten Wirkungsbeiträge dieses staatlichen Produktes. Bodenordnungsverfahren sind daher besonders geeignet für die Durchführung einer kombinierten Nutzenund Wirkungsanalyse ${ }^{792}$ Gerade in solchen staatlichen Handlungsbereichen besteht die Notwendigkeit, durch die Etablierung eines systematischen wirkungsorientierten Controllingprozesses, die Wirkungen staatlichen Handelns fortlaufend transparent zu machen.

Die Zielsetzung der Fallstudie besteht darin, die theoretisch hergeleiteten Inhalte dieser Arbeit - insbesondere zur Methodik der Wirkungsanalyse - in einen praktischen Anwendungsfall zu überführen. In diesem Kontext wird besonderes Augenmerk darauf gelegt, inwieweit sich die vorgestellten Vorgehensweisen und Strukturierungshilfen im Praxisfall bewähren. Im Gegensatz zu den eher einzelfallbezogenen Untersuchungen im Bereich der Evaluation soll die Verankerung der Wirkungsanalyse in einem systematischen Controllingprozess gelingen.

Die Fallstudie folgt dabei dem nachfolgenden Aufbau. Kapitel 5.1 erläutert zunächst die Grundlagen des staatlichen Flächenmanagements durch Bodenordnung. Hierbei werden die Ziele und Aufgaben, die gesetzlichen Verfahrensarten und die wesentlichen Ablaufschritte von Bodenordnungsverfahren, vorgestellt. Auf Grundlage des Projektes in Nordrhein-Westfalen beschreibt Kapitel 5.2 anschließend die Konzeption einer Nutzenanalyse zur differenzierten Beurteilung des Leistungsimpact. Die Anwendung der Methodik der Wirkungsanalyse erfolgt auf Grundlage der Projektergebnisse in Rheinland-Pfalz in Kapitel 5.3. Ausführlich wird das gesamtgesellschaftliche Wirkungsgefüge der Bodenordnung hergeleitet, um mit dessen Hilfe die Auswirkungen konkret betrachteter Flurbereinigungsverfahren möglichst umfassend zu identifizieren und zu bewerten. Die Fallstudie endet in Kapitel 5.4 mit der Analyse und Bewertung eines exemplarisch ausgewählten Bodenordnungsverfahrens.

\subsection{Grundlagen des Flächenmanagements durch Bodenordnung}

\subsubsection{Ziele und Aufgaben der ländlichen Bodenordnung}

Die ländliche Bodenordnung gehört zu den wichtigsten Instrumenten zur Neuordnung und Entwicklung des ländlichen Raumes. Ursprünglich zielten bodenordnerische Maßnahmen in erster Linie auf die reine Verbesserung der Agrarstruktur für die Land- und Forstwirtschaft ab. Im Zuge der stetigen Veränderungen der gesellschaftlichen Rahmenbedingungen ländlicher Regionen hat sich in den letzten Jahren auch die gesellschaftliche Bedeutung und damit einhergehend der Aufgabenbereich der Bodenordnung entscheidend gewandelt. ${ }^{793}$ Die zeitgemäße Flurbereinigung versteht sich dem-

\footnotetext{
$792 \mathrm{Vgl}$. hierzu auch Kapitel 4.2.3.

793 Für eine ausführliche und detaillierte Beschreibung der „ländlichen Entwicklung im Wandel der Zeit" vgl. insbesondere Schlosser (1999).
} 
nach vor allem als Instrument des staatlichen Flächenmanagements im ländlichen Raum (vgl. Abbildung 52). ${ }^{794}$

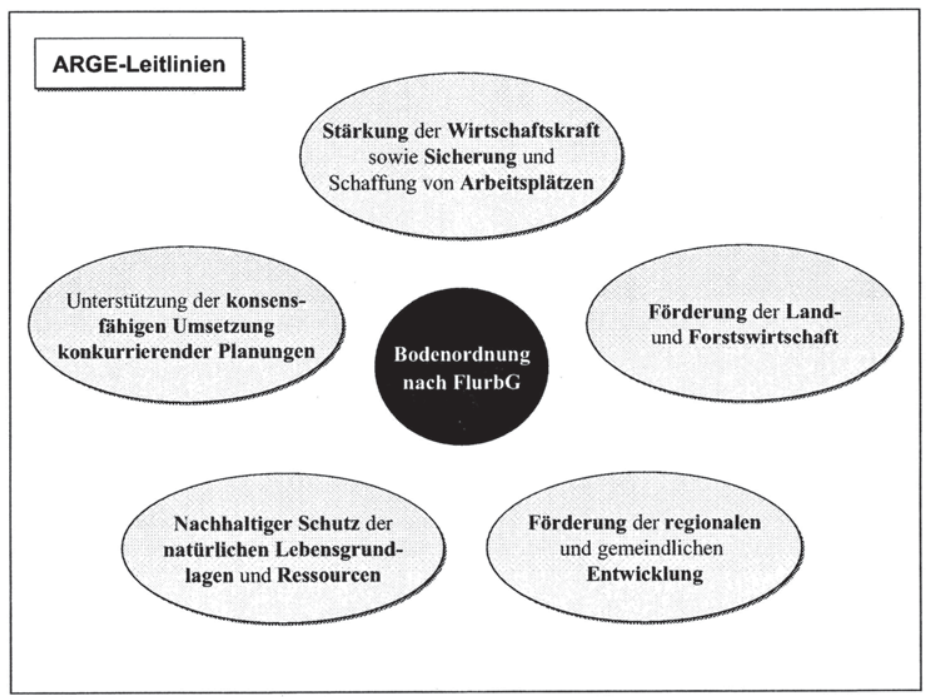

Abbildung 52: Ziele der Bodenordnung als Flächenmanagement

Die Bodenordnung besteht heute aus einem komplexen Maßnahmenbündel, das zunehmend zur integrierten Entwicklung ländlicher Räume eingesetzt wird. Die Aufgaben gehen demnach weit über die traditionelle Zielsetzung der Agrarstrukturverbesserung mit einer aus landwirtschaftlicher Sicht optimalen Flächenzusammenlegung und neuordnung hinaus. Bodenordnerische Maßnahmen zielen heute auf die Förderung und Stärkung ländlicher Gebiete als Ganzes ab. Durch Flächenmanagement werden ländliche Regionen bei ihrer Entwicklung zu wettbewerbsfähigen Lebens- und Wirtschaftsräumen unterstützt. Dazu bedarf es zum einen der Verbesserung der Produktions- und Arbeitsbedingungen, sowohl innerhalb als auch außerhalb der Agrarwirtschaft. Zum anderen gilt es, die natürlichen Lebensgrundlagen und Ressourcen nachhaltig zu schützen und damit eine wichtige Existenzgrundlage der ländlichen Regionen dauerhaft zu sichern. ${ }^{795}$

794 Vgl. hierzu die Leitlinien der ARGE Landentwicklung (2006).

795 Vgl. die Leitlinien der ARGE Landentwicklung (2006). Die Arbeitsgemeinschaft ist durch einen Beschluss der Agrarministerkonferenz vom 5. November 1976 als ArgeFlurb gebildet worden. Sie wurde durch einen Beschluss der Agrarministerkonferenz vom 16.09.1998 auf neue Ziele ausgerichtet und in ARGE Landentwicklung umbenannt. Sie hat vor allem die Aufgabe, grundsätzliche Angelegenheiten im Zusammenhang mit Verfahren nach dem Flurbereinigungsgesetz (FlurbG) und dem 8. Abschnitt des Landwirtschaftsanpassungsgesetzes (LwAnpG) zu erörtern und Empfehlungen für deren Durchführung zu erarbeiten. Mitglieder der Arbeitsgemeinschaft sind das zuständige Bundesministerium sowie Vertreter der jeweils zuständigen Landesministerien. 
Bei der Wahrnehmung dieser Zielsetzungen agiert die Flurbereinigungsbehörde in der Regel jedoch nicht als selbständiger Träger der unterschiedlichen Maßnahmen. Die Aufgabe besteht vielmehr darin, durch geschicktes Flächenmanagement die bestehenden Konflikte zwischen den regional konkurrierenden Fachplanungen und ihrem teilweise erheblichen Flächenbedarf aufzulösen. Beispiels-weise konkurrieren in einer Region häufig die Interessen der Land- und Forstwirtschaft mit den Planungen im Bereich Verkehrsinfrastruktur, der Ausweisung von Naturschutzräumen und den Bedarfen der Wasserwirtschaft. Hier gilt es, die Konflikte mit den betroffenen Eigentümern zu minimieren und gleichzeitig eine möglichst konsensfähige Realisierung der unterschiedlichen Maßnahmen zu gewährleisten. An die Flurbereinigungsbehörde wird in diesem Zusammenhang der Anspruch gestellt, ökonomische, ökologische, soziale und kulturelle Zielsetzungen sinnvoll miteinander in Einklang zu bringen und die zum Teil stark divergierenden Interessen aller Beteiligten an der Flächennutzung zu wahren. ${ }^{796}$

Die Anforderungen an das staatliche Flächenmanagement haben sich somit im Laufe der Zeit an die immer komplexer werdende gesellschaftliche Umwelt angepasst. Das umfangreiche und vielfältige Aufgabenspektrum der ländlichen Bodenordnung lässt sich demnach folgendermaßen charakterisieren:

- Trotz des insgesamt gewandelten Aufgabenfeldes stellt die Verbesserung der Produktions- und Arbeitsbedingungen in der Land-und Fortwirtschaft noch immer eine wichtige Aufgabe der Bodenordnung dar. Durch den Ausbau des land- und forstwirtschaftlichen Wegenetzes und die Neuordnung des Grundbesitzes wird hierzu ein Beitrag geleistet. Ziele sind die Existenzsicherung land- und forstwirtschaftlicher Betriebe und der hiermit verbundenen Arbeitsplätze, der Erhalt der Kulturlandschaft und die Stärkung einer nachhaltigen und ökologischen Flächenbewirtschaftung.

- Flächenintensive Infrastrukturmaßnahmen, wie beispielsweise der Bau überörtlicher Straßen, Eisenbahnstrecken oder Flughäfen haben in der Regel erhebliche Nutzungskonflikte mit den Eigentümern zur Folge. Durch eine begleitende Bodenordnung lassen sich der Flächenentzug und die Durchschneidung der Landschaft meist deutlich abmildern.

- Infrastrukturprojekte haben zusätzlich einen hohen Flächenbedarf für Ausgleichsund Ersatzmaßnahmen für die entstehenden Eingriffe in den Naturhaushalt. Die Bodenordnung kann diese Kompensationsflächen so ausweisen, dass Eigentumskonflikte vermieden werden und gleichzeitig ökologische Belange besser erfüllt werden.

- Die Bedeutung von Maßnahmen des Gewässer- und Hochwasserschutzes hat in den letzten Jahren stark zugenommen. Durch Flächenerwerb und Neuordnung im Rahmen von Bodenordnungsverfahren lassen sich hiermit verbundene Nutzungskonflikte häufig vermeiden. Flurbereinigungsverfahren unterstützen damit die Renaturierung von Gewässern und Gewässerauen, die Schaffung von Uferstreifen, die Verlegung von Deichen oder die Ausweisung von Rückhaltebecken.

796 Vgl. die Leitlinien der ARGE Landentwicklung (2006). 
- Durch Flächenerwerb und Flächentausch kann die Bodenordnung Nutzungskonflikte zwischen Naturschutz und Landschaftspflege und den Interessen der Landwirtschaft auflösen. Gleichzeitig werden auch Maßnahmen des Bodenschutzes durchgeführt. Für die landwirtschaftlich genutzten Flächen steht dabei der Erosionsschutz im Vordergrund.

- Aufgabe der Bodenordnung nach dem Flurbereinigungsgesetz sind auch Maßnahmen der Dorferneuerung. Um den nachteiligen Auswirkungen des Strukturwandels im ländlichen Raum zu begegnen, kann mit Hilfe der Bodenordnung eine bedarfsgerechte Infrastruktur geschaffen werden, welche zum Erhalt der Wirtschaftskraft und der Arbeitsplätze im ländlichen Raum beiträgt. Aufgabe ist hier die Flächenbereitstellung für kommunale Infrastruktureinrichtungen, Gewerbeflächen oder Fremdenverkehrseinrichtungen.

Den Rahmen zur Erfüllung dieses umfangreichen Aufgabenspektrums schafft das FlurbG und die dort verankerten Verfahrensarten der Bodenordnung.

\subsubsection{Gesetzliche Grundlagen und Verfahrensarten}

Im Fokus der weiteren Betrachtung der Fallstudie steht das staatliche Aufgabenfeld der Bodenordnung nach dem Flurbereinigungsgesetz (FlurbG). ${ }^{797}$ Die we-sentlichen, im Flurbereinigungsgesetz unterschiedenen Verfahrenstypen sind die Regelflurbereinigung nach $\S 1$ FlurbG, die Vereinfachte Flurbereinigung nach $\$ 86$ FlurbG, die Unternehmensflurbereinigung nach $\S 87$ FlurbG, die Beschleunigte Zusammenlegung nach \$91 FlurbG sowie der Freiwillige Landtausch nach §103a FlurbG (vgl. Abbildung 53).

\section{REGEL- \\ FLURBEREINIGUNG}

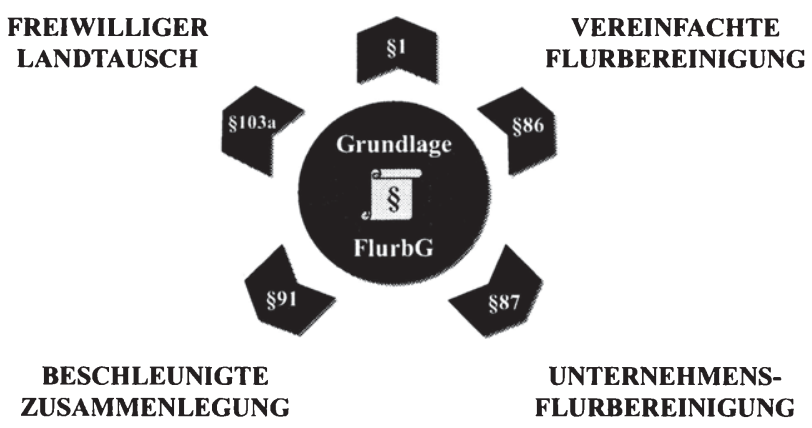

Abbildung 53: Verfahrensarten der Bodenordnung

797 Vgl. das Flurbereinigungsgesetz (FlurbG). 
\$1 FlurbG bestimmt den Begriff und den Hauptzweck der Bodenordnung:

- „Zur Verbesserung der Produktions- und Arbeitsbedingungen in der Land- und Forstwirtschaft sowie zur Förderung der allgemeinen Landeskultur und der Landentwicklung kann ländlicher Grundbesitz durch Maßnahmen nach diesem Gesetz neugeordnet werden (Flurbereinigung).“ 798

Laut Gesetz wird unter Flurbereinigung die Neuordnung ländlichen Grundbesitzes verstanden. In der Regelflurbereinigung zielt diese Neuordnung insbesondere auf die Verbesserung der Produktions- und Arbeitsbedingungen in der Land- und Forstwirtschaft ab. Dabei geht es darum, die Produktivität der land- und forstwirtschaftlichen Betriebe langfristig zu erhöhen sowie ihre Wirtschaftlichkeit nachhaltig zu verbessern, um ihre Wettbewerbsfähigkeit zu stärken und für die Zukunft zu sichern. ${ }^{799}$ Daneben hat die Regelflurbereinigung die Förderung der allgemeinen Landeskultur und Maßnahmen der Landentwicklung zum Ziel.

Demgegenüber sieht die Vereinfachte Flurbereinigung nach $§ 86$ FlurbG Vereinfachungen im Verfahrensablauf vor. Die Durchführung eines vereinfachten Bodenordnungsverfahrens ist jedoch an das Vorliegen bestimmter gesetzlicher Bedingungen geknüpft:

- „Eine vereinfachte Flurbereinigung kann eingeleitet werden, um

1. Maßnahmen der Landentwicklung, insbesondere Maßnahmen der Agrarstrukturverbesserung, der Siedlung, der Dorferneuerung, städtebauliche Maßnahmen, Maßnahmen des Umweltschutzes, der naturnahen Entwicklung von Gewässern, des Naturschutzes und der Landschaftspflege oder der Gestaltung des Orts- und Landschaftsbildes zu ermöglichen oder auszuführen,

2. Nachteile für die allgemeine Landeskultur zu beseitigen, die durch Herstellung, Änderung oder Beseitigung von Infrastrukturanlagen oder durch ähnliche Maßnahmen entstehen oder entstanden sind,

3. Landnutzungskonflikte auslösen oder

4. eine erforderlich gewordene Neuordnung des Grundbesitzes in Weilern, Gemeinden kleineren Umfanges, Gebieten mit Einzelhöfen sowie in bereits flurbereinigten Gemeinden durchzuführen.“" ${ }^{800}$

Die Besonderheiten und Vereinfachungen liegen zum einen in der Ermächtigung der Flurbereinigungsbehörde zur Anordnung des Verfahrens bzw. zur Anordnung von Teilflurbereinigungen. Zum anderen ermöglicht das Gesetz einen Verzicht auf einen förmlichen Planfeststellungsbeschluss. Der Gesetzgeber beabsichtigt mit diesen Regelungen nicht nur eine Erleichterung der Realisation der in $\$ 86$ (1) FlurbG genannten

\footnotetext{
798 § 1 FlurbG.

799 Vgl. Hegele et al. (1992), S. 2.

800 §86 FlurbG.
} 
Planungen, sondern in erster Linie die Vermeidung oder ggf. Beseitigung der aus der Durchführung der Maßnahmen resultierenden Nachteile für die allgemeine Landeskultur. ${ }^{801}$

Eine weitere Sondervorschrift des Flurbereinigungsgesetzes ist in $\S 87$ FlurbG geregelt. Werden ländliche Grundstücke für im öffentlichen Interesse liegende Großbauprojekte, wie beispielsweise Autobahnen, Bundes-, Landes- und Kreisstraßen, Bahntrassen oder ähnliches benötigt, ist zur Unterstützung dieser Bauvorhaben die Einleitung einer Unternehmensflurbereinigung möglich:

- „Ist aus besonderem Anlass eine Enteignung zulässig, durch die ländliche Grundstücke in großem Umfange in Anspruch genommen würden, so kann auf Antrag der Enteignungsbehörde ein Flurbereinigungsverfahren eingeleitet werden, wenn der den Betroffenen entstehende Landverlust auf einen größeren Kreis von Eigentümern verteilt oder Nachteile für die allgemeine Landeskultur, [...], vermieden werden sollen. $[\ldots]^{\text {“ } 802}$

Aus der Gesetzgebung lassen sich unmittelbar die mit der Durchführung einer Unternehmensflurbereinigung verbundenen Voraussetzungen herleiten. Die Einleitung einer Unternehmensflurbereinigung setzt neben der Zulässigkeit einer Enteignung die Möglichkeit voraus, den vermeintlichen Landverlust auf einen größeren Kreis von Eigentümern zu verteilen oder die im Zuge der Realisierung des Bauvorhabens entstehenden landeskulturellen Nachteile zu beseitigen. ${ }^{803}$

Das Beschleunigte Zusammenlegungsverfahren dient der raschen Verbesserung der Produktions- und Arbeitsbedingungen in der Land- und Forstwirtschaft oder der Durchführung notwendiger Maßnahmen des Naturschutzes und der Landschaftspflege ${ }^{804}$ Es kann durchgeführt werden, wenn die Anlage eines neuen Wegenetzes oder sonstige Maßnahmen der ländlichen Entwicklung nicht erforderlich ist. Da im Rahmen des Verfahrens nach Möglichkeit ganze Flurstücke getauscht werden, sieht der Verfahrensablauf Vereinfachungen bei der Wertermittlung und den Vermessungsarbeiten vor. ${ }^{805}$ In ähnlicher Weise handelt es sich auch beim Freiwilligen Landtausch um ein schnelles und einfaches Verfahren zur Verbesserung der Agrarstruktur oder aus Gründen des Naturschutzes und der Landschaftspflege. ${ }^{806}$ Der freiwillige Landtausch setzt voraus, dass sich die Eigentümer über die Tauschbedingungen einig sind und auf dieser Grundlage bei der Flurbereinigungsbehörde den Tausch beantragen. Er ist vor allem dann geeignet, wenn eine begrenzte Besitzzersplitterung behoben werden soll und hierzu keine oder nur geringe Vermessungs- und Folgearbeiten nötig sind.

\footnotetext{
801 Vgl. Hegele et al. (1992), S. 349 f.

$802 \S 87$ FlurbG.

803 Vgl. Hegele et al. (1992), S. 357.

804 Vgl. \$91 FlurbG.

$805 \mathrm{Zu}$ den wesentlichen Ablaufschritten in der Bodenordnung vgl. Kapitel 5.1.4.

806 Vgl. §103a-§103i FlurbG.
} 


\subsubsection{Schematische Gegenüberstellung der Grundstücksverhältnisse}

Die Grundstücksverhältnisse vor und nach einer Bodenordnung lassen sich anschaulich am Beispiel eines Infrastrukturvorhabens verdeutlichen (vgl. Abbildung 54). Wird z.B. ein Straßenbauprojekt ohne begleitende Bodenordnung durchgeführt, durchschneidet die Trassenfläche die bestehenden Grundstücksstrukturen. Der notwendige Landentzug belastet einerseits die in der Trassenfläche befindlichen Eigentümer erheblich, andererseits werden durch die Durchschneidung viele Restparzellen unwirtschaftlich. Hierdurch entstehen nicht selten Existenzgefährdungen in der Landwirtschaft, so dass Konflikte mit den Eigentümern und gerichtliche Auseinandersetzungen die Folge sind, mit den entsprechenden Verzögerungen für das Straßenbauprojekt. ${ }^{807}$

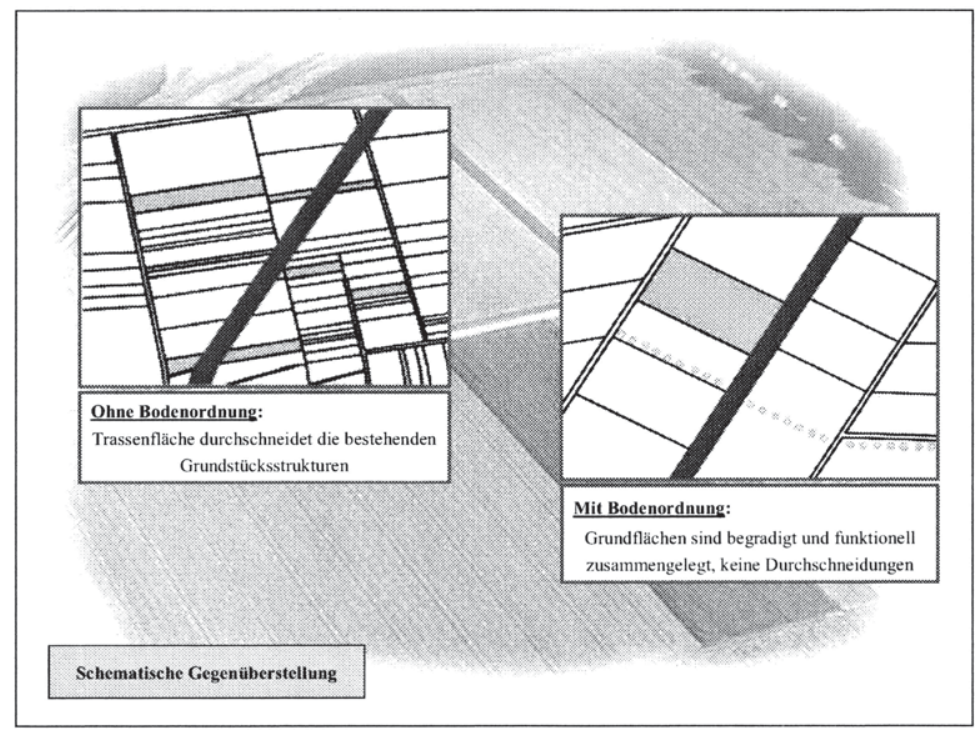

Abbildung 54: Grundstücksverhältnisse vor und nach Bodenordnung

Die Aufgabe eines begleitenden Bodenordnungsverfahrens besteht zunächst darin, den aus dem Projekt entstehenden Landverlust auf einen größeren Kreis von Eigentümern zu verteilen. D. h. der hohe Landverlust der direkt von der Trasse betroffenen Eigentümer wird abgemildert, indem ein geringerer Landentzug bei einer größeren Anzahl von Eigentümern in Trassennähe erfolgt. ${ }^{808}$ Das Verfahrensgebiet der Flurbereinigung ist dementsprechend groß - vergleichbar einem breiten Band um die Trassenfläche. Hier übernimmt die Flurbereinigungsbehörde sämtliche Grundstücke verkaufswilliger Eigentümer und tauscht diese Flächen gezielt in die Trassenfläche. Eigentümer, die

\footnotetext{
807 Vgl. BMS Consulting (2005).

${ }^{808} \mathrm{Vgl}$. beispielsweise $\$ 87$ FlurbG.
} 
sich innerhalb der Trassenfläche befinden und den Gesamtumfang ihres Grundbesitzes erhalten möchten, bekommen so neue Flächen außerhalb der Trasse. Hierdurch lassen sich die bestehenden Nutzungskonflikte häufig deutlich reduzieren und der Träger des Straßenprojektes kommt frühzeitig in den Besitz der Trassenfläche.

Weiterhin erfolgt innerhalb des Verfahrens eine komplette Neuordnung der Eigentumsverhältnisse und eine Zusammenlegung kleinerer Flächen (vgl. hierzu exemplarisch Abbildung 54). Hierdurch können Nachteile für die allgemeine Landeskultur vermieden werden. Die durch die Trasse erfolgte Zerschneidung landschaftlicher Strukturen, landwirtschaftlicher Nutzflächen und des vorhandenen Wegenetzes wird durch die Neuordnung der Flächen wieder weitestgehend geheilt und die Flächennutzung bleibt weiterhin wirtschaftlich. Gleichzeitig werden in Abstimmung mit den Eigentümern Maßnahmen des Naturschutzes und der Landschaftspflege umgesetzt.

\subsubsection{Wesentliche Ablaufschritte eines Bodenordnungsverfahrens}

In Abbildung 55 ist der idealtypische Ablauf eines Bodenordnungsverfahrens nach dem FlurbG mit den wesentlichen Prozessschritten und den entsprechenden Tätigkeitsschwerpunkten der öffentlichen Verwaltung dargestellt. ${ }^{809}$ Zunächst beginnt die Flurbereinigungsbehörde mit allgemeinen Vorarbeiten und Informationsveranstaltungen für die betroffenen Grundstückseigentümer zum anstehenden Verfahren. Durch den Flurbereinigungsbeschluss wird das Verfahren offiziell eingeleitet bzw. angeordnet. Die Grundstückseigentümer wählen anschließend den Vorstand der Teilnehmergemeinschaft, welcher ihre Interessen in der anstehenden Wertermittlung der Grundstücke und in den Planungen zum Ausbau der gemeinschaftlichen Anlagen ${ }^{810}$ vertritt. $^{811}$

Um dem Anspruch aller Teilnehmer auf wertgleiche Abfindung gerecht zu werden, ermittelt die zuständige Flurbereinigungsbehörde die legitimierten Beteiligten und ihre Rechte nach dem Grundbuch. ${ }^{812}$ Weiterhin ist die genaue Feststellung der Grundstücksgrößen nach dem Liegenschaftskataster oder durch Neuvermessung vorzunehmen. ${ }^{813}$ Die Wertermittlung des Bodens erfolgt in der Regel durch Sachverständige und orientiert sich am langfristigen, durchschnittlichen Ertragswert der betroffenen Flächen. ${ }^{814}$

809 Die Durchführung eines Bodenordnungsverfahrens ist von der jeweiligen Verfahrensart abhängig. Die nachfolgenden Ausführungen geben daher nur einen allgemeinen Überblick der sich ähnelnden Vorgehensweisen der Regelflurbereinigung, Vereinfachten Flurbereinigung und Unternehmensflurbereinigung.

Dabei handelt es sich um einen Plan nach $\$ 41$ FlurbG. Im Wesentlichen betrifft dies die Planung eines neuen Wegenetzes und Zuwegungen für landwirtschaftliche Nutzflächen sowie landschaftsgestaltender Anlagen, beispielsweise für Naturschutz und Landschaftspflege, im Verfahrensgebiet.

811 Die Teilnehmergemeinschaft - eine Körperschaft des öffentlichen Rechts - besteht aus allen Eigentümern und Erbbauberechtigten im Flurbereinigungsgebiet. Die Mitglieder der Teilnehmergemeinschaft wählen einen Vorstand, der die Geschäfte führt und die Interessen der Teilnehmer im Bodenordnungsverfahren vertritt. Vgl. §16 bis §18 FlurbG und §21 FlurbG.

812 Vgl. §10 FlurbG.

813 Vgl. \$30 FlurbG.

814 Vgl. §31 FlurbG. 


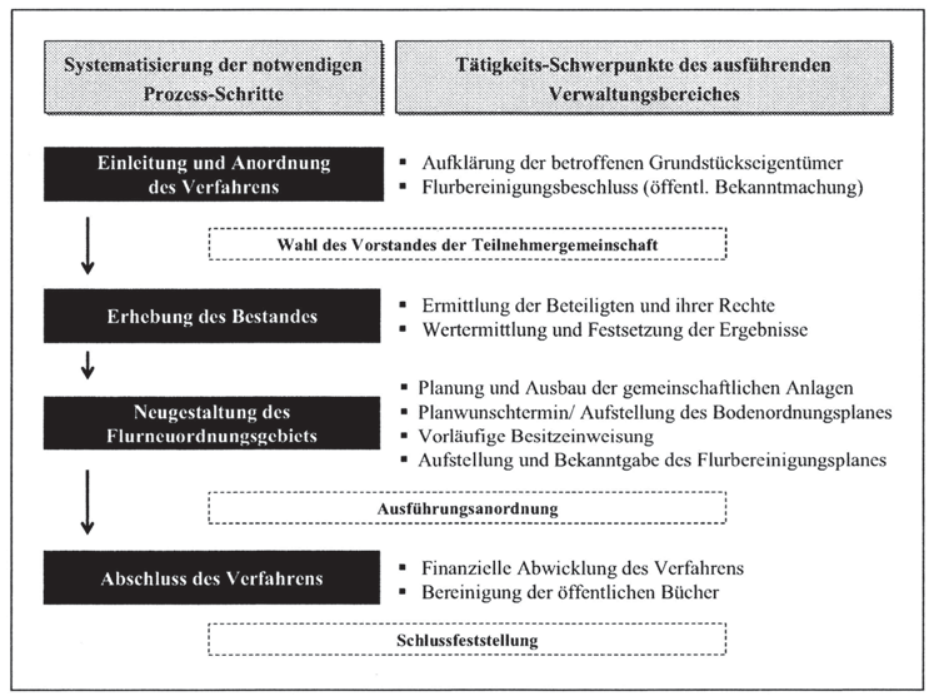

Abbildung 55: Prozessschritte eines Bodenordnungsverfahrens

Auf Basis der eingebrachten Vorschläge der Grundstückseigentümer im Planwunschtermin wird ein Flurbereinigungsplan ${ }^{815}$ zur beabsichtigten Flächenneuordnung bearbeitet. Auf der Grundlage des Ergebnisses des Planwunschtermins erstellt die Flurbereinigungsbehörde einen detaillierten Abfindungsentwurf, aus dem die Landabfindungen der Eigentümer, sowie die verschiedenen Geldausgleiche und sonstigen Festsetzungen hervorgehen ${ }^{816}$ Vor der endgültigen Festsetzung im Flurbereinigungsplan wird diese Rohplanung mit den Beteiligten erörtert und gegebenenfalls noch angepasst. Parallel zur Flächenarrondierung erfolgen die Verbesserung und der Neubau gemeinschaftlicher Anlagen sowie die Flächenbereitstellung für öffentliche Bedarfe, wie zum Beispiel Infrastrukturvorhaben oder kommunale Planungen. ${ }^{817}$

Sind sämtliche Widersprüche oder Klagen der betroffenen Grundeigentümer gegen den Flurbereinigungsplan ausgeräumt, ist der Plan unanfechtbar. Danach ordnet die Flurbereinigungsbehörde im Rahmen der Ausführungsanordnung die Ausführung des Flurbereinigungsplanes an und bestimmt damit den Zeitpunkt, an dem der neue Rechtszustand an die Stelle des bisherigen tritt. Durch eine vorläufige Besitzeinweisung erlangen die neuen Eigentümer allerdings schon frühzeitig die Möglichkeit, Vorhaben in den neu zugeteilten Flächen zu realisieren. ${ }^{818}$

${ }^{815}$ Beim Flurbereinigungsplan handelt es sich um einen Verwaltungsakt der Flurbereinigungsbehörde.

$816 \mathrm{Vgl}$. 444 bis $\$ 55$ FlurbG.

817 Hierbei handelt es sich um Wirtschaftswege, Gewässer und sonstige landschaftsgestaltende Anlagen entsprechend dem Wege- und Gewässerplan nach \$41 FlurbG.

818 Vgl. §65 bis §67 FlurbG. 
Das Bodenordnungsverfahren endet mit der Schlussfeststellung, d.h. der Feststellung, dass der Flurbereinigungsplan ordnungsgemäß ausgeführt wurde und den Beteiligten keine weiteren Ansprüche mehr zustehen. ${ }^{819}$ Zuvor wird das Verfahren allerdings $f i$ nanziell abgewickelt und es werden die im Flurbereinigungsplan zusammengefassten Ergebnisse der Bodenordnung durch die Flurbereinigungsbehörde in die öffentlichen Bücher übernommen.

\subsection{Nutzenanalyse: Impactbetrachtung des staatlichen Flächenmanage- ments durch Bodenordnung}

\subsubsection{Ausgangssituation und Zielsetzung des Projektes}

Im Rahmen des betrachteten Projektes wurde im Auftrag der Bezirksregierung Münster ein wirkungsorientierter Management- und Controllingansatz für Verfahren der Bodenordnung, im speziellen der Unternehmensflurbereinigung, entwickelt. ${ }^{820}$ Auf Grundlage des Modells der Ziel- und Ergebnisebenen öffentlicher Leistungserstellung $^{821}$ wurde innerhalb des Projektes eine systematische Analyse sämtlicher Ziel- und Ergebnisebenen vorgenommen. Dies schließt auch die differenzierte Analyse und Bewertung der Impactebene mit ein, wozu eine umfassende Kundenbefragung in Form einer Nutzenanalyse durchgeführt wurde. Die zentralen Ergebnisse der Nutzenanalyse stellt die folgende Fallstudie vor. Bevor sich die Ausführungen dem Aufbau und der Konzeption sowie den zentralen Ergebnissen der Kundenbefragung widmen, werden jedoch zunächst kurz die organisatorischen Rahmenbedingungen des betrachteten Verwaltungsbereiches erläutert. ${ }^{822}$

Die Bezirksregierung Münster ist eine von fünf Bezirksregierungen in NordrheinWestfalen. ${ }^{823}$ Mit rund 1.300 Beschäftigten ist sie die größte Mittelbehörde des Landes. Die Bezirksregierung besitzt einerseits die Aufgabe eines regionalen Moderators. Sie koordiniert und kontrolliert in ihrem Bezirk, ob gesetzliche Aufgaben und landespolitische Programme verwirklicht werden. Andererseits sorgt sie für einen regionalen Konsens und vertritt im Sinne einer kommunalfreundlichen Behörde die Interessen der Kreise und Kommunen gegenüber der Landesregierung. ${ }^{824}$ Durch die Umsetzung des 2. Modernisierungsgesetzes zu Beginn des Jahres 2001 wurde unter anderem das Landesamt für Agrarordnung (Obere Flurbereinigungsbehörde) in die Bezirksregierung Münster integriert, wodurch diese auch landesweite Zuständigkeiten erlangte.

\footnotetext{
819 Vgl. §149 FlurbG.

820 Vgl. ausführlich BMS Consulting (2005) und zusammenfassend Seyer/Pieper/Kasten/Mosiek (2006).

821 Vgl. Mosiek et al. (2003), S. 29 oder die Ausführungen des Kapitels 3.3.2.3.

822 Die nachfolgenden Ausführungen zur Organisationsstruktur der Bezirksregierung Münster und der Agrarordnungsverwaltung in Nordrhein-Westfalen beziehen sich auf den Zeitraum des Projektes bis Mitte 2005. Die Organisationsstruktur der Agrarordnungsverwaltung in NRW ist starken Änderungen unterworfen.

823 Ihren Ursprung hat die Bezirksregierung Münster in der am 1. Dezember 1803 gegründeten Kriegs- und Domänenkammer des Freiherrn vom und zum Stein. Sie ist als staatliche Behörde auf der mittleren Ebene in einem dreistufigen Verwaltungsaufbau Bestandteil der Landesverwaltung in Nordrhein-Westfalen.
}

824 Vgl. hierzu das Leitbild der Bezirksregierung Münster. 
Der organisatorische Aufbau der Agrarordnungsverwaltung in NRW ist mehrstufig und erinnert konzeptionell an eine Holding-Struktur. Die politische Führung der Agrarordnungsverwaltung obliegt dem Ministerium für Umwelt und Naturschutz, Landwirtschaft und Verbraucherschutz des Landes Nordrhein-Westfalen („AufsichtsratFunktion“). Die fachliche Führung (,Management-Funktion“) obliegt der Oberen Flurbereinigungsbehörde, heute Abteilung 9 der Bezirksregierung Münster. Der eigentliche operative Aufgabenvollzug wird in acht regional zuständigen Ämtern für Agrarordnung ausgeführt. Im Fokus des Verwaltungshandelns der Agrarordnungsverwaltung steht die Verbesserung der Standortqualität des ländlichen Raumes ${ }^{825}$ in Nordrhein-Westfalen. Zur Erreichung dieser Zielsetzung nehmen die Ämter für Agrarordnung eine Vielzahl unterschiedlicher Aufgaben wahr. Hierzu gehören im Wesentlichen das Flächenmanagement durch Bodenordnung, Maßnahmen zur Förderung der Dorfentwicklung und die Erarbeitung integrierter ländlicher Entwicklungskonzepte.

\subsubsection{Aufbau und Konzeption der Kundenbefragung}

Entsprechend dem Modell der Ziel- und Ergebnisebenen öffentlicher Leistungserstellung stellt die Zufriedenheit des Leistungsempfängers mit den Dienstleistungen der öffentlichen Verwaltung ein wesentliches Zielkriterium staatlichen Handelns dar ${ }^{826} \mathrm{Im}$ Fokus einer Nutzenanalyse steht die Messung der Zufriedenheit des Leistungsempfängers mit den Dienstleistungen des staatlichen Verwaltungshandelns, d.h. die subjektive Wahrnehmung der Dienstleistungsqualität durch einzelne Kunden und Klienten (subjektive Effektivität) ${ }^{827}$ Im Rahmen des betrachteten Projektes besteht die Aufgabe der Impactbetrachtung darin, das Ausmaß der wahrgenommenen Kundenorientierung der Agrarordnungsverwaltung in NRW bei der Durchführung von Bodenordnungsverfahren zu bewerten.

Zur Bewertung des Impacts wurde im Rahmen des Projektes eine schriftliche Kundenbefragung vorgenommen. ${ }^{828}$ In einem ersten Schritt wurden dazu die relevanten Anspruchsgruppen des Verwaltungsproduktes "Bodenordnung" identifiziert. Dabei handelt es sich vor allem um die vom Unternehmensflurbereinigungsverfahren betroffenen Grundstückseigentümer (GE), die verantwortlichen Träger des Bauprojekts (Projektträger), die Vorstände der Teilnehmergemeinschaften (VTG) sowie sonstige Träger öffentlicher Belange (TÖB). Die Befragung zeichnet sich demnach durch eine differenzierte Ansprache von vier unterschiedlichen Kundengruppen aus, wobei insgesamt mehr als 600 Fragebögen versendet wurden. Die differenzierte Ansprache der Akteure ermöglicht eine mehrdimensionale Bewertung unterschiedlicher Kundenperspektiven und die Berücksichtigung der teilweise gegenläufigen Zielsetzungen und Ansprüche.

\footnotetext{
825 Auszug aus dem Leitbild der Agrarordnungsverwaltung NRW.

$826 \mathrm{Vgl}$. Mosiek et al. (2003), S.

827 Vgl. Kapitel 4.2.4.2.

${ }^{828}$ Um die Ergebnisse zu validieren, wurde anschließend eine Reihe von Gesprächen mit fachlichen Experten geführt.
} 
Jeder Fragebogen wurde beim Aufbau in drei Themenkomplexe gegliedert (siehe $A b$ bildung 56). Im Bereich der allgemeinen Angaben wurden größtenteils statistische Daten zum Bodenordnungsverfahren sowie zum Befragten selbst erhoben. Hiermit wurden anschließend spezifische Auswertungen zu bestimmten Verfahrenstypen und Empfängergruppen ermöglicht. Im zweiten Themenbereich stand die Einschätzung der Dienstleistungs- und Servicequalität der Ämter für Agrarordnung im Mittelpunkt der Betrachtung. Hier wurde nach der Zufriedenheit der Kunden zu einzelnen Leistungskomponenten des Amtes (z.B. Dauer des Verfahrens/ Nachvollziehbarkeit von Entscheidungen/ Interessensausgleich) gefragt. Neben der Leistungszufriedenheit wurde zusätzlich die Zufriedenheit mit den Mitarbeitern (z.B. Freundlichkeit, Sachverstand, Engagement) separat bewertet. Die hier gewonnen Erkenntnisse lieferten zahlreiche Anhaltspunkte zur Optimierung der Bearbeitungsprozesse sowie zur Verbesserung der Kundenkommunikation. Im dritten Themenkomplex wurde abschließend mit differenzierten Fragen zu den Verfahrenszielen und Verfahrenswirkungen eine Einschätzung der Wirksamkeit der Bodenordnung erbeten. Hierbei treten die bereits thematisierten Überschneidungen zu den Outcomes auf, die subjektiven Nutzenvorstellungen einzelner Leistungsempfänger können bei ausreichender Repräsentativität der Erhebung auch zur Bewertung gesamtgesellschaftlicher Auswirkungen herangezogen werden. ${ }^{829}$

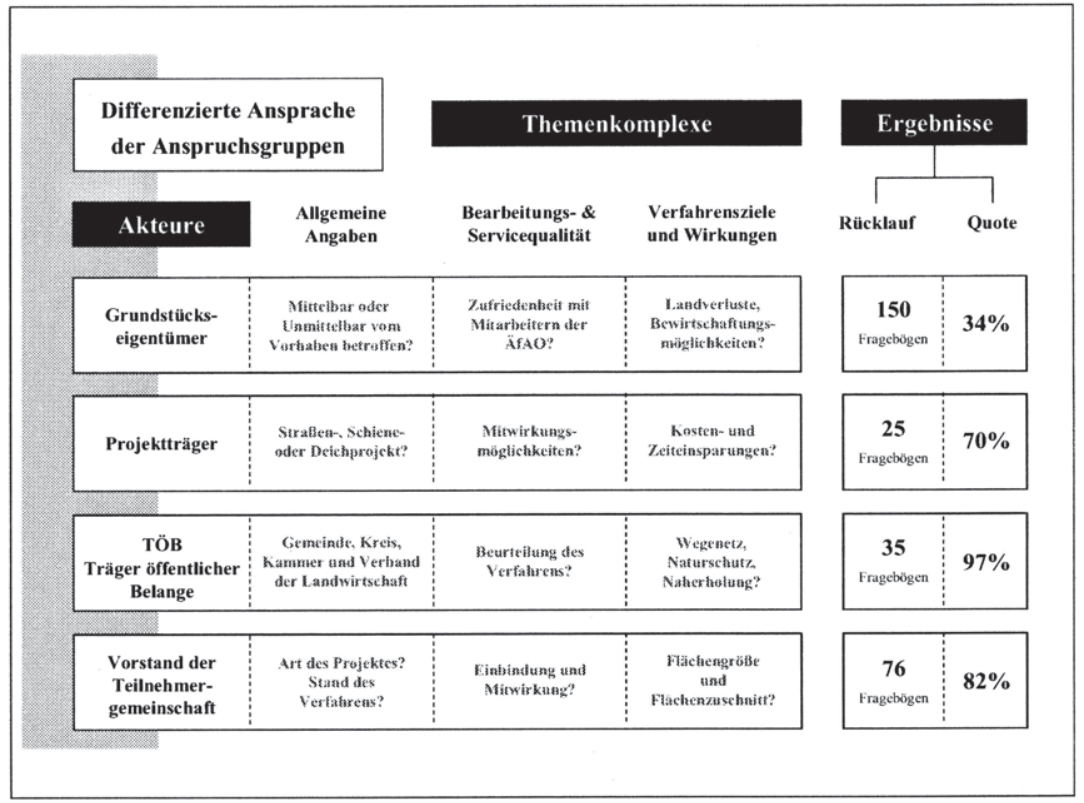

Abbildung 56: Konzeption und Rücklauf der Befragung

829 Vgl. Kapitel 4.2.1. 
Die inhaltliche Konzeption der Fragebögen folgte dem direkten merkmalsorientierten Ansatz, wobei die wahrgenommene Dienstleistungsqualität durch eine direkte Frage nach der Zufriedenheit des Kunden erhoben wurde. ${ }^{830}$ Allen Fragebögen lag dabei ein einheitliches, fünf-stufiges Antwortschema zugrunde, welches durch einfaches Ankreuzen beantwortet werden konnte, beispielsweise von „sehr zufrieden“ bis „sehr unzufrieden“ bzw. von „trifft voll zu“ bis „trifft nicht zu“. Neben anspruchsgruppenspezifischen Fragestellungen enthielten die Fragebögen auch zahlreiche gleichartige Abfragen, was entsprechende Querschnittsvergleiche zwischen den Akteuren ermöglicht.

Die Kundenbefragung fand im Zeitraum November 2004 bis Februar 2005 statt, wobei insgesamt 608 Fragebögen an die vier unterschiedlichen Kundengruppen verschickt wurden. Die deutliche Mehrheit der Fragebögen entfiel dabei auf die betroffenen Grundstückseigentümer von fünf zufällig ausgewählten Unternehmensflurbereinigungsverfahren in NRW mit insgesamt 443 Fragebögen. Je 36 Fragebögen wurden an die Gruppe der Projektträger und die Vorstände der Teilnehmergemeinschaften versandt. Insgesamt 93 Fragebögen gingen an die Träger öffentlicher Belange. Im Gegensatz zu den Grundstückseigentümern konnten aufgrund des geringeren Umfangs bei den anderen Kundengruppen sämtliche laufenden Unternehmensverfahren in NRW berücksichtigt werden.

Der Rücklauf betrug 286 Fragebögen und damit durchschnittlich 47\% (vgl. Abbildung 56). Die Mehrheit entfiel mit 150 Fragebögen auf die Grundstückseigentümer. ${ }^{831}$ Von den Projektträgern wurden 25, von den Vorständen der Teilnehmergemeinschaften 35 Fragebögen zurückgesandt. Die Träger öffentlicher Belange schickten 76 ausgefüllte Bögen zurück. Die hohen Rücklaufquoten, insbesondere in den kleineren Segmenten, sichern die repräsentative Aussagequalität der Daten. Hierdurch gewinnen die Befragungsergebnisse empirische Evidenz, so dass die Aussagen der Befragten zur Wirksamkeit der betreffenden Bodenordnungsverfahren auch Rückschlüsse für eine Wirkungsanalyse der Bodenordnung zulassen. ${ }^{832}$

Ein wichtiges Ergebnis des allgemeinen Befragungsteils ist beispielsweise die Feststellung, dass eine große Mehrheit der betroffenen Grundstückseigentümer $(72,7 \%)$ die eingebrachten Eigentumsflächen im Rahmen des Verfahrens nicht veräußert. ${ }^{833}$ Bei $38 \%$ der befragten Eigentümer handelte es sich um landwirtschaftliche Betriebe, die die betroffenen Eigentumsflächen selbst bewirtschaften und meist zusätzlich Pachtflächen im Verfahrensgebiet bewirtschaften. Nur eine Minderheit der Eigentümer ist in der Regel an einer Flächenveräußerung interessiert. Hiermit lassen sich auch die teilweise erheblichen Nutzungskonflikte erklären, die durch eine begleitende Bodenordnung abgemildert werden sollen.

${ }^{830} \mathrm{Vgl}$. hierzu ausführlich Kapitel 4.2.4.2.

${ }^{831}$ Eine vollständige Auswertung sämtlicher Fragebögen findet sich in der Anlage der Projektdokumentation. Vgl. BMS Consulting (2005).

${ }^{832} \mathrm{Vgl}$. hierzu auch Kapitel 4.2.2.

833 Dies wurde auch durch die Expertengespräche bestätigt. Eine deutliche Mehrzahl der betroffenen Eigentümer möchte demnach die bestehenden Eigentumsflächen behalten. Vgl. BMS Consulting (2005), S. $125 \mathrm{ff}$. 


\subsubsection{Befragungsergebnisse zur Dienstleistungs- und Servicequalität}

In diesem Abschnitt werden die Befragungsergebnisse des zweiten Themenkomplexes zur Servicequalität der Agrarordnungsverwaltung vorgestellt. Im Fokus der Betrachtung steht insbesondere der Wahrnehmungsvergleich der Dienstleistungsqualität aus den unterschiedlichen Perspektiven der Kundengruppen. ${ }^{834}$

$\mathrm{Zu}$ Beginn des Themenkomplexes wurden die Kunden der Ämter für Agrarordnung um die Einschätzung der Verfahrensabwicklung, und hier insbesondere um die Beurteilung der Informations- und Kommunikationspolitik, gebeten. Wichtig ist in diesem Kontext, inwieweit sich die Befragten zu Beginn über Ablauf und Ziele des Verfahrens von den Ämtern informiert fühlten und welche Mitwirkungsmöglichkeiten an den Entscheidungen des jeweiligen Amtes bestanden. Abbildung 57 zeigt die entsprechenden Befragungsergebnisse der Kunden.

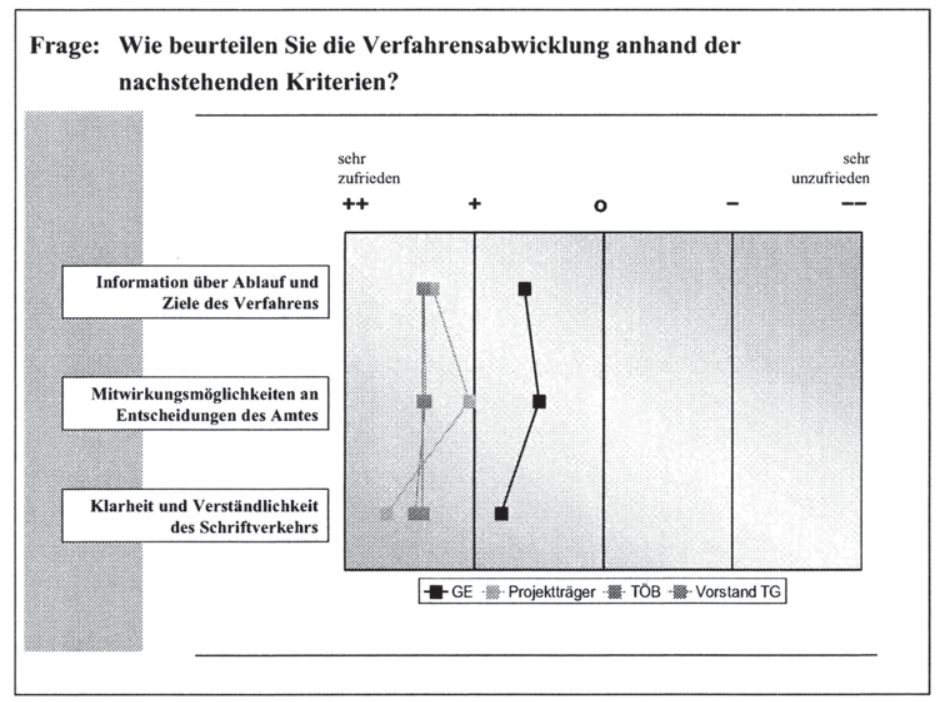

Abbildung 57: Beurteilung der Abwicklungsqualität

Generell lässt sich feststellen, dass sämtliche Kundengruppen die Verfahrensabwicklung in allen Kriterien positiv beurteilen. Hierbei fält jedoch auf, dass die Grundstückseigentümer im Vergleich zu den anderen Kundengruppen die Abwicklung insgesamt etwas schlechter beurteilen. Hervorzuheben ist, dass die Klarheit und Verständlichkeit des Schriftverkehrs - trotz des juristisch komplexen Verfahrensablaufs - von allen Kriterien am besten bewertet wird. Die Mitwirkungsmöglichkeiten an den Entscheidungen des Amtes werden dagegen von den Projektträgern und den Grundstücks-

$834 \mathrm{Zu}$ den vorgestellten Ergebnissen vgl. insbesondere BMS Consulting (2005), S. $112 \mathrm{ff}$. 
eigentümern etwas kritischer gesehen. Möglicherweise besteht für diese Gruppen ein besonderes Interesse zur Durchsetzung der eigenen Zielsetzungen im Verfahrensablauf, so dass über das Ergebnis tendenziell eher Anlass zur Unzufriedenheit besteht.

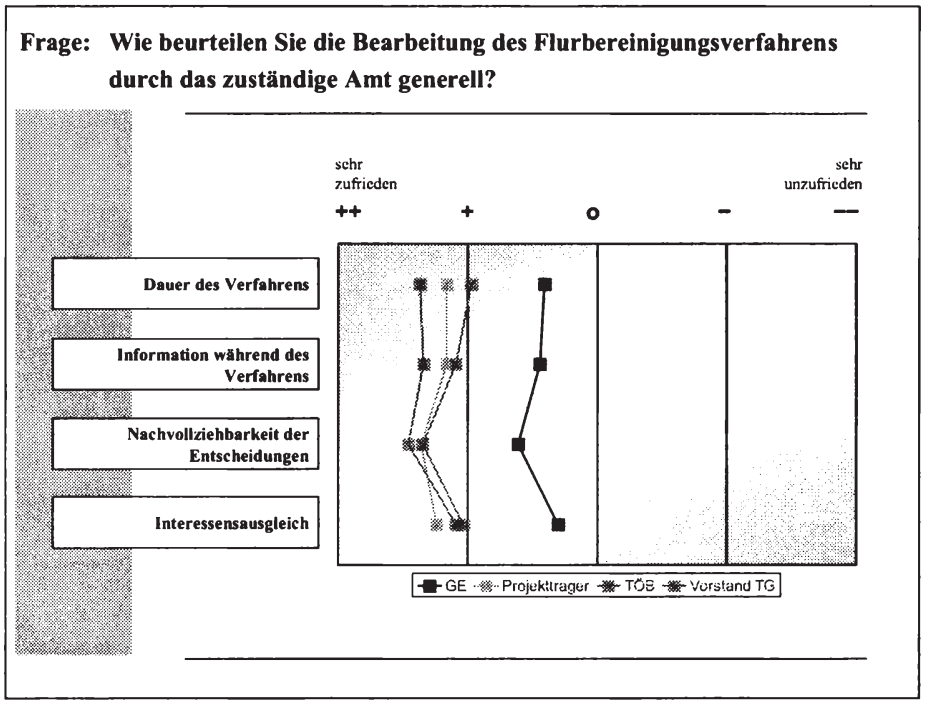

Abbildung 58: Beurteilung der Bearbeitungsqualität

Ein vergleichbares Gesamtbild ergibt sich auch bei der Betrachtung des zweiten Fragenblocks. Die Bearbeitungsqualität des Bodenordnungsverfahrens durch die zuständigen Ämter für Agrarordnung wurde durchweg positiv beurteilt. Die Befragten bewerteten die Verfahrensbearbeitung anhand der Merkmale «Dauer des Verfahrens«, "Information während des Verfahrens«, »Nachvollziehbarkeit von Entscheidungen« sowie »Interessenausgleich« zwischen den Beteiligten. Auch hier fällt das Urteil der Grundstückseigentümer insgesamt ungefähr eine Abstufung schlechter aus als bei den anderen Kundengruppen. Auffällig ist zudem, dass alle Kundengruppen die Nachvollziehbarkeit der Entscheidungen des Amtes am besten bewerten. Die Gesamtdauer des Verfahrens wird dagegen eher kritischer beurteilt. Viele Kunden wünschen sich demnach eine schnellere Abwicklung des Flurbereinigungsverfahrens (vgl. Abbildung 58).

In einem weiteren Fragenblock sollten die Kundengruppen die Servicequalität der Mitarbeiter der Ämter für Agrarordnung bewerten (vgl. Abbildung 59). Im Einzelnen wurden dabei die Kriterien Freundlichkeit, Sachverstand, Engagement, Erreichbarkeit sowie Information und Beratung abgefragt. Auch hier ergibt sich ein sehr positives Gesamtbild, welches tendenziell besser ausfällt als die bisherigen Ergebnisse. Gleichzeitig beurteilen die Grundstückseigentümer auch in diesem Befragungsabschnitt wiederum sämtliche Kriterien etwas kritischer. Im direkten Merkmalsvergleich wird der 
Aspekt Information und Beratung von allen Kunden am Besten bewertet, was auf eine recht hohe Kundenorientierung der Mitarbeiter der Ämter für Agrarordnung hindeutet.

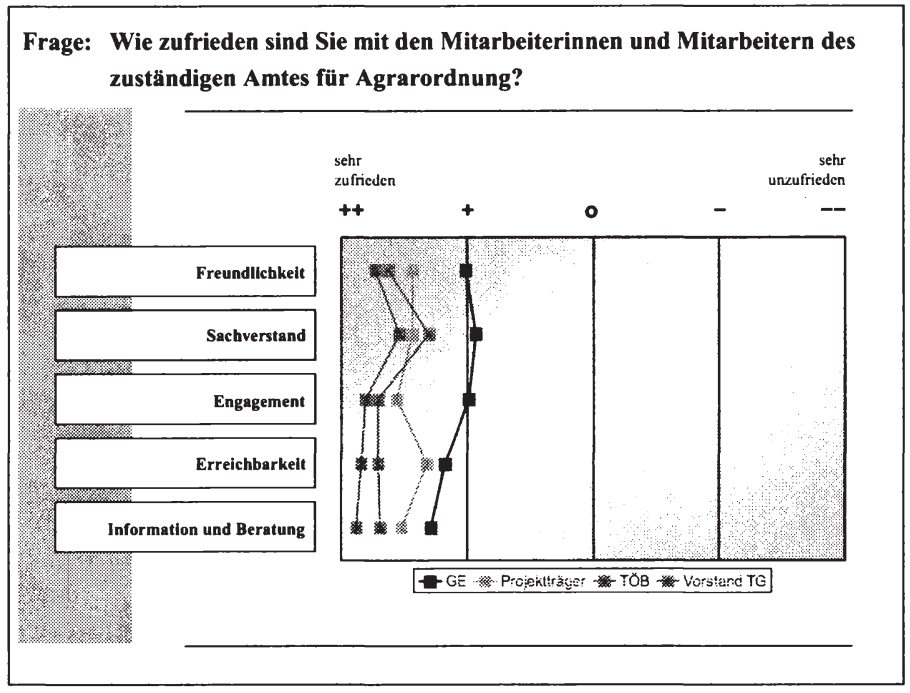

Abbildung 59: Beurteilung der Servicequalität der Mitarbeiter

Eine Einzelbetrachtung der Grundstückseigentümer macht deutlich, dass ein Sockel unzufriedener Eigentümer existiert. Diese beurteilen sämtliche Kriterien der Verfahrensabwicklung und -bearbeitung sowie die Servicequalität der Mitarbeiter durchgängig negativ. Das Antwortverhalten ist bei den Grundstückseigentümern daher stark zweigeteilt, eine Mehrheit von ca. $80 \%$ urteilt in der Regel positiv, eine Minderheit von knapp $20 \%$ dagegen durchgängig negativ. Belegt wird diese Beobachtung durch Korrelationen im Antwortverhalten der Grundstückseigentümer, die sich zwischen Werten von 0,75 bis 0,80 bewegen. Somit lässt sich auch die durchgängig festgestellte negative Abweichung im Antwortverhalten bei der Kundengruppe der Grundstückseigentümer erklären.

Die insgesamt gute Beurteilung der Arbeit der Ämter für Agrarordnung spiegelt sich auch in der abschließenden Fragestellung nach der generellen Zufriedenheit mit dem zuständigen Amt wider, welche in diesem Zusammenhang als "Globalzufriedenheit» interpretiert werden kann. Von den Grundstückseigentümern geben mehr als zwei Drittel der Befragten an, mit dem zuständigen Amt für Agrarordnung zufrieden bzw. sehr zufrieden zu sein (vgl. Abbildung 60). Bei den anderen Kundengruppen fällt das Ergebnis noch deutlicher aus. Hier geben fast alle Befragten an, mit dem zuständigen Amt für Agrarordnung entweder sehr zufrieden zu sein bzw. zufrieden zu sein. 


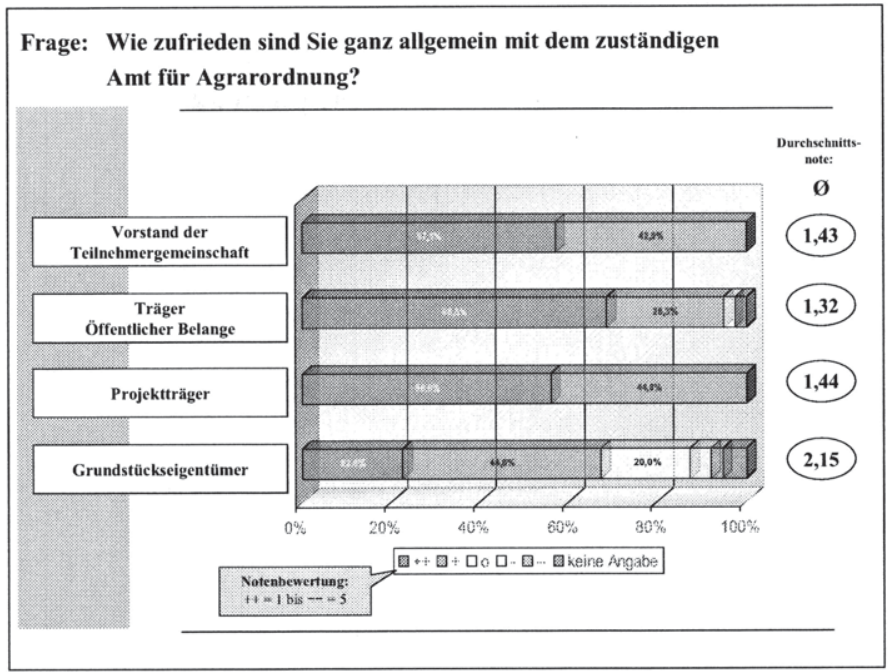

Abbildung 60: Beurteilung der Globalzufriedenheit

Insgesamt lässt sich somit eine recht positive Beurteilung des Themenkomplexes Dienstleistungs- und Servicequalität durch die Kunden der Agrarordnungsverwaltung in Nordrhein-Westfalen feststellen. Sicherlich gibt es in der Gesamtbetrachtung auch teilweise negative Beurteilungen und kritische Stimmen. Die Gesamtergebnisse sind allerdings immer vor dem Hintergrund einer Vollzugsbehörde zu bewerten, die wesentlich in die Eigentumsrechte der betroffenen Grundstückseigentümer eingreift. Zudem zeigen die freien Kommentare der Eigentümer - immerhin ein Drittel der Befragten hat diese Möglichkeit genutzt - dass die negative Beurteilung sich häufig auf die Infrastrukturmaßnahme selbst bezieht und nicht auf das Flächenmanagement der Bodenordnung.

\subsubsection{Befragungsergebnisse zu Verfahrenszielen und Wirkungen}

Im dritten Themenkomplex Verfahrensziele und Wirkungen wurde von den einzelnen Anspruchsgruppen erfragt, für wie wichtig sie die verschiedenen möglichen Zielsetzungen von Flurbereinigungsverfahren erachten. Des Weiteren sollten die Kunden beurteilen, inwieweit diese Zielsetzungen in ihrem Verfahren auch erreicht werden konnten. Die Betrachtung folgt damit einer direkten zielbezogenen Wirksamkeitsbeurteilung - im Sinne eines Soll-Ist-Vergleichs. ${ }^{835}$ Die Wirkungen werden damit aus der jeweiligen subjektiven Sicht des Leistungsempfängers beurteilt, aufgrund der Repräsentativi-

835 Alternativ lässt sich die Wirksamkeit staatlichen Handelns auch anhand des Beitrags zur allgemeinen Wohlfahrt - verstanden als gesamtgesellschaftlicher Wertschöpfungsbeitrag für die Gesellschaft - bewerten. Vgl. Mosiek/Gerhardt (2003), S. 293 f.; Pieper et al. (2006) und insbesondere Kapitel 4.1.1.1 dieser Arbeit. 
tät der Befragung lassen sich die Ergebnisse aber auch für eine Wirkungsanalyse nutzbar machen. ${ }^{836}$ Weiterhin wurden von spezifischen Kundengruppen auch Einschätzungen zu speziellen Wirkungskomponenten eingeholt, beispielsweise zur Form, Größe und Erreichbarkeit landwirtschaftlicher Nutzflächen durch die Landwirtschaft. Hierdurch können die in der Wirkungsanalyse vorzunehmenden Hypothesen zu bestimmten gesellschaftlichen Auswirkungen schrittweise empirische Evidenz erfahren.

Als Gründe für die Initiierung eines Flurbereinigungsverfahrens werden seitens des Trägers des Bauprojektes eine erwartete Zeitersparnis und die deutlich höhere Akzeptanz für das Bauprojekt in der Region genannt (jeweils 64,0\%). Für immerhin die Hälfte der Befragten Projektträger spielt auch die Erwartung einer Kostenersparnis, beispielsweise beim Grunderwerb, eine wichtige Rolle. Wichtigster Grund für die Verfahrenseinleitung ist aber der Wunsch der Träger öffentlicher Belange nach einer begleitenden Bodenordnung (76,0\%). Bei den Trägern öffentlicher Belange handelt es sich um die örtlich zuständigen Stellen bei den Gemeinden, Städten oder Kreisen. Dies deckt sich auch mit den entsprechenden Befragungsergebnissen. Mehr als $95 \%$ der Träger öffentlicher Belange halten die Flurbereinigung für ein sehr gutes bzw. gutes Instrument zur Flächenbeschaffung für das anstehende Bauprojekt. ${ }^{837}$

Im Fragenkomplex zur generellen Wichtigkeit unterschiedlicher Verfahrensziele wurden den vier Kundengruppen acht mögliche Ziele zur Auswahl gestellt:

- Vermeidung von Verlusten an Eigentumsfläche

- Behebung der durch das Bauprojekt entstandenen Bewirtschaftungsnachteile

- Erhalt landwirtschaftlicher Betriebe

- Verbesserung der Erreichbarkeit und der Bewirtschaftungsmöglichkeiten landwirtschaftlicher Nutzflächen

- Allgemeine Akzeptanz des Bauprojektes

- Beschleunigung des Bauprojektes

- Naturschutz und Landschaftspflege

- Berücksichtigung des Bodenschutzes (z.B. Reduzierung Bodenerosionen)

Dabei ist im Ergebnis festzuhalten, dass von den Befragten sämtliche Ziele im Durchschnitt als wichtig bis sehr wichtig eingestuft wurden. Dennoch lässt sich aus dem Antwortverhalten eine Zielpräferenz für jede Anspruchsgruppe ableiten. Abbildung 61 zeigt entsprechend die jeweils wichtigsten Ziele der Akteure. Auffällig ist in diesem Zusammenhang, dass das Ziel des Erhaltes landwirtschaftlicher Betriebe sich bei allen Anspruchsgruppen unter den drei wichtigsten Zielen wieder findet. Insgesamt wird Zielsetzungen, welche die Landwirtschaft betreffen, von allen Beteiligten eine hohe Bedeutung eingeräumt.

\footnotetext{
836 Vgl. Kapitel 4.2.2.

837 Vgl. BMS Consulting (2005), S. $110 \mathrm{ff}$.
} 


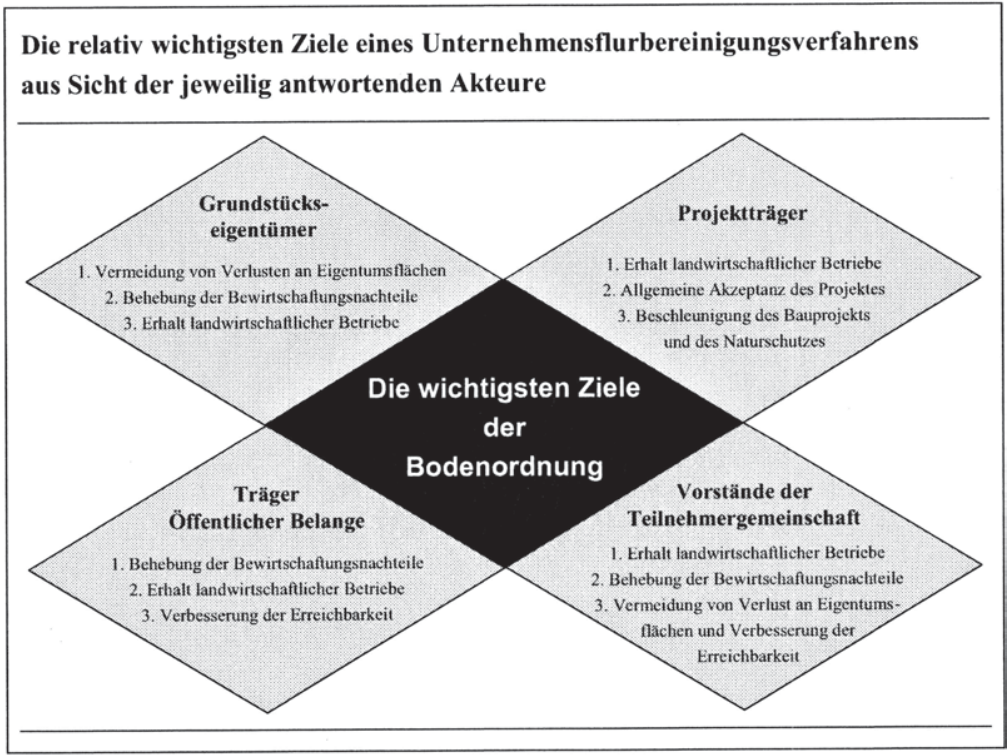

Abbildung 61: Die wichtigsten Verfahrensziele aus Kundensicht

Bei den Trägern Öffentlicher Belange und den Vorständen der Teilnehmergemeinschaft besitzen entsprechende Ziele ausnahmslos die höchste Priorität. Für die Grundstückseigentümer stellt zudem die Vermeidung von Verlusten an Eigentumsflächen das wichtigste Verfahrensziel dar. Bei einer deutlichen Mehrheit der Fälle sind die vom Infrastrukturprojekt betroffenen Eigentümer also nicht bereit, sich von Eigentumsflächen zu trennen. ${ }^{838}$ Für den Projektträger stehen daher auch Verfahrensziele im Vordergrund, die der Realisierung des Projektes zuträglich sind. Dies ist zum einen die allgemeine Akzeptanz des Projektes vor Ort. Zum anderen betrifft dies Belange des Naturschutzes - auch um hier Widerstände zu reduzieren - sowie der Erhalt landwirtschaftlicher Betriebe, die durch das Bauprojekt ansonsten in ihrer Existenz bedroht sind und mit hohen Entschädigungen abgefunden werden müssten.

Von Interesse ist allerdings nicht nur die Priorisierung einzelner Zielsetzungen, sondern auch, inwieweit diese Ziele im jeweiligen Verfahren auch erreicht werden konnten. Zur Verdeutlichung des Sachverhaltes wurde sich in Abbildung 62 einer sog. Portfoliodarstellung bedient. Dieser Darstellung liegt die grundsätzliche Hypothese zugrunde, dass jedes Ziel auch nur entsprechend seiner relativen Wichtigkeit erfüllt werden sollte. D.h. die Zielerfüllung durch das zuständige Amt für Agrarordnung entspricht genau der jeweiligen Wertzumessung durch den Kunden. Bei einer idealtypi-

838 Dies führt gerade bei großen Bauvorhaben, mit hohem Flächenbedarf, die ohne begleitende Bodenordung durchgeführt werden, immer wieder zu erheblichen Konflikten, gerichtlichen Auseinandersetzungen und damit zu Verzögerungen des Projektes. 
schen Zielerreichung wären demnach sämtliche Verfahrensziele auf einer Geraden durch den Ursprung (45-Grad-Linie) angeordnet. Ergeben sich stattdessen größere Abweichungen, kann dies auf zwei Ursachen zurückgeführt werden. Zum einen besteht die Möglichkeit, dass Ziele in höherem Maße erfüllt wurden, als ihnen vom Kunden an Bedeutung zukam (Zielübererfüllung). Zum anderen besteht die Möglichkeit, dass Ziele nicht entsprechend ihrer Wichtigkeit erfüllt wurden (Zieluntererfüllung). ${ }^{839}$

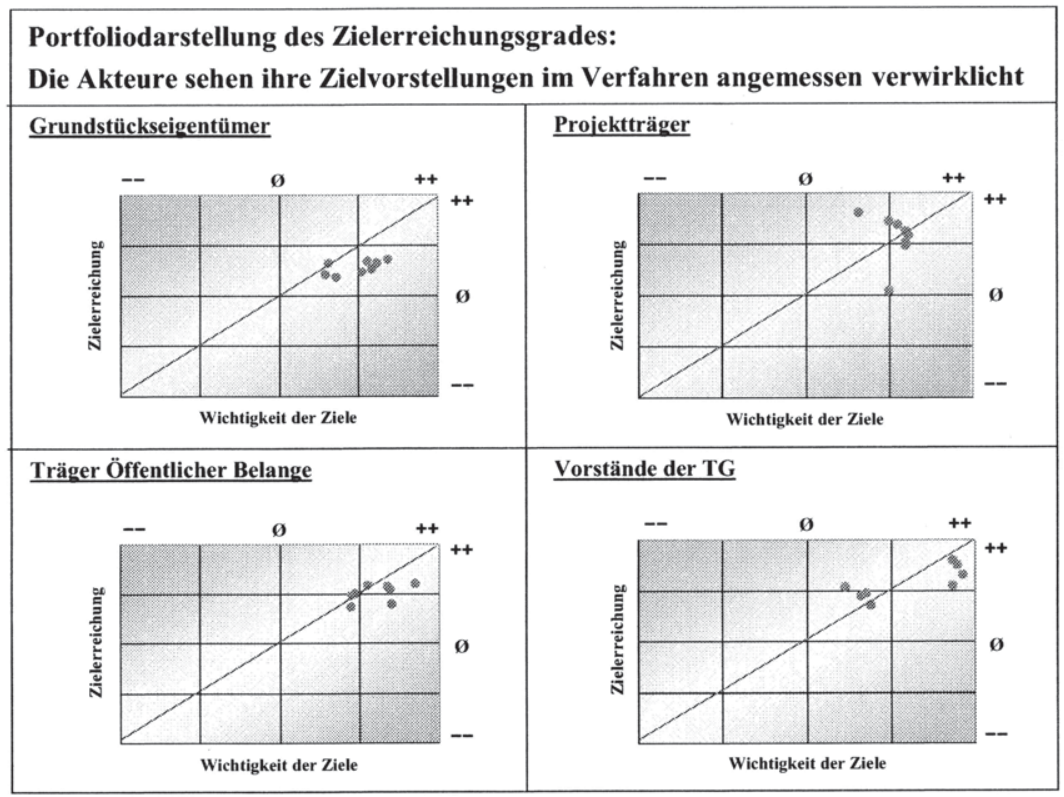

\section{Abbildung 62: Portfoliodarstellung der relativen Zielerfüllung allgemeiner Zielsetzungen}

Wie sich den einzelnen Portfolios in Abbildung 62 entnehmen lässt, ergeben sich für die Kunden der Agrarordnungsverwaltung keine wesentlichen Abweichungen von den dargestellten Geraden, d.h. die Zielerfüllung entspricht weitestgehend ihrer Wichtigkeit. Kleinere Auffälligkeiten zeigt einmal das Portfolio der Projektträger, mit einer leichten Zielübererfüllung im Bereich der Flächenerreichbarkeit und einer Zieluntererfüllung im Bereich Bodenschutz. ${ }^{840}$ Zum anderen zeigt das Portfolio der Vorstände der Teilnehmergemeinschaft, gemessen an der relativen Wichtigkeit, eine deutliche Häufung zweier Zielgruppen. Dies sind einerseits Ziele, die primär den Grundstückseigen-

839 Vgl. BMS Consulting (2005), S. 121 f.

840 Es ist jedoch wenig überraschend, dass aus Sicht der Projektträger das Ziel einer Verbesserung der Flächenerreichbarkeit in Bodenordnungsverfahren regelmäßig übererfüllt wird. Der Nutzen aus verstärktem Wegebau kommt primär den regionalen Landwirten zugute, die Kosten verbleiben i.d.R. beim Projektträger. 
tümern bzw. der Landwirtschaft zugute kommen sowie andererseits die sonstigen Verfahrensziele. ${ }^{841}$ Die letzte Auswertung betrifft schließlich die Kundenbeurteilung, inwieweit die originären gesetzlichen Zielsetzungen im Verfahren erreicht werden konnten. Als die intendierten Zielsetzungen des Gesetzgebers, bei der Ausarbeitung der entsprechenden Regelungen, kommt diesen Zielen natürlich besondere Bedeutung zu. Die Analyse der gesetzlich verankerten Zielsetzungen sind Ausdruck des politischen Willens und damit auch Ausgangspunkt der Wirkungsanalyse.

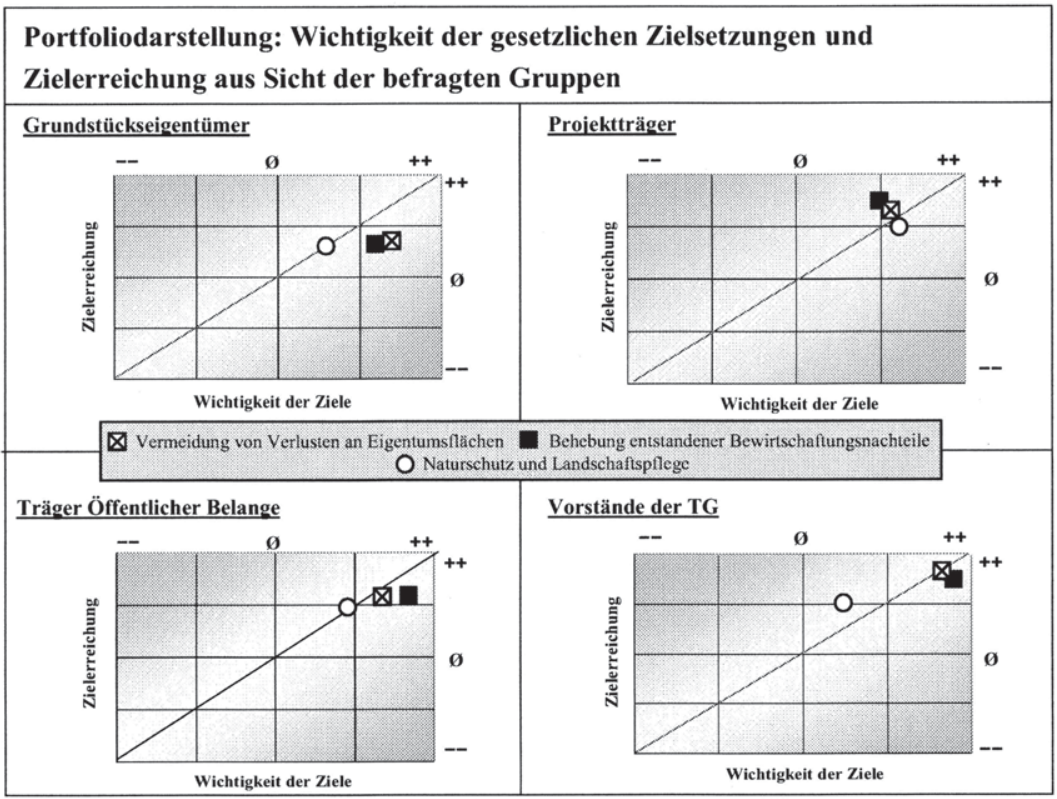

Abbildung 63: Portfoliodarstellung der relativen Zielerfüllung gesetzlicher Zielsetzungen

Einen ersten Überblick über die intendierten Wirkungen einer Unternehmensflurbereinigung bietet die entsprechende Gesetzesgrundlage. Gemäß $§ 87$ FlurbG wird mit der Verfahrenseinleitung zum einen die Verteilung des im Zuge der Realisierung des Infrastrukturvorhabens entstehenden Landverlustes auf einen größeren Kreis von Eigentümern bezweckt. ${ }^{842}$ Zum anderen sollen Nachteile für die allgemeine Landeskultur vermieden werden. Während klassische Bodenordnungen primär auf eine geringe Be-

841 Auch diese Ergebnisse sind wenig überraschend. Beim Vorstand der Teilnehmergemeinschaft handelt es sich um ein gewähltes Organ, welches die Interessen der Grundstückseigentümer und damit die Belange vieler Landwirte vertritt. Ziele, die dieser Gruppe nutzen, besitzen daher eine sehr hohe Bedeutung. Andere Ziele, wie z.B. die allgemeine Akzeptanz des Bauprojektes oder des Naturschutzes stehen dahinter zurück.

842 Vgl. $\$ 87$ FlurbG. 
einträchtigung der landwirtschaftlichen Nutzungs- und Bewirtschaftungsmöglichkeiten ausgerichtet waren, spielen im Zuge des gestiegenen Umweltbewusstseins heute auch ökologische Überlegungen eine bedeutende Rolle. ${ }^{843}$ Um aussagekräftige Befragungsergebnisse zu erhalten, wurden auf Grundlage des Gesetzes drei Teilziele formuliert die Vermeidung von Verlusten an Eigentumsflächen, die Behebung der durch das Bauprojekt entstandenen Bewirtschaftungsnachteile sowie Naturschutz und Landschaftspflege (vgl. auch Abbildung 63). ${ }^{844}$

Im Rahmen der Kundenbefragung wurde ermittelt, welche Bedeutung die Anspruchsgruppen diesen gesetzlichen Zielsetzungen beimessen und inwieweit diese das Bodenordnungsverfahren erfüllen konnte. Wie die Ergebnisse in Abbildung 63 zeigen, beurteilen alle befragten Gruppen den Zielerreichungsgrad in Bezug auf die gesetzlich induzierten Wirkungen als hoch bis sehr hoch, so dass die Agrarordnungsverwaltung aus Sicht ihrer Kunden ihren gesetzlichen Auftrag äußerst zufriedenstellend erfüllt. Als Gesamtergebnis lässt sich feststellen, dass die schriftliche Kundenbefragung ein positives Bild der Agrarordnungsverwaltung in Nordrhein-Westfalen zeichnet. Dies betrifft sowohl die Qualität der Verfahrensbearbeitung als auch die Servicequalität der Mitarbeiter. Die von den Kunden in die Verwaltung gerichteten Erwartungen über Ziele und Wirkungen eines Bodenordnungsverfahrens werden von der Agrarordnungsverwaltung in hohem Maße erfüllt.

Die Nutzenanalyse von Bodenordnungsverfahren zeigt, dass das Urteil der Kunden eine aussagekräftige und damit steuerungsrelevante Größe für die öffentliche Verwaltung darstellt. Im Rahmen regelmäßiger Messungen sollte das Controlling daher auch zukünftig ein überschaubares Set von Indikatoren erheben. Die Vielzahl individueller Wahrnehmungen der Verfahrenswirkungen durch einzelne Leistungsempfänger ermöglicht bei einer entsprechenden Repräsentativität letztlich auch eine objektive Einschätzung gesellschaftlicher Wirkungen. Durch die Ausweitung der Betrachtung auf die gesamtgesellschaftlich ausgelösten Wirkungen lassen sich im Rahmen der Wirkungsanalyse auch Rückschlüsse auf die Effektivität, d.h. die Wirksamkeit des Verwaltungshandelns, gewinnen.

\subsection{Wirkungsanalyse in der Konzeptionsphase: Aufbau der Analyse- und Bewertungsschemata}

\subsubsection{Ausgangssituation und Zielsetzung des Projektes}

Konzeption und Verfahren der Wirkungsanalyse sollen im Folgenden anhand eines Projektes zum staatlichen Flächenmanagement durch Bodenordnung in RheinlandPfalz ausführlich vorgestellt werden. ${ }^{845}$ Auf Grundlage der im theoretischen Teil der Arbeit vorgestellten Methodik, wurde innerhalb des Projektes eine Wirkungsanalyse

843 Vgl. Hegele et al. (1992), S. 357.

844 Für eine detaillierte Beschreibung der Vorgehensweise vgl. BMS Consulting (2005), S. 136ff.

845 Vgl. hierzu ausführlich Mosiek/Pieper/Kasten (2006) sowie Lorig/Kasten/Mosiek/Pieper (2006), S. $458 \mathrm{ff}$. 
von Bodenordnungsverfahren vorgenommen. Wesentliche Zielsetzung des Projektes war die weitgehende und möglichst umfassende Identifikation, Systematisierung und Bewertung der gesamtgesellschaftlichen Auswirkungen der Bodenordnung. Weiterhin war es ein erklärtes Projektziel, sämtliche gesetzliche Verfahrenstypen der Bodenordnung in die Analyse einzubeziehen ${ }^{846}$ Die zentralen Ergebnisse stellt die folgende Fallstudie vor.

Das Bundesland Rheinland-Pfalz betreibt seit mehreren Jahren eine konsequente Modernisierung der Verwaltungsprozesse. In diesem Kontext ist das Ministerium für Wirtschaft, Verkehr, Landwirtschaft und Weinbau des Landes Rheinland-Pfalz (MWVLW) unter anderem verantwortlich für die Verwaltungsmodernisierung der in seinem Geschäftsbereich ressortierenden sechs Dienstleistungszentren Ländlicher Raum (DLR). Der Gesamtprozess der Neuausrichtung wird unterstützt und begleitet durch die Aufsichts- und Dienstleistungsdirektion Trier (ADD), welche die Dienstaufsicht über die DLRs Inne hat. ${ }^{847}$ Das MWVLW Rheinland-Pfalz ist bestrebt, nicht nur die Effizienz, sondern auch die Effektivität des eigenen Verwaltungshandelns weiter zu erhöhen, insb. im Rahmen der strategischen Führung der ihm unterstellten Verwaltungsbereiche. Dazu soll im Geschäftsbereich "Bodenordnung" der Dienstleistungszentren Ländlicher Raum ein funktionsfähiges Wirkungsmanagement etabliert werden. Bodenordnungsverfahren stoßen allerdings ein komplexes Gefüge aus ökonomischen, ökologischen, sozialen und kulturellen Wirkungen an. Die Komplexität wirkungsorientierter Steuerungsprozesse macht es daher erforderlich, durch ein wirkungsorientiertes Controlling eine sachgerechte Führungsunterstützung zu implementieren. Mit Hilfe der Wirkungsanalyse müssen sämtliche Auswirkungen der Bodenordnung identifiziert und durch geeignete Messverfahren operationalisiert werden, um sie im Rahmen der Wirkungsrechnung einer Steuerung zugänglich zu machen. Das MWVLW RheinlandPfalz beabsichtigt, auf dieser Grundlage eine kombinierte Kosten- und Wirkungsprognose einzuführen und so zur Wirkungsoptimierung des Verwaltungshandelns beizutragen. Aufgrund des umfangreichen Adressatenkreises und der hohen individuellen Verfahrenskomplexität ist eine transparente Darstellung der relevanten Wirkungsbeiträge in der Bodenordnung besonders geboten - die Mitarbeiter der Verwaltung können erheblichen Einfluss auf die gesamtgesellschaftlichen Wirkungen nehmen. ${ }^{848}$

Die Fallstudie zur Wirkungsanalyse folgt dabei der im theoretischen Teil der Arbeit vorgestellten Vorgehenssystematik. Nach einer kurzen Darstellung der organisatorischen Rahmenbedingungen wird zunächst die Methodik der Wirkungsanalyse für Bodenordnungsverfahren erläutert. Der erste Schritt der Konzeptionsphase besteht dann in der Herleitung des gesamtgesellschaftlichen Wirkungsgefüges der Bodenordnung, der zweite Schritt in einer Operationalisierung und Bewertung der einzelnen Wirkungskomponenten. Die Anwendung der Wirkungsrechnung erfolgt schließlich anhand eines exemplarischen Beispiels.

\footnotetext{
${ }^{846}$ Nach dem Flurbereinigungsgesetz werden fünf wichtige Verfahrenstypen unterschieden. Vgl. Kapitel 5.1.2.

${ }^{847} \mathrm{Vgl}$. Mosiek/Pieper/Kasten (2006), S. $15 \mathrm{f}$.

${ }^{848}$ Zur Typisierung staatlicher Handlungsbereiche und der Eignung einer Wirkungsanalyse vgl. Kapitel 4.2.3. 


\subsubsection{Bodenordnungsverfahren als Dienstleistung des Landes Rheinland- Pfalz}

Nachfolgend werden die aktuellen organisatorischen Rahmenbedingungen und das Aufgabenspektrum der Landesverwaltung in Rheinland-Pfalz vorgestellt. Von besonderem Interesse im Rahmen dieser Untersuchung ist dabei das Aufgabenfeld des staatlichen Flächenmanagements durch Bodenordnung als ein wesentlicher Bestandteil der Landespolitik zur integrierten ländlichen Entwicklung. Die Landesverwaltung in Rheinland-Pfalz gliedert sich in oberste Landesbehörden (Staatskanzlei und Ministerien), in obere Landesbehörden (z.B. Aufsichts- und Dienstleistungsdirektion, Struktur- und Genehmigungsdirektionen oder das Landesuntersuchungsamt) und in untere Landesbehörden (z.B. Finanzämter, Vermessungsämter, Forstämter oder Dienstleistungszentren Ländlicher Raum). Daneben gibt es eigenständige Landesbetriebe, die spezielle Aufgaben der Landesverwaltung erfüllen (z.B. Landesbetrieb Straßen) ${ }^{849}$

Als oberste Landesbehörde ist das Ministerium für Wirtschaft, Verkehr, Landwirtschaft und Weinbau mit Sitz in Mainz für Bodenordnungsverfahren zuständig. Insgesamt besitzt das MWVLW die folgenden Aufgaben: ${ }^{850}$

- Wirtschaftspolitik und -ordnung, berufliche Bildung und Europa

- Wirtschaftsförderung, Mittelstand, Tourismus, Außenwirtschaft

- Innovation, Informations-/ Kommunikationswirtschaft, Energieaufsicht

- Weinbau, Landwirtschaft, Verbraucherschutz

- Landentwicklung, Agrarpolitik und Markt

- Verkehr und Straßenbau

Das MWVLW ist in diesem Kontext die für die Bodenordnung nach dem Flurbereinigungsgesetz zuständige oberste Flurbereinigungsbehörde ${ }^{851}$ Die Zuständigkeit für diese Funktion ist in der Abteilung 6 »Landentwicklung, Agrarpolitik und Markt« angesiedelt. Darüber hinaus ist in der Abteilung 6 des MWVLW auch eine Spruchstelle für Flurbereinigung eingerichtet. Sie entscheidet über Widersprüche gegen die Feststellungen im Rahmen von Bodenordnungsverfahren, wie z.B. die Ergebnisse der Wertermittlung oder über Widersprüche gegen den Flurbereinigungsplan.

Die Aufsichts- und Dienstleistungsdirektion mit Sitz in Trier ist obere Landesbehörde und zuständig für die Bereiche: ${ }^{852}$

- Kommunale und hoheitliche Aufgaben, Soziales

- Schulen

- Landwirtschaft, Weinbau und Wirtschaftsrecht

849 Zur Organisation und zum strukturellen Aufbau des öffentlichen Sektors vgl. auch Kapitel 2.1.2.

850 Stand: Februar 2007.

851 Vgl. §2 FlurbG.

852 Stand: Februar 2007. 
Hinsichtlich des Aufgabenspektrums der Bodenordnung ist die Abteilung 4 der Aufsichts- und Dienstleistungsdirektion Mittelbehörde für die Bereiche Landwirtschaft, Weinbau und Wirtschaftsrecht. Sie nimmt damit auch die Aufgabe als obere Flurbereinigungsbehörde nach dem FlurbG wahr ${ }^{853}$ Diese Zuständigkeit ist im Referat 44 "Ländliche Entwicklung/ Ländliche Bodenordnung" angesiedelt. Entsprechend dem mehrstufigen Verwaltungsaufbau sind die Dienstleistungszentren Ländlicher Raum untere Flurbereinigungsbehörden nach dem FlurbG und damit für die operative Leistungserstellung im Bereich des staatlichen Flächenmanagements durch Bodenordnung zuständig (vgl. Abbildung 64).

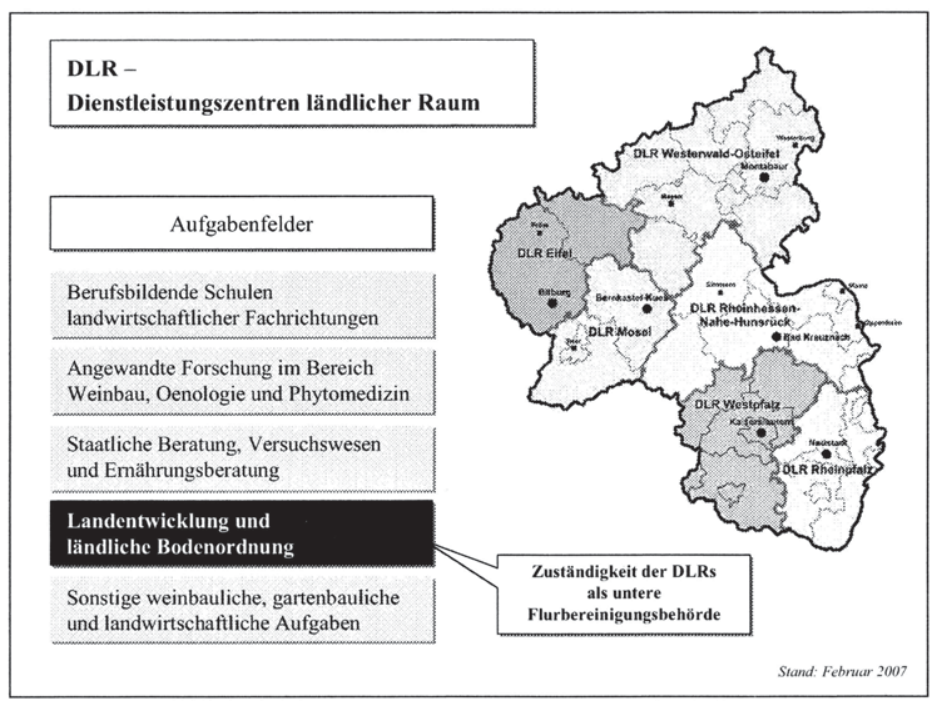

Abbildung 64: Struktur und Aufgaben der DLR

Im Rahmen der rheinland-pfälzischen Agrarverwaltungsreform wurden im Jahr 2003 zahlreiche untere Landesbehörden aufgelöst und zu den sechs DLRs zusammengefasst. ${ }^{854}$ Die Dienstleistungszentren Ländlicher Raum verfügen daher über ein breites Aufgabenfeld mit den Schwerpunkten "Ländliche Entwicklung" und "Agrarwirtschaft«. Im Einzelnen sind sie zuständig für die Bereiche Landentwicklung, ländliche Bodenordnung und Siedlung, die Berufsbildenden Schulen landwirtschaftlicher Fachrichtung, die staatliche Beratung, das Versuchswesen, die Ernährungsberatung, die angewandte Forschung im Weinbau, Oenologie, Phytomedizin sowie für andere weinbauliche, gartenbauliche und landwirtschaftliche Aufgaben. ${ }^{855}$

\footnotetext{
853 Vgl. \$2 FlurbG.

$854 \mathrm{Vgl}$. Mosiek/Pieper/Kasten (2006), S. 29.

855 Stand: Februar 2007.
} 
Die Amtsbezirke der DLRs decken die gesamte Landesfläche in Rheinland-Pfalz ab. Es handelt sich um selbständig agierende Behörden, deren Größe von einem eher kleinen Amt mit gut einhundert Beschäftigten bis zu einem großen Amt mit mehr als dreihundert Beschäftigten reicht. Insgesamt sind derzeit ca. 1.250 Mitarbeiter bei den Dienstleistungszentren beschäftigt, wobei ca. 530 Mitarbeiter in der Abteilung Landentwicklung angesiedelt sind. ${ }^{856}$ Der Tätigkeitsschwerpunkt der Abteilung Landentwicklung liegt in der Zuständigkeit als untere Flurbereinigungsbehörde in der operativen Durchführung von Bodenordnungsverfahren.

\subsubsection{Methodik der Wirkungsanalyse von Bodenordnungsverfahren}

Zielsetzung der vorliegenden Fallstudie ist die vollständige Identifikation und Bewertung der gesamtgesellschaftlichen Auswirkungen von Bodenordnungsverfahren nach dem FlurbG. Dazu ist es notwendig - im Rahmen einer Marginalbetrachtung - sämtliche Effekte zu analysieren, die in der Gesellschaft daraus erwachsen, dass eine Bodenordnung durchgeführt wurde. ${ }^{857}$ Im Fokus der Untersuchung stehen also die Veränderungen im gesamtgesellschaftlichen Wirkungsgefüge, die daraus resultieren, dass ein Flurbereinigungsverfahren eingeleitet wurde (vgl. Abbildung 65). ${ }^{858}$

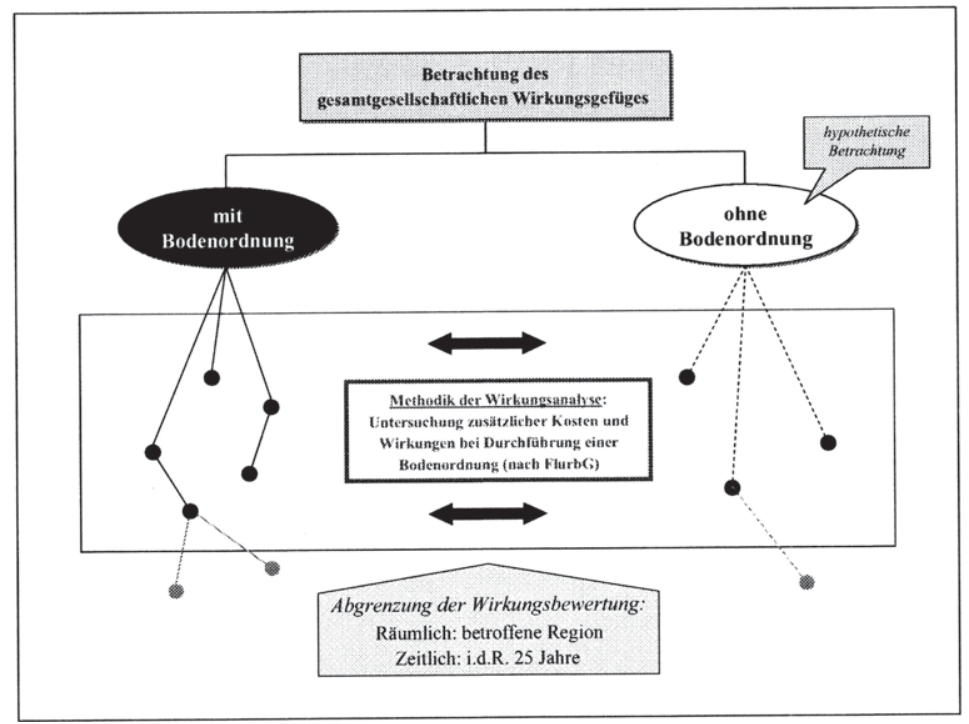

Abbildung 65: Methodik der Wirkungsanalyse von

Bodenordnungsverfahren

\footnotetext{
$856 \mathrm{Vgl}$. Mosiek/Pieper/Kasten (2006), S. $28 \mathrm{f}$.

$857 \mathrm{Vgl}$. Mosiek/Pieper/Kasten (2006), S. 34.

${ }^{858}$. Vgl. hierzu auch Kapitel 4.3.2.1.3.
} 
Grundsätzlich wird damit im weiteren Verlauf der Wirkungsanalyse unterstellt, dass sämtliche staatlichen Vorhaben sowohl mit, als auch ohne ein begleitendes Flurbereinigungsverfahren realisiert werden könnten. Zielsetzung der folgenden Analyse ist daher, die aus der Bodenordnung resultierenden zusätzlichen Kosten und Wirkungen für die Gesellschaft zu ermitteln. Damit basiert die Wirkungsanalyse zu einem großen Teil auf hypothetischen Überlegungen. ${ }^{859}$ Die untersuchungsleitende Fragestellung der Wirkungsanalyse für Bodenordnungsverfahren lautet demnach: „Welche gesamtgesellschaftlichen Veränderungen haben sich dadurch ergeben, dass ein Bodenordnungsverfahren durchgeführt wurde?"

Um die Bodenordnung hinsichtlich ihrer Wirksamkeit beurteilen zu können, ist die Herleitung eines möglichst umfassenden, in sich konsistenten Wirkungsgefüges der Bodenordnung erforderlich. ${ }^{860}$ Zur Identifikation der gesellschaftlichen Auswirkungen stehen der Wirkungsanalyse unterschiedliche Instrumente zur Verfügung. Neben der reinen Beobachtung von Veränderungen im Verfahrensgebiet, können hierzu einerseits die vom Gesetzgeber intendierten Wirkungen und andererseits die Erkenntnisse bereits durchgeführter Studien herangezogen werden (Dokumentenanalyse). Weitere Erkenntnisse können auch aus Kundenbefragungen, sofern hier eine Repräsentativität gewährleistet ist, sowie aus flankierenden Mitarbeiter- und Expertengesprächen gewonnen werden (Befragung).

Zur Wirkungsbewertung ist das Untersuchungsfeld sowohl in zeitlicher als auch in räumlicher Hinsicht abzugrenzen (vgl. Abbildung 65). Beim staatlichen Flächenmanagement durch Bodenordnung handelt es sich um räumliche beschränkte Verfahren, so dass die abgegrenzte Untersuchung der betroffenen Region angemessen erscheint. In zeitlicher Hinsicht wird ein Bewertungshorizont herangezogen, der in der Regel 25 Jahre beträgt. ${ }^{861}$ Es wird damit unterstellt, dass die identifizierten Auswirkungen regelmäßig für diesen Zeitraum ihre Wirkung in der Gesellschaft entfalten und danach ihre Wirksamkeit verlieren.

\subsubsection{Identifikation der Auswirkungen von Bodenordnungsverfahren}

\subsubsection{Gesetzlich intendierte Wirkungen der Bodenordnung}

Die Identifikation der relevanten Wirkungsbeiträge von Bodenordnungsverfahren erfordert zunächst eine Analyse der gesetzlichen Rahmenbedingungen. Entsprechend der Modellkonzeption staatlichen Handelns ${ }^{862}$ lassen sich intendierte, am Zielsystem des politisch-administrativen Systems ausgerichtete Wirkungen und nicht intendierte $\mathrm{Ne}$ benwirkungen unterscheiden. Ein wichtiger Anhaltspunkt im Hinblick auf die politisch intendierten Zielsetzungen von Bodenordnungsverfahren bietet die entsprechende Ge-

\footnotetext{
${ }^{859}$ Zur Bewertungssystematik der Wirkungsanalyse vgl. auch Kapitel 4.3.2.2.1.

${ }^{860}$ Zur systematischen Herleitung eines gesamtgesellschaftlichen Wirkungsgefüges vgl. Kapitel 4.3.2.1.

${ }^{861}$ Vgl. hierzu auch Kapitel 5.3.6.

${ }^{862} \mathrm{Vgl}$. ausführlich Kapitel 4.1.2.2.
} 
setzesgrundlage. Hierzu ist eine differenzierte Auseinandersetzung mit dem Flurbereinigungsgesetz erforderlich. Die nachfolgenden Ausführungen beziehen sich auf die Regelflurbereinigung nach $\S 1$ FlurbG, die Vereinfachte Flurbereinigung nach $\S 86$ FlurbG und die Unternehmensflurbereinigung nach $\$ 87$ FlurbG ${ }^{863}$ Hierbei handelt es sich um die gesetzlichen Verfahrensarten, die in der praktischen Anwendung der Flurbereinigungsbehörden sehr häufig genutzt werden. ${ }^{864}$ Diese Verfahrensarten stellen damit den Rahmen der weiteren Untersuchung dar.

Der Hauptzweck der Regelflurbereinigung besteht nach $\$ 1$ FlurbG in der Verbesserung der Produktions- und Arbeitsbedingungen in der Land- und Forstwirtschaft sowie in der Förderung der allgemeinen Landeskultur und der Landentwicklung. ${ }^{865}$ Unter der Verbesserung der Produktions- und Arbeitsbedingungen in der Land- und Forstwirtschaft lassen sich die folgenden gesetzlichen Zielsetzungen subsumieren: ${ }^{866}$

- Verbesserung der Produktions- und Arbeitsleistung

- Steigerung der Produktivität und Wirtschaftlichkeit

- Nachhaltige Stärkung und Sicherung der Wettbewerbsfähigkeit

Eine weitere gesetzliche Zielsetzung der Regelflurbereinigung besteht darin, die allgemeine Landeskultur sowie die Landentwicklung zu fördern. Traditionelle Bodenordnungsverfahren haben bei der Förderung der allgemeinen Landeskultur fast ausschließlich auf agrarstrukturelle Verbesserungen im ländlichen Raum abgezielt, d.h. im Vordergrund der Planungen stand in erster Linie die nachhaltige Verbesserung der Fruchtbarkeit und Ertragssicherheit landwirtschaftlicher Nutzflächen ${ }^{867}$ Das inhaltliche Verständnis des Begriffs »Landeskultur« hat sich jedoch insbesondere in den letzten Jahrzehnten entscheidend gewandelt und kann heute folgendermaßen interpretiert werden: ${ }^{868}$

- Landschaftspflege unter Berücksichtigung der ökologischen Ausgleichsfunktion

- Verbesserung der Wohn-, Wirtschafts- und Erholungsfunktion des ländlichen Raumes

- Dauerhafte Verbesserung der Lebensverhältnisse

Das klassische Aufgabenfeld der Bodenordnung hat demnach in den letzten Jahren einen starken Wandel erlebt und erfüllt heute vielfach auch ökologische und landespflegerische Zielsetzungen.

\footnotetext{
$863 \mathrm{Zu}$ den gesetzlichen Verfahrensarten der Bodenordnung vgl. Kapitel 5.1.2.

$864 \mathrm{Vgl}$. Mosiek/Pieper/Kasten (2006), S. 75.

865 Vgl. §1 FlurbG.

$866 \mathrm{Vgl}$. Hegele et al. (1992), S. 2 ff. und Mosiek/Pieper/Kasten (2006), S. 76 ff.

$867 \mathrm{Vgl}$. Hegele et al. (1992), S. 3.

868 Vgl. Hegele et al. (1992), S. 4 und Mosiek/Pieper/Kasten (2006), S. 78 f.
} 
Bodenordnungsverfahren dienen den unterschiedlichsten Zielsetzungen und Maßnahmen der ländlichen Entwicklung. ${ }^{869}$ Die Rahmenbedingungen für die Durchführung solcher Bodenordnungsverfahren sind im zweiten Absatz von §37 FlurbG festgelegt:

- „Die Flurbereinigungsbehörde hat [...] die öffentlichen Interessen zu wahren, vor allem den Erfordernissen der Raumplanung, der Landesplanung und einer geordneten städtebaulichen Entwicklung, des Umweltschutzes, des Naturschutzes und der Landschaftspflege, des Denkmalschutzes, der Erholung, der Wasserwirtschaft einschließlich Wasserversorgung und Abwasserbeseitigung, der Fischerei, des Jagdwesens, der Energieversorgung, des öffentlichen Verkehrs, der landwirtschaftlichen Siedlung, der Kleinsiedlung, des Kleingartenwesens und der Gestaltung des Ortsund Landschaftsbildes sowie einer möglichen bergbaulichen Nutzung und der Erhaltung und Sicherung mineralischer Rohstoffvorkommen Rechnung zu tragen. ${ }^{4870}$

Die Durchführung der Agrarstrukturverbesserung sowie der in $\$ 37$ (2) FlurbG genannten Maßnahmen ist gesetzlich nicht vorgeschrieben. ${ }^{871}$ Jede einzelne Maßnahme rechtfertigt für sich alleine die Einleitung eines Bodenordnungsverfahrens ${ }^{872}$ Grundsätzlich gilt es, die verschiedenen Belange gegeneinander abzuwägen und das Wohl der Allgemeinheit zu fördern. ${ }^{873}$ Dabei sind nicht nur die Interessen der Beteiligten, sondern ebenfalls die Interessen der allgemeinen Landeskultur sowie der Landentwicklung zu berücksichtigen. Zudem schreibt das Gesetz vor, die jeweilige Landesstruktur bei der Neuordnung des ländlichen Raumes zu beachten, d.h. die natürlichen Gegebenheiten der Landschaft sollten aus Gründen des Naturschutzes erhalten werden. ${ }^{874}$ Die Realisierung der in $\$ 37$ (2) genannten Maßnahmen erfolgt häufig in Verbindung mit der Vereinfachten Flurbereinigung entsprechend $\S 86$ FlurbG. Das Gesetz sieht bei der Durchführung solcher Flurbereinigungen Vereinfachungen im Verfahrensablauf vor. Das vereinfachte Flurbereinigungsverfahren findet häufig bei künstlichen Eingriffen in den ländlichen Raum Anwendung, bspw. bei städtebaulichen Maßnahmen, Infrastrukturprojekten, Maßnahmen des Umweltschutzes und der naturnahen Entwicklung von Gewässern oder im Rahmen von Bodenreform- oder Siedlungsverfahren. ${ }^{875}$ Als wesentliche Zielsetzungen des Gesetzgebers lassen sich die nachfolgenden Punkte festhalten: ${ }^{876}$

- Vermeidung/ Beseitigung der Nachteile für die allgemeine Landeskultur

- Erleichterung der Durchführung der Ausbaumaßnahmen

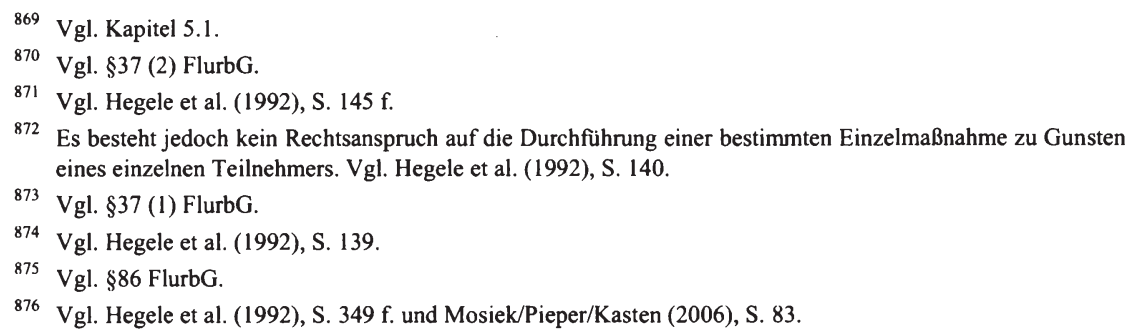


Eine weitere Sondervorschrift der Flurbereinigungsgesetzgebung ist die Unternehmensflurbereinigung nach $\S 87$ FlurbG. ${ }^{877}$ Werden ländliche Grundstücke für im öffentlichen Interesse liegende Großbauprojekte in Anspruch genommen, kann durch Bodenordnung der Landverlust auf einen größeren Kreis von Eigentümern verteilt werden und es lassen sich die landeskulturellen Nachteile des Projektes beseitigen ${ }^{878}$ Die gesetzlich intendierten Wirkungen der Unternehmensflurbereinigung lassen sich demnach wie folgt beschreiben: ${ }^{879}$

- Verringerung/Vermeidung von Eigentumsverlusten

- Behebung der entstehenden Bewirtschaftungsnachteile

- Förderung von Naturschutz und Landschaftspflege

Der Gesetzgeber bezweckt also eine möglichst geringe Beeinträchtigung der von den baulichen Planungen betroffenen Grundstückseigentümer, so dass die Unternehmensflurbereinigung für die Betroffenen sicherlich das mildere und verhältnismäßigere Mittel bei Enteignungen darstellt. $\S 87$ FlurbG steht folglich im Einklang mit dem Verfassungsgebot des geringstmöglichen Eingriffs. ${ }^{880}$ Zusätzlich bezweckt eine Unternehmensflurbereinigung auch die Beseitigung der Nachteile für die allgemeine Landeskultur. Dies bezieht sich einerseits auf Beeinträchtigung der Bewirtschaftungsmöglichkeiten landwirtschaftlicher Nutzflächen sowie andererseits auf Beeinträchtigung der Leistungs- und Funktionsfähigkeit des Naturhaushaltes und des Landschaftsbildes. ${ }^{881}$

Der Einblick in die intendierten Zielsetzungen des Flurbereinigungsgesetzes lässt bereits erahnen, wie vielfältig die Auswirkungen von Bodenordnungsverfahren sein können. Allerdings ist es Zielsetzung der Wirkungsanalyse, nicht nur die gesetzlich intendierten Wirkungsbeiträge abzubilden, sondern ebenfalls die darüber hinausgehenden Neben- und Folgewirkungen der Bodenordnung zu erfassen. ${ }^{822}$ Deshalb sind weitere Analysen notwendig. Als nächstes werden die bestehenden Forschungsergebnisse zu den Auswirkungen der Bodenordnung in den Untersuchungsablauf integriert.

\footnotetext{
877 Vgl. §87 FlurbG oder Kapitel 5.2.

878 Vgl. §87 FlurbG oder Hegele et al. (1992), S. 357.

879 Vgl. BMS Consulting (2005), S. 136 ff. und Mosiek/Pieper/Kasten (2006), S. 84 f.

${ }^{880}$ Für die Festsetzung der Höhe der Eigentumsentschädigung gewährt §88 Nr.7 FlurbG den betroffenen Landeigentümern den ordentlichen Rechtsweg, so dass ebenfalls die Konformität des Flurbereinigungsgesetzes mit Artikel 14 Absatz 3 Satz 3 GG gewährleistet ist. Artikel 14 des Grundgesetzes regelt allgemein den besonderen Schutz des Eigentums. Vgl. hierzu auch die Ausführungen zur Regelflurbereinigung in diesem Kapitel.

${ }^{882}$ Vgl. auch Kapitel 4.3.2.1.
} 


\subsubsection{Inhaltsanalyse bestehender Forschungsergebnisse}

Der folgende Literaturüberblick beschränkt sich auf eine kurze Beschreibung des jeweiligen Untersuchungsgegenstandes einiger ausgewählter Studien. ${ }^{83}$ Dabei soll im Wesentlichen zwischen Untersuchungen unterschieden werden, die die Auswirkungen von Bodenordnungsverfahren möglichst allgemein analysieren bzw. darauf abzielen, ein allgemeines Bild der durch die Bodenordnung ausgelösten Wirkungen zu zeichnen (vgl. Tabelle 2). Weiterhin werden Studien und Forschungsergebnisse betrachtet und in die vorliegende Wirkungsanalyse integriert, die sich auf spezifische Wirkungskomponenten der Bodenordnung fokussieren. In der weiteren Darstellung wird zunächst mit einer Analyse der relevanten Ergebnisse der eher allgemein angelegten Wirkungsstudien begonnen.

\begin{tabular}{ll}
\hline Studie & Untersuchungsgegenstand/ -ziel \\
\hline $\begin{array}{l}\text { BMS Consulting/ } \\
\text { BezReg Münster (2005) }\end{array}$ & $\begin{array}{l}\text { Umfassende Wirkungsanalyse für Bodenordnungsver- } \\
\text { fahren am Beispiel der Unternehmensflurbereinigung. } \\
\text { FAL (2003) }\end{array}$ \\
$\begin{array}{l}\text { Bewertung des aktuellen Zielerreichungsgrades des } \\
\text { NRW-Programms Ländlicher Raum. }\end{array}$ \\
$\begin{array}{l}\text { Entwicklung eines Ansatzes zur Bewertung von Pro- } \\
\text { (2002) }\end{array}$ \\
$\begin{array}{l}\text { jekten der ländlichen Entwicklung. } \\
\text { Kokermann/Buhse/ }\end{array}$ \\
$\begin{array}{l}\text { Analyse der Wirksamkeit integrierter ländlicher Ent- } \\
\text { wicklung im Bundesland Hessen. }\end{array}$ \\
Schlosser (1999) & $\begin{array}{l}\text { Analyse der Wirksamkeit der Flurbereinigung unter } \\
\text { Berücksichtigung des zeitlichen Wandels. }\end{array}$ \\
Weiss/Kremer/ & Kombination von Nutzwert- und Kostenwirksamkeits- \\
Strang (1996) & analyse zur Effizienzmessung in der Flurbereinigung. \\
Oberholzer (1993) & Optimierung der Flurbereinigung als Instrument inte- \\
& grierter Landentwicklung. \\
Kroés (1971) & Analyse der sozialökonomischen Auswirkungen und \\
& Kosten von Flurbereinigungsmaßnahmen. \\
\hline
\end{tabular}

Tabelle 2: Übersicht allgemeiner Wirkungsanalysen der Bodenordnung

\footnotetext{
${ }^{883}$ Für eine zusammenfassende Darstellung und Bewertung der Besonderheiten wichtiger Untersuchungen und Gutachten zur Bodenordnung vgl. auch Grüneberger (2003), S. 87 f.

${ }^{884}$ Für eine prägnante Zusammenfassung der Studie von Oberholzer (1993) vgl. Henkes (1998).

${ }^{885}$ Für eine prägnante Zusammenfassung der Untersuchung von Kroés (1971) vgl. ebenfalls Henkes (1998). 
Ziel der von der BEZIRKSREGIERUNG MüNSTER bei der BMS CONSUlTING (2005) in Auftrag gegebenen Untersuchung war die Herleitung eines möglichst umfassenden Wertschöpfungssystems, auf dessen Grundlage exemplarisch ausgewählte Unternehmensflurbereinigungen in Nordrhein-Westfalen im Hinblick auf ihre Effizienz und Effektivität beurteilt werden können. ${ }^{886}$ Die monetäre Quantifizierung der einzelnen Wirkungsbeiträge bildet dabei den Schwerpunkt der Analyse, um eine weitgehende Vergleichbarkeit von Wirkungen und Kosten der Unternehmensflurbereinigung zu gewährleisten. Die Vorteilhaftigkeit der betrachteten Bodenordnungsverfahren wird schließlich im Rahmen einer sog. Wertschöpfungsbilanz überprüft, indem der notwendige Ressourcenverzehr den monetarisierten Auswirkungen gegenübergestellt wird.

Das NRW-PROGRAMM LÄNDLICHER RAUM zielt primär auf „die Entwicklung einer ökologisch und ökonomisch stabilen Land- und Forstwirtschaft als Grundlage für einen attraktiven ländlichen Raum ${ }^{4887} \mathrm{ab}$. Zur Verwirklichung dieses Hauptziels der integrierten Landentwicklung bedarf es der Verfolgung einer Vielzahl sehr unterschiedlicher, teils sogar konkurrierender Teilziele. ${ }^{888}$ Aufgabe der Studie der FAL (2003) ist es, den bisherigen Erfolg des Programms in Hinblick auf die Erfüllung dieser vielfältigen Teilziele zu überprüfen.

SCHULTE ET AL. (2002) entwickeln zunächst eine verfahrensspezifische Wirkungssystematik, die sowohl ökonomische als auch soziale Zielsetzungen beinhaltet. ${ }^{889}$ Auf Grundlage diese Zielsystems wird anschließend der Nutzwert eines exemplarisch ausgewählten Projektes der ländlichen Entwicklung ermittelt.

BOKERMANN ET AL. (2000) zeigen das methodische Vorgehen im Rahmen einer Kosten-Wirksamkeits-Analyse für die integrierte Entwicklung am Beispiel ausgewählter Projekte in einer ländlichen Region Hessens auf. Im Zuge dieses Verfahrens werden die gesamtgesellschaftlich ausgelösten Wirkungen der einzelnen Landentwicklungsmaßnahmen entweder in physischen Einheiten ermittelt oder qualitativ beschrieben und den Projektkosten vergleichend gegenüber gestellt. Auf eine monetäre Quantifizierung wird in diesem Zusammenhang dagegen fast gänzlich verzichtet. ${ }^{890}$

SCHLOSSER (1999) untersucht die Auswirkungen von Flurbereinigungsverfahren auf die Entwicklung bayerischer Gemeinden. Dabei berücksichtigt er insbesondere den „Wandel der Flurbereinigung von einem Maßnahmenbündel zur überwiegend agrarischen Förderung zu einem vielseitigen Instrument der Landentwicklung ${ }^{6891}$ und zeigt die Verbindung dieses Wandels mit den gesellschaftlichen Veränderungen auf. Seine

\footnotetext{
886 Vgl. BMS Consulting (2005).

887 FAL (2003), Kapitel 1, S. 1.

888 Vgl. FAL (2003), Kapitel 9.

889 Vgl. Schulte/Bokermann (2002).

$890 \mathrm{Vgl}$. Bokermann/Buhse/Kaufmann (2000).

891 Schlosser (1999), S. 134.
} 
Analyse beschränkt sich demnach nicht nur auf den landwirtschaftlichen Sektor, sondern stützt sich zusätzlich auf ökologische Überlegungen. ${ }^{892}$

WEIB ET AL. (1996) stellen in ihrer Untersuchung ein sehr komplexes Zielsystem auf, das eine Beurteilung ,unterschiedlicher Maßnahmen und Maßnahmenbündel in der Flurbereinigung ${ }^{4{ }^{693}}$ hinsichtlich ihrer Effizienz ermöglichen soll. $\mathrm{Zu}$ diesem Zweck werden den einzelnen Teilzielen innerhalb des Systems geeignete Gewichtungsfaktoren und Messkriterien zugeordnet ${ }^{894}$ Auf dieser Grundlage lassen sich dann umfassende Nutzwertanalysen von Bodenordnungsverfahren durchführen.

Für OBERHOLZER (1993) stellt die Bodenordnung mit ihrer „bedeutsamen integralen Gestaltungsaufgabe in Richtung ganzheitlicher Landentwicklung ${ }^{6895}$ eines der wichtigsten Instrumente der Landentwicklungsverwaltung dar. In seinem Beitrag stellt er zahlreiche „Zielkriterien zur ganzheitlichen Simultanplanung der Kulturlandschaft“\$896 auf. Im Rahmen eines Flurbereinigungsverfahrens sollte seiner Ansicht nach unter Berücksichtigung möglicher Zielkonflikte stets eine optimale Zielerfüllung angestrebt werden.

KROÉS (1971) führt sehr umfangreiche Nutzen-Kosten-Analysen für drei exemplarisch ausgewählte Bodenordnungsverfahren in Nordrhein-Westfalen ex-post durch. Im Fokus seiner Untersuchung steht eine möglichst umfassende monetäre Bewertung der flurbereinigungsbedingten gesamtgesellschaftlichen Wirkungsbeiträge. ${ }^{897}$

Alle bisher erwähnten Studien setzen sich in sehr unterschiedlicher Weise mit der Beurteilung von Bodenordnungsverfahren auseinander. Die Bandbreite der verwendeten Verfahren reicht von einer einfachen Wirksamkeitsanalyse über die Nutzwertanalyse bis hin zu einer komplizierten Nutzen-Kosten-Analyse. Kombinationen aus unterschiedlichen Bewertungsansätzen sind dabei nicht ausgeschlossen. Dennoch ist allen Untersuchungen gemein, dass sie eine möglichst umfassende Identifikation der Wirkungsbeiträge der Bodenordnung anstreben. Zusätzlich existiert in der Literatur zu Flurbereinigungsverfahren eine Vielzahl von Beiträgen, die sich auf die gezielte Analyse eines ausgewählten Wirkungsbereichs der Bodenordnung fokussieren. In nachfolgender Tabelle 3 werden einige wichtige dieser Untersuchungen kurz vorgestellt.

Im Ergebnis liefert die im Auftrag der FAL von KLARE ET AL. (2005) durchgeführte Studie Faustzahlen zur Ermittlung der Kosteneinsparungen für landwirtschaftliche Betriebe. ${ }^{898}$ Diese Kosteneinsparungen entstehen durch die Neuordnung und Zusammenlegung von Flächen im Zuge der Bodenordnung. Auf Basis dieser Faustzahlen lassen

\footnotetext{
892 Vgl. Schlosser (1999).

893 Weiß/Kremer/Strang (1996), S. 9.

${ }^{894} \mathrm{Vgl}$. Weiß/Kremer/Strang (1996), S. $68 \mathrm{ff}$.

895 Oberholzer (1993), S.7.

${ }^{896}$ Oberholzer (1993), S. 8.

897 Vgl. Kroés (1971).

898 Vgl. Klare et al. (2005).
} 
sich beispielsweise die variablen Bewirtschaftungskosten in Abhängigkeit der Schlaglänge, der Schlaggröße und der durchschnittlichen Hof-Feld-Entfernung pauschal für unterschiedliche Regionen in Niedersachsen kalkulieren. ${ }^{899}$

Das BILDUNGSSEMINAR FÜR DIE AGRARVERWALTUNG RHEINLAND-PFALZ (1997) untersucht speziell die Auswirkungen von Bodenordnungsmaßnahmen auf den Steillagenweinbau. Dazu gehören zum einen Effekte, die sich unmittelbar aus der Zusammenlegung unwirtschaftlicher Parzellen ergeben. Die Parzellengröße spielt eine wesentliche Rolle für die Möglichkeit einer rentablen Bewirtschaftung eines Weinanbaugebietes. Zum anderen müssen aber auch die Effekte baulicher Maßnahmen in den Steillagen berücksichtigt werden, die eine stärkere Mechanisierung des Weinanbaus ermöglichen. ${ }^{900}$

\begin{tabular}{|c|c|}
\hline Studie & Untersuchungsgegenstand/ -ziel \\
\hline Klare et al. (2005) & $\begin{array}{l}\text { Entwicklung eines Instrumentariums zur Unterstützung } \\
\text { bei der Auswahl neuer Flurbereinigungsprojekte. }\end{array}$ \\
\hline $\begin{array}{l}\text { Bildungsseminar RLP } \\
\text { (1997) }\end{array}$ & $\begin{array}{l}\text { Bewertung von Rationalisierungsmaßnahmen im } \\
\text { Steillagenweinbau. }\end{array}$ \\
\hline $\begin{array}{l}\text { Burgmaier/Miersch/ } \\
\text { Donié (1995) }\end{array}$ & $\begin{array}{l}\text { Ermittlung von flurbereinigungsbedingten Agrarstruk- } \\
\text { turveränderungen. }\end{array}$ \\
\hline MLWF RLP (1991) & $\begin{array}{l}\text { Auswirkungen von Bodenordnungsverfahren auf die } \\
\text { Bewirtschaftung landwirtschaftlicher Betriebe in RLP. }\end{array}$ \\
\hline $\begin{array}{l}\text { Keymer/Linhart/Rinteln } \\
\text { Stumpf/Widermann } \\
\text { (1989) }\end{array}$ & $\begin{array}{l}\text { Auswirkungen von Bodenordnungsverfahren auf die } \\
\text { Bewirtschaftung landwirtschaftlicher Betriebe in Bayern. }\end{array}$ \\
\hline Karmann (1988) & $\begin{array}{l}\text { Bestimmung der Einflussfaktoren der Bodenpreise in } \\
\text { der bayerischen Flurbereinigung. }\end{array}$ \\
\hline Heinzlmeir (1983) & $\begin{array}{l}\text { Einfluss der Flurbereinigung auf den landwirtschaft- } \\
\text { lichen Bodenmarkt und den Bodenpreis. }\end{array}$ \\
\hline $\begin{array}{l}\text { Bauer/Franke/ } \\
\text { Gätschenberger } \\
\text { (1979) }\end{array}$ & $\begin{array}{l}\text { Empirische Untersuchung der Auswirkungen der Flur- } \\
\text { bereinigung auf den Erholungswert einer Landschaft. }\end{array}$ \\
\hline
\end{tabular}

Tabelle 3: Übersicht spezifischer Wirkungsanalysen der Bodenordnung

899 Unter den Begriffen "Schlaglänge und Schlaggröße« versteht man den Zuschnitt und die Größe von landwirtschaftlichen Nutzflächen.

900 Vgl. Bildungsseminar RLP (1997). 
BURGMAIER ET AL. (1995) zeigen an einem exemplarischen Beispiel, dass in Folge einer Bodenordnung eine Verbesserung der Agrarstruktur eintritt, wie beispielsweise eine Vergrößerung bzw. Verlängerung der Schlaglänge oder eine Verkürzung der HofFeld-Entfernung. Diese positiven agrarstrukturellen Veränderungen führen ihrerseits wiederum zu einer Verbesserung der Einkommenssituation in der Landwirtschaft. ${ }^{901}$

Das MinisteriUm FÜr LANDWIRTSCHAFT, WEINBAU UND FORSTEN RHEINLANDPFALZ (1991) stellt einen pauschalen Ansatz zur Bewertung flurbereinigungsbedingter Bewirtschaftungsvorteile für das Bundesland Rheinland-Pfalz zur Verfügung. Dabei werden u.a. Veränderungen der Schlaglängen, der Feldrandlängen, der mittleren HofFeld-Entfernungen sowie der Ernte- und Ausbringungsmengen berücksichtigt. Zusätzlich werden die Einsparungen bei der Arbeitszeit sowie beim Maschineneinsatz in der Bewertung berücksichtigt. ${ }^{902}$

KEYMER ET AL. (1989) ermitteln ebenfalls den Einfluss von Flurbereinigungsverfahren auf die Bewirtschaftung landwirtschaftlicher Betriebe. Die Untersuchung beschränkt sich allerdings auf zehn Regelflurbereinigungsverfahren in Bayern. ${ }^{903}$ Es konnten sowohl positive Effekte in Folge der Flächenzusammenlegung sowie durch die Verbesserung des regionalen Wegenetzes nachgewiesen werden.

KARMANN (1988) bestimmt mit Hilfe ökonometrischer Verfahren Faktoren, die sowohl den Bodenpreis als auch den Bodenmarkt landwirtschaftlich genutzter Flächen in der bayerischen Flurbereinigung berücksichtigen. Zu diesen Einflussgrößen ${ }^{904}$ zählen beispielsweise der Zeitpunkt des Landerwerbs, die Art des Flurbereinigungsverfahrens, die Flächengrößen sowie die Betriebsstruktur.

HEINZLMEIR (1983) geht ebenfalls der Frage nach, ,ob und in welchem Ausmaß der Verkehrswert des Bodens durch eine Flurbereinigung ${ }^{“ 905}$ beeinflusst wird. Für den niederbayerischen Landkreis Kelheim findet er beispielhaft heraus, dass sich sowohl der Ertrags- und Verkehrswert des landwirtschaftlich genutzten Bodens, als auch der Pachtpreis in Folge einer Bodenordnung, erhöhen.

BAUER ET AL. (1979) widerlegen in ihrer Untersuchung die auch heute noch weit verbreitete Meinung, dass Landschaften bzw. das Landschaftsbild generell durch bodenordnerische Maßnahmen an Attraktivität verlieren. Den Nachweis erbringen sie, indem sie vergleichbare geordnete und ungeordnete Gebiete hinsichtlich ihrer Vielseitigkeit, Neuartigkeit, Natürlichkeit, Zugänglichkeit und Übersichtlichkeit von Besuchern und Bewohnern beurteilen lassen. Mittels Varianzanalysen kann belegt werden, dass Regionen mit Bodenordnung tendenziell vielfältiger und natürlicher wirken. ${ }^{906}$

\footnotetext{
901 Vgl. Burgmaier/Miersch/Donié (1995).

$902 \mathrm{Vgl}$. MLWF RLP (1991).

$903 \mathrm{Vgl}$. Keymer/Linhart/Rinteln/Stumpf/Widermann (1989).

904 Vgl. Karmann (1988), S. 57.

905 Vgl. Heinzlmeir (1983), S. 75.

906 Vgl. Bauer/ Franke/Gätschenberger (1979).
} 
Der Literaturüberblick zeigt, dass mittlerweile zahlreiche Untersuchungen über die Wirksamkeit und Effektivität von Flurbereinigungsmaßnahmen vorliegen. Eine einheitliche Vorgehensweise existiert allerdings nicht. Die einzelnen Studien unterscheiden sich in ihren Zielsetzungen, ihrer Methodik und daraus resultierend auch in ihren Ergebnissen. Viele Untersuchungen beschränken sich lediglich auf einen ausgewählten Wirkungsbereich der Bodenordnung, meist im Bereich der Land- und Forstwirtschaft. Andere Studien sind dagegen breiter angelegt. Die Erkenntnisse der vorgestellten Forschungsergebnisse werden gezielt in den weiteren Untersuchungsaufbau der Wirkungsanalyse integriert.

\subsubsection{Durchführung von Befragungen}

Zur instrumentellen Unterstützung der Wirkungsanalyse und insbesondere der Wirkungsidentifikation wurden im betrachteten Projekt auch unterschiedliche Erhebungsmethoden der Befragung angewendet: ${ }^{907}$

- Mitarbeiterbefragungen: Im Rahmen des Projektes wurden eine Vielzahl informeller Einzelgespräche und zahlreiche Gruppendiskussionen mit den für die Bearbeitung von Bodenordnungsverfahren zuständigen Mitarbeitern der DLR geführt. Mitarbeitergespräche stellen in diesem Zusammenhang ein wichtiges und unverzichtbares Instrument zur explorativen Erkundung möglicher Auswirkungen im gesellschaftlichen Umfeld dar.

- Expertengespräche: Darüber hinaus wurden zahlreiche Gespräche mit Sachverständigen zur Bodenordnung bzw. zu spezifischen Themenstellungen geführt und die Ergebnisse in die Wirkungsanalyse integriert. Durch die Strukturierung der Interviews mit einem Gesprächsleitfaden lässt sich eine Expertenbefragung so gestalten, dass valide und substantielle Ergebnisse für die Untersuchung gewonnen werden können. In Anknüpfung an die Ergebnisse der Expertengespräche zur Unternehmensflurbereinigung ${ }^{908}$ in NRW lag der Schwerpunkt der Gespräche in RheinlandPfalz in den Bereichen Straßenbau, Wasserwirtschaft sowie Naherholung und Tourismus. ${ }^{909}$

- Kundenbefragungen: Im Gegensatz zum NRW-Projekt wurde im Rahmen des Projektes in Rheinland-Pfalz keine Kundenbefragung durchgeführt. Zur Beurteilung der Servicequalität konnte allerdings auf die Ergebnisse einer älteren Befragung aus dem Jahr 2002 zurückgegriffen werden. ${ }^{910}$ Für die Wirkungsanalyse sind zudem die umfassenden Ergebnisse der Kundenbefragung in NRW ${ }^{911}$ nutzbar und werden daher in den Untersuchungsablauf integriert.

\footnotetext{
907 Zum Instrument der »Befragung in der Wirkungsanalyse vgl. auch Kapitel 4.3.2.1.4.

908 In NRW wurden 12 Expertengespräche geführt. Zu den Ergebnissen vgl. BMS Consulting (2005), S. 125 ff.

909 Zu den Ergebnissen der Gespräche in Rheinland-Pfalz vgl. Mosiek/Pieper/Kasten (2006), S. $67 \mathrm{f}$.

910 Zu den Ergebnissen der Kundenbefragung vgl. Mosiek/Pieper/Kasten (2006), S. 62 ff.

911 Vgl. hierzu ausführlich Kapitel 5.2.
} 
Mit Hilfe der Analyse der intendierten Wirkungen des Flurbereinigungsgesetzes, des Literaturüberblicks bestehender Wirkungsuntersuchungen sowie umfangreicher Befragungen erfolgt nun die systematische Identifikation der Auswirkungen der Bodenordnung. Dazu wird im folgenden Kapitel das gesamtgesellschaftliche Wirkungsgefüge der Bodenordnung hergeleitet, indem zunächst eine geeignete Strukturierung der Auslöser- und Empfängerebene vorgenommen wird.

\subsubsection{Herleitung eines Wirkungsgefüges der Bodenordnung}

\subsubsection{Systematisierung der Auslöserebene}

Der erste Schritt zur Herleitung eines gesamtgesellschaftlichen Wirkungsgefüges der Bodenordnung besteht in einer geeigneten Systematisierung der Auslöserebene. ${ }^{912} \mathrm{Da}$ es sich bei Bodenordnungsverfahren um sehr umfangreiche staatliche Maßnahmen handelt, mit Bearbeitungszeiten von teilweise mehr als zehn Jahren, wird der "Produktbereich Ländliche Bodenordnung nach dem FlurbG« in wichtige Teilprozesse gegliedert. Abbildung 66 gibt einen Überblick über die vorgenommene Strukturierung der Auslöserebene.

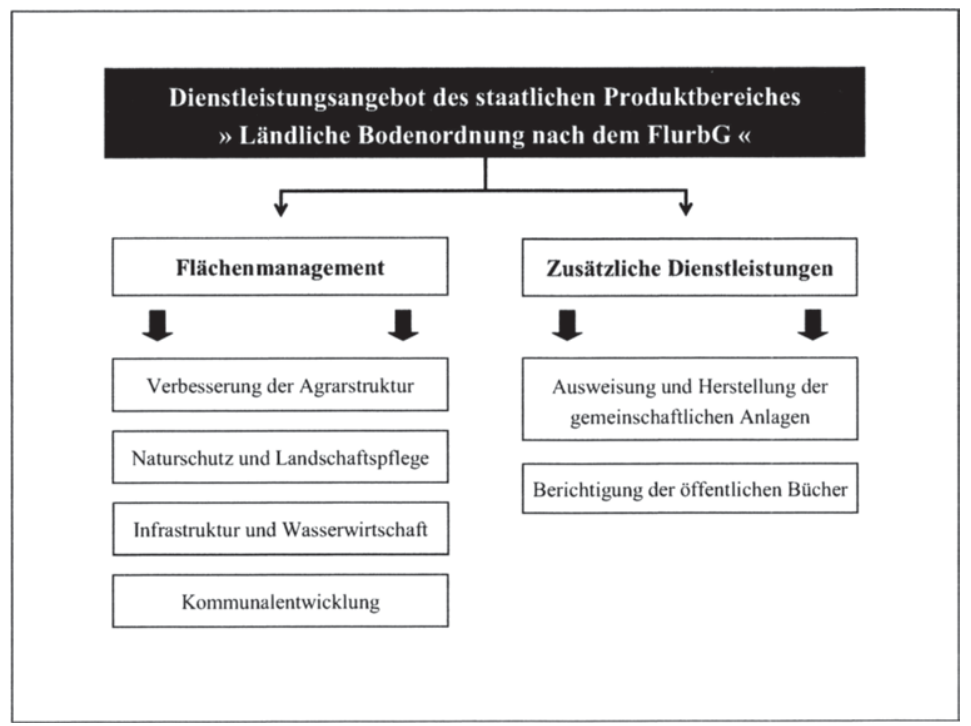

Abbildung 66: Auslöserebene der Bodenordnung

Demnach werden die Teilprozesse im Bereich der Bodenordnung zunächst in Dienstleistungen des Flächenmanagements und in zusätzliche Dienstleistungen der Verwal-

912 Zum pragmatischen Analysemodell der Auslöserebene vgl. Kapitel 4.3.2.1.1. 
tung eingeteilt. ${ }^{913}$ Aufgabe des Flächenmanagements durch Bodenordnung ist nicht die isolierte Entwicklung und Förderung von Teilbereichen, wie bspw. der Landwirtschaft, dem Verkehrswesen oder der Wasserwirtschaft. Die Aufgabe besteht vielmehr darin, die verschiedenen - teilweise stark konkurrierenden - Fachplanungen und Zielsetzungen im ländlichen Raum nach Möglichkeit zu harmonisieren und miteinander zu vereinbaren. Entsprechend werden die Dienstleistungen des Flächenmanagements nach den wesentlichen Zielsetzungen von Projekten im ländlichen Raum strukturiert. Dies betrifft insbesondere Agrarstrukturverbesserungen, Naturschutz und Landschaftspflege, Infrastruktur und Wasserwirtschaft sowie die Kommunalentwicklung. ${ }^{914}$ Zusätzlich $\mathrm{zu}$ diesen Hauptdienstleistungen leistet die Verwaltung noch Unterstützung bei der Herstellung und Ausweisung gemeinschaftlicher Anlagen sowie bei der Berichtigung und Bereinigung der öffentlichen Bücher (z.B. Grundbuch).

Die Strukturierung der Auslöserebene gewährleistet eine bessere Übersichtlichkeit bei der Zuordnung von Wirkungskomponenten zu einzelnen Dienstleistungen der Verwaltung. Ansätze zur Prozessverbesserung und zu einer gesteigerten Wirksamkeit des Verwaltungshandelns werden transparent. Gleichzeitig lässt sich die vorgenommene Strukturierung flexibel ergänzen oder erweitern, so dass der Komplexität und den Spezifika einzelner Bodenordnungsverfahren in der Wirkungsanalyse angemessen Rechnung getragen werden kann.

\subsubsection{Systematisierung der Empfängerebene}

Der zweite Schritt zur Herleitung eines gesamtgesellschaftlichen Wirkungsgefüges der Bodenordnung besteht in der Systematisierung der Empfängerebene. ${ }^{915}$ Entsprechend dem pragmatischen Analysemodell der Empfängerebene werden auf der Makroebene zunächst die zentralen gesellschaftlichen Subsysteme differenziert - Bürger, Wirtschaft, Staat und Umwelt. In Abbildung 67 ist die weitere Aufgliederung dieser gesellschaftlichen Subsysteme auf der Mesoebene dargestellt. Um den notwendigen Analyseumfang zu begrenzen, wurde auf eine Untersuchung der Mikroebene verzichtet. Bodenordnungsverfahren zeichnen sich in der Regel durch eine sehr hohe Zahl einzelner Wirkungsempfänger aus, so dass die Betrachtung der Mikroebene den Komplexitätsgrad der Wirkungsanalyse unangemessen erhöht hätte. ${ }^{916}$

Unter dem Begriff Bürger werden im Zusammenhang mit der Bodenordnung zum einen die betroffenen Grundstückseigentümer und zum anderen die regional betroffene Allgemeinheit differenziert. Der regionalen Allgemeinheit werden alle Wirkungskomponenten zugeordnet, die sich auf keine spezifische gesellschaftliche Bürgergruppe auswirken, sondern die Region als Gesamtheit betreffen - im Sinne der Auswirkungen

913 Vgl. auch Mosiek/Pieper/Kasten (2006), S. $92 \mathrm{ff}$.

$914 \mathrm{Vgl}$. hierzu auch die gesetzlichen Zielsetzungen der Bodenordnung in Kapitel 5.3.4.1.

915 Zum pragmatischen Analysemodell der Empfängerebene vgl. Kapitel 4.3.2.1.2.

916 Exemplarisch kann hier auf die Ergebnisse der Kundenbefragung in NRW zurückgegriffen werden, in der konkrete Wirkungsbeurteilungen auf der Mikroebene vorgenommen wurden. Vgl. auch Kapitel 5.2.4. 
auf das Gemeinwohl. Im Bereich Wirtschaft werden bei der Bodenordnung der landund forstwirtschaftliche Sektor ${ }^{917}$ sowie der Industrie- und Dienstleistungssektor separat betrachtet. ${ }^{918}$

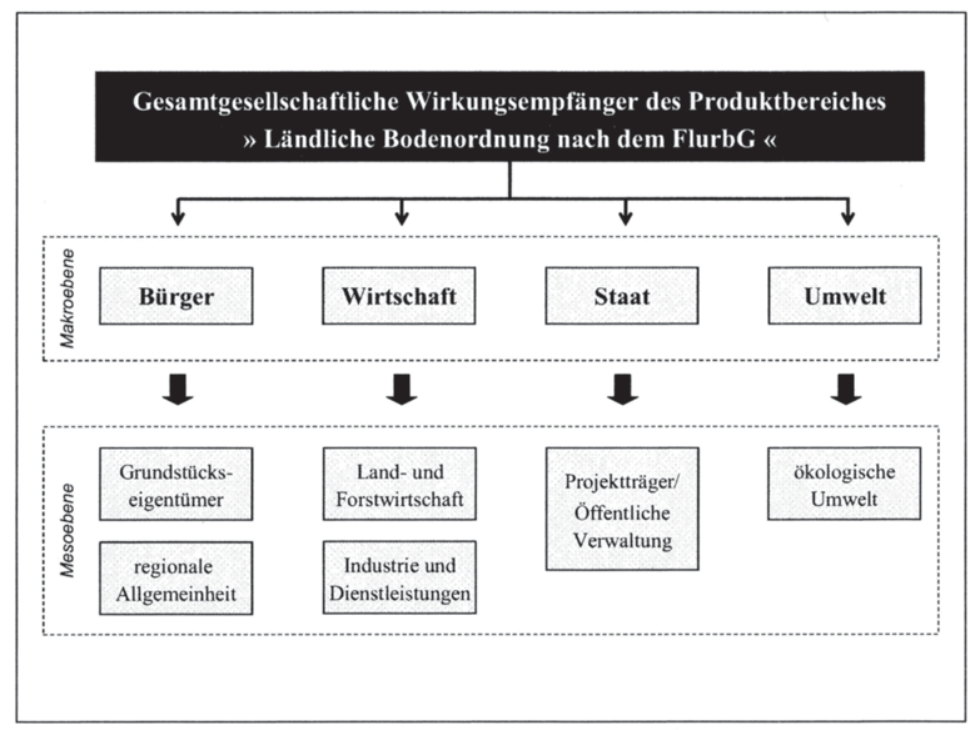

Abbildung 67: Empfängerebene der Bodenordnung

Der Staat fungiert im Rahmen der Bodenordnung häufig als Träger von Projekten, beispielsweise als Träger von Straßenbauprojekten oder von Hochwasserschutzmaßnahmen. Der Staat umfasst dabei begrifflich sämtliche Ausführungsebenen, d.h. sowohl den Bund, die Länder als auch die Kommunen. ${ }^{919}$ Sind im Zusammenhang mit der Bodenordnung Auswirkungen auf staatliche Vollzugsebenen bzw. einzelne Behörden zu erwarten, werden sie an dieser Stelle in die Untersuchung integriert. Die letzte wesentliche Empfängerebene stellt die Umwelt dar, wo sämtliche ökologischen Effekte auf die regionale Flora, Fauna oder das allgemeine Landschaftsbild erfasst werden.

\subsubsection{Identifikation der relevanten Wirkungskomponenten}

Nachfolgend sollen die gesamtgesellschaftlichen Auswirkungen der Bodenordnung möglichst umfassend identifiziert und dabei detailliert hergeleitet und beschrieben werden. Zur besseren Nachvollziehbarkeit der einzelnen Wirkungskomponenten orien-

917 Zum land- und forstwirtschaftlichen Sektor werden nicht nur die Agrar- und Forstwirtschaft, sondern auch der Weinbau gezählt, welcher in Rheinland-Pfalz eine bedeutende Rolle spielt.

918 Vgl. Mosiek/Pieper/Kasten (2006), S. 94 f.

919 Zum Aufbau des öffentlichen Sektors in Deutschland vgl. Kapitel 2.1.2. 
tiert sich die Reihenfolge der Darstellung an der Empfängerdimension des gesellschaftlichen Wirkungsgefüges der Bodenordnung. Die inhaltlichen Ausführungen fassen die Erkenntnisse der vorausgegangenen Untersuchungsschritte - Analyse der intendierten Zielsetzungen des Gesetzgebers, Integration der bisherigen Forschungsergebnisse und die erzielten Befragungsergebnisse - zusammen.

\subsection{Auswirkungen der Bodenordnung auf die Bürger}

\begin{tabular}{|ll|}
\hline Wirkung: & Verringerung oder Vermeidung von Eigentumsverlusten sowie Ein- \\
& sparung ansonsten anfallender Transaktionskosten \\
Empfänger: & Grundstückseigentümer \\
Wirkungstyp: & gesetzlich intendierte Wirkung \\
\hline
\end{tabular}

Der Gesetzgeber legt fest, dass bei der Neugestaltung eines Flurbereinigungsgebietes die Interessen aller Beteiligten zu berücksichtigen bzw. gegebenenfalls gegeneinander abzuwägen sind. ${ }^{920}$ Daraus ergibt sich die Forderung, Beeinträchtigungen der betroffenen Grundstückseigentümer zu minimieren. Die Durchführung einer Unternehmensflurbereinigung setzt sogar explizit die Möglichkeit voraus, den bei Großprojekten „den Betroffenen entstehenden Landverlust auf einen größeren Kreis von Eigentümern verteilen zu können“"921.

Häufig gelingt es im Rahmen der Bodenordnung sogar, Verluste an Eigentumsflächen gänzlich zu vermeiden, indem den betroffenen Eigentümern gleichwertige oder unter Umständen sogar qualitativ hochwertigere Ersatzflächen beschafft werden. Ohne die Unterstützung der Flurbereinigungsverwaltung ist ein derartiger Landtausch zwischen den Eigentümern und den Trägern der geplanten Maßnahmen in der Regel nicht möglich, insbesondere nicht, wenn die Umsetzung der Planung mit einem hohen Flächenbedarf verbunden ist. Dies ist vor allem für im öffentlichen Interesse liegende Infrastrukturprojekte, beispielsweise im Straßenbau, der Fall.

Seitens der Grundstückseigentümer besteht jedoch häufig der Wunsch nach Ersatz der entzogenen Flächen. ${ }^{922}$ Diesem Wunsch kann bei Verzicht auf eine begleitende Bodenordnung nur durch den Kauf neuer Flächen entsprochen werden. Beim käuflichen Erwerb von Flächen fallen grundsätzlich Notargebühren und Gebühren für Grundbucheintragungen an. Beim Kauf von Teilflächen entstehen zusätzlich Vermessungsgebühren und teilweise sind auch noch Maklergebühren zu entrichten. Als positive Folgewirkung eines Bodenordnungsverfahrens können entsprechende Gebühren durch die Bereitstellung von Ersatzland häufig eingespart werden.

920 Vgl. §1 FlurbG.

$921 \$ 87$ (1) FlurbG.

$922 \mathrm{Zu}$ den Ergebnissen der Befragung der Grundstückseigentümer im Projekt in Nordrhein-Westfalen vgl. Kapitel 5.2 dieser Arbeit. 


\section{Wirkung: $\quad$ Erhöhung der Rechtssicherheit des Eigentums sowie Verringerung hieraus resultierender Rechtsstreitigkeiten \\ Empfänger: Grundstückseigentümer \\ Wirkungstyp: positive Folgewirkung}

Zur Festlegung der exakten Besitzstücke werden die Gebiete in der Bodenordnung in der Regel komplett neu vermessen. Die Ergebnisse werden in modernen Karten- und Vermessungswerken dargestellt, die als Grundlage für die Berichtigung der öffentlichen Bücher dienen. ${ }^{923}$ Die ursprünglichen Grundbuch- und Katasterunterlagen weisen häufig erhebliche Ungenauigkeiten auf, insbesondere wenn ihre Erstellung noch auf Urvermessungen basiert. Diese Ungenauigkeiten können zu zeitintensiven und kostspieligen Rechtsstreitigkeiten zwischen den Eigentümern führen, die durch eine zeitgemäße Aktualisierung der Bücher im Rahmen der Flurbereinigung in großem Umfang vermieden werden können. Das genaue Ausmaß dieses Effektes hängt jedoch zweifellos entscheidend von der Qualität des zugrunde liegenden Liegenschaftskatasters ab. ${ }^{924}$ Festzuhalten bleibt damit, dass die Durchführung von Bodenordnungsverfahren im Allgemeinen eine wesentliche Erhöhung der Rechtssicherheit in Bezug auf die Eigentumsverhältnisse an Grund und Boden zur Folge hat und so zu einer Verringerung hieraus resultierender Rechtsstreitigkeiten beiträgt.

\section{Wirkung: $\quad$ Steigerung bzw. Sicherung des Bodenwertes \\ Empfänger: Grundstückseigentümer \\ Wirkungstyp: gesetzlich intendierte Wirkung/ positive Nebenwirkung ${ }^{925}$}

Der Wert des Bodens und damit eng verbunden auch der Pachtpreis ländlicher Grundstücke hängen entscheidend von der gegenwärtigen und der zukünftig zu erwartenden Nutzbarkeit der Grundstücke ab:

- „Der Verkehrswert (Marktwert) wird durch den Preis bestimmt, der in dem Zeitpunkt, auf den sich die Ermittlung bezieht, im gewöhnlichen Geschäftsverkehr nach den rechtlichen Gegebenheiten und tatsächlichen Eigenschaften, der sonstigen Beschaffenheit und der Lage des Grundstücks und des sonstigen Gegenstands der Wertermittlung ohne Rücksicht auf ungewöhnliche oder persönliche Verhältnisse zu erzielen wäre. ${ }^{\text {"9926 }}$

923 Vgl. Keymer et al. (1989), S. 12.

Vgl. BMS Consulting (2005), S. $159 \mathrm{ff}$.

925 Der Wirkungstyp ist vom konkreten gesetzlichen Verfahrenstyp der Bodenordnung abhängig. Nur bei der Regelflurbereinigung kann direkt von einer gesetzlich intendierten Steigerung des Ertragswertes und damit des Bodenwertes der landwirtschaftlichen Nutzflächen gesprochen werden. Vgl. auch Kapitel 5.1.2.

926 §194 Baugesetzbuch (BauGB). 
Die Regelflurbereinigung hat explizit die „Verbesserung der Arbeits- und Produktionsbedingungen in der Land- und Forstwirtschaft" zum Ziel ${ }^{927}$ Die Wirtschaftlichkeit und Wettbewerbsfähigkeit land- und forstwirtschaftlicher Betriebe wird sicherlich von zahlreichen Einflussfaktoren bestimmt. Eine besondere Bedeutung besitzt jedoch die rationelle Gestaltung landwirtschaftlicher Nutzflächen in Bezug auf ihre Form und Größe sowie ihrer Erschließbarkeit. Dies bildet die Grundlage für eine effiziente Flächenbewirtschaftung. Der positive Einfluss der Bodenordnung auf die Bewirtschaftung landwirtschaftlicher Betriebe wurde bereits im Rahmen zahlreicher Untersuchungen nachgewiesen ${ }^{928}$ Im Zuge der Neuordnung des Flurbereinigungsgebietes werden in der Regel die Schlaggröße und die Schlagform der Nutzflächen optimiert, die bestehenden Hof-Feld-Entfernungen reduziert sowie neue Wirtschaftswege angelegt oder das bestehende Wegenetz ausgebaut. Dadurch wird der Arbeitsaufwand bei der Bewirtschaftung des landwirtschaftlichen Bodens vermindert und sein Ertragswert langfristig gesichert und in der Regel sogar erhöht. In der Folge lassen sich aufgrund des gesteigerten Ertragswertes auch höhere Boden- oder Pachtpreise erzielen. Bodenordnungsmaßnahmen tragen somit grundsätzlich zur Sicherung/ Steigerung des Bodenwertes bei.

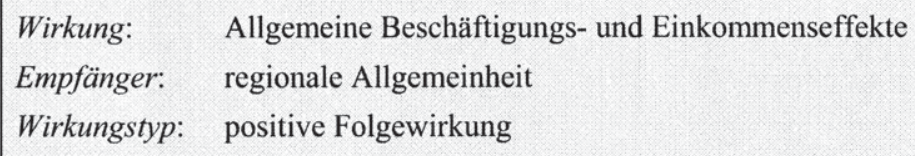

Wie bereits im Zusammenhang mit der Steigerung des Bodenwertes erläutert, führt eine Bodenordnung meist zu agrarstrukturellen Verbesserungen. Mit einer aus betriebswirtschaftlicher Sicht optimalen Flächengestaltung und der Verbesserung der Wegequalität sind i.d.R. erhebliche Arbeitszeiteinsparungen verbunden, insbesondere wenn durch die Neugestaltung der Grundstücke der Einsatz größerer Maschinen ermöglicht wird. In Verbindung mit weiteren Rationalisierungsmaßnahmen in Folge einer Bodenordnung wird nicht selten eine höhere Effizienz in der Flächenbewirtschaftung erzielt, die sich wiederum positiv auf die allgemeine Einkommens- und Beschäftigungssituation im regionalen Agrarsektor auswirkt. Durch die Möglichkeit einer effizienteren Bewirtschaftung kann das jährliche Betriebseinkommen erhöht und im Zuge einer verbesserten Wettbewerbsfähigkeit können Arbeitsplätze gesichert oder gegebenenfalls sogar neu geschaffen werden. Die flexible Flächenausweisung in der Bodenordnung kann zusätzlich zum Erhalt von existenzbedrohten Betrieben sowie der hiermit verbundenen Arbeitsplätze beitragen. Teilweise werden Flächen sogar erst im Zuge der Bodenordnung neu erschlossen und damit erstmalig einer wirtschaftlichen Nutzung zugänglich.

927 Vgl. §1 FlurbG.

928 Vgl. beispielsweise Keymer et al. (1989), Burgmaier et al. (1995) oder Klare et al. (2005). 


\section{Wirkung: Beschleunigung oder Realisierung von Infrastrukturprojekten \\ Empfänger: regionale Allgemeinheit \\ Wirkungstyp: intendierte gesetzliche Wirkung/ positive Nebenwirkung}

Die Realisierung öffentlicher Infrastrukturprojekte kann sich in vielerlei Hinsicht positiv auf eine Volkswirtschaft auswirken. ${ }^{929}$ Der Neu- und Ausbau von Straßen- oder Schienenwegen kann beispielsweise zu verringerten Fahrtzeiten, geringerer Staugefahr, verminderter Unfallgefahr, weniger Lärmbelästigung und reduzierten Immissionsbelastungen führen. ${ }^{930} \mathrm{Im}$ Allgemeinen ist die Einleitung eines Bodenordnungsverfahrens jedoch keine notwendige Bedingung für die Durchführung von Infrastrukturmaßnahmen, insbesondere dann nicht, wenn es sich um große Bauvorhaben handelt, die im Interesse der Öffentlichkeit liegen.

Durch die Unterstützung der Flurbereinigungsverwaltung kann die Umsetzung der Planungen jedoch in der Regel beschleunigt werden. Dieser Beschleunigungseffekt ergibt sich im Wesentlichen daraus, dass die Bodenordnung eine flexiblere Gestaltung des Flächenerwerbs für das Infrastrukturvorhaben ermöglicht. Während die Flurbereinigungsverwaltung die Möglichkeit zur kompletten Neugestaltung des Verfahrensgebietes und zum freihändigen Tausch der Grundstücke hat, ist der Projektträger ansonsten gezwungen, die direkt in der Trasse liegenden Eigentumsflächen zu kaufen. Die Wahrscheinlichkeit, dass sich die Projektverwirklichung durch eventuelle Einsprüche oder Klagen der Grundeigentümer verzögert, ist bei Verzicht auf die Durchführung einer Flurbereinigung demnach wesentlich höher. Falls sogar ein gerichtliches Enteignungsverfahren notwendig ist, kann diese Verzögerung beträchtliche Dimensionen annehmen. ${ }^{931}$

Eine Befragung der Grundstückseigentümer hat ergeben, dass die Einleitung eines begleitenden Bodenordnungsverfahrens zudem zu einer Erhöhung der Akzeptanz von Infrastrukturmaßnahmen führt. ${ }^{932}$ Demnach ist davon auszugehen, dass durch geringere Widerstände und das Flächenmanagement der Bodenordnung Bauprojekte früher realisiert werden können. Der aus einer früheren Projektrealisierung resultierende gesellschaftliche Wert kann aber nicht in vollem Umfang der Bodenordnung zugerechnet werden. Als Wirkungsbeitrag ist lediglich der Teil der Auswirkungen zu bewerten, der auf die beschleunigte Flächenbereitstellung für das Infrastrukturprojekt zurückzuführen ist. In Einzelfällen ist aber auch denkbar, dass die Umsetzung von Infrastrukturmaßnahmen erst durch eine begleitende Bodenordnung ermöglicht wird, vor allem wenn es sich um kleinere, sehr spezifische Planungen handelt. In diesen Fällen könnte sogar der gesamte gesellschaftliche Projektwert der Bodenordnung zugerechnet werden.

\footnotetext{
929 Vgl. BMS Consulting (2005), S. 155.

${ }^{930} \mathrm{Zu}$ den positiven Wirkungen des Verkehrs vgl. Hartwig et al. (2005).

${ }^{931}$ Ein Enteignungsverfahren dauert i.d.R. drei bis fünf Jahre. Vgl. Mosiek/Pieper/Kasten (2006), S. 100 f.

$932 \mathrm{Vgl}$. BMS Consulting (2005), S. $104 \mathrm{ff}$.
} 


\section{Wirkung: Unterstützung oder Realisierung von Naturschutzprojekten \\ Empfänger: regionale Allgemeinheit \\ Wirkungstyp: intendierte gesetzliche Wirkung/ positive Nebenwirkung}

Die Umsetzung von Naturschutzmaßnahmen ist im Vergleich zu Infrastrukturprojekten wesentlich häufiger an die Durchführung eines Bodenordnungsverfahrens gebunden, da außerhalb des Flächenmanagements einer Bodenordnung eine ökologisch sinnvolle Flächenausweisung sehr schwierig ist. Falls ökologische Planungen allerdings auch ohne die Unterstützung der Flurbereinigungsverwaltung verwirklicht werden können, ist jedoch im Rahmen der Bodenordnung zumindest von einer kostengünstigeren Umsetzung auszugehen. ${ }^{933}$ Hier wirken wiederum die Dienstleistungen des Flächenmanagements.

\section{Wirkung: Beschleunigung oder Realisierung kommunaler Projekte \\ Empfänger: regionale Allgemeinheit \\ Wirkungstyp: intendierte gesetzliche Wirkung/ positive Nebenwirkung}

Für kommunale Planungen gelten in Abhängigkeit der Projektart die vorangegangen Ausführungen zur Ermöglichung, Beschleunigung und Unterstützung von Infrastruktur- und Naturschutzprojekten. Es muss stets zwischen Maßnahmen unterschieden werden, die im Zuge der Flurbereinigung unterstützt oder beschleunigt werden und solchen, die ohne begleitende Bodenordnung nicht durchgeführt werden könnten. Im ersten Fall ist nur der Wirkungsbeitrag aus der Beschleunigung in Ansatz zu bringen. Im zweiten Fall können die gesamten gesellschaftlichen Effekte aus der Projektrealisierung der Bodenordnung zugerechnet werden.

$$
\begin{array}{ll}
\text { Wirkung: } & \text { Vorteile aus der Verbesserung des regionalen Wegenetzes } \\
\text { Empfänger: } & \text { regionale Allgemeinheit } \\
\text { Wirkungstyp: } & \text { intendierte gesetzliche Wirkung }
\end{array}
$$

Planung und Ausbau der gemeinschaftlichen Anlagen und damit des Wegenetzes gehören zu den gesetzlich intendierten Zielsetzungen der Bodenordnung. ${ }^{934}$ Die flurbereinigungsbedingte Verbesserung der Wegequalität wirkt sich im Wesentlichen auf drei verschiedene Bereiche aus. Zum einen sind durch den Aus- und Neubau des Wegenetzes positive Effekte für die Allgemeinheit, insbesondere für die Bewohner der betreffenden Region, zu erwarten. $\mathrm{Zu}$ diesen positiven Effekten können beispielsweise die

$933 \mathrm{Vgl}$. Mosiek/Pieper/Kasten (2006), S. 101.

934 Vgl. §41 FlurbG. 
Steigerung des Freizeit- und Erholungswertes gehören, da die Kulturlandschaft in Ausflügen bereist werden kann. Zudem können die Anwohner Zeitersparnisse durch Umwegeeinsparungen realisieren. Diese Auswirkungen der Bodenordnung werden an dieser Stelle in Ansatz gebracht. Zum anderen ist die Verbesserung des Wegenetzes häufig mit Zeitvorteilen für die Landwirte bei der Bewirtschaftung verbunden. ${ }^{935}$ Des Weiteren profitiert in der Regel auch die regionale Tourismusbranche von einer Erneuerung des Wegenetzes, da Gäste die Kulturlandschaft bereisen können. ${ }^{936}$ Im Allgemeinen kann davon ausgegangen werden, dass die Wegebaumaßnahmen ohne bodenordnerische Unterstützung nicht durchgeführt worden wären, so dass die daraus resultierenden positiven Effekte in vollem Umfang als Wirkung der Bodenordnung gezählt werden kann. Um eine Über- bzw. Doppelbewertung der Effekte zu vermeiden, wird unterstellt, dass sich die Wirkung zu etwa gleichen Teilen auf die genannten Bereiche - regionale Allgemeinheit, Land- und Forstwirtschaft sowie Tourismus - verteilen.

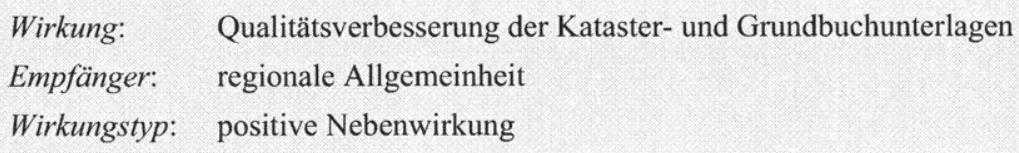

Die Neuordnung eines Flurbereinigungsgebietes macht in der Regel eine vollständige Neuvermessung des Verfahrensgebietes erforderlich, damit die rechtlichen Ansprüche der betroffenen Grundstückseigentümer auf Sicherung der gesetzlichen Landabfindungsansprüche gewahrt werden können. Darüber hinaus muss sichergestellt sein, dass die Flurbereinigung den Ansprüchen genügt, um vorübergehend als amtliches Verzeichnis der Grundstücke zu dienen. ${ }^{937}$ Der hiermit einhergehenden „Erneuerung des Liegenschaftskatasters kommt insbesondere in Zeiten, in denen eine systematische Katastererneuerung durch die Kataster- und Vermessungsverwaltungen kaum noch betrieben wird, eine erhebliche Bedeutung zu“. ${ }^{938}$ Der Gesetzgeber fordert jedoch, das Liegenschaftskataster entsprechend den gesellschaftlichen Anforderungen regelmäßig zu aktualisieren. ${ }^{939}$ Die Bodenordnung leistet einen wesentlichen Beitrag zur Erfüllung des gesetzlichen Auftrags nach dem Vermessungs- und Katastergesetz. ${ }^{940}$ Daher kann ein Teil der entstehenden Vermessungskosten als Wirkungskomponente der Bodenordnung angesehen werden.

\footnotetext{
935 Vgl. hierzu detailliert die Auswirkungen des Wegenetzes auf die Landwirtschaft in Kapitel 5.3.5.3.2.

$936 \mathrm{Vgl}$. hierzu detailliert die Auswirkungen des Wegenetzes auf die Tourismusbranche in Kapitel 5.3.5.3.2.

937 Vgl. \$2 (2) Grundbuchordnung (GBO).

938 Vgl. Prell et al. (2003), S. 30.

$939 \mathrm{Vgl}$. 1 (3)Vermessungs- und Katastergesetz (VermKatG).

940 Vgl. BMS Consulting (2005), S. $183 \mathrm{ff}$.
} 


$$
\begin{array}{ll}
\text { Wirkung: } & \begin{array}{l}
\text { Beschäftigungs- und Einkommenseffekte durch Infrastruktur- } \\
\text { investitionen in die gemeinschaftlichen Anlagen }
\end{array} \\
\text { Empfänger: } & \text { regionale Allgemeinheit } \\
\text { Wirkungstyp: } & \text { positive Folgewirkung }
\end{array}
$$

Infrastrukturinvestitionen wirken sich im Allgemeinen positiv auf die Einkommensund Beschäftigungssituation in einer Volkswirtschaft aus. ${ }^{941} \mathrm{Zu}$ unterscheiden sind im Wesentlichen direkte Einkommens- und Beschäftigungseffekte für die mit der Umsetzung des Bauvorhabens beauftragten Unternehmen sowie indirekte Auswirkungen auf ihre Vorleistungs- und Investitionsgüterlieferanten. Die Einkommenssteigerung in der Baubranche kann zusätzlich positive Wachstumseffekte im produzierenden Gewerbe auslösen, wenn davon ausgegangen wird, dass ein höheres verfügbares Einkommen einen erhöhten Konsum zur Folge hat. ${ }^{942}$ Der gesamte hieraus resultierende gesellschaftliche Vorteil lässt sich nur bei den Investitionen in die gemeinschaftlichen Anlagen direkt der Bodenordnung zuschreiben. Ansonsten ist ein Ansatz nur in Ausnahmefällen möglich, nämlich genau dann, wenn das Bauprojekt ohne Unterstützung der Bodenordnung nicht realisiert werden könnte.

\section{Wirkung: Verbesserung des überregionalen Hochwasserschutzes \\ Empfänger: regionale Allgemeinheit \\ Wirkungstyp: intendierte gesetzliche Wirkung/ positive Nebenwirkung}

$\S 1$ i.V.m. $\$ 37$ (2) FlurbG bestimmt, dass bei der Neuordnung und Entwicklung des ländlichen Raumes die Belange der Wasserwirtschaft zu berücksichtigen sind. ${ }^{943}$ Dies beinhaltet ebenfalls die mit der Verabschiedung des Wasserhaushaltsgesetzes (WHG) hinzugekommenen Bestimmungen. §31d WHG legt fest, dass in Abhängigkeit der Erforderlichkeit Hochwasserschutzpläne mit dem Ziel aufzustellen sind, „die Gefahren, die mindestens von einem statistisch einmal in 100 Jahren $\mathrm{zu}$ erwartenden Hochwasser ausgehen, so weit wie möglich und verhältnismäßig zu minimieren“.944 "In die Hochwasserschutzpläne sind insbesondere Maßnahmen zum Erhalt und zur Rückgewinnung von Rückhalteflächen, zu deren Flutung und Entleerung nach den Anforderungen des optimierten Hochwasserabflusses in Flussgebietseinheiten, zur Rückverlegung von Deichen, zum Erhalt oder zur Wiederherstellung von Auen sowie zur Rückhaltung von Niederschlagswasser, aufzunehmen. ${ }^{\text {“945 }}$ Die Bodenordnung kann vor allem in Hinblick auf eine sinnvolle und zeitnahe Flächenausweisung einen wertvollen Beitrag zur Um-

\footnotetext{
941 Vgl. Hartwig et al. (2005), S. $48 \mathrm{ff}$.

$942 \mathrm{Vgl}$. Hartwig et al. (2005), S. $52 \mathrm{ff}$.

$943 \mathrm{Vgl}$ \$1 und $\$ 37$ (2) FlurbG.

944 Vgl. §31d Wasserhaushaltsgesetz (WHG).

945 Vgl. §31d Wasserhaushaltsgesetz (WHG).
} 
setzung dieser überregionalen Hochwasserschutzmaßnahmen leisten. Die wasserwirtschaftlichen Belange rechtfertigen auch für sich alleine die Einleitung und Durchführung eines Bodenordnungsverfahrens. Grundsätzlich sind wieder zwei alternative flurbereinigungsbedingte Effekte zu erwarten. Entweder ein Beschleunigungseffekt bei der Projektrealisierung oder im Extremfall sogar die Ermöglichung der geplanten wasserwirtschaftlichen Maßnahmen durch das Flächenmanagement innerhalb der Bodenordnung. ${ }^{946}$
Wirkung: Vermeidung örtlicher Hochwasserschäden
Empfänger: regionale Allgemeinheit
Wirkungstyp: positive Nebenwirkung

Wie bereits im Rahmen des verbesserten überregionalen Hochwasserschutzes erläutert, hat die Flurbereinigung die Interessen wasserwirtschaftlicher Planungen zu wahren. In diesem Zusammenhang bestehen die wesentlichen Aufgaben der Bodenordnung darin, unter Berücksichtigung des deutschen Wasserhaushaltsgesetzes nachhaltige Veränderungen des mengenmäßigen und chemischen Zustands des Grundwassers zu vermeiden sowie ein Gleichgewicht zwischen Wasserentnahme und -neubildung zu gewährleisten. ${ }^{947}$ Aus einer nachteiligen Grundwassersituation resultieren örtlich häufig gravierende finanzielle Schäden. Die Nutzbarkeit betroffener Flächen kann stark eingeschränkt sein oder nur unter erheblichem finanziellen Aufwand erfolgen. Des Weiteren zieht ein zu feuchter Untergrund Gebäudeschäden nach sich. Zudem verursachen starke Regenfälle und Überschwemmungen in den Ortslagen nicht unerhebliche Schäden. Durch gezielte Maßnahmen trägt die Bodenordnung dazu bei, derartige Gefahren zu beseitigen bzw. sie beschleunigt ihre Beseitigung. Beispielsweise werden lokal Rückhalteräume ausgewiesen, so dass entsprechende örtliche Hochwasserschäden nicht mehr auftreten oder zumindest verringert werden. ${ }^{948}$

\begin{tabular}{|ll} 
Wirkung: & $\begin{array}{l}\text { Erhalt und Förderung der Kulturlandschaft und des } \\
\text { allgemeinen Landschaftsbildes }\end{array}$ \\
Empfänger: & regionale Allgemeinheit \\
Wirkungstyp: & gesetzlich intendierte Wirkung
\end{tabular}

Die traditionelle Bodenordnung war primär auf die Verbesserung der agrarstrukturellen Gegebenheiten in ländlichen Gebieten ausgerichtet. ${ }^{949}$ Ökologische und land-

\footnotetext{
$946 \mathrm{Vgl}$. Mosiek/Pieper/Kasten (2006), S. $103 \mathrm{f}$.

947 Vgl. Wasserhaushaltsgesetz (WHG).

$948 \mathrm{Vgl.} \mathrm{Mosiek/Pieper/Kasten} \mathrm{(2006),} \mathrm{S.} 105$.

949 Zum Aufgabenwandel der Bodenordnung vgl. ausführlich Kapitel 5.1.1.
} 
schaftspflegerische Zielsetzungen spielten damals eher eine untergeordnete Rolle. Mit zunehmendem Umweltbewusstsein hielten sie jedoch Einzug in die Flurbereinigungsgesetzgebung. Mittlerweile gehören Maßnahmen zum Schutz des Naturhaushaltes und zum Erhalt oder der Wiederherstellung des ökologischen Gleichgewichts sowie zur Landschaftspflege zu den wichtigsten Aufgaben der Bodenordnung. Es kann also von positiven Wirkungen der Bodenordnung auf den Erlebniswert einer Landschaft ausgegangen werden, insbesondere wenn die vielfältigen, oftmals konträr wirkenden Fachplanungen in einer Region optimal aufeinander abzustimmen sind. Durch Unterstützung der Flurbereinigungsverwaltung können die negativen ökologischen Auswirkungen dieser Projekte in der Regel vermieden werden. Zusätzlich erleichtert bzw. ermöglicht das Flächenmanagement unmittelbar die Verwirklichung landschaftspflegerischer und ökologischer Maßnahmen. BAUER ET AL. (1979) konnten mittels Varianzanalysen sogar quantitativ nachweisen, dass Regionen nach einer Bodenordnung tendenziell als natürlicher und in landschaftlicher Hinsicht vielfältiger empfunden werden als vergleichbare Gebiete ohne Bodenordnung. ${ }^{950}$

\begin{tabular}{|ll|}
\hline Wirkung: & Verbesserung der Naherholung und des Freizeitwertes \\
Empfänger: & regionale Allgemeinheit \\
Wirkungstyp: & positive Folgewirkung \\
\hline
\end{tabular}

Mit den positiven Auswirkungen der Flurbereinigung auf die natürliche Umwelt, die regionale Kulturlandschaft und das allgemeine Landschaftsbild, ist im Regelfall auch eine Steigerung des Naherholungs- und Freizeitwertes verbunden. Attraktivität und Erlebniswert der Landschaft werden durch den Ausbau des regionalen Rad- und Wanderwegenetzes zusätzlich erhöht. Entsprechende Maßnahmen würden ohne Unterstützung der Bodenordnung nicht realisiert.

\subsection{Auswirkungen der Bodenordnung auf die Wirtschaft}
Wirkung:
Bewirtschaftungsvorteile durch Flächenneuordnung
Empfänger:
Land- und Forstwirtschaft
Wirkungstyp: intendierte gesetzliche Wirkung/ positive Nebenwirkung

Der wirtschaftliche Erfolg landwirtschaftlicher Betriebe hängt entscheidend von der rationellen Gestaltung der zu bearbeitenden Nutzfläche ab. Die gesetzliche Hauptzielsetzung der Regelflurbereinigung besteht in einer Flächenneuordnung, um Bewirtschaftungsvorteile für die ansässigen landwirtschaftlichen Betriebe $\mathrm{zu}$ realisieren. ${ }^{951}$

\footnotetext{
$950 \mathrm{Vgl}$. Bauer et al. (1979), S. $56 \mathrm{ff}$.

951 Vgl. §l FlurbG.
} 
Ein weiteres Aufgabenfeld der Bodenordnung ist die Beseitigung der agrarstrukturellen Beeinträchtigungen bei der Umsetzung großer Infrastrukturvorhaben im ländlichen Raum. ${ }^{952}$ Bei entsprechenden Verfahren können die Produktions- und Arbeitsbedingungen im Zuge der Flächenneuordnung häufig sogar über den vorherigen Zustand hinaus verbessert werden. ${ }^{953}$ Im Rahmen der Flächenneuordnung werden die Flurstücke im Verfahrensgebiet durch Zusammenlegen nach Form und Größe optimiert und so der Einsatz größerer und effizienterer Maschinen ermöglicht. In Rheinland-Pfalz spielt zudem die Weinbergflurbereinigung eine bedeutende Rolle. ${ }^{954}$ Im Weinbau werden durch die Bodenordnung oftmals erst die Voraussetzungen für eine maschinelle Bewirtschaftung geschaffen. Der Ersatz der Handarbeit hat erhebliche Rationalisierungseffekte zur Folge, ohne die der Weinbau allerdings nicht mehr wettbewerbsfähig wäre.
Wirkung:
Bewirtschaftungsvorteile durch das verbesserte Wegenetz
Empfänger:
Land- und Forstwirtschaft
Wirkungstyp:
intendierte gesetzliche Wirkung

Zusätzliche Arbeitszeitersparnisse bei der Flächenbewirtschaftung ergeben sich durch die Verbesserung des Wegenetzes. Im Rahmen des Bodenordnungsverfahrens wird die Qualität des Wegenetzes verbessert, so dass die durchschnittliche Hof-Feld-Entfernung sinkt und sich die Felderschließung verbessert, weshalb auch größere und schwerere Maschinen zum Einsatz kommen können. ${ }^{955}$

\section{Wirkung: $\quad$ Optimierung der Flächenränder \\ Empfänger: Land- und Forstwirtschaft \\ Wirkungstyp: positive Nebenwirkung}

Im Zuge der flurbereinigungsbedingten Neuordnung der Flächen kommt es nicht nur durch die Vergrößerung der Schläge zu Bewirtschaftungsvorteilen, sondern häufig können auch Feldrandverluste vermieden werden. Insbesondere die Optimierung von Waldrändern spielt eine bedeutende Rolle, da Rheinland-Pfalz ein sehr waldreiches Bundesland ist. Infolge der Realteilung ist das Landschaftsbild häufig durch lang gezogene schmale Waldstücke geprägt. ${ }^{956}$ Die Gefahr, dass sich an den langen Grenzen der schmalen bewaldeten Landstreifen astige Randstämme bilden, die die Arbeitsbedingungen erschweren und dadurch die Erträge schmälern, ist enorm hoch. Zusätzlich

952 Vgl. beispielsweise $\S 87$ FlurbG.

953 Vgl. BMS Consulting (2005), S. 149.

954 Vgl. Mosiek/Pieper/Kasten (2006), S. 106.

$955 \mathrm{Vgl}$. Weiß (1982), S. $100 \mathrm{ff}$.

956 Bei der Realteilung handelt es sich um eine Erbfolgeregelung, bei der die Eigentumsflächen gleichwertig unter den Erben aufgeteilt werden. Hierdurch entsteht tendenziell eine Zersplitterung des Grundbesitzes. 
verhindern lange Grenzlinien eine effiziente Nutzung der Flächen, da die Einhaltung bestimmter Abstände zwischen den Grenzen gesetzlich vorgeschrieben ist. Mit Hilfe der Bodenordnung können derartige Probleme erheblich reduziert werden. Die Verbreiterung und Begradigung von Gehölzstreifen wirkt sich auch positiv auf die Nutzbarkeit angrenzender Ackerflächen aus, da es zu einer Verminderung der Schattenwirkung führt. ${ }^{957}$

$\begin{array}{ll}\text { Wirkung: } & \text { Verringerung der Bürokratiekosten bei Prämienkontrollen } \\ \text { Empfänger: } & \text { Land- und Forstwirtschaft } \\ \text { Wirkungstyp: } & \text { positive Folgewirkung }\end{array}$

Der strukturelle Anpassungsprozess der Landwirtschaft wird von der EU, Bund und Ländern mit öffentlichen Mitteln gefördert. Die Beantragung der Fördermittel setzt einen genauen Flächennachweis der landwirtschaftlichen Betriebe voraus. Dabei ist der Antragsteller grundsätzlich verpflichtet, die Unternehmensfläche bei Kontrollen durch Katasterunterlagen nachzuweisen. Durch die qualitative Verbesserung und Verkleinerung der Katasterunterlagen reduziert sich der zeitliche Aufwand der Prämienbeantragung bei gleichzeitig erhöhter Rechtssicherheit. Die Rechtmäßigkeit der Vergabe der Fördermittel wird zudem stichprobenartig überprüft. Diese Prämienkontrollen sind mit einem nicht unerheblichen Zeitaufwand verbunden, welcher von der Qualität der Katasterunterlagen und der Anzahl der betroffenen Flurstücke abhängt.

\section{Wirkung: Positive wirtschaftliche Effekte durch Folgeinvestitionen \\ Empfänger: Industrie- und Dienstleistungsunternehmen \\ Wirkungstyp: positive Folgewirkung}

Die agrarstrukturellen Verbesserungen in der Land- und Forstwirtschaft haben nicht unerhebliche Ertragswertsteigerungen der landwirtschaftlichen Nutzflächen zur Folge. Um die Arbeitszeiteinsparungen in vollem Umfang zu realisieren, ist häufig der Einsatz größerer und leistungsfähigerer Maschinen notwendig. Erst durch die Bodenordnung sind Investitionen in entsprechende Maschinen oder in Kapazitätsausweitungen für die Land- und Fortwirtschaft wieder rentabel. Im Weinanbau wird durch eine Bodenordnung der maschinelle Einsatz überhaupt erstmalig ermöglicht und damit eine längerfristige wettbewerbsfähige Existenzgrundlage geschaffen. In Folge von Bodenordnungsverfahren ist daher mit Investitionen der betroffenen land- und forstwirtschaftlichen Betriebe zu rechnen, mit den entsprechenden positiven Folgewirkungen ${ }^{958}$

$957 \mathrm{Vgl}$. Mosiek/Pieper/Kasten (2006), S. $106 \mathrm{f}$.

958 Experten gehen in diesem Zusammenhang von flurbereinigungsbedingten Wachstumseffekten in Höhe von $10-15 \%$ aus. Der genaue Beitrag der Bodenordnung zur Förderung des Wirtschaftswachstums im produzierenden Bereich des landwirtschaftlichen Maschinensektors lässt sich allerdings nicht genau erfassen. 
für die in diesem Sektor tätigen Industrie- und Dienstleistungsunternehmen. Durch die verbesserte Auftragslage kann das jährliche Betriebseinkommen erhöht und damit Arbeitsplätze gesichert oder gegebenenfalls sogar neu geschaffen werden.

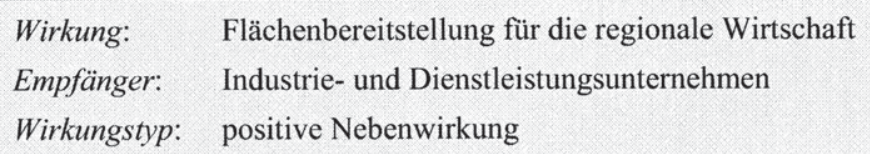

Das Flächenmanagement der Flurbereinigungsverwaltung kann sich positiv auf die Umsetzung privatwirtschaftlicher Planungen der regionalen Industrie- und Dienstleistungsunternehmen auswirken. Die Ausweisung der benötigten Flächen zur Realisierung dieser Planungen wird in der Regel beträchtlich vereinfacht oder im Extremfall sogar erst ermöglicht. Grundsätzlich ist davon auszugehen, dass privatwirtschaftliche Unternehmen beim Flächenerwerb innerhalb einer Bodenordnung von Kosteneinsparungen profitieren. Im Regelfall können Personalkosten für den Grunderwerb, Entschädigungsleistungen, Vermessungsgebühren, Gebühren für Notar und Grundbucheintragungen, eingespart werden. ${ }^{959}$

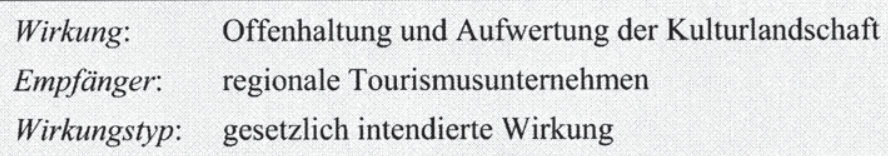

Ein strukturiertes Landschaftsbild und eine interessante Kulturlandschaft gehören zu den wichtigsten Voraussetzungen für die Entwicklung der Tourismusbranche im ländlichen Raum. Durch die Offenhaltung und Aufwertung der Kulturlandschaft leistet die Bodenordnung demnach auch einen Beitrag zum Erfolg des regionalen Tourismus in Rheinland-Pfalz. Wie bereits erläutert, kann sich durch die Bodenordnung der Freizeitund Erholungswert einer Region bedeutend erhöhen ${ }^{960}$ Mit einem erhöhten Freizeitund Erholungswert gewinnt das betreffende Gebiet häufig auch an Attraktivität für den Fremdenverkehr.

959 Vgl. BMS Consulting (2005), S. 155.

${ }^{960}$ Vgl. hierzu die Ausführungen zum Landschaftsbild in Kapitel 5.3.5.3.1. 


$$
\begin{array}{ll}
\text { Wirkung: } & \text { Realisierung spezieller touristischer Attraktionen } \\
\text { Empfänger: } & \text { regionale Tourismusunternehmen } \\
\text { Wirkungstyp: } & \text { positive Nebenwirkung }
\end{array}
$$

Zudem können mit Hilfe der Bodenordnung unter Umständen auch direkte Effekte auf die regionalen Besucherzahlen von Touristen erzielt werden, indem die Umsetzung besonderer verfahrensspezifischer touristischer Attraktionen ermöglicht wird, die dann Besucher in die Region locken. ${ }^{961}$

$$
\begin{array}{ll}
\text { Wirkung: } & \text { Vorteile für den Rad- und Wanderwegetourismus } \\
\text { Empfänger: } & \text { regionale Tourismusunternehmen } \\
\text { Wirkungstyp: } & \text { positive Nebenwirkung }
\end{array}
$$

Ein weiterer touristischer Vorteil ergibt sich aus dem Aus- und Neubau des regionalen Wegenetzes. Ein verbessertes Wegenetz kommt nicht nur der Allgemeinheit und der Landwirtschaft zu Gute, sondern fördert vor allem auch den Rad- und Wanderwegetourismus, welcher in Rheinland-Pfalz eine bedeutende Rolle spielt. Es wird in diesem Zusammenhang davon ausgegangen, dass die Allgemeinheit der Region, die Landwirtschaft und die regionale Tourismusbranche zu je einem Drittel vom realisierten Wegenetz profitieren.

\subsection{Auswirkungen der Bodenordnung auf den Staat}

\begin{tabular}{|ll}
\hline Wirkung: & $\begin{array}{l}\text { Vereinbarkeit unterschiedlicher Projekte und Erhöhung der } \\
\text { Realisierungschancen der Gesamtplanung }\end{array}$ \\
Empfänger: & $\begin{array}{l}\text { Projektträger der öffentlichen Verwaltung } \\
\text { Wirkungstyp: }\end{array}$ \\
gesetzlich intendierte Wirkung
\end{tabular}

Die Realisierungschancen von unterschiedlichen Projekten in ländlichen Gebieten können sich durch das Flächenmanagement im Rahmen eines begleitenden Bodenordnungsverfahrens beträchtlich erhöhen. Die Möglichkeit einer flexiblen Flächenausweisung erleichtert die notwendige Flächenbeschaffung der Projektträger für Infrastruktur, Hochwasserschutz, Naturschutz sowie für kommunale Planungen. Im Extremfall wird die Umsetzung einzelner Maßnahmen sogar erst durch die Einleitung eines Bodenordnungsverfahrens ermöglicht, da ansonsten die notwendige Akzeptanz der betroffenen Eigentümer für das Projekt fehlt. Es gehört zu den gesetzlichen Zielen der Flurbereinigung, die vielfältigen, teils miteinander konkurrierenden Fachplanungen in einer Region bestmöglich miteinander in Einklang zu bringen.

961 Dies ist vor allem in landschaftlich attraktiven und touristisch erschlossenen Regionen von Bedeutung. 


\section{Wirkung: Kostenersparnisse im Zuge der Flächenbereitstellung \\ Empfänger: Projektträger der öffentlichen Verwaltung \\ Wirkungstyp: positive Nebenwirkung}

Erfolgt das Flächenmanagement und damit die Flächenbereitstellung für geplante Projekte durch die Flurbereinigungsverwaltung, lassen sich an anderer Stelle der öffentlichen Verwaltung nicht unerhebliche Kostenersparnisse realisieren. ${ }^{962}$ Diese werden nachfolgend detailliert aufgeführt und beschrieben:

- Grunderwerbskosten: Wie bereits erläutert, können den vom Landentzug betroffenen Grundstückseigentümern im Rahmen einer Flurbereinigung häufig Ersatzflächen angeboten werden. Diese werden vom Projektträger oder der Teilnehmergemeinschaft der Bodenordnung an beliebiger Stelle des Verfahrensgebietes erworben. Innerhalb der Bodenordnung lässt sich der Flächenerwerb also wesentlich flexibler gestalten, da in größerem Ausmaß potenzielle Verkaufsflächen zur Verfügung stehen und damit auch die Wahrscheinlichkeit höher ist, verkaufswillige Landeigentümer zu finden. Im Gegensatz dazu wäre der Projektträger ohne Bodenordnung dazu gezwungen, genau die benötigten (Trassen-)Flächen zu erwerben. Hierdurch steigt in der Regel auch der Verkaufspreis der Flächen, da ein Aufschlag auf den üblichen Verkehrswert der Flächen bezahlt wird. Die Bodenordnung ermöglicht also in der Regel einen kostengünstigeren Grunderwerb für den Projektträger. ${ }^{963}$

- Entschädigungsleistungen: Die Beeinträchtigung landwirtschaftlicher Nutzflächen, vor allem An- und Durchschneidungen zusammenhängender Besitzstücke, die bei der Umsetzung öffentlicher Bauvorhaben - insbesondere bei der Realisierung großer Trassenprojekte - auftreten, lassen sich mit Hilfe der Bodenordnung häufig wesentlich verringern bzw. im Idealfall sogar vollständig vermeiden. ${ }^{964}$ Diese An- und Durchschneidungsschäden dienen als Grundlage zur Berechnung der zu entrichtenden Entschädigungszahlungen an die betroffenen Eigentümer. Dem stehen allerdings die im Bodenordnungsverfahren anfallenden Nutzungsausfallentschädigungen gegenüber. Außerhalb der Flurbereinigung werden Nutzungs- und Eigentumsentzug im Regelfall zeitgleich vollzogen. Im Rahmen der Bodenordnung erfolgt typischerweise zuerst der Nutzungsentzug, um den Projektträger frühzeitig in den Besitz der Flächen zu bringen. Falls bis dahin nicht in ausreichendem Maße Ersatzflächen zur Verfügung stehen, sind an die betroffenen Landwirte Nutzungsausfallentschädigungen zu zahlen. In der Regel sind die zu leistenden Entschädigungszahlungen jedoch wesentlich geringer, wenn eine begleitende Bodenordnung durchgeführt wird. ${ }^{965}$

- Personal- und Sachkosten für den Grunderwerb: Bei Verzicht auf eine begleitende Bodenordnung liegt die Zuständigkeit für den Grunderwerb ausschließlich bei den Projektträgern. Diese verfügen entweder über eine eigene Grunderwerbsabteilung,

\footnotetext{
962 Vgl. BMS Consulting (2005), S. $151 \mathrm{ff}$.

$963 \mathrm{Vgl}$. Mosiek/Pieper/Kasten (2006), S. 109.

964 Vgl. BMS Consulting (2005), S. 152.

965 Vgl. Hegele et al. (1997), S. 361.
} 
wie zum Beispiel die Straßenbauverwaltung, oder beauftragen externe Ingenieurbüros mit dieser Aufgabe. Innerhalb einer begleitenden Bodenordnung werden häufig wesentliche Teile des Grunderwerbs von der zuständigen Flurbereinigungsverwaltung übernommen. Hieraus resultieren beim Projektträger Einsparungen von ansonsten anfallenden Personal- und Sachkosten bzw. externer Kosten für das beauftragte Ingenieurbüro. Der Umfang dieser Kosteneinsparungen hängt im Wesentlichen vom Zeitpunkt der Einleitung des Bodenordnungsverfahrens ab. Je frühzeitiger die Einbindung der Flurbereinigungsverwaltung in den Planungsprozess erfolgt, desto höher ist im Regelfall die Kostenersparnis. ${ }^{966}$

- Kataster- und Notargebühren: Im Rahmen von Bodenordnungsverfahren ist der Grunderwerb durch die Flurbereinigungsbehörde gemäß §52 FlurbG ohne Beteiligung eines Notars möglich, die entsprechenden Gebühren entfallen daher. ${ }^{967}$ Des Weiteren gilt gemäß $§ 108$ (1) Flurbereinigungsgesetz die Gebührenfreiheit für Eintragungen in das Grundbuch und Liegenschaftskataster. ${ }^{968}$

- Kosten der Vermessung: Die für die Umsetzung der geplanten Maßnahmen benötigten Flächen gehen normalerweise in das Eigentum der Projektträger über. Um einen sicheren und rechtmäßigen Eigentumsübergang gewährleisten zu können, sind die betreffenden Flächen genau zu identifizieren. Ohne Bodenordnung sind dafür regelmäßig Fortführungsvermessungen notwendig, für die die Projektträger die entsprechenden Kosten zu tragen haben. Da innerhalb der Bodenordnung eine komplette Neuvermessung des Verfahrensgebietes durchgeführt wird, entfallen die Kosten für Fortführungsvermessungen. Die Vermessungskosten der Bodenordnung sind Bestandteil der Ausführungs- und Verfahrenskosten und sind beim Ressourcenverzehr des Verfahrens berücksichtigt. Aus diesem Grund müssen die ansonsten entstehenden Kosten für potenzielle Fortführungsvermessungen als Wirkungsbeitrag der Bodenordnung gewertet werden. ${ }^{969}$

$$
\begin{array}{ll}
\text { Wirkung: } & \text { Baukostenersparnisse durch eine beschleunigte Projektrealisierung } \\
\text { Empfänger: } & \text { Projektträger der öffentlichen Verwaltung } \\
\text { Wirkungstyp: } & \text { positive Folgewirkung }
\end{array}
$$

Wenn zur Unterstützung öffentlicher Bauvorhaben ein Bodenordnungsverfahren eingeleitet wird, kann durch das Flächenmanagement und die vorzeitige Besitzeinweisung in der Regel zeitnäher mit dem Bau begonnen werden. ${ }^{970}$ Grds. steigen die inflationsbereinigten Baupreise im Zeitverlauf, so dass eine beschleunigte Projektrealisierung letztlich auch geringere reale Baukosten für den ausführenden Träger zur Folge hat.

966 Vgl. BMS Consulting (2005), S. 153.
967 Vgl. §52 FlurbG.
968 Vgl. §108 (1) FlurbG.
969 Vgl. BMS Consulting (2005), S. $153 \mathrm{f}$.
970 Zum Ablauf eines Bodenordnungsverfahrens und zur vorläufigen Besitzeinweisung vgl. Kapitel 5.1.4. 


\section{Wirkung: Baukostenersparnisse durch spezielle Maßnahmen der Bodenordnung \\ Empfänger: Projektträger der öffentlichen Verwaltung \\ Wirkungstyp: positive Nebenwirkung}

Durch das Flächenmanagement der Bodenordnung lassen sich einzelne Fachplanungen im ländlichen Raum flexibler und effektiver gestalten sowie meist besser aufeinander abstimmen. Hierdurch lässt sich teilweise die Anzahl anzulegender Wege, Brücken oder Mauern reduzieren oder eine finanziell vorteilhaftere Bauweise wählen. ${ }^{971}$ So können bei Straßenbauprojekten durch den flexiblen Flächentausch innerhalb der Bodenordnung nicht selten ansonsten notwendige Wirtschaftswegebrücken eingespart werden. ${ }^{972}$ Die sich daraus ergebenden Kosteneinsparungen müssen im Rahmen der Wirkungsanalyse als Wirkungskomponente der Bodenordnung berücksichtigt werden.

\section{Wirkung: $\quad$ Kostenersparnisse bei der Unterhaltung gemeinschaftlicher Anlagen \\ Empfänger: Projektträger der öffentlichen Verwaltung \\ Wirkungstyp: positive Folgewirkung}

Unter Umständen wird die Unterhaltung gemeinschaftlicher Anlagen in einem Flurbereinigungsgebiet zeitweise von der zuständigen Teilnehmergemeinschaft übernommen. Die in diesem Fall von der Kommune eingesparten Unterhaltungskosten können als Wirkungsbeitrag der Bodenordnung bewertet werden. ${ }^{973}$ Zudem ist es möglich, dass im Zuge der Flächenneuordnung und des Umbaus des Wegenetzes die Unterhaltungskosten für bestimmte gemeinschaftliche Anlagen dauerhaft gesenkt werden, beispielsweise in Folge einer naturnäheren Gestaltung eines Baches. ${ }^{974}$ Diese dauerhaften Kosteneinsparungen müssen ebenfalls als positive Auswirkung der Bodenordnung berücksichtigt werden.

\footnotetext{
971 Vgl. Mosiek/Pieper/Kasten (2006), S. 111.

972 Vgl. BMS Consulting (2005), S. 154.

973 Vgl. Mosiek/Pieper/Kasten (2006), S. 111.

$974 \mathrm{Vgl}$. Mosiek/Pieper/Kasten (2006), S. 111.
} 


\section{Wirkung: Verringerung der Verwaltungskosten Kataster- und Grundbuchamt Empfänger: Projektträger der öffentlichen Verwaltung Wirkungstyp: positive Folgewirkung}

Als ein positiver Wirkungsbeitrag der Bodenordnung wurde bereits die Verbesserung der Qualität der öffentlichen Bücher identifiziert. ${ }^{975}$ Im Rahmen der Flurbereinigung erfolgt eine Aktualisierung und Berichtigung der öffentlichen Bücher. Die hierzu notwendigen Arbeiten werden komplett von der Flurbereinigungsverwaltung übernommen, die vorübergehend die amtlichen Verzeichnisse führt. Durch die Zusammenlegung der Flächen wird der Umfang der zu führenden Bücher zudem verkleinert, so dass in der Folge mit einer Verringerung des Verwaltungsaufwandes in den entsprechenden Ämtern und Behörden zu rechnen ist. ${ }^{976}$ In den Katasterämtern ist im Vergleich zu den Grundbuchämtern aufgrund des hohen Automatisierungsgrades eher mit geringen Einsparungen an Verwaltungskosten zu rechnen, so dass im Rahmen der Wirkungsanalyse lediglich die Ersparnisse bei der Grundbuchführung berücksichtigt werden. $^{977}$

\section{Wirkung: $\quad$ Verringerung der Verwaltungskosten der Katasternutzer \\ Empfänger: Projektträger der öffentlichen Verwaltung \\ Wirkungstyp: positive Folgewirkung}

Die Vorteile der Landwirtschaft bei der Einsparung von Bürokratiekosten im Zuge der Prämienkontrollen wurden bereits als Wirkungsbeitrag der Bodenordnung identifiziert. ${ }^{978}$ Wie bereits erläutert, hängt der Aufwand der Antragsstellung entscheidend von der Anzahl und Größe der einzelnen Parzellen der landwirtschaftlichen Betriebe sowie von der Qualität der zugrunde liegenden Katasterunterlagen ab. Im Zuge von Prämienkontrollen profitieren daher nicht nur die Landwirte von der Qualitätsverbesserung und Verringerung der Katasterunterlagen, sondern auch der staatliche Prüfdienst, der die Kontrollen durchführt. In der Regel werden die Kontrollen von jeweils zwei Prüfern einem Erstprüfer, der über eine fundierte landwirtschaftliche oder weinbauliche Ausbildung verfügt und einem Zweitprüfer, der als Vermesser ausgebildet ist - durchgeführt. Analog zu den betroffenen Landwirten ergibt sich auch beim Prüfdienst eine Zeitersparnis und damit eine Reduzierung bürokratischer Kosten.

Des Weiteren kommt die flurbereinigungsbedingte Berichtigung der öffentlichen Bücher auch noch einer Vielzahl weiterer Katasternutzer zu Gute. Tabelle 4 gibt einen Überblick über die potenziellen Katasternutzer, für die durch die verbesserte Qualität

\footnotetext{
$975 \mathrm{Vgl}$. hierzu Kapitel 5.3.5.3.1.

976 Vgl. Kroés (1971), S. 80 f.

977 Vgl. Mosiek/Pieper/Kasten (2006), S. 112.

$978 \mathrm{Vgl}$. Kapitel 5.3.5.3.2.
} 
der Unterlagen und die Reduzierung der Flurstücke eine Verringerung des eigenen Verwaltungsaufwandes zu erwarten ist.

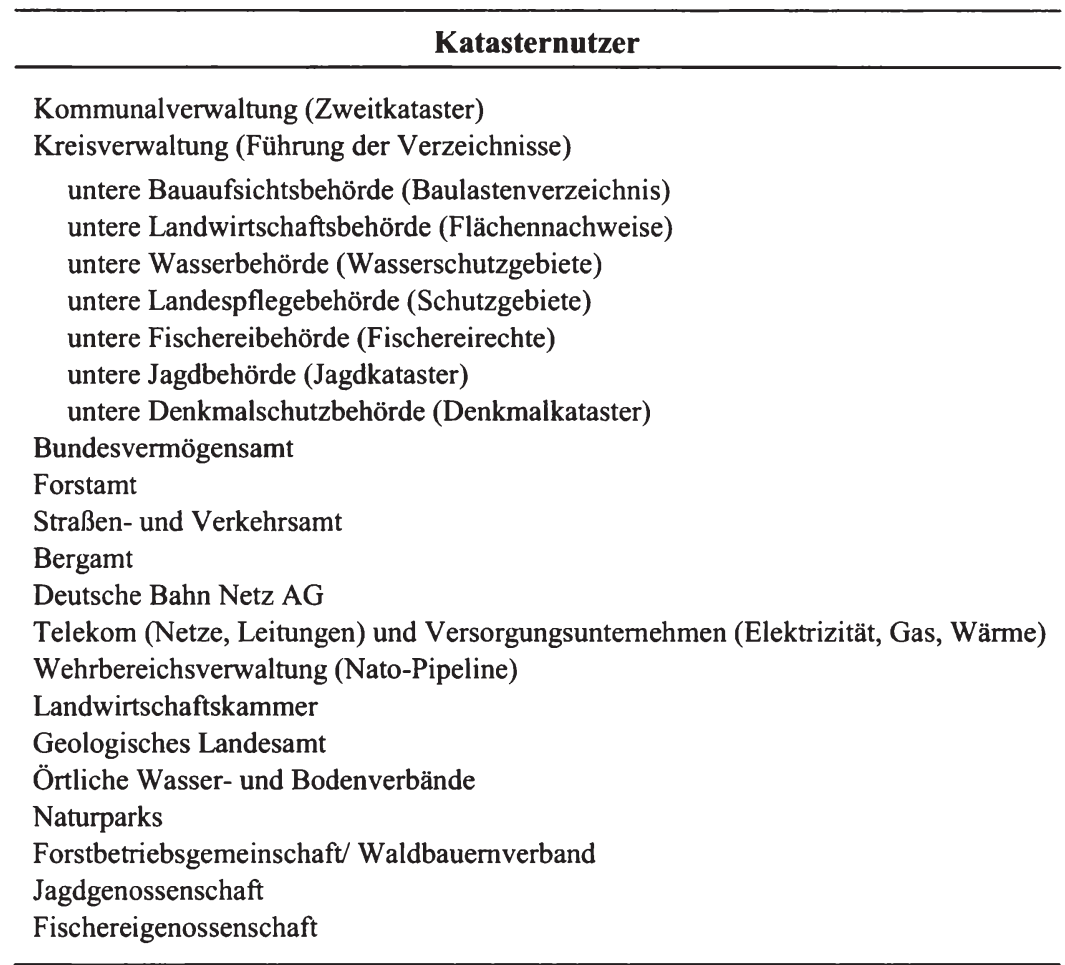

Tabelle 4: Übersicht potenzieller Katasternutzer

\subsection{Auswirkungen der Bodenordnung auf die Umwelt}

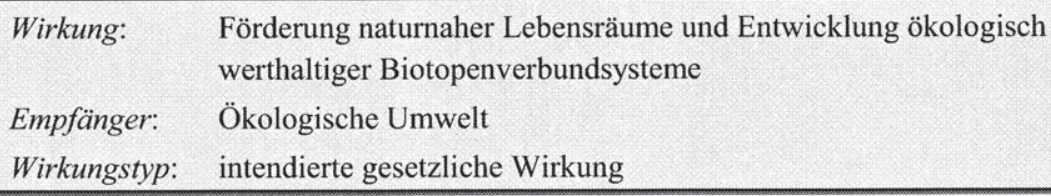

Insbesondere in den letzten Jahrzehnten hat sich in der Gesellschaft ein stärkeres Umweltbewusstsein entwickelt. Neben der ökonomischen Nutzbarkeit der natürlichen Umwelt wird zunehmend auch der ökologische Wert als gesellschaftlich förderungs- 
würdig erkannt. ${ }^{979} \mathrm{Im}$ Zuge dieser Entwicklung haben sich auch die Anforderungen an die Bodenordnung geändert. Seit der Novellierung des Flurbereinigungsgesetzes im Jahr 1976 werden landschaftspflegerische und ökologische Maßnahmen gezielt gefördert. ${ }^{980}$ Durch die Flächenneuordnung im ländlichen Raum können in der Regel bessere Voraussetzung für die Umsetzung von Planungen zum Erhalt oder der Wiederherstellung des natürlichen Gleichgewichts sowie zur Förderung naturnaher Lebensräume geschaffen werden. Die ökologischen Kompensationsflächen für Infrastrukturvorhaben lassen sich im Rahmen einer Bodenordnung meist bündeln, so dass die Entwicklung ökologisch werthaltiger Biotopenverbundsysteme ermöglicht wird. ${ }^{981}$

$\begin{array}{ll}\text { Wirkung: } & \text { Verringerung der Bodenerosion } \\ \text { Empfänger: } & \text { Ökologische Umwelt } \\ \text { Wirkungstyp: } & \text { positive Folgewirkung }\end{array}$

Die Bodenordnung ermöglicht jedoch nicht nur eine funktionellere Ausweisung von Kompensationsflächen zum Zwecke des Naturschutzes, sondern sie fördert ebenfalls den Bodenschutz und leistet damit einen bedeutenden Beitrag zur Vermeidung von Bodenerosionen. Natur- und Bodenschutz bedingen sich häufig gegenseitig. Die Qualität des Bodens ist ausschlaggebend für die Schaffung eines ökologisch sinnvollen Biotopverbundsystems. Umgekehrt beeinflusst der Zustand der natürlichen Umgebung und die Schaffung naturnaher Lebensräume auch die Qualität des Bodens. ${ }^{982}$
Wirkung: Verbesserung der Gewässerstrukturgüte und der Gewässerqualität
Empfänger: Ökologische Umwelt
Wirkungstyp: intendierte gesetzliche Wirkung

Gemäß $§ 37$ (2) FlurbG sind bei der Durchführung von Bodenordnungsverfahren auch wasserwirtschaftliche Belange $\mathrm{zu}$ berücksichtigen. ${ }^{983}$ Das Bundesnaturschutzgesetz legt klare Grundsätze zum Schutz natürlicher und naturnaher Gewässer fest. In \$2 (1) Nr. 4 BNatSchG heißt es dazu:

- „[...] Natürliche und naturnahe Gewässer sowie deren Uferzonen und natürliche Rückhaltebecken sind zu erhalten, zu entwickeln oder wiederherzustellen. Änderungen des Grundwasserspiegels, die zu einer Zerstörung oder nach-haltigen Beeinträchtigungen schutzbedürftiger Biotope führen können, sind zu vermeiden [...].“ 984

\footnotetext{
979 Vgl. Bauer et al. (1979), S. 2.

980 Vgl. FlurbG.

$981 \mathrm{Vgl}$. Mosiek/Pieper/Kasten (2006), S. 112.

$982 \mathrm{Vgl}$. Mosiek/Pieper/Kasten (2006), S. 113.

983 Vgl. $\$ 37$ (2) FlurbG.

$984 \S 2(1)$ Nr.4 BNatSchG.
} 
$\mathrm{Zu}$ den Aufgaben der Bodenordnung zählt demnach auch der Schutz des Wassers als unverzichtbare Lebensgrundlagen sowie die nachhaltige Sicherung der Nutzungsfähigkeiten von Gewässern. Dazu gehören insbesondere der Schutz vor Verunreinigungen, die Bewahrung der natürlichen Eigenschaften sowie die Sicherstellung eines in Menge und Qualität funktionsfähigen Wasserhaushaltes. Bodenordnerische Maßnahmen sind somit nicht ausschließlich auf die Verbesserung der allgemeinen Wasserqualität ausgerichtet, sondern können auch Strukturgüteverbesserungen an Gewässern, z.B. in Folge von Sohlanhebungen, Laufverlängerungen oder durch das Entfernen oder den Umbau von Rohrdurchlässen, zum Ziel haben. ${ }^{985}$

Nach einer ausführlichen Herleitung und Beschreibung der einzelnen Wirkungskomponenten der Bodenordnung, gilt es nun, diese den auslösenden Dienstleistungen der Flurbereinigungsverwaltung zuzuordnen und damit das gesamtgesellschaftliche Wirkungsgefüge der Bodenordnung abbilden zu können. Das Wirkungsgefüge enthält sämtliche potenzielle Auswirkungen von Bodenordnungsverfahren und dient damit als Analyseinstrument der Identifikation der tatsächlichen Auswirkungen konkret betrachteter Verfahren in der Anwendungsphase der Wirkungsanalyse. ${ }^{986}$

\footnotetext{
985 Vgl. Mosiek/Pieper/Kasten (2006), S. 113.
}

986 Zur Anwendungsphase der Wirkungsanalyse von Bodenordnungsverfahren vgl. Kapitel 5.4. 


\subsubsection{Abbildung des gesamtgesellschaftlichen Wirkungsgefüges}

Die Dienstleistungen der Flurbereinigungsverwaltung bilden die horizontale Zuordnungsebene und die Empfängerebene stellt die vertikale Dimension dar. Die komplette Systematik des Wirkungsgefüges ist in Abbildung 68 dargestellt.
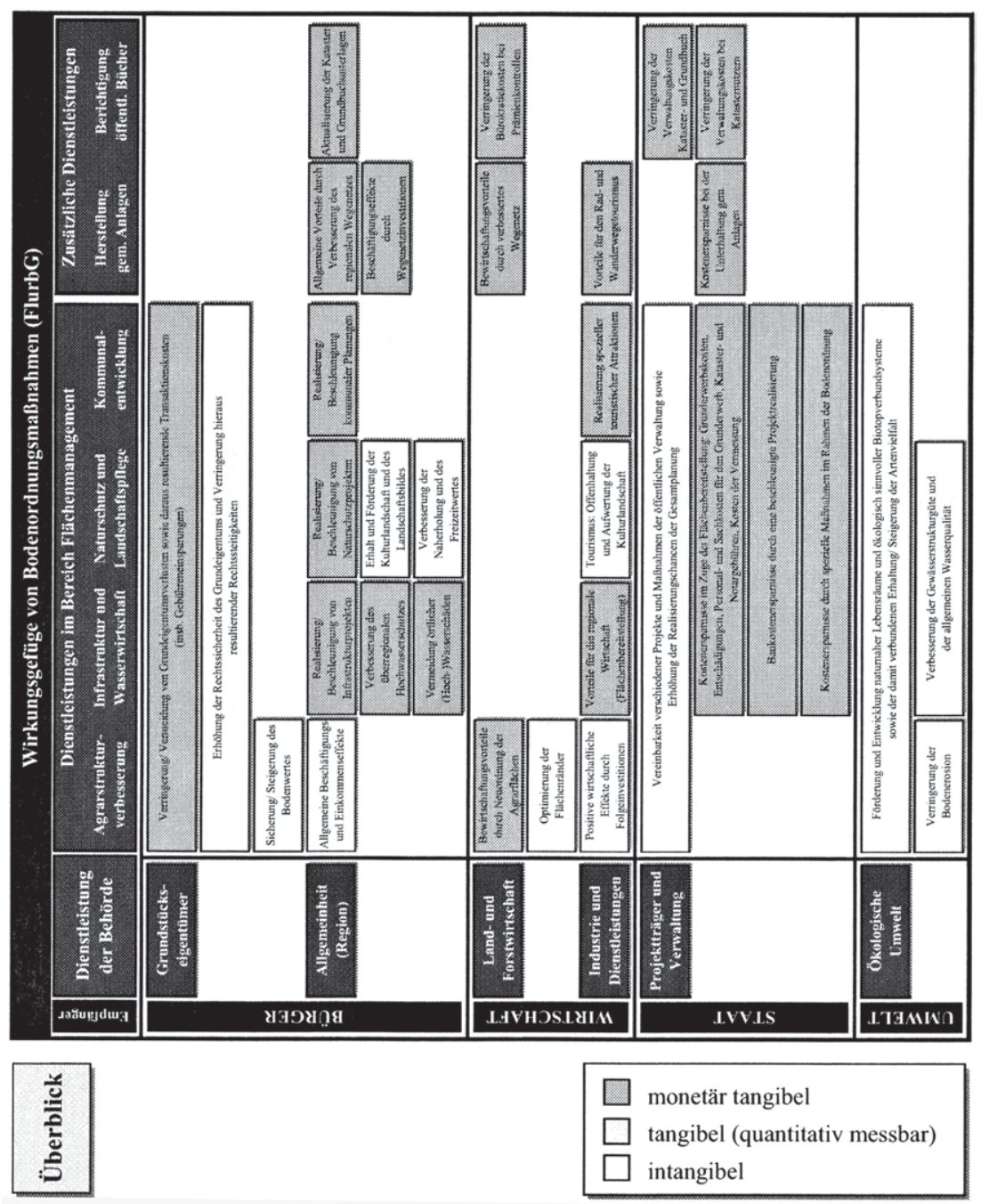

Abbildung 68: Gesellschaftliches Wirkungsgefüge der Bodenordnung 


\subsubsection{Bewertung der Wirkungskomponenten der Bodenordnung}

Der zweite Schritt einer Wirkungsanalyse besteht in der Operationalisierung und Bewertung der identifizierten Wirkungsbeiträge. Der Literaturüberblick bestehender Forschungsergebnisse macht deutlich, dass es bislang keinen umfassenden Ansatz zur Bewertung der Maßnahmen der ländlichen Bodenordnung gibt. Lediglich in Teilbereichen, wie zum Beispiel für die Bewirtschaftungsvorteile der Landwirtschaft, sind ausgereifte Bewertungsansätze entwickelt worden, auf die die Wirkungsanalyse der Bodenordnung zurückgreifen kann. ${ }^{977}$ Soweit möglich, werden diese Untersuchungsergebnisse zur Operationalisierung einzelner Wirkungsbeiträge in den weiteren Analyseschritten berücksichtigt bzw. übernommen. Ansonsten stellen Befragungen, und hier insbesondere Expertengespräche, ein geeignetes Mittel dar, die zur Bewertung notwendigen Annahmen und Prämissen zu validieren. ${ }^{988}$

Bei der Darstellung des gesamtgesellschaftlichen Wirkungsgefüges der Bodenordnung (vgl. Abbildung 68) ist bereits die Trennung der Bewertungsformen der einzelnen Wirkungsbeiträge vorgenommen worden. ${ }^{989}$ Grundsätzlich können in diesem Zusammenhang drei Bewertungsformen unterschieden werden. Soweit möglich, sind die einzelnen Wirkungskomponenten der Bodenordnung durch monetäre Bewertungsansätze zu quantifizieren. Den Wirkungsbeiträgen wird dazu mit Hilfe abgesicherter Näherungsund Umrechnungsverfahren ein gesellschaftlich akzeptierter Marktpreis zugeordnet. ${ }^{990}$ Gelingt die monetäre Bewertung tangibler Wirkungen nicht, sind alternativ geeignete Wirkungsindikatoren zu erheben. Diese machen die zuvor theoretisch beschriebenen Sachverhalte quantitativ messbar und damit einer wirkungsorientierten Steuerung zugänglich. Bei intangiblen Wirkungsbeiträgen gelingt auch dies nicht. Ausprägung und Intensität der Auswirkungen müssen dann so präzise wie möglich verbal beschrieben werden.

Nachfolgend werden die unterschiedlichen Bewertungsansätze und Operationalisierungen der identifizierten Auswirkungen der Bodenordnung detailliert erläutert. In diesem Zusammenhang wurde besonderes Augenmerk darauf gelegt, vorsichtige Bewertungsansätze zur Monetarisierung der einzelnen Wirkungsbeiträge zu wählen. Die im Folgenden ausgewiesenen Bewertungen stellen daher tendenziell Untergrenzen dar.

\footnotetext{
987 Vgl. Grüneberger (2003), S. 87 und die Ausführungen des Kapitels 5.3.4.2.

$988 \mathrm{Zu}$ den durchgeführten Befragungen vgl. Kapitel 5.3.4.3.

$989 \mathrm{Zu}$ den Bewertungsformen der Auswirkungen staatlichen Handelns vgl. Kapitel 4.3.2.2.2.

$990 \mathrm{Zu}$ den monetären Bewertungsansätzen vgl. ausführlich Kapitel 4.3.2.2.3.
} 


\subsubsection{Bewertung der Auswirkungen auf den Bürger}

\begin{tabular}{|ll|}
\hline Wirkung: & Verringerung oder Vermeidung von Eigentumsverlusten sowie \\
& Einsparung ansonsten anfallender Transaktionskosten \\
Empfänger: & Grundstückseigentümer \\
Bewertung: & monetär tangibel [Alternativkostenmethode] \\
\hline
\end{tabular}

Die Grundstückseigentümer profitieren vom Flächenmanagement der Bodenordnung durch eine Verringerung bzw. die vollständige Vermeidung von Eigentumsverlusten. Außerhalb einer Bodenordnung würden ansonsten Transaktionskosten für den Erwerb von Ersatzflächen anfallen. ${ }^{91}$ Als Gebühreneinsparungen in Folge einer Bodenordnung werden in diesem Zusammenhang pauschal 3\% der Re-Investitionssumme ${ }^{992}$ angesetzt. Es wird angenommen, dass durchschnittlich Zweidrittel der landwirtschaftlichen Nutzfläche des Verfahrensgebietes zum Betriebsvermögen der betroffenen Landwirte gehören und sich die restlichen Nutzflächen im Privatbesitz befinden (vgl. Abbildung 69).

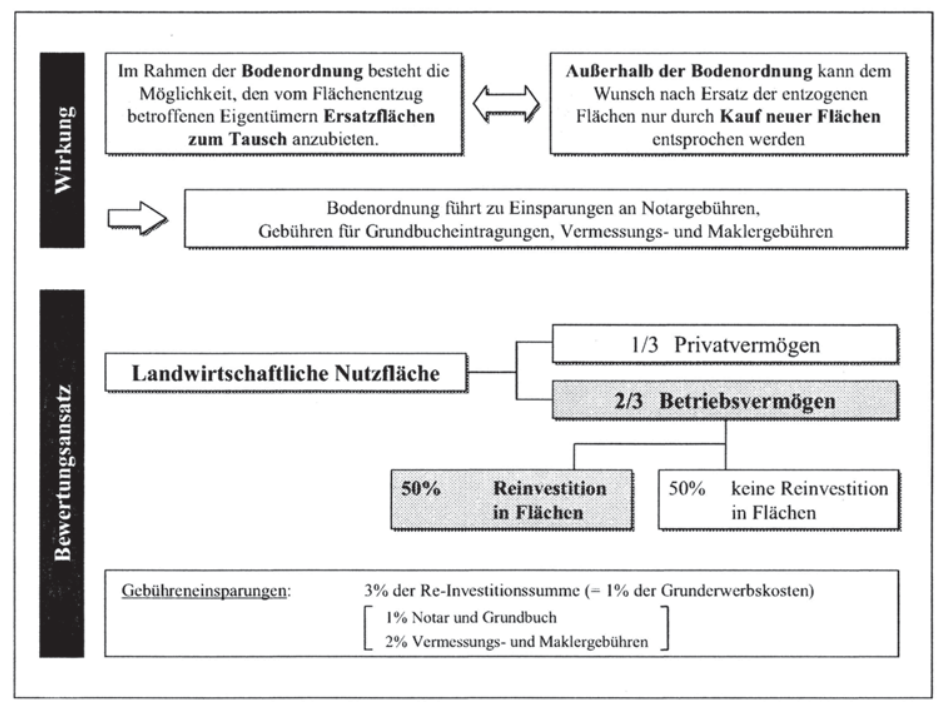

\section{Abbildung 69: Eingesparte Transaktionskosten der Eigentümer}

Weiterhin wird angenommen, dass die Hälfte der Grundstückseigentümer mit Nutzflächen im Betriebsvermögen nach einem erzwungenen Landverkauf außerhalb der Bo-

991 Vgl. BMS Consulting (2005), S. 169 f. sowie die Ausführungen in Kapitel 5.3.5.3.1.

992 Es wird dabei unterstellt, dass durchschnittlich $1 \%$ der Grunderwerbskosten für Notargebühren und Gebühren für Grundbucheintragungen anfallen sowie $2 \%$ der Grunderwerbskosten als notwendige Vermessungsund Maklergebühren. 
denordnung die Verkaufssumme in neue Flächen reinvestiert. ${ }^{993}$ Damit beträgt die ReInvestitionssumme genau ein Drittel der Grunderwerbskosten für den Flächenankauf. Als Einsparung der Transaktionskosten $\left(\mathrm{Tk}^{\mathrm{c}}\right)$ sind demnach 1\% der Grunderwerbskosten in Ansatz zu bringen, welche als Flächenverbrauch für Infrastruktur- oder Naturschutzprojekte benötigt werden.

\begin{tabular}{|ll|}
\hline Wirkung: & $\begin{array}{l}\text { Erhöhung der Rechtssicherheit des Eigentums sowie Verringerung } \\
\text { hieraus resultierender Rechtsstreitigkeiten }\end{array}$ \\
$\begin{array}{ll}\text { Empfänger: } & \text { Grundstückseigentümer } \\
\text { Bewertung: } & \text { tangibel [Wirkungsindikator: Landentzug in \%] }\end{array}$ \\
\hline
\end{tabular}

Zur Operationalisierung der erhöhten Rechtssicherheit in Bezug auf das Grundeigentum innerhalb einer Bodenordnung kann die Kennzahl "Landabzug in \%« als Wirkungsindikator herangezogen werden. Dabei ist von der Flurbereinigungsverwaltung ein möglichst geringer Landabzug anzustreben, welcher im Idealfall sogar $0 \%$ beträgt. Als Vergleichswert könnte zudem der Anteil des erzwungenen Landverkaufs außerhalb der Bodenordnung angegeben werden.

\section{Wirkung: $\quad$ Sicherung bzw. Steigerung des Bodenwertes \\ Empfänger: Grundstückseigentümer \\ Bewertung: intangibel}

Eine Steigerung bzw. Sicherung des Bodenwertes resultiert aus der Neuordnung des Verfahrensgebietes und der hiermit verbundenen Zusammenlegung der Flur-stücke. Hieraus erwachsen bei der landwirtschaftlichen Flächennutzung Kosteneinsparungen bei der Bewirtschaftung, was wiederum den Ertragswert und damit den Gesamtwert des Bodens steigert oder sich in steigenden Pachtpreisen ausdrückt. Die monetäre Bewertung erfolgt allerdings nicht an dieser Stelle, sondern bei der Quantifizierung der Bewirtschaftungsvorteile. Um den Gesamteffekt nicht zu überschätzen, bleibt die Bewertung an dieser Stelle intangibel.

993 Einerseits haben die Befragungsergebnisse gezeigt, dass eine deutliche Mehrzahl der Grundstückseigentümer nicht an einer Flächenveräußerung interessiert ist. Vgl. Kapitel 5.2. Andererseits bestehen auch steuerliche Anreize, den finanziellen Mittelzufluss der Flächenveräußerung zu reinvestieren. 


$$
\begin{array}{ll}
\text { Wirkung: } & \text { Allgemeine Beschäftigungs- und Einkommenseffekte } \\
\text { Empfänger: } & \text { regionale Allgemeinheit } \\
\text { Bewertung: } & \text { intangibel }
\end{array}
$$

Die Bodenordnung verbessert die Wettbewerbsfähigkeit der regionalen Landwirtschaft. Dies wirkt sich positiv auf die allgemeine Einkommens- und Beschäftigungssituation im Agrarsektor und damit auch auf die örtlichen Wirtschaftsbereiche aus. Aufgrund der schwierigen Quantifizierbarkeit dieses Wirkungsbeitrags wird nur eine intangible Bewertung vorgenommen.

$$
\begin{array}{ll}
\text { Wirkung: } & \text { Beschleunigung oder Realisierung von Infrastrukturprojekten } \\
\text { Empfänger: } & \text { regionale Allgemeinheit } \\
\text { Bewertung: } & \text { monetär tangibel [Zeitersparnisse] }
\end{array}
$$

Bei der Bewertung des Beitrags der Bodenordnung zur Unterstützung geplanter Infrastrukturprojekte sind grundsätzlich zwei Fälle zu unterscheiden:

- Trassenfläche des Infrastrukturprojektes liegt im Verfahrensgebiet, so dass die Bodenordnung zu einer unmittelbaren Beschleunigung beiträgt.

- Trassenfläche des Infrastrukturprojektes liegt außerhalb des Verfahrensgebietes, allerdings werden Flächen für notwendige ökologische Ausgleichsmaßnahmen beschafft und das Projekt mittelbar beschleunigt.

Die Quantifizierung dieses Beitrags erfolgt auf der Grundlage von verkehrsträgerspezifischen Nutzen-Kosten-Faktoren für den Straßen-, Schienenwege- und Wasserwegebau $\left(\mathrm{NKF}_{\mathrm{j}}\right.$ mit $\mathrm{j}=$ Straße, Schiene, Wasserweg). Diese wurden im Rahmen einer von HARTWIG ET AL. (2005) durchgeführten Analyse der „Volkswirtschaftlichen Effekte unterlassener Verkehrsinfrastrukturinvestitionen“ ermittelt. ${ }^{994}$ Auf Basis dieser durchschnittlichen Nutzen-Kosten-Relationen lassen sich in Abhängigkeit der jeweiligen Investitionen $\left(\mathrm{I}_{\mathrm{j}}\right)$ in das Infrastrukturprojekt $\mathrm{j}$ die entsprechenden Barwerte des Projektnettonutzens wie folgt berechnen: ${ }^{995}$

$$
\text { Barwert des Nettonutzens }=I_{j} \cdot N K F_{j}
$$

Als Wirkungsbeitrag der Bodenordnung ist jedoch lediglich der Teil des Projektnettonutzens in Ansatz zu bringen, der auf die frühzeitige Inanspruchnahme der verbesser-

994 Vgl. Hartwig et al. (2005), S. 43. Als Ergebnis weisen HARTWIG ET AL. für Straßenbauprojekte einen durchschnittlichen $\mathrm{NKF}=4,2$ aus. Für Schienenwegeprojekte beträgt der $\mathrm{NKF}=2,1$, für Wasserwege beträgt der $\mathrm{NKF}=3$,9. Die Ergebnisse sind aus dem Bundesverkehrswegeplan abgeleitet.

995 Vgl. auch Pieper et al. (2006), S. 251. 
ten Infrastruktur zurückzuführen ist und damit dem Zinsertrag aus dieser Beschleunigung $\left(\mathrm{ZE}_{\mathrm{j}}\right)$ entspricht:

$$
Z E_{j}=\left(I_{j} \cdot N K F_{j}\right) \cdot(1+i)^{b}-\left(I_{j} \cdot N K F_{j}\right)
$$

Hierbei stellt $\mathrm{i}$ den Zinssatz zur Diskontierung und $\mathrm{b}$ die durchschnittliche Beschleunigung in Jahren dar. Analog zu den der Untersuchung von HARTWIG ET AL. zugrunde liegenden Berechnungen im Bundesverkehrswegeplan wird an dieser Stelle der Wirkungsanalyse eine Diskontierungsrate von $\mathrm{i}=3 \%$ unterstellt. Was die Einschätzung der durchschnittlichen Beschleunigung betrifft, werden für die Wirkungsanalyse die folgenden Annahmen getroffen: ${ }^{996}$

- Erfolgt die Bodenordnung unmittelbar und begleitend zum Infrastrukturprojekt, wird eine Beschleunigung von 6 Monaten unterstellt $(b=0,5) .{ }^{997}$

- Erfolgt die Bodenordnung erst nach Abschluss der infrastrukturellen Planungen, beispielsweise um landschaftliche Durchschneidungen $\mathrm{zu}$ heilen, wird kein Beschleunigungseffekt in Ansatz gebracht $(b=0)$.

- Erfolgt die Bodenordnung mittelbar, bspw. für spezielle ortsgebundene ökologische Kompensationsmaßnahmen, wird eine Beschleunigung von 3 Jahren angenommen $(\mathrm{b}=3){ }^{.98}$

- Erfolgt die Bodenordnung mittelbar für ortsungebundene ökologische Kompensationsmaßnahmen, ist lediglich von einer Beschleunigung von 3 Monaten auszugehen $(\mathrm{b}=0,25)$.

996 Die Kundenbefragung im Rahmen des NRW-Projektes ergab bei der Befragung der Projektträger eine Beschleunigung zwischen 6 und 24 Monaten. Es wurde ein Durchschnittswert von 9,4 Monaten ermittelt. Vgl. BMS Consulting (2005), S. 181.

Da hierzu keine Befragungsergebnisse in Rheinland-Pfalz vorlagen, wurde vereinfachend der Mindestwert der NRW-Untersuchung unterstellt. Zudem werden in Rheinland-Pfalz fast sämtliche größeren Infrastrukturprojekte von Bodenordnungsverfahren begleitet, so dass die Annahme eher vorsichtig eingeschätzt ist.

Expertengespräche haben bestätigt, dass insbesondere die Flächenbeschaffung für ortsgebundene Kompensationsmaßnahmen mit hohen Widerständen verbunden ist. Vgl. Mosiek/Pieper/Kasten (2006), S. $67 \mathrm{f}$. Bei mittelbaren Beschleunigungen durch ökologische Kompensationsmaßnahmen außerhalb des Verfahrensgebietes wird nur der Teil der Investitionssumme in Ansatz gebracht, der auch dem Flächenanteil der Kompensationsmaßnahme im Verhältnis zu den gesamten Kompensationsflächen entspricht. 


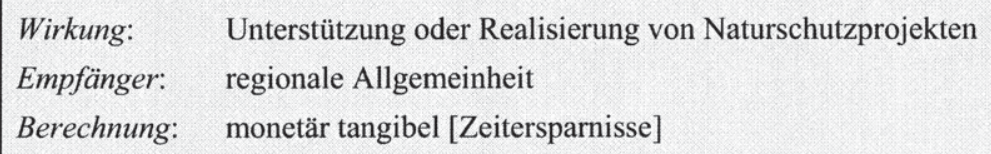

Die Umsetzung von Naturschutzmaßnahmen ist im Vergleich zu Infrastrukturprojekten noch stärker an eine begleitende Bodenordnung gebunden. Dementsprechend kann auch hier ein monetärer Zinsertrag $\left(\mathrm{ZE}_{\mathrm{j}}\right)$ aus der Beschleunigung von Naturschutzprojekten geltend gemacht werden. Analog zur Ausweisung ortsgebundener Kompensationsmaßnahmen wird hier eine Beschleunigung von 3 Jahren unterstellt $(b=3)$. Als Barwert des Nettonutzens kann in diesem Zusammenhang hilfsweise die Investitionssumme $\left(I_{j}\right)$ angesetzt werden. ${ }^{999}$

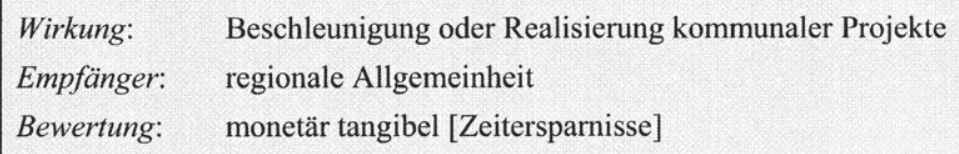

Da auch kommunale Planungen in der Regel infrastrukturelle oder naturschutzfachliche Maßnahmen umfassen, kann wiederum der Zinsertrag aus der Projektbeschleunigung in Ansatz gebracht werden $\left(\mathrm{ZE}_{\mathrm{j}}\right)$. Abhängig vom spezifischen kommunalen Projekt sind die Einschätzungen zum Projektnettonutzen sowie der Beschleunigung zu treffen. Grundsätzlich können sich die Annahmen an den bereits dargestellten Werten orientieren. In spezifischen Fällen lassen sich aber auch Werte aus Expertengesprächen mit Vertretern der Kommunen herleiten. ${ }^{1000}$

\section{Wirkung: Vorteile aus der Verbesserung des regionalen Wegenetzes \\ Empfänger: regionale Allgemeinheit \\ Bewertung: monetär tangibel [Zeitersparnisse]}

Die Investitionen in den Aus- und Neubau des regionalen Wegenetzes haben positive Wirkungen für die regionale Allgemeinheit, z.B. durch Umwegeeinsparungen. Unter der Annahme einer Nutzen-Kosten-Relation von 1:1 entspricht die Gesamtwirkung genau der Summe aus den getätigten Investitionen in den Aus- und Neubau der Wirt-

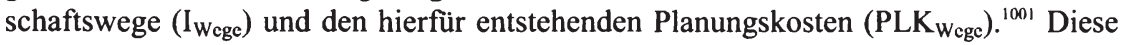
Gesamtwirkung wird allerdings nur zu einem Drittel der regionalen Allgemeinheit zu-

999 Im Vergleich zu Infrastrukturprojekten wird damit ein Nutzen-Kosten-Verhältnis von 1:1 unterstellt.

1000 Vgl. ausführlich Mosiek/Pieper/Kasten (2006), S. 117 f.

1001 Alternativ zur Investitionssumme kann auch der Sachwert der Wirtschaftswege in Ansatz gebracht werden. In der Wirkungsanalyse wurden die Sachwerte nach der Sachwertmethode ( $\$ \S 21-25$ WertV) bestimmt. 
gerechnet $\left(\mathrm{VV}_{\text {Wege }}\right)$. Ein weiteres Drittel dieser Summe gilt als Vorteil der regionalen Tourismusbranche aus der Nutzung des Wegenetzes für den Rad- und Wanderwegetourismus. Das letzte Drittel wird bei der Land- und Forstwirtschaft in Ansatz gebracht, die erhebliche Zeiteinsparungen bei der verbesserten Anfahrt der Nutzflächen realisiert.

$$
V V_{\text {Wege }}=\frac{1}{3} \cdot\left(I_{\text {Wege }}+P L K_{\text {Wege }}\right)
$$

Zur Ermittlung der beim Wegebau anfallenden Planungskosten (PLK $\mathrm{K}_{\text {Wege }}$ ) wird die Verordnung über die Honorare und Leistungen der Architekten und der Ingenieure (HOAI), Teil VII „Leistungen bei Ingenieurbauwerken und Verkehrsanlagen“, herangezogen. ${ }^{1002}$ Planungsleistungen beim Wirtschaftswegebau fallen gemäß $§ 54$ (2) HOAI allgemein in die Honorarzone I. ${ }^{1003}$ Die Planung von landwirtschaftlichen Wirtschaftswegen im Rahmen der Bodenordnung ist den in $\$ 55$ (1) HOAI kodifizierten Leistungsphasen 1 bis 5 und 9 zuzuordnen. ${ }^{1004}$ Diese Leistungen umfassen ca. $70 \%$ des Gesamthonorars der Architekten und Ingenieure. Um auch die Planungskosten für Ausgleichsmaßnahmen abzudecken, werden bei der Kalkulation der Gesamtplanungskosten die in $§ 56$ (2) HOAI hinterlegten Höchstsätze in Ansatz gebracht. ${ }^{1005}$ Diese Höchstsätze werden in Abhängigkeit der Herstellungskosten der Wirtschaftswege $\left(\mathrm{HK}_{\mathrm{Wcgc}}\right)$, die im Gesetzestext als anrechenbare Kosten bezeichnet werden, festgelegt. Um die Herleitung der Planungshonorare $(\mathrm{H})$ zu erleichtern, werden die anrechenbaren Kosten der Höhe nach in vier Kategorien eingeteilt. Diesen Kategorien ist jeweils ein pauschaler Prozentwert (p) für das Planungshonorar zugeordnet: ${ }^{1006}$

- Kategorie I:

bis $75.000 €$;

$$
\begin{aligned}
& p=11,50 \% \\
& p=7,25 \% \\
& p=4,00 \% \\
& p=2,75 \%
\end{aligned}
$$

- Kategorie II:

$75.000 €-750.000 €$;

- Kategorie III:

$750.000 €-7,5$ Mio. $€$;

- Kategorie IV:

ab 7,5 Mio. $€$;

Bei Herstellungskosten für das Wegenetz bis einschließlich $75.000 €$ sind die Planungshonorare beispielsweise mit 11,5\% dieser Kosten in Ansatz zu bringen, bei Herstellungskosten über 7,5 Mio. $€$ allerdings nur noch mit $2,75 \%$. Wie bereits erwähnt, fallen beim Wegebau im Rahmen der Bodenordnung rund 70\% dieser Honorare an, so dass sich Planungskosten in folgender Höhe ergeben:

$$
P L K_{\text {Wege }}=0,7 \cdot H K_{\text {Wege }} \cdot p
$$

\footnotetext{
1002 Vgl. HOAI (Verordnung über die Honorare und Leistungen der Architekten und der Ingenieure).

1003 Vgl. \$54 (2) HOAI.

$1004 \mathrm{Vgl}$. 55 (1) HOAI.

1005 Eine zusätzliche Ermittlung der Planungshonorare für die Ausgleichsmaßnahmen kann somit entfallen.

1006 Die Ermittlung der Kategorien und Prozentwerte erfolgt auf Basis von Modellrechnungen.
} 


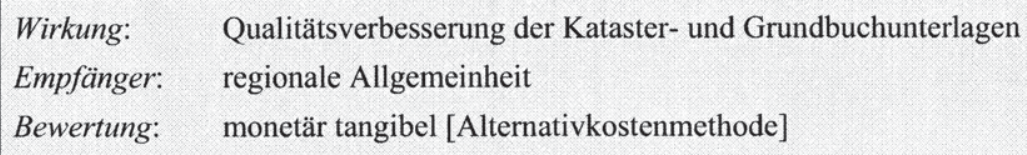

Die Bodenordnung leistet einen Beitrag zur Erneuerung der Katasterunterlagen im Verfahrensgebiet und damit zur Erfüllung des gesetzlichen Auftrags der regelmäßigen Aktualisierung des Liegenschaftskatasters. ${ }^{1007}$ Die monetäre Quantifizierung dieses Wirkungsbeitrags $\left(A_{K}\right)$ erfolgt auf Grundlage der Kosten, die außerhalb der Bodenordnung für eine Katastererneuerung angefallen wären (VK). Es wird dabei unterstellt, dass das gesellschaftliche Interesse an einer Erneuerung des Liegenschaftskatasters und die damit verbundene Zahlungsbereitschaft wesentlich durch den Zustand der zugrunde liegenden Unterlagen bestimmt werden. Da das gesellschaftliche Interesse an einer regelmäßigen und systematischen Erneuerung der Katasterunterlagen jedoch aufgrund der relativ hohen Kosten eher gering ist, werden höchstens $50 \%$ der ermittelten Vermessungskosten angesetzt. Dies ist der Fall, wenn das vorhandene Kataster noch auf Urvermessungen beruht, ansonsten werden höhere Abschläge vorgenommen: ${ }^{1008}$

- Urvermessung: $\quad 50 \%$

- Neuvermessung vor 1960: $\quad 30 \%$

- Neuvermessung zwischen 1960 und 1980: $\quad 20 \%$

- Neuvermessung nach 1980: $\quad 10 \%$

Die Kosten zur Aktualisierung der Katasterunterlagen werden auf Grundlage der Vermessungskosten (VK) ermittelt, die außerhalb der Bodenordnung im Rahmen einer Erneuerung der öffentlichen Bücher anfallen würden. Hierzu sind die entsprechenden Tagessätze $\left(\mathrm{ts}_{\mathrm{k}}\right)$ für den Außendienst der Vermessung für Ingenieure $(\mathrm{k}=1)$ und Messgehilfen $(\mathrm{k}=2)$ zu berechnen. ${ }^{1009}$ Unter Berücksichtigung des notwendigen Zeitaufwandes (ZA) für die Neuvermessung pro Tag und ha sowie die Neuvermessungsfläche in ha $\left(\mathrm{FL}_{\text {neu }}\right)$ ergeben sich die Kosten des Außendienstes. Zuzüglich eines $15 \%$ igen Aufschlags für die Arbeiten im Innendienst ergeben sich die Kosten einer Katastererneuerung wie folgt: ${ }^{1010}$

$$
V K=1,15 \cdot\left[\sum_{k=1}^{2} t s_{k} \cdot Z A \cdot F L_{n e u}\right]
$$

\footnotetext{
1007 Vgl. §l (3) VermKatG und Kapitel 5.3.5.3.1.

1008 Vgl. BMS Consulting (2005), S. 185.

1009 Die Tagessätze für Ingenieurleistungen und Messgehilfen sind in der HOAI geregelt.

1010 Vgl. Mosiek/Pieper/Kasten (2006), S. 120 f.
} 


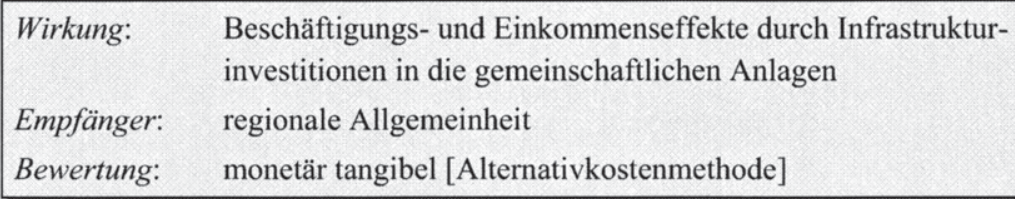

Investitionen in die Infrastruktur und das regionale Wegenetz haben in der Regel positive Einkommens- und Beschäftigungseffekte zur Folge. HARTWIG ET AL. haben auf Grundlage der Input-Output-Analysen des Statistischen Bundesamtes die durchschnittlichen Beschäftigungseffekte ermittelt, die durch eine Infrastrukturinvestition in Höhe von $1 \mathrm{Mrd}$. $€$ ausgelöst werden. ${ }^{1011}$ Demnach führt eine Investition von $1 \mathrm{Mrd} . €$ für die Auftragnehmer der Bauwirtschaft zu einem Beschäftigungseffekt in Höhe von durchschnittlich 11.860 Erwerbstätigenjahren (direkter Effekt). Im Bereich der Vorleistungsund Investitionsgüterlieferanten können im Mittel 6.480 Arbeitsplätze (indirekter Effekt) und in sonstigen Bereichen 3.204 Arbeitsplätze (induzierter Effekt) geschaffen werden. ${ }^{1012}$ Daraus ergibt sich ein Gesamteffekt von 21.544 Erwerbstätigenjahren. ${ }^{1013}$ Der Beschäftigungseffekt aus der Bodenordnung lässt sich anhand der Investitionen in die örtliche Infrastruktur und das regionale Wegenetzes $\left(I_{\text {gesamt }}\right)$ wie folgt ermitteln:

$$
\text { Beschäftigungseffekt }=\frac{I_{\text {gesumt }}}{1.000 .000 .000} \cdot 21.544
$$

Die monetäre Bewertung dieses Wirkungsbeitrags erfolgt auf Grundlage der gesamtfiskalischen Kosten der Arbeitslosigkeit in Deutschland. ${ }^{1014}$ BACH ET AL. berücksichtigen im Rahmen ihrer Modellrechnung sowohl die Ausgabenseite des Staates, als auch die Belastungen der öffentlichen Haushalte auf der Einnahmeseite. Dabei wählen sie einen Minimalkostenansatz, der die direkten Ausgaben für Arbeitslosengeld und -hilfe sowie andere Sozialleistungen berücksichtigt und auch Mindereinahmen an Steuern und Sozialbeiträgen kalkuliert. Andere Mindereinnahmen, wie z.B. bei der Mehrwertsteuer, bleiben unberücksichtigt. ${ }^{1015} \mathrm{Im}$ Durchschnitt ergeben sich damit staatliche Gesamtkosten der Arbeitslosigkeit in Höhe von $18.986 €$ pro Person und Jahr. ${ }^{1016}$ Auf dieser Grundlage lässt sich der monetäre Beschäftigungseffekt einer Infrastruktur- oder Wegenetzinvestition $(\mathrm{BESCH})$ wie folgt ermitteln:

$$
B E S C H=\frac{I_{\text {gesam }}}{1.000 .000 .000} \cdot 21.544 \cdot 18.986 €
$$

1011 Vgl. Hartwig et al. (2005), S. $54 \mathrm{ff}$.

1012 Die Anzahl der Arbeitsplätze ist auch jeweils in Erwerbstätigenjahren gemessen.

1013 Hierbei wurden lediglich neu geschaffene Arbeitsplätze berücksichtigt. Vgl. Hartwig et al. (2005), S. 48 ff.

1014 Vgl. Bach et al. (2003).

1015 Eine hohe Arbeitslosenquote ist im Allgemeinen mit einer Reduktion der Kaufkraft verbunden, was wiederum zu Mindereinnahmen im Bereich der Mehrwertsteuer führt. Vgl. Bach et al. (2003), S. 5.

1016 Die Kalkulation der Durchschnittskosten basiert auf den entsprechenden Daten des IAB für die Jahre 1998 bis 2004. Vgl. IAB (2004). 


\section{Wirkung: Verbesserung des überregionalen Hochwasserschutzes \\ Empfänger: regionale Allgemeinheit \\ Bewertung: monetär tangibel [schadenskompensierende Güter]}

Die Realisierung wasserrechtlicher Planungen zum überregionalen Hochwasserschutz wird mit Hilfe von Bodenordnungsverfahren wesentlich beschleunigt, im Extremfall wird die Realisierung überhaupt erst ermöglicht. ${ }^{1017}$ Im Rahmen der Wirkungsanalyse wird ein vorsichtiger Ansatz gewählt und eine durchschnittliche Beschleunigung von drei Jahren $(b=3)$ unterstellt. ${ }^{1018}$ Der Wirkungsbeitrag der Bodenordnung $\left(\mathrm{BE}_{\mathrm{HW}}\right)$ besteht somit in der frühzeitigen Verbesserung des Hochwasserschutzes und die damit verbundene vorzeitige Schadensvermeidung $\left(\mathrm{S}^{\mathfrak{c}}\right)$. Das jährliche Einsparpotenzial an vermiedenen Hochwasserschäden $\left(S^{e}\right)$ ergibt sich aus der Multiplikation der Schadenseintrittswahrscheinlichkeit mit dem verfahrensspezifischen Erwartungswert für Hochwasserschäden. Diese lassen sich aus speziellen Untersuchungen zu den Schadenspotenzialen von 100-jährigen Hochwassern in den betroffenen Regionen in RheinlandPfalz ermitteln. ${ }^{1019}$

$$
B E_{H W}=b \cdot S^{e}
$$

\section{Wirkung: Vermeidung örtlicher Hochwasserschäden \\ Empfänger: regionale Allgemeinheit \\ Bewertung: monetär tangibel [schadenskompensierende Güter]}

Kleinere, lokal wirkende Hochwassermaßnahmen können häufig nur im Rahmen eines Flurbereinigungsverfahrens realisiert werden, so dass der gesamte Barwert der vermiedenen Schäden bzw. der Barwert der Schadensbeseitigungskosten $\left(\mathrm{BW}^{\mathrm{S}}\right)$ als Wirkungskomponente der Bodenordnung gewertet werden kann. Die Höhe der durchschnittlich jährlich vermiedenen Schäden bzw. die durchschnittlichen Kosten der Schadensbeseitigung (S) können in der Regel von Vertretern der örtlichen Kommunen abgeschätzt werden. Die Kalkulation des monetären Wirkungsbeitrags erfolgt unter der Annahme einer Gesamtwirkungsdauer von 50 Jahren $(T=50)$ und einer Diskontierungsrate in Höhe von $4 \%(i=0,04):{ }^{1020}$

$$
B W^{S}=S \cdot \sum_{t=0}^{T-1}(1+i)^{-t}
$$

1017 Vgl. das Expertengespräch mit Vertretern der Wasserwirtschaft in Mosiek/Pieper/Kasten (2006), S. 67.

1018 Der Zeitansatz orientiert sich damit wiederum an der Mindestdauer gerichtlicher Enteignungsverfahren.

$1019 \mathrm{Zu}$ den jeweiligen Schadenspotenzialen vgl. detailliert Mosiek/Pieper/Kasten (2006), S. $122 \mathrm{ff}$.

1020 Entsprechend den üblichen Kalkulationsverfahren in der Wasserwirtschaft wurde an dieser Stelle eine Wirkungsdauer von 50 Jahren sowie eine Diskontrate von $4 \%$ unterstellt. 


\begin{tabular}{|ll}
\hline Wirkung: & Erhalt der Kulturlandschaft und des Landschaftsbildes/ \\
& Verbesserung der Naherholung und des Freizeitwertes \\
Empfänger: & regionale Allgemeinheit \\
Bewertung: & intangibel
\end{tabular}

Die positiven Auswirkungen der Bodenordnung auf den Erhalt der Kulturlandschaft und die Verbesserung der Naherholung und des Freizeitwertes wurde in Studien nachgewiesen. ${ }^{1021}$ Aufgrund des subjektiven Charakters dieser Wirkungen muss die Bewertung allerdings intangibel bleiben.

\subsubsection{Bewertung der Auswirkungen auf die Wirtschaft}

$$
\begin{array}{ll}
\text { Wirkung: } & \text { Bewirtschaftungsvorteile durch Flächenneuordnung } \\
\text { Empfänger: } & \text { Land- und Forstwirtschaft } \\
\text { Bewertung: } & \text { monetär tangibel [Kosten- und Zeitersparnisse] }
\end{array}
$$

Zur monetären Bewertung der Bewirtschaftungsvorteile, die sich im Zuge der Flächenneuordnung und -zusammenlegung in der Bodenordnung ergeben, sind in Rheinland-Pfalz grundsätzlich drei Bereiche zu unterscheiden:

- Bewirtschaftungsvorteile in der Landwirtschaft

- Bewirtschaftungsvorteile im Weinbau

- Bewirtschaftungsvorteile in der Forstwirtschaft

Im Bereich der Landwirtschaft erfolgt die Bewertung auf Grundlage der Ergebnisse einer im Auftrag des Bundesministeriums für Ernährung, Landwirtschaft und Verbraucherschutz durchgeführten Studie zur „Effizienz staatlich geförderter Flurneuordnungsverfahren nach dem Flurbereinigungsgesetz". ${ }^{1022}$ Im Rahmen dieser Untersuchung wurden Pauschalwerte für die Kosteneinsparungen bei der Bewirtschaftung von Acker- und Grünflächen hergeleitet. Dabei wurde in insgesamt neun Flurbereinigungsverfahren auf einzelbetrieblicher Ebene das Einsparpotenzial an variablen Maschinenkosten, Feldrandverlusten und Arbeitszeit analysiert. ${ }^{1023}$ Die Gesamteinsparung (EP) ist abhängig vom Zusammenlegungsverhältnis in der Bodenordnung, so dass drei Kategorien unterschieden werden:

- geringe Zusammenlegung (bis 2:1)

Ackerland: $51 € \quad$ Grünland: $137 €$

- mittlere Zusammenlegung (bis 3:1)

Ackerland: $86 € \quad$ Grünland: $232 €$

- hohe Zusammenlegung (ab 3:1)

Ackerland: $107 € \quad$ Grünland: $287 €$

\footnotetext{
1021 Vgl. Bauer et al. (1979), S. 56 ff. sowie die Ausführungen des Kapitels 5.3.5.3.1.

1022 Vgl. BMELV (2006).

1023 Vgl. BMELV (2006), S. 58 f. und S. 74 f.
} 
Als Gesamtvorteil der Flächenneuordnung wird der Barwert des jeweiligen Einsparpotenzials (EP x NFL mit NFL = landwirtschaftliche Nutzfläche in ha) in Ansatz gebracht, wobei eine Wirkungsdauer von 25 Jahren $(T=25)$ sowie die in der Landwirtschaft übliche Diskontierung von $4 \%\left(i_{L}=0,04\right)$ unterstellt wird:

$$
B V_{\text {Land }}=E P \cdot N F L \cdot \sum_{t=0}^{T-1}\left(1+i_{L}\right)^{-t}
$$

Im Bereich des Weinbaus, welcher in Rheinland-Pfalz ein bedeutender Wirtschaftsfaktor ist, erfolgt die Bewertung ebenfalls auf Grundlage von einzelbetrieblichen Untersuchungen. ${ }^{1024}$ Bei der Ermittlung der Bewirtschaftungsvorteile $\left(\mathrm{BV}_{\text {Wein }}\right)$ die aus einer Rebflurbereinigung resultieren, ist eine Unterscheidung zwischen Weinbau in der Ebene, Weinbau in Hanglagen, Weinbau in Steillagen und Steillagenweinbau in Flusstälern notwendig. Für die vier unterschiedenen Anbaugebiete werden somit Durchschnittswerte für das jährliche Einsparpotenzial (EP) durch die Bodenordnung je ha hergeleitet: ${ }^{1025}$

- Weinbau in der Ebene:

$475 €$ pro Jahr und ha

- Weinbau in Hanglagen:

$600 €$ pro Jahr und ha

- Weinbau in Steillagen (Zweitbereinigung):

$600 €$ pro Jahr und ha

- Steillagenweinbau in Flusstälern:

$2.500 €$ pro Jahr und ha

Der monetäre Bewirtschaftungsvorteil entspricht dem Barwert der Gesamtkostenersparnis (EP x RFL mit RFL = Rebfläche in ha). Dabei wird analog zu den landwirtschaftlichen Effekten eine Wirkungsdauer von 25 Jahren $(\mathrm{T}=25)$ sowie eine Diskontierungsrate von $4 \%\left(i_{L}=0,04\right)$ unterstellt:

$$
B V_{W e i n}=E P \cdot R F L \cdot \sum_{t=0}^{T-1}\left(1+i_{L}\right)^{-t}
$$

Im Bereich der Forstwirtschaft ist es Aufgabe der Bodenordnung, den Wald sowohl für eine forstwirtschaftliche Nutzung zugänglich zu machen, als auch ihn in seiner Schutz- und Erholungsfunktion zu stärken. Die Förderung dieser Funktionen hat wiederum positive Folgewirkungen auf die regionale Beschäftigungssituation, die Erschließung des Waldes, die regionale Tourismusbranche und auf den Naturschutz bzw. die Landschaftspflege.

1024 Vgl. Adams (1995) und Bildungsseminar der Agrarverwaltung Rheinland-Pfalz (1997).

1025 Dabei wurden auch die Arbeitskostenersparnisse in Folge einer Verbesserung des Wegenetzes berücksichtigt. $\mathrm{Zu}$ den monetären Ergebnissen vgl. Adams (1995), S. 35 ff. und Bildungsseminar der Agrarverwaltung Rheinland-Pfalz (1997), S. 68 f. 
Der Wirkungsbeitrag der Waldflurbereinigung ( $\left.\mathrm{BV}_{\mathrm{Wald}}\right)$ hängt zum einen vom Erschlossenheitsgrad des Waldes ab. Zum anderen ist es für die Ermittlung der monetären Effekte aber auch von zentraler Bedeutung, ob der Wald bereits vor der Durchführung der Bodenordnung bewirtschaftet oder erst im Zuge der Flurbereinigung einer wirtschaftlich sinnvollen Nutzung zugänglich gemacht wurde. Zudem hat der Waldtyp einen wichtigen Einfluss auf die Höhe des monetären Wirkungsbeitrags der Bodenordnung. Der Bewertungsansatz wurde in Zusammenarbeit mit Fachvertretern aus dem Bereich der Forstwirtschaft entwickelt. Demnach können folgende Bewertungen (EP) in Ansatz gebracht werden: ${ }^{1026}$

- Unerschlossener Laubwald:

- Unerschlossener Nadelwald:

- Teilerschlossener Wald:
$120 €$ pro Jahr und ha

$108 €$ pro Jahr und ha

$72 €$ pro Jahr und ha

Der monetäre Bewirtschaftungsvorteil entspricht dem Barwert der Gesamtkostenersparnis (EP x WFL mit WFL = Waldfläche in ha). Dabei wird analog zu den bisherigen Einschätzungen eine Wirkungsdauer von 25 Jahren $(\mathrm{T}=25)$ sowie eine Diskontierungsrate von $4 \%\left(i_{L}=0,04\right)$ unterstellt:

$$
B V_{\text {Wald }}=E P \cdot W F L \cdot \sum_{t=0}^{T-1}\left(1+i_{L}\right)^{-t}
$$

$\begin{array}{ll}\text { Wirkung: } & \text { Bewirtschaftungsvorteile durch das verbesserte Wegenetz } \\ \text { Empfänger: } & \text { Land- und Forstwirtschaft } \\ \text { Bewertung: } & \text { monetär tangibel [Zeitersparnisse] }\end{array}$

Aus der Verbesserung des regionalen Wegenetzes resultieren Kostenersparnisse für die Land- und Forstwirtschaft. ${ }^{1027}$ Diese Kostenersparnisse sind grundsätzlich zur monetären Bewertung geeignet. Die aus der Verbesserung des Wegenetzes resultierenden $\mathrm{Be}$ wirtschaftungsvorteile sind allerdings bereits in den oben beschriebenen Bewertungsansätzen für die Land- und Forstwirtschaft sowie den Weinbau berücksichtigt, so dass ein differenzierter Ausweis der Effekte im Rahmen dieser Wirkungsanalyse unterbleibt.

\footnotetext{
1026 Für die weitestgehend unerschlossenen und nicht bewirtschafteten Waldflächen führt eine Waldflurbereinigung zu einem erwartenden Ertrag von 6 Festmetern Holz pro Jahr und ha. Bei teilerschlossenen Waldflächen reduzieren sich die Bewirtschaftungskosten durch kürzere Transportwege sowie einer verbesserten Erschließung der Grundstücke. Zudem erfolgt eine Zusammenlegung von Streuparzellen, so dass der Wald in Summe wirtschaftlich genutzt werden kann. Die Werte der Kosteneinsparungen sind nach Abzug der Holzerntekosten ausgewiesen.
}

1027 Vgl. Kapitel 5.3.5.3.2. 


$$
\begin{array}{ll}
\text { Wirkung: } & \text { Optimierung der Flächenränder } \\
\text { Empfänger: } & \text { Land- und Forstwirtschaft } \\
\text { Bewertung: } & \text { tangibel [Wirkungsindikator: opt. Flächenränder in \%] }
\end{array}
$$

Zur Operationalisierung der optimierten Flächenränder innerhalb einer Bodenordnung kann als Kennzahl der Anteil der »opt. Flächenränder in \%« als Wirkungsindikator herangezogen werden. Dabei ist von der Flurbereinigungsverwaltung ein möglichst hoher Anteil optimierter Flächenränder anzustreben.

\begin{tabular}{|ll|}
\hline Wirkung: & Verringerung der Bürokratiekosten bei Prämienkontrollen \\
Empfänger: & Land- und Forstwirtschaft \\
Bewertung: & monetär tangibel [Kostenersparnisse] \\
\hline
\end{tabular}

Aus der Qualitätsverbesserung der öffentlichen Bücher im Rahmen eines Bodenordnungsverfahrens resultieren für die Landwirtschaft im Wesentlichen zwei positive Effekte und zu einer Entlastung an Bürokratiekosten:

- geringere Bürokratiekosten bei der Beantragung von Flächenprämien $\left(V^{e}{ }_{L w}\right)$

- geringere Bürokratiekosten bei der staatlichen Prämienkontrolle (VA $\left.{ }_{\mathrm{PK}}^{\mathrm{e}}\right)$

Der zeitliche Aufwand der Landwirte bei der Beantragung von Flächenprämien hängt entscheidend von der Betriebsgröße, von der Anzahl und Größe der einzelnen Parzellen sowie von der Nutzungsart der Flächen ab. Das spezifische Einsparpotenzial wird daher mit durchschnittlich einem Arbeitstag pro Jahr und Landwirt (Lw) angenommen. ${ }^{1028}$ Zugleich wird eine tägliche Arbeitszeit von $8 \mathrm{~h}$ und ein Kostensatz von $15 €$ in Ansatz gebracht. Auf dieser Grundlage ergibt sich bei einer unterstellten Wirkungsdauer von 25 Jahren $(T=25)$ und einer Diskontierungsrate von $4 \%(i=0,04)$ folgender Gesamteffekt $\left(\mathrm{VA}_{\mathrm{Lw}}^{\mathrm{e}}\right)$ :

$$
V A_{L w}^{e}=[L w \cdot 8 h \cdot 15 € / h] \cdot \sum_{t=1}^{T-1}(1+i)^{-t}
$$

Zusätzlich verringert sich auch im Rahmen von Prämienkontrollen der zeitliche Aufwand der Landwirte. In Rheinland-Pfalz werden nach Angaben des zuständigen Prüfdienstes jedes Jahr ungefähr 8\% aller landwirtschaftlichen Betriebe kontrolliert. Nach Abschätzung des Prüfdienstes dauert die Prüfung eines Betriebes im Mittel einen Tag, d.h. in etwa acht Stunden. ${ }^{1029}$ Aufgrund der Flächenzusammenlegung und der Verbesserung der Katasterunterlagen ist im Durchschnitt eine Zeitersparnis von $50 \%$ bzw.

1028 Die Annahme beruht auf Expertenschätzungen.

1029 Dabei handelt es sich um einen Referenzbetrieb in Rheinland-Pfalz mit 40 ha Nutzfläche. 
von 4 Stunden pro Betrieb zu erwarten. Da die Landwirte den Prüfern während der Kontrolle zur Verfügung stehen müssen, ist auch ihnen diese zeitliche Ersparnis als Wirkungsbeitrag anzurechnen $\left(\mathrm{VA}_{\mathrm{PK}}^{\mathrm{c}}\right)$. Zur Ermittlung der Anzahl der landwirtschaftlichen Betriebe wird die Nutzfläche des Verfahrensgebietes (NFL) durch die Größe eines Referenzbetriebes (40 ha) geteilt. Der Gesamteffekt lässt sich bei einer unterstellten Wirkungsdauer der Flurbereinigung von 25 Jahren $(T=25)$, einer Diskontierungsrate von $4 \%(i=0,04)$ und einem Stundenkostensatz von $15 €$ wie folgt ermitteln:

$$
V A_{P K}^{e}=\left[0,08 \cdot \frac{N F L}{40} \cdot 4 h \cdot 15 € / h\right] \cdot \sum_{t=1}^{T-1}(1+i)^{-t}
$$

Wirkung: $\quad$ Positive wirtschaftliche Effekte durch Folgeinvestitionen

Empfänger: Industrie- und Dienstleistungsunternehmen

Bewertung: intangibel

Die agrarstrukturellen Verbesserungen in der Land- und Forstwirtschaft machen in der Regel den Einsatz größerer und leistungsfähigerer Maschinen rentabel. Die nicht unerheblichen Folgeinvestitionen in die entsprechende Mechanisierung und Kapazitätsausweitung haben positive Einkommens- und Beschäftigungseffekte in Vorleistungsbranchen zur Folge. Der Gesamteffekt ist jedoch schwierig quantifizierbar, weshalb der Wirkungsbeitrag im Rahmen der Wirkungsanalyse nur intangibel bewertet wird.

$$
\begin{array}{ll}
\text { Wirkung: } & \text { Flächenbereitstellung für die regionale Wirtschaft } \\
\text { Empfänger: } & \text { Industrie- und Dienstleistungsunternehmen } \\
\text { Bewertung: } & \text { monetär tangibel [Kostenersparnisse] }
\end{array}
$$

Im Rahmen des Flächenmanagements der Bodenordnung werden häufig auch benötigte Flächen für Unternehmen beschafft, beispielsweise für Umsiedlungen oder für Erweiterungen. In der Regel profitiert das Unternehmen in diesem Zusammenhang von denselben Kostenersparnissen $\left(\mathrm{FBK}^{\mathrm{c}}\right)$ wie ein Projektträger der öffentlichen Verwaltung. Dies sind Ersparnisse bei den Grunderwerbskosten, bei den eigenen Personalund Sachkosten für den Grunderwerb, bei den Kataster- und Notargebühren und eventuell entstehenden Vermessungskosten. Da in der Regel jedoch staatliche Projekte durch Bodenordnungsverfahren begleitet werden, sollen die einzelnen Bewertungsansätze zur Ermittlung dieser Einspareffekte bei der staatlichen Empfängerebene näher erläutert werden. ${ }^{1030}$ 


$$
\begin{array}{ll}
\text { Wirkung: } & \text { Offenhaltung und Aufwertung der Kulturlandschaft } \\
\text { Empfänger: } & \text { regionale Tourismusunternehmen } \\
\text { Bewertung: } & \text { intangibel }
\end{array}
$$

Ein strukturiertes Landschaftsbild stellt eine der Grundvoraussetzungen für eine wettbewerbsfähige regionale Tourismusbranche dar. Die Bodenordnung leistet im Allgemeinen einen wertvollen Beitrag zur Offenhaltung und Aufwertung der regionalen Kulturlandschaft und fördert damit auch den Erfolg der regionalen Tourismusbranche. ${ }^{1031}$ Eine Quantifizierung dieses Wirkungsbeitrags zum wirtschaftlichen Erfolg der regionalen Tourismusunternehmen ist allerdings außerordentlich schwierig, so dass die Bewertung zunächst intangibel bleibt.

\begin{tabular}{ll}
\hline Wirkung: & Realisierung spezieller touristischer Attraktionen \\
Empfänger: & regionale Tourismusunternehmen \\
Bewertung: & monetär tangibel [Abfrage der Zahlungsbereitschaft]
\end{tabular}

Die Wirkungen spezieller touristischer Attraktionen $\left(\mathrm{TV}_{\text {Projektc }}\right)$ lassen sich ausschließlich verfahrensspezifisch bewerten. Die monetäre Quantifizierung dieses Wirkungsbeitrags sollte vorzugsweise über eine Abfrage der Zahlungsbereitschaft erfolgen. Entweder werden direkt Eintrittsgelder (z.B. Museumsstätte) verlangt oder Nutzer werden danach befragt. In Abhängigkeit der Besucherzahlen lässt sich so ein monetärer Gesamtwert bestimmen. Alternativ können auch komplementäre Privatleistungen in Ansatz gebracht werden, bspw. indem man die Anfahrtskosten der Besucher auswertet. ${ }^{1032}$ Nur in Ausnahmenfällen sollte als approximativer Wert die Investitionssumme des touristischen Projekts herangezogen werden. Hierbei wird ein Nutzen-Kosten-Verhältnis von 1:1 unterstellt, so dass der Barwert der positiven Effekte die Kosten deckt. Grundsätzlich liegt allen Ansätzen die zentrale Annahme zugrunde, dass die touristischen Planungen ohne Unterstützung der Bodenordnung nicht realisiert worden wären. ${ }^{1033}$

$$
\begin{array}{ll}
\text { Wirkung: } & \text { Vorteile für den Rad- und Wanderwegetourismus } \\
\text { Empfänger: } & \text { regionale Tourismusunternehmen } \\
\text { Bewertung: } & \text { monetär tangibel [substituive Leistungen] }
\end{array}
$$

Der Neu- und Ausbau des regionalen Wegenetzes hat positive Auswirkungen auf den Rad- und Wanderwegetourismus und damit auf die regionalen Tourismusunternehmen.

1031 Vgl. Kapitel 5.3.5.3.2.

$1032 \mathrm{Zu}$ den monetären Bewertungsansätzen der Wirkungsanalyse vgl. ausführlich Kapitel 4.3.2.2.3.

1033 Falls diese Annahme nicht zutrifft, lässt sich zumindest ein Beschleunigungseffekt in Ansatz bringen. 
Es wird in diesem Zusammenhang die Annahme getroffen, dass die Nutzung der erschlossenen Wege zu etwa einem Drittel auf touristische Zwecke zurückzuführen ist. Bei einem Nutzen-Kosten-Verhältnis von 1:1 lässt sich demnach ungefähr ein Drittel der Summe aus den Investitionen $\left(\mathrm{I}_{\mathrm{Wegc}_{\mathrm{c}}}\right)$ und Planungskosten $\left(\mathrm{PLK}_{\mathrm{Wegc}}\right)$ in das Wegenetz als touristischer Wirkungsbeitrag $\left(\mathrm{TV}_{\mathrm{Wegc}}\right)$ der Bodenordnung in Ansatz bringen: ${ }^{1034}$

$$
T V_{\text {wege }}=\frac{1}{3} \cdot\left(I_{\text {wege }}+P L K_{\text {wege }}\right)
$$

\subsubsection{Bewertung der Auswirkungen auf den Staat}

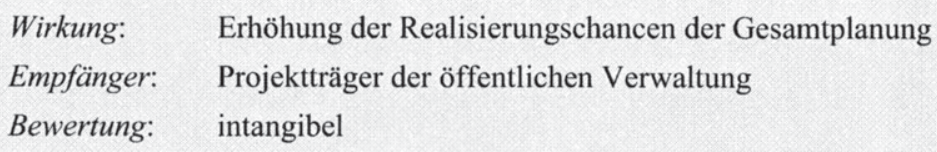

Ein besonders wichtiger Wirkungsbeitrag der Bodenordnung ist Vereinbarkeit verschiedener - teilweise um den Flächenbedarf konkurrierender - Projekte im ländlichen Raum und die Erhöhung der Realisierungschancen der Gesamtplanung. Eine Quantifizierung dieser Wirkungskomponente erscheint allerdings sehr schwierig, so dass die Bewertung intangibel bleibt.

\section{Wirkung: Kostenersparnisse im Zuge der Flächenbereitstellung \\ Empfänger: Projektträger der öffentlichen Verwaltung \\ Bewertung: monetär tangibel [Kostenersparnisse]}

Die Flächenbereitstellung für geplante Projekte durch die Flurbereinigungsverwaltung führen an anderer Stelle der öffentlichen Verwaltung zu Kostenersparnissen. Diese können wie folgt monetär bewertet werden: ${ }^{1035}$

- Grunderwerbskosten: Der Grunderwerb lässt sich im Rahmen einer Bodenordnung in der Regel flexibler, zeitnäher und kostengünstiger vollziehen. ${ }^{1036}$ Konkrete empirische Ergebnisse zur Höhe der Einsparungen liegen allerdings bislang nicht vor. Da aber sowohl sachliche Gründe, als auch Expertenbefragungen das Einsparpotenzial bei den Grunderwerbskosten $\left(\mathrm{GK}^{\mathrm{c}}\right)$ bestätigen, wird in der Wirkungsanalyse ein

\footnotetext{
${ }^{1034}$ Vgl. hierzu auch die Bewertung des Wegenetzes bei der regionalen Allgemeinheit in Kapitel 5.3.6.1.

${ }^{1035}$ Vgl. Kapitel 5.3.5.3.3.

1036 Vgl. BMS Consulting (2005), S. 170 und Mosiek/Pieper/Kasten (2006), S. 135 f.
} 
vorsichtiger Ansatz in Höhe von 10\% der anfallenden Grunderwerbskosten (GK) gewählt.

$$
G K^{e}=0,1 \cdot G K
$$

- Entschädigungsleistungen: Die Einsparung an Entschädigungen $\left(\mathrm{E}^{\mathfrak{c}}\right)$ ergibt sich als Differenz der Entschädigungsleistungen $\left(E_{\text {kalk }}\right)$, die die Träger der jeweiligen Planung bei Verzicht auf die Einleitung einer Flurbereinigung zu entrichten hätten, und den tatsächlich geleisteten Entschädigungen (E) innerhalb der Bodenordnung. Grundlage der Kalkulation der potenziellen Entschädigungsleistungen außerhalb der Bodenordnung bildet die Entschädigungsrichtlinie (EntschR) für Rheinland-Pfalz. Im Wesentlichen werden dabei Entschädigungen für An- und Durchschneidungsschäden der Grundstücke sowie für Um- bzw. Mehrwege berücksichtigt. Je kleingliedriger die Grundstücksstruktur, desto höher sind die Einsparungen an Entschädigungsleistungen: ${ }^{1037}$

$$
E^{e}=E_{k a l k}-E
$$

- Personal- und Sachkosten für den Grunderwerb: Für die Kalkulation der Personalund Sachkosten für den Grunderwerb wird unterstellt, dass ein Mitarbeiter in der Grunderwerbsabteilung eines Projektträgers, wie z.B. dem Landesbetrieb Straßenbau in Rheinland-Pfalz, im Durchschnitt 80 Grunderwerbs-fälle pro Jahr $\left(\mathrm{KV}_{\mathrm{Jahr}}\right)$ bearbeitet, d.h. es gilt $\mathrm{KV}_{\mathrm{Jahr}}=80$. Der Quotient aus der Anzahl der innerhalb der Bodenordnung getätigten Grunderwerbsfälle (KV) und der durchschnittlichen Anzahl an Kaufverträgen pro Jahr und Mitarbeiter $\left(\mathrm{KV}_{\mathrm{Jahr}}\right)$ stellt den Personalbedarf (PB) dar, der sich für den jeweiligen Projektträger bei Verzicht auf eine Bodenordnung ergeben würde. Die monetäre Quantifizierung dieses potenziellen Personalbedarfs basiert auf den durchschnittlichen laufbahnbezogenen Jahrespersonalkosten des gehobenen Dienstes (55.000 $€$ ) zuzüglich einer Vorleistungsquote von $30 \%$ für Zentralbereiche und einer Sachkostenquote von $20 \%$. Insgesamt ergeben sich daraus im Durchschnitt Personalkosten (PK) in Höhe von $85.800 € .^{1038}$ Die Einsparungen an Personal- und Sachkosten berechnen sich auf dieser Grundlage wie folgt:

$$
P K^{e}=P B \cdot P K \quad \text { mit } \quad P B=\frac{K V}{\overline{K V}} \text { Jahr }
$$

- Kataster- und Notargebühren: Im Rahmen von Bodenordnungsverfahren erfolgt der Grunderwerb auf Grundlage von Landverzichtserklärungen gemäß §52 FlurbG. Nach $\$ 52$ (3) FlurbG wird zur Absicherung dieses Grunderwerbs ein Verfügungs-

1037 Vgl. hierzu auch Mosiek/Pieper/Kasten (2006), S. 137.

1038 Zur Herleitung dieser Kostenwerte vgl. BMS Consulting (2005), S. 172 f. 
verbot in das Grundbuch gebührenfrei eingetragen. ${ }^{1039}$ Beim Grunderwerb außerhalb der Bodenordnung würden sowohl Notarkosten als auch Gebühren für notwendige Grundbucheintragungen anfallen. Die Gebühreneinsparungen $\left(\mathrm{Gb}^{\mathrm{c}}\right)$ können demnach als Wirkungsbeitrag der Bodenordnung betrachtet werden. Der Umfang der Einsparungen beträgt nach detaillierten Untersuchungen im Rahmen des NRWProjektes durchschnittlich $1 \%$ des jeweiligen Kaufpreises und wird entsprechend in Ansatz gebracht: ${ }^{1040}$

$$
G b^{e}=0,01 \cdot G K
$$

- Kosten der Vermessung: Außerhalb von Bodenordnungsverfahren fallen zusätzliche Vermessungskosten für Fortführungsvermessungen an, beispielsweise bei der Schlussvermessung eines Straßenbauwerkes. Diese Kosten werden durch die Bodenordnung eingespart. ${ }^{1041}$ Die Kalkulation des Einspareffektes erfolgt auf Grundlage der Landesverordnung über Gebühren der Vermessungs- und Katasterbehörden des Landes Rheinland-Pfalz. Die durchschnittlichen Kosten für Fortführungsvermessungen betragen demnach für langgestreckte Anlagen $17.500 €$ pro km. ${ }^{1042}$ Als Wirkungsbeitrag der Bodenordnung können die gesamten Kosten der Fortführungsvermessung in Ansatz gebracht werden, die sich aus der Multiplikation der Durchschnittskosten pro km und der Gesamtlänge der Anlage in km (L) ergeben:

$$
V K_{\text {Fort }}=17.500 € / \mathrm{km} \cdot \mathrm{L}
$$

\section{Wirkung: Baukostenersparnisse durch eine beschleunigte Projektrealisierung Empfänger: Projektträger der öffentlichen Verwaltung Bewertung: monetär tangibel [Kostenersparnisse]}

Durch eine begleitende Bodenordnung können große Bauprojekte in der Regel beschleunigt werden. Da Baukosten im Zeitverlauf realen Steigerungsraten unterworfen sind, entstehen dem Projektträger Kostenersparnisse. Zur Bestimmung der Kosteneinsparung in Folge des zeitnäheren Baubeginns $\left(\mathrm{K}_{\text {Bau }}^{\mathrm{c}}\right)$ wird die Differenz zwischen den tatsächlich gezahlten Baukosten $\left(\mathrm{K}_{\mathrm{Bau}}\right)$ und den um die geschätzten sechs Monate $(\mathrm{b}=$ 0,5) abgezinsten Baukosten gebildet. ${ }^{1043}$ Als Diskontierungssatz wird die durchschnitt-

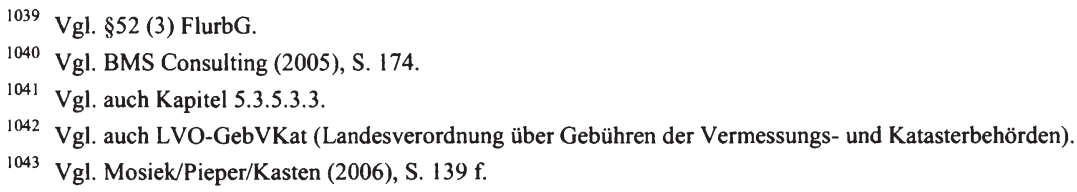


liche Wachstumsrate der realen Baupreise $(w=0,46 \%)$ im Zeitraum von 1999 bis 2005 gewählt: ${ }^{1044}$

$$
K_{B u u}^{e}=K_{B u u}-\frac{K_{B u u}}{(1+w)^{b}}
$$
Wirkung:
Baukostenersparnisse durch spezielle Maßnahmen
Empfänger:
Projektträger der öffentlichen Verwaltung
Bewertung:
monetär tangibel [Kostenersparnisse]

Für die Kostenersparnisse durch spezielle Maßnahmen der Bodenordnung lässt sich kein standardisierter Bewertungsansatz festlegen, da sie wesentlich durch die Struktur und Eigenschaften des jeweiligen Flurbereinigungsgebietes sowie durch verfahrensspezifische Besonderheiten bestimmt werden. ${ }^{1045}$ Die monetäre Bewertung des Effektes $\left(\mathrm{K}_{\text {Bo }}^{\mathrm{e}}\right)$ kann daher immer nur anhand des speziellen Projektes vorgenommen werden. Eine Abschätzung der Kostenersparnisse sollte da-bei in Zusammenarbeit mit den Trägern der betroffenen Projekte erfolgen.
Wirkung:
Kostenersparnisse bei der Unterhaltung gemeinschaftlicher Anlagen
Empfänger: Projektträger der öffentlichen Verwaltung
Bewertung: monetär tangibel [Kostenersparnisse]

Bei der Unterhaltung gemeinschaftlicher Anlagen treten im Rahmen von Bodenordnungsverfahren sowohl temporäre, als auch dauerhafte Kostenersparnisse auf. Die temporären Kostenersparnisse $\left(\mathrm{UK}^{\mathrm{c}}\right)$ durch die zeitweise Unterhaltung der gemeinschaftlichen Anlagen durch die zuständige Teilnehmergemeinschaft ergeben sich als Produkt aus der Dauer der Unterhaltung (d) in Monaten und dem monatlichen Pflegebzw. Unterhaltungskosten (UK): ${ }^{1046}$

$$
U K^{e}=d \cdot U K
$$

Werden dagegen dauerhaft Unterhaltungskosten eingespart, bspw. durch ein neu gestaltetes Wegenetz, wird der Barwert der Kosten $\left(\mathrm{BW}^{\mathrm{UK}}\right)$ in Ansatz gebracht:

$$
B W^{U K}=U K^{e} \cdot \sum_{t=0}^{T-1}(1+i)^{-t}
$$

\footnotetext{
1044 Dies entspricht der durchschnittlichen realen Verteuerung der Baupreise auf Bundesebene.

1045 Vgl. Kapitel 5.3.5.3.3.

$1046 \mathrm{Vgl}$. Mosiek/Pieper/Kasten (2006), S. 141.
} 


\section{Wirkung: Verringerung der Verwaltungskosten im Grundbuchamt \\ Empfänger: Projektträger der öffentlichen Verwaltung \\ Bewertung: monetär tangibel [Kostenersparnisse]}

Die Ersparnisse durch einen verringerten Verwaltungsaufwand im Grundbuchamt werden auf Grundlage der Untersuchungsergebnisse von KROÉS berechnet. Demnach ergeben sich in Folge der Bereinigung des Grundbuches im Rahmen der Bodenordnung durchschnittliche jährliche Einsparungen im Grundbuchamt in Höhe von 1,75€ pro ha Verfahrensfläche (VFL). ${ }^{1047}$ Auf eine inflationsbereinigte Anpassung des ermittelten Wertes wurde bewusst verzichtet, da unterstellt wird, dass sich die Verteuerungsrate und Effizienzgewinne durch den gestiegenen Automatisierungsgrad ungefähr ausgleichen. Als Wirkungsbeitrag wird der Barwert der eingesparten Verwaltungskosten $\left(\mathrm{BW}^{\text {Grundbuch }}\right)$ angesetzt. Dabei wird ein Diskontierungssatz i von 3\% und eine Laufzeit $\mathrm{T}$ von 25 Jahren unterstellt:

$$
B W^{\text {(innundhac' }}=1,75 \cdot V F L \cdot \sum_{t=0}^{T-1}(1+i)^{-t}
$$

Wirkung: Verringerung der Verwaltungskosten der Katasternutzer

Empfänger: Projektträger der öffentlichen Verwaltung

Bewertung: monetär tangibel [Kostenersparnisse]

Die Bürokratiekostenersparnisse der Landwirte bei Prämienkontrollen führen auch bei der kontrollierenden Verwaltungseinheit zu Kostenersparnissen. ${ }^{1048}$ In Rheinland-Pfalz ist der Agrarförderungsdienst der $A D D$ für die Prämienkontrollen zuständig. Jährlich werden $8 \%$ aller Betriebe kontrolliert. Die Prüfung eines 40 ha-Referenzbetriebes durch ein Prüfteam, bestehend aus einem Erst- und einem Zweitprüfer, dauert durchschnittlich acht Stunden. In Folge einer Bodenordnung lässt sich der Zeitbedarf auf vier Stunden halbieren. ${ }^{1049}$ Die monetäre Bewertung der Zeitersparnis des Prüfdienstes erfolgt auf Grundlage der durchschnittlichen Personalkostensätze des öffentlichen Dienstes. Für den Erstprüfer wird ein durchschnittlicher Tagessatz für den gehobenen Dienst $\left(\mathrm{ts}_{\mathrm{gD}}\right)$ und für den Zweitprüfer für den mittleren Dienst $\left(\mathrm{ts}_{\mathrm{mD}}\right)$ in Ansatz gebracht, wobei der Tagessatz im gehobenen Dienst derzeit bei $289 €$ und im mittleren Dienst bei $216 €$ liegt. Bei einer Wirkungsdauer von 25 Jahren $(T=25)$ und einer Diskontierungsrate von $4 \%(i=0,04)$ ergeben sich folgende Kostenersparnisse $\left(\mathrm{KK}_{\mathrm{PD}}^{\mathrm{c}}\right)$ :

$$
K K_{P D}^{e}=\left[\frac{1}{2} \cdot\left(0,08 \cdot \frac{N F L}{40} \cdot\left(t s_{g D}+t s_{m D}\right)\right)\right] \cdot \sum_{t=0}^{T-1}(1+i)^{-t}
$$

\footnotetext{
1047 Vgl. Kroés (1971), S. 109.

1048 Vgl. Kapitel 5.3.6.2.
}

1049 Die Annahme beruht auf Expertenschätzungen des Agrarförderungsdienstes der ADD. 


\subsubsection{Bewertung der Auswirkungen auf die Umwelt}

\begin{tabular}{|ll|}
\hline Wirkung: & Förderung naturnaher Lebensräume und Entwicklung ökologisch \\
& werthaltiger Biotopenverbundsysteme \\
Empfänger: & Ökologische Umwelt \\
Bewertung: & intangibel
\end{tabular}

Die Bodenordnung leistet einen bedeutenden Beitrag zur Umsetzung von Naturschutzplanungen sowie zur Förderung naturnaher Lebensräume. Zugleich werden Kompensationsflächen für Infrastrukturvorhaben zu ökologisch werthaltigen Biotopenverbundsystemen entwickelt. ${ }^{1050}$ Der Naturschutz besitzt heute eine hohe gesellschaftliche und politische Wertschätzung, dennoch lassen sich Effekte nur schwierig quantifizieren, so dass die Bewertung zunächst intangibel bleibt.

\begin{tabular}{|ll|}
\hline Wirkung: & Verringerung der Bodenerosion \\
Empfänger: & Ökologische Umwelt \\
Bewertung: & intangibel \\
\hline
\end{tabular}

Die Bodenordnung verbessert den Bodenschutz und leistet damit einen bedeutenden Beitrag zur Vermeidung von Bodenerosionen. ${ }^{1051}$ Doch auch dieser Wirkungsbeitrag lässt sich nur schwierig quantifizieren und bleibt damit intangibel.

$$
\begin{array}{ll}
\text { Wirkung: } & \text { Verbesserung der Gewässerqualität } \\
\text { Empfänger: } & \text { Ökologische Umwelt } \\
\text { Bewertung: } & \text { tangibel [Wirkungsindikator: Gewässerstrukturgüte] }
\end{array}
$$

Bodenordnungsverfahren führen häufig zu einer Verbesserung der Strukturgüte sowie der allgemeinen Qualität von Gewässern. Die Naturnähe bzw. der ökologische Wert eines Gewässers lässt sich mit Hilfe einer 7-stufigen Skala tangibel bewerten. Die Skala der Gewässerstrukturgüte kann so als Wirkungsindikator zum Einsatz kommen. Unveränderte Gewässerabschnitte werden dabei in Güteklasse I und vollständig veränderte Abschnitte in Klasse VII eingestuft. ${ }^{1052}$

\footnotetext{
1050 Vgl. Mosiek/Pieper/Kasten (2006), S. 112.

1051 Vgl. Kapitel 5.3.5.3.4.

1052 Vgl. Mosiek/Pieper/Kasten (2006), S. 187.
} 


\subsubsection{Anwendung der hergeleiteten Analyse- und Bewertungsschemata}

Die zweite Säule einer Wirkungsanalyse bildet die Anwendungsphase. ${ }^{1053}$ Nach dem Aufbau der allgemeinen Analyse- und Bewertungssystematik für den staatlichen Produktbereich »Bodenordnung « innerhalb der Konzeptionsphase, ist es Aufgabe der Anwendungsphase einer Wirkungsanalyse, die laufende operative Erfolgskontrolle im Rahmen eines systematischen Controllingprozesses vorzunehmen. Dementsprechend verfolgt die Flurbereinigungsverwaltung in Rheinland-Pfalz ${ }^{1054}$ das Ziel, auf Grundlage der vorgestellten Systematik, für sämtliche Bodenordnungsverfahren das Verfahren der Wirkungsrechnung fest zu etablieren. ${ }^{1055}$ So soll zukünftig vor Einleitung eines Flurbereinigungsverfahrens regelmäßig eine Wirkungsprognose erfolgen. Die Ergebnisse der Wirkungsprognose werden mit den geplanten Kosten des Verfahrens verglichen, um auf dieser Grundlage weitere Entscheidungen zur Gestaltung und Priorisierung der bodenordnerischen Maßnahmen zu treffen.

In einem derart gestaltenden wirkungsorientierten Controllingprozess kann es gelingen, die Wirkungen des staatlichen Handelns fortlaufend und systematisch transparent zu machen. Im Gegensatz zu den eher einzelfallbezogenen Untersuchungen im Bereich der Evaluation, handelt es sich hierbei erstmalig um eine flächendeckende Wirkungsanalyse sämtlicher durchgeführter bzw. durchzuführender Bodenordnungsverfahren. Anstatt im Rahmen einer Outcome-Evaluation die Wirksamkeit einiger weniger, exemplarisch ausgewählter Bodenordnungsverfahren durch externe Institute beurteilen $\mathrm{zu}$ lassen, zeichnen sich interne Controllingprozesse durch eine vollständige Erfassung sämtlicher Flurbereinigungsverfahren aus. Zudem unterstützt das Controlling die Verwaltungsführung, indem kontinuierlich entscheidungsrelevante Wirkungsinformationen zur Verfügung gestellt werden. Damit entfaltet die Wirkungsanalyse direkten Steuerungsnutzen in der Flurbereinigungsverwaltung und stellt nicht nur den wirtschaftlichen, sondern zugleich auch den wirksamen Einsatz der staatlichen verwendeten Ressourcen sicher.

Die konkrete Anwendung der hergeleiteten Analyse- und Bewertungsschemata zur Untersuchung der Auswirkungen von Bodenordnungsverfahren soll im Folgenden anhand der Wirkungsrechnung eines exemplarisch ausgewählten Flurbereinigungsverfahrens aufgezeigt werden.

\footnotetext{
$1053 \mathrm{Vgl}$. hierzu auch Kapitel 4.2.3.

1054 Zum derzeitigen Aufbau der Flurbereinigungsverwaltung in Rheinland-Pfalz vgl. Kapitel 5.3.2.

1055 Vgl. auch Mosiek/Pieper/Kasten (2006), S. $193 \mathrm{ff}$.
} 


\subsection{Wirkungsanalyse in der Anwendungsphase: Wirkungsrechnung eines exemplarisch ausgewählten Bodenordnungsverfahrens}

\subsubsection{Beschreibung des betrachteten Bodenordnungsverfahrens}

Für den Ausbau der Autobahn A63 und die Umsetzung der geforderten naturschutzrechtlichen Kompensationsmaßnahmen wurden in großem Umfang ländliche Grundstücke in Anspruch genommen. Um den Landverlust auf einen größeren Kreis betroffener Eigentümer zu verteilen und die aufgrund der baulichen Maßnahmen entstehenden Nachteile für die allgemeine Landeskultur zu beseitigen, wurde am 19. November 1999 die Unternehmensflurbereinigung Kaiserslautern-Ost-Mehlingen nach §87ff. FlurbG eingeleitet. Nebenzweck des Verfahrens ist die Unterstützung des Baus der Landstraße L382 sowie die Umsetzung landschaftspflegerischer Begleitmaßnahmen, insbesondere aus dem Bereich der Wasserwirtschaft. Die Planfeststellung erfolgt voraussichtlich im Laufe des Jahres 2007. Die Ausführungsanordnung des Verfahrens ist für 2009 geplant. Tabelle 5 stellt die wichtigsten Verfahrensdaten zusammen.

\begin{tabular}{ll}
\hline \multicolumn{1}{c}{ Allgemeine Verfahrensdaten } \\
\hline Verfahrensart: & Unternehmensflurbereinigung nach §87 FlurbG \\
Zuständiges Amt: & DLR Westpfalz \\
Hauptgrund des Verfahrens: & Flächenmanagement für die Autobahn A63 \\
Nebenziele des Verfahrens: & Agrarstrukturverbesserung/ Landespflege/ \\
& Flächenmanagement für die Landstraße L383 \\
Datum der Verfahrenseinleitung: & 19. November 1999 \\
Größe des Verfahrensgebietes: & 1.370 ha \\
Anzahl der legitimierten Eigentümer: & 1.020 \\
Anzahl der betroffenen Flurstücke: & 1.685 \\
Qualität des vorgefundenen Katasters: & überwiegend Urkataster \\
Geschäftsvorfälle im Grunderwerb: & 90 Grunderwerbsfälle \\
Kosten des Grunderwerbs: & ca. 3.300.000 $€$ \\
Neugeordnete Ackerflächen: & 600 ha \\
Neugeordnete Grünlandflächen: & 123 ha \\
Lokaler Hochwasserschutz: & Bau zweier Rückhaltebecken \\
Naturschutz und Landespflege: & Ausweisung eines Gewässerrandstreifens \\
\hline
\end{tabular}

Tabelle 5: Verfahrensbrief „Kaiserslautern-Ost-Mehlingen« 


\subsubsection{Ergebnisse der Kostenanalyse}

Für das Bodenordnungsverfahren "Kaiserslautern-Ost-Mehlingen« soll im Folgenden eine umfassende Kosten- und Wirkungsanalyse vorgenommen werden. Entsprechend dem Modell der Ziel- und Ergebnisebenen öffentlicher Leistungserstellung besteht der erste Untersuchungsschritt in einer Bewertung des gesamten staatlichen Ressourceneinsatzes. Hierzu sind zunächst die relevanten Kostenbestandteile abzugrenzen, welche in die Analyse einbezogen werden müssen. Im Rahmen der Flurbereinigung unterscheidet man in diesem Zusammenhang zwischen Verfahrens- und Ausführungskosten (vgl. Abbildung 70). ${ }^{1056}$

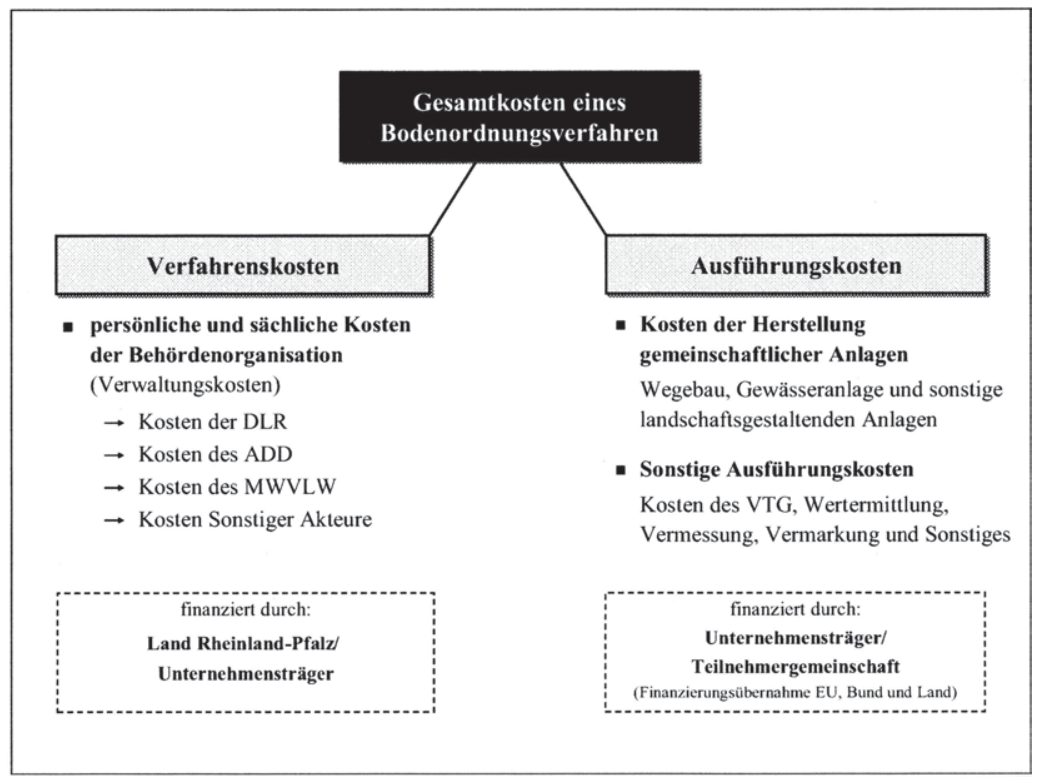

Abbildung 70: Verfahrens- und Ausführungskosten der Bodenordnung

Bei den Verfahrenskosten handelt es sich um die persönlichen und sächlichen Kosten der Behördenorganisation, dies sind insbesondere Verwaltungskosten. Hierbei ist zu beachten, dass sämtliche, an der Verfahrensausführung direkt bzw. indirekt beteiligten staatlichen Vollzugsebenen in die Analyse einbezogen werden müssen. Dazu zählen in der Flurbereinigungsverwaltung Rheinland-Pfalz die operativ verantwortlichen Dienstleistungszentren Ländlicher Raum, die zuständige Mittelinstanz, die ADD in Trier sowie das zuständige Ministerium für Wirtschaft, Verkehr, Landwirtschaft und Weinbau. ${ }^{1057}$ Die Ausführungskosten sind Kosten für die Herstellung der sog. gemeinschaft-

\footnotetext{
$1056 \mathrm{Vgl}$. Mosiek/Pieper/Kasten (2006), S. $43 \mathrm{f}$.

1057 Vgl. hierzu auch Kapitel 5.3.2.
} 
lichen Anlagen ${ }^{1058}$ sowie Kosten im Zusammenhang mit der Vermessung, Vermarkung und Wertermittlung der vom Verfahren betroffenen Grundstücke. Zusätzlich fällt eine Kostenumlage für den Verband der Teilnehmergemeinschaften (VTG) an, welcher für die Bauausführung der gemeinschaftlichen Anlagen verantwortlich ist.

Zur Bestimmung der Verfahrenskosten der Bodenordnung "Kaiserslautern-OstMehlingen« werden zunächst die Verwaltungskosten des zuständigen DLR analysiert. Hierzu werden die Personalkapazitäten für einzelne Bearbeitungsschritte über den gesamten Zeitraum des Bodenordnungsverfahrens erfasst. Da das Verfahren noch nicht abgeschlossen ist, enthalten die ausgewiesenen Beträge anteilige Planwerte. Auf Grundlage der Laufbahngruppen der beteiligten Mitarbeiter ergeben sich für die direkte Bearbeitung prognostizierte 2.300 Personeneinsatztage ${ }^{1059}$ mit Personalkosten in Höhe von $611.875 €$. Zusätzlich ist ein Personalgemeinkostenzuschlag in Höhe von $12,1 \% \mathrm{zu}$ berücksichtigen, der die indirekten Bereiche der allgemeinen Amtsverwaltung enthält. Weiterhin ergibt sich im DLR Westpfalz eine Sachkostenquote von $16,7 \%$, die auf das Bodenordnungsverfahren verrechnet wird, so dass die folgenden Gesamtkosten entstehen: ${ }^{1060}$

- Verfahrenskosten des DLR Westpfalz: $863.837 €$

Neben dem DLR sind die übergeordneten Verwaltungsebenen der ADD und des MWVLW in die Ressourcenbetrachtung aufzunehmen. Die hier entstehenden Verfahrenskosten der Bodenordnung werden als Gemeinkostenzuschlag auf das Verfahren »Kaiserslautern-Ost-Mehlingen « umgelegt. Der Zuschlagssatz der ADD beträgt in diesem Zusammenhang 4,48\% und der Zuschlagssatz des MWVLW beträgt 2,63\%, so dass die folgenden Verfahrenskosten entstehen: ${ }^{1061}$

- Verfahrenskosten der ADD: $38.700 €$

- Verfahrenskosten des MWVLW: $22.719 €$

Die Ausführungskosten der Bodenordnung können verfahrensspezifisch erhoben werden. Sie betragen für das Verfahren »Kaiserslautern-Ost-Mehlingen« gemäß Finanzplan 1,5 Mio. $€$ für die Herstellung der gemeinschaftlichen Anlagen und $490.000 €$ sonstige Ausführungskosten, wobei es sich im Wesentlichen um die Kosten der Vermessung im Verfahrensgebiet handelt: ${ }^{1062}$

- Ausführungskosten des Verfahrens: $1.990 .000 €$

\footnotetext{
1058 Bei den gemeinschaftlichen Anlagen handelt es sich insb. um kleine Nebenstraßen bzw. befestigte Feldwege, welche im Flurbereinigungsgebiet angelegt werden und sonstige landschaftsgestaltende Maßnahmen.

1059 In der Kapazitätsanalyse wurden Fehlzeiten, wie z.B. Krankheit oder Urlaub, berücksichtigt.

1060 Vgl. Mosiek/Pieper/Kasten (2006), S. 46 ff.

$1061 \mathrm{Vgl}$. Mosiek/Pieper/Kasten (2006), S. $51 \mathrm{ff}$.

$1062 \mathrm{Vgl}$. Mosiek/Pieper/Kasten (2006), S. 55 f.
} 


\subsubsection{Ergebnisse der Wirkungsanalyse}

\subsubsection{Identifikation der verfahrensspezifischen Auswirkungen}

Der zweite Untersuchungsschritt besteht in einer umfassenden Wirkungsanalyse des Bodenordnungsverfahrens. Zur Identifikation der gesellschaftlichen Auswirkungen des ausgewählten Flurbereinigungsverfahrens kann das zuvor erarbeitete allgemeine Wirkungsgefüge der Bodenordnung herangezogen werden. ${ }^{1063}$ Das Bodenordnungsverfahren "Kaiserslautern-Ost-Mehlingen" stellt mit insgesamt 1.370 ha Fläche und 1.020 legitimierten Eigentümern ein eher großes Flurbereinigungsverfahren im Land Rheinland-Pfalz dar. Da die Unternehmensflurbereinigung erst nach dem Ausbau der A63 durchgeführt wurde, sind keine nennenswerten Einsparungen an Entschädigungsleistungen zu erwarten. Von einem Beschleunigungseffekt durch das Bodenordnungsverfahren ist dennoch auszugehen, da durch die Bekanntgabe der Durchführung einer Unternehmensflurbereinigung mit großer Wahrscheinlichkeit Enteignungen vermieden werden konnten. Diese hätten den Bau einiger Teilstücke der A63 erheblich verzögert. Für den Bau der Landstraße L382 ist in Folge des Flächenmanagements der Bodenordnung ebenfalls ein positiver zeitlicher Effekt zu erwarten.

Die wasserrechtlichen Schutzmaßnahmen, die im Rahmen der Unternehmensflurbereinigung »Kaiserslautern-Ost-Mehlingen« realisiert wurden, bestehen zum einen in der Flächenausweisung für einen $10 \mathrm{~m}$ breiten Gewässerrandstreifen und zum anderen in der Anlage zweier Rückhaltebecken, zur Sicherung der Ortslage Mehlingen. Letztere Maßnahme trägt unmittelbar zur Hochwasserschadensvermeidung bei und kann daher auf Basis der durchschnittlichen Kosten der Schadensbeseitigung monetär bewertet werden. Der Wirkungsbeitrag des Gewässerrandstreifens liegt insbesondere in der Verbesserung der Gewässerstrukturgüte und der allgemeinen Wasserqualität. Die Ausweisung des Randstreifens ermöglicht eine konfliktfreie Entwicklung des Gewässers, da die Bewirtschafter angrenzender Nutzflächen gezwungen sind, einen größeren Abstand zum Gewässer einzuhalten. Infolgedessen werden Schadstoffeinleitungen durch Dünge- und Spritzmittel reduziert. Insgesamt leistet die Flurbereinigung damit einen wertvollen Beitrag zur Landschaftspflege. Die Ausweisung eines Gewässerrandstreifens macht zudem Bepflanzungen mit ausreichenden Grenzabständen möglich. Hierdurch sinken die Unterhaltungskosten des Gewässers, da an Uferstücken keine Abtragungen mehr entstehen.

Das Flächenmanagement der Bodenordnung trägt in dieser Weise zur Erhöhung der Realisierungschancen der Gesamtplanung und zu einer konfliktfreien Umsetzung der Teilplanungen in den Bereichen Infrastruktur, Wasserwirtschaft und Naturschutz bei. Die Projektträger profitieren von der Flächenbeschaffung innerhalb der Bodenordnung. So wurden für den Bau der Landstraße und für die Kompensationsflächen der Autobahn insgesamt neunzig Grunderwerbsfälle mit einem Gesamtvolumen von 3,3 Mio. $€$ abgewickelt. Hierdurch entstehen den Projektträgern Kostenersparnisse im $\mathrm{Zu}$ sammenhang mit dem Grunderwerb.

$1063 \mathrm{Vgl}$. Kapitel 5.3.5.4. 
Für die Grundstückseigentümer ist das Unternehmensflurbereinigungsverfahren mit einer Verringerung von Eigentumsverlusten verbunden. Ansonsten entstehende Transaktionskosten zum Flächenneuerwerb fallen nicht an. Die Rechtssicherheit des Eigentums wird insgesamt erhöht, insbesondere können Rechtsstreitigkeiten, bis hin zu gerichtlichen Enteignungsverfahren, vermieden werden.

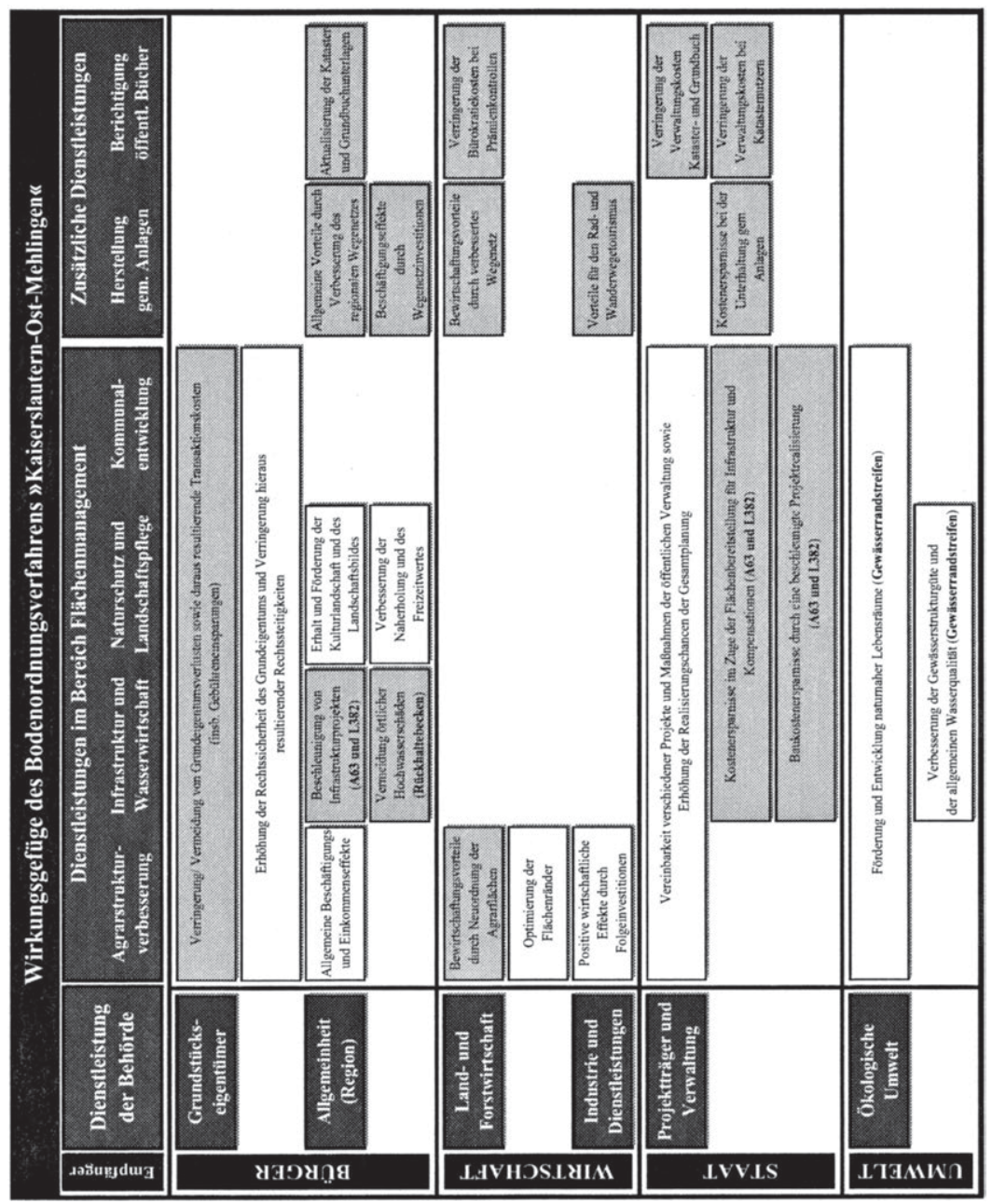

Abbildung 71: Gesellschaftliches Wirkungsgefüge des Verfahrens "Kaiserslautern-Ost-Mehlingen" 
Durch eine komplette Neuvermessung des Verfahrensgebietes wird zudem die Qualität des Liegenschaftskatasters verbessert. In Folge der Flächenneuordnung und Flächenzusammenlegung reduzieren sich die Verwaltungskosten bei den Kataster- und Grundbuchämtern. Auch die Landwirtschaft profitiert von der Berichtigung der öffentlichen Bücher. Hier verringern sich Bürokratiekosten bei der Beantragung und der Kontrolle von Flächenprämien. In gleicher Weise reduziert sich auch der notwendige staatliche Verwaltungsaufwand bei den Katasternutzern.

Durch die Verbesserung der Agrarstruktur und den Ausbau des Wegenetzes entstehen den Landwirten Bewirtschaftungsvorteile. Die Schläge wurden auf 7 ha vergrößert und damit der Einsatz von Lohnunternehmen ermöglicht. Gleichzeitig konnten die Voraussetzungen für eine Aussiedelung eines Haupterwerbbetriebes geschaffen und so dessen Existenz gesichert werden. Positive Effekte für den regionalen Rad- und Wanderwegetourismus ergeben sich im Zuge der Bodenordnung »Kaiserslautern-Ost-Mehlingen « in erster Linie aufgrund des Aus- und Neubaus des Wegenetzes. Gleichzeitig profitieren auch die Anwohner von der Verbesserung des Wegenetzes, indem Umwege eingespart werden. Die durch den Straßenbau entstehenden Nachteile für das Landschaftsbild wurden innerhalb des Verfahrens beseitigt und ein Beitrag zum Erhalt der Kulturlandschaft geleistet. Das Wegenetz ermöglicht den Bewohnern der Region zudem die Nutzung der Landschaft zur Naherholung. Das gesellschaftliche Wirkungsgefüge des Verfahrens ist in Abbildung 71 dargestellt.

\subsubsection{Bewertung der verfahrensspezifischen Auswirkungen}

Nachdem die Wirkungen des Verfahrens "Kaiserslautern-Ost-Mehlingen« vollständig erfasst wurden, ist in einem weiteren Schritt die verfahrensspezifische Bewertung der einzelnen Wirkungsbeiträge vorzunehmen. Hierzu wird auf die hergeleiteten Bewertungsansätze der Bodenordnung zurückgegriffen. ${ }^{1064}$

\section{Empfängerebene: Bürger}

Im Bodenordungsverfahren »Kaiserslautern-Ost-Mehlingen« wurden Flächen für insgesamt 3,3 Mio. $€$ vom DLR erworben und den unterschiedlichen Projektträgern zur Verfügung gestellt. Annahmegemäß können die betroffenen Grundstückseigentümer $1 \%$ dieser Summe als Transaktionskosten einsparen:

- Einsparung an Transaktionskosten $\left(\mathrm{TK}^{\mathrm{c}}\right): 33.000 €$

Zum Schutz der Gemeinde Mehlingen vor Hochwasser wurden im Rahmen des Verfahrens zwei Rückhaltebecken angelegt. Hierdurch wird nach Aussage der Kommune ein durchschnittlicher jährlicher Schaden (S) in Höhe von $7.000 €$ vermieden. Bei einer unterstellten Laufzeit von 50 Jahren und einer Diskontierungsrate von $4 \%$ resultiert daraus der folgende Wirkungsbeitrag:

- Vermeidung lokaler Hochwasserschäden $\left(\mathrm{BW}^{\mathrm{S}}\right): 156.390 €$

1064 Vgl. hierzu und im Folgenden Kapitel 5.3.6. 
Aufgabe der betrachteten Bodenordnung ist es, den Bau der A63 sowie den Bau der L382 zu unterstützen. Die verfahrensbezogenen Investitionen $\left(I_{j}\right)$ in den Bau der A63 umfassen 50 Mio. $€$, in den Ausbau der L382 insgesamt 47.000 $€$. Unter der Annahme, dass die baulichen Maßnahmen durch das Flurbereinigungsverfahren beschleunigt wurden, kann ein gesellschaftlicher Zinsertrag aus der frühzeitigen Inanspruchnahme geltend gemacht werden. Das Flächenmanagement für die Kompensationen haben den Autobahnbau um drei Monate $(b=0,25)$ und den Ausbau der Landstraße um ein halbes Jahr $(b=0,5)$ beschleunigt. Unter Beachtung der spezifischen Nutzen-KostenFaktoren und einer Diskontierungsrate von 3\% ergibt sich folgender Gesamteffekt:

- Beschleunigung des Baus der A63 $\left(\mathrm{ZE}_{\mathrm{A} 63}\right): 1.557 .585 €$

- Beschleunigung des Ausbaus der L382 (ZE $\left.\mathrm{Z}_{\mathrm{L} 382}\right): 2.939 €$

In den Neu- und Ausbau des regionalen Wegenetzes werden im Verfahren "Kaiserslautern-Ost-Mehlingen« insgesamt $1.158 .000 €$ investiert $\left(\mathrm{I}_{\text {Wege }}\right)$. Die reinen Herstellungskosten des Wegenetzes $\left(\mathrm{HK}_{\text {Wege }}\right)$ betragen in etwa 1,1 Mio. $€$, so dass Planungskosten $\left(\mathrm{PLK}_{\mathrm{Wegc}}\right)$ in Höhe von $30.800 €$ geltend gemacht werden können. Ein Drittel des Gesamtbetrages entspricht annahmegemäß dem gesellschaftlichen Vorteil der regionalen Allgemeinheit:

- Allgemeiner Vorteil des ausgebauten Wegenetzes $\left(V_{W_{\text {Wege }}}\right): 396.267 €$

Weiterhin resultieren aus den Investitionen in das regionale Wegenetz Einkommensund Beschäftigungseffekte. Die Investitionen in den Neu- und Ausbau des Wegenetzes in Höhe von 1.158.000 $€$ haben einen zusätzlichen Beschäftigungseffekt von 24,95 Erwerbstätigenjahren. Unter Beachtung der gesamtfiskalischen Kosten der Arbeitslosigkeit in Höhe von $18.986 €$ pro Person und Jahr in Deutschland ergibt sich folgender Wirkungsbeitrag der Bodenordnung:

- Beschäftigungseffekte aus Wegenetzinvestitionen (Besch): $473.662 €$

Durch die komplette Neuvermessung des Verfahrensgebietes trägt die Bodenordnung zur Qualitätsverbesserung der Katasterunterlagen bei. Die Kosten einer Neuvermessung außerhalb der Bodenordnung (VK) würden für das Verfahren »KaiserslauternOst-Mehlingen « insgesamt $402.666 €$ betragen. Da die Qualität der vorgefundenen Katasterunterlagen überwiegend aus Urkataster besteht, können als gesellschaftlicher Wirkungsbeitrag 50\% dieser Summe in Ansatz gebracht werden:

- Aktualisierung Kataster- und Grundbuch $\left(\mathrm{A}_{\mathrm{K}}\right): 201.333 €$

Für die Empfängerebene Bürger ergibt sich hiermit ein monetär bewerteter Gesamtwirkungsbeitrag in Höhe von 2.821.176€. 


\section{Empfängerebene: Wirtschaft}

Die Neuordnung des Verfahrensgebietes und die Verbesserung des regionalen Wegenetzes führen $\mathrm{zu}$ einer Verbesserung der Agrarstruktur und damit zu Bewirtschaftungsvorteilen für die ansässige Landwirtschaft. Insgesamt wurden innerhalb des Verfahrens 600 ha Ackerland und 123 ha Grünland neu geordnet. Dabei konnte ein $\mathrm{Zu}-$ sammenlegungsverhältnis von 3:1 erzielt werden, wodurch die jährlichen Kosteneinsparungen $107 €$ je Hektar Ackerfläche und $287 €$ je Hektar Grünlandfläche betragen. Bei einer unterstellten Laufzeit von 25 Jahren und einer Diskontierung mit 4\% resultiert daraus der folgende Wirkungsbeitrag:

- Bewirtschaftungsvorteile Ackerland $\left(\mathrm{BV}_{\mathrm{Acker}}\right): 1.043 .055 €$

- Bewirtschaftungsvorteile Grünland $\left(\mathrm{BV}_{\mathrm{Grün}}\right): 573.534 €$

Die Flächenzusammenlegung und die Verbesserung des Katasters verringern die Bürokratiekosten der Landwirte. Im vorliegenden Verfahren profitieren 35 Betriebe (Lw) von diesen Kosteneinsparungen. Dies betrifft zum einen Einsparungen bei der jährlichen Beantragung der Flächenprämie, zum anderen Einsparungen bei Prämienkontrollen. Bei einer unterstellten Wirkungsdauer von insgesamt 25 Jahren und einem Stundenlohn von $15 €$ ergeben sich folgende Effekte:

- Bürokratiekosteneinsparung Prämienbeantragung (VA $\left.{ }_{\mathrm{LW}}^{\mathrm{c}}\right): 68.237 €$

- Bürokratiekosteneinsparung Prämienkontrolle (VA $\left.{ }_{\mathrm{PK}}^{\mathrm{e}}\right): 1.413 €$

Der Aus- und Neubau des Wegenetzes wirkt sich zudem positiv auf den regionalen Rad- und Wanderwegetourismus aus. Annahmegemäß kann ein Drittel des Gesamtbetrages aus Investitions- und Planungskosten des Wegenetzes als Wirkungsbeitrag für die regionale Tourismusbranche in Ansatz gebracht werden:

- Vorteil des Rad- und Wanderwegetourismus (TV Wege $): 396.267 €$

Für die Empfängerebene Wirtschaft ergibt sich hiermit ein monetär bewerteter Gesamtwirkungsbeitrag in Höhe von 2.082.506 $€$.

\section{Empfängerebene: Staat}

Durch den Grunderwerb im Rahmen der Bodenordnung profitieren die Projektträger von unterschiedlichen Kosteneinsparungen. Die Grunderwerbskosten im Verfahren »Kaiserslautern-Ost-Mehlingen« belaufen sich auf 3,3 Mio. $€$, wobei 90 Erwerbsfälle vorliegen. Annahmegemäß betragen die Einsparungen bei den Grunderwerbskosten $10 \%$ dieser Summe und bei den Notargebühren 1\% dieser Summe. Entschädigungsleistungen an die Grundeigentümer wurden nicht eingespart, allerdings Personal- und Sachkosten für die 90 Kaufverträge sowie die Kosten der ansonsten notwendigen Fortführungsvermessung $(20 \mathrm{~km})$ :

- Grunderwerbskosten $\left(\mathrm{Gk}^{\mathrm{c}}\right): 330.000 €$

- Notar- und Katastergebühren $\left(\mathrm{Gb}^{\mathrm{c}}\right): 33.000 €$ 
- Personal- und Sachkosten für den Grunderwerb $\left(\mathrm{Pk}^{\mathrm{e}}\right): 96.525 €$

- Kosten der Fortführungsvermessung $\left(\mathrm{VK}_{\text {Fort }}\right): 350.000 €$

Durch den beschleunigten Baubeginn der A63 und der L382 können die Projektträger Baukosten einsparen. Die verfahrensbezogenen Baukosten betragen für den Bau der Autobahn 50 Mio. $€$ und für den Ausbau der Landstraße 47.000 €. Bei einer unterstellten jährlichen Kostensteigerung (real) von $w=0,46 \%$ und einer Beschleunigung von drei Monaten bzw. einem halben Jahr ergeben sich die folgenden Gesamteinsparungen:

- Baukosteneinsparung A63 ( $\left.\mathrm{K}_{\text {Bau }}^{\mathrm{c}}\right): 57.335 €$

- Baukosteneinsparung L382 ( $\left.\mathrm{K}_{\text {Bau }}^{\mathrm{e}}\right): 108 €$

Die Unterhaltungskosten der gemeinschaftlichen Anlagen im Verfahrensgebiet betragen ungefähr $5.000 €$ pro Jahr. Diese Unterhaltungskosten wurden für ein halbes Jahr vom zuständigen DLR übernommen:

- Einsparung temporärer Unterhaltungskosten $\left(\mathrm{UK}^{\mathrm{c}}\right): 2.500 €$

Durch die Maßnahmen im Rahmen der Bodenordnung wird der Verwaltungsaufwand der Grundbuchführung dauerhaft reduziert. Bei einer Verfahrensgesamtfläche (VFL) von 1.370 ha und einer Laufzeit von 25 Jahren ergibt sich bei einem Diskontierungssatz von $3 \%$ folgender Wirkungsbeitrag:

- Verwaltungskosteneinsparung Grundbuch (BW $\left.{ }^{\text {Grundbuch }}\right): 43.009 €$

Schließlich reduzieren sich durch die Neuordnung und Zusammenlegung der Flächen auch die Verwaltungskosten der Katasternutzer. Bei einer landwirtschaftlichen Nutzfläche von insgesamt 723 ha befinden sich ungefähr 18 Referenzbetriebe im Verfahrensgebiet, von denen jährlich $8 \%$ geprüft werden. Die Kontrollkosten des Agrarförderungsprüfdienstes reduzieren sich in Folge eines Bodenordnungsverfahrens annahmegemäß um die Hälfte:

- Verwaltungskosten der Prämienkontrolle ( $\left.\mathrm{KK}_{\mathrm{PD}}^{\mathrm{e}}\right): 5.930 €$

Für die Empfängerebene Staat ergibt sich hiermit ein monetär bewerteter Gesamtwirkungsbeitrag in Höhe von $918.407 €$.

\section{Empfängerebene: Umwelt}

Für die Empfängerebene Umwelt ergibt sich kein monetär bewerteter Wirkungsbeitrag. Die Ergebnisse der Kostenanalyse und der Wirkungsanalyse der Unternehmensflurbereinigung "Kaiserslautern-Ost-Mehlingen« sollen im Folgenden anhand einer Wertschöpfungsbilanz übersichtlich dargestellt werden. ${ }^{1065}$

1065 Vgl. hierzu ausführlich Kapitel 4.4.3. 


\subsubsection{Wertschöpfungsbilanz des Bodenordnungsverfahrens}

Aus Abbildung 72 wird ersichtlich, dass das betrachtete Unternehmensflurbereinigungsverfahren "Kaiserslautern-Ost-Mehligen« Gesamtkosten von insgesamt gut 2,9 Mio. $€$ verursacht hat. Der staatliche Ressourcenverzehr entsteht in diesem Zusammenhang hauptsächlich auf der Ebene des zuständigen DLR. Zudem wird deutlich, dass im betrachteten Verfahren die Ausführungskosten die Verfahrenskosten deutlich übersteigen. Den staatlichen Vorleistungen steht ein monetärer Wirkungsbeitrag in Höhe von 5,82 Mio. $€$ gegenüber - intangible Wirkungsbeiträge sind hier noch nicht enthalten, wobei die gesellschaftliche Wertzumessung dieser Wirkungen eine höhere Bedeutung besitzen kann. Damit hat das betrachtete Bodenordnungsverfahren einen positiven Wertschöpfungsbeitrag für die Gesellschaft erbracht. Hieraus ergibt sich ein bewerteter gesellschaftlicher Kosten-Wirkungsfaktor von ungefähr 2. Die Hauptwirkungsempfänger der betrachteten Unternehmensflurbereinigung sind die regionale Allgemeinheit, die Landwirtschaft und die Träger der Straßenbauprojekte.

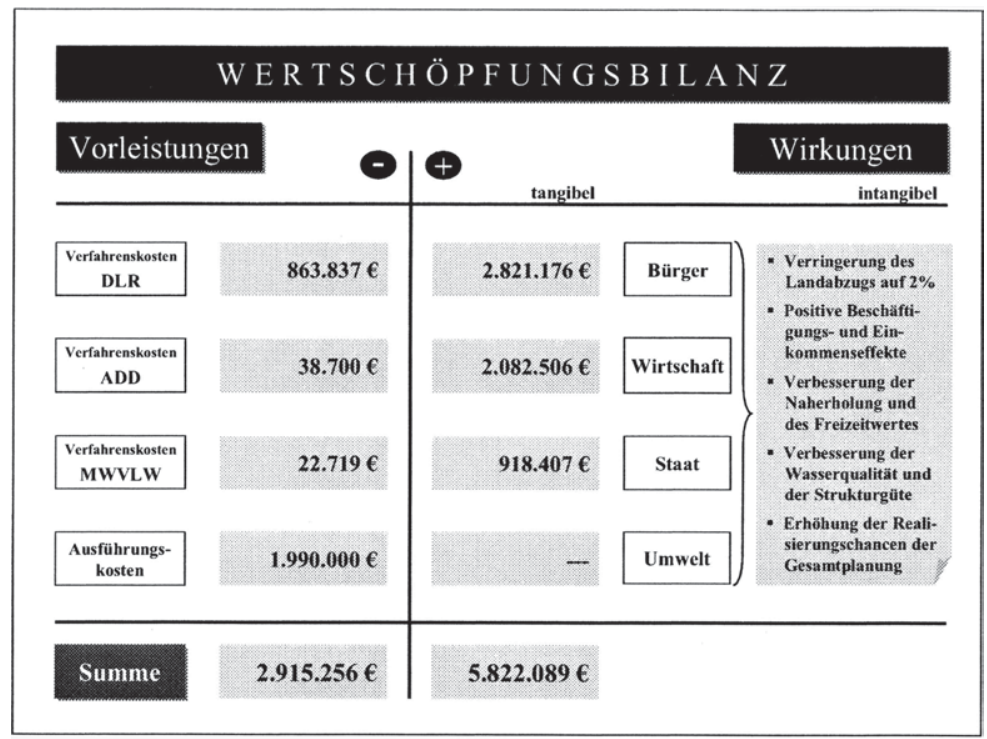

Abbildung 72: Wertschöpfungsbilanz »Kaiserslautern-Ost-Mehlingen»

Abbildung 72 zeigt zudem, welche intangiblen Wirkungsbeiträge im betrachteten Verfahren von Relevanz sind. Zu diesen Effekten gehören allgemeine Einkommens- und Beschäftigungseffekte, die Verbesserung der Wasserqualität und der Gewässerstrukturgüte durch den ausgewiesenen Gewässerrandstreifen sowie der Erhalt der Kulturlandschaft. Die Investitionen in das Wegenetz schaffen weiterhin Verbesserungen im Naherholungs- und Freizeitbereich. Grundsätzlich hat das Flächenmanagement der Bodenordnung auch die Realisierungschancen der Gesamtplanung im Verfahrensge- 
biet erhöht. Bei den quantitativ messbaren Wirkungsindikatoren konnte der Landverlust der Eigentümer im Rahmen der Bodenordnung auf 14 ha bzw. 2\% der landwirtschaftlichen Nutzfläche begrenzt werden. Weiterhin ist es gelungen, die Anzahl der ungeklärten Eigentumsverhältnisse von 54 auf $4 \mathrm{zu}$ reduzieren. ${ }^{1066}$

Die bilanzielle Gegenüberstellung von Kosten und Wirkungen einer Bodenordnungsmaßnahme zeigt das Potenzial eines systematischen wirkungsorientierten Controllingprozesses, bei den Akteuren im politisch-administrativen System Sensibilität für angemessene Kosten-Wirkungsrelationen zu schaffen. Durch die Identifikation der bestimmenden Kostentreiber und Wirkungshebel werden direkte Anknüpfungspunkte zur Steigerung der Wirtschaftlichkeit und Wirksamkeit staatlicher Maßnahmen aufgezeigt. Die Wirkungsanalyse des Bodenordnungsverfahrens "Kaiserslautern-Ost-Mehligen" macht deutlich, dass es auch ohne aufwändige einzelfallbezogene Evaluierungen und hochanalytische Messverfahren gelingen kann, den Wertschöpfungsbeitrag staatlichen Handelns transparent zu machen. Mit Hilfe pragmatischer Ansätze und eher standardisierter Analyse- und Bewertungsschemata werden im Rahmen einer Wirkungsanalyse wichtige Kausalzusammenhänge in einem fortlaufenden Controllingprozess identifiziert und beurteilt.

Die Wertschöpfungsbilanz bietet zugleich das Potenzial, ein wichtiges Informationsinstrument der politischen Ebene darzustellen. Der Politik werden relevante Wirkungsinformationen in einem hohen Verdichtungsgrad zugänglich gemacht. Die operative Verwaltungsebene hat gleichzeitig die Möglichkeit, die gesellschaftliche Wertschöpfung ihres Handelns zu dokumentieren und in dieser Form analytische Politikberatung $\mathrm{zu}$ betreiben. Inwieweit die bereitgestellten Wirkungsinformationen tatsächlich Eingang in den politischen Zielbildungsprozess finden, bleibt indes abzuwarten. Die Flurbereinigungsverwaltung in Rheinland-Pfalz hat zumindest die Voraussetzungen hierfür geschaffen, so dass in diesem konkreten Fall zu hoffen bleibt, dass sich auch die politische Ebene der Erkenntnisse annimmt und in ihren Entscheidungen angemessen berücksichtigt. 
Thorsten Pieper - 978-3-631-75332-3

Downloaded from PubFactory at 01/11/2019 06:04:26AM

via free access 


\section{Schlussbetrachtung}

Staaten stehen als Anbieter von Standorten in einem weltweiten Wettbewerb um Unternehmensansiedlungen, Kapitalinvestitionen und Wissen. Ein entscheidendes Wettbewerbselement ist in diesem Zusammenhang die Leistungsfähigkeit des öffentlichen Sektors. Staatliches Handeln steht daher - national wie international - unter dem Druck fortlaufender Modernisierungsbestrebungen. Bisherige Reformansätze beschränken sich allerdings vielfach auf die einseitige Verbesserung der Wirtschaftlichkeit des öffentlichen Ressourceneinsatzes, mit dem Ziel, die Verwaltungsleistungen so effizient wie möglich anzubieten. Grundsätzliche Legitimation erfahren öffentliche Leistungen aber nicht durch eine möglichst günstige Erstellung, sondern durch das Auslösen objektiver Wirkungen in der Gesellschaft, d.h. durch ihre Wirksamkeit. Werden Wirkungen nicht in die Entscheidungsprozesse einbezogen, besteht permanent die Gefahr, die falschen - da unwirksamen - Verwaltungsprodukte äußerst effizient herzustellen. Zur Steigerung seiner Leistungsfähigkeit muss der Staat sein Handeln daher systematisch an den daraus resultierenden Wirkungen in der Gesellschaft ausrichten.

Sowohl die bisherigen Erfahrungen der Wirkungsorientierung in der Praxis, als auch die theoretische Aufarbeitung der Thematik offenbaren aber die bestehenden Herausforderungen. Hierzu gehört die geringe Bereitschaft der Politik, klare und messbare Wirkungsziele zu vereinbaren. Weiterhin existieren methodische Herausforderungen zur Messung der Wirkungszielerreichung und die korrekte Dokumentation, welche Verwaltungsprodukte welche Wirkungen erzeugen. Eine wesentliche Problemstellung ist also die Beschaffung der fehlenden Informationen zur wirkungsoptimierenden Steuerung des staatlichen Handelns.

Das Ziel dieser Arbeit ist daher, einen Beitrag zur Entwicklung eines praktikablen Instrumentariums zur systematischen und strukturierten Analyse der durch staatliches Handeln im gesellschaftlichen Umfeld ausgelösten Wirkungen zu leisten. Hierzu wird durch die Konstruktion eines allgemeinen Beschreibungsmodells einerseits eine aus theoretischer Sicht wünschenswerte Strukturierung der wesentlichen Modellvariablen der Wirkungen staatlichen Handelns vorgenommen. Andererseits wird auf dieser Grundlage eine aus praktischer Sicht wünschenswerte Methodik der Wirkungsanalyse entwickelt, welche mit Hilfe geeigneter Instrumente eine möglichst umfassende Identifikation und Bewertung der gesamtgesellschaftlichen Auswirkungen konkret betrachteter Verwaltungsprodukte erlaubt. In dieser Weise leistet die Arbeit einen Beitrag, die bestehenden methodischen Probleme und Defizite bei der Analyse der Wirkungen staatlicher Maßnahmen - zumindest in Ansätzen - zu überwinden. Die Ausführungen dienen damit der Vervollständigung einer umfassenden wirkungsorientierten Controllingkonzeption zur sachgerechten Unterstützung der wirkungsoptimierenden Steuerung staatlichen Handelns. Letztlich wird mit einer solchen Controllingkonzeption die Absicht verfolgt, nicht nur die Wirtschaftlichkeit, sondern auch die Wirksamkeit des öffentlichen Ressourceneinsatzes zu erhöhen. 
$\mathrm{Zu}$ diesem Zweck wird auf Grundlage der Problemstellung in Kapitel 1 zunächst die Zielsetzung der vorliegenden Arbeit spezifiziert, bevor die hieraus abzuleitende Vorgehensweise zur Überwindung der Problemstellung erläutert wird. In diesem Kontext wird vom Verfasser ein zweistufiger Forschungsprozess angestrebt. Die erste Stufe orientiert sich an einer theoretisch ausgerichteten Grundlagenforschung, mit dem Ziel, dass methodische Grundlagenwissen im Bereich der Wirkungsanalyse staatlichen Handelns zu verbreitern. Die zweite Stufe beinhaltet die Anwendung der theoretischen Erkenntnisse im Rahmen einer praktischen Fallstudie, welche die systematische Identifikation und Bewertung der gesellschaftlichen Wirkungen für einen spezifischen staatlichen Leistungsbereich vornimmt.

In Kapitel 2 werden die relevanten Grundlagen des öffentlichen Sektors in Deutschland und ausgewählte Aspekte des staatlichen Handelns dargestellt. Durch die Analyse der allgemeinen Rahmenbedingungen sowie der bestehenden Steuerungsansätze im politisch-administrativen System wird damit zu Beginn der Arbeit ein einheitliches Grundverständnis der Thematik sichergestellt. Hierzu werden die grundlegenden Ursachen und Aufgaben der Staatstätigkeit erläutert, wie zum Beispiel die Schaffung eines einheitlichen Ordnungsrahmens, die Sicherstellung eines fairen Wettbewerbs oder der Eingriff bei Marktversagen. Staatliches Handeln ist in diesem Zusammenhang auf eine Steigerung des Gemeinwohls bezogen und daher primär an Sachzielen orientiert, d.h. an der Erzielung bestimmter Wirkungen in der Gesellschaft. Zur Durchsetzung seiner Ziele trifft der Staat rechtliche Regelungen, erhebt Steuern und Abgaben und stellt mit Hilfe dieser Ressourcen (Inputs), Güter und Dienstleistungen bereit (Outputs), welche wiederum die gewünschten Wirkungen (Outcomes) in der Gesellschaft auslösen sollen - verstanden als Prozess der öffentlichen Leistungserstellung.

Die Steuerung öffentlicher Leistungserstellungsprozesse unterliegt derzeit einem hohen Wandel und einem fortlaufenden Modernisierungsdruck. Doch obwohl sich die öffentliche Verwaltung im Zuge des New Public Management seit Beginn der 90er Jahre im Umbruch befindet, sind Bürokratie als administratives Steuerungsmodell und Kameralistik als Instrument der Finanzmittelallokation nach wie vor die dominierenden Systeme im deutschen Staatswesen. Auf dem bürokratischen Verwaltungsmodell lastet allerdings ein hoher Veränderungsdruck, da es bei den heutigen gesellschaftlichen Erfordernissen und Umweltbedingungen zunehmend Schwächen zeigt. Im Kontext internationaler Entwicklungen wird auch in Deutschland zunehmend ein neues Steuerungsleitbild umgesetzt, mit einer Neubewertung notwendiger Staatsaufgaben und der Neuorganisation der Aufgabenerledigung. Wichtige Elemente dieses Leitbildes sind die Etablierung dezentraler Führungs- und Organisationsstrukturen, die Einführung einer Outputsteuerung über definierte Verwaltungsprodukte, die Schaffung von Wettbewerb und Marktdruck sowie die Reorganisation des öffentlichen Rechnungswesens und die Einführung von Kosten- und Leistungsrechnungen.

Ein entsprechend ausgestalteter Steuerungsansatz des politisch-administrativen Systems besitzt ein hohes Potenzial, die Wirtschaftlichkeit des staatlichen Ressourceneinsatzes zu erhöhen. Die möglichst effiziente Erstellung von öffentlichen Leistungen 
stellt allerdings keinen Selbstzweck dar, denn die hierdurch ausgelösten Wirkungen in der Gesellschaft sind das eigentliche Ziel der Leistungserstellung. Die Realisierung eines wirkungsorientierten Steuerungsansatzes ist für das politisch-administrative System mit vielfältigen Chancen verbunden, weshalb auch in Deutschland der Pfad zur Wirkungsorientierung eingeschlagen werden sollte. Neben den Verwaltungsprodukten sollten zunehmend auch die hiermit verbundenen Wirkungen in das Zentrum des Interesses rücken, d.h. es wird vermehrt nach der eigentlichen Wirksamkeit des staatlichen Ressourceneinsatzes gefragt.

Das die Wirkungsorientierung bislang faktisch noch keinen Eingang in die bestehenden Steuerungskreisläufe von Politik und Verwaltung gefunden hat, ist auf bisher ungelöste Problembereiche zurückzuführen. Im Rahmen dieser Arbeit wurden sowohl methodische Bewertungshindernisse, als auch die klare Zuweisung von Verantwortlichkeiten sowie die Einbindung der politischen Ebene als zentrale Problembereiche der Wirkungsorientierung identifiziert. Der Erfolg der wirkungsorientierten Steuerung als praxistaugliches Konzept und die Realisierung der damit verbundenen Chancen liegt demnach in einer schrittweisen Überwindung der aufgezeigten Problemstellungen. Ein hohes Potenzial - zumindest die methodischen Herausforderungen ansatzweise zu überwinden - wird in diesem Zusammenhang dem Verwaltungscontrolling zugesprochen. Die Frage nach den Wirkungen staatlichen Handelns bildet damit den Ausgangspunkt zur Weiterentwicklung des Verwaltungscontrollings zu einem wirkungsorientierten Controlling. Entsprechend setzt sich Kapitel 3 intensiv mit den bestehenden Bezugsrahmen wirkungsorientierter Controllingkonzeptionen auseinander. Eine vergleichende Bewertung der Modellkonzeptionen führt zu dem Schluss, dass insbesondere das Modell der Ziel- und Ergebnisebenen öffentlicher Leistungserstellung hinsichtlich Umfang und Differenzierungsgrad der berücksichtigten Analyseperspektiven einen geeigneten Bezugsrahmen zur Ausgestaltung des wirkungsorientierten Controllings darstellt.

Mit einem solchen Bezugsrahmen gelingt es zunächst, den komplexen Prozess der öffentlichen Leistungserstellung sowie die relevanten Betrachtungsebenen und Bewertungskriterien angemessen zu strukturieren. Fraglich ist nun, inwieweit das auf den einzelnen Ziel- und Ergebnisebenen bereitgestellte Controlling-Instrumentarium die beteiligten Akteure dazu in die Lage versetzt, die Bewertungsebenen des Leistungsprozesses sachgerecht abzubilden. Eine eingehende Analyse der Modellkonzeptionen offenbart bestehende methodische und instrumentelle Gestaltungsdefizite bei der systematischen Identifikation und Bewertung der Wirkungen staatlichen Handelns. Zwar wird in diesem Zusammenhang häufig der Einsatz von Evaluationen vorgeschlagen. Da es sich hierbei aber in der Regel um eher wissenschaftlich geprägte Einzelfalluntersuchungen durch externe Institute handelt, die zudem mit einem hohen Kosten- und Zeitaufwand verbunden sind, wird sich das wirkungsorientierte Verwaltungscontrolling in der Breite nicht hierauf stützen können. Darüber hinaus verhindert der geringe Standardisierungsgrad von Evaluationen die Etablierung eines kontinuierlichen und systematischen Controllingprozesses, welcher regelmäßig steuerungsrelevante Wirkungsdaten zur Verfügung stellt. 
Zur Überwindung der aufgezeigten methodischen Defizite wird in Kapitel 4 eine eigene Methodik der Wirkungsanalyse staatlichen Handelns entwickelt. Das Ziel besteht darin, dem Verwaltungscontrolling ein möglichst praktikables Instrumentarium bereitzustellen, welches die systematische Analyse und Bewertung der in der Gesellschaft ausgelösten Wirkungen erlaubt. Dazu wird zunächst ein allgemeines Beschreibungsmodell der Wirkungen staatlichen Handelns entwickelt, um die hochkomplexen Wirkungszusammenhänge der Realiät beherrschbar zu machen. Ausgangspunkt der Betrachtung ist eine staatliche Maßnahme als verursachende, erklärende Variable und die gesamtgesellschaftlichen Wirkungen als anhängige, zu erklärende Variablen. Staatliches Handeln wird in diesem Kontext einer Auslöserebene, die ausgelösten Wirkungen einer Empfängerebene zugeordnet. Das Gesamtmodell ist weiter ausdifferenziert und berücksichtigt auf der Auslöserebene unterschiedliche staatliche Maßnahmen und exogene Effekte als erklärende Faktoren. Auf der Empfängerebene werden sämtliche intendierte, am Zielsystem ausgerichtete Wirkungen sowie alle erwünschten und unerwünschten Nebenwirkungen und wesentliche Folgewirkungen als zu erklärende Faktoren erfasst. Zwischen diesen Strukturelementen der Modellkonzeption bestehen zudem vielfältige statische und dynamische Wirkungsbeziehungen. Als statische Aspekte lassen sich die Wirkungsrelevanz, die Wirkungsstärke und die Wirkungsinteraktion beschreiben, als dynamische Aspekte Wirkungsketten, die Wirkungsverzögerung und die Wirkungsdauer.

Mit Hilfe dieses Beschreibungsmodells gelingt zunächst eine sinnvolle Strukturierung der für die Wirkungsanalyse relevanten Elemente und Wirkungsbeziehungen. Weiterhin ist eine eindeutige Abgrenzung der Analyseperspektiven vorzunehmen. Die Charakteristik staatlichen Handelns bringt es mit sich, dass hierbei mit dem Impact und dem Outcome zwei unterschiedliche Wirkungsdimensionen von Relevanz sind. Die Perspektive des Impact bewertet Wirkungen als subjektive Anspruchserfüllung aus Sicht der Leistungsempfänger. Im Rahmen einer Nutzenanalyse werden die Einwirkungen öffentlicher Leistungen auf die Kunden aus einer individuellen personalen Perspektive analysiert, in der Regel durch Kundenbefragungen. Die Perspektive des Outcome beurteilt Wirkungen dagegen als eher objektive gesamtgesellschaftliche Auswirkungen öffentlicher Leistungen, verstanden als Beitrag zum Gemeinwohl der Gesellschaft. Hier ist es Aufgabe der Wirkungsanalyse, diese Auswirkungen möglichst umfassend zu erfassen und zu bewerten.

Hieran anknüpfend wird eine eigene Vorgehenssystematik der Wirkungsanalyse vorgestellt, die der systematischen Identifikation und Bewertung der ausgelösten Wirkungen staatlichen Handelns dient. Der erste Schritt dieser Vorgehenssystematik besteht in einer sog. Konzeptionsphase. Innerhalb der Konzeptionsphase werden die notwendigen Analyse- und Bewertungsschemata eines betrachteten staatlichen Handlungsbereiches entwickelt. Hierzu stellt die Arbeit dem Controlling pragmatische Strukturierungshilfen der Auslöser- und Empfängerebene zur Verfügung, welche sich zu einer übersichtlichen Darstellung in Form einer Analysematrix - dem gesamtgesellschaftlichen Wirkungsgefüge - verbinden lassen. Mit Hilfe des Wirkungsgefüges soll eine möglichst umfassende Identifikation der ausgelösten Wirkungen gelingen. Zur Operationalisie- 
rung der identifizierten Wirkungskomponenten werden dem Controlling zudem unterschiedliche Bewertungsinstrumente zur Verfügung gestellt. In Abhängigkeit von der Bewertungsform kann so entweder eine monetäre Quantifizierung durch geeignete Bewertungsansätze, eine Quantifizierung durch Wirkungsindikatoren oder eine qualitativ-verbale Beschreibung der einzelnen Wirkungsbeiträge erfolgen.

Der zweite Schritt der Vorgehenssystematik besteht in einer sog. Anwendungsphase. Nach dem Aufbau der Analyse- und Bewertungsschemata für einen bestimmten staatlichen Produktbereich, findet innerhalb der Anwendungsphase die eigentliche operative Erfolgskontrolle des Verwaltungsoutputs im Rahmen eines systematischen Controllingprozesses statt. Hierbei handelt es sich um die verschiedenen Verfahren der Wirkungsrechnung. In Abhängigkeit vom Einsatzzeitpunkt im Rahmen des Controllingprozesses lassen sich hier die Wirkungsprognose (ex-ante), das Wirkungsmonitoring (on-going) und die Wirksamkeitsanalyse (ex-post) unterscheiden. Fundierte Kenntnisse über tatsächlich eingetretene Wirkungen sowie das zunehmende Verständnis der Wirkungszusammenhänge erfordern zusätzlich kontinuierliche Rückkopplungsschleifen aus dem operativen Einsatz. Hierdurch kann eine laufende Anpassung der verwendeten Analyse- und Bewertungsschemata erfolgen, ausgebildet als Lernschleife zwischen der Konzeptions- und Anwendungsphase.

Der theoretische Teil der Arbeit endet mit einer Eingliederung der Wirkungsanalyse in die bestehenden Controllingprozesse. Die institutionelle Ausgestaltung eines wirkungsorientierten Controllings muss sich an den jeweiligen Informationserfordernissen der Entscheidungsträger im politisch-administrativen System orientieren. Auf der Ebene der operativen Leistungserstellung ist das operativ-taktische Wirkungscontrolling zur Sicherstellung eines wirksamen und wirtschaftlichen Verwaltungshandelns angesiedelt. Controlling bezieht sich im politisch-administrativen System zusätzlich auf die Exekutive und die Legislative, wobei im Führungszyklus allerdings eine spezielle politische Rationalität beachtet werden muss. Hieraus ergibt sich die Notwendigkeit zur Abgrenzung eines strategischen Controllings, welches eher der administrativen Regierungsebene zuzuordnen ist und eines politischen Controllings, welches eher der politischen Ebene zuzuordnen ist.

Kapitel 5 beinhaltet die Anwendung der theoretischen Erkenntnisse im Rahmen einer Fallstudie. Die theoretisch erarbeiteten Inhalte zur Wirkungsanalyse staatlichen Handelns werden so an einem praktischen Anwendungsfall auf ihre Eignung überprüft. Die Fallstudie beschäftigt sich ausführlich mit der adressatenbezogenen Systematisierung und Bewertung der Wirkungsbeiträge des staatlichen Flächenmanagements durch Bodenordnung. Die Ergebnisse der Fallstudie verdeutlichen das Potenzial, durch einen systematischen wirkungsorientierten Controllingprozess, Sensibilität für angemessene Kosten-Wirkungsrelationen zu schaffen. Durch die Identifikation der bestimmenden Kostentreiber und Wirkungshebel werden innerhalb des operativ-taktischen Wirkungscontrollings direkte Anknüpfungspunkte zur Steigerung der Wirtschaftlichkeit und Wirksamkeit der staatlichen Maßnahme aufgezeigt. Das strategische Wirkungscontrolling erhält zudem die notwendigen Informationen zur Priorisierung staatlicher Maß- 
nahmen und zur Verteilung der Budgets an die Verwaltungseinheiten. Zugleich muss sich das politische Wirkungscontrolling der Politik zuwenden und relevante Wirkungsinformationen in einem hohen Verdichtungsgrad zugänglich machen. In diesem $\mathrm{Zu}$ sammenhang bleibt zu hoffen, dass die bereitgestellten Informationen auch tatsächlich Eingang in den politischen Zielbildungsprozess finden und angemessen in politischen Entscheidungen berücksichtigt werden.

Die Komplexität wirkungsorientierter Steuerungsprozesse zeigt aber auch, dass in der Praxis eine schrittweise Umsetzung erfolgen sollte, im Sinne organisch aufeinander aufbauender Evolutionsstufen. Bevor den Akteuren des politisch-administrativen Systems Verantwortung für das Erreichen bestimmter Wirkungen zugewiesen werden kann, sollten die zugrunde liegenden Analyse- und Bewertungsschemata eine hohe Evidenz aufweisen. Das wirkungsorientierte Controlling sollte zunächst umfangreiche Erfahrungen mit den identifizierten Wirkungen sowie den zugrunde liegenden Messmethoden sammeln. Erst bei hinreichender Bewährung der erarbeiteten Wirkungshypothesen trifft die Wirkungsorientierung auf die notwendige Akzeptanz der Akteure im politisch-administrativen System - sowohl bei den Mitarbeitern und der Verwaltungsführung, als auch bei der Politik und dem interessierten Bürger.

Für den weiteren Forschungsprozess ergeben sich auf Grundlage dieser Arbeit verschiedene Anknüpfungspunkte. In näherer Zukunft bedarf es vor allem der inhaltlichen Auseinandersetzung mit dem wirkungsorientierten Controlling und der Methodik der Wirkungsanalyse durch weitere Fallstudien. Durch Kooperation von wissenschaftlicher Forschung und der Verwaltungspraxis sind in gemeinsamen Projekten weitere Erfahrungen mit der Wirkungsorientierung zu sammeln. Diese Projekte können später als Referenzmodelle für einen verbreiteten Einsatz in der Verwaltungspraxis dienen und gleichzeitig den weiteren methodischen Forschungsbedarf aufzeigen. In diesem Kontext ist es auch von Bedeutung, zunächst die staatlichen Handlungsfelder zu identifizieren, die sich für die Durchführung systematischer Wirkungsanalysen besonders gut eignen. Die im Rahmen dieser Arbeit vorgenommene grobe Typisierung staatlicher Aufgabenbereiche kann dazu nur erste Hinweise geben. Zukünftig müssen hier eindeutige Kriterien entwickelt werden, aus den sich strukturiert ableiten lässt, ob eine Leistungs-, Nutzen- oder Wirkungsanalyse zum Einsatz kommen sollte bzw. einer geeignete Kombination aus diesen Instrumenten.

Weiterhin besteht ein großer Bedarf an theoretischer Forschung zur Methodik der Wirkungsanalyse. So ist zunächst eine weitere Ausdifferenzierung des entwickelten Beschreibungsmodells denkbar, um den Realitätsbezug zu erhöhen. Die entwickelten Strukturierungshilfen zur Wirkungsidentifikation, d.h. die Systematisierung der Auslöser- und Empfängerebene und das gesamtgesellschaftliche Wirkungsgefüge sind auf ihre Tauglichkeit in unterschiedlichen staatlichen Handlungsbereichen zu überprüfen. Zudem wäre der Aufbau einer Systematik der Wirkungsarten, beispielsweise in Abhängigkeit des gesellschaftlichen Vorteils, wünschenswert sowie vertiefende Forschungen zur Wirkungsbewertung mit Hilfe von Wirkungsindikatoren und monetärer Bewertungsansätze. 
Schließlich ist grundsätzlich in allen Bereichen der Gefahr der einseitigen Fokussierung auf monetär bewertete Wirkungsbeiträge zu begegnen. Es ist davon auszugehen, dass in einem großen Teil der staatlichen Handlungsfelder bedeutende Wirkungskomponenten intangibel bleiben und daher nur verbal beschrieben werden können. Wichtig ist daher, dass diese Wirkungsbeiträge bei einer Beurteilung der staatlichen Maßnahme nicht untergehen. Hier sind einerseits Ansätze zu entwickeln, wie intangible Wirkungen in einem Berichtswesen angemessen gewürdigt werden können. Andererseits sind auch Methoden - vergleichbar einer Nutzwertanalyse - denkbar, die eine Zusammenführung intangibler Wirkungsbeiträge und monetärer Bewertungsansätze unterstützen. 
Thorsten Pieper - 978-3-631-75332-3

Downloaded from PubFactory at 01/11/2019 06:04:26AM

via free access 


\section{Literaturverzeichnis}

Adam, D. (2000): Investitionscontrolling, 3., völlig neu bearb. und wesentlich erw. Aufl., München/Wien 2000.

Adam, D./Backhaus, K./Bauer, M./Dinge, A./Johannwille, U./Voeth, M./Welker, M. (1998): Koordination betrieblicher Entscheidungen, 2. Auflage, Berlin et al. 1998.

Adams, K. (1995): Die Auswirkungen der Bodenordnung im Weinbau (Rebflurbereinigung) auf die Rebflächenstruktur, die Mechanisierung, die Kosten der Arbeitserledigung und die Wirtschaftlichkeit der Weinbaubetriebe: Abschlussbericht, Mainz 1995.

Albert, H. (1980): Traktat der kritischen Vernunft, 4. Auflage, Tübingen 1980.

Amshoff, B. (1993): Controlling in deutschen Unternehmungen: Realtypen, Kontext und Effizienz, 2. Aufl., Wiesbaden 1993.

Andel, N. (1977): Nutzen-Kosten-Analysen, in: Andel, N./Haller, H./Neumark, F. (Hrsg.): Handbuch der Finanzwissenschaft, Band I, 3. Aufl., Tübingen 1977, S. 475-518.

ARGE Landentwicklung (2006): Leitlinien Landentwicklung - Zukunft im ländlichen Raum gemeinsam gestalten, Schriftenreihe der Bund-Länder-Arbeitsgemeinschaft ARGE Landentwicklung, Heft 18, Münster 2006.

von Arnim, H.H. (1989): Finanzkontrolle im Wandel: Vorträge und Diskussionsbeiträge der 15. Verwaltungswissenschaftlichen Arbeitstagung 1988 des Forschungsinstituts für öffentliche Verwaltung der Hochschule für Verwaltungswissenschaften Speyer, Berlin 1989.

Arnold, V. (1980): Nutzen-Kosten-Analyse II: Anwendung, in: Albers, W. (Hrsg.): Handwörterbuch der Wirtschaftswissenschaften, Band 5, Stuttgart et al. 1980, S. 382-399.

Aucoin, P. (1995): The New Public Management - Canada in Comparative Perspective, Montreal 1995.

Bähr, U. (2002): Controlling in der öffentlichen Verwaltung, Sternenfels 2002.

Bach, H.-U./Spitznagel, E. (2003): Gesamtfiskalische Modellrechnungen - Was kostet uns die Arbeitslosigkeit?, IAB Kurzbericht, Ausgabe Nr. 10, Nürnberg 2003. 
Bals, H./Reichhard, C. (2000): Das neue kommunale Haushalts- und Rechnungswesen, in: Budäus, D./Küpper, H.-U./Streitferdt, L. (Hrsg.): Neues öffentliches Rechnungswesen. Stand und Perspektiven. Festschrift für Klaus Lüder zum 65. Geburtstag, Wiesbaden 2000, S. 203-233.

Balzer, K. (2005): Produkte als Informationsträger, in: Blanke, B. et al. (Hrsg.): Handbuch zur Verwaltungsreform, 3. Aufl., Wiesbaden 2005, S. 422-430.

Banner, G. (2001): Kommunale Verwaltungsmodernisierung: Wie erfolgreich waren die letzten 10 Jahre, in: Schröter, E. (Hrsg.): Empirische Policy- und Verwaltungsforschung, Opladen 2001, S. 279-303.

Baßeler, U./Heinrich, J./Utecht, B. (2006): Grundlagen und Probleme der Volkswirtschaft, 18., überarbeitete Auflage, Stuttgart 2006.

Bauer, F./Franke, J./Gätschenberger, K. (1979): Flurbereinigung und Erholungslandschaft - Empirische Studie zur Wirkung der Flurbereinigung auf den Erholungswert der Landschaft, Schriftenreihe des Bundesministers für Ernährung, Landwirtschaft und Forsten, Reihe B: Flurbereinigung, Heft 68, MünsterHiltrup 1979.

Baumgärtner, S. (2003): Warum Messung und Bewertung biologischer Vielfalt nicht unabhängig voneinander möglich sind, in: Weimann, J./Hoffmann, A./Hoffmann, S. (Hrsg.): Messung und ökonomische Bewertung von Biodiversität, Marburg 2003, S. 43-66.

Beck, J. (2002): Wirkungsorientiertes Management in der Landesverwaltung zur Optimierung von Förderprogrammen, in: Rechnungswesen und Controlling in der öffentlichen Verwaltung, Loseblattsammlung 3/2002, Berlin, S. 260-291.

Becker, B. (1997): Entscheidungen in der öffentlichen Verwaltung, in: König, K./Siedentopf, H. (Hrsg.): Öffentliche Verwaltung in Deutschland, BadenBaden 1997.

Beesley, M. E. (1965): The value of time spent in travelling: Some new evidence, in: Economica, 32 (1965), S. 174-185.

Behrendt, H. (1995): Wirkungsanalyse von Technologie- und Gründerzentren in Westdeutschland, Heidelberg 1995.

Berens, W./Bertelsmann, R. (2002): Controlling, in: Küpper, H.-U./Wagenhofer, A. (Hrsg.): Handwörterbuch Unternehmensrechnung und Controlling, 4. Auflage 2002, Sp. 280-288.

Berens, W./Bücker, H.-H./Finken, T. (1998): Einführung der Kosten- und Leistungsrechnung in Landesbildungseinrichtungen Nordrhein-Westfalens, in: krp, 42. Jg. (1998), Heft 6, S. 373-380. 
Berens, W./Budäus, D./Buschor, E./Fischer, E./Lüder, K./Streim, H. (2005): Eckpunkte für die Grundsätze ordnungsmäßiger Buchführung im öffentlichen Haushaltsund Rechnungswesen auf Basis der Integrierten Verbundrechnung, in: Die Wirtschaftsprüfung, 58. Jg. (2005), Heft 16, S. 887-890.

Berens, W./Delfmann, W. (1995): Quantitative Planung: Konzeption, Methoden und Anwendungen, 2. überarb. Aufl., Stuttgart 1995.

Berens, W./Hoffjan, A. (2004): Controlling in der öffentlichen Verwaltung: Grundlagen, Fallstudien, Lösungen, Stuttgart 2004.

Berens, W./Hoffjan, A./Strack, M. (1995): Ökologiebezogenes Controlling: Umweltorientierte Koordination in kommunalen Versorgungsunternehmen, in: Zeitschrift für öffentliche und gemeinwirtschaftliche Unternehmen, Bd. 18 (1995), S. 143-160.

Berens, W./Mosiek, T./Röhrig, A./Gerhardt, B. (2004): Outcome-orientiertes Management in der öffentlichen Verwaltung: Evolutionspfade zu einem wirkungsorientierten Controlling, in: BFuP, Heft 4/2004, S. 323-341.

Bertelsmann, R. (2005): Entwicklung einer Controlling-Konzeption im verallgemeinerten Neuen Steuerungsmodell in Trägerorganisationen der gesetzlichen Unfallversicherung, Frankfurt a.M. 2005.

Beyer, L./Kienzle, H.G. (2005): Öffentliches Rechnungswesen: Kameralistik oder Doppik?, in: Blanke, B. et al. (Hrsg.): Handbuch zur Verwaltungsreform, 3. Auflage, Wiesbaden 2005, S. 351-360.

Bildungsseminar für die Agrarverwaltung Rheinland-Pfalz (1997): Chancen im Steillagenweinbau durch Rationalisierung, Emelshausen 1997.

BMELV (2006): Effizienz staatlich geförderter Flurneuordnungsverfahren nach dem Flurbereinigungsgesetz (FlurbG), Tiersdorf 2006.

BMS Consulting (2004): Wirkungsorientiertes Controlling: Entwicklung eines Konzeptes zur Wertschöpfungsanalyse der Bezirksregierung Münster/ Versorgungsverwaltung NRW, Düsseldorf 2004.

BMS Consulting (2005): Wirkungsorientiertes Controlling: Gesamtwirtschaftliche Wertschöpfungsanalyse von Bodenordnungsverfahren der Verwaltung für Agrarordnung am Beispiel der Bodenordnung nach $\$ 87$ FlurbG (Unternehmensflurbereinigung), Düsseldorf 2005.

Böhret, C./Konzendorf, G. (2000): Moderner Staat - Moderne Verwaltung, Leitfaden zur Gesetzesfolgenabschätzung, Bundesministerium des Innern (Hrsg.), Berlin 2000. 
Böhret, C./Konzendorf, G. (2001): Handbuch Gesetzesfolgenabschätzung (GFA) Gesetze, Verordnungen, Verwaltungsvorschriften; im Auftrag des Bundesministeriums des Inneren (Hrsg.), Berlin 2001.

Böllhoff, D./Wewer, G. (2002): Zieldefinition in der Verwaltung, in: Eichhorn et al. (Hrsg.): Verwaltungslexikon, 3. neu bearbeitete Auflage, Baden-Baden 2002.

Bogumil, J. (1999): Modernisierung der Landesverwaltung - Institutionelle Ausgangslage, Implementationsstand und Zukunftsperspektiven, in: Bogumil, J. (Hrsg.): Modernisierung der Landesverwaltungen, polis Nr. 42, Hagen 1999, S. 5-14.

Bogumil, J. (2001): Modernisierung lokaler Politik. Kommunale Entscheidungsprozesse im Spannungsfeld zwischen Parteienwettbewerb, Verhandlungszwängen und Ökonomisierung, Baden-Baden 2001.

Bogumil, J./Holtkamp, L./Wollmann, H. (2003): Öffentlicher Sektor und private Akteure in der Stadt der Zukunft, Berlin, Hagen 2003.

Bogumil, J./Kuhlmann, S. (2004): Zehn Jahre kommunale Verwaltungsmodernisierung: Ansätze einer Wirkungsanalyse, in: Jann, W. et al (Hrsg.): Status-Report Verwaltungsreform: Eine Zwischenbilanz nach zehn Jahren, Berlin 2004, S. 51-63.

Bokermann, R./ Kaufmann, J./Buhse, B. (2000): Ausprägung und Wirksamkeit integrierter Entwicklung in einer ländlichen Region Hessens, in: Zeitschrift für Kulturtechnik und Landentwicklung, Heft 2 (2000), S. 62-67.

Borins, S./Grüning, G. (1998): New Public Management - Theoretische Grundlagen und problematische Aspekte der Kritik, in: Budäus, D./Conrad, P./Schreyögg, G. (Hrsg.): New Public Management, Managementforschung 8, Berlin/New York 1998, S. 11-53.

Bortz, J./Döring, N. (2006): Forschungsmethoden und Evaluation für Human- und Sozialwissenschaftler, 4., überarbeitete Auflage, Heidelberg 2006.

Bräunig, D. (2004): Rechnungswesen und Steuerung öffentlicher Verwaltungen, in: Controlling in der Verwaltung und in öffentlich-rechtlichen Unternehmen, BFuP 4/2004, S. 309-322.

Bräunlein, T. (2004): Integration der Gesetzesfolgenabschätzung in das politischadministrative System der Bundesrepublik Deutschland, Frankfurt am Main 2004.

Bramsemann, R./Köster, M. (1998): Controlling in der öffentlichen Verwaltung, Münster 1998. 
Braun, G. (1988): Ziele in öffentlicher Verwaltung und privatem Betrieb - Vergleich zwischen öffentlicher Verwaltung und privatem Betrieb sowie eine Analyse der Einsatzbedingungen betriebswirtschaftlicher Planungsmethoden in der öffentlichen Verwaltung, Baden-Baden 1988.

Brecht, U. (1999): Potentiale und Blockaden der kommunalen Leistungserstellung: eine Kritik des Neuen Steuerungsmodells, München 1999.

Brede, H. (2001): Grundzüge der öffentlichen Betriebswirtschaftslehre, München 2001.

Brinckmann, H. (1995): Strategien für eine effektivere und effizientere Verwaltung, in: Naschold, F./Pröhl, M. (Hrsg.): Produktivität öffentlicher Dienstleistungen, 3. Aufl., Gütersloh 1995, S. 167-242.

Brockhaus (2006a): Brockhaus Enzyklopädie, Nr. 19, 21., völlig neu bearbeitete Auflage, Leipzig/Mannheim 2006.

Brockhaus (2006b): Brockhaus Enzyklopädie, Nr. 21, 21., völlig neu bearbeitete Auflage, Leipzig/Mannheim 2006.

Brockhaus (2006c): Brockhaus Enzyklopädie, Nr. 24, 21., völlig neu bearbeitete Auflage, Leipzig/Mannheim 2006.

Broekmate, L./Dahrendorf, K./Dunker, K. (2001): Qualitätsmanagement in der öffentlichen Verwaltung, München 2001.

Brüggemeier, M. (1998): Controlling in der öffentlichen Verwaltung: Ansätze, Probleme und Entwicklungstendenzen eines betriebswirtschaftlichen Steuerungskonzeptes, 3. Aufl., München 1998.

Brüggemeier, M. (2004): Von der Kunst, erfolgreich zu scheitern - Wirkungsorientiertes Controlling in öffentlichen Verwaltungen, in: Kuhlmann, S./ Bogumil, J./Wollmann, H. (Hrsg.): Leistungsmessung und -vergleich in Politik und Verwaltung: Konzepte und Praxis, Wiesbaden 2004.

Brühlmeier, D./Haldemann, T./Mastronardi, P./Schedler, K. (2001): Politische Planung: Mittelfristige Steuerung in der wirkungsorientierten Verwaltungsführung, Bern/Stuttgart/Wien 2001.

Brümmerhoff, D. (1988): Finanzwissenschaft, 3. völlig überarb. und erw. Aufl., München 1988.

Bruhn, M. (2007): Marketing: Grundlagen für Studium und Praxis, 8., überarbeitete Auflage, Wiesbaden 2007. 
Bühler, B. M. (2002): Von Outputs zu Outcomes: Internationale Erfahrungen mit outcome-orientierter Steuerung, in: Verwaltung und Management, 8. Jg. (2002), Heft 5, S. 273-278.

Buchholtz, K. (2000): Controllingorientierte Kosten- und Leistungsrechnung für ein Neues Public Management, Wiesbaden 2000.

Buchholtz, K. (2001): Verwaltungssteuerung mit Kosten- und Leistungsrechnung: Internationale Erfahrungen, Anforderungen und Konzepte, Wiesbaden 2001.

Budäus, D. (1995): Public Management: Konzepte und Verfahren zur Modernisierung öffentlicher Verwaltungen, 3. Auflage, Berlin 1995.

Budäus, D. (1998): Von der bürokratischen Steuerung zum New Public Management Eine Einführung, in: Budäus, D./Conrad, P./Schreyögg, G. (Hrsg.): New Public Management, Managementforschung 8, Berlin/New York 1998, S. 1-9.

Budäus, D. (1999a): Von der Dominanz der Sachziele im Öffentlichen Sektor zum System von Formalzielen als Grundlage zukünftiger Reformentwicklungen, in: Bräunig, D./Greiling, D. (Hrsg.): Stand und Perspektiven der Öffentlichen Betriebswirtschaftslehre, Festschrift für Prof. Dr. Peter Eichhorn zur Vollendung des 60. Lebensjahres, Berlin 1999, S. 55-65.

Budäus, D. (1999b): Neues Öffentliches Rechnungswesen - Notwendigkeiten, Probleme und Perspektiven, in: Budäus, D./Gronbach, P. (Hrsg.): Umsetzung neuer Rechnungs- und Informationssysteme in innovativen Verwaltungen, Freiburg i. Br. 1999, S. 321-341.

Budäus, D. (2000a): Vom Neuen Kommunalen Rechungswesen zum öffentlichen Management-Informationssystem: Grundlage eines Verwaltungscontrolling, in: Verwaltung und Management, 6. Jg. (2000), Heft 2, S. 68-76.

Budäus, D. (2000b): Aktuelle Bestrebungen um Leistungserfassung und leistungsorientierte Ressourcensteuerung in öffentlichen Verwaltungen, in: Budäus, D. (Hrsg.): Leistungserfassung und Leistungsmessung in öffentlichen Verwaltungen, Wiesbaden 2000, S. 11-21.

Budäus, D. (2001): Public Management Grundlagen für die Reform des öffentlichen Sektors aus ökonomischer Sicht - Kompendium der neuen BWL, in: Frankfurter Allgemeine Zeitung, Nr. 233 vom 8. Oktober 2001.

Budäus, D. (2002a): Operatives und strategisches Verwaltungscontrolling im aktuellen Reformprozess des öffentlichen Sektors (Teil 1), in: Controlling, 14. Jg. (2002), Heft 4/5, S. 205-211. 
Budäus, D. (2002b): Operatives und strategisches Verwaltungscontrolling im aktuellen Reformprozess des öffentlichen Sektors (Teil 2), in: Controlling, 14. Jg. (2002), Heft 7, S. 389-394.

Budäus, D. (2004): Modernisierung des öffentlichen Haushalts- und Rechnungswesens, in: Jann, W. et al. (Hrsg.): Status-Report Verwaltungsreform: Eine Zwischenbilanz nach zehn Jahren, Berlin 2004, S. 75-86.

Budäus, D. (2005): Umsetzung gesellschaftlicher Verantwortung von Unternehmen durch Kooperation mit dem öffentlichen Sektor im Zeitalter der Globalisierung, in: Governance von Profit- und Nonprofit-Organisationen in gesellschaftlicher Verantwortung, Wiesbaden 2005.

Budäus, D./Buchholtz, K. (1997): Konzeptionelle Grundlagen des Controlling in öffentlichen Verwaltungen, in: Die Betriebswirtschaft, Jg. 57 (1997), Heft 3, S. 322-337.

Budäus, D./Eichhorn, P. (1997): Public Private Partnership, Baden-Baden 1997.

Budäus, D./Grüning, G. (1998): New Public Management - Entwicklung und Grundlagen einer „Revolution“ des öffentlichen Sektors, in: Zeitschrift Führung + Organisation, 67 Jg. (1998), S. 4-9.

Bundesrechnungshof (1998): Erfolgskontrolle finanzwirksamer Maßnahmen in der öffentlichen Verwaltung, Stuttgart 1998.

Burgmaier, K./Miersch, K./Donié, M. (1995): Auswirkungen einer Flurbereinigung auf agrarstrukturelle Faktoren der Flurstruktur, in: Zeitschrift für Kulturtechnik und Landentwicklung, Heft 1 (1995), S. 18-21.

Burr, W./Seidlmeier, H. (1998): Benchmarking in der öffentlichen Verwaltung: Anwendungspotenziale und Grenzen aus theoretischer und empirischer Sicht, in: Budäus, D./Conrad, P./Schreyögg, G. (Hrsg.): New Public Management, Managementforschung 8, Berlin/New York 1998, S. 55-92.

Buschor, E. (1993): Zwanzig Jahre Haushaltsreform - Eine verwaltungswissenschaftliche Bilanz, in: Brede, H./Buschor, E. (Hrsg.): Das neue Öffentliche Rechnungswesen, Baden-Baden 1993, S. 199-269.

Buschor, E. (2002): Evaluation und New Public Management, in: Zeitschrift für Evaluation, 1. Jg. (2002), Heft 1, S. 61-73.

Buschor, E./Lüder, K. (1994): Thesen zur künftigen Gestaltung des öffentlichen Rechnungswesens, in: Lüder, K. (Hrsg.): Öffentliches Rechnungswesen 2000, Berlin 1994, S. 163-188. 
Bussmann, W. (1995): Instrumente der Erfolgskontrolle, in: Verwaltung-OrganisationPersonal, 17. Jg. (1995), Heft 6, S. 345-351.

Caiden, G.E./Caiden, N.J. (1999): Überwachung, Messung und Evaluierung der Leistung öffentlicher Programme: Ansätze und Maßstäbe, in: Verwaltung und Management, 5. Jg. (1999), Heft 3, S. 138-146.

Chan, M./Nizette, M./La Rance, L./Broughton, C./Russell, D. (2002) : Australia, in: OECD Journal on Budgeting, Vol. 1, No. 4 (2002), S. 35-69.

Chelimsky, E. (1995): New dimensions in evaluation, in: World Bank Operations Evaluations Department (Hrsg.): Evaluation and Development: Proceedings of the 1994 World Bank Conference, Washington D.C. 1995, S. 3-11.

Chmielewicz, K. (1985): Anmerkungen zur Öffentlichen Betriebswirtschaftslehre, in: Eichhorn, P. (Hrsg.): Betriebswirtschaftliche Erkenntnisse für Regierung, Verwaltung und öffentliche Unternehmen, Baden-Baden 1985, S. 27-33.

Conings, V. (2006): Are state structure and form of political executive indicators for content and functions of budget reform?: Conclusions from the Belgian, Dutch and Swedish cases, Paper for the conference: $2^{\text {nd }}$ Transatlantic Dialogue, Leuven (Belgium) 2006.

Cooter, R./Ulen, T. (1988): Law and Economics, Glenview (Illinois) 1988.

Corsten, H. (1990): Betriebswirtschaftslehre der Dienstleistungsunternehmen, 2. Aufl., München/Wien 1990.

Cranach, M./Frenz, H.G. (1969): Systematische Beobachtung, in: Graumann, C.F. (Hrsg.): Handbuch der Psychologie: Sozialpsychologie, Göttingen 1969, S. 269-330.

Curristine, T. (2005): Performance Information in the Budget Process: Results of the OECD 2005 Questionnaire, in: OECD Journal on Budgeting, Vol. 5, No. 2 (2005), S. 87-132.

Daschmann, H.-A. (1994): Erfolgsfaktoren mittelständischer Unternehmen: Ein Beitrag zur Erfolgsfaktorenforschung, Stuttgart 1994.

DeGEval (2002): Deutsche Gesellschaft für Evaluation: Standards für Evaluation, Köln 2002.

Diamond, P.A./ Hausman, J.A. (1994): Contingent Valuation: Is Some Number Better than No Number?, in: The Journal of Economic Perspectives, Vol. 8 (1994), S. 45-64. 
Diederich, H. (1989): Ziele öffentlicher Unternehmen, in: Chmielewicz, K./Eichhorn, P. (Hrsg.): Handwörterbuch der öffentlichen Betriebswirtschaft, Stuttgart 1989, Sp. 1856-1867.

DIN (1997): Deutsche Industrie Norm: Leittechnik - Regelungstechnik und Steuerungstechnik, Berlin/Wien/Zürich 1997.

Downs, A. (1967): Inside Bureaucracy, Boston 1967.

Duden (2002): Duden: Das Bedeutungswörterbuch, 2., neu bearbeitete und erweiterte Auflage, Band 10, Mannheim/Leipzig/Wien/Zürich 2002.

Eckstein, O. (1961): Water-Resource Development. The Economics of Project Evaluation, Harvard Economic Studies, Vol. CIV, Harvard University Press, Cambridge 1961 .

Econcept (2002): Evaluation der Verwaltungsreform wif!, Schlussbericht im Auftrag des Regierungsrats des Kantons Zürich, 2002.

Egli, H.-P./ Käch, U. (1995): Instrumente der neuen Verwaltungsführung im Projekt Wirkungsorientierte Verwaltung (WOV) des Kantons Luzern, in: Hablützel et al. (1995), S. 165-184.

Eichhorn, P. (1987): Allgemeine und Öffentliche Betriebswirtschaftslehre, insbesondere Doppik und Kameralistik; in: Eichhorn, P. (Hrsg.): Doppik und Kameralistik, Baden-Baden 1987, S. 48-62.

Eichhorn, P. (1991): Immaterielle Leistungsanreize im öffentlichen Dienst, in: VOP 3/1991, S. $152-156$.

Eichhorn, P. (1997): Öffentliche Betriebswirtschaftslehre: Beiträge zur BWL der öffentlichen Verwaltungen und öffentlichen Unternehmen, Baden-Baden 1997.

Eichhorn, P. (1999): Verwaltungen im Umbruch, Klagenfurt 1999.

Eichhorn, P. (2002): Öffentliche Verwaltung, in: Eichhorn et al. (Hrsg.): Verwaltungslexikon, 3. neu bearbeitete Auflage, Baden-Baden 2002.

Eichhorn, P./Wiechers, M. (2001): Strategisches Management für Kommunalverwaltungen, Baden-Baden 2001.

Ellis, K./Mitchell, S. (2002): Outcome-focused Management in the United Kingdom, in: OECD Journal on Budgeting, Vol. 1, No. 4 (2002), S. 111-128.

Faber, M. (1986): Mikroökonomische Methoden der Präferenzerfassung bei Freizeiteinrichtungen, München 1986. 
FAL: Bundesforschungsanstalt für Landwirtschaft (2003): Halbzeitbewertung des NRW-Programms Ländlicher Raum, Braunschweig 2003.

Fiedler, J./Vernau, K. (2001): Strategisches Management als fehlendes Teilchen im Puzzle des Neuen Steuerungsmodells, in: Eichhorn, P./Wiechers, M. (Hrsg.): Strategisches Management für Kommunalverwaltungen, Baden-Baden 2001, S. 28-51.

Forsthoff, E. (1973): Verfassungsrechtliche Grenzen einer Reform des öffentlichen Dienstrechts, in: Forsthoff et al. (Hrsg.): Verfassungsrechtliche Grenzen einer Reform des öffentlichen Dienstrechts, Baden-Baden 1973.

Frantzke, A. (1999): Grundlagen der Volkswirtschaftslehre: Mikroökonomische Theorie und Aufgaben des Staates in der Marktwirtschaft, in: Praxisnahes Wirtschaftsstudium, Pietschmann, B. P./Vahs, D. (Hrsg.), Stuttgart 1999.

Franz, K.-P. (2004): Die Ergebniszielorientierung des Controlling als Unterstützungsfunktion, in: Scherm, E./Pietsch, G. (Hrsg.): Controlling: Theorien und Konzeptionen, München 2004, S. 271-288.

Frey, H.-E. (1994): Agonie des Bürokratiemodells?, in: Steger, U. (Hrsg.): Lean Administration - die Krise der öffentlichen Verwaltung als Chance, Frankfurt/New York 1994, S. 23-47.

Friedrichs, J. (1973): Methoden empirischer Sozialforschung, Reinbek 1973.

Gans, O./Marggraf, R. (1997): Kosten-Nutzen-Analyse und ökonomische Politikbewertung: Wohlfahrtsmessung und betriebswirtschaftliche Investitionskriterien, Band 1, Berlin u.a. 1997.

Gornas, J. (1992): Grundzüge einer Verwaltungskostenrechnung: Die Kostenrechnung als Instrument zur Planung und Kontrolle der Wirtschaftlichkeit in der öffentlichen Verwaltung, 2. Aufl., Baden-Baden 1992.

Groszyk, W. (2002): Outcome-focused Management in the United States, in: OECD Journal on Budgeting, Vol. 1, No. 4 (2002), S. 129-148.

Grüneberger, U. (2003): Zur Quantifizierung von Wertschöpfungsbeiträgen der Flurneuordnungsbehörde, in: Nachrichtenblatt, Heft 39 (2003), S. 87-109.

Grüning, G. (2000): Grundlagen des New Public Management: Entwicklung, theoretischer Hintergrund und wissenschaftliche Bedeutung des New Public Management aus der Sicht der politisch-administrativen Wissenschaften der USA, Münster/Hamburg/London 2000. 
Günther, T./Niepel, M./Schill, O. (2002): Herausforderungen an die Umsetzung des Neuen Steuerungsmodells aus der Perspektive des Controlling, in: Controlling, 14. Jg. (2002), Heft 4/5, S. 219-231.

Haenecke, H. (2002): Methodenorientierte Systematisierung der Kritik an der Erfolgsfaktorenforschung, in: ZfB, 72. Jg. (2002), Heft 2, S. 166-183.

Haiber, T. (1997): Controlling für öffentliche Unternehmen: Konzeption und instrumentelle Umsetzung aus der Perspektive des New Public Management, München 1997.

Haldemann, T. (1998): Zur Konzeption wirkungsorientierter Planung und Budgetierung in Politik und Verwaltung, in: Budäus, D./Conrad, P./Schrey-ögg, G. (Hrsg.): New Public Management, Managementforschung 8, Berlin/New York 1998, S. 191-215.

Haldemann, T./Marek, D. (2001): Evaluationsstudien für ein tatsächliches NPM, in: LesGes: Gesetzgebung und Evaluation, Nr. 2 (2001), S. 41-62.

Hanusch, H. (1994): Nutzen-Kosten-Analyse, 2., überarbeitete Aufl., München 1994.

Hanusch, H./Kuhn, Th./Cantner, U. (2002): Volkswirtschaftslehre 1 - Grundlegende Mikro- und Makroökonomik, 6. Auflage, Berlin 2002.

Hartwig, K.-H./Armbrecht, H. (2005): Volkswirtschaftliche Effekte unterlassener Infrastrukturinvestitionen: Studie im Auftrag des Bundesverbandes der deutschen Zementindustrie, des Hauptverbandes der Deutschen Bauindustrie und des Verbandes der Automobilindustrie, Düsseldorf 2005.

Hatry, H.P. (1999): Performance Measurement: Getting Results, Washington 1999.

Hatry, H.P./Morley, E./Rossman, S.B./Wholey, J.S. (2003): How Federal Programs Use Outcome Information: Opportunities for Federal Managers, in: IBM Endowment for the Business of Government (Hrsg.): Managing for Results Series, May 2003.

Hegele, H./Schoof, E./Schwantag, F. (1992): Flurbereinigungsgesetz - Kommentar, 6. durchgesehene Auflage, Münster 1992.

Heinz, R. (2000): Kommunales Management: Überlegungen zu einem KGSt-Ansatz, Stuttgart 2000.

Heinzlmeir, A. (1983): Landwirtschaftlicher Bodenpreis - ein Beitrag zur Klärung des Einflusses der Flurbereinigung, München 1983.

Henkes, E. (1998): Wirkungen der Verfahren nach dem FlurbG, in: Nachrichten aus der Landeskulturverwaltung Rheinland-Pfalz, 17. Jg. (1998), Heft 29, S. 23-34. 
Hesse, T./Ellwein, J. (1997): Das Regierungssystem der Bundesrepublik Deutschland, Bd. 1, 8. Auflage, Wiesbaden 1997.

Heun, W. (1989): Staatshaushalt und Staatsleitung: Das Haushaltsrecht im parlamentarischen Regierungssystem des Grundgesetzes, Baden-Baden 1989.

Hieber, F. (1999): Öffentliche Betriebswirtschaftslehre - Grundlagen für das Management in der öffentlichen Verwaltung, 3. Auflage, Berlin 1999.

Hildebrandt, L. (1992): Kausalanalyse mit Strukturgleichungsmodellen als Untersuchungsansatz, in: Hildebrandt, L./Rudinger, G./Schmidt, P. (Hrsg.): Kausalanalysen in der Umweltforschung: Beiträge eines Methodenworkshops am Wissenschaftszentrum Berlin, Stuttgart 1992, S. 3-14.

Hildebrandt, L. (1999): Hypothesenbildung und empirische Überprüfung, in: Herrmann, A./Homburg, C. (Hrsg.): Marktforschung: Methoden, Anwendungen, Praxisbeispiele, Wiesbaden 1999, S. 33-57.

Hill, H. (1996): Strategisches Controlling in der Kommunalverwaltung, in: Controlling, 8. Jg. (1996), Heft 4, S. 232-237.

Hill, H. (2000): Verwaltung als Adressat und Akteur: Einführung in das 3. Kolloquium, in: Hill, H./Hof, H. (Hrsg.): Wirkungsforschung zum Recht II: Verwaltung als Adressat und Akteur, Baden-Banden 2000, S. 27-39.

Hill, H. (2002): Indikator Lebensqualität, Gütersloh 2002.

Hoffjan, A. (1998): Entwicklung einer verhaltensorientierten Controlling-Konzeption für die Arbeitsverwaltung, 2. Auflage, Wiesbaden 1998.

Hoffjan, A. (1999): Der praktische Fall: Nutzen-Kosten-Untersuchung bei der thermischen Abfallbehandlung, in: Verwaltungsrundschau Nr. 7/99, S. 251-255.

Homburg, C./Rudolph, B. (1997): Theoretische Perspektiven der Kundenzufriedenheit, in: Simon, H./Homburg, C. (Hrsg.): Kundenzufriedenheit: Konzepte - Methoden - Erfahrungen, 2. Aufl., Wiesbaden 1997, S. 33-51.

Hood, C. (1991): A Public Management for all Seasons? In: Public Administration Vol. 69, Nr. 1 (1991), S. 3-19.

Horváth, P. (2001): Controlling, 8. vollständig überarbeitete Auflage, München 2001.

Horváth, P./Kühnle, B.A. (2002): Die Balanced Scorecard als Konzeption und Instrument zur Umsetzung politischer Programme und Strategien: Am Beispiel eines Bundeslandes, in: Verwaltung und Management, 8. Jg. (2002), Nr. 6, S. 329-334. 
Hunold, C. (2003): Kommunale Kostenrechnung. Gestaltung, Nutzung und Erfolgsfaktoren, Wiesbaden 2003.

IMF (1997): World Economic Outlook, International Monetary Fund (Hrsg.), Washington 1997.

IOFEZ (2004): Policy Budgets and Policy Accountability: Evaluation Lessons from practice, Interministerial Consultations for Financial and Economic Affairs (IOFEZ), Ministry of Finance 2004.

Irwin, T. (1996): An Analysis of New Zealand's New System of Public Sector Management, in: OECD Public Management Occasional Papers No. 9: Performance Measurement in Government - Contemporary Illustrations, Paris 1996, S. 7-32.

Jackson, J.H. (1949): The Comptroller - His Functions and Organization, Cambridge, Mass 1949.

Jann, W. (2002): Neues Steuerungsmodell, in: Eichhorn, P. (Hrsg.): Verwaltungslexikon, 3. neu bearbeitete Auflage, Baden-Baden 2002, S. 74-84.

Jann, W. (2004): Einleitung: Instrumente, Resultate und Wirkungen - die deutsche Verwaltung im Modernisierungsschub?, in: Jann, W. et al. (Hrsg.): StatusReport Verwaltungsreform: Eine Zwischenbilanz nach zehn Jahren, Berlin 2004, S. 9-21.

Jann, W. (2004): Verwaltungsmodernisierung auf Bundesebene, in: Jann, W. et al. (Hrsg.): Status-Report Verwaltungsreform: Eine Zwischenbilanz nach zehn Jahren, Berlin 2004, S. 100-111.

Jenner, T. (2000): Zur Messung des Unternehmenserfolges, in: WISU - Das Wirtschaftsstudium, Heft 3, 29. Jg. (2000), S. 326-331.

Johannson, P.O. (1993): Cost-Benefit-Analysis of Environmental Change, Cambridge 1993.

Jung, H. (2002): Allgemeine Betriebswirtschaftslehre, 8. Auflage, München, Wien 2002.

Kaplan, R./Norton, D. (1996): The Balanced Scorecard: translating strategy into action, Boston/Massachusetts 1996.

Karmann, H. (1988): Ökonometrische Bestimmung der Einflußgrößen auf Bodenpreis und Bodenmarkt landwirtschaftlich genutzter Flächen in der bayerischen Flurbereinigung, München 1988.

Mc Kevitt, D./Lawton, A. (1994): Public Sector Management - Theory, Critique \& Practice, London/Thousands Oaks/New Delhi 1994. 
Keymer, U./Linhart, C./Rintelen, P.-M./Stumpf, M./Widermann, R. (1989): Der Einfluss der Flurbereinigung auf die Bewirtschaftung landwirtschaftlicher Betriebe in Bayern; in: Bayerisches Staatsministerium für Ernährung, Landwirtschaft und Forsten (Hrsg.): Materialien zur Flurbereinigung; Heft 16, München 1989.

KGSt (1991): Kommunale Gemeinschaftsstelle (Hrsg.): Dezentrale RessourcenVerantwortung: Überlegungen zu einem Neuen Steuerungsmodell, Bericht Nr. 12/1991, Köln 1991.

KGSt (1993): Kommunale Gemeinschaftsstelle (Hrsg.): Das Neue Steuerungsmodell. Begründung, Konturen, Umsetzung, Bericht Nr. 5/1993, Köln 1993.

KGSt (1995): Kommunale Gemeinschaftsstelle (Hrsg.): Das Neue Steuerungsmodell Erste Zwischenbilanz, Bericht Nr. 10/1995, Köln 1995.

KGSt (2000): Kommunale Gemeinschaftsstelle (Hrsg.): Strategisches Management III: Zielbezogene Budgetierung, Bericht Nr. 10/2000, Köln 2000.

KGSt (2001): Kommunale Gemeinschaftsstelle (Hrsg.): Steuern mit Zielen: Ziele entwickeln und präzisieren, Bericht Nr. 3/2001, Köln 2001.

Kibblewhite, A./Ussher, C. (2002): Outcome-focused Management in New Zealand, in: OECD Journal on Budgeting, Vol. 1, No. 4 (2002), S. 85-109.

Kiesel, B (2005): Wirkungsorientierte Steuerung einer Landesverwaltung: Strategisches Controllingkonzept für ein Bundesland, Wiesbaden 2005.

Kirsch, W. (1971): Entscheidungsprozesse, Band 3, Entscheidungen in Organisationen, Wiesbaden 1971.

Kissling-Näf, I./Knoepfel, P. (1993): Kontrollinstrumente zur erfolgreichen Implementation von Politiken: Impulse aus der Umweltbeobachtung für ein integriertes Policy-Monitoring, in: Schweizerisches Jahrbuch für Politische Wissenschaft, Nr. 33 (1993), S. 277-294.

Klages, H. (2003): Nachhaltige Verwaltungsmodernisierung: Eine Bilanz nach zwölf Jahren Neuer Steuerung, in: Verwaltung und Management, 9. Jg. (2003), S. 4-12.

Klare, K./Roggendorf, W./Tietz, A./ Wollenweber, I. (2005): Untersuchungen über Nutzen und Wirkungen der Flurbereinigung in Niedersachsen, in: FAL: Bundesforschungsanstalt für Landwirtschaft (Hrsg.): Arbeitsberichte des Bereichs Agrarökonomie 01/2005, Braunschweig 2005.

Klatt, E./Dietrich, R. (1983): Langenscheidts Taschenwörterbuch der englischen und deutschen Sprache, Berlin u.a. 1983. 
Klingebiel, N. (1999): Controlling in öffentlichen Institutionen, in: Die Betriebswirtschaft, 59. Jg. (1999), Heft 3, S. 378-396.

Klöti, U. (1997): Inhaltliche und methodische Anforderungen an wissenschaftliche Politikevaluationen, in: Bussmann, W./Klöti, U./Knoepfel, P. (Hrsg.): Einführung in die Politikevaluation, Basel 1997, S. 39-57.

Klöti, U./Widmer, T. (1997): Untersuchungsdesigns, in: Bussmann, W./Klöti, U./Knoepfel, P. (Hrsg.): Einführung in die Politikevaluation, Basel 1997, S. 185-213.

Kniola, F. (1998): Neue Steuerung für die Landesverwaltung, in: VOP Nr. 7-8/1998, S. $8-10$.

König, K. (1997): Öffentliche Verwaltungen im vereinigten Deutschland, in: König, K./Siedentopf, H. (Hrsg.): Öffentliche Verwaltung in Deutschland, BadenBaden 1997.

Konzendorf, G. (2005): Gesetzesfolgenabschätzung, in: Blanke, B. et al. (Hrsg.): Handbuch zur Verwaltungsreform, 3. Auflage, Wiesbaden 2005, S. 460-469.

Kosiol, E. (1974): Die Unternehmung als wirtschaftliches Aktionszentrum, Reinbek 1974.

Kosiol, E. (1976): Organisation der Unternehmung, 2. Aufl., Wiesbaden 1976.

Kramer, U./Neulau, M. (1998): Simulationstechnik, München/Wien 1998.

Kristensen, J. K./Grozyk, W. S./Bühler, B. (2002): Outcome-focused Management and Budgeting, in: OECD Journal on Budgeting, Vol. 1, No. 4 (2002), S. 7-35.

Kroés, G. (1971): Der Beitrag der Flurbereinigung zur regionalen Entwicklung: Sozialökonomische Auswirkungen, Kosten, Konsequenzen, in: Bundesministerium für Ernährung, Landwirtschaft und Forsten (Hrsg.): Schriftenreihe für Flurbereinigung, Heft 55, Münster-Hiltrup 1971.

Kroker-Stille, S./Kölling, M. (2001): Pilotprojekt zum Querschnittscontrolling: Praxisbeispiel für wirkungsorientierte Steuerung aus dem Berliner Reformprozess, in: VOP, 23. Jg. (2001), Heft 7/8, S. 32-35.

Kückelhaus, M. (1999): Ergebnisorientierte Führung in Politik und Verwaltung: ein integratives Modell, Wiesbaden 1999.

Küpper, H.-U. (1988): Koordination und Interdependenz als Bausteine einer konzeptionellen und theoretischen Fundierung des Controlling, in: Lücke, W. v. (Hrsg.): Betriebswirtschaftliche Steuerungs- und Kontrollprobleme, Wiesbaden 1988 , S. 163-183. 
Küpper, H.-U. (2005): Controlling: Konzeption, Aufgaben, Instrumente, 4., überarbeitete Auflage, Stuttgart 2005.

Küpper, H.-U./Weber, J./Zünd, A. (1990): Zum Verständnis und Selbstverständnis des Controlling: Thesen zur Konsensbildung, in: ZfB, Nr. 3 (1990), S. 281-293.

Küpper, H.-U./Winckler, B./Zhang, S. (1990): Planungsverfahren und Planungsinformationen als Instrumente des Controlling, in: DBW, 50. Jg. (1990), S. 435-458.

Laatz, W. (1993): Empirische Methoden: Ein Lehrbuch für Sozialwissenschaftler, Thun und Frankfurt am Main 1993.

Laux, E. (1993): Vom Verwalten. Beiträge zur Staatsorganisation und zum Kommunalwesen, Baden-Baden 1993.

Linder, S.H./Peters, G.B. (1989): Implementation as a guide to policy formulation: a question of "when" rather than "whether", in: International Review of Administration Sciences, Vol. 55 (1989), S. 631-652.

Liner, B. et al. (2001): Making Results-based State Government Work, Washington 2001.

Lingnau, V. (1998): Geschichte des Controllings, in: WiSt, 27. Jg. (1998), S. 274-281.

von Loesch, A. (1997): Die öffentlichen Unternehmen Deutschlands, in: König, K./Siedentopf, H. (Hrsg.): Öffentliche Verwaltung in Deutschland, BadenBaden 1997.

Lorig, A./ Kasten, T./ Mosiek, T./ Pieper, T. (2006): Zur Objektivierung von Wertschöpfungen, in: Forum, Heft 4, 32. Jg. (2006), S. 454-469.

Lüder, K. (1989): Öffentliche Verwaltung, in: Chmielewicz, K./Eichhorn, P. (Hrsg.): Handwörterbuch der öffentlichen Betriebswirtschaftslehre, Stuttgart 1989, S. 1152-1164.

Lüder, K. (1999): Konzeptionelle Grundlagen des Neuen Kommunalen Rechnungswesens (Speyerer Verfahren), 2. Auflage, Stuttgart 1999.

Lüder, K. (2001): Neues öffentliches Haushalts- und Rechnungswesen, Berlin 2001.

Luft, M.R. (2006): Systematik: Die universale Systemtheorie, Berlin 2006.

Mäder, H./Schedler, K. (1994): Die Entwicklung des öffentlichen Rechnungswesens in der Schweiz vor dem Hintergrund der spezifischen nationalen Rahmenbedingungen, in: Lüder, K. (Hrsg.): Öffentliches Rechnungswesen 2000, Berlin 1994, S. 49-68. 
Männel, W. (1988): Besonderheiten der internen Rechnungslegung öffentlicher Unternehmungen und Verwaltungen, in: ZfB, 58. Jg. (1988), S. 839-857.

Maurer, H. (2000): Allgemeines Verwaltungsrecht, 13. überarbeitete und ergänzte Auflage, München 2000.

Mayer, F. (1972): Allgemeines Verwaltungsrecht - Eine Einführung, 3. Aufl., Stuttgart, München, Hannover 1972.

Mayntz, R. (1978): Soziologie der öffentlichen Verwaltung, Heidelberg 1978.

Mayntz, R. (1997): Soziologie der öffentlichen Verwaltung, 4. Aufl., Heidelberg 1997.

Meffert, H./Bruhn, M. (2003): Dienstleistungsmarketing. Grundlagen - Konzepte Methoden, 4. Auflage, Wiesbaden 2003.

Mertens, D.M. (1998): Research methods in education and psychology: Integrating diversity with quantitative and qualitative approaches, Thousands Oaks, CA 1998.

Meyer, A./Dornach, F. (2001): Kundenmonitor Deutschland - Qualität und Kundenorientierung: Jahrbuch der Kundenorientierung in Deutschland 2001, München 2001.

Mintzberg, H. (1996): Managing Government - Governing Management, in: Harvard Business Review, 1996, S. 75-83.

MLWF RLP: Ministerium für Landwirtschaft, Weinbau und Forsten Rheinland-Pfalz (1991): Der Einfluß von Bodenordnungsverfahren nach dem Flurbereinigungsgesetz auf die Bewirtschaftung landwirtschaftlicher Betriebe in RheinlandPfalz, 2. Auflage, Mainz 1991.

Mosiek, T. (2002): Interne Kundenorientierung des Controlling, Frankfurt 2002.

Mosiek, T./Gerhardt, B. (2003): Outcome-orientiertes Verwaltungsmanagement, in: Verwaltung und Management, 9. Jg. (2003), Heft 6, S. 288-294.

Mosiek, T./Gerhardt, B./Wirtz, A./Berens, W. (2003): Wirkungsorientiertes Controlling: Wertschöpfungsanalyse in der öffentlichen Verwaltung am Beispiel der Bezirksregierung Münster, in: Controlling, Heft 1/2003, S. 27-35.

Mosiek, T./ Pieper, T./ Kasten, T. (2006): Wirkungsorientiertes Controlling: Entwicklung und Einführung eines Konzeptes zur Wirkungsanalyse und -Prognose für Bodenordnungsverfahren in Rheinland-Pfalz, in: Ministerium für Wirtschaft, Verkehr, Landwirtschaft und Weinbau des Landes Rheinland-Pfalz (Hrsg.): Nachrichtenblatt, Sonderheft 17/2006. 
Mühlenkamp, H. (1994): Öffentliche Unternehmen, München, Wien 1994.

Müller, M. (2005): Verwaltungsmanagement: Thesen zur Entwicklung der öffentlichen Verwaltung in der Schweiz, in: UBS outlook: Impulse zur Unternehmensführung, Zürich 2005.

Müller, U. (1995): Controlling als Steuerungsinstrument der öffentlichen Verwaltung: Von der Ordnungsmäßigkeitskontrolle zur Bewertung von Controllingverfahren, in: Aus Politik und Zeitgeschichte, Band 5 (1995), S. 11-19.

Müller, U. (2004): Controlling aus verwaltungswissenschaftlicher Perspektive: Ein Beitrag zur Verwaltungsreform, Wiesbaden 2004.

Müller, W. (1974): Die Koordination von Informationsbedarf und Informationsbeschaffung als zentrale Aufgabe des Controlling, in: zfbf, Nr. 26 (1974), S. 683-693.

Naschold, F. (1995): Ergebnissteuerung, Wettbewerb, Qualitätspolitik: Entwicklungspfade des öffentlichen Sektors in Europa, Berlin 1995.

Naschold, F. et al. (1996): Leistungstiefe im öffentlichen Sektor: Erfahrungen, Konzepte, Methoden, Berlin 1996.

Naschold, F./Bogumil, J. (2000): Modernisierung des Staates: New Public Management in deutscher und internationaler Perspektive, 2. Auflage, Opladen 2000.

Niemand, S./Rassat, T. (1997): Marktorientiertes Dienstleistungsmanagement - ein qualitäts- und kostenorientierter Ansatz, in: krp 1/1997, S. 41-49.

Niskanen, W. A. (1971): Bureaucracy and Representative Government, Chicago 1971.

Norman, R. (2006): Managing for outcomes while accounting for outputs: Defining "public value" in New Zealand's performance management system, Paper for the conference: $2^{\text {nd }}$ Transatlantic Dialogue, Leuven (Belgium) 2006.

Nowotny, E. (1999): Der öffentliche Sektor: Einführung in die Finanzwissenschaft, 4. neubearb. und erw. Aufl., Berlin 1999.

Nullmeier, F. (1998): Input, Output, Outcome, Effektivität und Effizienz, in: Blanke, B. et al. (Hrsg.): Handbuch der Verwaltungsreform, Opladen 1998, S. 314-322.

Oberholzer, G. (1993): Landentwicklung als angewandte Systemforschung, in: Nachrichten aus der Landeskulturverwaltung Rheinland-Pfalz, 12. Jg. (1993), Heft 19, S. 3-12.

OECD (2002): OECD Journal on Budgeting, Vol. 1, No. 4, Paris 2002.

OECD (2005): OECD Journal on Budgeting, Vol. 5, No. 2, Paris 2005. 
Oettle, K./Thiemeyer, T. (1969): Thesen über die Unterschiede zwischen privatunternehmerischen und öffentlich-wirtschaftlichen Zielen, in: Die öffentliche Wirtschaft, Heft 1, 1969, S. 5-7.

Osborne, D./Gaebler, T. (1992): Reinventing Government: How the Entrepreneurial Spirit is transforming the Public Sector, Reading 1992.

o.V. (2006): Die erstaunliche Wandlung eines häßlichen Entleins, Welt am Sonntag Nr. 31, 30. Juli 2006, S. 20.

Parsons, W. (1995): Public Policy: An Introduction of the Theory and Practice of Policy-Analysis, Cheltenham 1995.

Pede, L. (1999): Externe, wirkungsorientierte Prüfung der öffentlichen Verwaltung im Sinne des New Public Managements, St. Gallen 1999.

Peffekoven, R. (1996): Einführung in die Grundbegriffe der Finanzwissenschaft, 3. überarb. Aufl., Darmstadt 1996.

Peters, G./Savoie, D. (1994): Civil Service Reform: Misdiagnosing the Patient, in: Public Administration Review, 54. Jg. (1994), S. 418-425.

Pfohl, H.C./Zettelmeyer, B. (1987): Strategisches Controlling?, in: ZfB, Nr. 2 (1987), S. 145-175.

Pieper, T./Kasten, T./Mosiek, T./Gerhardt, B. (2006): Wirkungsorientiertes Verwaltungsmanagement und -controlling: Am Beispiel der ländlichen Bodenordnung in Nordrhein-Westfalen, in: Verwaltung und Management, 12. Jg. (2006), Heft 5, S. 245-252.

Pietsch, G. (2003): Reflexionsorientiertes Controlling: Konzeption und Gestaltung, Wiesbaden 2003.

Pietsch, G./Scherm, E. (2001): Die Reflexionsaufgabe im Zentrum des Controlling, in: krp, Nr. 45 (2001), S. 307-313.

Pollitt, C. (2003): Joined-Up Government: a Survey, in: Political Studies Review, Vol. 1 (2003), S. 34-49.

Pook, M./Fischer, E. (2002): Controlling in der öffentlichen Verwaltung: Entwicklungsstand und Perspektiven für die Kommunalverwaltung, in: Kostenrechnungspraxis, Sonderheft 2 (2002), S. 43-53.

Pook, M./Tebbe, G. (2002): Berichtswesen und Controlling, Berlin/München 2002.

Popper, K. R. (2005): Logik der Forschung, 11. Auflage, Tübingen 2005. 
Porter, M. E. (1999): Wettbewerbsvorteile (Competitive Advantage): Spitzenleistungen erreichen und behaupten, 5. Auflage, Frankfurt am Main 1999.

Prell, K.-M./Rodig, C. (2003): Vermessungen in Flurbereinigungsverfahren und ihr Anteil an der Wertschöpfung der Flurbereinigung, in: DVW-Hessen/ DVWThüringen Mitteilungen, Heft 1 (2003), S. 27-31.

Proeller, I. (2006): Stand der strategischen Steuerung im internationalen Vergleich, in: Bertelsmann Stiftung (Hrsg.): Strategische Steuerung: Dokumentation eines Expertendialogs im Rahmen der Projektinitiative „Staat der Zukunft“, Gütersloh 2006, S. 12-21.

Promberger, K. (1995): Controlling für Politik und öffentliche Verwaltung, Wien 1995.

Püttner, G. (1995): Allgemeines Verwaltungsrecht: Ein Studienbuch, 7. Auflage, Düsseldorf 1995.

Reichard, C. (1987): Betriebswirtschaftslehre der öffentlichen Verwaltung, 2. Aufl., New York 1987.

Reichard, C. (1994): Internationale Ansätze eines „New Public Management“, in: Hofmann, M./Al-Ani, A. (Hrsg.): Neue Entwicklungen im Management, Heidelberg 1994, S. 135-164.

Reichard, C. (1995): Von Max Weber zum „New Public Management“ - Verwaltungsmanagement im 20. Jahrhundert, in: Halblützel, P. et al (Hrsg.): Umbruch in Politik und Verwaltung: Ansichten und Erfahrungen zum New Public Management in der Schweiz, Bern/Stuttgart/Wien 1995, S. 57-80.

Reichard, C. (1996): Die „New Public-Management“-Debatte im internationalen Kontext, in: Reichard, C./Wollmann, H. (Hrsg.): Kommunalverwaltung im Modernisierungsschub?, Basel u.a. 1996, S. 241-274.

Reichard, C. (2001): Verwaltungsmodernisierung in Deutschland in internationaler Perspektive, in: Wallerath, M. (Hrsg.): Verwaltungserneuerung: Eine Zwischenbilanz der Modernisierung öffentlicher Verwaltungen, Baden-Baden 2001, S. 13-35.

Reichard, C. (2002): Outcome-based service delivery: Some experiences from Germany and Switzerland, in: Wyk, B./van der Molen, K./Van Rooyen, A. (Hrsg.): Outcome-based Governance: Assessing the Results, Sandown 2002, S. 23-38.

Reichard, C. (2004): Verwaltungsmodernisierung in den Bundesländern, in: Jann, W. et al. (Hrsg.): Status-Report Verwaltungsreform: Eine Zwischenbilanz nach zehn Jahren, Berlin 2004, S. 87-99. 
Reichmann, T. (1993): Controlling mit Kennzahlen und Managementberichten: Grundlagen einer systemgestützten Controlling-Konzeption, 3. überarb. und erw. Aufl., München 1993.

Reinermann, H. (1993): Ein neues Paradigma für die öffentliche Verwaltung? - Was Max Weber heute empfehlen würde -, Speyerer Arbeitshefte 97, Speyer 1993.

Reinermann, H. (2000): Neues Politik- und Verwaltungsmanagement: Leitbild und theoretische Grundlagen, Speyerer Arbeitshefte 130, Speyer 2000.

Richter, M. (2000): Controlling-Konzeption für öffentliche Verwaltungsbetriebe, Hamburg 2000.

Rieder, S. (2006): Bilanz der wirkungsorientierten Steuerung in der Schweiz - Ein Erfahrungsbericht, in: Bertelsmann Stiftung (Hrsg.): Strategische Steuerung: Dokumentation eines Expertendialogs im Rahmen der Projektinitiative „Staat der Zukunft", Gütersloh 2006, S. 22-28.

Röhrig, A. (2008): Wirkungsorientiertes Controlling im politisch-administrativen System unter besonderer Berücksichtigung der Gestaltungsmöglichkeiten von öffentlichen Verwaltungen, Frankfurt a. M. 2008.

Roschmann, Ch. (2005): Public-Private-Partnerships: Versuch der Bestimmung eines Begriffes und seiner Operationalisierung, in: Stember, J. (Hrsg.): Public Private Partnerships - Zukunftsmodelle für die öffentliche Verwaltung, Ostbevern 2005, S. 36-53.

Rossi et al. (1988): Programm Evaluation: Einführung in die Methoden angewandter Sozialforschung, Stuttgart 1988.

Rossi. P. H./ Freeman, H. (1993): Evaluation: a systematic approach, 5th edition, Newbury Park 1993.

Rupp, T. (2002): Multiperspektivisches Controlling für öffentliche Verwaltungen, Hamburg 2002.

Sachverständigenrat (2005): Begutachtung der gesamtwirtschaftlichen Entwicklung: Jahresgutachten 2005/06, Stuttgart 2005.

Schaefer, M. (2003): Wörterbuch der Ökologie, 4. neu bearbeitete und erweiterte Auflage, Heidelberg/Berlin 2003.

Scharpf, H. (1982): Die ökologische Risikoanalyse als Beitrag zur Umweltverträglichkeitsprüfung in der Landwirtschaft, Hannover 1982. 
Schatz, H. (1991): Lehren aus Ansätzen zu Verwaltungs-Informationssystemen in der Vergangenheit (2), in: Reinermann, H. (Hrsg.): Führung und Information: Chancen der Informationstechnik für die Führung in Politik und Verwaltung, Heidelberg 1991, S. 49-66.

Schauer, R. (1984): Öffentliche Verwaltungen, Band 1, Linz 1984.

Schedler, K. (1993): Anreizsysteme in der öffentlichen Verwaltung, Bern/Stuttgart/Wien 1993.

Schedler, K. (1996): Ansätze einer wirkungsorientierten Verwaltungsführung: Von der Idee des New Public Management (NPM) zum konkreten Gestaltungsmodell: Fallbeispiel Schweiz, 2. Auflage, Bern/Stuttgart/Wien 1996.

Schedler, K./Proeller, I. (2000): New Public Management, Bern/Stuttgart/Wien 2000.

Schedler, K./Proeller, I. (2003): New Public Management, Bern/Stuttgart/Wien 2003.

Schenker-Wicki, A. (1996): Evaluationen von Hochschulleistungen: Leistungsindikatoren und Performance Measurements, Wiesbaden 1996.

Scherer, A.G./Alt, J.M. (Hrsg.): Balanced Scorecard in Verwaltung und Non-ProfitOrganisationen, Stuttgart 2002.

Scherm, E./Pietsch, G. (2001): Neue Controlling-Konzeptionen, in: WISU, 30. Jg. (2001), Nr. 2, S. 206-213.

Schierenbeck, H. (2003): Grundzüge der Betriebswirtschaftslehre, 16., vollständig überarbeitete und erweiterte Auflage, München 2003.

Schlosser, F. (1999): Ländliche Entwicklung im Wandel der Zeit - Zielsetzungen und Wirkungen, in: Magel, H. (Hrsg.): Materialsammlung der Technischen Universität München, Lehrstuhl für Bodenordnung und Landentwicklung, Heft 21, München 1999.

Schmidberger, J. (1994): Controlling für öffentliche Verwaltungen, 2. aktualisierte Aufl., Wiesbaden 1994.

Schmitting, W./Siemes, A. (2003): Konzeption eines Risikomanagement-Modells: Begriffsrahmen und IT-Umsetzung, in: Controller Magazin, Heft Nr. 6 (2003), S. 533-540.

Schnell, R./Hill, P./Esser, E. (1999): Methoden der empirischen Sozialforschung, 6., völlig überarb. und erw. Aufl., München/Wien 1999. 
Schröder, J./Kettiger, D. (2001): Wirkungsorientierte Steuerung in der sozialen Arbeit: Ergebnisse einer internationalen Recherche in den USA, den Niederlanden und der Schweiz, Schriftenreihe des Bundesministeriums für Familie, Senioren, Frauen und Jugend, Band 229, Stuttgart 2001.

Schröter, E./Wollmann, H. (2001): New Public Management, in: Blanke, B. et al. (Hrsg.): Handbuch zur Verwaltungsreform, 2. Auflage, Opladen 2001, S. 71-80.

Schröter, E./Wollmann, H. (2002): New Public Management, in: Eichhorn, P. (Hrsg.): Verwaltungslexikon, 3. neu bearbeitete Auflage, Baden-Baden 2002, S. 63-74.

Schubert, K./Bandelow, N.C. (2003): Lehrbuch der Politikfeldanalyse, München 2003.

Schulte, A./Bokermann, R. (2002): Ein Bewertungsansatz für Projekte der ländlichen Entwicklung, in: Landnutzung und Landentwicklung (43), Heft 5 (2002), S. 198-204.

Schuster, F. (2001): Benchmarking als Ersatz für Wettbewerb: Können interkommunale Leistungsvergleiche ein Motor für Veränderungen sein?, in: Edeling et al. (Hrsg.): Reorganisationsstrategien in Wirtschaft und Verwaltung, Opladen 2001, S. 201-228.

Scriven, M. (1973): Goal-free Evaluation, in: House, E. (Hrsg.): School Evaluation: The Politics and Process, Berkeley 1973, S. 319-328.

Seifert, K. (1998): Prozessmanagement für die öffentliche Verwaltung, Wiesbaden 1998.

Seyer, G./ Pieper, T./Kasten, T./ Mosiek, T. (2006): Gesamtwirtschaftliche Wertschöpfungsbeiträge durch Unternehmensflurbereinigungen, in: fub - Flächenmanagement und Bodenordnung, Heft 1/2006, S. 39-48.

Siems, C. (2005): Public Target Costing: Zielkostenmanagement als Controllinginstrument für die öffentliche Verwaltung, Frankfurt a.M. 2005.

Smith, A. (1776): An Inquiry into the Nature and Causes of the Wealth of Nations, in: Campbell R.H./Skinner, A.S. (Hrsg.): The Glasgow Edition of the Works and Correspondence of Adam Smith, Vol. II, Oxford 1976.

Speier, F. (2002): Die Einführung der Kosten- und Leistungsrechnung in die Kommunalverwaltung: Eine empirische Untersuchung der Auswirkungen auf die Beschäftigten und personeller Erfolgsfaktoren, Verlag im Internet 2002.

Statistisches Bundesamt (2006): Personal des öffentlichen Dienstes: Jahresdaten der Personalstandsstatistik, Daten zum 30.06.2004, Statistisches Bundesamt 2006. 
Steinle, C./Bruch, H. (2003): Controlling: Kompendium für Ausbildung und Praxis, 3., überarbeitete und erweiterte Auflage, Stuttgart 2003.

Sterck, M./Bouckaert, G. (2006): The impact of performance budgeting on the role of parliament: a four-country study, Paper for the conference: $2^{\text {nd }}$ Transatlantic Dialogue, Leuven (Belgium) 2006.

Stiglitz, J.E. (1999): Volkswirtschaftslehre, 2. Auflage, München 1999.

Stockmann, R. (2004): Evaluation in Deutschland, in: Stockmann, R. (Hrsg.): Evaluationsforschung: Grundlagen und ausgewählte Forschungsfelder, 2., überarbeitete und aktualisierte Auflage, Opladen 2004, S. 13-44.

Sturm, A. (2000): Performance Measurement und Environmental Performance Measurement: Entwicklung eines Controllingmodells zur unternehmensinternen Messung der betrieblichen Umweltleistung, Verlag im Internet 2000.

Teubner, A. (2002): Theoretische Grundlagen des Software Engineering, in: WISU Das Wirtschaftsstudium, Heft 5, 31. Jg. (2002), S. 690-697.

Teufel, E. (1997): Die wirtschaftliche Bedeutung der Verwaltungsreform, in: VOP Nr. 4/1997, S. 9-11.

Thieme, W. (1984): Verwaltungslehre, 4. erweiterte und völlig neubearbeitete Auflage, Köln u.a. 1984.

Thoenig, J. C. (2003): Learning from evaluation practice: The case of public-sector reforms, in: Wollmann, H. (Hrsg.): Evaluation in Public Sector Reforms: Concepts and Practice in International Perspective, Aldershot 2003.

Ulrich, H./Sidler, F. (1977): Ein Management-Modell für die öffentliche Hand, Bern/Stuttgart 1977.

Vedung, E. (1999): Evaluation im öffentlichen Sektor, Wien/Köln/Graz 1999.

Wagener, F. (1983): Diskussionsbeitrag in den Veröffentlichungen der Vereinigung der Deutschen Staatsrechtslehrer, Band 41, 1983.

Weber, J. (2004): Einführung in das Controlling, 10., überarbeitete und aktualisierte Auflage, Stuttgart 2004.

Weber, J./Schäffer, U. (1999): Sicherstellung der Rationalität von Führung als Aufgabe des Controlling?, in: DBW, Jg. 59 (1999), S. 731-747.

Weber, J./Schäffer, U. (2002): Herausforderungen für das Dienstleistungscontrolling, in: krp Sonderheft Nr. 2 (2002), S. 5-13. 
Weber, J./Weise, F.-J. (2004): Rolle des Controllings für die Umgestaltung der Bundesagentur für Arbeit, in: BFuP, Heft 4 (2004), S. 355-368.

Weber, M. (1976): Wirtschaft und Gesellschaft, Grundriss der verstehenden Soziologie, 5. Auflage, Tübingen 1976.

Wegener, A. (2002): Die Kriterien zu Good Governance, in: Pröhl, M. (Hrsg.): Good Governance für Lebensqualität vor Ort: Internationale Praxisbeispiele für Kommunen, Gütersloh 2002, S. 16-115.

Weiß, E. (1982): Zur Entwicklung der ländlichen Bodenordnung im Lande NordrheinWestfalen, Akademie für Raumforschung und Landesplanung, Band 63, Hannover 1982 .

Weiß, E./Kremer, S./Strang, H. (1996): Effizienz der Flurbereinigung - Praxisreife Fortentwicklung der Erfolgskontrolle, Schriftenreihe des Bundesministeriums für Ernährung, Landwirtschaft und Forsten, Heft 82, Bonn 1996.

Widmer, T. (2004): Qualität der Evaluation - Wenn Wissenschaft zur praktischen Kunst wird, in: Stockmann (Hrsg.): Evaluationsforschung: Grundlagen und ausgewählte Forschungsfelder, 2., überarbeitete und aktualisierte Auflage, Opladen 2004, S. 205-231.

wif! (2002): Wirkungsorientierte Verwaltungsführung - Die Verwaltungsreform wif! des Kantons Zürich und ihre Instrumente, Zürich 2002.

Wilde, K.D. (1989): Bewertung von Produkt-Markt-Strategien: Theorie und Methoden, Berlin 1989.

Wöhe, G. (2005): Einführung in die Betriebswirtschaftslehre, 22. neu bearb. Aufl., München 2005.

Wollmann, H. (2002): Verwaltungspolitische Reformdiskurse und -verläufe im internationalen Vergleich, in: König, K. (Hrsg.): Deutsche Verwaltung in der Wende zum 21. Jahrhundert, Baden-Baden 2002, S. 489-524.

Wollmann, H. (2004a): Leistungsmessung (,performance measurement“) in Politik und Verwaltung: Phasen, Typen und Ansätze im internationalen Überblick, in: Kuhlmann, S./Bogumil, J./Wollmann, H. (Hrsg.): Leistungsmessung und vergleich in Politik und Verwaltung: Konzepte und Praxis, Wiesbaden 2004, S. 21-46.

Wollmann, H. (2004b): Evaluation und Verwaltungspolitik: Konzepte und Praxis in Deutschland und im internationalen Kontext, in: Stockmann (Hrsg.): Evaluationsforschung: Grundlagen und ausgewählte Forschungsfelder, 2., überarbeitete und aktualisierte Auflage, Opladen 2004, S. 205-231. 
Zielinski, H. (2000): Lokalpolitische Modernisierungsprozesse in Wissenschaft und Praxis, in: Zielinski, H. (Hrsg.): Die Modernisierung der Städte, Wiesbaden 2000.

Ziener, M. (1985): Controlling in multinationalen Unternehmen, Landsberg am Lech 1985.

Zimmermann, H./Henke, K.-D. (1994): Finanzwissenschaft, 7., völlig überarbeitete und erweiterte Aufl., München 1997.

Zünd, A. (1985): Der Controller Bereich (Controllership), Randbemerkungen zur Institutionalisierung der Controller Funktion, in: Controlling und Unternehmensführung, Probst, G. J. B./Schmitz-Dräger R. (Hrsg.), Bern 1985, S. 28-40. 


\section{Quellenverzeichnis}

\section{RECHTSQUELLEN}

BauGB: Baugesetzbuch, vom 8. Dezember 1986 (BGB1. I, S. 2253), zuletzt geändert am 23. November 1994 (BGB1. I, S. 3486).

BNatSchG: Gesetz über Naturschutz und Landschaftspflege (Bundesnaturschutzgesetz), vom 25. März 2002, zuletzt geändert durch Gesetz vom 21. Juni 2005 (BGB1. I Nr. 22 S. 1818).

FlurbG: Flurbereinigungsgesetz, in der Bekanntmachung vom 16. März 1976 (BGB1. I S. 546), zuletzt geändert durch Gesetz vom 20. Dezember 2001 (BGB1. I S. 3987).

GBO: Grundbuchordnung, in der Fassung der Bekanntmachung vom 26. Mai 1994 (BGB1. I S. 1114)., zuletzt geändert durch Gesetz vom 09. Dezember 2004 (BGB1. I S. 3220) m.W.v. 01. Januar 2005.

GG: Grundgesetz für die Bundesrepublik Deutschland vom 23.05.1949 (BGB1. I S. 1), zuletzt geändert durch zwei Gesetze zur Änderung des Grundgesetzes am 26.07.2002 (BGB1. I S. 2862/2863).

HOAI: Verordnung über die Honorare für Leitungen der Architekten und Ingenieure, in der Fassung vom 14. November 2001.

VermKatG: Vermessungs- und Katastergesetz, in der Fassung vom 1. März 2005.

WHG: Gesetz zur Ordnung des Wasserhaushalts - Wasserhaushaltsgesetz, vom 19. August 2002, (BGB1. I Nr. 59, S. 3245).

\section{INTERNETQUELLEN}

IAB (2004): Gesamtfiskalische Kosten der Arbeitslosigkeit in Deutschland (hhtp://doku.iab.de/presse/2005/info_kostenALO2004.pdf, Abruf: 12. Februar 2007).

HM Treasury (2006): Public Services Performance Index, (http://www.hm-treasury. gov.uk, Abruf: 26. September 2006).

Türke, R. (2002): Strategisches Controlling in Berlin - Konzeption und Umsetzung am Beispiel der Integration von Langzeitarbeitslosen, Präsentationsfolien, Speyer 2002(http://www.hfv-speyer.de/hill/tagungen/Tagungen-2002/Moderne-Verwaltung.htm, Abruf: 01. September 2006). 
wif! (2006): Die Verwaltungsreform wif! des Kantons Zürich und ihre Instrumente: Offizielle Internetseite der Verwaltungsreform des Kantons Zürich, Zürich 2006 (http://www.wif.zh.ch, Abruf: 17. Oktober 2006). 


\title{
Beiträge zum Controlling
}

\author{
Herausgegeben von Wolfgang Berens
}

Band 1 Wolfgang Berens / Joachim Strauch: Due Diligence bei Unternehmensakquisitionen - eine empirische Untersuchung. Unter Mitarbeit von Thorsten Behrens und Julia Lescher. 2002.

Band 2 Andreas Siemes: Marktorientierte Kreditrisikobewertung. Eine empirische Untersuchung mittels Künstlicher Neuronaler Netze. 2002.

Band 3 Karl Christoph Heinen: Die Berücksichtigung von Kosten in der Konkurrenzanalyse. 2002.

Band 4 Thomas Mosiek: Interne Kundenorientierung des Controlling. 2002.

Band 5 Vera Südmeyer: Wettbewerbsvorteile durch strategisches Betriebsformenmanagement. Ein dynamischer Bezugsrahmen für Einzelhandelsunternehmen. 2003.

Band 6 Wolfgang Berens / Walter Schmitting (Hrsg.): Controlling im E-Business. Rückkehr zur Rationalität. 2004.

Band 7 René Bertelsmann: Entwicklung einer Controlling-Konzeption im verallgemeinerten Neuen Steuerungsmodell für Trägerorganisationen der gesetzlichen Unfallversicherung. 2005.

Band 8 Mirko Tillmann: Risikokapitalbasierte Steuerung in der Schaden- und Unfallversicherung. Konzeption einer modellgestützten Risikoanalyse. 2005.

Band 9 Andreas Hoffjan: Risikorechnung bel industrieller Auftragsfertigung. Theoretische Konzeption und Anwendung für die Bauwirtschaft. 2006.

Band 10 Pascal Nevries: Die Marketingwirkungen von Börsengängen. Eine konzeptionelle Analyse. 2006.

Band 11 Klaus Segbers: Die Geschäftsbeziehung zwischen mittelständischen Unternehmen und ihrer Hausbank. Eine ökonomische und verhaltenswissenschaftliche Analyse. 2007.

Band 12 Andreas Röhrig: Wirkungsorientiertes Controlling im politisch-administrativen System. Unter besonderer Berücksichtigung der Gestaltungsmöglichkeiten von öffentlichen Verwaltungen. 2008.

Band 13 Nino Raddao: Potenzialorientierte Kundensegmentierung zur Optimierung des Leistungsportfolios in der Firmenkundenbank. Konzeption und Implementierung einer efficient customization am Beisplel von Genossenschaftsbanken. 2008.

Band 14 Thorsten Pieper: Wirkungsorientiertes Controlling staatlichen Handelns. Systematische Identifikation und Bewertung der gesamtgesellschaftlichen Wirkungen staatlichen Handelns. 2009.

www.peterlang.de 


\section{Wirkungsorientiertes Controlling im politisch-administrativen System}

\section{Unter besonderer Berücksichtigung der Gestaltungsmöglichkeiten von öffentlichen Verwaltungen}

Frankfurt am Main, Berlin, Bern, Bruxelles, New York, Oxford, Wien, 2008. XIX, 291 S., zahlr. Tab. und Graf.

Beiträge zum Controlling. Herausgegeben von Wolfgang Berens. Bd. 12 ISBN 978-3-631-56068-6 · br. € 51.50*

In der Arbeit wird ein wirkungsorientierter Controlling-Bezugsrahmen für das politisch-administrative System entwickelt. Über diesen kann es gelingen, öffentliches Handeln bzw.den öffentlichen Aufgabenvollzug - mittels einer Integration von Wirkungs- und Ressourcenbetrachtung - im Sinne einer gesellschaftlichen Wertschöpfung interpretierbar und im Idealfall steuerbar zu machen. Ein derart verstandenes Controlling kann dazu beitragen, eine bislang fehlende Übersetzungsfunktion zwischen Politik und öffentlicher Verwaltung „auszufüllen"und damit das Zusammenspiel von Verwaltung und Politik im Sinne des Bürgers zu verbessern.

Aus dem Inhalt: Rahmenbedingungen und strukturelle Besonderheiten im Reformprozess der Verwaltungsmodernisierung • "Wirkung” als Zieldimension eines Controlling im politisch-administrativen System · Entwicklung und Darstellung des „Erweiterten Ziel- und Ergebnisebenemodells öffentlicher Leistungserstellung" als integrierter Bezugsrahmen für ein wirkungsorientiertes Controlling · Integration von Erkenntnissen in den Führungskreislauf des politisch-administrativen Systems

Frankfurt am Main - Berlin - Bern - Bruxelles · New York · Oxford · Wien

Auslieferung: Verlag Peter Lang AG

Moosstr. 1, $\mathrm{CH}-2542$ Pieterlen

Telefax 0041 (0) 32/3761727

*inklusive der in Deutschland gültigen Mehrwertsteuer Preisänderungen vorbehalten

Homepage http://www.peterlang.de 